NIST Special Publication 1500-3r2

\title{
NIST Big Data Interoperability Framework: Volume 3, Use Cases and General Requirements
}

\section{Version 3}

NIST Big Data Public Working Group Definitions and Taxonomies Subgroup 


\section{NIST Special Publication 1500-3r2}

\section{NIST Big Data Interoperability Framework: Volume 3, Use Cases and General Requirements}

\section{Version 3}

NIST Big Data Public Working Group Definitions and Taxonomies Subgroup

Information Technology Laboratory National Institute of Standards and Technology

Gaithersburg, MD 20899

This publication is available free of charge from:

https://doi.org/10.6028/NIST.SP.1500-3r2

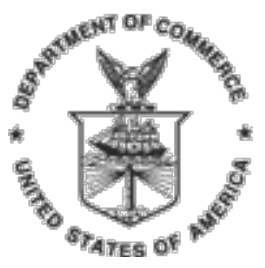

U.S. Department of Commerce Wilbur L. Ross, Jr., Secretary

National Institute of Standards and Technology Walter Copan, NIST Director and Undersecretary of Commerce for Standards and Technology 


\title{
National Institute of Standards and Technology (NIST) Special Publication 1500-3r2
} 360 pages (October 2019)

NIST Special Publication series 1500 is intended to capture external perspectives related to NIST standards, measurement, and testing-related efforts. These external perspectives can come from industry, academia, government, and others. These reports are intended to document external perspectives and do not represent official NIST positions.

Certain commercial entities, equipment, or materials may be identified in this document to describe an experimental procedure or concept adequately. Such identification is not intended to imply recommendation or endorsement by NIST, nor is it intended to imply that the entities, materials, or equipment are necessarily the best available for the purpose.

There may be references in this publication to other publications currently under development by NIST in accordance with its assigned statutory responsibilities. The information in this publication, including concepts and methodologies, may be used by federal agencies even before the completion of such companion publications. Thus, until each publication is completed, current requirements, guidelines, and procedures, where they exist, remain operative. For planning and transition purposes, federal agencies may wish to closely follow the development of these new publications by NIST.

Organizations are encouraged to review all publications during public comment periods and provide feedback to NIST. All NIST publications are available at http://www.nist.gov/publication-portal.cfm.

\section{Copyrights and Permissions}

Official publications of the National Institute of Standards and Technology are not subject to copyright in the United States. Foreign rights are reserved. Questions concerning the possibility of copyrights in foreign countries should be referred to the Office of Chief Counsel at NIST via email to nistcounsel@nist.gov.

\section{Comments on this publication may be submitted to Wo Chang}

\author{
National Institute of Standards and Technology \\ Attn: Wo Chang, Information Technology Laboratory \\ 100 Bureau Drive (Mail Stop 8900) Gaithersburg, MD 20899-8930 \\ Email: SP1500comments@nist.gov
}




\section{Reports on Computer Systems Technology}

The Information Technology Laboratory (ITL) at NIST promotes the U.S. economy and public welfare by providing technical leadership for the Nation's measurement and standards infrastructure. ITL develops tests, test methods, reference data, proof of concept implementations, and technical analyses to advance the development and productive use of information technology. ITL's responsibilities include the development of management, administrative, technical, and physical standards and guidelines for the cost-effective security and privacy of other than national security-related information in federal information systems. This document reports on ITL's research, guidance, and outreach efforts in Information Technology and its collaborative activities with industry, government, and academic organizations.

\section{Abstract}

Big Data is a term used to describe the large amount of data in the networked, digitized, sensor-laden, information-driven world. While opportunities exist with Big Data, the data can overwhelm traditional technical approaches and the growth of data is outpacing scientific and technological advances in data analytics. To advance progress in Big Data, the NIST Big Data Public Working Group (NBD-PWG) worked to develop consensus on important fundamental concepts related to Big Data. The results are reported in the NIST Big Data Interoperability Framework series of volumes. This volume, Volume 3, contains the original 51 Version 1 use cases gathered by the NBD-PWG Use Cases and Requirements Subgroup and the requirements generated from those use cases. The use cases are presented in their original and summarized form. Requirements, or challenges, were extracted from each use case, and then summarized over all the use cases. These generalized requirements were used in the development of the NIST Big Data Reference Architecture (NBDRA), which is presented in Volume 6. During the development of Version 2 of the NBDIF, the Use Cases and Requirements Subgroup and the Security and Privacy Subgroup identified the need for additional use cases to strengthen work of the NBD-PWG in Stage 3. The subgroup accepted additional use case submissions using the more detailed Use Case Template 2. The three additional use case submissions collected using Use Case Template 2 are presented and summarized in this volume.

\section{Keywords}

Big Data; Big Data Application Provider; Big Data characteristics; Big Data Framework Provider; Big Data taxonomy; Data Consumer; Data Provider; data science; Management Fabric; reference architecture; Security and Privacy Fabric; System Orchestrator; use cases. 


\section{Acknowledgements}

This document reflects the contributions and discussions by the membership of the NBD-PWG, cochaired by Wo Chang (NIST ITL), Bob Marcus (ET-Strategies), and Chaitan Baru (San Diego Supercomputer Center; National Science Foundation). For all versions, the Subgroups were led by the following people: Nancy Grady (SAIC), Natasha Balac (San Diego Supercomputer Center), and Eugene Luster (R2AD) for the Definitions and Taxonomies Subgroup; Geoffrey Fox (Indiana University) and Tsegereda Beyene (Cisco Systems) for the Use Cases and Requirements Subgroup; Arnab Roy (Fujitsu), Mark Underwood (Krypton Brothers; Synchrony Financial), and Akhil Manchanda (GE) for the Security and Privacy Subgroup; David Boyd (InCadence Strategic Solutions), Orit Levin (Microsoft), Don Krapohl (Augmented Intelligence), and James Ketner (AT\&T) for the Reference Architecture Subgroup; and Russell Reinsch (Center for Government Interoperability), David Boyd (InCadence Strategic Solutions), Carl Buffington (Vistronix), and Dan McClary (Oracle), for the Standards Roadmap Subgroup.

The editors for this document were the following:

- Version 1: Geoffrey Fox (Indiana University) and Wo Chang (NIST)

- Version 2: Geoffrey Fox (Indiana University) and Wo Chang (NIST)

- Version 3: Geoffrey Fox (Indiana University) and Wo Chang (NIST)

Laurie Aldape (Energetics Incorporated) and Elizabeth Lennon (NIST) provided editorial assistance across all NBDIF volumes.

NIST SP1500-3, Version 3 has been collaboratively authored by the NBD-PWG. As of the date of this publication, there are over six hundred NBD-PWG participants from industry, academia, and government. Federal agency participants include the National Archives and Records Administration (NARA), National Aeronautics and Space Administration (NASA), National Science Foundation (NSF), and the U.S. Departments of Agriculture, Commerce, Defense, Energy, Health and Human Services, Homeland Security, Transportation, Treasury, and Veterans Affairs.

NIST would like to acknowledge the specific contributions ${ }^{1}$ to this volume, during Version 1 , Version 2, and/or Version 3 activities, by the following NBD-PWG members:

Tsegereda Beyene
Cisco Systems
Deborah Blackstock
MITRE Corporation
David Boyd
InCadence Strategic Services
Scott Brim
Internet2
Pw McKenna Carey, III
Compliance Partners,
LLC/RALAND
Wo Chang
NIST

\section{Nancy Grady SAIC \\ Jay Greenberg The Boeing Company}

\section{Karen Guertler \\ Consultant}

Keith Hare

JCC Consulting, Inc.

Babak Jahromi
Microsoft
Pavithra Kenjige
PK Technologies
Donald Krapohl
Augmented Intelligence

Ashok Malhotra Oracle Corporation

Robert Marcus ET-Strategies

Gary Mazzaferro AlloyCloud, Inc.

William Miller
MaCT USA
Sanjay Mishra
Verizon
Doug Scrimager
Slalom Consulting
Cherry Tom
IEEE-SA

\footnotetext{
1 “Contributors" are members of the NIST Big Data Public Working Group who dedicated great effort to prepare and gave substantial time on a regular basis to research and development in support of this document.
} 
NIST Big DATA INTEROPERABILITY FRAMEWORK: Volume 3, USE CASES AND GENERAL REQUIREMENTS

Marge Cole

SGT, Inc.

Yuri Demchenko

University of Amsterdam

Safia Djennane

Cloud-Age-IT

Geoffrey Fox

Indiana University
Luca Lepori

Data Hold

Orit Levin

Microsoft

Eugene Luster

$D I S A / R 2 A D$
Wilco van Ginkel

Verizon

Timothy Zimmerlin

Consultant

Alicia Zuniga-Alvarado

Consultant 


\section{TABLE OF CONTENTS}

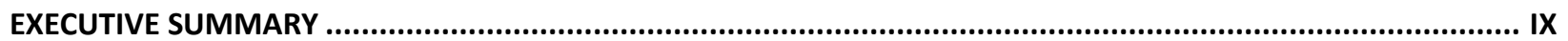

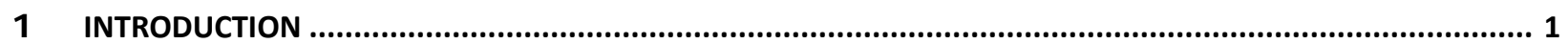

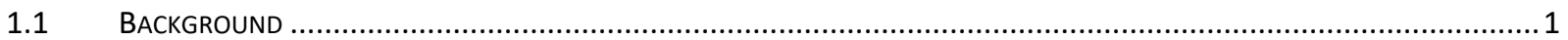

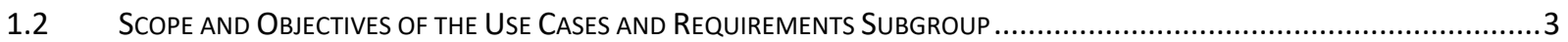

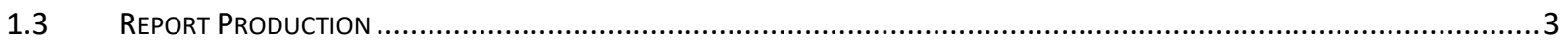

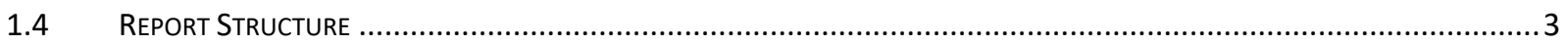

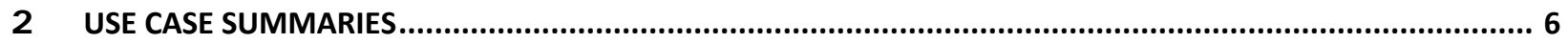

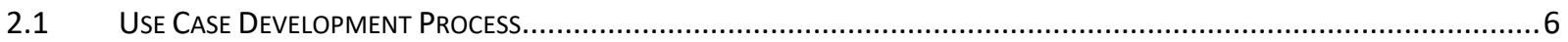

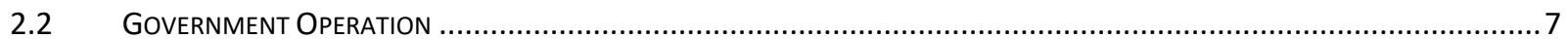

2.2.1 Use Case 1: Census 2010 and 2000-Title 13 Big Data ............................................................... 7

2.2.2 Use Case 2: NARA Accession, Search, Retrieve, Preservation ........................................................... 7

2.2.3 Use Case 3: Statistical Survey Response Improvement .................................................................

2.2.4 Use Case 4: Non-Traditional Data in Statistical Survey Response Improvement (Adaptive Design).........8

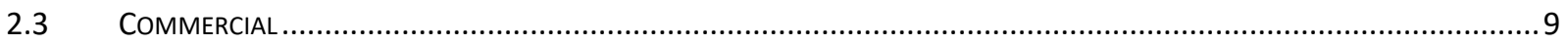

2.3.1 Use Case 5: Cloud Eco-System for Financial Industries ......................................................................

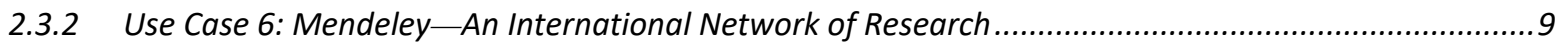

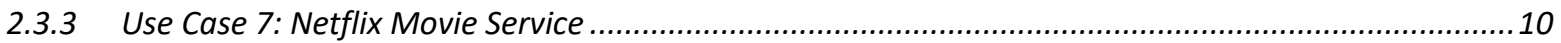

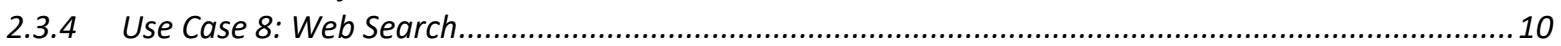

2.3.5 Use Case 9: Big Data Business Continuity and Disaster Recovery Within a Cloud Eco-System..............11

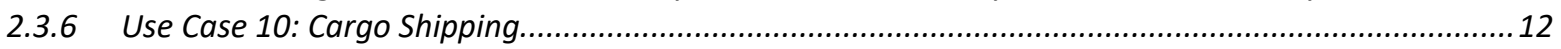

2.3.7 Use Case 11: Materials Data for Manufacturing ............................................................................13

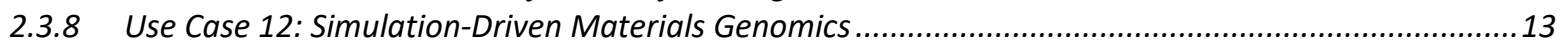

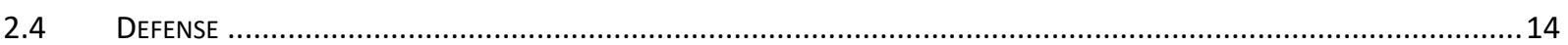

2.4.1 Use Case 13: Cloud Large-Scale Geospatial Analysis and Visualization................................................14

2.4.2 Use Case 14: Object Identification and Tracking from Wide-Area Large Format Imagery or Full Motion

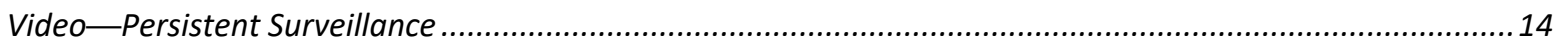

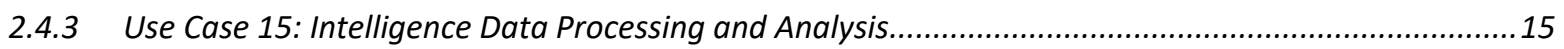

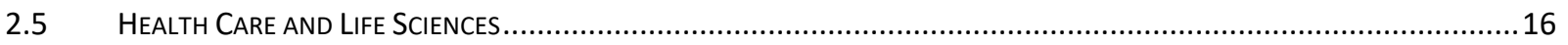

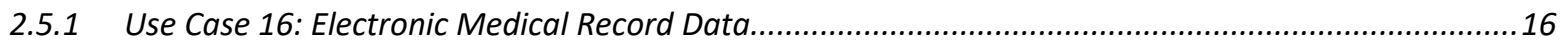

2.5.2 Use Case 17: Pathology Imaging/Digital Pathology .....................................................................17

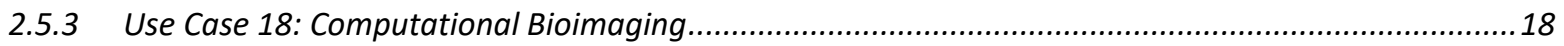

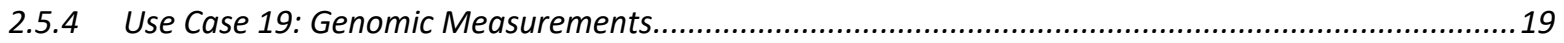

2.5.5 Use Case 20: Comparative Analysis for Metagenomes and Genomes.............................................19

2.5.6 Use Case 21: Individualized Diabetes Management ......................................................................20

2.5.7 Use Case 22: Statistical Relational Artificial Intelligence for Health Care...........................................20

2.5.8 Use Case 23: World Population-Scale Epidemiological Study ...........................................................21

2.5.9 Use Case 24: Social Contagion Modeling for Planning, Public Health, and Disaster Management.......21

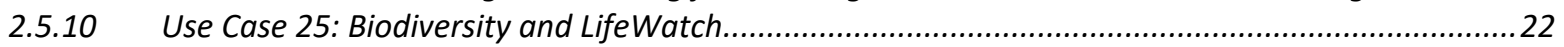

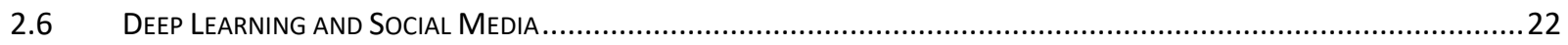

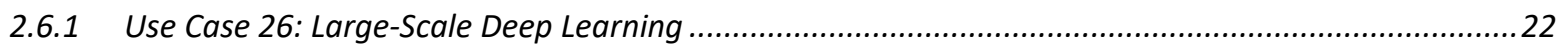

2.6.2 Use Case 27: Organizing Large-Scale, Unstructured Collections of Consumer Photos ...........................23

2.6.3 Use Case 28: Truthy —Information Diffusion Research from Twitter Data...........................................24

2.6.4 Use Case 29: Crowd Sourcing in the Humanities as Source for Big and Dynamic Data ........................24

2.6.5 Use Case 30: CINET_Cyberinfrastructure for Network (Graph) Science and Analytics .......................25 
NIST Big Data InTERoperability Framework: Volume 3, Use Cases and General ReQuiRements

2.6.6 Use Case 31: NIST Information Access Division—Analytic Technology Performance Measurements,

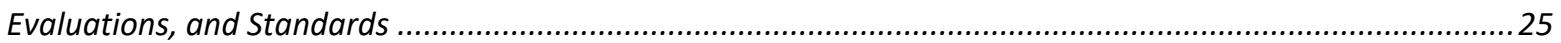

2.6.7 Use Case 2-3: Urban context-aware event management for Smart Cities - Public safety ......................26

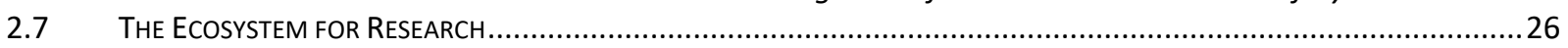

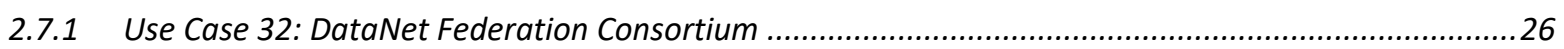

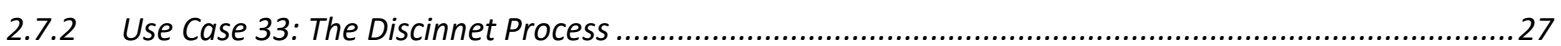

2.7.3 Use Case 34: Semantic Graph Search on Scientific Chemical and Text-Based Data ............................28

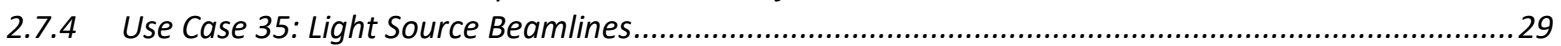

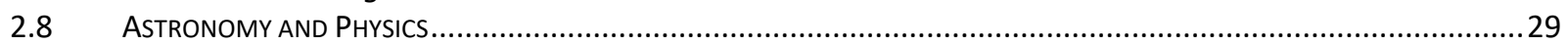

2.8.1 Use Case 36: Catalina Real-Time Transient Survey: A Digital, Panoramic, Synoptic Sky Survey............29

2.8.2 Use Case 37: DOE Extreme Data from Cosmological Sky Survey and Simulations.................................31

2.8.3 Use Case 38: Large Survey Data for Cosmology ............................................................................ 31

2.8.4 Use Case 39: Particle Physics—Analysis of Large Hadron Collider Data: Discovery of Higgs Particle ...32

2.8.5 Use Case 40: Belle II High Energy Physics Experiment .....................................................................34

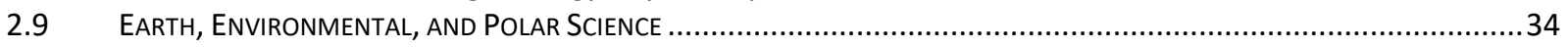

2.9.1 Use Case 41: European Incoherent Scatter Scientific Association 3D Incoherent Scatter Radar System...

2.9.2 Use Case 42: Common Operations of Environmental Research Infrastructure...................................35

2.9.3 Use Case 43: Radar Data Analysis for the Center for Remote Sensing of Ice Sheets ............................40

2.9.4 Use Case 44: Unmanned Air Vehicle Synthetic Aperture Radar (UAVSAR) Data Processing, Data

Product Delivery, and Data Services .................................................................................................. 42

2.9.5 Use Case 45: NASA Langley Research Center/ Goddard Space Flight Center iRODS Federation Test Bed.

2.9.6 Use Case 46: MERRA Analytic Services (MERRA/AS)

2.9.7 Use Case 47: Atmospheric Turbulence - Event Discovery and Predictive Analytics................................44

2.9.8 Use Case 48: Climate Studies Using the Community Earth System Model at the U.S. Department of

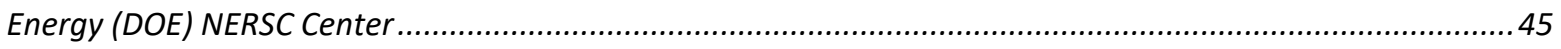

2.9.9 Use Case 49: DOE Biological and Environmental Research (BER) Subsurface Biogeochemistry Scientific

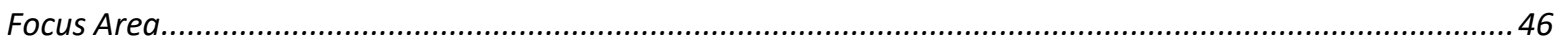

2.9.10 Use Case 50: DOE BER AmeriFlux and FLUXNET Networks.......................................................47

2.9.11 Use Case 2-1: NASA Earth Observing System Data and Information System (EOSDIS) ......................47

2.9.12 Use Case 2-2: Web-Enabled Landsat Data (WELD) Processing ...................................................48

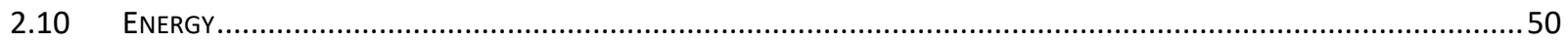

2.10.1 Use Case 51: Consumption Forecasting in Smart Grids ............................................................50

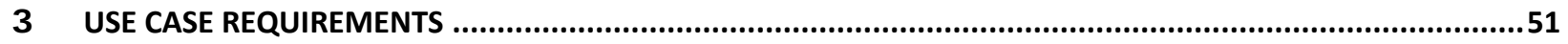

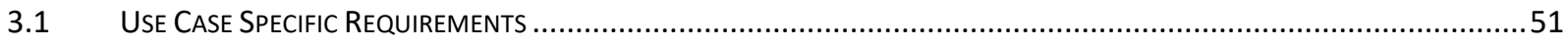

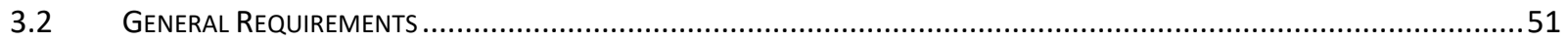

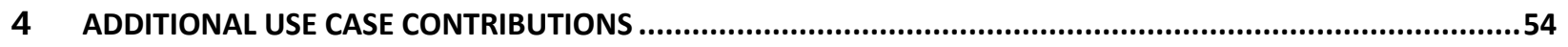

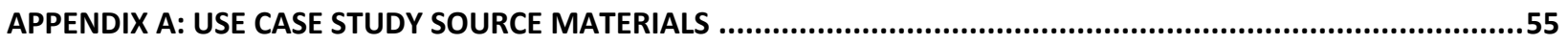

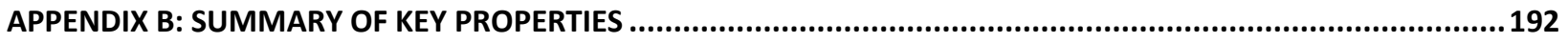

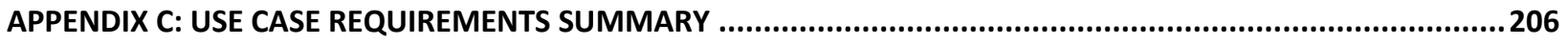

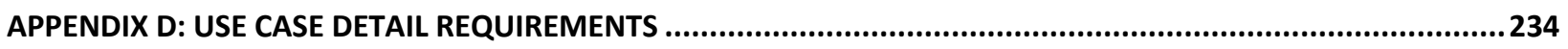

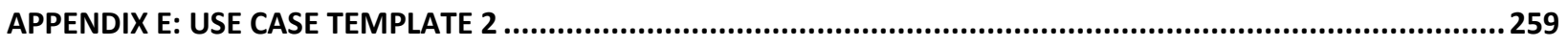

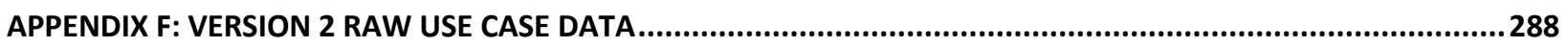

F.1 USE CASE 2-1: NASA EARTH ObSERVING SYSTEM DATA AND INFORMATION SYSTEM (EOSDIS) ...............................28

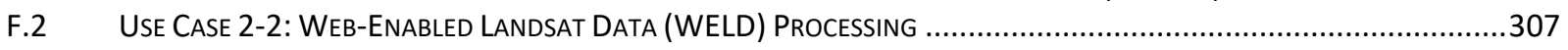

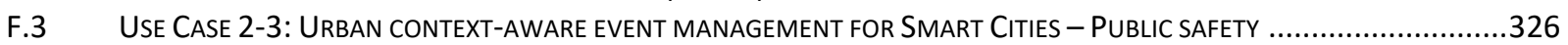


NIST Big DATA INTEROPERABILITY FRAMEWORK: Volume 3, USE CASES AND GENERAL REQUIREMENTS

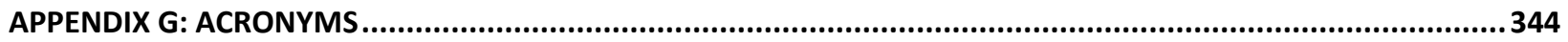

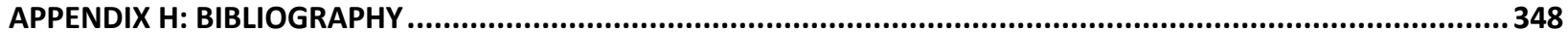

\section{FI GURES}

Figure 1: NBDif Documents Navigation Diagram Provides Content Flow Between Volumes .......................................5

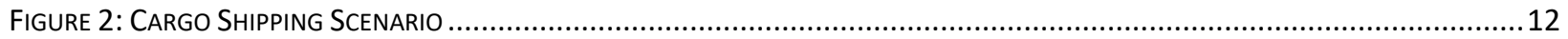

Figure 3: PAthology IMAging/Digital Pathology—EXamples of 2-D AND 3-D Pathology IMAgeS .................................17

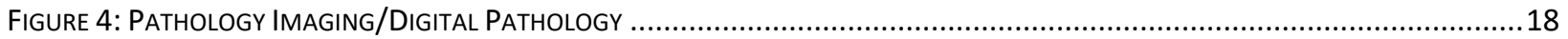

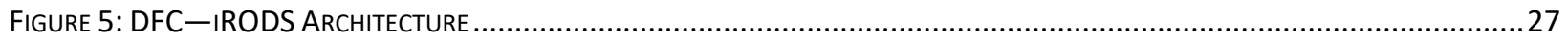

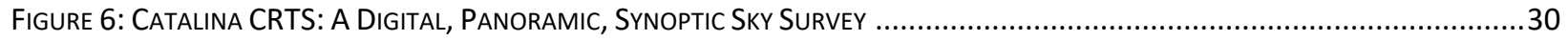

Figure 7: PARTICLE Physics: Analysis of LHC DATA: DisCovery of HIgGS PARTICLE-CERN LHC LOCATION ............................32

Figure 8: Particle Physics: Analysis of lhC Data: Discovery of Higgs Particle-The Multi-tier LhC Computing

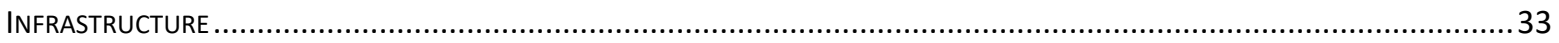

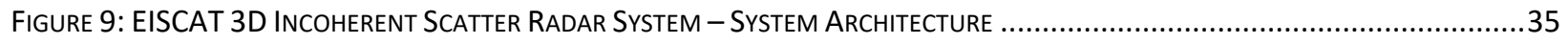

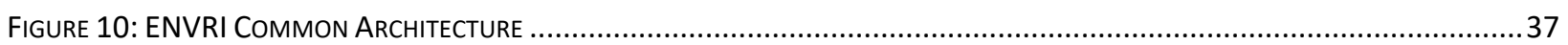

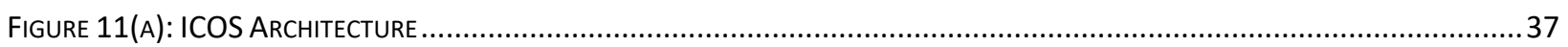

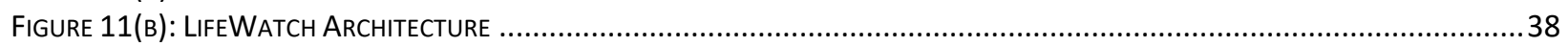

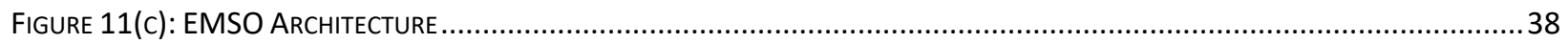

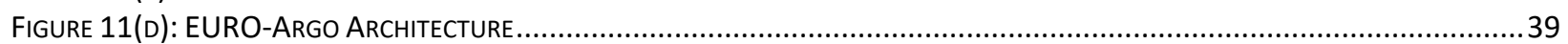

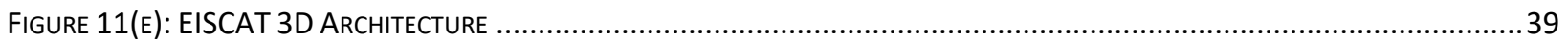

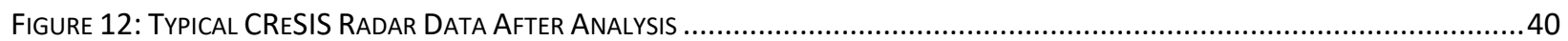

Figure 13: Radar Data Analysis for CReSis Remote Sensing of ICE Sheets- Typical Flight Paths of Data Gathering IN

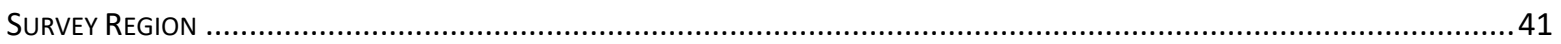

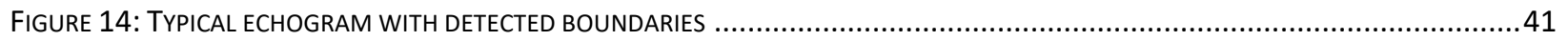

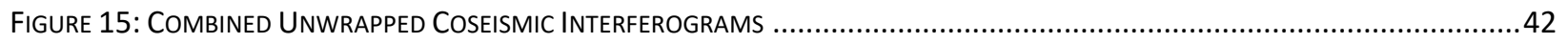

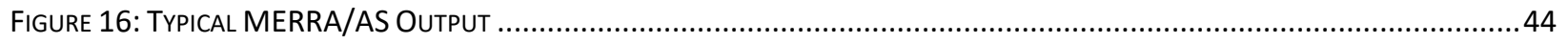

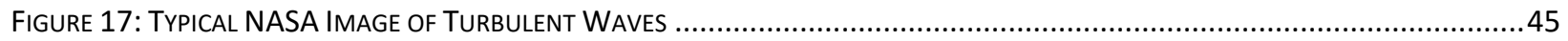

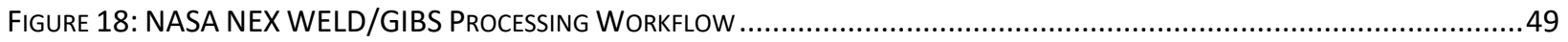

\section{TABLES}

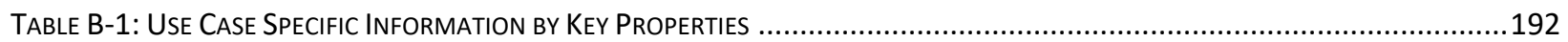

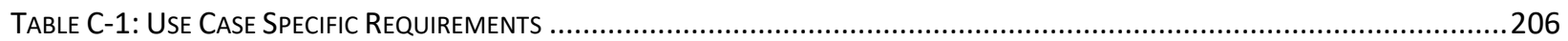

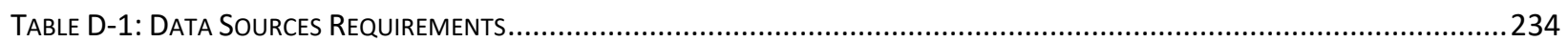

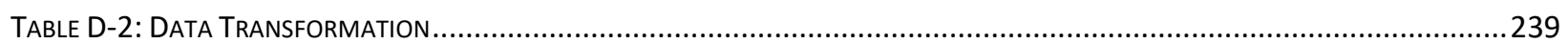

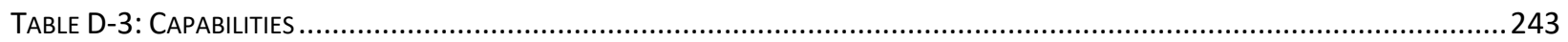

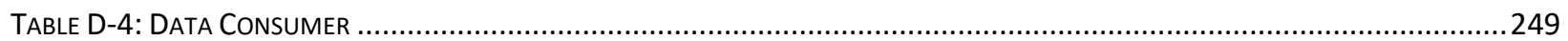

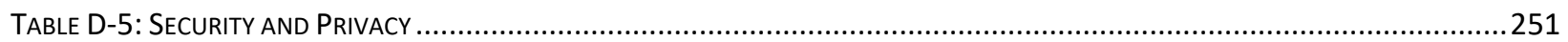

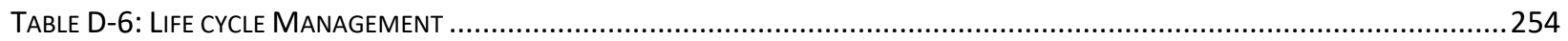

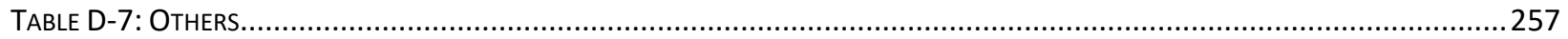


The NIST Big Data Interoperability Framework (NBDIF) was released in three versions, which correspond to the three stages of the NBD-PWG work. Version 3 (current version) of the NBDIF volumes resulted from Stage 3 work with major emphasis on the validation of the NBDRA Interfaces and content enhancement. Stage 3 work built upon the foundation created during Stage 2 and Stage 1 . The current effort documented in this volume reflects concepts developed within the rapidly evolving field of Big Data. The three stages (in reverse order) aim to achieve the following with respect to the NIST Big Data Reference Architecture (NBDRA).

Stage 3: Validate the NBDRA by building Big Data general applications through the general interfaces;

Stage 2: Define general interfaces between the NBDRA components; and

Stage 1: Identify the high-level Big Data reference architecture key components, which are technology-, infrastructure-, and vendor-agnostic.

The NBDIF consists of nine volumes, each of which addresses a specific key topic, resulting from the work of the NBD-PWG. The nine volumes are as follows:

- Volume 1, Definitions [1]

- Volume 2, Taxonomies [2]

- Volume 3, Use Cases and General Requirements (this volume)

- Volume 4, Security and Privacy [3]

- Volume 5, Architectures White Paper Survey [4]

- Volume 6, Reference Architecture [5]

- Volume 7, Standards Roadmap [6]

- Volume 8: Reference Architecture Implementation [7]

- Volume 9: Adoption and Modernization [8]

During Stage 1, Volumes 1 through 7 were conceptualized, organized, and written. The finalized Version 1 documents can be downloaded from the V1.0 Final Version page of the NBD-PWG website (https://bigdatawg.nist.gov/V1_output_docs.php).

During Stage 2, the NBD-PWG developed Version 2 of the NBDIF Version 1 volumes, with the exception of Volume 5, which contained the completed architecture survey work that was used to inform Stage 1 work of the NBD-PWG. The goals of Stage 2 were to enhance the Version 1 content, define general interfaces between the NBDRA components by aggregating low-level interactions into high-level general interfaces, and demonstrate how the NBDRA can be used. As a result of the Stage 2 work, the need for NBDIF Volume 8 and NBDIF Volume 9 were identified and the two new volumes were created. Version 2 of the NBDIF volumes, resulting from Stage 2 work, can be downloaded from the V2.0 Final Version page of the NBD-PWG website (https://bigdatawg.nist.gov/V2_output_docs.php).

This volume, NIST Big Data Interoperability Framework: Volume 3, Use Cases and General Requirements, was prepared by the NIST Big Data Public Working Group (NBD-PWG) Use Cases and Requirements Subgroup to document the collection of use cases and extraction of requirements. The Subgroup developed the first use case template with 26 fields that were completed by 51 users in the following broad areas:

- Government Operations (4)

- Commercial (8) 
- Defense (3)

- Healthcare and Life Sciences (10)

- Deep Learning and Social Media (6)

- The Ecosystem for Research (4)

- Astronomy and Physics (5)

- Earth, Environmental and Polar Science (10)

- Energy (1)

The use cases are, of course, only representative, and do not encompass the entire spectrum of Big Data usage. All the use cases were openly submitted and no significant editing was performed. While there are differences between the use cases in scope and interpretation, the benefits of free and open submission outweighed those of greater uniformity. The Use Cases and Requirements Subgroup examined the use cases, extracted specific and general requirements, and provided input to the other subgroups to inform their work as documented in the other NBDIF Volumes.

During the development of Version 2 of the NBDIF, the Use Cases and Requirements Subgroup and the Security and Privacy Subgroup identified the need for additional use cases to strengthen the future work of the NBD-PWG. These two subgroups collaboratively created the Use Case Template 2 (https://bigdatawg.nist.gov/ uploadfiles/M0621 v2 7345181325.pdf), which was used to collect additional use cases during Stage 2 and Stage 3 of the NBD-PWG work. The three use cases submitted with the Use Case Template 2 are presented in this document. Two use cases belong to the "Earth, Environmental and Polar Science" application domain and the third use case belongs to the "Deep Learning and Social Media” application domain.

This volume documents the process used by the Subgroup to collect the 51 use cases and extract requirements to form the NBDRA. Included in this document are summaries of the 51 Version 1 use cases, extracted requirements, the original, unedited 51 Version 1 use cases, the questions contained in Use Case Template 2, and the three Template 2 use cases submitted to date. The current effort documented in this volume reflects concepts developed within the rapidly evolving field of Big Data. 


\section{INTRODUCTION}

\subsection{BACKGROUND}

There is broad agreement among commercial, academic, and government leaders about the potential of Big Data to spark innovation, fuel commerce, and drive progress. Big Data is the common term used to describe the deluge of data in today's networked, digitized, sensor-laden, and information-driven world. The availability of vast data resources carries the potential to answer questions previously out of reach, including the following:

- How can a potential pandemic reliably be detected early enough to intervene?

- Can new materials with advanced properties be predicted before these materials have ever been synthesized?

- How can the current advantage of the attacker over the defender in guarding against cybersecurity threats be reversed?

There is also broad agreement on the ability of Big Data to overwhelm traditional approaches. The growth rates for data volumes, speeds, and complexity are outpacing scientific and technological advances in data analytics, management, transport, and data user spheres.

Despite widespread agreement on the inherent opportunities and current limitations of Big Data, a lack of consensus on some important fundamental questions continues to confuse potential users and stymie progress. These questions include the following:

- How is Big Data defined?

- What attributes define Big Data solutions?

- What is new in Big Data?

- What is the difference between Big Data and bigger data that has been collected for years?

- How is Big Data different from traditional data environments and related applications?

- What are the essential characteristics of Big Data environments?

- How do these environments integrate with currently deployed architectures?

- What are the central scientific, technological, and standardization challenges that need to be addressed to accelerate the deployment of robust, secure Big Data solutions?

Within this context, on March 29, 2012, the White House announced the Big Data Research and Development Initiative [9]. The initiative's goals include helping to accelerate the pace of discovery in science and engineering, strengthening national security, and transforming teaching and learning by improving analysts' ability to extract knowledge and insights from large and complex collections of digital data.

Six federal departments and their agencies announced more than $\$ 200$ million in commitments spread across more than 80 projects, which aim to significantly improve the tools and techniques needed to access, organize, and draw conclusions from huge volumes of digital data. The initiative also challenged industry, research universities, and nonprofits to join with the federal government to make the most of the opportunities created by Big Data.

Motivated by the White House initiative and public suggestions, the National Institute of Standards and Technology (NIST) accepted the challenge to stimulate collaboration among industry professionals to further the secure and effective adoption of Big Data. As one result of NIST's Cloud and Big Data Forum held on January 15-17, 2013, there was strong encouragement for NIST to create a public working group 
for the development of a Big Data Standards Roadmap. Forum participants noted that this roadmap should define and prioritize Big Data requirements, including interoperability, portability, reusability, extensibility, data usage, analytics, and technology infrastructure. In doing so, the roadmap would accelerate the adoption of the most secure and effective Big Data techniques and technology.

On June 19, 2013, the NIST Big Data Public Working Group (NBD-PWG) was launched with extensive participation by industry, academia, and government from across the nation. The scope of the NBD-PWG involves forming a community of interests from all sectors - including industry, academia, and government - with the goal of developing consensus on definitions, taxonomies, secure reference architectures, security and privacy, and, from these, a standards roadmap. Such a consensus would create a vendor-neutral, technology- and infrastructure-independent framework that would enable Big Data stakeholders to identify and use the best analytics tools for their processing and visualization requirements on the most suitable computing platform and cluster, while also allowing added value from Big Data service providers.

The NIST Big Data Interoperability Framework (NBDIF) was released in three versions, which correspond to the three stages of the NBD-PWG work. Version 3 (current version) of the NBDIF volumes resulted from Stage 3 work with major emphasis on the validation of the NBDRA Interfaces and content enhancement. Stage 3 work built upon the foundation created during Stage 2 and Stage 1 . The current effort documented in this volume reflects concepts developed within the rapidly evolving field of Big Data. The three stages (in reverse order) aim to achieve the following with respect to the NIST Big Data Reference Architecture (NBDRA).

Stage 3: Validate the NBDRA by building Big Data general applications through the general interfaces;

Stage 2: Define general interfaces between the NBDRA components; and

Stage 1: Identify the high-level Big Data reference architecture key components, which are technology-, infrastructure-, and vendor-agnostic.

The NBDIF consists of nine volumes, each of which addresses a specific key topic, resulting from the work of the NBD-PWG. The nine volumes are as follows:

- Volume 1, Definitions [1]

- Volume 2, Taxonomies [2]

- Volume 3, Use Cases and General Requirements (this volume)

- Volume 4, Security and Privacy [3]

- Volume 5, Architectures White Paper Survey [4]

- Volume 6, Reference Architecture [5]

- Volume 7, Standards Roadmap [6]

- Volume 8, Reference Architecture Interfaces [7]

- Volume 9, Adoption and Modernization [8]

During Stage 1, Volumes 1 through 7 were conceptualized, organized, and written. The finalized Version 1 documents can be downloaded from the V1.0 Final Version page of the NBD-PWG website (https://bigdatawg.nist.gov/V1 output docs.php).

During Stage 2, the NBD-PWG developed Version 2 of the NBDIF Version 1 volumes, with the exception of Volume 5, which contained the completed architecture survey work that was used to inform Stage 1 work of the NBD-PWG. The goals of Stage 2 were to enhance the Version 1 content, define general interfaces between the NBDRA components by aggregating low-level interactions into high-level general interfaces, and demonstrate how the NBDRA can be used. As a result of the Stage 2 work, the need for NBDIF Volume 8 and NBDIF Volume 9 were identified and the two new volumes were created. Version 2 of the NBDIF volumes, resulting from Stage 2 work, can be downloaded from the V2.0 Final Version page of the NBD-PWG website (https://bigdatawg.nist.gov/V2_output_docs.php). 


\subsection{SCOPE AND OBJECTIVES OF THE USE CASES AND REQUIREMENTS SUBGROUP}

This volume was prepared by the NBD-PWG Use Cases and Requirements Subgroup. The effort focused on forming a community of interest from industry, academia, and government, with the goal of developing a consensus list of Big Data requirements across all stakeholders. This included gathering and understanding various use cases from nine diversified areas (i.e., application domains.) To achieve this goal, the Subgroup completed the following tasks:

- Gathered input from all stakeholders regarding Big Data requirements;

- Analyzed and prioritized a list of challenging use case specific requirements that may delay or prevent adoption of Big Data deployment;

- Developed a comprehensive list of generalized Big Data requirements;

- Collaborated with the NBD-PWG Reference Architecture Subgroup to provide input for the NBDRA;

- Collaborated with the NBD-PWG Security and Privacy Subgroup to produce the Use Case Template 2, which helped gather valuable input to strengthen the work of the NBD-PWG; and

- Documented the findings in this report.

\subsection{REPORT PRODUCTION}

Version 1 of this report was produced using an open collaborative process involving weekly telephone conversations and information exchange using the NIST document system. The 51 Version 1 use cases, included herein, came from Subgroup members participating in the calls and from other interested parties informed of the opportunity to contribute.

The outputs from the use case process are presented in this report and online at the following locations:

- Index to all use cases: https://bigdatawg.nist.gov/usecases.php

- List of specific requirements versus use case: https://bigdatawg.nist.gov/uc reqs summary.php

- List of general requirements versus architecture component: https://bigdatawg.nist.gov/uc_reqs_gen.php

- List of general requirements versus architecture component with record of use cases giving requirements: https://bigdatawg.nist.gov/uc_reqs_gen_ref.php

- List of architecture components and specific requirements plus use case constraining the components: https://bigdatawg.nist.gov/uc_reqs_gen_detail.php

- General requirements: https://bigdatawg.nist.gov/uc_reqs_gen.php.

During development of Version 2 of this report, the subgroup focused on preparing the revised Use Case Template 2 (an outline of which is provided in Appendix E) and collaborating with other subgroups on content development for the other NBDIF volumes.

To achieve technical and high-quality document content, this document will go through a public comments period along with NIST internal review.

\subsection{REPORT STRUCTURE}

Following this introductory section, the remainder of this document is organized as follows:

- Section 2 presents the original (Version 1) 51 use cases and 2 new use cases gotten with updated Version 2 summary.

o Section 2.1 discusses the process that led to their production. of the use cases. 


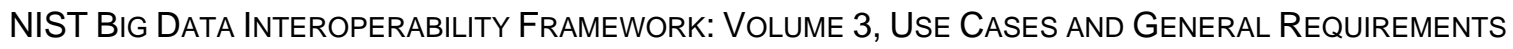

199

200

201

202

203

204

205

206

207

208

209

210

211

212

213

214

215

216

217

218

219

220

221

222

223

224

225

226

227

228

229

230

231

232

233

234

235

o Sections 2.2 through 2.10 provide summaries of the 53 use cases; each summary has three subsections: Application, Current Approach, and Future. The use cases are organized into the nine broad areas (application domains) listed below, with the number of associated use cases in parentheses:

- Government Operation (4)

- Commercial (8)

- Defense (3)

- Healthcare and Life Sciences (10)

- Deep Learning and Social Media (6)

- The Ecosystem for Research (4)

- Astronomy and Physics (5)

- Earth, Environmental, and Polar Science (10) plus 2 additional Version 2 use cases (12 total)

- Energy (1)

- Section 3 presents a more detailed analysis of requirements across use cases.

- Section 4 introduces the Version 2 use cases.

- Appendix A contains the original, unedited use cases.

- Appendix B summarizes key properties of each use case.

- Appendix C presents a summary of use case requirements.

- Appendix D provides the requirements extracted from each use case and aggregated general requirements grouped by characterization category.

- Appendix E presents the structure of the revised Use Case Template 2. The fillable pdf can be downloaded from https://bigdatawg.nist.gov/ uploadfiles/M0621 v2 7345181325.pdf.

- Appendix F contains the Version 2 use cases.

- Appendix G contains acronyms and abbreviations used in this document.

- Appendix H supplies the document references.

While each NBDIF volume was created with a specific focus within Big Data, all volumes are interconnected. During the creation of the volumes, information from some volumes was used as input for other volumes. Broad topics (e.g., definition, architecture) may be discussed in several volumes with each discussion circumscribed by the volume's particular focus. Arrows shown in Figure 1 indicate the main flow of information input and/or output from the volumes. Volumes 2, 3, and 5 (blue circles) are essentially standalone documents that provide output to other volumes (e.g., to Volume 6). These volumes contain the initial situational awareness research. During the creation of Volumes 4, 7, 8, and 9 (green circles), input from other volumes was used. The development of these volumes took into account work on the other volumes. Volumes 1 and 6 (red circles) were developed using the initial situational awareness research and continued to be modified based on work in other volumes. The information from these volumes was also used as input to the volumes in the green circles. 


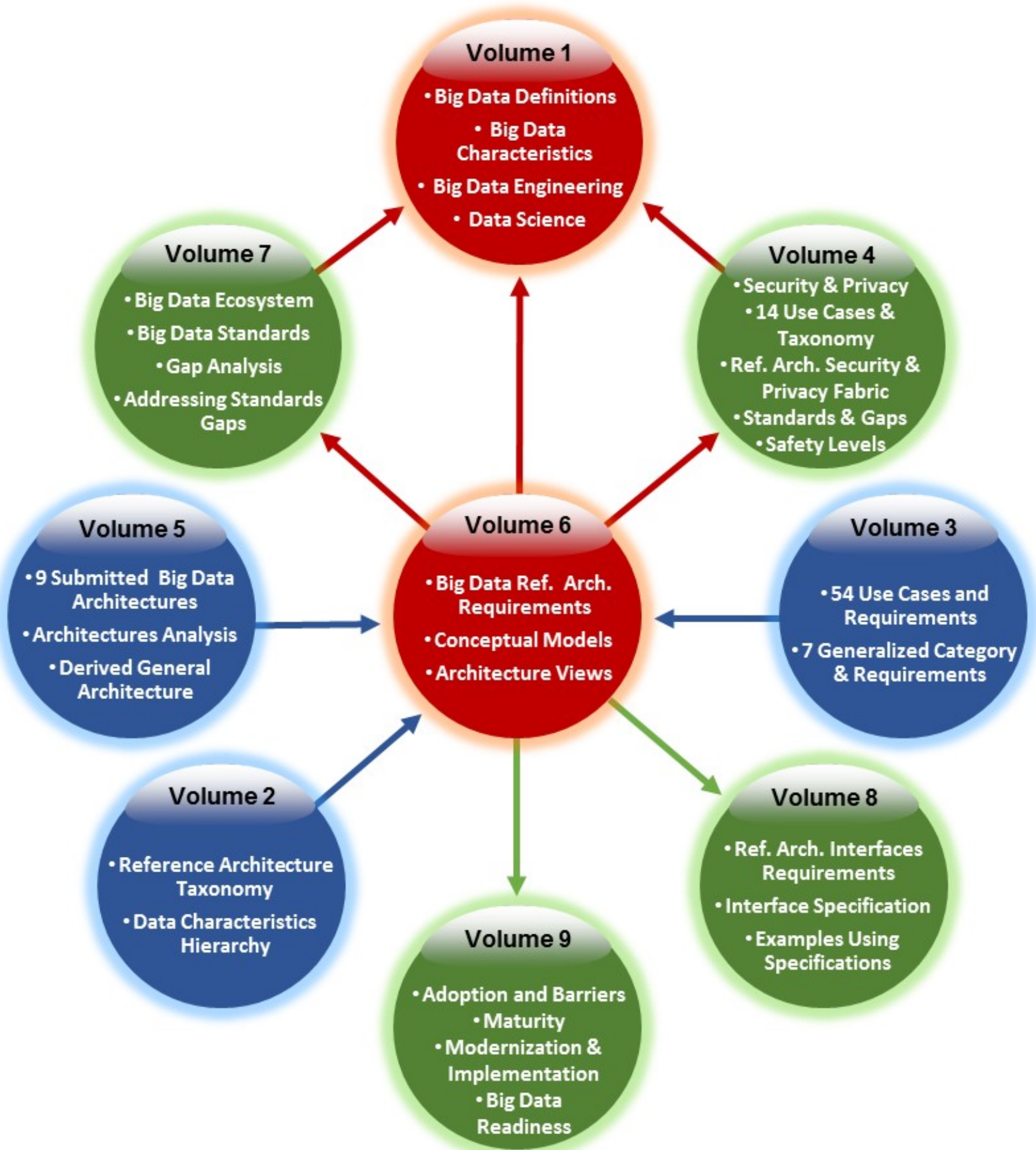

Figure 1: NBDIF Documents Navigation Diagram Provides Content Flow Between Volumes 


\section{USE CASE SUMMARIES}

\subsection{USE CASE DEVELOPMENT PROCESS}

A use case is a typical application stated at a high level for the purposes of extracting requirements or comparing usages across fields. In order to develop a consensus list of Big Data requirements across all stakeholders, the Subgroup began by collecting use cases. Publicly available information was collected for various Big Data architecture examples with special attention given to some areas including Healthcare and Government. After collection of 51 use cases, nine broad areas (i.e., application domains) were identified by the Subgroup members to better organize the collection of use cases. The list of application domains reflects the use cases submitted and is not intended to be exhaustive. If other application domains are proposed, they will be considered. Each example of Big Data architecture constituted one use case. The nine application domains were as follows:

- Government Operation;

- Commercial;

- Defense;

- Healthcare and Life Sciences;

- Deep Learning and Social Media;

- The Ecosystem for Research;

- Astronomy and Physics;

- Earth, Environmental, and Polar Science; and

- Energy.

As noted above, participants in the NBD-PWG Use Cases and Requirements Subgroup and other interested parties supplied the information for the use cases. The template used to collect use case information and provided at the front of Appendix A, was valuable for gathering consistent information that enabled the Subgroup to develop supporting analysis and comparison of the use cases. However, varied levels of detail and quantitative or qualitative information were received for each use case template section. The original, unedited use cases are also included in Appendix A and may be downloaded from the NIST document library (http://bigdatawg.nist.gov/usecases.php).

Beginning with Section 2.2 below, the following information is presented for each Big Data use case:

- Application: a high-level description of the use case;

- Current approach: the current manifestation of the use case; and

- Future: desired computational environment, if submitted.

For some application domains, several similar Big Data use cases are presented, providing a more complete view of Big Data requirements within that application domain.

The use cases are presented in this section with the information originally submitted. The original content has not been modified. Specific vendor solutions and technologies are mentioned in the use cases. However, the listing of these solutions and technologies does not constitute endorsement from the NBDPWG. The front matter (page ii) contains a general disclaimer. The use cases are numbered sequentially to facilitate cross-referencing between the use case summaries presented in this section, the original use cases (Appendix A), and the use case summary tables (Appendices B, C, and D). 


\subsection{GOVERNMENT OPERATION}

\subsubsection{Use Case 1: Census 2010 and 2000-TitLe 13 Big Data}

Submitted by Vivek Navale and Quyen Nguyen, National Archives and Records Administration (NARA)

\section{APPLICATION}

Census 2010 and 2000-Title 13 data must be preserved for several decades so they can be accessed and analyzed after 75 years. Data must be maintained 'as-is' with no access and no data analytics for 75 years, preserved at the bit level, and curated, which may include format transformation. Access and analytics must be provided after 75 years. Title 13 of the U.S. Code authorizes the U.S. Census Bureau to collect and preserve census related data and guarantees that individual and industry-specific data are protected.

\section{CURRENT APPROACH}

The dataset contains 380 terabytes (TB) of scanned documents.

\section{FUTURE}

Future data scenarios and applications were not expressed for this use case.

\subsubsection{Use Case 2: NaRA Accession, Search, Retrieve, Preservation}

Submitted by Vivek Navale and Quyen Nguyen, NARA

\section{APPLICATION}

This area comprises accession, search, retrieval, and long-term preservation of government data.

\section{CURRENT ApProACH}

The data are currently handled as follows:

1. Get physical and legal custody of the data

2. Pre-process data for conducting virus scans, identifying file format identifications, and removing empty files

3. Index the data

4. Categorize records (e.g., sensitive, non-sensitive, privacy data)

5. Transform old file formats to modern formats (e.g., WordPerfect to PDF)

6. Conduct e-discovery

7. Search and retrieve to respond to special requests

8. Search and retrieve public records by public users

Currently hundreds of TBs are stored centrally in commercial databases supported by custom software and commercial search products.

\section{FUTURE}

Federal agencies possess many distributed data sources, which currently must be transferred to centralized storage. In the future, those data sources may reside in multiple cloud environments. In this case, physical custody should avoid transferring Big Data from cloud to cloud or from cloud to data center. 


\title{
2.2.3 Use CASE 3: Statistical SuRVEy Response Improvement
}

\author{
Submitted by Cavan Capps, U.S. Census Bureau
}

\section{APPLICATION}

Survey costs are increasing as survey responses decline. The goal of this work is to increase the qualityand reduce the cost —of field surveys by using advanced 'recommendation system techniques.' These techniques are open and scientifically objective, using data mashed up from several sources and also historical survey para-data (i.e., administrative data about the survey.)

\section{CURRENT APPROACH}

This use case handles about a petabyte (PB) of data coming from surveys and other government administrative sources. Data can be streamed. During the decennial census, approximately 150 million records transmitted as field data are streamed continuously. All data must be both confidential and secure. All processes must be auditable for security and confidentiality as required by various legal statutes. Data quality should be high and statistically checked for accuracy and reliability throughout the collection process. Software used includes Hadoop, Spark, Hive, R, SAS, Mahout, Allegrograph, MySQL, Oracle, Storm, BigMemory, Cassandra, and Pig.

\section{FUTURE}

Improved recommendation systems are needed similar to those used in e-commerce (e.g., similar to the Netflix use case) that reduce costs and improve quality, while providing confidentiality safeguards that are reliable and publicly auditable. Data visualization is useful for data review, operational activity, and general analysis. The system continues to evolve and incorporate important features such as mobile access.

\subsubsection{USE Case 4: Non-Traditional Data in Statistical SuRVEy Response IMPROVEMENT (ADAPTIVE DESIGN)}

\author{
Submitted by Cavan Capps, U.S. Census Bureau
}

\section{APPLICATION}

Survey costs are increasing as survey response declines. This use case has goals similar to those of the Statistical Survey Response Improvement use case. However, this case involves non-traditional commercial and public data sources from the web, wireless communication, and electronic transactions mashed up analytically with traditional surveys. The purpose of the mashup is to improve statistics for small area geographies and new measures, as well as the timeliness of released statistics.

\section{CURRENT APPROACH}

Data from a range of sources are integrated including survey data, other government administrative data, web scrapped data, wireless data, e-transaction data, possibly social media data, and positioning data from various sources. Software, visualization, and data characteristics are similar to those in the Statistical Survey Response Improvement use case.

\section{FUTURE}

Analytics need to be developed that give more detailed statistical estimations, on a more near real-time basis, for less cost. The reliability of estimated statistics from such mashed-up sources still must be evaluated. 


\subsection{COMMERCIAL}

\subsubsection{USE CASE 5: Cloud ECO-SYSTEM for FInANCIAL INDUSTRIES}

Submitted by Pw Carey, Compliance Partners, LLC

\section{APPLICATION}

Use of cloud (e.g., Big Data) technologies needs to be extended in financial industries (i.e., banking, securities and investments, insurance) transacting business within the U.S.

\section{CURRENT APPROACH}

The financial industry is already using Big Data and Hadoop for fraud detection, risk analysis, assessments, as well as improving their knowledge and understanding of customers. At the same time, the industry is still using traditional client/server/data warehouse/relational database management system (RDBMS) for the handling, processing, storage, and archival of financial data. Real-time data and analysis are important in these applications.

\section{FUTURE}

Security, privacy, and regulation must be addressed. For example, the financial industry must examine SEC-mandated use of XBRL (extensible business-related markup language) and use of other cloud functions.

\subsubsection{Use CASE 6: Mendeley-An International Network of Research}

Submitted by William Gunn, Mendeley

\section{APPLICATION}

Mendeley has built a database of research documents and facilitates the creation of shared bibliographies. Mendeley collects and uses the information about research reading patterns and other activities conducted via their software to build more efficient literature discovery and analysis tools. Text mining and classification systems enable automatic recommendation of relevant research, improving research teams' performance and cost-efficiency, particularly those engaged in curation of literature on a particular subject.

\section{CURRENT APPROACH}

Data size is presently 15 TB and growing at a rate of about 1 TB per month. Processing takes place on Amazon Web Services (AWS) using the following software: Hadoop, Scribe, Hive, Mahout, and Python. The database uses standard libraries for machine learning and analytics, latent Dirichlet allocation (LDA, a generative probabilistic model for discrete data collection), and custom-built reporting tools for aggregating readership and social activities for each document.

\section{FUTURE}

Currently Hadoop batch jobs are scheduled daily, but work has begun on real-time recommendation. The database contains approximately 400 million documents and roughly 80 million unique documents, and receives 500,000 to 700,000 new uploads on a weekday. Thus, a major challenge is clustering matching documents together in a computationally efficient way (i.e., scalable and parallelized) when they are uploaded from different sources and have been slightly modified via third-party annotation tools or publisher watermarks and cover pages.

\section{RESOURCES}

- Mendeley. http://mendeley.com. Accessed March 3, 2015.

- Mendeley. http://dev.mendeley.com. Accessed March 3, 2015. 


\title{
2.3.3 Use Case 7: Netflix Movie Service
}

\author{
Submitted by Geoffrey Fox, Indiana University
}

\section{APPLICATION}

Netflix allows streaming of user-selected movies to satisfy multiple objectives (for different stakeholders) - but with a focus on retaining subscribers. The company needs to find the best possible ordering of a set of videos for a user (e.g., household) within a given context in real time, with the objective of maximizing movie consumption. Recommendation systems and streaming video delivery are core Netflix technologies. Recommendation systems are always personalized and use logistic/linear regression, elastic nets, matrix factorization, clustering, LDA, association rules, gradient-boosted decision trees, and other tools. Digital movies are stored in the cloud with metadata, along with individual user profiles and rankings for small fraction of movies. The current system uses multiple criteria: a contentbased recommendation system, a user-based recommendation system, and diversity. Algorithms are continuously refined with A/B testing (i.e., two-variable randomized experiments used in online marketing).

\section{CURRENT APPROACH}

Netflix held a competition for the best collaborative filtering algorithm to predict user ratings for filmsthe purpose of which was to improve ratings by $10 \%$. The winning system combined over 100 different algorithms. Netflix systems use SQL, NoSQL, and Map/Reduce on AWS. Netflix recommendation systems have features in common with e-commerce systems such as Amazon.com. Streaming video has features in common with other content-providing services such as iTunes, Google Play, Pandora, and Last.fm. Business initiatives such as Netflix-sponsored content have been used to increase viewership.

\section{FUTURE}

Streaming video is a very competitive business. Netflix needs to be aware of other companies and trends in both content (e.g., which movies are popular) and Big Data technology.

\section{RESOURCES}

- Building Large-scale Real-world Recommender Systems - Recsys2012 tutorial. http://www.slideshare.net/xamat/building-largescale-realworld-recommender-systemsrecsys2012-tutorial. Accessed March 3, 2015.

- RAD - Outlier Detection on Big Data. http://techblog.netflix.com/. Accessed March 3, 2015.

\subsubsection{Use Case 8: Web Search}

Submitted by Geoffrey Fox, Indiana University

\section{APPLICATION}

A web search function returns results in $\approx 0.1$ seconds based on search terms with an average of three words. It is important to maximize quantities such as "precision@10" for the number of highly accurate/appropriate responses in the top 10 ranked results.

\section{CURRENT APPROACH}

The current approach uses the following steps:

1. Crawl the web

2. Pre-process data to identify what is searchable (words, positions)

3. Form an inverted index, which maps words to their locations in documents

4. Rank the relevance of documents using the PageRank algorithm

5. Employ advertising technology, e.g., using reverse engineering to identify ranking models—or preventing reverse engineering 
6. Cluster documents into topics (as in Google News)

7. Update results efficiently

Modern clouds and technologies such as Map/Reduce have been heavily influenced by this application, which now comprises $\sim 45$ billion web pages total.

\section{FUTURE}

Web search is a very competitive field, so continuous innovation is needed. Two important innovation areas are addressing the growing segment of mobile clients, and increasing sophistication of responses and layout to maximize the total benefit of clients, advertisers, and the search company. The "deep web" (content not indexed by standard search engines, buried behind user interfaces to databases, etc.) and multimedia searches are also of increasing importance. Each day, 500 million photos are uploaded, and each minute, 100 hours of video are uploaded to YouTube.

\section{RESOURCES}

- Internet Trends D11 Conference. http://www.slideshare.net/kleinerperkins/kpcb-Internet-trends2013. Accessed March 3, 2015.

- Introduction to Search Engine Technology. http://webcourse.cs.technion.ac.il/236621/Winter2011-2012/en/ho_Lectures.html. Accessed March 3, 2015.

- Lecture "Information Retrieval and Web Search Engines" (SS 2011). http://www.ifis.cs.tubs.de/teaching/ss-11/irws. Accessed March 3, 2015.

- Recommender Systems Tutorial (Part 1)-Introduction. http://www.slideshare.net/beechung/recommender-systems-tutorialpart1intro. Accessed March 3, 2015.

- The size of the World Wide Web (The Internet). http://www.worldwidewebsize.com/. Accessed March 3, 2015.

\subsubsection{Use Case 9: Big Data Business Continuity and Disaster Recovery WITHIN A CLOUD ECO-SYSTEM}

Submitted by Pw Carey, Compliance Partners, LLC

\section{APPLICATION}

Business Continuity and Disaster Recovery (BC/DR) needs to consider the role that four overlaying and interdependent forces will play in ensuring a workable solution to an entity's business continuity plan and requisite disaster recovery strategy. The four areas are people (i.e., resources), processes (e.g., time/cost/return on investment [ROI]), technology (e.g., various operating systems, platforms, and footprints), and governance (e.g., subject to various and multiple regulatory agencies).

\section{CURRENT APPROACH}

Data replication services are provided through cloud ecosystems, incorporating IaaS and supported by Tier 3 data centers. Replication is different from backup and only moves the changes that took place since the previous replication, including block-level changes. The replication can be done quickly-with a fivesecond window-while the data are replicated every four hours. This data snapshot is retained for seven business days, or longer if necessary. Replicated data can be moved to a failover center (i.e., a backup system) to satisfy an organization's recovery point objectives (RPO) and recovery time objectives (RTO). There are some relevant technologies from VMware, NetApps, Oracle, IBM, and Brocade. Data sizes range from terabytes to petabytes. 


\section{FUTURE}

Migrating from a primary site to either a replication site or a backup site is not yet fully automated. The goal is to enable the user to automatically initiate the failover sequence. Both organizations must know which servers have to be restored and what the dependencies and inter-dependencies are between the primary site servers and replication and/or backup site servers. This knowledge requires continuous monitoring of both.

\section{RESOURCES}

- Disaster Recovery. http://www.disasterrecovery.org/. Accessed March 3, 2015.

\subsubsection{USE CASE 10: CARGO SHIPPING}

Submitted by William Miller, MaCT USA

\section{APPLICATION}

Delivery companies such as Federal Express, United Parcel Service (UPS), and DHL need optimal means of monitoring and tracking cargo.

\section{CURRENT APPROACH}

Information is updated only when items are checked with a bar code scanner, which sends data to the central server. An item's location is not currently displayed in real time. Figure 2 provides an architectural diagram.

\section{FUTURE}

Tracking items in real time is feasible through the Internet of Things application, in which objects are given unique identifiers and capability to transfer data automatically, i.e., without human interaction. A new aspect will be the item's status condition, including sensor information, global positioning system (GPS) coordinates, and a unique identification schema based upon standards under development (specifically International Organization for Standardization [ISO] standard 29161) from the ISO Joint Technical Committee 1, Subcommittee 31, Working Group 2, which develops technical standards for data structures used for automatic identification applications.

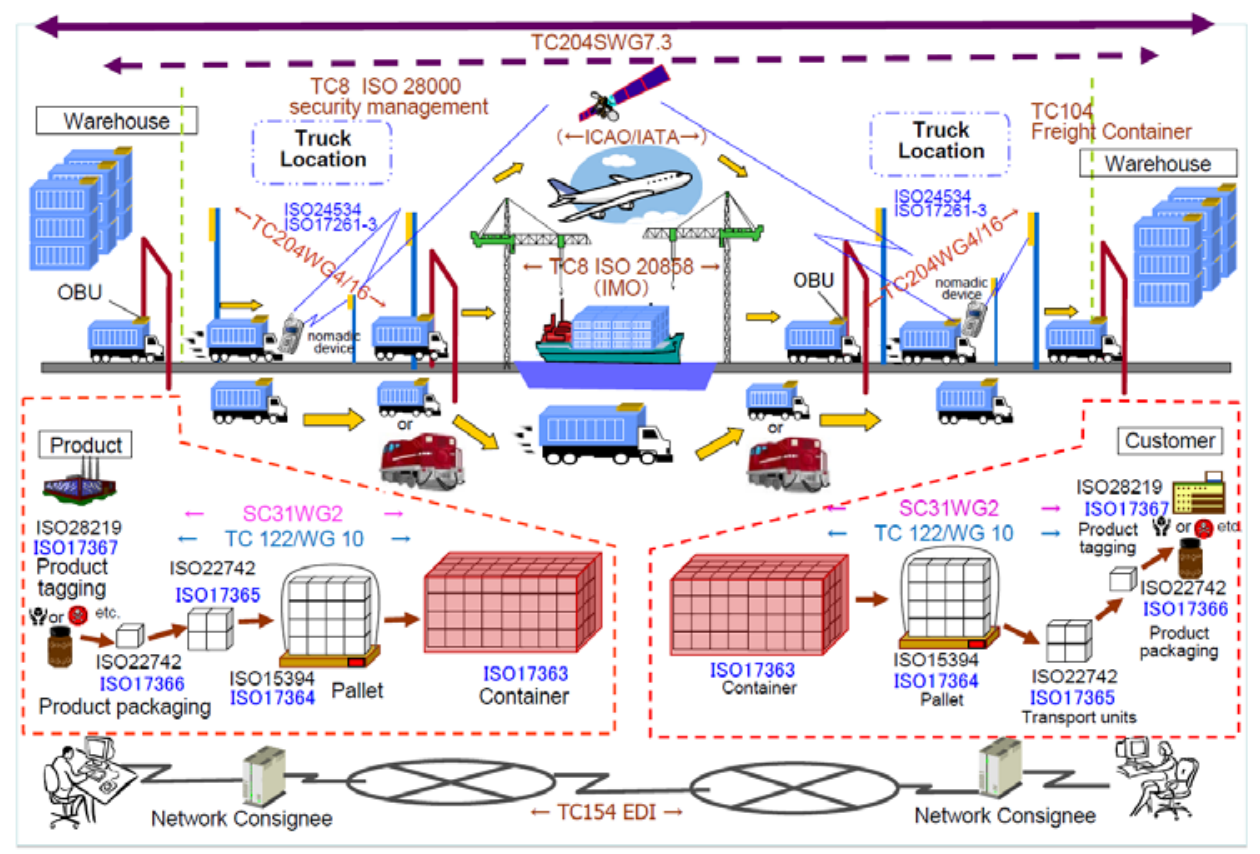

Figure 2: Cargo Shipping Scenario 


\title{
2.3.7 USE CASE 11: MATERIALS DATA FoR MANUfACtURING
}

Submitted by John Rumble, R\&R Data Services

\section{APPLICATION}

Every physical product is made from a material that has been selected for its properties, cost, and availability. This translates into hundreds of billions of dollars of material decisions made every year. However, the adoption of new materials normally takes decades (usually two to three decades) rather than a small number of years, in part because data on new materials are not easily available. To speed adoption time, accessibility, quality, and usability must be broadened, and proprietary barriers to sharing materials data must be overcome. Sufficiently large repositories of materials data are needed to support discovery.

\section{CURRENT APPROACH}

Decisions about materials usage are currently unnecessarily conservative, are often based on older rather than newer materials research and development data, and do not take advantage of advances in modeling and simulation.

\section{FUTURE}

Materials informatics is an area in which the new tools of data science can have a major impact by predicting the performance of real materials (in gram to ton quantities) starting at the atomistic, nanometer, and/or micrometer levels of description. The following efforts are needed to support this area:

- Establish materials data repositories, beyond the existing ones, that focus on fundamental data.

- Develop internationally accepted data recording standards that can be used by a very diverse materials community, including developers of materials test standards (e.g., ASTM International and ISO), testing companies, materials producers, and research and development labs.

- Develop tools and procedures to help organizations that need to deposit proprietary materials in data repositories to mask proprietary information while maintaining the data's usability.

- Develop multi-variable materials data visualization tools in which the number of variables can be quite high.

\section{RESOURCES}

- $\quad$ The Materials Project. http://www.materialsproject.org. Accessed March 3, 2015.

\subsubsection{Use CASE 12: Simulation-Driven Materials GenOMics}

\author{
Submitted by David Skinner, Lawrence Berkeley National Laboratory (LBNL)
}

\section{APPLICATION}

Massive simulations spanning wide spaces of possible design lead to innovative battery technologies. Systematic computational studies are being conducted to examine innovation possibilities in photovoltaics. Search and simulation is the basis for rational design of materials. All these require management of simulation results contributing to the materials genome.

\section{CURRENT APPROACH}

Survey results are produced using PyMatGen, FireWorks, VASP, ABINIT, NWChem, BerkeleyGW, and varied materials community codes running on large supercomputers, such as the Hopper at the National Energy Research Scientific Computing Center (NERSC), a 150,000-core machine that produces highresolution simulations.

\section{FUTURE}

Large-scale computing and flexible data methods at scale for messy data are needed for simulation science. The advancement of goal-driven thinking in materials design requires machine learning and 
knowledge systems that integrate data from publications, experiments, and simulations. Other needs include scalable key-value and object store databases; the current $100 \mathrm{~TB}$ of data will grow to $500 \mathrm{~TB}$ over the next five years.

\section{RESOURCES}

- The Materials Project. http://www.materialsproject.org. Accessed March 3, 2015.

\subsection{DEFENSE}

\subsubsection{Use Case 13: Cloud LaRge-Scale Geospatial Analysis and VISUALIZATION}

Submitted by David Boyd, Data Tactics

\section{APPLICATION}

Large-scale geospatial data analysis and visualization must be supported. As the number of geospatially aware sensors and geospatially tagged data sources increase, the volume of geospatial data requiring complex analysis and visualization is growing exponentially.

\section{CURRENT APPROACH}

Traditional geographic information systems (GISs) are generally capable of analyzing millions of objects and visualizing thousands. Data types include imagery (various formats such as NITF, GeoTiff, and CADRG) and vector (various formats such as shape files, KML [Keyhole Markup Language], and text streams). Object types include points, lines, areas, polylines, circles, and ellipses. Image registrationtransforming various data into one system - requires data and sensor accuracy. Analytics include principal component analysis (PCA) and independent component analysis (ICA) and consider closest point of approach, deviation from route, and point density over time. Software includes a server with a geospatially enabled RDBMS, geospatial server/analysis software (ESRI ArcServer or Geoserver), and visualization (either browser-based or using the ArcMap application).

\section{FUTURE}

Today's intelligence systems often contain trillions of geospatial objects and must visualize and interact with millions of objects. Critical issues are indexing, retrieval and distributed analysis (note that geospatial data requires unique approaches to indexing and distributed analysis); visualization generation and transmission; and visualization of data at the end of low-bandwidth wireless connections. Data are sensitive and must be completely secure in transit and at rest (particularly on handhelds).

\section{RESOURCES}

- OGC® Standards and Supporting Documents. http://www.opengeospatial.org/standards. Accessed March 3, 2015.

- GeoJSON. http://geojson.org/. Accessed March 3, 2015.

- Compressed ARC Digitized Raster Graphics (CADRG). http://earthinfo.nga.mil/publications/specs/printed/CADRG/cadrg.html. Accessed March 3, 2015.

\subsubsection{USE CASE 14: OBJECT IDENTIFICATION AND TRACKING FROM WIDE-AREA LARGE FORMAT IMAGERY OR FULL MOTION VIDEO-PERSISTENT SURVEILLANCE}

Submitted by David Boyd, Data Tactics 


\section{APPLICATION}

Persistent surveillance sensors can easily collect PB of imagery data in the space of a few hours. The data should be reduced to a set of geospatial objects (e.g., points, tracks) that can be easily integrated with other data to form a common operational picture. Typical processing involves extracting and tracking entities (e.g., vehicles, people, packages) over time from the raw image data.

\section{CURRENT APPROACH}

It is not feasible for humans to process these data for either alerting or tracking purposes. The data need to be processed close to the sensor, which is likely forward-deployed since it is too large to be easily transmitted. Typical object extraction systems are currently small (e.g., 1 to 20 nodes) graphics processing unit (GPU)-enhanced clusters. There are a wide range of custom software and tools, including traditional RDBMSs and display tools. Real-time data are obtained at Full Motion Video (FMV) - 30 to 60 frames per second at full-color 1080p resolution (i.e., 1920 x 1080 pixels, a high-definition progressive scan) or Wide-Area Large Format Imagery (WALF) -1 to 10 frames per second at 10,000 pixels x 10,000 pixels and full-color resolution. Visualization of extracted outputs will typically be as overlays on a geospatial (i.e., GIS) display. Analytics are basic object detection analytics and integration with sophisticated situation awareness tools with data fusion. Significant security issues must be considered; sources and methods cannot be compromised (i.e., "the enemy" should not know what we see).

\section{FUTURE}

A typical problem is integration of this processing into a large GPU cluster capable of processing data from several sensors in parallel and in near real time. Transmission of data from sensor to system is also a major challenge.

\section{RESOURCES}

- Persistent surveillance relies on extracting relevant data points and connecting the dots. http://www.militaryaerospace.com/topics/m/video/79088650/persistent-surveillance-relies-onextracting-relevant-data-points-and-connecting-the-dots.htm. Accessed March 3, 2015.

- Wide Area Persistent Surveillance Revolutionizes Tactical ISR. http://www.defencetalk.com/wide-area-persistent-surveillance-revolutionizes-tactical-isr-45745/. Accessed March 3, 2015.

\subsubsection{Use Case 15: Intelligence Data Processing and Analysis}

Submitted by David Boyd, Data Tactics

\section{APPLICATION}

Intelligence analysts need the following capabilities:

- Identify relationships between entities (e.g., people, organizations, places, equipment).

- Spot trends in sentiment or intent for either the general population or a leadership group such as state and non-state actors.

- Identify the locations and possibly timing of hostile actions including implantation of improvised explosive devices.

- Track the location and actions of potentially hostile actors.

- Reason against and derive knowledge from diverse, disconnected, and frequently unstructured (e.g., text) data sources.

- Process data close to the point of collection, and allow for easy sharing of data to/from individual soldiers, forward-deployed units, and senior leadership in garrisons. 


\section{CURRENT APPROACH}

Software includes Hadoop, Accumulo (Big Table), Solr, natural language processing (NLP), Puppet (for deployment and security), and Storm running on medium-size clusters. Data size ranges from tens of terabytes to hundreds of petabytes, with imagery intelligence devices gathering a petabyte in a few hours. Dismounted warfighters typically have at most one to hundreds of gigabytes (GBs), which is typically handheld data storage.

\section{FUTURE}

Data currently exist in disparate silos. These data must be accessible through a semantically integrated data space. A wide variety of data types, sources, structures, and quality will span domains and require integrated search and reasoning. Most critical data are either unstructured or maintained as imagery or video, which requires significant processing to extract entities and information. Network quality, provenance, and security are essential.

\section{RESOURCES}

- Program Overview: AFCEA Aberdeen Chapter Luncheon March 14 ${ }^{\text {th }}$, 2012. http://www.afceaaberdeen.org/files/presentations/AFCEAAberdeen_DCGSA_COLWells_PS.pdf. Accessed March 3, 2015.

- Horizontal Integration of Warfighter Intelligence Data: A Shared Semantic Resource for the Intelligence Community. http://stids.c4i.gmu.edu/papers/STIDSPapers/STIDS2012_T14_SmithEtAl_HorizontalIntegration OfWarfighterIntel.pdf. Accessed March 3, 2015.

- Integration of Intelligence Data through Semantic Enhancement. http://stids.c4i.gmu.edu/STIDS2011/papers/STIDS2011_CR T1_SalmenEtAl.pdf. Accessed March 3, 2015.

- DCGSA Standard Cloud. http://www.youtube.com/watch?v=l4Qii7T8zeg. Accessed March 3, 2015.

- Distributed Common Ground System - Army. http://dcgsa.apg.army.mil/. Accessed March 3, 2015.

\subsection{HEALTH CARE AND LIFE SCIENCES}

\subsubsection{USE CASE 16: EleCtRonic Medical Record DATA}

Submitted by Shaun Grannis, Indiana University

\section{APPLICATION}

Large national initiatives around health data are emerging. These include developing a digital learning health care system to support increasingly evidence-based clinical decisions with timely, accurate, and up-to-date patient-centered clinical information; using electronic observational clinical data to efficiently and rapidly translate scientific discoveries into effective clinical treatments; and electronically sharing integrated health data to improve healthcare process efficiency and outcomes. These key initiatives all rely on high-quality, large-scale, standardized, and aggregate health data. Advanced methods are needed for normalizing patient, provider, facility, and clinical concept identification within and among separate health care organizations. With these methods in place, feature selection, information retrieval, and enhanced machine learning decision-models can be used to define and extract clinical phenotypes from non-standard, discrete, and free-text clinical data. Clinical phenotype data must be leveraged to support cohort selection, clinical outcomes research, and clinical decision support. 


\title{
CURRENT APPROACH
}

The Indiana Network for Patient Care (INPC), the nation's largest and longest-running health information exchange, houses clinical data from more than 1,100 discrete logical operational healthcare sources. More than $20 \mathrm{~TB}$ of raw data, these data describe over 12 million patients and over 4 billion discrete clinical observations. Between 500,000 and 1.5 million new real-time clinical transactions are added every day.

\section{FUTURE}

Running on an Indiana University supercomputer, Teradata, PostgreSQL, and MongoDB will support information retrieval methods to identify relevant clinical features (e.g., term frequency-inverse document frequency [tf-idf], latent semantic analysis, mutual information). NLP techniques will extract relevant clinical features. Validated features will be used to parameterize clinical phenotype decision models based on maximum likelihood estimators and Bayesian networks. Decision models will be used to identify a variety of clinical phenotypes such as diabetes, congestive heart failure, and pancreatic cancer.

\section{RESOURCES}

- A universal code system for tests, measurements, and observations. http://loinc.org/. Accessed March 3, 2015.

\subsubsection{Use Case 17: Pathology Imaging/Digital Pathology}

\author{
Submitted by Fusheng Wang, Emory University
}

\section{APPLICATION}

Digital pathology imaging is an emerging field in which examination of high-resolution images of tissue specimens enables novel and more effective ways to diagnose diseases. Pathology image analysis segments massive spatial objects (e.g., millions of objects per image) such as nuclei and blood vessels, represented with their boundaries, along with many extracted image features from these objects. The derived information is used for many complex queries and analytics to support biomedical research and clinical diagnosis. Figure 3 presents examples of two- and three-dimensional (2D and 3D) pathology images.
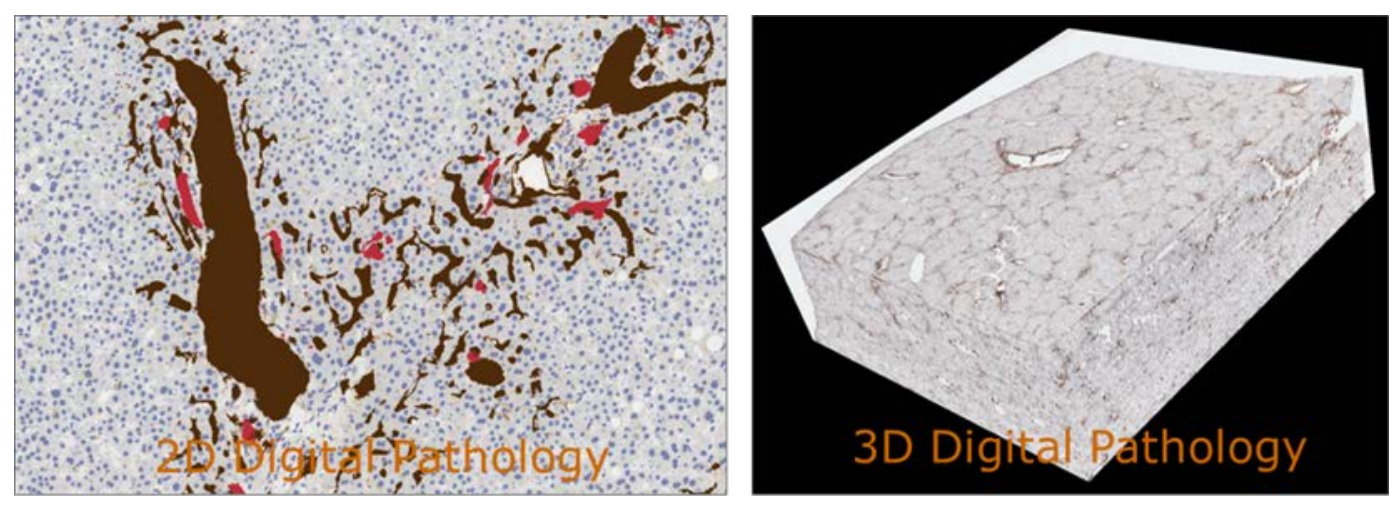

Figure 3: Pathology Imaging/Digital Pathology-Examples of 2-D and 3-D Pathology Images

\section{CURRENT APPROACH}

Each 2D image comprises $1 \mathrm{~GB}$ of raw image data and entails $1.5 \mathrm{~GB}$ of analytical results. Message Passing Interface (MPI) is used for image analysis. Data processing happens with Map/Reduce (a data processing program) and Hive (to abstract the Map/Reduce program and support data warehouse interactions), along with spatial extension on supercomputers and clouds. GPUs are used effectively for image creation. Figure 4 shows the architecture of Hadoop-GIS, a spatial data warehousing system, over Map/Reduce to support spatial analytics for analytical pathology imaging. 


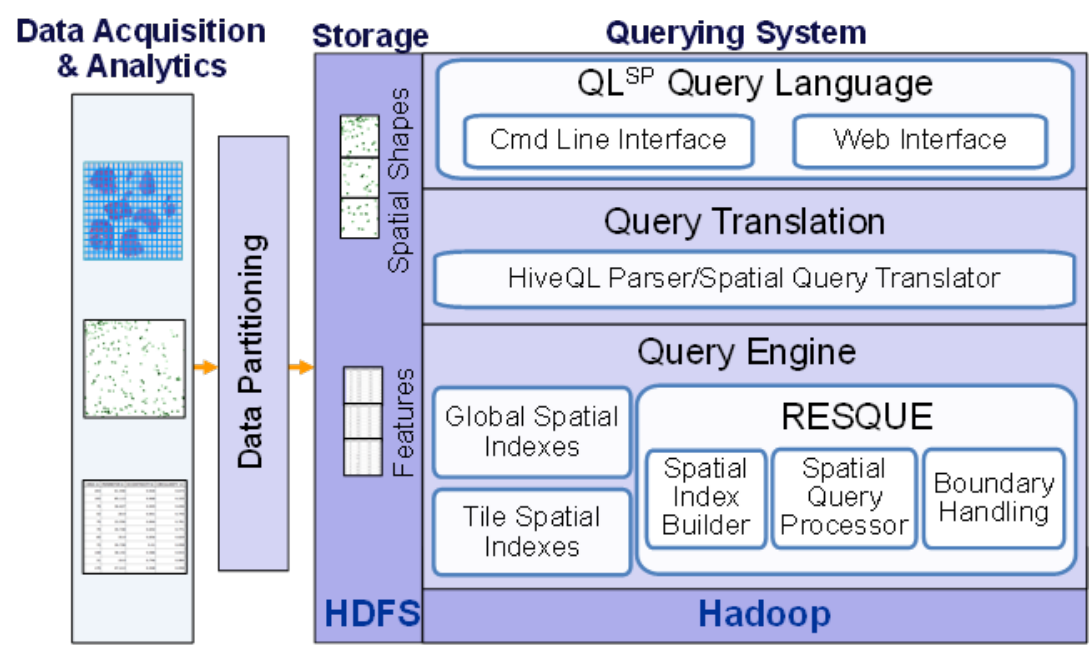

Figure 4: Pathology Imaging/Digital Pathology

\section{FUTURE}

Recently, 3D pathology imaging has been made possible using 3D laser technologies or serially sectioning hundreds of tissue sections onto slides and scanning them into digital images. Segmenting 3D microanatomic objects from registered serial images could produce tens of millions of 3D objects from a single image. This provides a deep 'map' of human tissues for next-generation diagnosis. 3D images can comprise $1 \mathrm{~TB}$ of raw image data and entail $1 \mathrm{~TB}$ of analytical results. A moderated hospital would generate $1 \mathrm{~PB}$ of data per year.

\section{RESOURCES}

- Pathology Analytical Imaging Standards. http://openpais.org. Accessed March 3, 2015.

- Hadoop-GIS: Spatial Big Data Solutions. http://hadoopgis.org/. Accessed March 3, 2015.

\subsubsection{USe Case 18: Computational Bioimaging}

Submitted by David Skinner, Joaquin Correa, Daniela Ushizima, and Joerg Meyer, LBNL

\section{APPLICATION}

Data delivered from bioimaging are increasingly automated, higher resolution, and multi-modal. This has created a data analysis bottleneck that, if resolved, can advance bioscience discovery through Big Data techniques.

\section{CURRENT APPROACH}

The current piecemeal analysis approach does not scale to situations in which a single scan on emerging machines is $32 \mathrm{~TB}$ and medical diagnostic imaging is annually around $70 \mathrm{~PB}$, excluding cardiology. A web-based, one-stop shop is needed for high-performance, high-throughput image processing for producers and consumers of models built on bio-imaging data.

\section{FUTURE}

The goal is to resolve that bottleneck with extreme-scale computing and community-focused science gateways, both of which apply massive data analysis toward massive imaging datasets. Workflow components include data acquisition, storage, enhancement, noise minimization, segmentation of regions of interest, crowd-based selection and extraction of features, and object classification, as well as organization and search. Suggested software packages are ImageJ, OMERO, VolRover, and advanced segmentation and feature detection software. 


\subsubsection{Use Case 19: Genomic MeAsurements}

Submitted by Justin Zook, National Institute of Standards and Technology

\section{APPLICATION}

The NIST Genome in a Bottle Consortium integrates data from multiple sequencing technologies and methods to develop highly confident characterization of whole human genomes as reference materials. The consortium also develops methods to use these reference materials to assess performance of any genome sequencing run.

\section{CURRENT APPROACH}

NIST's approximately 40 TB network file system (NFS) is full. The National Institutes of Health (NIH) and the National Center for Biotechnology Information (NCBI) are also currently storing PBs of data. NIST is also storing data using open-source sequencing bioinformatics software from academic groups (UNIX-based) on a 72-core cluster, supplemented by larger systems at collaborators.

\section{FUTURE}

DNA sequencers can generate $\approx 300 \mathrm{~GB}$ of compressed data per day, and this volume has increased much faster than Moore's Law gives for increase in computer processing power. Future data could include other 'omics' (e.g., genomics) measurements, which will be even larger than DNA sequencing. Clouds have been explored as a cost effective scalable approach.

\section{RESOURCES}

- Genome in a Bottle Consortium. http://www.genomeinabottle.org. Accessed March 3, 2015.

\subsubsection{Use Case 20: Comparative Analysis for Metagenomes and GENOMES}

Submitted by Ernest Szeto, LBNL, Joint Genome Institute

\section{APPLICATION}

Given a metagenomic sample this use case aims to do the following:

- Determine the community composition in terms of other reference isolate genomes;

- Characterize the function of its genes;

- Begin to infer possible functional pathways;

- Characterize similarity or dissimilarity with other metagenomic samples;

- Begin to characterize changes in community composition and function due to changes in environmental pressures; and

- Isolate subsections of data based on quality measures and community composition.

\section{CURRENT APPROACH}

The current integrated comparative analysis system for metagenomes and genomes is front-ended by an interactive web user interface (UI) with core data. The system involves backend precomputations and batch job computation submission from the UI. The system provides an interface to standard bioinformatics tools (e.g., BLAST, HMMER, multiple alignment and phylogenetic tools, gene callers, sequence feature predictors).

\section{FUTURE}

Management of heterogeneity of biological data is currently performed by a RDBMS (i.e., Oracle). Unfortunately, it does not scale for even the current volume, 50 TB of data. NoSQL solutions aim at 
providing an alternative, but unfortunately, they do not always lend themselves to real-time interactive use or rapid and parallel bulk loading, and sometimes they have issues regarding robustness.

\section{RESOURCES}

- IMG Data Management. http://img.jgi.doe.gov. Accessed March 3, 2015.

\subsubsection{USE CASE 21: INDIVIDUALIZED Diabetes MANAGEMENT}

Submitted by Ying Ding, Indiana University

\section{APPLICATION}

Diabetes is a growing illness in the world population, affecting both developing and developed countries. Current management strategies do not adequately take into account individual patient profiles, such as comorbidities and medications, which are common in patients with chronic illnesses. Advanced graph-based data mining techniques must be applied to electronic health records (EHRs), converting them into RDF (Resource Description Framework) graphs. These advanced techniques would facilitate searches for diabetes patients and allow for extraction of their EHR data for outcome evaluation.

\section{CURRENT APPROACH}

Typical patient data records are composed of 100 controlled vocabulary values and 1,000 continuous values. Most values have a timestamp. The traditional paradigm of relational row-column lookup needs to be updated to semantic graph traversal.

\section{FUTURE}

The first step is to compare patient records to identify similar patients from a large EHR database (i.e., an individualized cohort.) Each patient's management outcome should be evaluated to formulate the most appropriate solution for a given patient with diabetes. The process would use efficient parallel retrieval algorithms, suitable for cloud or high-performance computing (HPC), using the open source Hbase database with both indexed and custom search capability to identify patients of possible interest. The Semantic Linking for Property Values method would be used to convert an existing data warehouse at Mayo Clinic, called the Enterprise Data Trust (EDT), into RDF triples that enable one to find similar patients through linking of both vocabulary-based and continuous values. The time-dependent properties need to be processed before query to allow matching based on derivatives and other derived properties.

\subsubsection{Use CASE 22: Statistical Relational ARtificial INTELligence for HEALTH CARE}

Submitted by Sriram Natarajan, Indiana University

\section{APPLICATION}

The goal of the project is to analyze large, multi-modal medical data, including different data types such as imaging, EHR, and genetic and natural language. This approach employs relational probabilistic models that have the capability of handling rich relational data and modeling uncertainty using probability theory. The software learns models from multiple data types, and can possibly integrate information and reason about complex queries. Users can provide a set of descriptions, for instance: magnetic resonance imaging (MRI) images and demographic data about a particular subject. They can then query for the onset of a particular disease (e.g., Alzheimer's), and the system will provide a probability distribution over the possible occurrence of this disease.

\section{CURRENT ApProACH}

A single server can handle a test cohort of a few hundred patients with associated data of hundreds of GBs. 


\section{FUTURE}

A cohort of millions of patients can involve PB size datasets. A major issue is the availability of too much data (e.g., images, genetic sequences), which can make the analysis complicated. Sometimes, large amounts of data about a single subject are available, but the number of subjects is not very high (i.e., data imbalance). This can result in learning algorithms picking up random correlations between the multiple data types as important features in analysis. Another challenge lies in aligning the data and merging from multiple sources in a form that will be useful for a combined analysis.

\subsubsection{Use Case 23: World Population-Scale Epidemiological Study}

Submitted by Madhav Marathe, Stephen Eubank, and Chris Barrett, Virginia Tech

\section{APPLICATION}

There is a need for reliable, real-time prediction and control of pandemics similar to the 2009 H1N1 influenza. Addressing various kinds of contagion diffusion may involve modeling and computing information, diseases, and social unrest. Agent-based models can utilize the underlying interaction network (i.e., a network defined by a model of people, vehicles, and their activities) to study the evolution of the desired phenomena.

\section{CURRENT APPROACH}

There is a two-step approach: (1) build a synthetic global population; and (2) run simulations over the global population to reason about outbreaks and various intervention strategies. The current $100 \mathrm{~TB}$ dataset was generated centrally with an MPI-based simulation system written in Charm++. Parallelism is achieved by exploiting the disease residence time period.

\section{FUTURE}

Large social contagion models can be used to study complex global-scale issues, greatly increasing the size of systems used.

\subsubsection{Use Case 24: Social Contagion Modeling for Planning, Public HEALTH, AND DISASTER MANAGEMENT}

Submitted by Madhav Marathe and Chris Kuhlman, Virginia Tech

\section{APPLICATION}

Social behavior models are applicable to national security, public health, viral marketing, city planning, and disaster preparedness. In a social unrest application, people take to the streets to voice either unhappiness with or support for government leadership. Models would help quantify the degree to which normal business and activities are disrupted because of fear and anger, the possibility of peaceful demonstrations and/or violent protests, and the potential for government responses ranging from appeasement, to allowing protests, to issuing threats against protestors, to taking actions to thwart protests. Addressing these issues would require fine-resolution models (at the level of individual people, vehicles, and buildings) and datasets.

\section{CURRENT APPROACH}

The social contagion model infrastructure simulates different types of human-to-human interactions (e.g., face-to-face versus online media), and also interactions between people, services (e.g., transportation), and infrastructure (e.g., Internet, electric power). These activity models are generated from averages such as census data. 


\section{FUTURE}

One significant concern is data fusion (i.e., how to combine data from different sources and how to deal with missing or incomplete data.) A valid modeling process must take into account heterogeneous features of hundreds of millions or billions of individuals, as well as cultural variations across countries. For such large and complex models, the validation process itself is also a challenge.

\subsubsection{USE CASE 25: BIODIVERSITY AND LIFEWATCH}

Submitted by Wouter Los and Yuri Demchenko, University of Amsterdam

\section{APPLICATION}

Research and monitor different ecosystems, biological species, their dynamics, and their migration with a mix of custom sensors and data access/processing, and a federation with relevant projects in the area. Particular case studies include monitoring alien species, migrating birds, and wetlands. One of many efforts from the consortium titled Common Operations for Environmental Research Infrastructures (ENVRI) is investigating integration of LifeWatch with other environmental e-infrastructures.

\section{CURRENT APPROACH}

At this time, this project is in the preliminary planning phases and, therefore, the current approach is not fully developed.

\section{FUTURE}

The LifeWatch initiative will provide integrated access to a variety of data, analytical, and modeling tools as served by a variety of collaborating initiatives. It will also offer data and tools in selected workflows for specific scientific communities. In addition, LifeWatch will provide opportunities to construct personalized "virtual labs," allowing participants to enter and access new data and analytical tools. New data will be shared with the data facilities cooperating with LifeWatch, including both the Global Biodiversity Information Facility and the Biodiversity Catalogue, also known as the Biodiversity Science Web Services Registry. Data include 'omics', species information, ecological information (e.g., biomass, population density), and ecosystem data (e.g., carbon dioxide $\left[\mathrm{CO}_{2}\right]$ fluxes, algal blooming, water and soil characteristics.)

\subsection{DEEP LEARNING AND SOCIAL MEDIA}

\subsubsection{USE CASE 26: LARGE-SCALE DEEP LEARNING}

Submitted by Adam Coates, Stanford University

\section{APPLICATION}

There is a need to increase the size of datasets and models that can be tackled with deep learning algorithms. Large models (e.g., neural networks with more neurons and connections) combined with large datasets are increasingly the top performers in benchmark tasks for vision, speech, and NLP. It will be necessary to train a deep neural network from a large (e.g., much greater than 1 TB) corpus of data, which is typically comprised of imagery, video, audio, or text. Such training procedures often require customization of the neural network architecture, learning criteria, and dataset preprocessing. In addition to the computational expense demanded by the learning algorithms, the need for rapid prototyping and ease of development is extremely high.

\section{CURRENT APPROACH}

The largest applications so far are to image recognition and scientific studies of unsupervised learning with 10 million images and up to 11 billion parameters on a 64 GPU HPC Infiniband cluster. Both supervised (i.e., using existing classified images) and unsupervised applications are being investigated. 


\section{FUTURE}

Large datasets of $100 \mathrm{~TB}$ or more may be necessary to exploit the representational power of the larger models. Training a self-driving car could take 100 million images at megapixel resolution. Deep learning shares many characteristics with the broader field of machine learning. The paramount requirements are high computational throughput for mostly dense linear algebra operations, and extremely high productivity for researcher exploration. High-performance libraries must be integrated with high-level (e.g., Python) prototyping environments.

\section{RESOURCES}

- $\quad$ Scientists See Promise in Deep-Learning Programs. http://www.nytimes.com/2012/11/24/science/scientists-see-advances-in-deep-learning-a-part-ofartificial-intelligence.html. Accessed March 3, 2015.

- How Many Computers to Identify a Cat? 16,000. http://www.nytimes.com/2012/06/26/technology/in-a-big-network-of-computers-evidence-ofmachine-learning.html. Accessed March 3, 2015.

- Now You Can Build Google’s \$1M Artificial Brain on the Cheap. http://www.wired.com/wiredenterprise/2013/06/andrew ng/. Accessed March 3, 2015.

- Coates, A., Huval, B., Wang, T., Wu, D. J., Ng, A., Catanzaro, B. "Deep learning with COTS HPC systems.” Proceedings of the $30^{\text {th }}$ International Conference on Machine Learning, Atlanta, Georgia, USA, 2013. JMLR: W\&CP Volume 28. http://www.cs.stanford.edu/ acoates/papers/CoatesHuvalWangWuNgCatanzaro_icml2013.pdf . Accessed March 3, 2015.

- Unsupervised Feature Learning and Deep Learning. http://ufldl.stanford.edu/wiki/index.php/Main_Page. Accessed March 3, 2015.

- Welcome to Deep Learning. http://deeplearning.net/. Accessed March 3, 2015.

\subsubsection{Use CASE 27: Organizing LARge-Scale, Unstructured Collections OF CONSUMER PHOTOS}

Submitted by David Crandall, Indiana University

\section{APPLICATION}

Collections of millions to billions of consumer images are used to produce 3D reconstructions of scenes-with no a priori knowledge of either the scene structure or the camera positions. The resulting 3D models allow efficient and effective browsing of large-scale photo collections by geographic position. New images can be geolocated by matching them to 3D models, and object recognition can be performed on each image. The 3D reconstruction can be posed as a robust, non-linear, least squares optimization problem: observed or noisy correspondences between images are constraints, and unknowns are sixdimensional (6D) camera poses of each image and 3D positions of each point in the scene.

\section{CURRENT APPROACH}

The current system is a Hadoop cluster with 480 cores processing data of initial applications. Over 500 billion images are currently on Facebook, and over 5 billion are on Flickr, with over 500 million images added to social media sites each day.

\section{FUTURE}

Necessary maintenance and upgrades require many analytics including feature extraction, feature matching, and large-scale probabilistic inference. These analytics appear in many or most computer vision and image processing problems, including recognition, stereo resolution, and image denoising. Other needs are visualizing large-scale, 3D reconstructions and navigating large-scale collections of images that have been aligned to maps. 


\section{RESOURCES}

- Discrete-continuous optimization for large-scale structure from motion. http://vision.soic.indiana.edu/disco. Accessed March 3, 2015.

\subsubsection{USE CASE 28: TRUTHY-INFORMATION DIFFUSION RESEARCH FROM TWITTER DATA}

Submitted by Filippo Menczer, Alessandro Flammini, and Emilio Ferrara, Indiana University

\section{APPLICATION}

How communication spreads on socio-technical networks must be better understood, and methods are needed to detect potentially harmful information spread at early stages (e.g., deceiving messages, orchestrated campaigns, untrustworthy information).

\section{CURRENT APPROACH}

Twitter generates a large volume of continuous streaming data-about 30 TB a year, compressedthrough circulation of $\approx 100$ million messages per day. The increase over time is roughly 500 GB data per day. All these data must be acquired and stored. Additional needs include near real-time analysis of such data for anomaly detection, stream clustering, signal classification, and online-learning; and data retrieval, Big Data visualization, data-interactive web interfaces, and public application programming interfaces (APIs) for data querying. Software packages for data analysis include Python/ SciPy/ NumPy/ MPI. Information diffusion, clustering, and dynamic network visualization capabilities already exist.

\section{FUTURE}

Truthy plans to expand, incorporating Google+ and Facebook, and so needs to move toward advanced distributed storage programs, such as Hadoop/Indexed HBase and Hadoop Distributed File System (HDFS). Redis should be used as an in-memory database to be a buffer for real-time analysis. Solutions will need to incorporate streaming clustering, anomaly detection, and online learning.

\section{RESOURCES}

- Truthy: Information diffusion research at Indiana University. http://truthy.indiana.edu/. Accessed March 3, 2015.

- Truthy: Information Diffusion in Online Social Networks. http://cnets.indiana.edu/groups/nan/truthy. Accessed March 3, 2015.

- Detecting Early Signature of Persuasion in Information Cascades (DESPIC). http://cnets.indiana.edu/groups/nan/despic. Accessed March 3, 2015.

\subsubsection{Use Case 29: CRowd Sourcing in the Humanities as Source for Big AND DYNAMIC DATA}

Submitted by Sebastian Drude, Max-Planck-Institute for Psycholinguistics, Nijmegen, the Netherlands

\section{APPLICATION}

Information is captured from many individuals and their devices using a range of sources: manually entered, recorded multimedia, reaction times, pictures, sensor information. These data are used to characterize wide-ranging individual, social, cultural, and linguistic variations among several dimensions (e.g., space, social space, time).

\section{CURRENT APPROACH}

At this point, typical systems used are Extensible Markup Language (XML) technology and traditional relational databases. Other than pictures, not much multi-media is employed yet. 


\section{FUTURE}

Crowd sourcing is beginning to be used on a larger scale. However, the availability of sensors in mobile devices provides a huge potential for collecting large amount of data from numerous individuals. This possibility has not been explored on a large scale so far; existing crowd sourcing projects are usually of a limited scale and web-based. Privacy issues may be involved because of access to individuals' audiovisual files; anonymization may be necessary but not always possible. Data management and curation are critical. With multimedia, the size could be hundreds of terabytes.

\subsubsection{USE CASE 30: CINET_CYBERINFRASTRUCTURE FOR NETWORK (GRAPH) SCIENCE AND ANALYTICS}

Submitted by Madhav Marathe and Keith Bisset, Virginia Tech

\section{APPLICATION}

CINET provides a common web-based platform that allows the end user seamless access to the following:

- Network and graph analysis tools such as SNAP, NetworkX, and Galib;

- Real-world and synthetic networks;

- Computing resources; and

- Data management systems.

\section{CURRENT APPROACH}

CINET uses an Infiniband-connected HPC cluster with 720 cores to provide HPC as a service. The platform is being used for research and education. CINET is used in classes and to support research by social science and social networking communities

\section{FUTURE}

Rapid repository growth is expected to lead to at least 1,000 to 5,000 networks and methods in about a year. As more fields use graphs of increasing size, parallel algorithms will be important. Two critical challenges are data manipulation and bookkeeping of the derived data, as there are no well-defined and effective models and tools for unified management of various graph data.

\section{RESOURCES}

- Computational Network Sciences (CINET) GRANITE system. http://cinet.vbi.vt.edu/ . Accessed March 3, 2015.

\subsubsection{USE CASE 31: NIST INFORMATION ACCESS DIVISION-ANALYTIC TeChNology Performance Measurements, EVAluations, and STANDARDS}

Submitted by John Garofolo, NIST

\section{APPLICATION}

Performance metrics, measurement methods, and community evaluations are needed to ground and accelerate development of advanced analytic technologies in the areas of speech and language processing, video and multimedia processing, biometric image processing, and heterogeneous data processing, as well as the interaction of analytics with users. Typically, one of two processing models are employed: (1) push test data out to test participants, and analyze the output of participant systems, and (2) push algorithm test harness interfaces out to participants, bring in their algorithms, and test them on internal computing clusters. 


\section{CURRENT APPROACH}

There is a large annotated corpora of unstructured/semi-structured text, audio, video, images, multimedia, and heterogeneous collections of the above, including ground truth annotations for training, developmental testing, and summative evaluations. The test corpora exceed 900 million web pages occupying 30 TB of storage, 100 million tweets, 100 million ground-truthed biometric images, several hundred thousand partially ground-truthed video clips, and terabytes of smaller fully ground-truthed test collections.

\section{FUTURE}

Even larger data collections are being planned for future evaluations of analytics involving multiple data streams and very heterogeneous data. In addition to larger datasets, the future includes testing of streaming algorithms with multiple heterogeneous data. The use of clouds is being explored.

\section{RESOURCES}

- Information Access Division. http://www.nist.gov/itl/iad/. Accessed March 3, 2015.

\subsubsection{USE CASE 2-3: URBAN CONTEXT-AWARE EVENT MANAGEMENT FOR SMART CITIES - PUBLIC SAFETY}

Submitted by Olivera Kotevska, Gilad Kusne, Daniel Samarov, and Ahmed Lbath.

\section{APPLI CATION}

The real-world events are now being observed by multiple networked streams, where each is complementing the other with its characteristics, features, and perspectives. Many of these networked data streams are becoming digitalized, with some available to the public (open data initiative) and available for sense making.

The networked data streams provide an opportunity for their link identification, similarity, and time dynamics can aid in recognizing the evolving patterns in the inter-intra-city/community. The delivered information can help improve the understanding of how cities/communities work. The information can also be used to detect events and patterns that can facilitate a broad range of issues affecting the everyday lives of citizens and efficiency of cities. Providing the tools that can make this process easy and accessible to the city/community representatives will potentially impact traffic, event management, disaster management systems, health monitoring systems, air quality, and city/community planning.

\section{CURRENT APPROACH}

The current approach uses fixed and deployed computing clusters ranging from 10's to 100's of nodes. These employ NLP (Natural Language Processing) and custom applications in a variety of languages (e.g., R, Python, Java) using technologies such as Spark and Kafka. Visualization tools are important.

\section{FUTURE}

This type of analysis is just starting and the present vision given above is the expected future.

\subsection{THE ECOSYSTEM FOR RESEARCH}

\subsubsection{USE CASE 32: DATANET FEDERATION CONSORTIUM}

Submitted by Reagan Moore, University of North Carolina at Chapel Hill

\section{APPLICATION}

The DataNet Federation Consortium (DFC) promotes collaborative and interdisciplinary research through a federation of data management systems across federal repositories, national academic research 
initiatives, institutional repositories, and international collaborations. The collaboration environment runs at scale and includes petabytes of data, hundreds of millions of files, hundreds of millions of metadata attributes, tens of thousands of users, and a thousand storage resources.

\section{CURRENT APPROACH}

Currently, 25 science and engineering domains have projects that rely on the iRODS (Integrated RuleOriented Data System) policy-based data management system. Active organizations include the National Science Foundation, with major projects such as the Ocean Observatories Initiative (sensor archiving); Temporal Dynamics of Learning Center (cognitive science data grid); iPlant Collaborative (plant genomics); Drexel's engineering digital library; and H. W. Odum Institute for Research in Social Science (data grid federation with Dataverse). iRODS currently manages PB of data, hundreds of millions of files, hundreds of millions of metadata attributes, tens of thousands of users, and a thousand storage resources. It interoperates with workflow systems (e.g., National Center for Computing Applications' [NCSA's] Cyberintegrator, Kepler, Taverna), cloud, and more traditional storage models, as well as different transport protocols. Figure 4 presents a diagram of the iRODS architecture.

\section{FUTURE}

Future data scenarios and applications were not expressed for this use case.

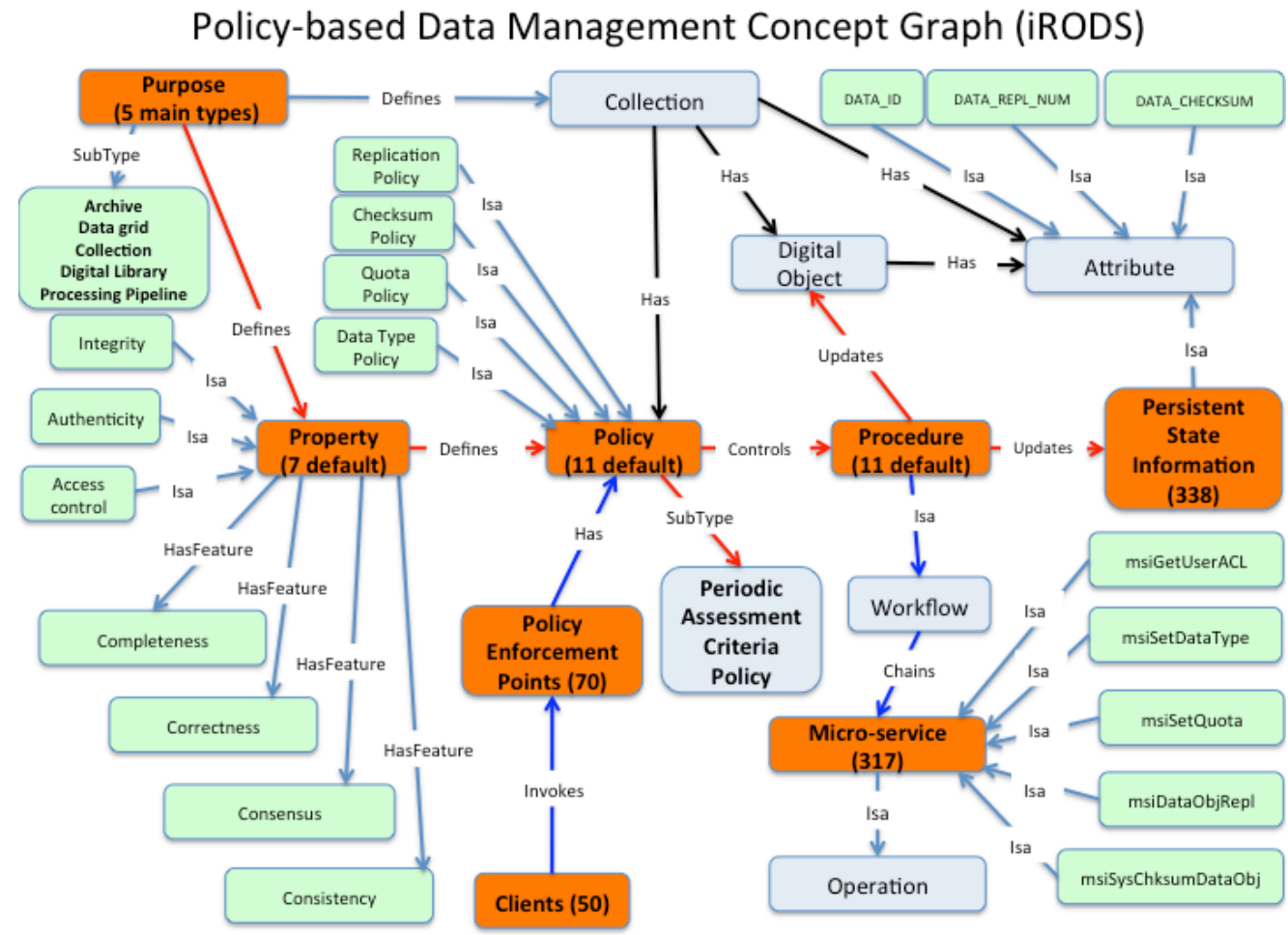

Figure 5: DFC—iRODS Architecture

\section{RESOURCES}

- DataNet Federation Consortium. http://renci.org/research/datanet-federation-consortium/. Accessed March 3, 2015.

\subsubsection{Use Case 33: The Discinnet Process}

Submitted by P. Journeau, Discinnet Labs 


\section{APPLICATION}

Discinnet has developed a Web 2.0 collaborative platform and research prototype as a pilot installation, which is now being deployed and tested by researchers from a growing number of diverse research fields. The goal is to reach a wide enough sample of active research fields, represented as clusters (i.e., researchers projected and aggregating within a manifold of mostly shared experimental dimensions) to test general, hence potentially interdisciplinary, epistemological models throughout the present decade.

\section{CURRENT APPROACH}

Currently, 35 clusters have been started, with close to 100 awaiting more resources. There is potential for many more to be created, administered, and animated by research communities. Examples of clusters include optics, cosmology, materials, microalgae, health care, applied math, computation, rubber, and other chemical products/issues.

\section{FUTURE}

Discinnet itself would not be Big Data but rather will generate metadata when applied to a cluster that involves Big Data. In interdisciplinary integration of several fields, the process would reconcile metadata from many complexity levels.

\section{RESOURCES}

- DiscInNet: Interdisciplinary Networking. http://www.discinnet.org. Accessed March 3, 2015.

\subsubsection{Use Case 34: Semantic Graph Search on Scientific Chemical and TEXT-BASED DATA}

Submitted by Talapady Bhat, NIST

\section{APPLICATION}

Social media-based infrastructure, terminology and semantic data-graphs are established to annotate and present technology information. The process uses root- and rule-based methods currently associated primarily with certain Indo-European languages, such as Sanskrit and Latin.

\section{CURRENT APPROACH}

Many reports, including a recent one on the Material Genome Project, find that exclusive top-down solutions to facilitate data sharing and integration are not desirable for multi-disciplinary efforts. However, a bottom-up approach can be chaotic. For this reason, there is need for a balanced blend of the two approaches to support easy-to-use techniques to metadata creation, integration, and sharing. This challenge is very similar to the challenge faced by language developers, so a recently developed method is based on these ideas. There are ongoing efforts to extend this method to publications of interest to the Material Genome Initiative [10], the Open Government movement [11], and the NIST Integrated Knowledge Editorial Net (NIKE) [12], a NIST-wide publication archive. These efforts are a component of the Research Data Alliance Metadata Standards Directory Working Group [13].

\section{FUTURE}

A cloud infrastructure should be created for social media of scientific information. Scientists from across the world could use this infrastructure to participate and deposit results of their experiments. Prior to establishing a scientific social medium, some issues must be resolved including the following:

- Minimize challenges related to establishing re-usable, interdisciplinary, scalable, on-demand, usecase, and user-friendly vocabulary.

- Adopt an existing or create new on-demand 'data-graph' to place information in an intuitive way, such that it would easily integrate with existing data-graphs in a federated environment, independently of details of data management. 
- Find relevant scientific data without spending too much time on the Internet.

Start with resources such as the Open Government movement, Material Genome Initiative, and Protein Databank. This effort includes many local and networked resources. Developing an infrastructure to automatically integrate information from all these resources using data-graphs is a challenge, but steps are being taken to solve it. Strong database tools and servers for data-graph manipulation are needed.

\section{RESOURCES}

- Facebook for molecules. http://www.eurekalert.org/pub_releases/2013-07/aiop-ffm071813.php. Accessed March 3, 2015.

- Chem-BLAST. http://xpdb.nist.gov/chemblast/pdb.pl. Accessed March 3, 2015.

\subsubsection{USE CASE 35: LIGHT SOURCE BeAMLINES}

Submitted by Eli Dart, LBNL

\section{APPLICATION}

Samples are exposed to X-rays from light sources in a variety of configurations, depending on the experiment. Detectors, essentially high-speed digital cameras, collect the data. The data are then analyzed to reconstruct a view of the sample or process being studied.

\section{CURRENT APPROACH}

A variety of commercial and open source software is used for data analysis. For example, Octopus is used for tomographic reconstruction, and Avizo (http://vsg3d.com) and FIJI (a distribution of ImageJ) are used for visualization and analysis. Data transfer is accomplished using physical transport of portable media, which severely limits performance, high-performance GridFTP, managed by Globus Online, or workflow systems such as SPADE (Support for Provenance Auditing in Distributed Environments, an open source software infrastructure).

\section{FUTURE}

Camera resolution is continually increasing. Data transfer to large-scale computing facilities is becoming necessary because of the computational power required to conduct the analysis on timescales useful to the experiment. Because of the large number of beamlines (e.g., 39 at the LBNL Advanced Light Source), aggregate data load is likely to increase significantly over coming years, as will the need for a generalized infrastructure for analyzing GB per second of data from many beamline detectors at multiple facilities.

\section{RESOURCES}

- Advanced Light Source. http://www-als.lbl.gov/. Accessed March 3, 2015.

- Advanced Photon Source. http://www.aps.anl.gov/. Accessed March 3, 2015.

\subsection{ASTRONOMY AND PHYSICS}

\subsubsection{Use Case 36: Catalina Real-Time Transient SuRVey: A Digital, PANORAMIC, SYNOPTIC SKY SURVEY}

Submitted by S. G. Djorgovski, Caltech

\section{APPLICATION}

Catalina Real-Time Transient Survey (CRTS) explores the variable universe in the visible light regime, on timescales ranging from minutes to years, by searching for variable and transient sources. It discovers a broad variety of astrophysical objects and phenomena, including various types of cosmic explosions (e.g., supernovae), variable stars, phenomena associated with accretion to massive black holes (e.g., 
active galactic nuclei) and their relativistic jets, and high proper motion stars. The data are collected from three telescopes (two in Arizona and one in Australia), with additional ones expected in the near future in Chile.

\section{CURRENT APPROACH}

The survey generates up to approximately $0.1 \mathrm{~TB}$ on a clear night with a total of approximately $100 \mathrm{~TB}$ in current data holdings. The data are preprocessed at the telescope and then transferred to the University of Arizona and Caltech for further analysis, distribution, and archiving. The data are processed in real time, and detected transient events are published electronically through a variety of dissemination mechanisms, with no proprietary withholding period (CRTS has a completely open data policy). Further data analysis includes classification of the detected transient events, additional observations using other telescopes, scientific interpretation, and publishing. This process makes heavy use of the archival data (several PBs) from a wide variety of geographically distributed resources connected through the virtual observatory (VO) framework.

\section{FUTURE}

CRTS is a scientific and methodological test bed and precursor of larger surveys to come, notably the Large Synoptic Survey Telescope (LSST), expected to operate in the 2020s and selected as the highestpriority ground-based instrument in the 2010 Astronomy and Astrophysics Decadal Survey. LSST will gather about 30 TB per night. Figure 6 illustrates the schematic architecture for a cyber infrastructure for time domain astronomy.

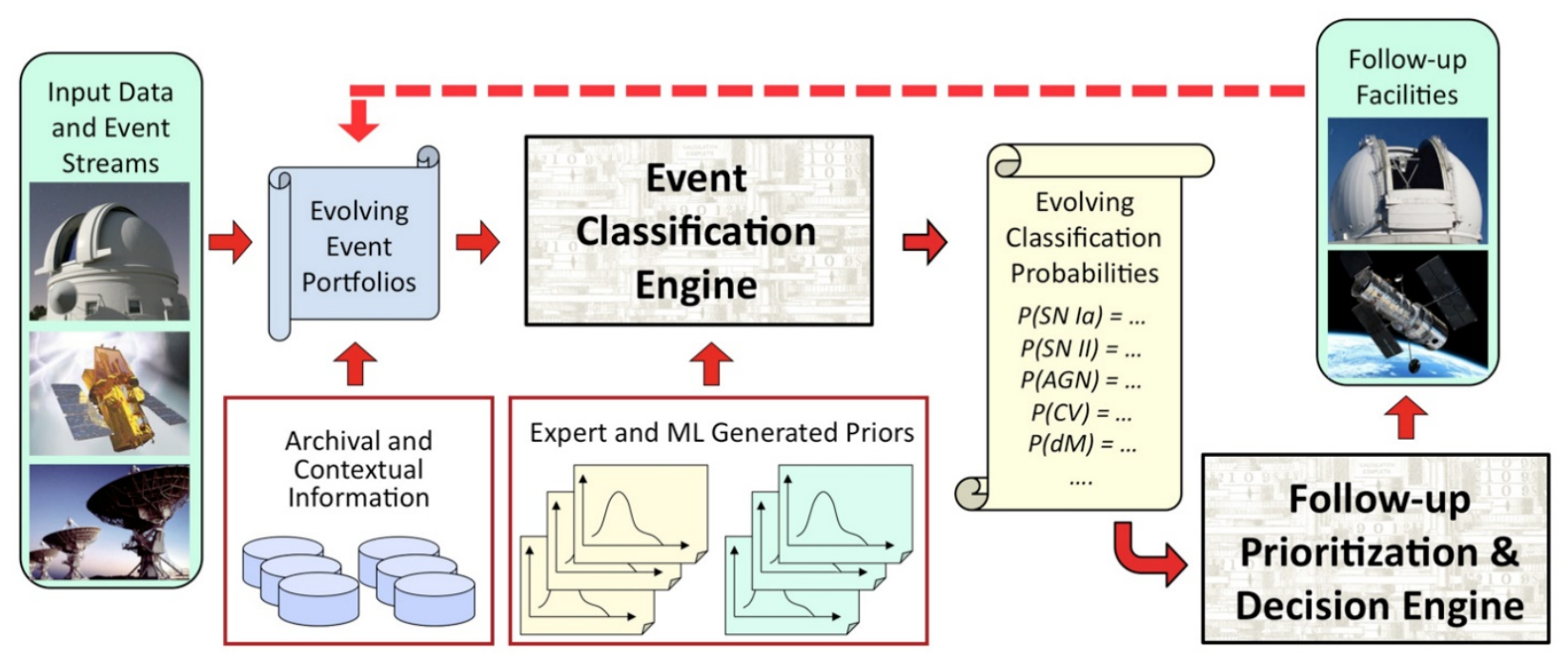

Figure 6: Catalina CRTS: A Digital, Panoramic, Synoptic Sky Survey

Survey pipelines from telescopes (on the ground or in space) produce transient event data streams, and the events, along with their observational descriptions, are ingested by one or more depositories, from which the event data can be disseminated electronically to human astronomers or robotic telescopes. Each event is assigned an evolving portfolio of information, which includes all available data on that celestial position. The data are gathered from a wide variety of data archives unified under the Virtual Observatory framework, expert annotations, etc. Representations of such federated information can be both humanreadable and machine-readable. The data are fed into one or more automated event characterization, classification, and prioritization engines that deploy a variety of machine learning tools for these tasks. The engines' output, which evolves dynamically as new information arrives and is processed, informs the follow-up observations of the selected events, and the resulting data are communicated back to the event portfolios for the next iteration. Users, either human or robotic, can tap into the system at multiple points, 
both for information retrieval and to contribute new information, through a standardized set of formats and protocols. This could be done in (near) real-time or in archival (i.e., not time-critical) modes.

\section{RESOURCES}

- Flashes in a Star Stream: Automated Classification of Astronomical Transient Events. http://arxiv.org/abs/1209.1681. Accessed March 3, 2015.

\subsubsection{USe CASE 37: DOE ExtReme Data fRom Cosmological SKy SuRVEy AND SimULATIONS}

Submitted by Salman Habib, Argonne National Laboratory; Andrew Connolly, University of Washington

\section{APPLICATION}

A cosmology discovery tool integrates simulations and observation to clarify the nature of dark matter, dark energy, and inflation-some of the most exciting, perplexing, and challenging questions facing modern physics, including the properties of fundamental particles affecting the early universe. The simulations will generate data sizes comparable to observation.

\section{CURRENT APPROACH}

At this time, this project is in the preliminary planning phases and, therefore, the current approach is not fully developed.

\section{FUTURE}

These systems will use huge amounts of supercomputer time- over 200 million hours. Associated data sizes are as follows:

- Dark Energy Survey (DES): 4 PB per year in 2015

- Zwicky Transient Factory (ZTF): 1 PB per year in 2015

- LSST (see CRTS discussion above): 7 PB per year in 2019

- Simulations: 10 PB per year in 2017

\section{RESOURCES}

- The New Sky. http://www.lsst.org/lsst/. Accessed March 3, 2015.

- National Energy Research Scientific Computing Center. http://www.nersc.gov/. Accessed March 3, 2015.

- Basic Research: Non-Accelerator Physics. http://science.energy.gov/hep/research/basicresearch/non-accelerator-physics/. Accessed March 3, 2015.

- Present and Future Computing Requirements for Computational Cosmology. http://www.nersc.gov/assets/Uploads/HabibcosmosimV2.pdf. Accessed March 3, 2015.

\subsubsection{Use CASE 38: LARge SuRVey Data for Cosmology}

Submitted by Peter Nugent, LBNL

\section{APPLICATION}

For DES, the data are sent from the mountaintop, via a microwave link, to La Serena, Chile. From there, an optical link forwards them to the NCSA and to NERSC for storage and 'reduction.' Here, galaxies and stars in both the individual and stacked images are identified and catalogued, and finally their properties are measured and stored in a database. 


\section{CURRENT APPROACH}

Subtraction pipelines are run using extant imaging data to find new optical transients through machine learning algorithms. Data technologies are Linux cluster, Oracle RDBMS server, Postgres PSQL, large memory machines, standard Linux interactive hosts, and the General Parallel File System (GPFS). HPC resources are needed for simulations. Software needs include standard astrophysics reduction software as well as Perl/Python wrapper scripts and Linux Cluster scheduling.

\section{FUTURE}

Techniques are needed for handling Cholesky decomposition for thousands of simulations with matrices of order one million on a side and parallel image storage. LSST will generate $60 \mathrm{~PB}$ of imaging data and 15 PB of catalog data and a correspondingly large (or larger) amount of simulation data. In total, over 20 TB of data will be generated per night.

\section{RESOURCES}

- $\quad$ Dark Energy Spectroscopic Instrument (DESI). http://desi.lbl.gov. Accessed March 3, 2015.

- Why is the universe speeding up? http://www.darkenergysurvey.org. Accessed March 3, 2015.

\subsubsection{Use Case 39: Particle Physics-Analysis of LaRge Hadron Collider DATA: Discovery of Higgs PARTICLE}

Submitted by Michael Ernst, Brookhaven National Laboratory (BNL); Lothar Bauerdick, Fermi National Accelerator Laboratory (FNAL); Geoffrey Fox, Indiana University; Eli Dart, LBNL

\section{APPLICATION}

Analysis is conducted on collisions at the European Organization for Nuclear Research (CERN) Large Hadron Collider (LHC) accelerator (Figure 7) and Monte Carlo producing events describing particleapparatus interaction.

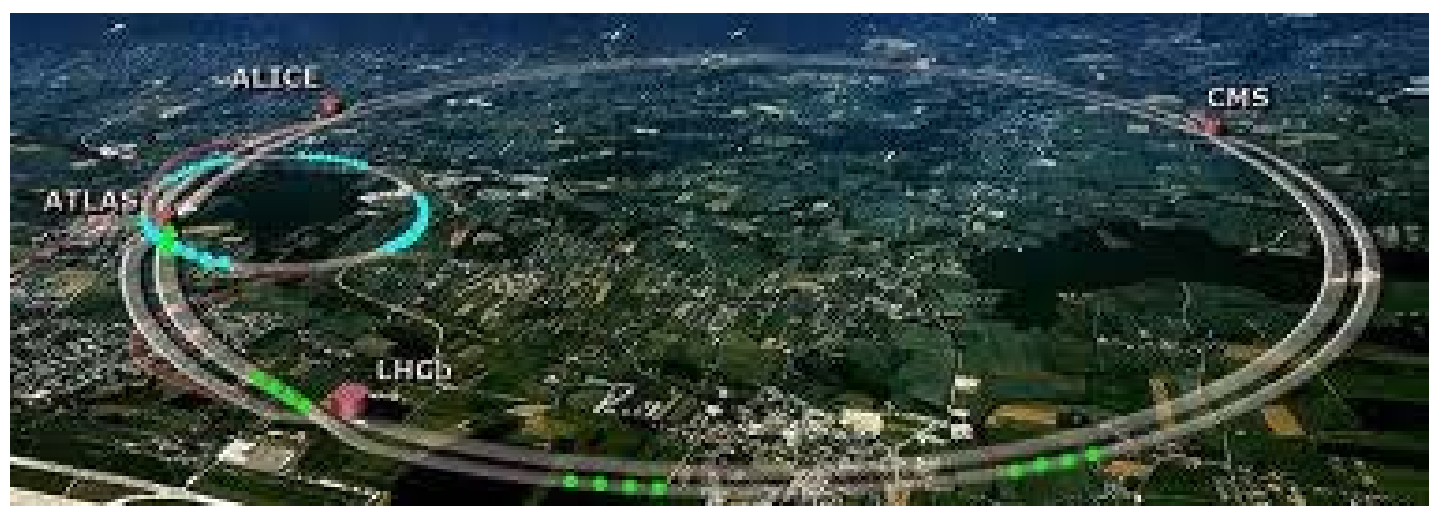

Figure 7: Particle Physics: Analysis of LHC Data: Discovery of Higgs Particle-CERN LHC Location

Processed information defines physics properties of events and generates lists of particles with type and momenta. These events are analyzed to find new effects—both new particles (e.g., Higgs), and present evidence that conjectured particles (e.g., Supersymmetry) have not been detected. A few major experiments are being conducted at LHC, including ATLAS and CMS (Compact Muon Solenoid). These experiments have global participants (e.g., CMS has 3,600 participants from 183 institutions in 38 countries), and so the data at all levels are transported and accessed across continents.

\section{CURRENT APPROACH}

The LHC experiments are pioneers of a distributed Big Data science infrastructure. Several aspects of the LHC experiments' workflow highlight issues that other disciplines will need to solve. These issues 
include automation of data distribution, high-performance data transfer, and large-scale high-throughput computing. Figure 8 shows grid analysis with 350,000 cores running near-continuously-over two million jobs per day arranged in three major tiers: CERN, Continents/Countries, and Universities. The analysis uses distributed, high-throughput computing (i.e., pleasing parallel) architecture with facilities integrated across the world by the Worldwide LHC Computing Grid (WLCG) and Open Science Grid in the U.S. Accelerator data and analysis generates 15 PB of data each year for a total of $200 \mathrm{~PB}$.

Specifically, in 2012, ATLAS had 8 PB on Tier1 tape and over 10 PB on Tier 1 disk at BNL and 12 PB on disk cache at U.S. Tier 2 centers. CMS has similar data sizes. Over half the resources are used for Monte Carlo simulations as opposed to data analysis.

\section{LHC Data Grid Hierarchy:}

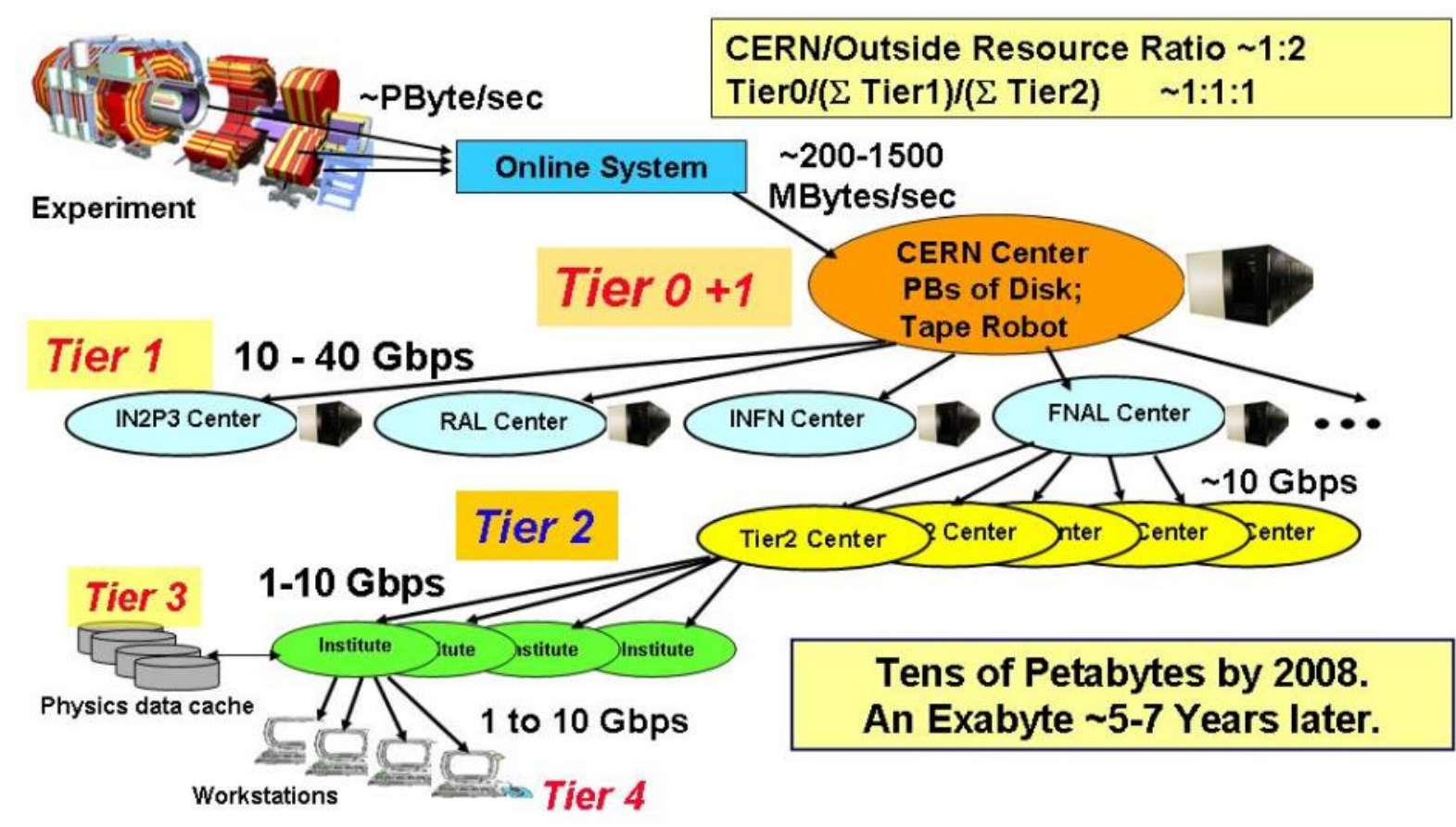

\section{Emerging Vision: A Richly Structured, Global Dynamic System}

Figure 8: Particle Physics: Analysis of LHC Data: Discovery of Higgs Particle-The Multi-tier LHC Computing Infrastructure

\section{FUTURE}

In the past, the particle physics community has been able to rely on industry to deliver exponential increases in performance per unit cost over time, as described by Moore's Law. However, the available performance will be much more difficult to exploit in the future since technology limitations, in particular regarding power consumption, have led to profound changes in the architecture of modern central processing unit (CPU) chips. In the past, software could run unchanged on successive processor generations and achieve performance gains that follow Moore's Law, thanks to the regular increase in clock rate that continued until 2006. The era of scaling sequential applications on an HEP (heterogeneous element processor) is now over. Changes in CPU architectures imply significantly more software parallelism, as well as exploitation of specialized floating-point capabilities. The structure and performance of HEP data processing software need to be changed such that they can continue to be adapted and developed to run efficiently on new hardware. This represents a major paradigm shift in HEP software design and implies large-scale re-engineering of data structures and algorithms. Parallelism 
needs to be added simultaneously at all levels: the event level, the algorithm level, and the sub-algorithm level. Components at all levels in the software stack need to interoperate, and therefore the goal is to standardize as much as possible on basic design patterns and on the choice of a concurrency model. This will also help to ensure efficient and balanced use of resources.

\section{RESOURCES}

- Where does all the data come from?

http://grids.ucs.indiana.edu/ptliupages/publications/Where\%20does\%20all\%20the\%20data\%20co me\%20from\%20v7.pdf. Accessed March 3, 2015.

- Enabling high throughput in widely distributed data management and analysis systems: Lessons from the LHC. http://www.es.net/assets/pubs_presos/High-throughput-lessons-from-the-LHCexperience.Johnston.TNC2013.pdf. Accessed March 3, 2015.

\subsubsection{USe CASE 40: Belle II High ENERgy Physics EXPERIMENT}

Submitted by David Asner and Malachi Schram, Pacific Northwest National Laboratory (PNNL)

\section{APPLICATION}

The Belle experiment is a particle physics experiment with more than 400 physicists and engineers investigating charge parity (CP) violation effects with B meson production at the High Energy Accelerator KEKB e+ e- accelerator in Tsukuba, Japan. In particular, numerous decay modes at the Upsilon(4S) resonance are sought to identify new phenomena beyond the standard model of particle physics. This accelerator has the largest intensity of any in the world, but the events are simpler than those from LHC, and so analysis is less complicated, but similar in style to the CERN accelerator analysis.

\section{CURRENT APPROACH}

At this time, this project is in the preliminary planning phases and, therefore, the current approach is not fully developed.

\section{FUTURE}

An upgraded experiment Belle II and accelerator SuperKEKB will start operation in 2015. Data will increase by a factor of 50 , with total integrated raw data of $\approx 120 \mathrm{~PB}$ and physics data of $\approx 15 \mathrm{~PB}$ and $\approx 100$ PB of Monte Carlo samples. The next stage will necessitate a move to a distributed computing model requiring continuous raw data transfer of $\approx 20$ GB per second at designed luminosity between Japan and the United States. Open Science Grid, Geant4, DIRAC, FTS, and Belle II framework software will be needed.

\section{RESOURCES}

- Belle II Collaboration. http://belle2.kek.jp. Accessed March 3, 2015.

\subsection{EARTH, ENVIRONMENTAL, AND POLAR SCIENCE}

\subsubsection{USE CASE 41: EUROPEAN INCOHERENT SCATTER SCIENTIFIC ASSOCIATION 3D INCOHERENT SCATTER RADAR SYSTEM}

Submitted by Yin Chen, Cardiff University; Ingemar Häggström, Ingrid Mann, and Craig Heinselman, European Incoherent Scatter Scientific Association (EISCAT)

\section{APPLICATION}

EISCAT conducts research on the lower, middle, and upper atmosphere and ionosphere using the incoherent scatter radar technique. This technique is the most powerful ground-based tool for these research applications. EISCAT studies instabilities in the ionosphere and investigates the structure and 
dynamics of the middle atmosphere. EISCAT operates a diagnostic instrument in ionospheric modification experiments with addition of a separate heating facility. Currently, EISCAT operates three of the ten major incoherent radar scattering instruments worldwide; their three systems are located in the Scandinavian sector, north of the Arctic Circle.

\section{CURRENT APPROACH}

The currently running EISCAT radar generates data at rates of terabytes per year. The system does not present special challenges.

\section{FUTURE}

The design of the next-generation radar, EISCAT_3D, will consist of a core site with transmitting and receiving radar arrays and four sites with receiving antenna arrays at some 100 kilometers from the core. The fully operational five-site system will generate several thousand times the number of data of the current EISCAT system, with 40 PB per year in 2022, and is expected to operate for 30 years. EISCAT_3D data e-Infrastructure plans to use high-performance computers for central site data processing and high-throughput computers for mirror site data processing. Downloading the full data is not time-critical, but operations require real-time information about certain pre-defined events, which would be sent from the sites to the operations center, and a real-time link from the operations center to the sites to set the mode of radar operation in real time. See Figure 9.

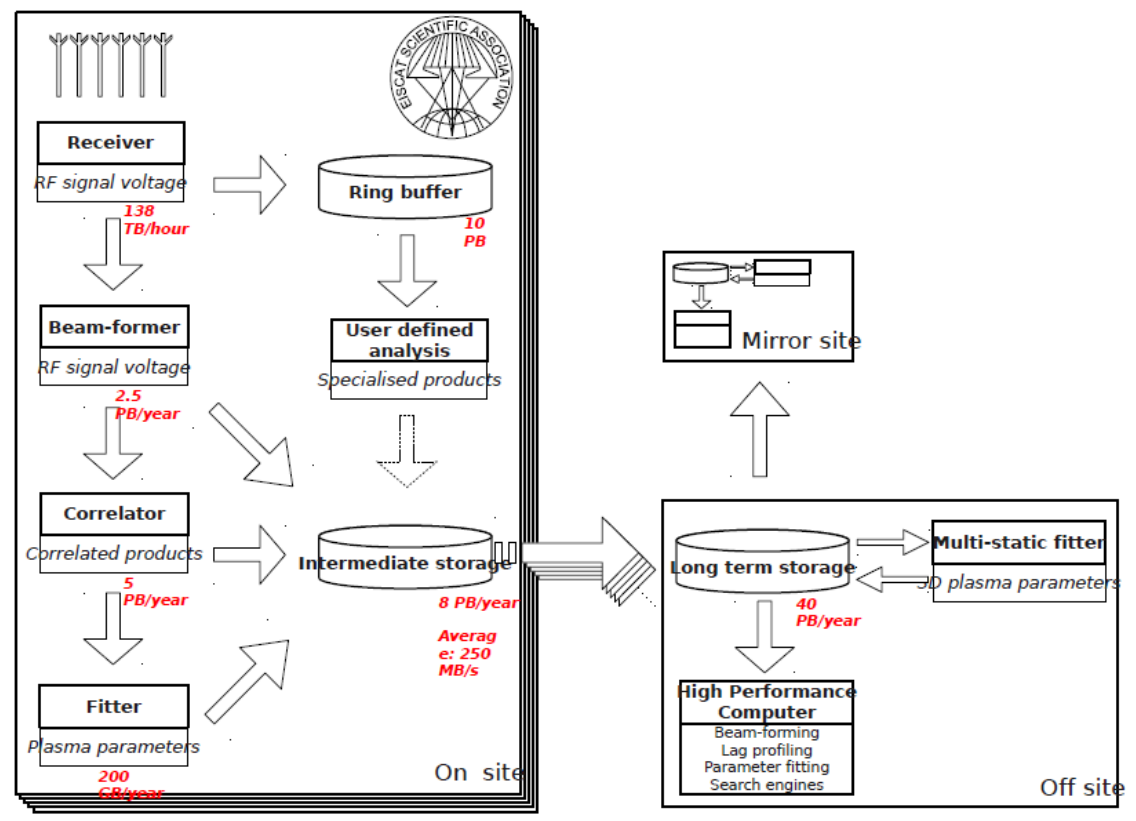

Figure 9: EISCAT 3D Incoherent Scatter Radar System - System Architecture

\section{RESOURCES}

- $\quad$ EISCAT 3D. https://www.eiscat3d.se/. Accessed March 3, 2015.

\subsubsection{Use CASE 42: Common Operations of EnVIRONMENTAL ReSEARCH INFRASTRUCTURE}

Submitted by Yin Chen, Cardiff University

\section{APPLICATION}

ENVRI (Common Operations of Environmental Research Infrastructures) addresses European distributed, long-term, remote-controlled observational networks focused on understanding processes, trends, 
thresholds, interactions, and feedbacks, as well as increasing the predictive power to address future environmental challenges. The following efforts are part of ENVRI:

- ICOS (Integrated Carbon Observation System) is a European distributed infrastructure dedicated to the monitoring of greenhouse gases (GHGs) through its atmospheric, ecosystem, and ocean networks.

- EURO-Argo is the European contribution to Argo, which is a global ocean observing system.

- EISCAT_3D (described separately) is a European new-generation incoherent scatter research radar system for upper atmospheric science.

- LifeWatch (described separately) is an e-science infrastructure for biodiversity and ecosystem research.

- EPOS (European Plate Observing System) is a European research infrastructure for earthquakes, volcanoes, surface dynamics, and tectonics.

- EMSO (European Multidisciplinary Seafloor and Water Column Observatory) is a European network of seafloor observatories for the long-term monitoring of environmental processes related to ecosystems, climate change, and geo-hazards.

- IAGOS (In-service Aircraft for a Global Observing System) is setting up a network of aircraft for global atmospheric observation.

- SIOS (Svalbard Integrated Arctic Earth Observing System) is establishing an observation system in and around Svalbard that integrates the studies of geophysical, chemical, and biological processes from all research and monitoring platforms.

\section{CURRENT APPROACH}

ENVRI develops a reference model (ENVRI RM) as a common ontological framework and standard for the description and characterization of computational and storage infrastructures. The goal is to achieve seamless interoperability between the heterogeneous resources of different infrastructures. The ENVRI RM serves as a common language for community communication, providing a uniform framework into which the infrastructure's components can be classified and compared. The ENVRI RM also serves to identify common solutions to common problems. Data sizes in a given infrastructure vary from GBs to petabytes per year.

\section{FUTURE}

ENVRI's common environment will empower the users of the collaborating environmental research infrastructures and enable multidisciplinary scientists to access, study, and correlate data from multiple domains for system-level research. Collaboration affects Big Data requirements coming from interdisciplinary research.

ENVRI analyzed the computational characteristics of the six European Strategy Forum on Research Infrastructures (ESFRI) environmental research infrastructures, and identified five common subsystems (Figure 10). They are defined in the ENVRI RM (http://www.envri.eu/rm) and below:

- Data acquisition: Collects raw data from sensor arrays, various instruments, or human observers, and brings the measurements (data streams) into the system.

- Data curation: Facilitates quality control and preservation of scientific data and is typically operated at a data center.

- Data access: Enables discovery and retrieval of data housed in data resources managed by a data curation subsystem.

- Data processing: Aggregates data from various resources and provides computational capabilities and capacities for conducting data analysis and scientific experiments.

- Community support: Manages, controls, and tracks users' activities and supports users in conduct of their community roles. 


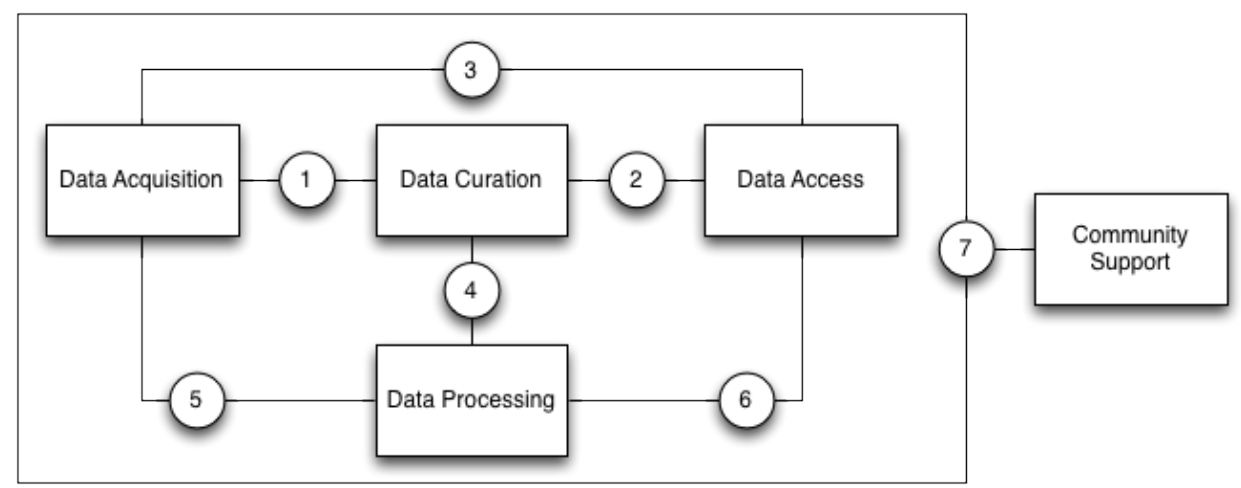

Figure 10: ENVRI Common Architecture

Figures 11(a) through 11(e) illustrate how well the five subsystems map to the architectures of the ESFRI environmental research infrastructures.

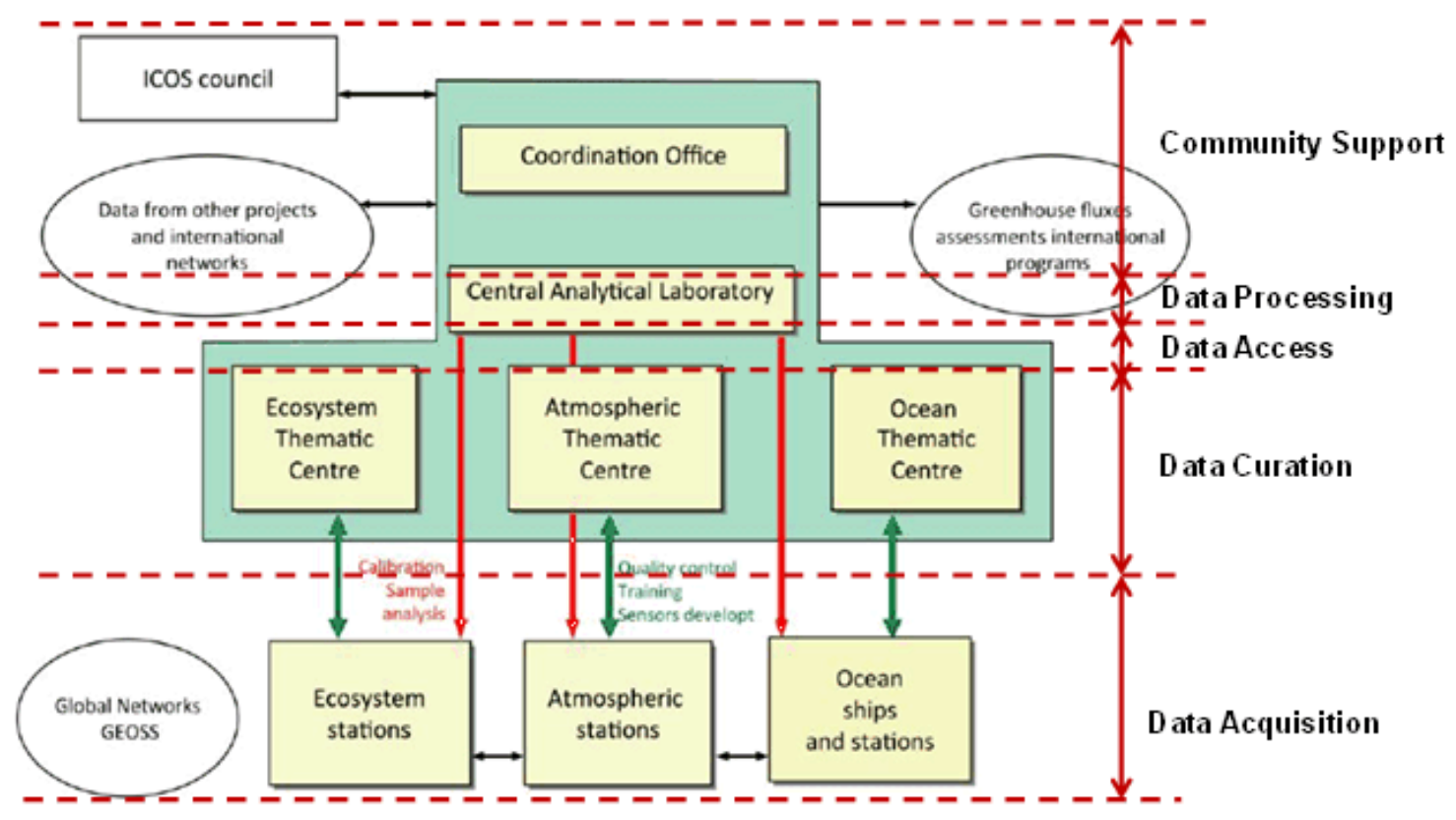

Figure 11(a): ICOS Architecture 


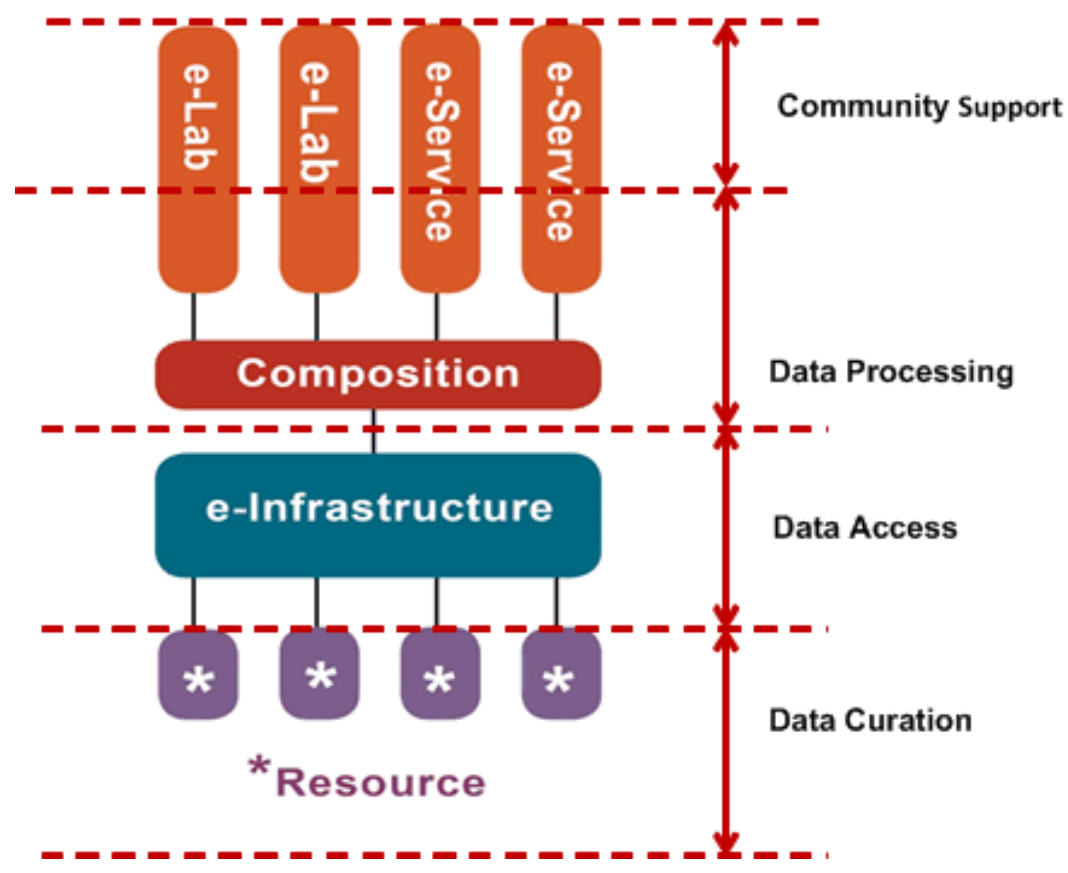

Figure 11(b): LifeWatch Architecture

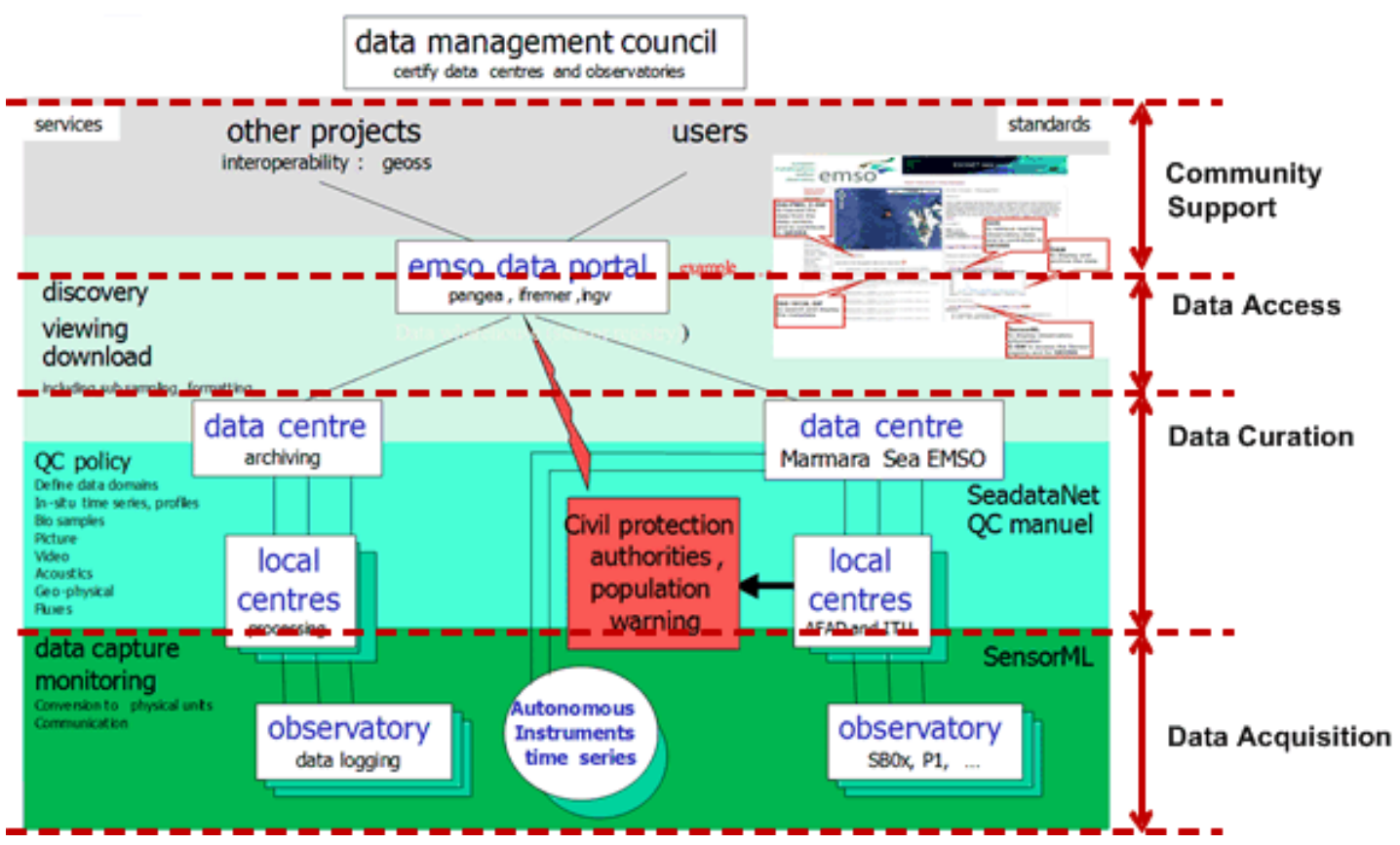

Figure 11(c): EMSO Architecture 


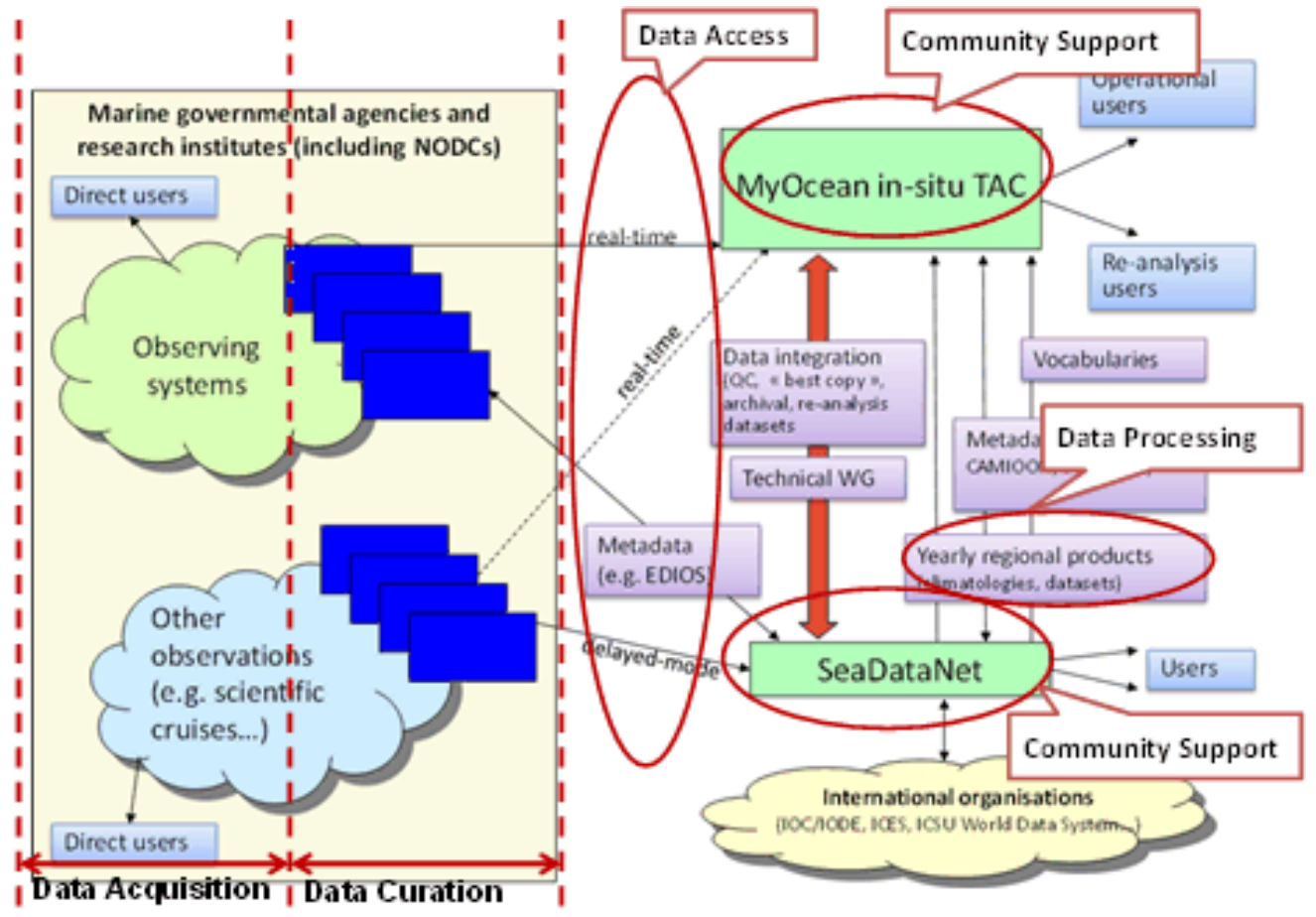

Figure 11(d): EURO-Argo Architecture

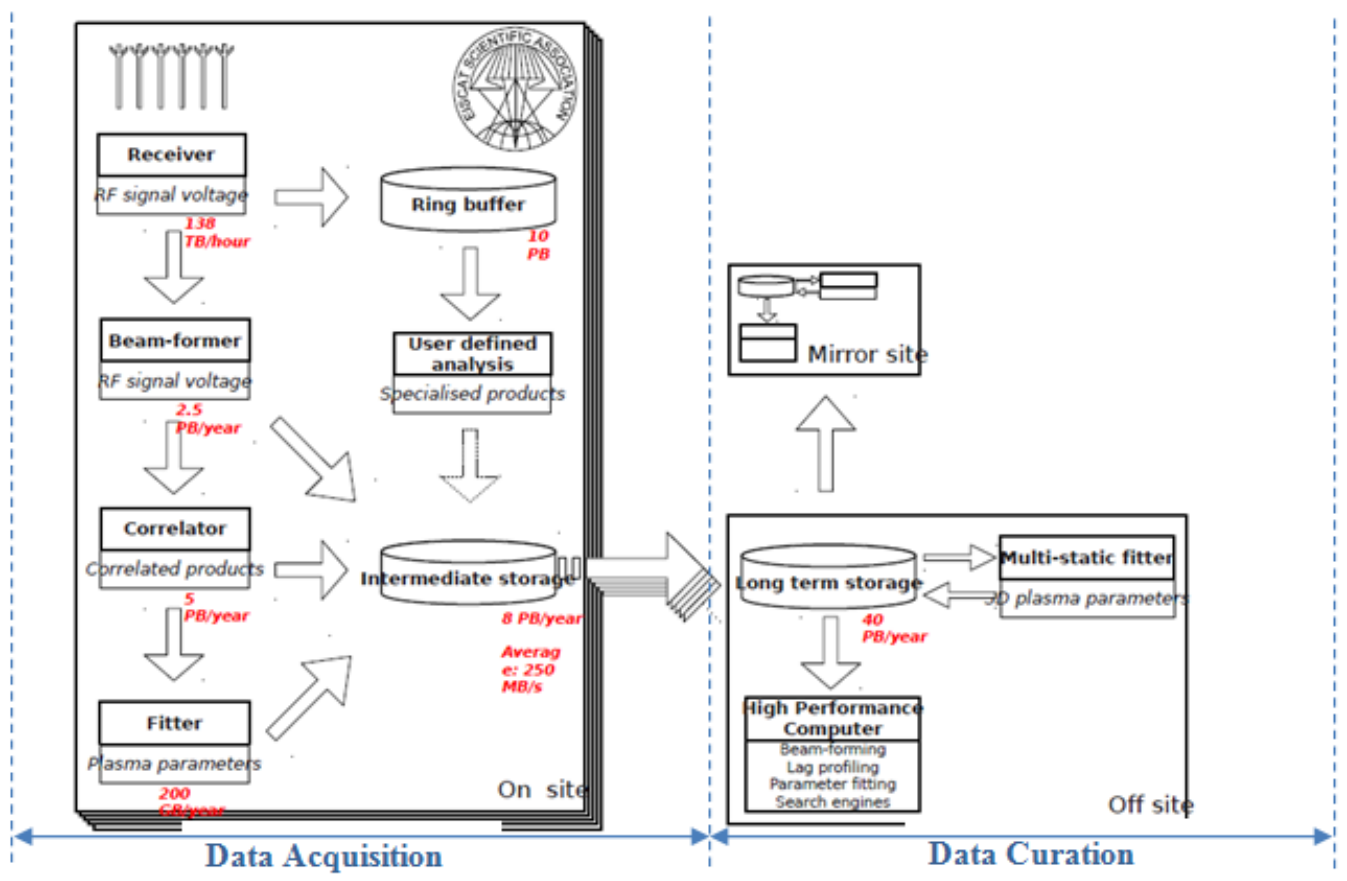

Figure 11(e): EISCAT 3D Architecture

\section{RESOURCES}

- Analysis of Common Requirements for Environmental Science Research Infrastructures. http://pos.sissa.it/archive/conferences/179/032/ISGC\%202013 032.pdf. Accessed March 3, 2015.

- Euro-Argo RI. http://www.euro-argo.eu/. Accessed March 3, 2015.

- $\quad$ EISCAT 3D. https://www.eiscat3d.se/. Accessed March 3, 2015. 
- $\quad$ LifeWatch. http://www.lifewatch.com/. Accessed March 3, 2015.

- European Multidisciplinary Seafloor \& Water Column Observatory (EMSO). http://www.emsoeu.org/. Accessed March 3, 2015.

\subsubsection{Use Case 43: Radar Data Analysis for the Center for Remote SENSING OF ICE SHEETS}

Submitted by Geoffrey Fox, Indiana University

\section{APPLICATION}

As illustrated in Figure 12, the Center for Remote Sensing of Ice Sheets (CReSIS) effort uses custom radar systems to measure ice sheet bed depths and (annual) snow layers at the North and South Poles and mountainous regions.

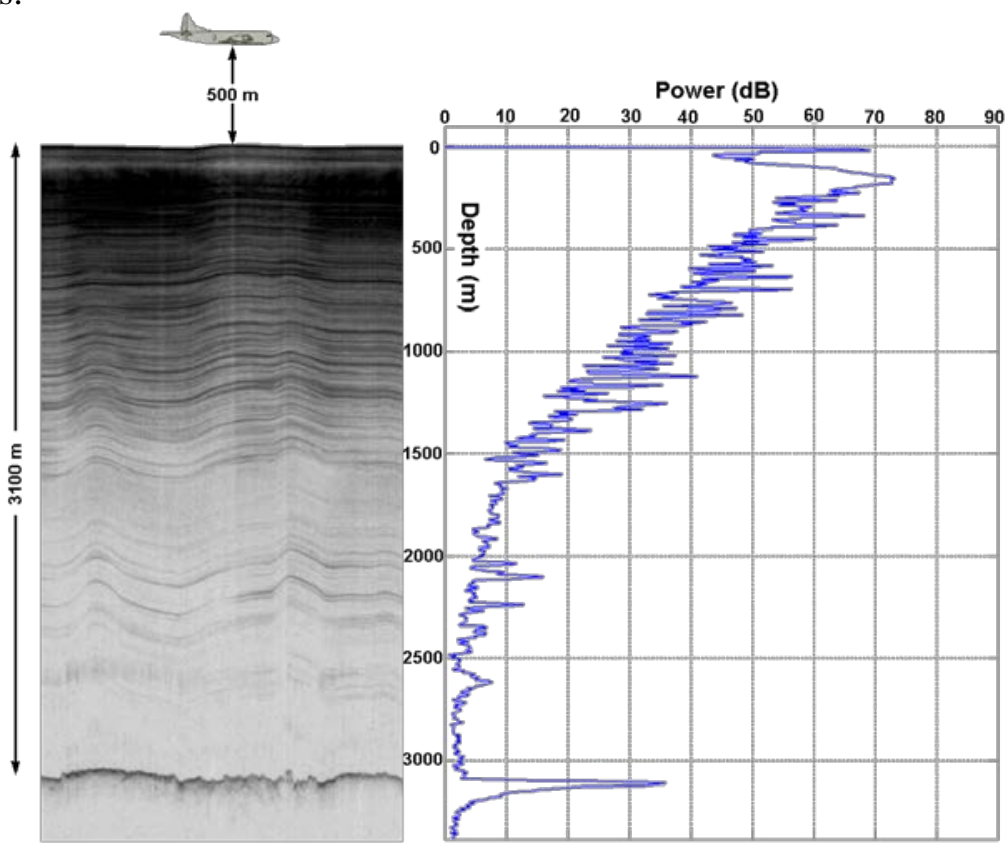

Figure 12: Typical CReSIS Radar Data After Analysis

Resulting data feed into the Intergovernmental Panel on Climate Change (IPCC). The radar systems are typically flown in by aircraft in multiple paths, as illustrated by Figure 13. 


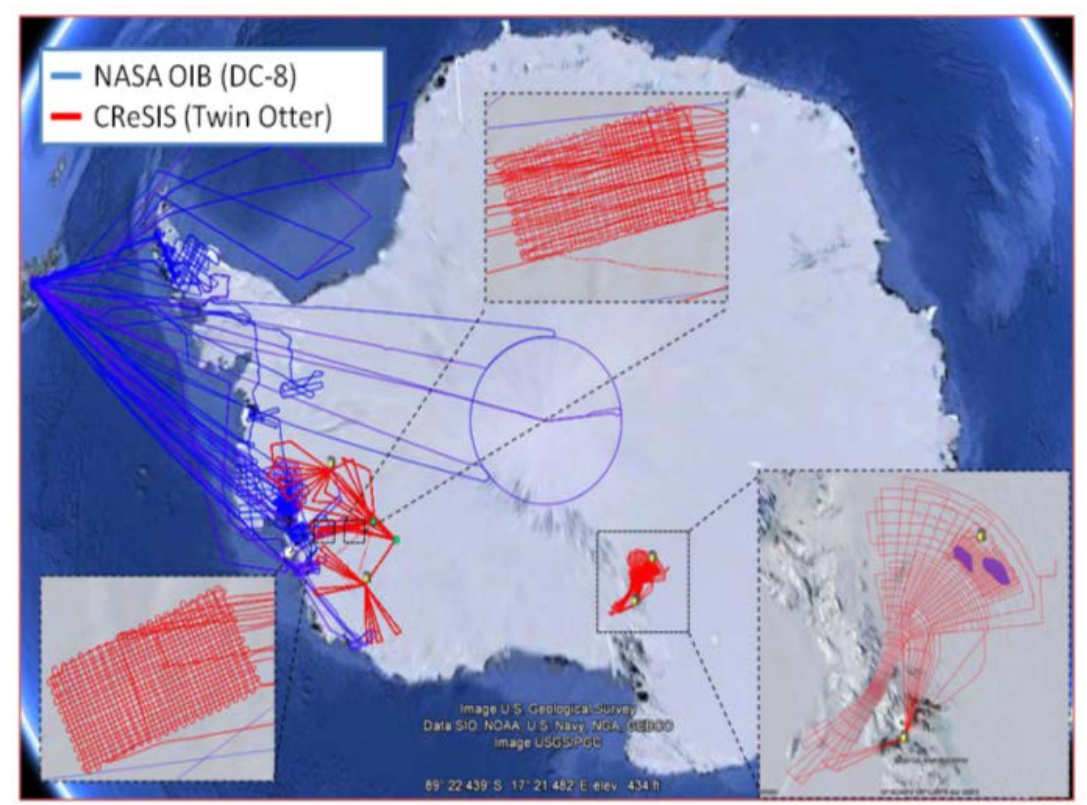

Figure 13: Radar Data Analysis for CReSIS Remote Sensing of Ice Sheets- Typical Flight Paths of Data Gathering in Survey Region

\section{CURRENT APPROACH}

The initial analysis uses MATLAB signal processing that produces a set of radar images. These cannot be transported from the field over the Internet and are typically copied onsite to a few removable disks that hold a terabyte of data, then flown to a laboratory for detailed analysis. Figure 14 illustrates image features (i.e., layers) found using image understanding tools with some human oversight. Figure 13 is a typical echogram with detected boundaries. The upper (green) boundary is between air and ice layers, while the lower (red) boundary is between ice and terrain. This information is stored in a database frontended by a geographical information system. The ice sheet bed depths are used in simulations of glacier flow. Each trip into the field, usually lasting a few weeks, results in 50 TB to 100 TB of data.

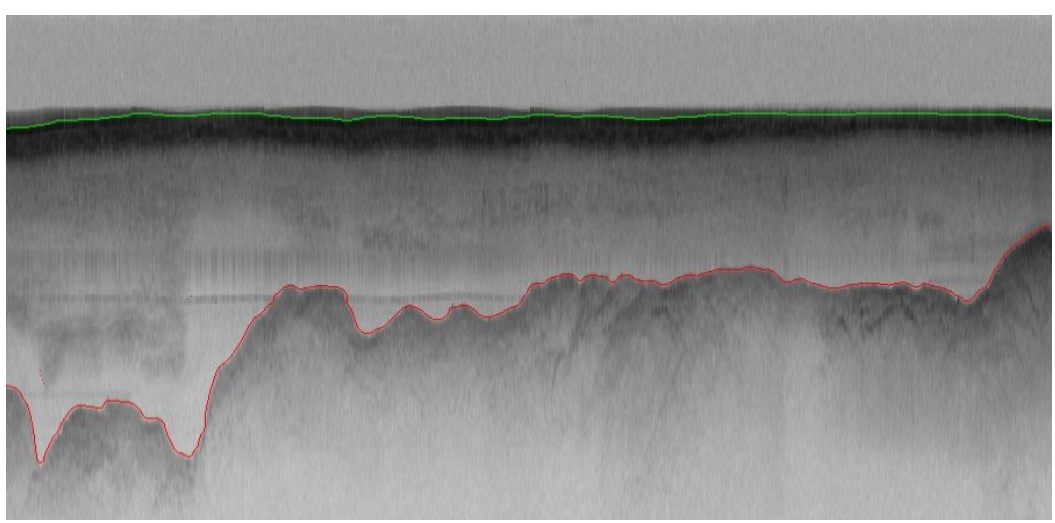

Figure 14: Typical echogram with detected boundaries

\section{FUTURE}

With improved instrumentation, an order of magnitude more data (a petabyte per mission) is projected. As the increasing field data must be processed in an environment with constrained power access, lowpower or low-performance architectures, such as GPU systems, are indicated. 


\section{RESOURCES}

- CReSIS. https://www.cresis.ku.edu. Accessed March 3, 2015.

- Polar Grid Multimedia Gallery, Indiana University. http://polargrid.org/gallery.html . Accessed March 3, 2015.

\subsubsection{Use CASE 44: UnmanNed AIR Vehicle SyNthetic ApertuRe RadAR (UAVSAR) Data Processing, Data Product Delivery, and Data SERVICES}

Submitted by Andrea Donnellan and Jay Parker, National Aeronautics and Space Administration (NASA) Jet Propulsion Laboratory

\section{APPLICATION}

Synthetic aperture radar (SAR) can identify landscape changes caused by seismic activity, landslides, deforestation, vegetation changes, and flooding. This function can be used to support earthquake science, as shown in Figure 14, as well as disaster management. Figure 15 shows the combined unwrapped coseismic interferograms for flight lines 26501, 26505, and 08508 for the October 2009 to April 2010 time period. End points where slip can be seen on the Imperial, Superstition Hills, and Elmore Ranch faults are noted. GPS stations are marked by dots and are labeled. This use case supports the storage, image processing application, and visualization of geo-located data with angular specification.

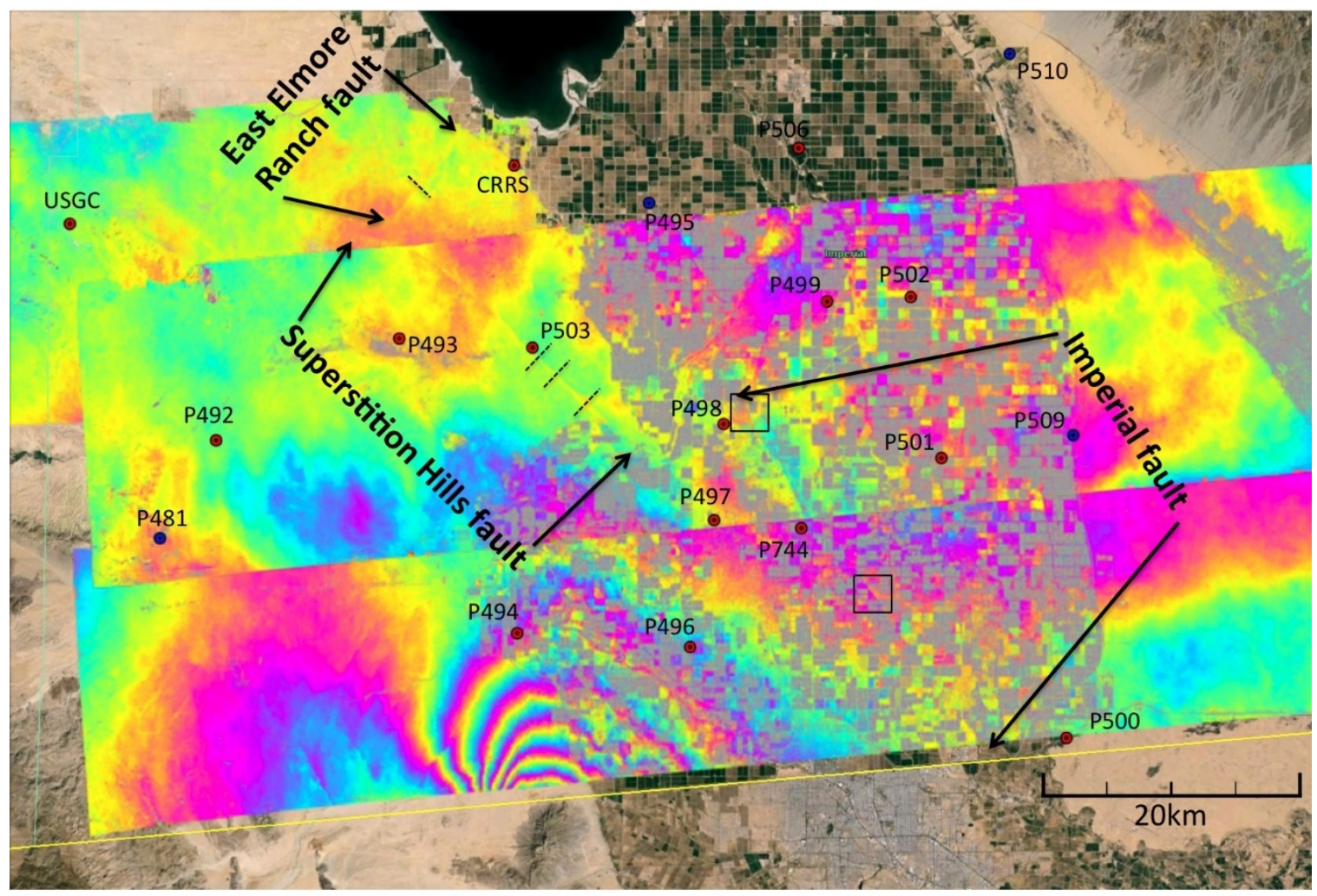

Figure 15: Combined Unwrapped Coseismic Interferograms

\section{CURRENT APPROACH}

Data from planes and satellites are processed on NASA computers before being stored after substantial data communication. The data are made public upon processing. They require significant curation owing to instrumental glitches. The current data size is approximately $150 \mathrm{~TB}$. 


\title{
FUTURE
}

The data size would increase dramatically if Earth Radar Mission launched. Clouds are suitable hosts but are not used today in production.

\section{RESOURCES}

- Uninhabited Aerial Vehicle Synthetic Aperture Radar. http://uavsar.jpl.nasa.gov/. Accessed March 3, 2015.

- Alaska Satellite Facility. http://www.asf.alaska.edu/program/sdc. Accessed March 3, 2015.

- QuakeSim: Understanding Earthquake Processes. http://quakesim.org. Accessed March 3, 2015.

\subsubsection{Use CASE 45: NASA LANGLEy Research CENTER/ GoddaRd SPACE FLIGHT CENTER IRODS FEDERATION TEST BED}

Submitted by Brandi Quam, NASA Langley Research Center

\section{APPLICATION}

NASA Center for Climate Simulation and NASA Atmospheric Science Data Center have complementary datasets, each containing vast amounts of data that are not easily shared and queried. Climate researchers, weather forecasters, instrument teams, and other scientists need to access data from across multiple datasets in order to compare sensor measurements from various instruments, compare sensor measurements to model outputs, calibrate instruments, look for correlations across multiple parameters, and more.

\section{CURRENT APPROACH}

Data are generated from two products: the Modern Era Retrospective Analysis for Research and Applications (MERRA, described separately) and NASA Clouds and Earth's Radiant Energy System (CERES) EBAF-TOA (Energy Balanced and Filled-Top of Atmosphere) product, which accounts for about $420 \mathrm{MB}$, and the EBAF-Surface product, which accounts for about $690 \mathrm{MB}$. Data numbers grow with each version update (about every six months). To analyze, visualize, and otherwise process data from heterogeneous datasets is currently a time-consuming effort. Scientists must separately access, search for, and download data from multiple servers, and often the data are duplicated without an understanding of the authoritative source. Often accessing data takes longer than scientific analysis. Current datasets are hosted on modest-sized (144 to 576 cores) Infiniband clusters.

\section{FUTURE}

Improved access will be enabled through the use of iRODS. These systems support parallel downloads of datasets from selected replica servers, providing users with worldwide access to the geographically dispersed servers. iRODS operation will be enhanced with semantically organized metadata and managed via a highly precise NASA Earth Science ontology. Cloud solutions will also be explored.

\subsubsection{Use CASE 46: MERRA ANalytic SeRVICEs (MERRA/AS)}

\author{
Submitted by John L. Schnase and Daniel Q. Duffy, NASA Goddard Space Flight Center
}

\section{APPLICATION}

This application produces global temporally and spatially consistent syntheses of 26 key climate variables by combining numerical simulations with observational data. Three-dimensional results are produced every six hours extending from 1979 to the present. The data support important applications such as IPCC research and the NASA/Department of Interior RECOVER wildfire decision support system; these applications typically involve integration of MERRA with other datasets. Figure 16 shows a typical MERRA/AS output. 


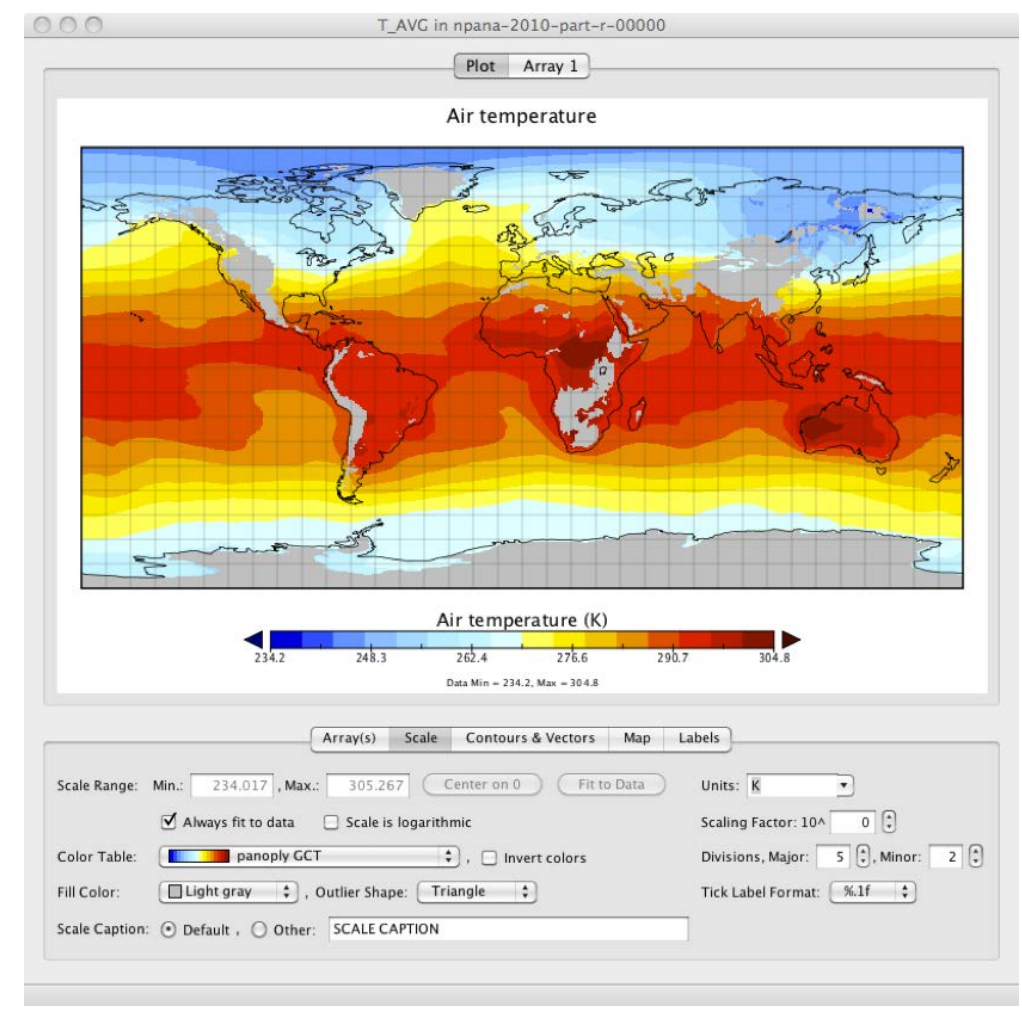

Figure 16: Typical MERRA/AS Output

\section{CURRENT APPROACH}

Map/Reduce is used to process a current total of $480 \mathrm{~TB}$. The current system is hosted on a 36-node Infiniband cluster.

\section{FUTURE}

Clouds are being investigated. The data is growing by one TB a month.

\subsubsection{Use CASE 47: AtMospheric TURBULENCE - EVENT DisCoVERY AND PREDICTIVE ANALYTICS}

Submitted by Michael Seablom, NASA headquarters

\section{APPLICATION}

Data mining is built on top of reanalysis products, including MERRA (described separately) and the North American Regional Reanalysis (NARR), a long-term, high-resolution climate dataset for the North American domain. The analytics correlate aircraft reports of turbulence (either from pilot reports or from automated aircraft measurements of eddy dissipation rates) with recently completed atmospheric reanalyses. The information is of value to aviation industry and to weather forecasters. There are no standards for reanalysis products, complicating systems for which Map/Reduce is being investigated. The reanalysis data are hundreds of terabytes, slowly updated, whereas the turbulence dataset is smaller in size and implemented as a streaming service. Figure 17 shows a typical turbulent wave image. 


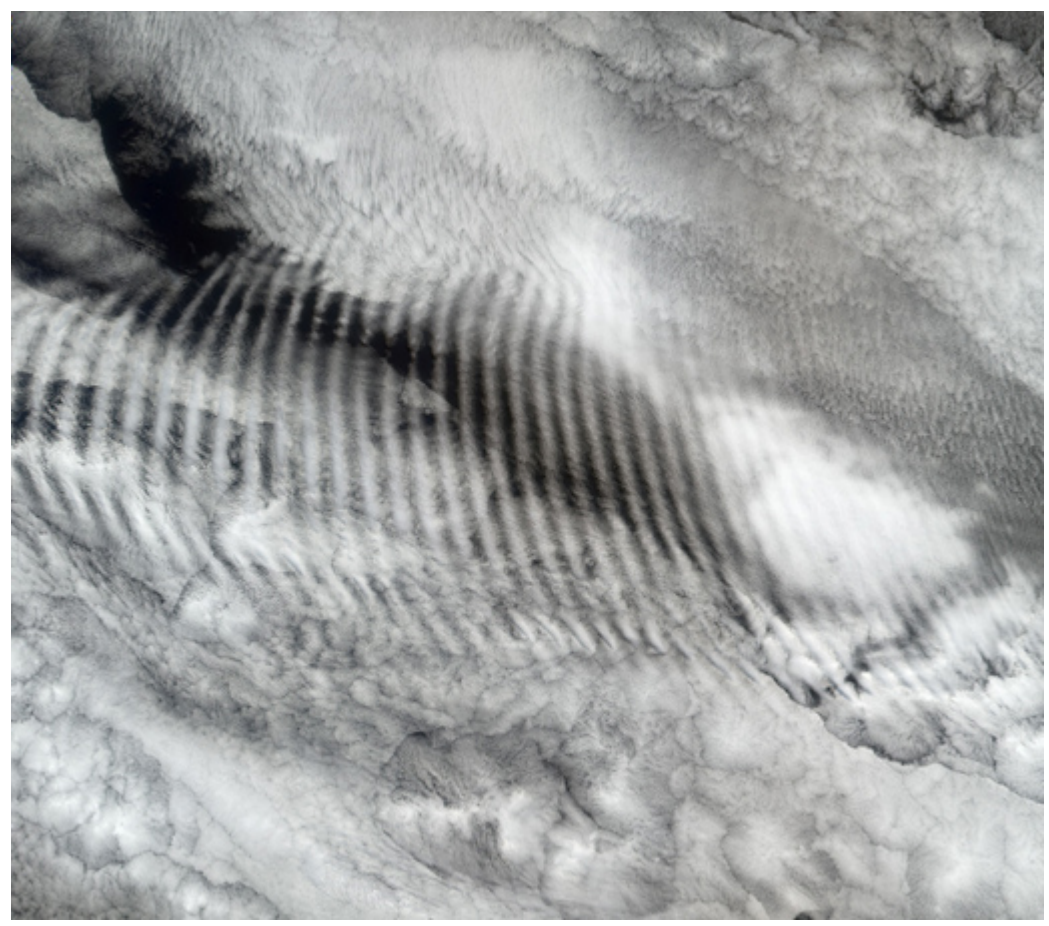

Figure 17: Typical NASA Image of Turbulent Waves

\section{CURRENT APPROACH}

The current 200 TB dataset can be analyzed with Map/Reduce or the like using SciDB or another scientific database.

\section{FUTURE}

The dataset will reach 500 TB in five years. The initial turbulence case can be extended to other ocean/atmosphere phenomena, but the analytics would be different in each case.

\section{RESOURCES}

- $\quad$ El Niño Teleconnections. http://oceanworld.tamu.edu/resources/oceanographybook/teleconnections.htm. Accessed March 3, 2015.

- Meet The Scientists Mining Big Data To Predict The Weather. http://www.forbes.com/sites/toddwoody/2012/03/21/meet-the-scientists-mining-big-data-topredict-the-weather/. Accessed March 3, 2015.

\subsubsection{Use Case 48: Climate Studies Using the Community Earth System MODEL AT THE U.S. DEPARTMENT OF ENERGY (DOE) NERSC CENTER}

Submitted by Warren Washington, National Center for Atmospheric Research

\section{APPLICATION}

Simulations with the Community Earth System Model (CESM) can be used to understand and quantify contributions of natural and anthropogenic-induced patterns of climate variability and change in the $20^{\text {th }}$ and $21^{\text {st }}$ centuries. The results of supercomputer simulations across the world should be stored and compared. 


\section{CuRrent Approach}

The Earth System Grid (ESG) enables global access to climate science data on a massive scalepetascale, or even exascale-with multiple petabytes of data at dozens of federated sites worldwide. The ESG is recognized as the leading infrastructure for the management and access of large distributed data volumes for climate change research. It supports the Coupled Model Intercomparison Project (CMIP), whose protocols enable the periodic assessments carried out by the IPCC.

\section{FUTURE}

Rapid growth of data is expected, with 30 PB produced at NERSC (assuming 15 end-to-end climate change experiments) in 2017 and many times more than this worldwide.

\section{RESOURCES}

- $\quad$ Earth System Grid (ESG) Gateway at the National Center for Atmospheric Research. http://www.earthsystemgrid.org. Accessed March 3, 2015.

- Welcome to PCMDI! http://www-pcmdi.llnl.gov/. Accessed March 3, 2015.

- National Energy Research Scientific Computing Center. http://www.nersc.gov/. Accessed March 3, 2015.

- Research: Climate and Environmental Sciences Division (CESD). http://science.energy.gov/ber/research/cesd/. Accessed March 3, 2015.

- Computational \& Information Systems Lab (CISL). http://www2.cisl.ucar.edu/. Accessed March 3, 2015.

\subsubsection{USE CASE 49: DOE BIological AND ENVIRONMENTAL RESEARCH (BER) SUBSURFACE BIOGEOCHEMISTRY SCIENTIFIC FOCUS AREA}

Submitted by Deb Agarwal, LBNL

\section{APPLICATION}

A genome-enabled watershed simulation capability (GEWaSC) is needed to provide a predictive framework for understanding the following:

- How genomic information stored in a subsurface microbiome affects biogeochemical watershed functioning.

- How watershed-scale processes affect microbial functioning.

- How these interactions co-evolve.

\section{CURRENT APPROACH}

Current modeling capabilities can represent processes occurring over an impressive range of scales-from a single bacterial cell to that of a contaminant plume. Data cross all scales from genomics of the microbes in the soil to watershed hydro-biogeochemistry. Data are generated by the different research areas and include simulation data, field data (e.g., hydrological, geochemical, geophysical), 'omics' data, and observations from laboratory experiments.

\section{FUTURE}

Little effort to date has been devoted to developing a framework for systematically connecting scales, as is needed to identify key controls and to simulate important feedbacks. GEWaSC will develop a simulation framework that formally scales from genomes to watersheds and will synthesize diverse and disparate field, laboratory, and simulation datasets across different semantic, spatial, and temporal scales. 


\subsubsection{USE CASE 50: DOE BER AMERIFLUX AND FLUXNET NETWORKS}

Submitted by Deb Agarwal, LBNL

\section{APPLICATION}

AmeriFlux and Flux Tower Network (FLUXNET) are U.S. and world collections, respectively, of sensors that observe trace gas fluxes (e.g., $\mathrm{CO}_{2}$, water vapor) across a broad spectrum of times (e.g., hours, days, seasons, years, and decades) and space. Moreover, such datasets provide the crucial linkages among organisms, ecosystems, and process-scale studies - at climate-relevant scales of landscapes, regions, and continents—-for incorporation into biogeochemical and climate models.

\section{CURRENT APPROACH}

Software includes EddyPro, custom analysis software, R, Python, neural networks, and MATLAB. There are approximately 150 towers in AmeriFlux and over 500 towers distributed globally collecting flux measurements.

\section{FUTURE}

Field experiment data-taking would be improved by access to existing data and automated entry of new data via mobile devices. Interdisciplinary studies integrating diverse data sources will be expanded.

\section{RESOURCES}

- AmeriFlux. http://Ameriflux.lbl.gov. Accessed March 3, 2015.

- Welcome to the Fluxdata.org web site. http://www.fluxdata.org. Accessed March 3, 2015.

\subsubsection{USE CASE 2-1: NASA EARTH OBSERVING SYSTEM DATA AND INFORMATION SYSTEM (EOSDIS)}

Submitted by Christopher Lynnes

\section{APPLICATION}

The Earth Observing System Data and Information System (EOSDIS) is the main system maintained by NASA for the archive and dissemination of Earth Observation data. The system comprises 12 disciplineoriented data systems spread across the United States. This network is linked together using interoperability frameworks such as the Common Metadata Repository, a file-level database that supports one-stop searching across EOSDIS. The data consist of satellite, aircraft, field campaign, and in situ data over a variety of disciplines related to Earth science, covering the Atmosphere, Hydrosphere, Cryosphere, Lithosphere, Biosphere, and Anthroposphere. Data are distributed to a diverse community ranging from Earth science researchers to applications to citizen science and educational users.

EOSDIS faces major challenges in both Volume and Variety. As of early 2017, the cumulative archive data volume is over 20 Petabytes. Higher-resolution space-borne instruments are expected to increase that volume by two orders of magnitude ( 200 PB) over the next 7 years. More importantly, the data distribution to users is equally high. In a given year, EOSDIS distributes a volume that is comparable to the overall cumulative archive volume.

Detailed topics include the following:

- Data Archiving: storing NASA's Earth Observation data;

- Data Distribution: disseminating data to end users in Research, Applications (e.g., water resource management) and Education;

- Data Discovery: search and access to Earth Observation data;

- Data Visualization: static browse images and dynamically constructed visualizations; 
- Data Customization: subsetting, reformatting, regridding, mosaicking, and quality screening on behalf of end users;

- Data Processing: routine production of standard scientific datasets, converting raw data to geophysical variables; and

- Data Analytics: end-user analysis of large datasets, such as time-averaged maps and areaaveraged time series.

\section{CURRENT APPROACH}

Standard data processing converts raw data to geophysical parameters. Though much of this is heritage custom Fortran or $\mathrm{C}$ code running, current prototypes are using cloud computing to scale up to rapid reprocessing campaigns.

EOSDIS support of end-user analysis currently uses high-performance software, such as the netCDF Command Operators. However, current prototypes are using cloud computing and data-parallel algorithms (e.g., Spark) to achieve an order of magnitude speed-up.

\section{FUTURE}

EOSDIS is beginning to migrate data archiving to the cloud to enable end users to bring algorithms to the data. We also expect to reorganize certain high-value datasets into forms that lend themselves to cloud data-parallel computing. Prototypes are under way to prove out storage schemes that are optimized for cloud analytics, such as space-time tiles stored in cloud databases and cloud file systems.

\section{RESOURCES}

- NASA, Earthdata: https://earthdata.nasa.gov/

- NASA Earthdata, Global Imagery Browse Services (GIBS): https://earthdata.nasa.gov/about/science-system-description/eosdis-components/global-imagerybrowse-services-gibs

- NASA Earthdata, Worldview: https://worldview.earthdata.nasa.gov/

\subsubsection{USE CASE 2-2: Web-EnABLED LANDSAT DATA (WELD) PROCESSING}

Submitted by Andrew Michaelis

\section{APPLICATION}

The use case shown in Figure18 is specific to the part of the project where data is available on the HPC platform and processed through the science workflow. It is a 32-stage processing pipeline of images from the Landsat 4, 5, and 7 satellites that includes two separate science products (Top-of-the-Atmosphere [TOA] reflectances and surface reflectances) as well as QA and visualization components which forms a dataset of science products of use to the land surface science community that is made freely available by NASA.

\section{CURRENT APPROACH}

This uses the High Performance Computing (HPC) system Pleiades at NASA Ames Research Center with storage in NASA Earth Exchange (NEX) NFS storage system for read-only data storage (2.5PB), Lustre for read-write access during processing (1PB), tape for near-line storage (50PB). The networking is InfiniBand partial hypercube internal interconnect within the HPC system; $1 \mathrm{G}$ to $10 \mathrm{G}$ connection to external data providers. The software is the NEX science platform for data management, workflow processing, provenance capture; the WELD science processing algorithms from South Dakota State University for visualization and time-series; the Global Imagery Browse Service (GIBS) data visualization platform; and the USGS data distribution platform. This is a custom-built application and libraries built on top of open-source libraries. 


\section{FUTURE}

Processing will be improved with newer and updated algorithms. This process may also be applied to future datasets and processing systems (Landsat 8 and Sentinel-2 satellites, for example).

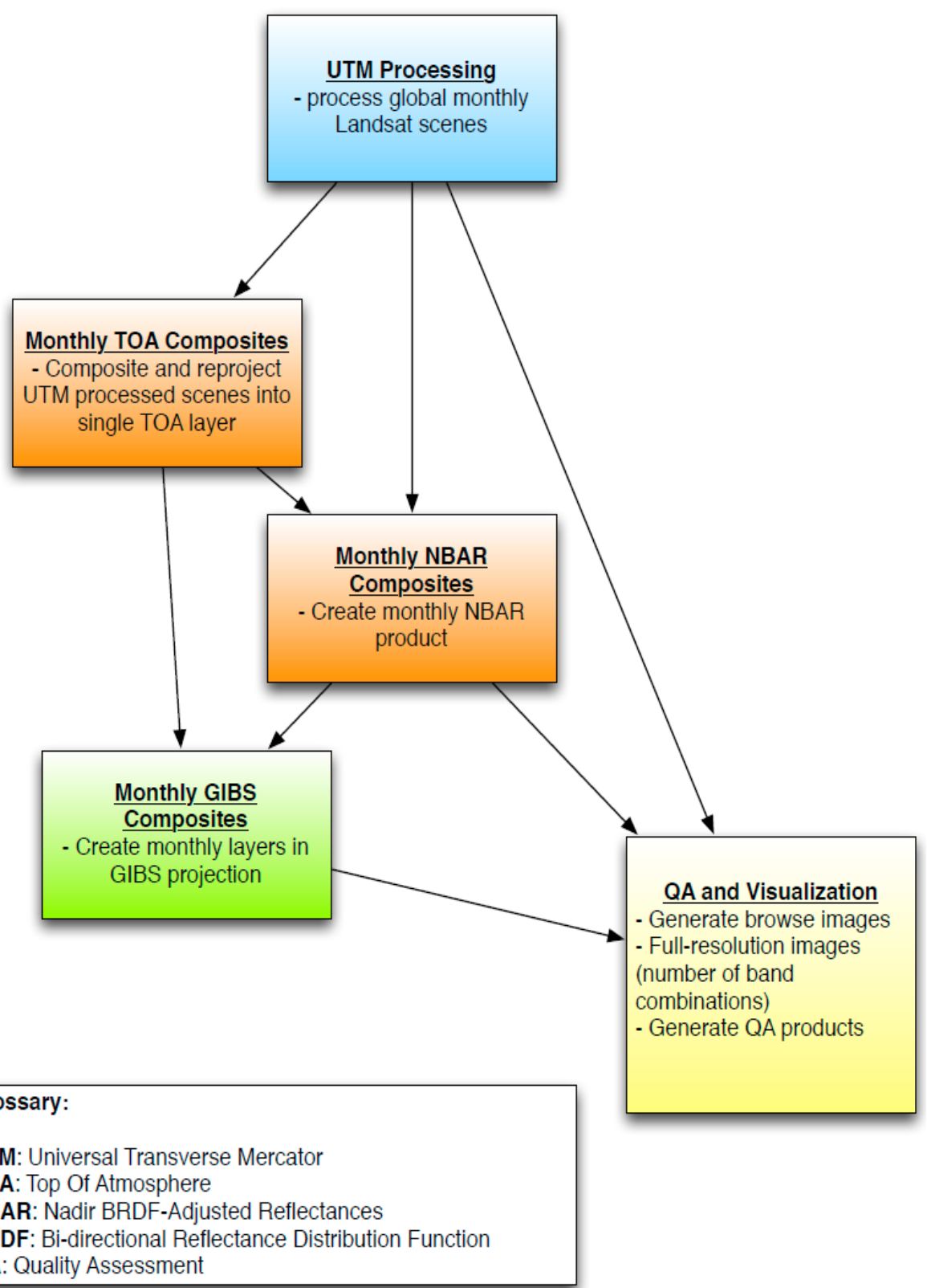

Figure 18: NASA NEX WELD/GIBS Processing Workflow

\section{RESOURCES}

- Global Web-Enabled Landsat Data, Geospatial Sciences Center of Excellence (GSCE), South Dakota State University: http://globalmonitoring.sdstate.edu/projects/weldglobal/gweld.html

- Global Web-Enabled Landsat Data, U.S. Geological Survey: http://globalweld.cr.usgs.gov/

- NASA Earth Exchange (NEX): https://nex.nasa.gov

- NASA High-End Computing Capability: http://www.nas.nasa.gov/hecc/resources/pleiades.html 


\subsection{ENERGY}

\subsubsection{USE CASE 51: Consumption Forecasting IN SMART GRIDS}

Submitted by Yogesh Simmhan, University of Southern California

\section{APPLICATION}

Smart meters support prediction of energy consumption for customers, transformers, substations and the electrical grid service area. Advanced meters provide measurements every 15 minutes at the granularity of individual consumers within the service area of smart power utilities. Data to be combined include the head end of smart meters (distributed), utility databases (customer information, network topology; centralized), U.S. Census data (distributed), NOAA weather data (distributed), micro-grid building information systems (centralized), and micro-grid sensor networks (distributed). The central theme is realtime, data-driven analytics for time series from cyber-physical systems.

\section{CURRENT APPROACH}

Forecasting uses GIS-based visualization. Data amount to around 4 TB per year for a city such as Los Angeles with 1.4 million sensors. The process uses R/Matlab, Weka, and Hadoop software. There are significant privacy issues requiring anonymization by aggregation. Real-time and historic data are combined with machine learning to predict consumption.

\section{FUTURE}

Advanced grid technologies will have wide-spread deployment. Smart grids will have new analytics integrating diverse data and supporting curtailment requests. New technologies will support mobile applications for client interactions.

\section{RESOURCES}

- $\quad$ USC Smart Grid. http://smartgrid.usc.edu. Accessed March 3, 2015.

- Smart Grid. http://ganges.usc.edu/wiki/Smart_Grid. Accessed March 3, 2015.

- Smart Grid L.A. https://www.ladwp.com/ladwp/faces/ladwp/aboutus/a-power/a-p-smartgridla. Accessed March 3, 2015.

- Cloud-Based Software Platform for Big Data Analytics in Smart Grids. http://ieeexplore.ieee.org/xpl/articleDetails.jsp?arnumber=6475927. Accessed March 3, 2015. 
Requirements are the challenges limiting further use of Big Data. After collection, processing, and review of the use cases, requirements within seven characteristic categories were extracted from the individual use cases. These use case specific requirements were then aggregated to produce high-level, general requirements, within the seven characteristic categories, that are vendor-neutral and technology-agnostic. Neither the use case nor the requirements lists are exhaustive.

\subsection{USE CASE SPECIFIC REQUIREMENTS}

Each use case was evaluated for requirements within the following seven categories. These categories were derived from Subgroup discussions and motivated by components of the evolving reference architecture at the time. The process involved several Subgroup members extracting requirements and iterating back their suggestions for modifying the categories.

1. Data source (e.g., data size, file formats, rate of growth, at rest or in motion);

2. Data transformation (e.g., data fusion, analytics);

3. Capabilities (e.g., software tools, platform tools, hardware resources such as storage and networking);

4. Data consumer (e.g., processed results in text, table, visual, and other formats);

5. Security and privacy;

6. Life cycle management (e.g., curation, conversion, quality check, pre-analytic processing); and

7. Other requirements.

Some use cases contained requirements in all seven categories while others included only requirements for a few categories. The complete list of specific requirements extracted from the use cases is presented in Appendix D. Section 2.1 of the NIST Big Data Interoperability Framework: Volume 6 Reference Architecture maps these seven categories to terms used in the reference architecture. The categories map in a one-to-one fashion but have slightly different terminology as the use case requirements analysis was performed before the reference architecture was finalized.

3.2 GENERAL REQUIREMENTS

Aggregation of the use case-specific requirements allowed formation of more generalized requirements under the seven categories. These generalized requirements are listed below by category.

\section{DATA SOURCE REQUI REMENTS (DSR)}

- DSR-1: Needs to support reliable real-time, asynchronous, streaming, and batch processing to collect data from centralized, distributed, and cloud data sources, sensors, or instruments.

- DSR-2: Needs to support slow, bursty, and high-throughput data transmission between data sources and computing clusters.

- DSR-3: Needs to support diversified data content ranging from structured and unstructured text, document, graph, web, geospatial, compressed, timed, spatial, multimedia, simulation, and instrumental data. 


\section{TRANSFormati ON PROVIDER ReQUI REMENTS (TPR)}

- TPR-1: Needs to support diversified compute-intensive, statistical and graph analytic processing, and machine learning techniques.

- TPR-2: Needs to support batch and real-time analytic processing.

- TPR-3: Needs to support processing large diversified data content and modeling.

- TPR-4: Needs to support processing data in motion (streaming, fetching new content, tracking, etc.).

\section{CAPABI LITY PROVIDER REQUI REMENTS (CPR)}

- CPR-1: Needs to support legacy and advanced software packages (software).

- CPR-2: Needs to support legacy and advanced computing platforms (platform).

- CPR-3: Needs to support legacy and advanced distributed computing clusters, co-processors, input output (I/O) processing (infrastructure).

- CPR-4: Needs to support elastic data transmission (networking).

- CPR-5: Needs to support legacy, large, and advanced distributed data storage (storage).

- CPR-6: Needs to support legacy and advanced executable programming: applications, tools, utilities, and libraries (software).

\section{DATA CONSUMER REQUI REMENTS (DCR)}

- DCR-1: Needs to support fast searches from processed data with high relevancy, accuracy, and recall.

- DCR-2: Needs to support diversified output file formats for visualization, rendering, and reporting.

- $\quad$ DCR-3: Needs to support visual layout for results presentation.

- DCR-4: Needs to support rich user interface for access using browser, visualization tools.

- DCR-5: Needs to support high-resolution, multidimension layer of data visualization.

- DCR-6: Needs to support streaming results to clients.

\section{SECURITY AND PRIVACY REQUI REMENTS (SPR)}

- SPR-1: Needs to protect and preserve security and privacy of sensitive data.

- SPR-2: Needs to support sandbox, access control, and multilevel, policy-driven authentication on protected data.

\section{LIFE CYCLE MANAGEMENT REQUI REMENTS (LMR)}

- LMR-1: Needs to support data quality curation including preprocessing, data clustering, classification, reduction, and format transformation.

- LMR-2: Needs to support dynamic updates on data, user profiles, and links.

- LMR-3: Needs to support data life cycle and long-term preservation policy, including data provenance.

- LMR-4: Needs to support data validation.

- LMR-5: Needs to support human annotation for data validation.

- LMR-6: Needs to support prevention of data loss or corruption.

- LMR-7: Needs to support multisite archives.

- LMR-8: Needs to support persistent identifier and data traceability.

- 'LMR-9: Needs to support standardizing, aggregating, and normalizing data from disparate sources. 
79

80

81

82

83

84

85

\section{OTHER REQUI REMENTS (OR)}

- OR-1: Needs to support rich user interface from mobile platforms to access processed results.

- OR-2: Needs to support performance monitoring on analytic processing from mobile platforms.

- OR-3: Needs to support rich visual content search and rendering from mobile platforms.

- OR-4: Needs to support mobile device data acquisition.

- OR-5: Needs to support security across mobile devices. 


\section{ADDITIONAL USE CASE CONTRIBUTIONS}

During the development of Version 2 of the NBDIF, the Use Cases and Requirements Subgroup and the Security and Privacy Subgroup identified the need for additional use cases to strengthen the future work of the NBD-PWG. These two subgroups collaboratively created the Use Case Template 2 with the aim of collecting specific and standardized information for each use case. In addition to questions from the original use case template, the Use Case Template 2 contains questions that provided a comprehensive view of security, privacy, and other topics for each use case.

The NBD-PWG invited the public to submit new use cases through the Use Case Template 2 PDF form (https://bigdatawg.nist.gov/_uploadfiles/M0621_v2_7345181325.pdf). Use cases were accepted until the end of Phase 3 work and were evaluated as they are submitted. The following three additional use cases were submitted using the new template:

- $\quad$ Use Case 2-1: NASA Earth Observing System Data and Information System (EOSDIS)

- Use Case 2-2: Web-Enabled Landsat Data (WELD) Processing

- Use Case 2-3: Urban context-aware event management for Smart Cities - Public safety 


\section{Appendix A: Use Case Study Source Materials}

105

Appendix A contains one blank use case template and the original completed use cases. The Use Case Studies Template 1 included in this Appendix is no longer being used to collect use case information. To submit a new use case, refer to Appendix E for the current Use Case Template 2.

These use cases were the source material for the use case summaries presented in Section 2 and the use case requirements presented in Section 3 of this document. The completed use cases have not been edited and contain the original text as submitted by the author(s). The use cases are as follows:

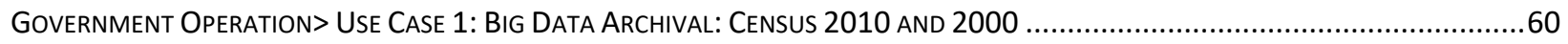

Government Operation> USE CASE 2: NARA ACCESSION, SEARCH, RETRIEVE, PRESERVATION..............................................61

GOVERNMENT OPERATION> USE CASE 3: STATISTICAL SURVEY RESPONSE IMPROVEMENT ...........................................................63

Government OPERATION> USE CASE 4: Non-TRAditional Data In STATISTICAL SURVEY ....................................................6

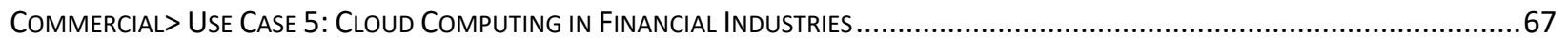

COMMERCIAL> USE CASE 6: MENDELEY—AN INTERNATIONAL NeTWORK OF RESEARCH ……..............................................76

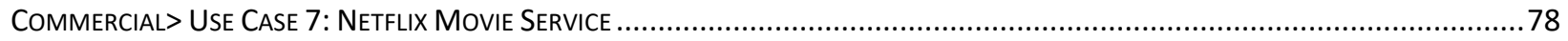

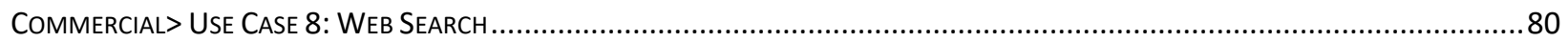

COMMERCIAL> USE CASE 9: CLOUD-BASED CONTINUITY AND DISASTER RECOVERY ............................................................

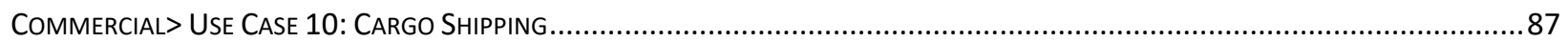

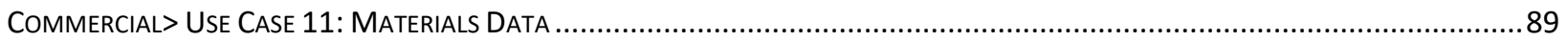

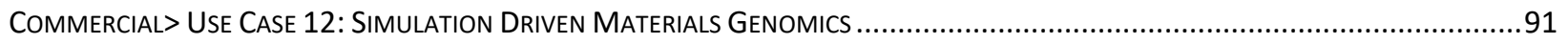

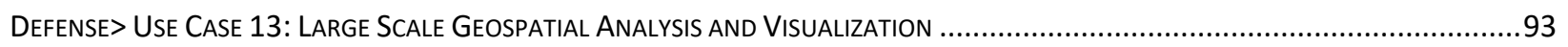

DefenSE> USE CASE 14: ObJeCt IDENTIFICATION AND TRACKING - PeRSISTENT SURVEILLANCE ................................................95

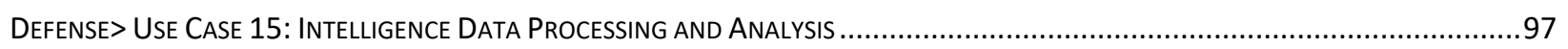

HealthCARE ANd LIFE SCIENCES> USE CASE 16: EleCtronic Medical ReCord DATA ......................................................100

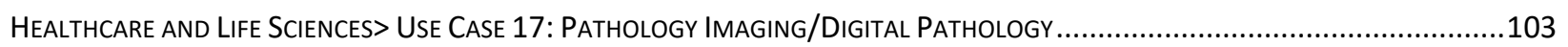

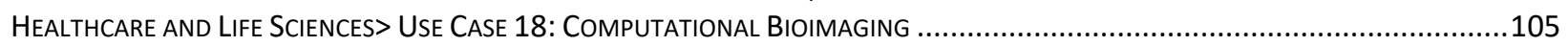

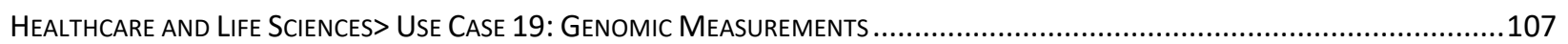

HealthCARE AND LIFE SCIENCES> USE CASE 20: Comparative AnALYSIS FOR (META) GeNOMES ............................................109

HeALTHCARE AND LIFE SCIENCES> USE CASE 21: INDIVIDUALIZEd DIABETES MANAGEMENT .......................................................112

HealthCARe And Life SCIences> Use CaSe 22: Statistical Relational Al for Health CARe .............................................114

HealthCARE AND LIFE SCIENCES> USE CASE 23: World Population SCALE EPIDEMIology ..................................................116

HEALTHCARE AND LIFE SCIENCES> USE CASE 24: SOCIAL CONTAGION MODELING ...............................................................118

HEALTHCARE AND LIFE SCIENCES> USE CASE 25: LIFEWATCH BIODIVERSITY ......................................................................120

Deep Learning AND SocIAL MediA> USE CASE 26: LARGE-SCALE DeEP LeARNING ...........................................................123

Deep Learning and Social Media> Use Case 27: Large SCale Consumer Photos Organization ......................................126

Deep Learning ANd SoCIAL Media> Use CASE 28: Truthy TWITTER Data ANalysis.......................................................128

Deep Learning ANd Social Media> Use CASE 29: Crowd Sourcing IN THe Humanities..................................................130

Deep Learning ANd Social Media> Use CASe 30: CINET Network SCIenCE Cyberinfrastructure ....................................132

DeEP Learning ANd Social Media> Use CaSe 31: NIST Analytic TeChnology Measurement And Evaluations.....................135

THE ECOSYSTEM FOR RESEARCH> USE CASE 32: DATANET FEDERATION CONSORTIUM (DFC) .................................................138

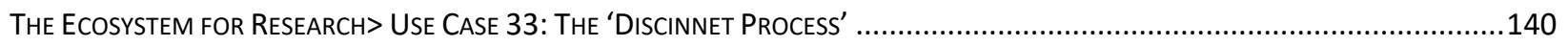

The ECOSYSTEM for ReSEARCH> USE CASE 34: GRAPH SEARCH ON SCIENTIFIC DATA .......................................................142

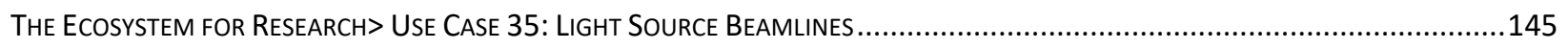

ASTRONomy AND PHYSICS> USE CASE 36: CATALINA DIGITAL SKY SURVEY FOR TRANSIENTS................................................147

ASTRONOMY AND PHYSICS> USE CASE 37: COSMOLOGICAL SKY SURVEY AND SIMULATIONS ....................................................150 
NIST Big Data Interoperability Framework: Volume 3, Use CaSes and General ReQuiRements

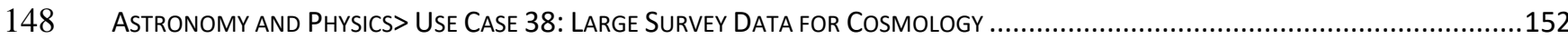

149 Astronomy AND PHYSICS> USE CASE 39: ANALYSIS Of LHC (LARGE HADRON ColLIDER) DATA ..........................................154

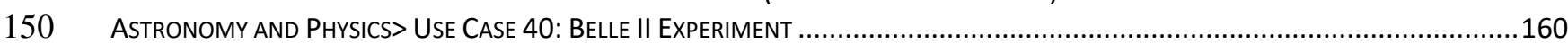

151 EARTH, ENVIRONMENTAL AND Polar SCIENCE> USE CASE 41: EISCAT 3D InCOHERENT SCATter RADAR SYSTEM ..........................162

152 EARTh, ENVIRONMENTAL AND Polar SCIENCE> USE CASE 42: Common ENVIRONMENTAL RESEARCH INFRASTRUCtURE .................165

153 EARTH, ENVIRONMENTAL AND Polar SCIENCE> USE CASE 43: RADAR DATA ANALYSIS FOR CRESIS..........................................171

154 EARTh, ENVIRONMENTAL AND Polar SCIENCE> USE CASE 44: UAVSAR DATA PROCESSING .................................................173

155 EARTH, ENVIRONMENTAL AND PolAR SCIENCE> USE CASE 45: NASA LARC/GSFC IRODS FEdERATION TESTBED .........................175

156 EARTh, ENVIRONMENTAL AND Polar SCIENCE> USE CASE 46: MERRA ANALYTIC SERVICES ...................................................179

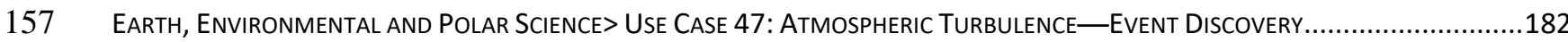

158 Earth, Environmental and Polar SCIence> Use CaSe 48: Climate Studies using the Community Earth System Model .....184

159 EARTH, ENVIRONMENTAL AND PolAR SCIENCE> USE CASE 49: SUBSURFACE BIOGEOCHEMISTRY ...............................................186

160 EARTh, ENVIRONMENTAL AND PolAR SCIENCE> USE CASE 50: AMERIFLUX AND FLUXXNET .................................................18

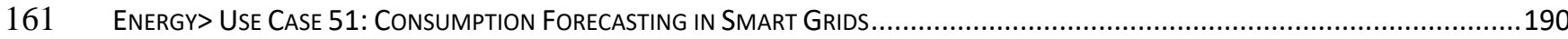

162

163 
NBD-PWg Use Case Studi es Template 1

\begin{tabular}{|c|c|c|}
\hline \multicolumn{3}{|l|}{ Use Case Title } \\
\hline \multirow{2}{*}{\multicolumn{3}{|c|}{$\begin{array}{r}\text { Vertical (area) } \\
\text { Author/Company/Email }\end{array}$}} \\
\hline & & \\
\hline \multicolumn{3}{|l|}{$\begin{array}{r}\text { Actors/ Stakeholders } \\
\text { and their roles and } \\
\text { responsibilities }\end{array}$} \\
\hline \multicolumn{3}{|l|}{ Goals } \\
\hline \multicolumn{3}{|l|}{ Use Case Description } \\
\hline \multirow{4}{*}{$\begin{array}{r}\text { Current } \\
\text { Solutions }\end{array}$} & Compute(System) & \\
\hline & Storage & \\
\hline & Networking & \\
\hline & Software & \\
\hline \multirow[t]{5}{*}{$\begin{array}{r}\text { Big Data } \\
\text { Characteristics }\end{array}$} & $\begin{array}{r}\text { Data Source } \\
\text { (distributed/centralized) }\end{array}$ & \\
\hline & Volume (size) & \\
\hline & $\begin{array}{r}\text { Velocity } \\
\text { (e.g. real time) }\end{array}$ & \\
\hline & $\begin{array}{r}\text { Variety } \\
\text { (multiple datasets, } \\
\text { mashup) }\end{array}$ & \\
\hline & $\begin{array}{r}\text { Variability (rate of } \\
\text { change) }\end{array}$ & \\
\hline \multirow{5}{*}{$\begin{array}{r}\text { Big Data Science } \\
\text { (collection, curation, } \\
\text { analysis, } \\
\text { action) }\end{array}$} & $\begin{array}{r}\text { Veracity (Robustness } \\
\text { Issues, semantics) }\end{array}$ & \\
\hline & Visualization & \\
\hline & Data Quality (syntax) & \\
\hline & Data Types & \\
\hline & Data Analytics & \\
\hline \multicolumn{3}{|l|}{$\begin{array}{l}\text { Big Data Specific } \\
\text { Challenges (Gaps) }\end{array}$} \\
\hline \multicolumn{3}{|l|}{$\begin{array}{r}\text { Big Data Specific } \\
\text { Challenges in Mobility }\end{array}$} \\
\hline \multicolumn{3}{|l|}{$\begin{array}{r}\text { Security and Privacy } \\
\text { Requirements }\end{array}$} \\
\hline \multicolumn{3}{|l|}{$\begin{array}{r}\text { Highlight issues for } \\
\text { generalizing this use } \\
\text { case (e.g. for ref. } \\
\text { architecture) }\end{array}$} \\
\hline \multicolumn{3}{|c|}{$\begin{array}{r}\text { More Information } \\
\text { (URLs) }\end{array}$} \\
\hline \multicolumn{3}{|c|}{ Note: <additional comments> } \\
\hline
\end{tabular}




\section{Comments on fields}

The following descriptions of fields in the template are provided to help with the understanding of both document intention and meaning of the 26 fields and also to indicate ways that they can be improved.

- Use Case Title: Title provided by the use case author

- Vertical (area): Intended to categorize the use cases. However, an ontology was not created prior to the use case submissions so this field was not used in the use case compilation.

- Author/Company/Email: Name, company, and email (if provided) of the person(s) submitting the use case.

- Actors/ Stakeholders and their roles and responsibilities: Describes the players and their roles in the use case.

- Goals: Objectives of the use case.

- Use Case Description: Brief description of the use case.

- Current Solutions: Describes current approach to processing Big Data at the hardware and software infrastructure level.

o Compute (System): Computing component of the data analysis system.

o Storage: Storage component of the data analysis system.

o Networking: Networking component of the data analysis system.

o Software: Software component of the data analysis system.

- Big Data Characteristics: Describes the properties of the (raw) data including the four major 'V's' of Big Data described in NIST Big Data Interoperability Framework: Volume 1, Big Data Definition of this report series.

o Data Source: The origin of data, which could be from instruments, Internet of Things, Web, Surveys, Commercial activity, or from simulations. The source(s) can be distributed, centralized, local, or remote.

o Volume: The characteristic of data at rest that is most associated with Big Data. The size of data varied drastically between use cases from terabytes to petabytes for science research (100 petabytes was the largest science use case for LHC data analysis), or up to exabytes in a commercial use case.

o Velocity: Refers to the rate of flow at which the data is created, stored, analyzed, and visualized. For example, big velocity means that a large quantity of data is being processed in a short amount of time.

o Variety: Refers to data from multiple repositories, domains, or types.

o Variability: Refers to changes in rate and nature of data gathered by use case.

- Big Data Science: Describes the high-level aspects of the data analysis process

o Veracity: Refers to the completeness and accuracy of the data with respect to semantic content. NIST Big Data Interoperability Framework: Volume 1, Big Data Definition discusses veracity in more detail.

o Visualization: Refers to the way data is viewed by an analyst making decisions based on the data. Typically, visualization is the final stage of a technical data analysis pipeline and follows the data analytics stage.

o Data Quality: This refers to syntactical quality of data. In retrospect, this template field could have been included in the Veracity field.

o Data Types: Refers to the style of data such as structured, unstructured, images (e.g., pixels), text (e.g., characters), gene sequences, and numerical.

o Data Analytics: Defined in NIST Big Data Interoperability Framework: Volume 1, Big Data Definition as "the synthesis of knowledge from information". In the context of these use cases, analytics refers broadly to tools and algorithms used in processing the data at any stage including the data to information or knowledge to wisdom stages, as well as the information to knowledge stage. 
- Big Data Specific Challenges (Gaps): Allows for explanation of special difficulties for processing Big Data in the use case and gaps where new approaches/technologies are used.

- Big Data Specific Challenges in Mobility: Refers to issues in accessing or generating Big Data from Smart Phones and tablets.

- Security and Privacy Requirements: Allows for explanation of security and privacy issues or needs related to this use case.

- Highlight issues for generalizing this use case: Allows for documentation of issues that could be common across multiple use-cases and could lead to reference architecture constraints.

- More Information (URLs): Resources that provide more information on the use case.

- Note: <additional comments>: Includes pictures of use-case in action but was not otherwise used. 


\section{Submi Tted Use Case Studies}

\section{Government Operation> Use Case 1: Big Data Archival: Census 2010 and 2000}

\begin{tabular}{|c|c|c|}
\hline Use Case Title & \multicolumn{2}{|c|}{ Big Data Archival: Census 2010 and 2000-Title 13 Big Data } \\
\hline Vertical (area) & \multicolumn{2}{|c|}{ Digital Archives } \\
\hline Author/Company/Email & \multicolumn{2}{|c|}{ Vivek Navale and Quyen Nguyen (NARA) } \\
\hline $\begin{array}{l}\text { Actors/Stakeholders } \\
\text { and their roles and } \\
\text { responsibilities }\end{array}$ & \multicolumn{2}{|c|}{$\begin{array}{l}\text { NARA's Archivists } \\
\text { Public users (after } 75 \text { years) }\end{array}$} \\
\hline Goals & \multicolumn{2}{|c|}{$\begin{array}{l}\text { Preserve data for a long term in order to provide access and perform analytics after } \\
75 \text { years. Title } 13 \text { of U.S. code authorizes the Census Bureau and guarantees that } \\
\text { individual and industry specific data is protected. }\end{array}$} \\
\hline Use Case Description & \multicolumn{2}{|c|}{$\begin{array}{l}\text { Maintain data "as-is". No access and no data analytics for } 75 \text { years. } \\
\text { Preserve the data at the bit-level. } \\
\text { Perform curation, which includes format transformation if necessary. } \\
\text { Provide access and analytics after nearly } 75 \text { years. }\end{array}$} \\
\hline \multirow{4}{*}{$\begin{array}{r}\text { Current } \\
\text { Solutions }\end{array}$} & Compute(System) & Linux servers \\
\hline & Storage & NetApps, Magnetic tapes. \\
\hline & Networking & \\
\hline & Software & \\
\hline \multirow[t]{5}{*}{$\begin{array}{r}\text { Big Data } \\
\text { Characteristics }\end{array}$} & $\begin{array}{r}\text { Data Source } \\
\text { (distributed/centralized) }\end{array}$ & Centralized storage. \\
\hline & Volume (size) & 380 Terabytes. \\
\hline & $\begin{array}{r}\text { Velocity } \\
\text { (e.g. real time) } \\
\end{array}$ & Static. \\
\hline & $\begin{array}{r}\text { Variety } \\
\text { (multiple datasets, } \\
\text { mashup) }\end{array}$ & Scanned documents \\
\hline & $\begin{array}{r}\text { Variability (rate of } \\
\text { change) }\end{array}$ & None \\
\hline \multirow{5}{*}{$\begin{array}{r}\text { Big Data Science } \\
\text { (collection, curation, } \\
\text { analysis, } \\
\text { action) }\end{array}$} & $\begin{array}{r}\begin{array}{r}\text { Veracity (Robustness } \\
\text { Issues) }\end{array} \\
\end{array}$ & Cannot tolerate data loss. \\
\hline & Visualization & TBD \\
\hline & Data Quality & Unknown. \\
\hline & Data Types & Scanned documents \\
\hline & Data Analytics & Only after 75 years. \\
\hline $\begin{array}{r}\text { Big Data Specific } \\
\text { Challenges (Gaps) }\end{array}$ & \multicolumn{2}{|c|}{ Preserve data for a long time scale. } \\
\hline $\begin{array}{r}\text { Big Data Specific } \\
\text { Challenges in Mobility }\end{array}$ & \multicolumn{2}{|l|}{ TBD } \\
\hline $\begin{array}{r}\text { Security and Privacy } \\
\text { Requirements }\end{array}$ & \multicolumn{2}{|l|}{ Title 13 data. } \\
\hline \multicolumn{3}{|l|}{$\begin{array}{r}\text { Highlight issues for } \\
\text { generalizing this use } \\
\text { case (e.g. for ref. } \\
\text { architecture) } \\
\end{array}$} \\
\hline $\begin{array}{r}\text { More Information } \\
\text { (URLs) }\end{array}$ & & \\
\hline
\end{tabular}




\section{Government Operation> Use Case 2: NARA Accession, Search, Retrieve, Preservation}

\begin{tabular}{|c|c|c|}
\hline Use Case Title & \multicolumn{2}{|c|}{$\begin{array}{l}\text { National Archives and Records Administration Accession NARA Accession, Search, } \\
\text { Retrieve, Preservation }\end{array}$} \\
\hline Vertical (area) & \multicolumn{2}{|c|}{ Digital Archives } \\
\hline Author/Company/Email & \multicolumn{2}{|c|}{ Quyen Nguyen and Vivek Navale (NARA) } \\
\hline $\begin{array}{r}\text { Actors/Stakeholders } \\
\text { and their roles and } \\
\text { responsibilities }\end{array}$ & \multicolumn{2}{|c|}{$\begin{array}{l}\text { Agencies' Records Managers } \\
\text { NARA's Records Accessioners } \\
\text { NARA's Archivists } \\
\text { Public users }\end{array}$} \\
\hline Goals & \multicolumn{2}{|c|}{ Accession, Search, Retrieval, and Long-Term Preservation of Big Data. } \\
\hline Use Case Description & \multicolumn{2}{|c|}{$\begin{array}{l}\text { 1) Get physical and legal custody of the data. In the future, if data reside in the } \\
\text { cloud, physical custody should avoid transferring Big Data from Cloud to Cloud } \\
\text { or from Cloud to Data Center. } \\
\text { 2) Pre-process data for virus scan, identifying file format identification, removing } \\
\text { empty files } \\
\text { 3) Index } \\
\text { 4) Categorize records (sensitive, unsensitive, privacy data, etc.) } \\
\text { 5) Transform old file formats to modern formats (e.g. WordPerfect to PDF) } \\
\text { 6) E-discovery } \\
\text { 7) Search and retrieve to respond to special request } \\
\text { 8) Search and retrieve of public records by public users }\end{array}$} \\
\hline \multirow{4}{*}{$\begin{array}{r}\text { Current } \\
\text { Solutions }\end{array}$} & Compute(System) & Linux servers \\
\hline & Storage & NetApps, Hitachi, Magnetic tapes. \\
\hline & Networking & \\
\hline & Software & $\begin{array}{l}\text { Custom software, commercial search products, } \\
\text { commercial databases. }\end{array}$ \\
\hline \multirow[t]{5}{*}{$\begin{array}{r}\text { Big Data } \\
\text { Characteristics }\end{array}$} & $\begin{array}{r}\text { Data Source } \\
\text { (distributed/centralized) }\end{array}$ & $\begin{array}{l}\text { Distributed data sources from federal agencies. } \\
\text { Current solution requires transfer of those data to a } \\
\text { centralized storage. } \\
\text { In the future, those data sources may reside in different } \\
\text { Cloud environments. }\end{array}$ \\
\hline & Volume (size) & Hundreds of Terabytes, and growing. \\
\hline & $\begin{array}{r}\text { Velocity } \\
\text { (e.g. real time) }\end{array}$ & $\begin{array}{l}\text { Input rate is relatively low compared to other use cases, } \\
\text { but the trend is bursty. That is the data can arrive in } \\
\text { batches of size ranging from GB to hundreds of TB. }\end{array}$ \\
\hline & $\begin{array}{r}\text { Variety } \\
\text { (multiple datasets, } \\
\text { mashup) }\end{array}$ & $\begin{array}{l}\text { Variety data types, unstructured and structured data: } \\
\text { textual documents, emails, photos, scanned documents, } \\
\text { multimedia, social networks, web sites, databases, etc. } \\
\text { Variety of application domains, since records come } \\
\text { from different agencies. } \\
\text { Data come from variety of repositories, some of which } \\
\text { can be cloud-based in the future. }\end{array}$ \\
\hline & $\begin{array}{r}\text { Variability (rate of } \\
\text { change) }\end{array}$ & $\begin{array}{l}\text { Rate can change especially if input sources are variable, } \\
\text { some having audio, video more, some more text, and } \\
\text { other images, etc. }\end{array}$ \\
\hline
\end{tabular}




\section{Government Operation> Use Case 2: NARA Accession, Search, Retrieve, Preservation}

\begin{tabular}{|c|c|c|}
\hline Use Case Title & \multicolumn{2}{|c|}{$\begin{array}{l}\text { National Archives and Records Administration Accession NARA Accession, Search, } \\
\text { Retrieve, Preservation }\end{array}$} \\
\hline \multirow{5}{*}{$\begin{array}{r}\text { Big Data Science } \\
\text { (collection, curation, } \\
\text { analysis, } \\
\text { action) }\end{array}$} & $\begin{array}{r}\text { Veracity (Robustness } \\
\text { Issues) }\end{array}$ & $\begin{array}{l}\text { Search results should have high relevancy and high } \\
\text { recall. } \\
\text { Categorization of records should be highly accurate. }\end{array}$ \\
\hline & Visualization & TBD \\
\hline & Data Quality & Unknown. \\
\hline & Data Types & $\begin{array}{l}\text { Variety data types: textual documents, emails, photos, } \\
\text { scanned documents, multimedia, databases, etc. }\end{array}$ \\
\hline & Data Analytics & $\begin{array}{l}\text { Crawl/index; search; ranking; predictive search. } \\
\text { Data categorization (sensitive, confidential, etc.) } \\
\text { Personally Identifiable Information (PII) data detection } \\
\text { and flagging. }\end{array}$ \\
\hline $\begin{array}{l}\text { Big Data Specific } \\
\text { Challenges (Gaps) }\end{array}$ & \multicolumn{2}{|c|}{$\begin{array}{l}\text { Perform preprocessing and manage for long-term of large and varied data. } \\
\text { Search huge amount of data. } \\
\text { Ensure high relevancy and recall. } \\
\text { Data sources may be distributed in different clouds in future. }\end{array}$} \\
\hline $\begin{array}{r}\text { Big Data Specific } \\
\text { Challenges in Mobility }\end{array}$ & \multicolumn{2}{|c|}{ Mobile search must have similar interfaces/results } \\
\hline $\begin{array}{r}\text { Security and Privacy } \\
\text { Requirements }\end{array}$ & \multicolumn{2}{|c|}{ Need to be sensitive to data access restrictions. } \\
\hline $\begin{array}{r}\text { Highlight issues for } \\
\text { generalizing this use } \\
\text { case (e.g. for ref. } \\
\text { architecture) } \\
\end{array}$ & & \\
\hline $\begin{array}{r}\text { More Information } \\
\text { (URLs) }\end{array}$ & & \\
\hline
\end{tabular}




\section{Government Operation> Use Case 3: Statistical Survey Response I mprovement}

\begin{tabular}{|c|c|c|}
\hline Use Case Title & \multicolumn{2}{|c|}{ Statistical Survey Response Improvement (Adaptive Design) } \\
\hline Vertical (area) & \multicolumn{2}{|c|}{ Government Statistical Logistics } \\
\hline Author/Company/Email & \multicolumn{2}{|c|}{ Cavan Capps: U.S. Census Bureau/cavan.paul.capps@census.gov } \\
\hline $\begin{array}{r}\text { Actors/Stakeholders } \\
\text { and their roles and } \\
\text { responsibilities }\end{array}$ & \multicolumn{2}{|c|}{$\begin{array}{l}\text { U.S. statistical agencies are charged to be the leading authoritative sources about the } \\
\text { nation's people and economy, while honoring privacy and rigorously protecting } \\
\text { confidentiality. This is done by working with states, local governments and other } \\
\text { government agencies. }\end{array}$} \\
\hline Goals & \multicolumn{2}{|c|}{$\begin{array}{l}\text { To use advanced methods, that are open and scientifically objective, the statistical } \\
\text { agencies endeavor to improve the quality, the specificity and the timeliness of } \\
\text { statistics provided while reducing operational costs and maintaining the } \\
\text { confidentiality of those measured. }\end{array}$} \\
\hline Use Case Description & \multicolumn{2}{|c|}{$\begin{array}{l}\text { Survey costs are increasing as survey response declines. The goal of this work is to } \\
\text { use advanced "recommendation system techniques" using data mashed up from } \\
\text { several sources and historical survey para-data to drive operational processes in an } \\
\text { effort to increase quality and reduce the cost of field surveys. }\end{array}$} \\
\hline \multirow{4}{*}{$\begin{array}{r}\text { Current } \\
\text { Solutions }\end{array}$} & Compute(System) & Linux systems \\
\hline & Storage & SAN and Direct Storage \\
\hline & Networking & Fiber, 10 gigabit Ethernet, Infiniband 40 gigabit. \\
\hline & Software & $\begin{array}{l}\text { Hadoop, Spark, Hive, R, SAS, Mahout, Allegrograph, } \\
\text { MySQL, Oracle, Storm, BigMemory, Cassandra, Pig }\end{array}$ \\
\hline \multirow[t]{5}{*}{$\begin{array}{r}\text { Big Data } \\
\text { Characteristics }\end{array}$} & $\begin{array}{r}\text { Data Source } \\
\text { (distributed/centralized) }\end{array}$ & $\begin{array}{l}\text { Survey data, other government administrative data, } \\
\text { geographical positioning data from various sources. }\end{array}$ \\
\hline & Volume (size) & $\begin{array}{l}\text { For this particular class of operational problem } \\
\text { approximately one petabyte. }\end{array}$ \\
\hline & $\begin{array}{r}\text { Velocity } \\
\text { (e.g. real time) }\end{array}$ & $\begin{array}{l}\text { Varies, paradata from field data streamed continuously, } \\
\text { during the decennial census approximately } 150 \text { million } \\
\text { records transmitted. }\end{array}$ \\
\hline & $\begin{array}{r}\text { Variety } \\
\text { (multiple datasets, } \\
\text { mashup) }\end{array}$ & $\begin{array}{l}\text { Data is typically defined strings and numerical fields. } \\
\text { Data can be from multiple datasets mashed together for } \\
\text { analytical use. }\end{array}$ \\
\hline & $\begin{array}{r}\text { Variability (rate of } \\
\text { change) }\end{array}$ & $\begin{array}{l}\text { Varies depending on surveys in the field at a given time. } \\
\text { High rate of velocity during a decennial census. }\end{array}$ \\
\hline \multirow[t]{5}{*}{$\begin{array}{r}\text { Big Data Science } \\
\text { (collection, curation, } \\
\text { analysis, } \\
\text { action) }\end{array}$} & $\begin{array}{l}\text { Veracity (Robustness } \\
\text { Issues, semantics) }\end{array}$ & $\begin{array}{l}\text { Data must have high veracity and systems must be very } \\
\text { robust. The semantic integrity of conceptual metadata } \\
\text { concerning what exactly is measured and the resulting } \\
\text { limits of inference remain a challenge }\end{array}$ \\
\hline & Visualization & $\begin{array}{l}\text { Data visualization is useful for data review, operational } \\
\text { activity and general analysis. It continues to evolve. }\end{array}$ \\
\hline & Data Quality (syntax) & $\begin{array}{l}\text { Data quality should be high and statistically checked for } \\
\text { accuracy and reliability throughout the collection } \\
\text { process. }\end{array}$ \\
\hline & Data Types & Pre-defined ASCII strings and numerical data \\
\hline & Data Analytics & $\begin{array}{l}\text { Analytics are required for recommendation systems, } \\
\text { continued monitoring and general survey improvement. }\end{array}$ \\
\hline $\begin{array}{r}\text { Big Data Specific } \\
\text { Challenges (Gaps) }\end{array}$ & \multicolumn{2}{|c|}{$\begin{array}{l}\text { Improving recommendation systems that reduce costs and improve quality while } \\
\text { providing confidentiality safeguards that are reliable and publicly auditable. }\end{array}$} \\
\hline $\begin{array}{r}\text { Big Data Specific } \\
\text { Challenges in Mobility }\end{array}$ & \multicolumn{2}{|c|}{ Mobile access is important. } \\
\hline Security and Privacy & \multicolumn{2}{|c|}{ All data must be both confidential and secure. All processes must be auditable for } \\
\hline
\end{tabular}




\section{Government Operation> Use Case 3: Statistical Survey Response I mprovement}

\begin{tabular}{|r|l|}
\hline Requirements & security and confidentiality as required by various legal statutes. \\
\hline Highlight issues for & Recommender systems have features in common to e-commerce like Amazon, \\
generalizing this use & Netflix, UPS etc. \\
case (e.g. for ref. & \\
architecture) & \\
\hline $\begin{array}{r}\text { More Information } \\
\text { (URLs) }\end{array}$ & \\
\hline
\end{tabular}




\section{Government Operation> Use Case 4: Non-Traditional Data in Statistical Survey}

\begin{tabular}{|c|c|c|}
\hline Use Case Title & \multicolumn{2}{|c|}{ Non-Traditional Data in Statistical Survey Response Improvement (Adaptive Design) } \\
\hline Vertical (area) & \multicolumn{2}{|c|}{ Government Statistical Logistics } \\
\hline Author/Company/Email & \multicolumn{2}{|c|}{ Cavan Capps: U.S. Census Bureau / cavan.paul.capps@census.gov } \\
\hline $\begin{array}{r}\text { Actors/Stakeholders } \\
\text { and their roles and } \\
\text { responsibilities }\end{array}$ & \multicolumn{2}{|c|}{$\begin{array}{l}\text { U.S. statistical agencies are charged to be the leading authoritative sources about the } \\
\text { nation's people and economy, while honoring privacy and rigorously protecting } \\
\text { confidentiality. This is done by working with states, local governments and other } \\
\text { government agencies. }\end{array}$} \\
\hline Goals & \multicolumn{2}{|c|}{$\begin{array}{l}\text { To use advanced methods, that are open and scientifically objective, the statistical } \\
\text { agencies endeavor to improve the quality, the specificity and the timeliness of } \\
\text { statistics provided while reducing operational costs and maintaining the } \\
\text { confidentiality of those measured. }\end{array}$} \\
\hline Use Case Description & \multicolumn{2}{|c|}{$\begin{array}{l}\text { Survey costs are increasing as survey response declines. The potential of using non- } \\
\text { traditional commercial and public data sources from the web, wireless } \\
\text { communication, electronic transactions mashed up analytically with traditional } \\
\text { surveys to improve statistics for small area geographies, new measures and to } \\
\text { improve the timeliness of released statistics. }\end{array}$} \\
\hline \multirow{4}{*}{$\begin{array}{r}\text { Current } \\
\text { Solutions }\end{array}$} & Compute(System) & Linux systems \\
\hline & Storage & SAN and Direct Storage \\
\hline & Networking & Fiber, 10 gigabit Ethernet, Infiniband 40 gigabit. \\
\hline & Software & $\begin{array}{l}\text { Hadoop, Spark, Hive, R, SAS, Mahout, Allegrograph, } \\
\text { MySQL, Oracle, Storm, BigMemory, Cassandra, Pig }\end{array}$ \\
\hline \multirow[t]{5}{*}{$\begin{array}{r}\text { Big Data } \\
\text { Characteristics }\end{array}$} & $\begin{array}{r}\text { Data Source } \\
\text { (distributed/centralized) }\end{array}$ & $\begin{array}{l}\text { Survey data, other government administrative data, web } \\
\text { scrapped data, wireless data, e-transaction data, } \\
\text { potentially social media data and positioning data from } \\
\text { various sources. }\end{array}$ \\
\hline & Volume (size) & TBD \\
\hline & $\begin{array}{r}\text { Velocity } \\
\text { (e.g. real time) } \\
\end{array}$ & TBD \\
\hline & $\begin{array}{r}\text { Variety } \\
\text { (multiple datasets, } \\
\text { mashup) }\end{array}$ & $\begin{array}{l}\text { Textual data as well as the traditionally defined strings } \\
\text { and numerical fields. Data can be from multiple datasets } \\
\text { mashed together for analytical use. }\end{array}$ \\
\hline & $\begin{array}{r}\text { Variability (rate of } \\
\text { change) }\end{array}$ & TBD. \\
\hline \multirow[t]{5}{*}{$\begin{array}{r}\text { Big Data Science } \\
\text { (collection, curation, } \\
\text { analysis, } \\
\text { action) }\end{array}$} & $\begin{array}{l}\text { Veracity (Robustness } \\
\text { Issues, semantics) }\end{array}$ & $\begin{array}{l}\text { Data must have high veracity and systems must be very } \\
\text { robust. The semantic integrity of conceptual metadata } \\
\text { concerning what exactly is measured and the resulting } \\
\text { limits of inference remain a challenge }\end{array}$ \\
\hline & Visualization & $\begin{array}{l}\text { Data visualization is useful for data review, operational } \\
\text { activity and general analysis. It continues to evolve. }\end{array}$ \\
\hline & Data Quality (syntax) & $\begin{array}{l}\text { Data quality should be high and statistically checked for } \\
\text { accuracy and reliability throughout the collection } \\
\text { process. }\end{array}$ \\
\hline & Data Types & $\begin{array}{l}\text { Textual data, pre-defined ASCII strings and numerical } \\
\text { data }\end{array}$ \\
\hline & Data Analytics & $\begin{array}{l}\text { Analytics are required to create reliable estimates using } \\
\text { data from traditional survey sources, government } \\
\text { administrative data sources and non-traditional sources } \\
\text { from the digital economy. }\end{array}$ \\
\hline
\end{tabular}




\section{Government Operation> Use Case 4: Non-Traditional Data in Statistical Survey}

\begin{tabular}{|r|l|}
\hline $\begin{array}{r}\text { Big Data Specific } \\
\text { Challenges (Gaps) }\end{array}$ & $\begin{array}{l}\text { Improving analytic and modeling systems that provide reliable and robust statistical } \\
\text { estimated using data from multiple sources that are scientifically transparent and } \\
\text { while providing confidentiality safeguards that are reliable and publicly auditable. }\end{array}$ \\
\hline $\begin{array}{r}\text { Big Data Specific } \\
\text { Challenges in Mobility }\end{array}$ & Mobile access is important. \\
\hline $\begin{array}{r}\text { Security and Privacy } \\
\text { Requirements }\end{array}$ & $\begin{array}{r}\text { All data must be both confidential and secure. All processes must be auditable for } \\
\text { security and confidentiality as required by various legal statutes. }\end{array}$ \\
\hline $\begin{array}{r}\text { Highlight issues for } \\
\text { generalizing this use } \\
\text { case (e.g. for ref. } \\
\text { architecture) }\end{array}$ & $\begin{array}{r}\text { Statistical estimation that provide more detail, on a more near real time basis for less } \\
\text { cost. The reliability of estimated statistics from such "mashed up" sources still must } \\
\text { be evaluated. }\end{array}$ \\
\hline $\begin{array}{r}\text { More Information } \\
\text { (URLs) }\end{array}$ & \\
\hline
\end{tabular}




\section{Commercial> Use Case 5: Cloud Computing in Financial Industries}

\begin{tabular}{|c|c|}
\hline Use Case Title & $\begin{array}{l}\text { This use case represents one approach to implementing a BD (Big Data) strategy, within } \\
\text { a Cloud Eco-System, for FI (Financial Industries) transacting business within the United } \\
\text { States. }\end{array}$ \\
\hline Vertical (area) & $\begin{array}{l}\text { The following lines of business (LOB) include: } \\
\text { Banking, including: Commercial, Retail, Credit Cards, Consumer Finance, Corporate } \\
\text { Banking, Transaction Banking, Trade Finance, and Global Payments. } \\
\text { Securities and Investments, such as; Retail Brokerage, Private Banking/Wealth } \\
\text { Management, Institutional Brokerages, Investment Banking, Trust Banking, Asset } \\
\text { Management, Custody and Clearing Services } \\
\text { Insurance, including; Personal and Group Life, Personal and Group Property/Casualty, } \\
\text { Fixed and Variable Annuities, and Other Investments } \\
\text { Please Note: Any Public/Private entity, providing financial services within the } \\
\text { regulatory and jurisdictional risk and compliance purview of the United States, are } \\
\text { required to satisfy a complex multilayer number of regulatory governance, risk } \\
\text { management, and compliance (GRC)/ confidentiality, integrity, and availability (CIA) } \\
\text { requirements, as overseen by various jurisdictions and agencies, including; Fed., State, } \\
\text { Local and cross-border. }\end{array}$ \\
\hline Author/Company/Email & Pw Carey, Compliance Partners, LLC, pwc.pwcarey@email.com \\
\hline $\begin{array}{r}\text { Actors/Stakeholders } \\
\text { and their roles and } \\
\text { responsibilities }\end{array}$ & $\begin{array}{l}\text { Regulatory and advisory organizations and agencies including the; SEC (Securities } \\
\text { and Exchange Commission), FDIC (Federal Deposit Insurance Corporation), CFTC } \\
\text { (Commodity Futures Trading Commission), US Treasury, PCAOB (Public Company } \\
\text { Accounting and Oversight Board), COSO, CobiT, reporting supply chains and } \\
\text { stakeholders, investment community, shareholders, pension funds, executive } \\
\text { management, data custodians, and employees. } \\
\text { At each level of a financial services organization, an inter-related and inter- } \\
\text { dependent mix of duties, obligations and responsibilities are in-place, which are } \\
\text { directly responsible for the performance, preparation and transmittal of financial data, } \\
\text { thereby satisfying both the regulatory GRC and CIA of their organizations financial data. } \\
\text { This same information is directly tied to the continuing reputation, trust and } \\
\text { survivability of an organization's business. }\end{array}$ \\
\hline Goals & $\begin{array}{l}\text { The following represents one approach to developing a workable BD/FI strategy } \\
\text { within the financial services industry. Prior to initiation and switch-over, an } \\
\text { organization must perform the following baseline methodology for utilizing BD/FI } \\
\text { within a Cloud Eco-system for both public and private financial entities offering } \\
\text { financial services within the regulatory confines of the United States; Federal, State, } \\
\text { Local and/or cross-border such as the UK, EU and China. } \\
\text { Each financial services organization must approach the following disciplines } \\
\text { supporting their BD/FI initiative, with an understanding and appreciation for the impact } \\
\text { each of the following four overlaying and inter-dependent forces will play in a workable } \\
\text { implementation. } \\
\text { These four areas are: } \\
\text { 1. People (resources), } \\
\text { 2. Processes (time/cost/ROI), } \\
\text { 3. Technology (various operating systems, platforms and footprints) and } \\
\text { 4. Regulatory Governance (subject to various and multiple regulatory agencies). } \\
\text { In addition, these four areas must work through the process of being; identified, } \\
\text { analyzed, evaluated, addressed, tested, and reviewed in preparation for attending to } \\
\text { the following implementation phases: } \\
\text { 1. Project Initiation and Management Buy-in } \\
\text { 2. Risk Evaluations and Controls } \\
\text { 3. Business Impact Analysis }\end{array}$ \\
\hline
\end{tabular}




\section{Commercial> Use Case 5: Cloud Computing in Financial Industries}

\begin{tabular}{|c|c|c|}
\hline & \multicolumn{2}{|c|}{$\begin{array}{l}\text { 4. Design, Development and Testing of the Business Continuity Strategies } \\
\text { 5. Emergency Response and Operations (aka; Disaster Recovery) } \\
\text { 6. Developing and Implementing Business Continuity Plans } \\
\text { 7. Awareness and Training Programs } \\
\text { 8. Maintaining and Exercising Business Continuity, (aka: Maintaining Regulatory } \\
\text { Currency) } \\
\text { Please Note: Whenever appropriate, these eight areas should be tailored and } \\
\text { modified to fit the requirements of each organizations unique and specific corporate } \\
\text { culture and line of financial services. }\end{array}$} \\
\hline Use Case Description & \multicolumn{2}{|c|}{$\begin{array}{l}\text { Big Data as developed by Google was intended to serve as an Internet Web site } \\
\text { indexing tool to help them sort, shuffle, categorize and label the Internet. At the } \\
\text { outset, it was not viewed as a replacement for legacy information technology (IT) data } \\
\text { infrastructures. With the spin-off development within OpenGroup and Hadoop, Big } \\
\text { Data has evolved into a robust data analysis and storage tool that is still undergoing } \\
\text { development. However, in the end, Big Data is still being developed as an adjunct to } \\
\text { the current IT client/server/big iron data warehouse architectures which is better at } \\
\text { some things, than these same data warehouse environments, but not others. } \\
\text { Currently within Fl, BD/Hadoop is used for fraud detection, risk analysis and } \\
\text { assessments as well as improving the organizations knowledge and understanding of } \\
\text { the customers via a strategy known as....'know your customer', pretty clever, eh? } \\
\text { However, this strategy still must be following a well thought out taxonomy that } \\
\text { satisfies the entities unique, and individual requirements. One such strategy is the } \\
\text { following formal methodology which address two fundamental yet paramount } \\
\text { questions; "What are we doing"? and "Why are we doing it"? } \\
\text { 1). Policy Statement/Project Charter (Goal of the Plan, Reasons and } \\
\text { Resources....define each), } \\
\text { 2). Business Impact Analysis (how does effort improve our business services), } \\
\text { 3). Identify System-wide Policies, Procedures and Requirements, } \\
\text { 4). Identify Best Practices for Implementation (including Change Management/ } \\
\text { Configuration Management) and/or Future Enhancements, } \\
\text { 5). Plan B-Recovery Strategies (how and what will need to be recovered, if } \\
\text { necessary), } \\
\text { 6). Plan Development (Write the Plan and Implement the Plan Elements), } \\
\text { 7). Plan buy-in and Testing (important everyone Knows the Plan, and Knows What to } \\
\text { Do), and } \\
\text { 8). Implement the Plan (then identify and fix gaps during first } 3 \text { months, } 6 \text { months, } \\
\text { and annually after initial implementation) } \\
\text { 9). Maintenance (Continuous monitoring and updates to reflect the current } \\
\text { enterprise environment) } \\
\text { 10). Lastly, System Retirement }\end{array}$} \\
\hline $\begin{array}{r}\text { Current } \\
\text { Solutions }\end{array}$ & Compute(System) & $\begin{array}{l}\text { Currently, Big Data/Hadoop within a Cloud Eco-system } \\
\text { within the FI is operating as part of a hybrid system, with } \\
\text { BD being utilized as a useful tool for conducting risk and } \\
\text { fraud analysis, in addition to assisting in organizations in } \\
\text { the process of ('know your customer'). These are three } \\
\text { areas where BD has proven to be good at; } \\
\text { 1. detecting fraud, } \\
\text { 2. associated risks and a } \\
\text { 3. 'know your customer' strategy. } \\
\text { At the same time, the traditional client/server/data } \\
\text { warehouse/RDBMS are used for the handling, processing, }\end{array}$ \\
\hline
\end{tabular}




\section{Commercial> Use Case 5: Cloud Computing in Financial Industries}

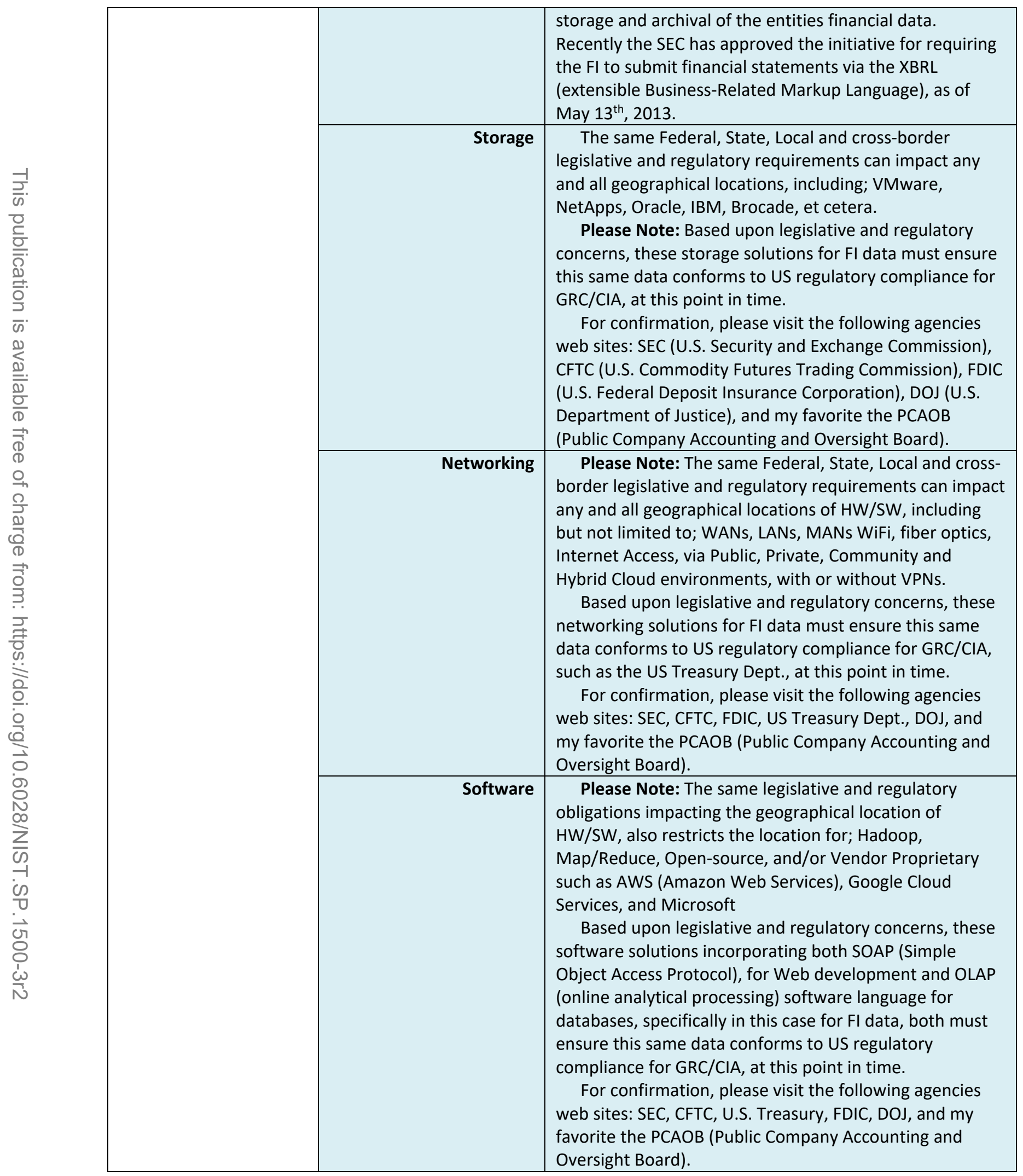




\section{Commercial> Use Case 5: Cloud Computing in Financial I ndustries}

\begin{tabular}{|c|c|c|}
\hline \multirow[t]{5}{*}{$\begin{array}{r}\text { Big Data } \\
\text { Characteristics }\end{array}$} & $\begin{array}{r}\text { Data Source (distributed/ } \\
\text { centralized) }\end{array}$ & $\begin{array}{l}\text { Please Note: The same legislative and regulatory } \\
\text { obligations impacting the geographical location of } \\
\text { HW/SW, also impacts the location for; both } \\
\text { distributed/centralized data sources flowing into HA/DR } \\
\text { Environment and HVSs (Hosted Virtual Servers), such as } \\
\text { the following constructs: DC1---> VMWare/KVM (Clusters, } \\
\text { w/Virtual Firewalls), Data link-Vmware Link-Vmotion Link- } \\
\text { Network Link, Multiple PB of NaaS (Network as a Service), } \\
\text { DC2--->, VMWare/KVM (Clusters w/Virtual Firewalls), } \\
\text { DataLink (Vmware Link, Vmotion Link, Network Link), } \\
\text { Multiple PB of NaaS, (Requires Fail-Over Virtualization), } \\
\text { among other considerations. } \\
\text { Based upon legislative and regulatory concerns, these } \\
\text { data source solutions, either distributed and/or } \\
\text { centralized for FI data, must ensure this same data } \\
\text { conforms to US regulatory compliance for GRC/CIA, at this } \\
\text { point in time. } \\
\text { For confirmation, please visit the following agencies } \\
\text { web sites: SEC, CFTC, US Treasury, FDIC, DOJ, and my } \\
\text { favorite the PCAOB (Public Company Accounting and } \\
\text { Oversight Board). }\end{array}$ \\
\hline & Volume (size) & $\begin{array}{l}\text { Tera-bytes up to Peta-bytes. } \\
\text { Please Note: This is a 'Floppy Free Zone'. }\end{array}$ \\
\hline & $\begin{array}{r}\text { Velocity } \\
\text { (e.g. real time) }\end{array}$ & $\begin{array}{l}\text { Velocity is more important for fraud detection, risk } \\
\text { assessments and the 'know your customer' initiative } \\
\text { within the BD FI. } \\
\text { Please Note: However, based upon legislative and } \\
\text { regulatory concerns, velocity is not at issue regarding BD } \\
\text { solutions for FI data, except for fraud detection, risk } \\
\text { analysis and customer analysis. } \\
\text { Based upon legislative and regulatory restrictions, } \\
\text { velocity is not at issue, rather the primary concern for FI } \\
\text { data, is that it must satisfy all US regulatory compliance } \\
\text { obligations for GRC/CIA, at this point in time. }\end{array}$ \\
\hline & $\begin{array}{r}\text { Variety } \\
\text { (multiple datasets, mash- } \\
\text { up) }\end{array}$ & $\begin{array}{l}\text { Multiple virtual environments either operating within } \\
\text { a batch processing architecture or a hot-swappable } \\
\text { parallel architecture supporting fraud detection, risk } \\
\text { assessments and customer service solutions. } \\
\text { Please Note: Based upon legislative and regulatory } \\
\text { concerns, variety is not at issue regarding BD solutions for } \\
\text { FI data within a Cloud Eco-system, except for fraud } \\
\text { detection, risk analysis and customer analysis. } \\
\text { Based upon legislative and regulatory restrictions, } \\
\text { variety is not at issue, rather the primary concern for FI } \\
\text { data, is that it must satisfy all US regulatory compliance } \\
\text { obligations for } \mathrm{GRC} / \mathrm{CIA} \text {, at this point in time. }\end{array}$ \\
\hline & $\begin{array}{r}\text { Variability (rate of } \\
\text { change) }\end{array}$ & $\begin{array}{l}\text { Please Note: Based upon legislative and regulatory } \\
\text { concerns, variability is not at issue regarding BD solutions } \\
\text { for FI data within a Cloud Eco-system, except for fraud } \\
\text { detection, risk analysis and customer analysis. }\end{array}$ \\
\hline
\end{tabular}




\section{Commercial> Use Case 5: Cloud Computing in Financial I ndustries}

\begin{tabular}{|c|c|c|}
\hline & & $\begin{array}{l}\text { Based upon legislative and regulatory restrictions, } \\
\text { variability is not at issue, rather the primary concern for } \\
\text { FI data, is that it must satisfy all US regulatory compliance } \\
\text { obligations for GRC/CIA, at this point in time. } \\
\text { Variability with BD FI within a Cloud Eco-System will } \\
\text { depending upon the strength and completeness of the } \\
\text { SLA agreements, the costs associated with (CapEx), and } \\
\text { depending upon the requirements of the business. }\end{array}$ \\
\hline \multirow[t]{4}{*}{$\begin{array}{r}\text { Big Data Science } \\
\text { (collection, curation, } \\
\text { analysis, } \\
\text { action) }\end{array}$} & $\begin{array}{r}\text { Veracity (Robustness } \\
\text { Issues) }\end{array}$ & $\begin{array}{l}\text { Please Note: Based upon legislative and regulatory } \\
\text { concerns, veracity is not at issue regarding BD solutions } \\
\text { for FI data within a Cloud Eco-system, except for fraud } \\
\text { detection, risk analysis and customer analysis. } \\
\text { Based upon legislative and regulatory restrictions, } \\
\text { veracity is not at issue, rather the primary concern for FI } \\
\text { data, is that it must satisfy all US regulatory compliance } \\
\text { obligations for GRC/CIA, at this point in time. } \\
\text { Within a Big Data Cloud Eco-System, data integrity is } \\
\text { important over the entire life cycle of the organization } \\
\text { due to regulatory and compliance issues related to } \\
\text { individual data privacy and security, in the areas of CIA } \\
\text { and GRC requirements. }\end{array}$ \\
\hline & Visualization & $\begin{array}{l}\text { Please Note: Based upon legislative and regulatory } \\
\text { concerns, visualization is not at issue regarding BD } \\
\text { solutions for FI data, except for fraud detection, risk } \\
\text { analysis and customer analysis, FI data is handled by } \\
\text { traditional client/server/data warehouse big iron servers. } \\
\text { Based upon legislative and regulatory restrictions, } \\
\text { visualization is not at issue, rather the primary concern } \\
\text { for FI data, is that it must satisfy all US regulatory } \\
\text { compliance obligations for GRC/CIA, at this point in time. } \\
\text { Data integrity within BD is critical and essential over } \\
\text { the entire life-cycle of the organization due to regulatory } \\
\text { and compliance issues related to CIA and GRC } \\
\text { requirements. }\end{array}$ \\
\hline & Data Quality & $\begin{array}{l}\text { Please Note: Based upon legislative and regulatory } \\
\text { concerns, data quality will always be an issue, regardless } \\
\text { of the industry or platform. } \\
\text { Based upon legislative and regulatory restrictions, } \\
\text { data quality is at the core of data integrity, and is the } \\
\text { primary concern for FI data, in that it must satisfy all US } \\
\text { regulatory compliance obligations for GRC/CIA, at this } \\
\text { point in time. } \\
\text { For BD/FI data, data integrity is critical and essential } \\
\text { over the entire life-cycle of the organization due to } \\
\text { regulatory and compliance issues related to CIA and GRC } \\
\text { requirements. }\end{array}$ \\
\hline & Data Types & $\begin{array}{l}\text { Please Note: Based upon legislative and regulatory } \\
\text { concerns, data types are important in that it must have a } \\
\text { degree of consistency and especially survivability during } \\
\text { audits and digital forensic investigations where the data } \\
\text { format deterioration can negatively impact both an audit }\end{array}$ \\
\hline
\end{tabular}




\section{Commercial> Use Case 5: Cloud Computing in Financial I ndustries}

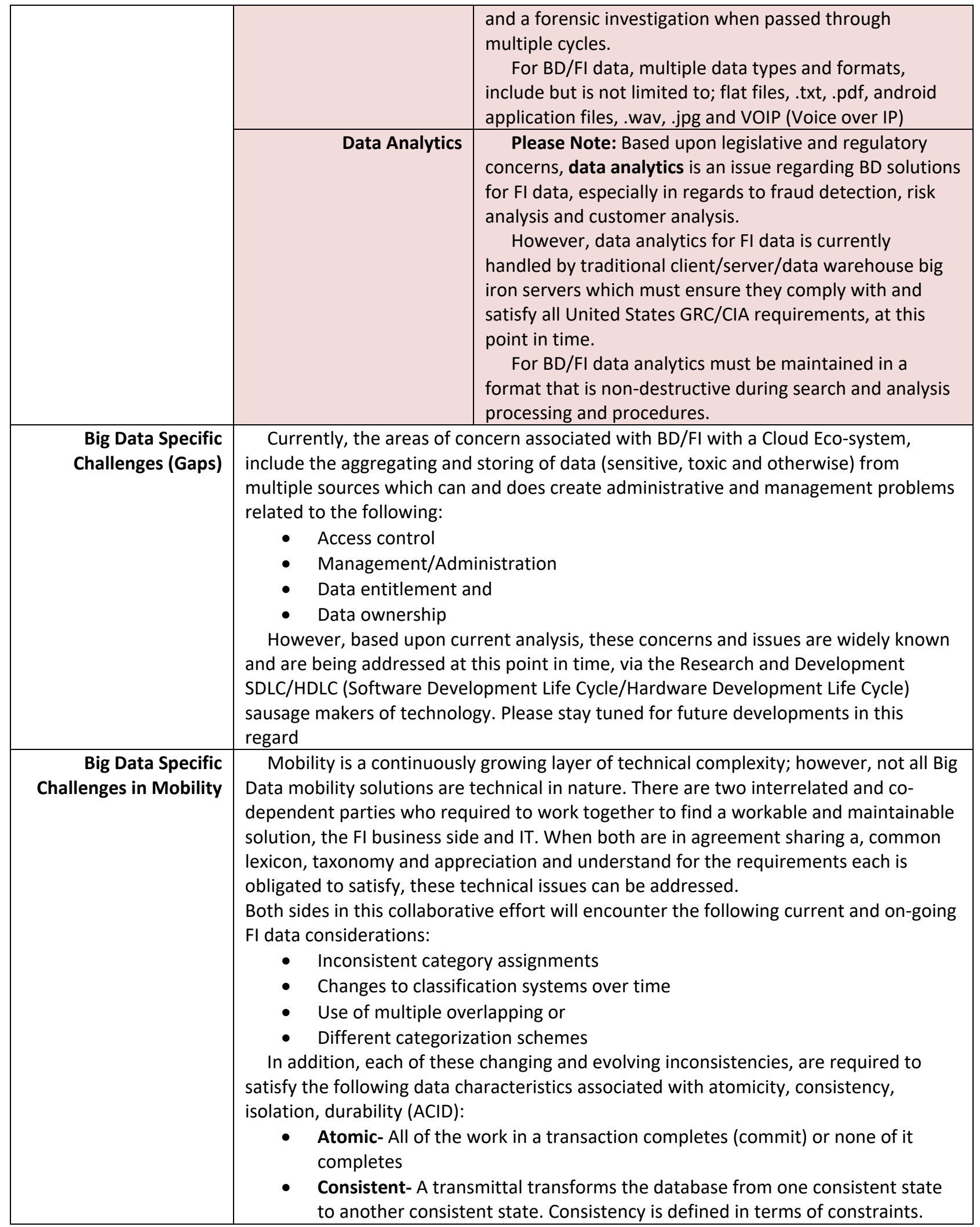




\section{Commercial> Use Case 5: Cloud Computing in Financial I ndustries}

\begin{tabular}{|c|c|}
\hline & $\begin{array}{l}\text { - Isolated- The results of any changes made during a transaction are not visible } \\
\text { until the transaction has committed. } \\
\text { - Durable- The results of a committed transaction survive failures. } \\
\text { When each of these data categories is satisfied, well, it's a glorious thing. } \\
\text { Unfortunately, sometimes glory is not in the room, however, that does not mean we } \\
\text { give up the effort to resolve these issues. }\end{array}$ \\
\hline $\begin{array}{r}\text { Security and Privacy } \\
\text { Requirements }\end{array}$ & $\begin{array}{l}\text { No amount of security and privacy due diligence will make up for the innate } \\
\text { deficiencies associated with human nature that creep into any program and/or } \\
\text { strategy. Currently, the BD/FI must contend with a growing number of risk buckets, } \\
\text { such as: } \\
\text { - AML-Anti-Money Laundering } \\
\text { - CDD- Client Due Diligence } \\
\text { - Watch-lists } \\
\text { - FCPA - Foreign Corrupt Practices Act } \\
\text {...to name a few. } \\
\text { For a reality check, please consider Mr. Harry M. Markopolos' nine-year effort to get } \\
\text { the SEC among other agencies to do their job and shut down Mr. Bernard Madoff's } \\
\text { billion dollar Ponzi scheme. } \\
\text { However, that aside, identifying and addressing the privacy/security requirements of } \\
\text { the FI, providing services within a BD/Cloud Eco-system, via continuous improvements } \\
\text { in: } \\
\text { 1. technology, } \\
\text { 2. processes, } \\
\text { 3. procedures, } \\
\text { 4. people and } \\
\text { 5. regulatory jurisdictions } \\
\text {...is a far better choice for both the individual and the organization, especially when } \\
\text { considering the alternative. } \\
\text { Utilizing a layered approach, this strategy can be broken down into the following sub } \\
\text { categories: } \\
\text { 1. Maintaining operational resilience } \\
\text { 2. Protecting valuable assets } \\
\text { 3. Controlling system accounts } \\
\text { 4. Managing security services effectively, and } \\
\text { 5. Maintaining operational resilience } \\
\text { For additional background security and privacy solutions addressing both security } \\
\text { and privacy, we'll refer you to the two following organizations: } \\
\text { - ISACA (International Society of Auditors and Computer Analysts) } \\
\text { - isc2 (International Security Computer and Systems Auditors) }\end{array}$ \\
\hline $\begin{array}{r}\text { Highlight issues for } \\
\text { generalizing this use case } \\
\text { (e.g. for ref. } \\
\text { architecture) }\end{array}$ & $\begin{array}{l}\text { Areas of concern include the aggregating and storing data from multiple sources can } \\
\text { create problems related to the following: } \\
\text { - Access control } \\
\text { - Management/Administration } \\
\text { - Data entitlement and } \\
\text { Each of these areas is being improved upon, yet they still must be considered and } \\
\text { addressed, via access control solutions, and SIEM (Security Incident/Event } \\
\text { Management) tools. }\end{array}$ \\
\hline
\end{tabular}




\section{Commercial> Use Case 5: Cloud Computing in Financial I ndustries}

\begin{tabular}{|c|c|}
\hline & $\begin{array}{l}\text { I don't believe we're there yet, based upon current security concerns mentioned } \\
\text { whenever Big Data/Hadoop within a Cloud Eco-system is brought up in polite } \\
\text { conversation. } \\
\text { Current and on-going challenges to implementing BD Finance within a Cloud Eco, as } \\
\text { well as traditional client/server data warehouse architectures, include the following } \\
\text { areas of Financial Accounting under both US GAAP (U.S. Generally Accepted Accounting } \\
\text { Practices) or IFRS (International Financial Reporting Standards): } \\
\text { XBRL (extensible Business-Related Markup Language) } \\
\text { Consistency (terminology, formatting, technologies, regulatory gaps) } \\
\text { SEC mandated use of XBRL (extensible Business-Related Markup Language) for } \\
\text { regulatory financial reporting. } \\
\text { SEC, GAAP/IFRS and the yet to be fully resolved new financial legislation impacting } \\
\text { reporting requirements are changing and point to trying to improve the } \\
\text { implementation, testing, training, reporting and communication best practices } \\
\text { required of an independent auditor, regarding: } \\
\text { Auditing, Auditor's reports, Control self-assessments, Financial audits, GAAS / ISAs, } \\
\text { Internal audits, and the Sarbanes-Oxley Act of } 2002 \text { (SOX). }\end{array}$ \\
\hline More Information (URLs) & $\begin{array}{l}\text { 1. Cloud Security Alliance Big Data Working Group, "Top } 10 \text { Challenges in Big Data } \\
\text { Security and Privacy", } 2012 . \\
\text { 2. The IFRS, Securities and Markets Working Group, http://www.xbrl-eu.org } \\
\text { 3. IEEE Big Data conference } \\
\text { http://www.ischool.drexel.edu/bigdata/bigdata2013/topics.htm } \\
\text { 4. Map/Reduce http://www.mapreduce.org. } \\
\text { 5. PCAOB http://www.pcaob.org } \\
\text { 6. http://www.ey.com/GL/en/Industries/Financial-Services/Insurance } \\
\text { 7. http://www.treasury.gov/resource-center/fin-mkts/Pages/default.aspx } \\
\text { 8. CFTC http://www.cftc.org } \\
\text { 9. SEC http://www.sec.gov } \\
\text { 10. FDIC http://www.fdic.gov } \\
\text { 11. COSO http://www.coso.org } \\
\text { 12. isc2 International Information Systems Security Certification Consortium, Inc.: } \\
\text { http://www.isc2.org } \\
\text { 13. ISACA Information Systems Audit and Control Association: http://www.isca.org } \\
\text { 14. IFARS http://www.ifars.org } \\
\text { 15. Apache http://www.opengroup.org } \\
\text { 16. http://www.computerworld.com/s/article/print/9221652/IT must prepare for H } \\
\text { adoop security issues?tax ... } \\
\text { 17. "No One Would Listen: A True Financial Thriller" (hard-cover book). Hoboken, NJ: } \\
\text { John Wiley \& Sons. March 2010. Retrieved April 30, 2010. ISBN 978-0-470-55373-2 } \\
\text { 18. Assessing the Madoff Ponzi Scheme and Regulatory Failures (Archive of: } \\
\text { Subcommittee on Capital Markets, Insurance, and Government Sponsored } \\
\text { Enterprises Hearing) (http://financialserv.edgeboss.net/wmedia/ } \\
\text { financialserv/hearing020409.wvx) (Windows Media). U.S. House Financial Services } \\
\text { Committee. February 4, 2009. Retrieved June 29, 2009. } \\
\text { 19. COSO, The Committee of Sponsoring Organizations of the Treadway Commission } \\
\text { (COSO), Copyright@ 2013, http://www.coso.org. } \\
\text { 20. (ITIL) Information Technology Infrastructure Library, Copyright@ 2007-13 APM } \\
\text { Group Ltd. All rights reserved, Registered in England No. 2861902, http://www.itil- } \\
\text { officialsite.com. } \\
\text { 21. CobiT, Ver. 5.0, 2013, ISACA, Information Systems Audit and Control Association, (a } \\
\text { framework for IT Governance and Controls), http://www.isaca.org. }\end{array}$ \\
\hline
\end{tabular}




\section{Commercial> Use Case 5: Cloud Computing in Financial I ndustries}

22. TOGAF, Ver. 9.1, The Open Group Architecture Framework (a framework for IT architecture), http://www.opengroup.org.

23. ISO/IEC 27000:2012 Info. Security Mgt., International Organization for Standardization and the International Electrotechnical Commission, http://www.standards.iso.org/

Note: Please feel free to improve our INITIAL DRAFT, Ver. 0.1, August 25 ${ }^{\text {th }}, \mathbf{2 0 1 3}$.....as we do not consider our efforts to be pearls, at this point in time...... Respectfully yours, Pw Carey, Compliance Partners, 


\section{Commercial> Use Case 6: Mendeley-An International Network of Research}

\begin{tabular}{|c|c|c|}
\hline Use Case Title & \multicolumn{2}{|c|}{ Mendeley - An International Network of Research } \\
\hline Vertical (area) & \multicolumn{2}{|c|}{ Commercial Cloud Consumer Services } \\
\hline Author/Company/Email & \multicolumn{2}{|c|}{ William Gunn / Mendeley / william.gunn@mendeley.com } \\
\hline $\begin{array}{r}\text { Actors/Stakeholders } \\
\text { and their roles and } \\
\text { responsibilities }\end{array}$ & \multicolumn{2}{|c|}{ Researchers, librarians, publishers, and funding organizations. } \\
\hline Goals & \multicolumn{2}{|c|}{$\begin{array}{l}\text { To promote more rapid advancement in scientific research by enabling researchers } \\
\text { to efficiently collaborate, librarians to understand researcher needs, publishers to } \\
\text { distribute research findings more quickly and broadly, and funding organizations to } \\
\text { better understand the impact of the projects they fund. }\end{array}$} \\
\hline Use Case Description & \multicolumn{2}{|c|}{$\begin{array}{l}\text { Mendeley has built a database of research documents and facilitates the creation of } \\
\text { shared bibliographies. Mendeley uses the information collected about research } \\
\text { reading patterns and other activities conducted via the software to build more } \\
\text { efficient literature discovery and analysis tools. Text mining and classification } \\
\text { systems enables automatic recommendation of relevant research, improving the } \\
\text { cost and performance of research teams, particularly those engaged in curation of } \\
\text { literature on a particular subject, such as the Mouse Genome Informatics group at } \\
\text { Jackson Labs, which has a large team of manual curators who scan the literature. } \\
\text { Other use cases include enabling publishers to more rapidly disseminate } \\
\text { publications, facilitating research institutions and librarians with data management } \\
\text { plan compliance, and enabling funders to better understand the impact of the work } \\
\text { they fund via real-time data on the access and use of funded research. }\end{array}$} \\
\hline \multirow{4}{*}{$\begin{array}{r}\text { Current } \\
\text { Solutions }\end{array}$} & Compute(System) & Amazon EC2 \\
\hline & Storage & HDFS Amazon S3 \\
\hline & Networking & $\begin{array}{l}\text { Client-server connections between Mendeley and end } \\
\text { user machines, connections between Mendeley offices } \\
\text { and Amazon services. }\end{array}$ \\
\hline & Software & Hadoop, Scribe, Hive, Mahout, Python \\
\hline \multirow[t]{5}{*}{$\begin{array}{r}\text { Big Data } \\
\text { Characteristics }\end{array}$} & $\begin{array}{r}\text { Data Source } \\
\text { (distributed/centralized) }\end{array}$ & Distributed and centralized \\
\hline & Volume (size) & 15TB presently, growing about $1 \mathrm{~TB} /$ month \\
\hline & $\begin{array}{r}\text { Velocity } \\
\text { (e.g. real time) }\end{array}$ & $\begin{array}{l}\text { Currently Hadoop batch jobs are scheduled daily, but } \\
\text { work has begun on real-time recommendation }\end{array}$ \\
\hline & $\begin{array}{r}\text { Variety } \\
\text { (multiple datasets, } \\
\text { mashup) }\end{array}$ & $\begin{array}{l}\text { PDF documents and log files of social network and client } \\
\text { activities }\end{array}$ \\
\hline & $\begin{array}{r}\text { Variability (rate of } \\
\text { change) }\end{array}$ & $\begin{array}{l}\text { Currently a high rate of growth as more researchers sign } \\
\text { up for the service, highly fluctuating activity over the } \\
\text { course of the year }\end{array}$ \\
\hline \multirow[t]{4}{*}{$\begin{array}{r}\text { Big Data Science } \\
\text { (collection, curation, } \\
\text { analysis, } \\
\text { action) }\end{array}$} & $\begin{array}{r}\text { Veracity (Robustness } \\
\text { Issues) }\end{array}$ & $\begin{array}{l}\text { Metadata extraction from PDFs is variable, it's } \\
\text { challenging to identify duplicates, there's no universal } \\
\text { identifier system for documents or authors (though } \\
\text { ORCID proposes to be this) }\end{array}$ \\
\hline & Visualization & $\begin{array}{l}\text { Network visualization via Gephi, scatterplots of } \\
\text { readership vs. citation rate, etc. }\end{array}$ \\
\hline & Data Quality & $\begin{array}{l}90 \% \text { correct metadata extraction according to } \\
\text { comparison with Crossref, Pubmed, and Arxiv }\end{array}$ \\
\hline & Data Types & $\begin{array}{l}\text { Mostly PDFs, some image, spreadsheet, and } \\
\text { presentation files }\end{array}$ \\
\hline
\end{tabular}




\section{Commercial> Use Case 6: Mendeley-An International Network of Research}

\begin{tabular}{|r|l|l|}
\hline & Data Analytics & $\begin{array}{l}\text { Standard libraries for machine learning and analytics, } \\
\text { LDA, custom built reporting tools for aggregating } \\
\text { readership and social activities per document }\end{array}$ \\
\hline $\begin{array}{r}\text { Big Data Specific } \\
\text { Challenges (Gaps) }\end{array}$ & $\begin{array}{l}\text { The database contains } \approx 400 \mathrm{M} \text { documents, roughly 80M unique documents, and } \\
\text { receives 5-700k new uploads on a weekday. Thus, a major challenge is clustering } \\
\text { matching documents together in a computationally efficient way (scalable and } \\
\text { parallelized) when they're uploaded from different sources and have been slightly } \\
\text { modified via third-part annotation tools or publisher watermarks and cover pages }\end{array}$ \\
\hline $\begin{array}{r}\text { Big Data Specific } \\
\text { Challenges in Mobility }\end{array}$ & $\begin{array}{l}\text { Delivering content and services to various computing platforms from Windows } \\
\text { desktops to Android and iOS mobile devices }\end{array}$ \\
\hline $\begin{array}{r}\text { Security and Privacy } \\
\text { Requirements }\end{array}$ & $\begin{array}{l}\text { Researchers often want to keep what they're reading private, especially industry } \\
\text { researchers, so the data about who's reading what has access controls. }\end{array}$ \\
\hline $\begin{array}{r}\text { Highlight issues for } \\
\text { generalizing this use } \\
\text { case (e.g. for ref. } \\
\text { architecture) }\end{array}$ & $\begin{array}{l}\text { This use case could be generalized to providing content-based recommendations to } \\
\text { various scenarios of information consumption }\end{array}$ \\
\hline $\begin{array}{r}\text { More Information } \\
\text { (URLs) }\end{array}$ & http://mendeley.com http://dev.mendeley.com \\
\hline
\end{tabular}




\section{Commercial> Use Case 7: Netflix Movie Service}

\begin{tabular}{|c|c|c|}
\hline Use Case Title & \multicolumn{2}{|l|}{ Netflix Movie Service } \\
\hline Vertical (area) & \multicolumn{2}{|c|}{ Commercial Cloud Consumer Services } \\
\hline Author/Company/Email & \multicolumn{2}{|c|}{ Geoffrey Fox, Indiana University gcf@indiana.edu } \\
\hline $\begin{array}{r}\text { Actors/Stakeholders } \\
\text { and their roles and } \\
\text { responsibilities }\end{array}$ & \multicolumn{2}{|c|}{$\begin{array}{l}\text { Netflix Company (Grow sustainable Business), Cloud Provider (Support streaming } \\
\text { and data analysis), Client user (Identify and watch good movies on demand) }\end{array}$} \\
\hline Goals & \multicolumn{2}{|c|}{$\begin{array}{l}\text { Allow streaming of user selected movies to satisfy multiple objectives (for different } \\
\text { stakeholders) -- especially retaining subscribers. Find best possible ordering of a set } \\
\text { of videos for a user (household) within a given context in real time; maximize movie } \\
\text { consumption. }\end{array}$} \\
\hline Use Case Description & \multicolumn{2}{|c|}{$\begin{array}{l}\text { Digital movies stored in cloud with metadata; user profiles and rankings for small } \\
\text { fraction of movies for each user. Use multiple criteria - content based recommender } \\
\text { system; user-based recommender system; diversity. Refine algorithms continuously } \\
\text { with A/B testing. }\end{array}$} \\
\hline \multirow{4}{*}{$\begin{array}{r}\text { Current } \\
\text { Solutions }\end{array}$} & Compute(System) & Amazon Web Services AWS \\
\hline & Storage & Uses Cassandra NoSQL technology with Hive, Teradata \\
\hline & Networking & $\begin{array}{l}\text { Need Content Delivery System to support effective } \\
\text { streaming video }\end{array}$ \\
\hline & Software & Hadoop and Pig; Cassandra; Teradata \\
\hline \multirow[t]{5}{*}{$\begin{array}{r}\text { Big Data } \\
\text { Characteristics }\end{array}$} & $\begin{array}{r}\text { Data Source } \\
\text { (distributed/centralized) }\end{array}$ & $\begin{array}{l}\text { Add movies institutionally. Collect user rankings and } \\
\text { profiles in a distributed fashion }\end{array}$ \\
\hline & Volume (size) & $\begin{array}{l}\text { Summer } 2012.25 \text { million subscribers; } 4 \text { million ratings } \\
\text { per day; } 3 \text { million searches per day; } 1 \text { billion hours } \\
\text { streamed in June } 2012 \text {. Cloud storage } 2 \text { petabytes (June } \\
\text { 2013) }\end{array}$ \\
\hline & $\begin{array}{r}\text { Velocity } \\
\text { (e.g. real time) }\end{array}$ & $\begin{array}{l}\text { Media (video and properties) and Rankings continually } \\
\text { updated }\end{array}$ \\
\hline & $\begin{array}{r}\text { Variety } \\
\text { (multiple datasets, } \\
\text { mashup) }\end{array}$ & $\begin{array}{l}\text { Data varies from digital media to user rankings, user } \\
\text { profiles and media properties for content-based } \\
\text { recommendations }\end{array}$ \\
\hline & $\begin{array}{r}\text { Variability (rate of } \\
\text { change) }\end{array}$ & $\begin{array}{l}\text { Very competitive business. Need to aware of other } \\
\text { companies and trends in both content (which Movies } \\
\text { are hot) and technology. Need to investigate new } \\
\text { business initiatives such as Netflix sponsored content }\end{array}$ \\
\hline \multirow{5}{*}{$\begin{array}{r}\text { Big Data Science } \\
\text { (collection, curation, } \\
\text { analysis, } \\
\text { action) }\end{array}$} & $\begin{array}{r}\text { Veracity (Robustness } \\
\text { Issues) }\end{array}$ & Success of business requires excellent quality of service \\
\hline & Visualization & $\begin{array}{l}\text { Streaming media and quality user-experience to allow } \\
\text { choice of content }\end{array}$ \\
\hline & Data Quality & $\begin{array}{l}\text { Rankings are intrinsically "rough" data and need robust } \\
\text { learning algorithms }\end{array}$ \\
\hline & Data Types & Media content, user profiles, "bag" of user rankings \\
\hline & Data Analytics & $\begin{array}{l}\text { Recommender systems and streaming video delivery. } \\
\text { Recommender systems are always personalized and } \\
\text { use logistic/linear regression, elastic nets, matrix } \\
\text { factorization, clustering, latent Dirichlet allocation, } \\
\text { association rules, gradient boosted decision trees and } \\
\text { others. Winner of Netflix competition (to improve } \\
\text { ratings by } 10 \% \text { ) combined over } 100 \text { different } \\
\text { algorithms. }\end{array}$ \\
\hline
\end{tabular}




\section{Commercial> Use Case 7: Netflix Movie Service}

\begin{tabular}{|r|l|}
\hline $\begin{array}{r}\text { Big Data Specific } \\
\text { Challenges (Gaps) }\end{array}$ & Analytics needs continued monitoring and improvement. \\
\hline $\begin{array}{r}\text { Big Data Specific } \\
\text { Challenges in Mobility }\end{array}$ & Mobile access important \\
\hline $\begin{array}{r}\text { Security and Privacy } \\
\text { Requirements }\end{array}$ & Need to preserve privacy for users and digital rights for media. \\
\hline $\begin{array}{r}\text { Highlight issues for } \\
\text { generalizing this use } \\
\text { case (e.g. for ref. } \\
\text { architecture) }\end{array}$ & $\begin{array}{l}\text { Recommender systems have features in common to e-commerce like Amazon. } \\
\text { Streaming video has features in common with other content providing services like } \\
\text { iTunes, Google Play, Pandora and Last.fm }\end{array}$ \\
\hline $\begin{array}{r}\text { More Information } \\
\text { (URLs) }\end{array}$ & $\begin{array}{l}\text { http://www.slideshare.net/xamat/building-largescale-realworld-recommender- } \\
\text { systems-recsys2012-tutorial by Xavier Amatriain } \\
\text { http://techblog.netflix.com/ }\end{array}$ \\
\hline
\end{tabular}

242 


\section{Commercial> Use Case 8: Web Search}

\begin{tabular}{|c|c|c|}
\hline Use Case Title & \multicolumn{2}{|c|}{ Web Search (Bing, Google, Yahoo...) } \\
\hline Vertical (area) & \multicolumn{2}{|c|}{ Commercial Cloud Consumer Services } \\
\hline Author/Company/Email & \multicolumn{2}{|c|}{ Geoffrey Fox, Indiana University gcf@indiana.edu } \\
\hline $\begin{array}{r}\text { Actors/Stakeholders } \\
\text { and their roles and } \\
\text { responsibilities }\end{array}$ & \multicolumn{2}{|c|}{$\begin{array}{l}\text { Owners of web information being searched; search engine companies; advertisers; } \\
\text { users }\end{array}$} \\
\hline Goals & \multicolumn{2}{|c|}{$\begin{array}{l}\text { Return in } \approx 0.1 \text { seconds, the results of a search based on average of } 3 \text { words; } \\
\text { important to maximize "precision@ @10"; number of great responses in top } 10 \text { ranked } \\
\text { results }\end{array}$} \\
\hline Use Case Description & \multicolumn{2}{|c|}{$\begin{array}{l}\text { 1) Crawl the web; 2) Pre-process data to get searchable things (words, positions); } \\
\text { 3) Form Inverted Index mapping words to documents; 4) Rank relevance of } \\
\text { documents: PageRank; 5) Lots of technology for advertising, "reverse engineering } \\
\text { ranking" "preventing reverse engineering"; 6) Clustering of documents into topics (as } \\
\text { in Google News) 7) Update results efficiently }\end{array}$} \\
\hline \multirow{4}{*}{$\begin{array}{r}\text { Current } \\
\text { Solutions }\end{array}$} & Compute(System) & Large Clouds \\
\hline & Storage & $\begin{array}{l}\text { Inverted Index not huge; crawled documents are } \\
\text { petabytes of text - rich media much more }\end{array}$ \\
\hline & Networking & $\begin{array}{l}\text { Need excellent external network links; most operations } \\
\text { pleasingly parallel and } \mathrm{I} / \mathrm{O} \text { sensitive. High performance } \\
\text { internal network not needed }\end{array}$ \\
\hline & Software & $\begin{array}{l}\text { Map/Reduce + Bigtable; Dryad + Cosmos. PageRank. } \\
\text { Final step essentially a recommender engine }\end{array}$ \\
\hline \multirow[t]{5}{*}{$\begin{array}{r}\text { Big Data } \\
\text { Characteristics }\end{array}$} & $\begin{array}{r}\text { Data Source } \\
\text { (distributed/centralized) } \\
\end{array}$ & Distributed web sites \\
\hline & Volume (size) & $\begin{array}{l}\text { 45B web pages total, } 500 \mathrm{M} \text { photos uploaded each day, } \\
100 \text { hours of video uploaded to YouTube each minute }\end{array}$ \\
\hline & $\begin{array}{r}\text { Velocity } \\
\text { (e.g. real time) }\end{array}$ & Data continually updated \\
\hline & $\begin{array}{r}\text { Variety } \\
\text { (multiple datasets, } \\
\text { mashup) }\end{array}$ & $\begin{array}{l}\text { Rich set of functions. After processing, data similar for } \\
\text { each page (except for media types) }\end{array}$ \\
\hline & $\begin{array}{r}\text { Variability (rate of } \\
\text { change) }\end{array}$ & Average page has life of a few months \\
\hline \multirow{5}{*}{$\begin{array}{r}\text { Big Data Science } \\
\text { (collection, curation, } \\
\text { analysis, } \\
\text { action) }\end{array}$} & $\begin{array}{r}\text { Veracity (Robustness } \\
\text { Issues) }\end{array}$ & $\begin{array}{l}\text { Exact results not essential but important to get main } \\
\text { hubs and authorities for search query }\end{array}$ \\
\hline & Visualization & Not important although page layout critical \\
\hline & Data Quality & A lot of duplication and spam \\
\hline & Data Types & $\begin{array}{l}\text { Mainly text but more interest in rapidly growing image } \\
\text { and video }\end{array}$ \\
\hline & Data Analytics & $\begin{array}{l}\text { Crawling; searching including topic based search; } \\
\text { ranking; recommending }\end{array}$ \\
\hline $\begin{array}{r}\text { Big Data Specific } \\
\text { Challenges (Gaps) }\end{array}$ & \multicolumn{2}{|c|}{$\begin{array}{l}\text { Search of "deep web" (information behind query front ends) } \\
\text { Ranking of responses sensitive to intrinsic value (as in Pagerank) as well as } \\
\text { advertising value } \\
\text { Link to user profiles and social network data }\end{array}$} \\
\hline $\begin{array}{r}\text { Big Data Specific } \\
\text { Challenges in Mobility }\end{array}$ & \multicolumn{2}{|c|}{ Mobile search must have similar interfaces/results } \\
\hline $\begin{array}{r}\text { Security and Privacy } \\
\text { Requirements }\end{array}$ & \multicolumn{2}{|c|}{ Need to be sensitive to crawling restrictions. Avoid Spam results } \\
\hline
\end{tabular}




\section{Commercial> Use Case 8: Web Search}

\begin{tabular}{|c|c|}
\hline $\begin{array}{r}\text { Highlight issues for } \\
\text { generalizing this use } \\
\text { case (e.g. for ref. } \\
\text { architecture) }\end{array}$ & Relation to Information retrieval such as search of scholarly works. \\
\hline $\begin{array}{r}\text { More Information } \\
\text { (URLs) }\end{array}$ & $\begin{array}{l}\text { http://www.slideshare.net/kleinerperkins/kpcb-Internet-trends-2013 } \\
\text { http://webcourse.cs.technion.ac.il/236621/Winter2011-2012/en/ho_Lectures.html } \\
\text { http://www.ifis.cs.tu-bs.de/teaching/ss-11/irws } \\
\text { http://www.slideshare.net/beechung/recommender-systems-tutorialpart1intro } \\
\text { http://www.worldwidewebsize.com/ }\end{array}$ \\
\hline
\end{tabular}




\section{Commercial> Use Case 9: Cloud-based Continuity and Disaster Recovery}

\begin{tabular}{|c|c|}
\hline Use Case Title & $\begin{array}{l}\text { laaS (Infrastructure as a Service) Big Data BC/DR Within a Cloud Eco-System provided } \\
\text { by Cloud Service Providers (CSPs) and Cloud Brokerage Service Providers (CBSPs) }\end{array}$ \\
\hline Vertical (area) & Large Scale Reliable Data Storage \\
\hline Author/Company/Email & Pw Carey, Compliance Partners, LLC, pwc.pwcarey@email.com \\
\hline $\begin{array}{r}\text { Actors/Stakeholders } \\
\text { and their roles and } \\
\text { responsibilities }\end{array}$ & $\begin{array}{l}\text { Executive Management, Data Custodians, and Employees responsible for the integrity, } \\
\text { protection, privacy, confidentiality, availability, safety, security and survivability of a } \\
\text { business by ensuring the 3-As of data accessibility to an organizations services are } \\
\text { satisfied; anytime, anyplace and on any device. }\end{array}$ \\
\hline Goals & $\begin{array}{l}\text { The following represents one approach to developing a workable BC/DR strategy. } \\
\text { Prior to outsourcing an organizations BC/DR onto the backs/shoulders of a CSP or CBSP, } \\
\text { the organization must perform the following Use Case, which will provide each } \\
\text { organization with a baseline methodology for BC/DR best practices, within a Cloud Eco- } \\
\text { system for both Public and Private organizations. } \\
\text { Each organization must approach the ten disciplines supporting BC/DR, with an } \\
\text { understanding and appreciation for the impact each of the following four overlaying } \\
\text { and inter-dependent forces will play in ensuring a workable solution to an entity's } \\
\text { business continuity plan and requisite disaster recovery strategy. The four areas are; } \\
\text { people (resources), processes (time/cost/ROI), technology (various operating systems, } \\
\text { platforms and footprints) and governance (subject to various and multiple regulatory } \\
\text { agencies). } \\
\text { These four concerns must be; identified, analyzed, evaluated, addressed, tested, } \\
\text { reviewed, addressed during the following ten phases: } \\
\text { 1. Project Initiation and Management Buy-in } \\
\text { 2. Risk Evaluations and Controls } \\
\text { 3. Business Impact Analysis } \\
\text { 4. Design, Development and Testing of the Business Continuity Strategies } \\
\text { 5. Emergency Response and Operations (aka; Disaster Recovery } \\
\text { 6. Developing and Implementing Business Continuity Plans } \\
\text { 7. Awareness and Training Programs } \\
\text { 8. Maintaining and Exercising Business Continuity Plans, (aka: Maintaining } \\
\text { 9. Public Relations (PR) and Crises Management Plans } \\
\text { 10. Coordination with Public Agencies } \\
\text { requirements of the organization. }\end{array}$ \\
\hline Use Case Description & $\begin{array}{l}\text { Big Data as developed by Google was intended to serve as an Internet Web site } \\
\text { indexing tool to help them sort, shuffle, categorize and label the Internet. At the outset, } \\
\text { it was not viewed as a replacement for legacy IT data infrastructures. With the spin-off } \\
\text { development within OpenGroup and Hadoop, Big Data has evolved into a robust data } \\
\text { analysis and storage tool that is still undergoing development. However, in the end, Big } \\
\text { Data is still being developed as an adjunct to the current IT client/server/big iron data } \\
\text { warehouse architectures which is better at some things, than these same data } \\
\text { warehouse environments, but not others. } \\
\text { As a result, it is necessary, within this business continuity/disaster recovery use case, } \\
\text { we ask good questions, such as; why are we doing this and what are we trying to } \\
\text { accomplish? What are our dependencies upon manual practices and when can we } \\
\text { leverage them? What systems have been and remain outsourced to other } \\
\text { organizations, such as our Telephony and what are their DR/BC business functions, if } \\
\text { any? Lastly, we must recognize the functions that can be simplified and what are the }\end{array}$ \\
\hline
\end{tabular}




\section{Commercial> Use Case 9: Cloud-based Continuity and Disaster Recovery}

\begin{tabular}{|c|c|c|}
\hline & \multicolumn{2}{|c|}{$\begin{array}{l}\text { preventative steps we can take that do not have a high cost associated with them such } \\
\text { as simplifying business practices. } \\
\text { We must identify what are the critical business functions that need to be recovered, } \\
\text { 1st, } 2 \text { nd, } 3^{\text {rd }} \text { in priority, or at a later time/date, and what is the Model of a Disaster } \\
\text { we're trying to resolve, what are the types of disasters more likely to occur realizing } \\
\text { that we don't need to resolve all types of disasters. When backing up data within a } \\
\text { Cloud Eco-system is a good solution, this will shorten the fail-over time and satisfy the } \\
\text { requirements of RTO/RPO. In addition, there must be 'Buy-in', as this is not just an IT } \\
\text { problem; it is a business services problem as well, requiring the testing of the Disaster } \\
\text { Plan via formal walk-throughs, et cetera. There should be a formal methodology for } \\
\text { developing a BC/DR Plan, including: 1). Policy Statement (Goal of the Plan, Reasons and } \\
\text { Resources....define each), 2). Business Impact Analysis (how does a shutdown impact } \\
\text { the business financially and otherwise), 3). Identify Preventive Steps (can a disaster be } \\
\text { avoided by taking prudent steps), 4). Recovery Strategies (how and what you will need } \\
\text { to recover), 5). Plan Development (Write the Plan and Implement the Plan Elements), } \\
\text { 6). Plan buy-in and Testing (very important so that everyone knows the Plan and knows } \\
\text { what to do during its execution), and 7). Maintenance (Continuous changes to reflect } \\
\text { the current enterprise environment) }\end{array}$} \\
\hline \multirow[t]{4}{*}{$\begin{array}{r}\text { Current } \\
\text { Solutions }\end{array}$} & Compute(System) & $\begin{array}{l}\text { Cloud Eco-systems, incorporating laaS (Infrastructure as a } \\
\text { Service), supported by Tier } 3 \text { Data Centers....Secure Fault } \\
\text { Tolerant (Power).... for Security, Power, Air Conditioning } \\
\text { et cetera...geographically off-site data recovery } \\
\text { centers...providing data replication services, Note: } \\
\text { Replication is different from Backup. Replication only } \\
\text { moves the changes since the last time a replication, } \\
\text { including block level changes. The replication can be done } \\
\text { quickly, with a five second window, while the data is } \\
\text { replicated every four hours. This data snap shot is } \\
\text { retained for seven business days, or longer if necessary. } \\
\text { Replicated data can be moved to a Fail-over Center to } \\
\text { satisfy the organizations RPO (Recovery Point Objectives) } \\
\text { and RTO }\end{array}$ \\
\hline & Storage & VMware, NetApps, Oracle, IBM, Brocade, \\
\hline & Networking & $\begin{array}{l}\text { WANs, LANs, WiFi, Internet Access, via Public, Private, } \\
\text { Community and Hybrid Cloud environments, with or } \\
\text { without VPNs. }\end{array}$ \\
\hline & Software & $\begin{array}{l}\text { Hadoop, Map/Reduce, Open-source, and/or Vendor } \\
\text { Proprietary such as AWS (Amazon Web Services), Google } \\
\text { Cloud Services, and Microsoft }\end{array}$ \\
\hline \multirow[t]{2}{*}{$\begin{array}{r}\text { Big Data } \\
\text { Characteristics }\end{array}$} & $\begin{array}{r}\text { Data Source (distributed } \\
\text { /centralized) }\end{array}$ & $\begin{array}{l}\text { Both distributed/centralized data sources flowing into } \\
\text { HA/DR Environment and HVSs, such as the following: } \\
\text { DC1---> VMWare/KVM (Clusters, w/Virtual Firewalls), } \\
\text { Data link-VMware Link-Vmotion Link-Network Link, } \\
\text { Multiple PB of NaaS, DC2--->, VMWare/KVM (Clusters } \\
\text { w/Virtual Firewalls), DataLink (VMware Link, Motion Link, } \\
\text { Network Link), Multiple PB of NaaS, (Requires Fail-Over } \\
\text { Virtualization) }\end{array}$ \\
\hline & Volume (size) & Terabytes up to Petabytes \\
\hline
\end{tabular}




\section{Commercial> Use Case 9: Cloud-based Continuity and Disaster Recovery}

\begin{tabular}{|c|c|c|}
\hline & $\begin{array}{r}\text { Velocity } \\
\text { (e.g. real time) }\end{array}$ & $\begin{array}{l}\text { Tier } 3 \text { Data Centers with Secure Fault Tolerant (Power) for } \\
\text { Security, Power, and Air Conditioning. laaS (Infrastructure } \\
\text { as a Service) in this example, based upon NetApps. } \\
\text { Replication is different from Backup; replication requires } \\
\text { only moving the CHANGES since the last time a } \\
\text { REPLICATION was performed, including the block level } \\
\text { changes. The Replication can be done quickly as the data } \\
\text { is Replicated every four hours. These replications can be } \\
\text { performed within a } 5 \text { second window, and this Snap Shot } \\
\text { will be kept for seven business days, or longer if necessary } \\
\text { to a Fail-Over Center......at the RPO and RTO.... }\end{array}$ \\
\hline & $\begin{array}{r}\text { Variety } \\
\text { (multiple datasets, mash- } \\
\text { up) }\end{array}$ & $\begin{array}{l}\text { Multiple virtual environments either operating within a } \\
\text { batch processing architecture or a hot-swappable parallel } \\
\text { architecture. }\end{array}$ \\
\hline & $\begin{array}{r}\text { Variability (rate of } \\
\text { change) }\end{array}$ & $\begin{array}{l}\text { Depending upon the SLA agreement, the costs (CapEx) } \\
\text { increases, depending upon the RTO/RPO and the } \\
\text { requirements of the business. }\end{array}$ \\
\hline $\begin{array}{r}\text { Big Data Science } \\
\text { (collection, curation, } \\
\text { analysis, } \\
\text { action) }\end{array}$ & $\begin{array}{r}\begin{array}{r}\text { Veracity (Robustness } \\
\text { Issues) }\end{array}\end{array}$ & $\begin{array}{l}\text { Data integrity is critical and essential over the entire life- } \\
\text { cycle of the organization due to regulatory and } \\
\text { compliance issues related to data CIA and GRC data } \\
\text { requirements. }\end{array}$ \\
\hline & Visualization & $\begin{array}{l}\text { Data integrity is critical and essential over the entire life- } \\
\text { cycle of the organization due to regulatory and } \\
\text { compliance issues related to data CIA and GRC data } \\
\text { requirements. }\end{array}$ \\
\hline & Data Quality & $\begin{array}{l}\text { Data integrity is critical and essential over the entire life- } \\
\text { cycle of the organization due to regulatory and } \\
\text { compliance issues related to data CIA and GRC data } \\
\text { requirements. }\end{array}$ \\
\hline & Data Types & $\begin{array}{l}\text { Multiple data types and formats, including but not limited } \\
\text { to; flat files, .txt, .pdf, android application files, .wav, .jpg } \\
\text { and VOIP (Voice over IP) }\end{array}$ \\
\hline & Data Analytics & $\begin{array}{l}\text { Must be maintained in a format that is non-destructive } \\
\text { during search and analysis processing and procedures. }\end{array}$ \\
\hline $\begin{array}{r}\text { Big Data Specific } \\
\text { Challenges (Gaps) }\end{array}$ & \multicolumn{2}{|c|}{$\begin{array}{l}\text { The complexities associated with migrating from a Primary Site to either a Replication } \\
\text { Site or a Backup Site is not fully automated at this point in time. The goal is to enable } \\
\text { the user to automatically initiate the Fail Over Sequence, moving Data Hosted within } \\
\text { Cloud requires a well-defined and continuously monitored server configuration } \\
\text { management. In addition, both organizations must know which servers have to be } \\
\text { restored and what are the dependencies and inter-dependencies between the Primary } \\
\text { Site servers and Replication and/or Backup Site servers. This requires a continuous } \\
\text { monitoring of both, since there are two solutions involved with this process, either } \\
\text { dealing with servers housing stored images or servers running hot all the time, as in } \\
\text { running parallel systems with hot-swappable functionality, all of which requires } \\
\text { accurate and up-to-date information from the client. }\end{array}$} \\
\hline $\begin{array}{r}\text { Big Data Specific } \\
\text { Challenges in Mobility }\end{array}$ & \multicolumn{2}{|c|}{$\begin{array}{l}\text { Mobility is a continuously growing layer of technical complexity; however, not all } \\
\text { DR/BC solutions are technical in nature, as there are two sides required to work } \\
\text { together to find a solution, the business side and the IT side. When they are in } \\
\text { agreement, these technical issues must be addressed by the BC/DR strategy }\end{array}$} \\
\hline
\end{tabular}




\section{Commercial> Use Case 9: Cloud-based Continuity and Disaster Recovery}

\begin{tabular}{|c|c|}
\hline & $\begin{array}{l}\text { implemented and maintained by the entire organization. One area, which is not limited } \\
\text { to mobility challenges, concerns a fundamental issue impacting most BC/DR solutions. } \\
\text { If your Primary Servers }(A, B, C) \text { understand } X, Y, Z \text {.... but your Secondary Virtual } \\
\text { Replication/Backup Servers }(a, b, c) \text { over the passage of time, are not properly } \\
\text { maintained (configuration management) and become out of sync with your Primary } \\
\text { Servers, and only understand } X \text {, and } Y, \text { when called upon to perform a Replication or } \\
\text { Back-up, well "Houston, we have a problem...." } \\
\text { Please Note: Over time all systems can and will suffer from sync-creep, some more } \\
\text { than others, when relying upon manual processes to ensure system stability. }\end{array}$ \\
\hline $\begin{array}{r}\text { Security and Privacy } \\
\text { Requirements }\end{array}$ & $\begin{array}{l}\text { Dependent upon the nature and requirements of the organization's industry verticals, } \\
\text { such as; Finance, Insurance, and Life Sciences including both public and/or private } \\
\text { entities, and the restrictions placed upon them by; regulatory, compliance and legal } \\
\text { jurisdictions. }\end{array}$ \\
\hline $\begin{array}{l}\text { Highlight issues for } \\
\text { generalizing this use } \\
\text { case (e.g. for ref. } \\
\text { architecture) }\end{array}$ & $\begin{array}{l}\text { Challenges to Implement BC/DR, include the following: } \\
\text { 1) Recognition, a). Management Vision, b). Assuming the issue is an IT issue, when it is } \\
\text { not just an IT issue, 2). People: a). Staffing levels - Many SMBs are understaffed in IT for } \\
\text { their current workload, b). Vision - (Driven from the Top Down) Can the business and IT } \\
\text { resources see the whole problem and craft a strategy such a 'Call List' in case of a } \\
\text { Disaster, c). Skills - Are there resources that can architect, implement and test a BC/DR } \\
\text { Solution, d). Time - Do Resources have the time and does the business have the } \\
\text { Windows of Time for constructing and testing a DR/BC Solution as DR/BC is an } \\
\text { additional Add-On Project the organization needs the time and resources. 3). Money - } \\
\text { This can be turned in to an OpEx Solution rather than a CapEx Solution which and can } \\
\text { be controlled by varying RPO/RTO, a). Capital is always a constrained resource, b). BC } \\
\text { Solutions need to start with "what is the Risk" and "how does cost constrain the } \\
\text { solution"? 4). Disruption - Build BC/DR into the standard "Cloud" infrastructure (laaS) of } \\
\text { the SMB, a). Planning for BC/DR is disruptive to business resources, b). Testing BC is } \\
\text { also disruptive..... }\end{array}$ \\
\hline $\begin{array}{r}\text { More Information } \\
\text { (URLs) }\end{array}$ & $\begin{array}{l}\text { 1. http://www.disasterrecovery.org/, (March 2013). } \\
\text { 2. BC_DR From the Cloud, Avoid IT Disasters EN POINTE Technologies and dinCloud, } \\
\text { Webinar Presenter Barry Weber, http://www.dincloud.com. } \\
\text { 3. COSO, The Committee of Sponsoring Organizations of the Treadway Commission } \\
\text { (COSO), Copyright@ 2013, http://www.coso.org. } \\
\text { 4. ITIL Information Technology Infrastructure Library, CopyrightC 2007-13 APM } \\
\text { Group Ltd. All rights reserved, Registered in England No. 2861902, http://www.itil- } \\
\text { officialsite.com. } \\
\text { 5. CobiT, Ver. 5.0, 2013, ISACA, Information Systems Audit and Control Association, (a } \\
\text { framework for IT Governance and Controls), http://www.isaca.org. } \\
\text { 6. TOGAF, Ver. 9.1, The Open Group Architecture Framework (a framework for IT } \\
\text { architecture), http://www.opengroup.org. } \\
\text { 7. ISO/IEC 27000:2012 Info. Security Mgt., International Organization for } \\
\text { Standardization and the International Electrotechnical Commission, } \\
\text { http://www.standards.iso.org/. } \\
\text { 8CAOB, Public Company Accounting and Oversight Board, } \\
\text { http://www.pcaobus.org. }\end{array}$ \\
\hline \multicolumn{2}{|c|}{$\begin{array}{l}\text { Note: Please feel free to improve our INITIAL DRAFT, Ver. } 0.1, \text { August } 10^{\text {th }}, 2013 \ldots . . . \text { as we do not consider our } \\
\text { efforts to be pearls, at this point in time.......Respectfully yours, Pw Carey, Compliance Partners, } \\
\text { LLC_pwc.pwcarey@gmail.com }\end{array}$} \\
\hline
\end{tabular}


NIST Big Data Interoperability Framework: Volume 3, Use CaSes and General ReQuiRements 


\section{Commercial> Use Case 10: Cargo Shipping}

\begin{tabular}{|c|c|c|}
\hline Use Case Title & \multicolumn{2}{|l|}{ Cargo Shipping } \\
\hline Vertical (area) & \multicolumn{2}{|l|}{ Industry } \\
\hline Author/Company/Email & \multicolumn{2}{|c|}{ William Miller/MaCT USA/mact-usa@att.net } \\
\hline $\begin{array}{r}\text { Actors } / \text { Stakeholders } \\
\text { and their roles and } \\
\text { responsibilities }\end{array}$ & \multicolumn{2}{|c|}{$\begin{array}{l}\text { End-users (Sender/Recipients) } \\
\text { Transport Handlers (Truck/Ship/Plane) } \\
\text { Telecom Providers (Cellular/SATCOM) } \\
\text { Shippers (Shipping and Receiving) }\end{array}$} \\
\hline Goals & \multicolumn{2}{|c|}{ Retention and analysis of items (Things) in transport } \\
\hline Use Case Description & \multicolumn{2}{|c|}{$\begin{array}{l}\text { The following use case defines the overview of a Big Data application related to the } \\
\text { shipping industry (i.e., FedEx, UPS, DHL, etc.). The shipping industry represents } \\
\text { possible the largest potential use case of Big Data that is in common use today. It } \\
\text { relates to the identification, transport, and handling of item (Things) in the supply } \\
\text { chain. The identification of an item begins with the sender to the recipients and for } \\
\text { all those in between with a need to know the location and time of arrive of the items } \\
\text { while in transport. A new aspect will be status condition of the items which will } \\
\text { include sensor information, GPS coordinates, and a unique identification schema } \\
\text { based upon a new ISO } 29161 \text { standards under development within ISO JTC1 SC31 } \\
\text { WG2. The data is in near real time being updated when a truck arrives at a depot or } \\
\text { upon delivery of the item to the recipient. Intermediate conditions are not currently } \\
\text { known; the location is not updated in real time, items lost in a warehouse or while in } \\
\text { shipment represent a problem potentially for homeland security. The records are } \\
\text { retained in an archive and can be accessed for xx days. }\end{array}$} \\
\hline \multirow{4}{*}{$\begin{array}{r}\text { Current } \\
\text { Solutions }\end{array}$} & Compute(System) & Unknown \\
\hline & Storage & Unknown \\
\hline & Networking & LAN/T1/Internet Web Pages \\
\hline & Software & Unknown \\
\hline \multirow[t]{5}{*}{$\begin{array}{r}\text { Big Data } \\
\text { Characteristics }\end{array}$} & $\begin{array}{r}\text { Data Source } \\
\text { (distributed/centralized) }\end{array}$ & Centralized today \\
\hline & Volume (size) & Large \\
\hline & $\begin{array}{r}\text { Velocity } \\
\text { (e.g. real time) } \\
\end{array}$ & The system is not currently real time. \\
\hline & $\begin{array}{r}\text { Variety } \\
\text { (multiple datasets, mashup) }\end{array}$ & $\begin{array}{l}\text { Updated when the driver arrives at the depot and } \\
\text { download the time and date the items were picked } \\
\text { up. This is currently not real time. }\end{array}$ \\
\hline & Variability (rate of change) & $\begin{array}{l}\text { Today the information is updated only when the } \\
\text { items that were checked with a bar code scanner are } \\
\text { sent to the central server. The location is not } \\
\text { currently displayed in real time. }\end{array}$ \\
\hline \multirow{5}{*}{$\begin{array}{r}\text { Big Data Science } \\
\text { (collection, curation, } \\
\text { analysis, } \\
\text { action) }\end{array}$} & $\begin{array}{r}\begin{array}{r}\text { Veracity (Robustness } \\
\text { Issues) }\end{array} \\
\end{array}$ & \\
\hline & Visualization & NONE \\
\hline & Data Quality & YES \\
\hline & Data Types & Not Available \\
\hline & Data Analytics & YES \\
\hline $\begin{array}{r}\text { Big Data Specific } \\
\text { Challenges (Gaps) }\end{array}$ & \multicolumn{2}{|c|}{$\begin{array}{l}\text { Provide more rapid assessment of the identity, location, and conditions of the } \\
\text { shipments, provide detailed analytics and location of problems in the system in real } \\
\text { time. }\end{array}$} \\
\hline
\end{tabular}




\section{Commercial> Use Case 10: Cargo Shipping}

\begin{tabular}{|r|l|}
\hline $\begin{array}{r}\text { Big Data Specific } \\
\text { Challenges in Mobility }\end{array}$ & Currently conditions are not monitored on-board trucks, ships, and aircraft \\
\hline $\begin{array}{r}\text { Security and Privacy } \\
\text { Requirements }\end{array}$ & Security need to be more robust \\
\hline $\begin{array}{r}\text { Highlight issues for } \\
\text { generalizing this use } \\
\text { case (e.g. for ref. } \\
\text { architecture) }\end{array}$ & $\begin{array}{r}\text { This use case includes local data bases as well as the requirement to synchronize } \\
\text { with the central server. This operation would eventually extend to mobile device and } \\
\text { on-board systems which can track the location of the items and provide real-time } \\
\text { update of the information including the status of the conditions, logging, and alerts } \\
\text { to individuals who have a need to know. }\end{array}$ \\
\hline $\begin{array}{r}\text { More Information } \\
\text { (URLs) }\end{array}$ & \\
\hline
\end{tabular}

See Figure 1: Cargo Shipping - Scenario. 
Commercial> Use Case 11: Materials Data

\begin{tabular}{|c|c|c|}
\hline Use Case Title & \multicolumn{2}{|l|}{ Materials Data } \\
\hline Vertical (area) & \multicolumn{2}{|c|}{ Manufacturing, Materials Research } \\
\hline Author/Company/Email & \multicolumn{2}{|c|}{ John Rumble, R\&R Data Services; jumbleusa@earthlink.net } \\
\hline $\begin{array}{r}\text { Actors/Stakeholders } \\
\text { and their roles and } \\
\text { responsibilities }\end{array}$ & \multicolumn{2}{|c|}{$\begin{array}{l}\text { Product Designers (Inputters of materials data in CAE) } \\
\text { Materials Researchers (Generators of materials data; users in some cases) } \\
\text { Materials Testers (Generators of materials data; standards developers) } \\
\text { Data distributors (Providers of access to materials, often for profit) }\end{array}$} \\
\hline Goals & \multicolumn{2}{|c|}{$\begin{array}{l}\text { Broaden accessibility, quality, and usability; Overcome proprietary barriers to sharing } \\
\text { materials data; Create sufficiently large repositories of materials data to support } \\
\text { discovery }\end{array}$} \\
\hline Use Case Description & \multicolumn{2}{|c|}{$\begin{array}{l}\text { Every physical product is made from a material that has been selected for its } \\
\text { properties, cost, and availability. This translates into hundreds of billion dollars of } \\
\text { material decisions made every year. } \\
\text { In addition, as the Materials Genome Initiative has so effectively pointed out, the } \\
\text { adoption of new materials normally takes decades (two to three) rather than a small } \\
\text { number of years, in part because data on new materials is not easily available. } \\
\text { All actors within the materials life cycle today have access to very limited } \\
\text { quantities of materials data, thereby resulting in materials-related decision that are } \\
\text { non-optimal, inefficient, and costly. While the Materials Genome Initiative is } \\
\text { addressing one major and important aspect of the issue, namely the fundamental } \\
\text { materials data necessary to design and test materials computationally, the issues } \\
\text { related to physical measurements on physical materials ( from basic structural and } \\
\text { thermal properties to complex performance properties to properties of novel } \\
\text { (nanoscale materials) are not being addressed systematically, broadly (cross- } \\
\text { discipline and internationally), or effectively (virtually no materials data meetings, } \\
\text { standards groups, or dedicated funded programs). } \\
\text { One of the greatest challenges that Big Data approaches can address is predicting } \\
\text { the performance of real materials (gram to ton quantities) starting at the atomistic, } \\
\text { nanometer, and/or micrometer level of description. } \\
\text { As a result of the above considerations, decisions about materials usage are } \\
\text { unnecessarily conservative, often based on older rather than newer materials } \\
\text { research and development data, and not taking advantage of advances in modeling } \\
\text { and simulations. Materials informatics is an area in which the new tools of data } \\
\text { science can have major impact. }\end{array}$} \\
\hline \multirow{4}{*}{$\begin{array}{r}\text { Current } \\
\text { Solutions }\end{array}$} & Compute(System) & None \\
\hline & Storage & Widely dispersed with many barriers to access \\
\hline & Networking & Virtually none \\
\hline & Software & $\begin{array}{l}\text { Narrow approaches based on national programs (Japan, } \\
\text { Korea, and China), applications (EU Nuclear program), } \\
\text { proprietary solutions (Granta, etc.) }\end{array}$ \\
\hline \multirow[t]{3}{*}{$\begin{array}{r}\text { Big Data } \\
\text { Characteristics }\end{array}$} & $\begin{array}{r}\text { Data Source } \\
\text { (distributed/centralized) }\end{array}$ & $\begin{array}{l}\text { Extremely distributed with data repositories existing } \\
\text { only for a very few fundamental properties }\end{array}$ \\
\hline & Volume (size) & $\begin{array}{l}\text { It has been estimated (in the } 1980 \text { s) that there were } \\
\text { over } 500,000 \text { commercial materials made in the last } \\
\text { fifty years. The last three decades has seen large } \\
\text { growth in that number. }\end{array}$ \\
\hline & $\begin{array}{r}\text { Velocity } \\
\text { (e.g. real time) }\end{array}$ & $\begin{array}{l}\text { Computer-designed and theoretically design materials } \\
\text { (e.g., nanomaterials) are growing over time }\end{array}$ \\
\hline
\end{tabular}




\section{Commercial> Use Case 11: Materials Data}

\begin{tabular}{|c|c|c|}
\hline & $\begin{array}{r}\text { Variety } \\
\text { (multiple datasets, } \\
\text { mashup) }\end{array}$ & Many datasets and virtually no standards for mashups \\
\hline & $\begin{array}{r}\text { Variability (rate of } \\
\text { change) }\end{array}$ & $\begin{array}{l}\text { Materials are changing all the time, and new materials } \\
\text { data are constantly being generated to describe the } \\
\text { new materials }\end{array}$ \\
\hline \multirow[t]{5}{*}{$\begin{array}{r}\text { Big Data Science } \\
\text { (collection, curation, } \\
\text { analysis, } \\
\text { action) }\end{array}$} & $\begin{array}{r}\text { Veracity (Robustness } \\
\text { Issues) }\end{array}$ & $\begin{array}{l}\text { More complex material properties can require many } \\
\text { (100s?) of independent variables to describe } \\
\text { accurately. Virtually no activity no exists that is trying to } \\
\text { identify and systematize the collection of these } \\
\text { variables to create robust datasets. }\end{array}$ \\
\hline & Visualization & $\begin{array}{l}\text { Important for materials discovery. Potentially } \\
\text { important to understand the dependency of properties } \\
\text { on the many independent variables. Virtually } \\
\text { unaddressed. }\end{array}$ \\
\hline & Data Quality & $\begin{array}{l}\text { Except for fundamental data on the structural and } \\
\text { thermal properties, data quality is poor or unknown. } \\
\text { See Munro's NIST Standard Practice Guide. }\end{array}$ \\
\hline & Data Types & Numbers, graphical, images \\
\hline & Data Analytics & Empirical and narrow in scope \\
\hline $\begin{array}{r}\text { Big Data Specific } \\
\text { Challenges (Gaps) }\end{array}$ & \multicolumn{2}{|c|}{$\begin{array}{l}\text { 1. Establishing materials data repositories beyond the existing ones that focus on } \\
\text { fundamental data } \\
\text { 2. Developing internationally-accepted data recording standards that can be used } \\
\text { by a very diverse materials community, including developers materials test } \\
\text { standards (such as ASTM and ISO), testing companies, materials producers, and } \\
\text { research and development labs } \\
\text { 3. Tools and procedures to help organizations wishing to deposit proprietary } \\
\text { materials in data repositories to mask proprietary information, yet to maintain } \\
\text { the usability of data } \\
\text { Multi-variable materials data visualization tools, in which the number of } \\
\text { variables can be quite high }\end{array}$} \\
\hline $\begin{array}{r}\text { Big Data Specific } \\
\text { Challenges in Mobility }\end{array}$ & \multicolumn{2}{|c|}{ Not important at this time } \\
\hline $\begin{array}{r}\text { Security and Privacy } \\
\text { Requirements }\end{array}$ & \multicolumn{2}{|c|}{ Proprietary nature of many data very sensitive. } \\
\hline $\begin{array}{r}\text { Highlight issues for } \\
\text { generalizing this use } \\
\text { case (e.g. for ref. } \\
\text { architecture) }\end{array}$ & \multicolumn{2}{|c|}{$\begin{array}{l}\text { Development of standards; development of large scale repositories; involving } \\
\text { industrial users; integration with CAE (don't underestimate the difficulty of this - } \\
\text { materials people are generally not as computer savvy as chemists, bioinformatics } \\
\text { people, and engineers) }\end{array}$} \\
\hline $\begin{array}{r}\text { More Information } \\
\text { (URLs) }\end{array}$ & & \\
\hline
\end{tabular}




\section{Commercial> Use Case 12: Simulation Driven Materials Genomics}

\begin{tabular}{|c|c|c|}
\hline Use Case Title & \multicolumn{2}{|c|}{ Simulation driven Materials Genomics } \\
\hline Vertical (area) & \multicolumn{2}{|c|}{ Scientific Research: Materials Science } \\
\hline Author/Company/Email & \multicolumn{2}{|c|}{ David Skinner/LBNL/deskinner@lbl.gov } \\
\hline $\begin{array}{r}\text { Actors/Stakeholders } \\
\text { and their roles and } \\
\text { responsibilities }\end{array}$ & \multicolumn{2}{|c|}{$\begin{array}{l}\text { Capability providers: National labs and energy hubs provide advanced materials } \\
\text { genomics capabilities using computing and data as instruments of discovery. } \\
\text { User Community: DOE, industry and academic researchers as a user community } \\
\text { seeking capabilities for rapid innovation in materials. }\end{array}$} \\
\hline Goals & \multicolumn{2}{|c|}{$\begin{array}{l}\text { Speed the discovery of advanced materials through informatically driven simulation } \\
\text { surveys. }\end{array}$} \\
\hline Use Case Description & \multicolumn{2}{|c|}{$\begin{array}{l}\text { Innovation of battery technologies through massive simulations spanning wide } \\
\text { spaces of possible design. Systematic computational studies of innovation } \\
\text { possibilities in photovoltaics. Rational design of materials based on search and } \\
\text { simulation. }\end{array}$} \\
\hline \multirow[t]{4}{*}{$\begin{array}{r}\text { Current } \\
\text { Solutions }\end{array}$} & Compute(System) & $\begin{array}{l}\text { Hopper.nersc.gov (150K cores), omics-like data } \\
\text { analytics hardware resources. }\end{array}$ \\
\hline & Storage & GPFS, MongoDB \\
\hline & Networking & $10 \mathrm{~Gb}$ \\
\hline & Software & $\begin{array}{l}\text { PyMatGen, FireWorks, VASP, ABINIT, NWChem, } \\
\text { BerkeleyGW, varied community codes }\end{array}$ \\
\hline \multirow[t]{5}{*}{$\begin{array}{r}\text { Big Data } \\
\text { Characteristics }\end{array}$} & $\begin{array}{r}\text { Data Source } \\
\text { (distributed/centralized) }\end{array}$ & $\begin{array}{l}\text { Gateway-like. Data streams from simulation surveys } \\
\text { driven on centralized peta/exascale systems. Widely } \\
\text { distributed web of dataflows from central gateway to } \\
\text { users. }\end{array}$ \\
\hline & Volume (size) & $\begin{array}{l}\text { 100TB (current), 500TB within } 5 \text { years. Scalable key- } \\
\text { value and object store databases needed. }\end{array}$ \\
\hline & $\begin{array}{r}\text { Velocity } \\
\text { (e.g. real time) }\end{array}$ & $\begin{array}{l}\text { High throughput computing (HTC), fine-grained tasking } \\
\text { and queuing. Rapid start/stop for ensembles of tasks. } \\
\text { Real-time data analysis for web-like responsiveness. }\end{array}$ \\
\hline & $\begin{array}{r}\text { Variety } \\
\text { (multiple datasets, } \\
\text { mashup) }\end{array}$ & $\begin{array}{l}\text { Mashup of simulation outputs across codes and levels } \\
\text { of theory. Formatting, registration and integration of } \\
\text { datasets. Mashups of data across simulation scales. }\end{array}$ \\
\hline & $\begin{array}{r}\text { Variability (rate of } \\
\text { change) }\end{array}$ & $\begin{array}{l}\text { The targets for materials design will become more } \\
\text { search and crowd-driven. The computational backend } \\
\text { must flexibly adapt to new targets. }\end{array}$ \\
\hline \multirow{5}{*}{$\begin{array}{r}\text { Big Data Science } \\
\text { (collection, curation, } \\
\text { analysis, } \\
\text { action) }\end{array}$} & $\begin{array}{r}\text { Veracity (Robustness } \\
\text { Issues, semantics) }\end{array}$ & $\begin{array}{l}\text { Validation and UQ of simulation with experimental data } \\
\text { of varied quality. Error checking and bounds estimation } \\
\text { from simulation inter-comparison. }\end{array}$ \\
\hline & Visualization & $\begin{array}{l}\text { Materials browsers as data from search grows. Visual } \\
\text { design of materials. }\end{array}$ \\
\hline & Data Quality (syntax) & $\begin{array}{l}\text { UQ in results based on multiple datasets. } \\
\text { Propagation of error in knowledge systems. }\end{array}$ \\
\hline & Data Types & Key value pairs, JSON, materials file formats \\
\hline & Data Analytics & $\begin{array}{l}\text { Map/Reduce and search that join simulation and } \\
\text { experimental data. }\end{array}$ \\
\hline $\begin{array}{l}\text { Big Data Specific } \\
\text { Challenges (Gaps) }\end{array}$ & \multicolumn{2}{|c|}{$\begin{array}{l}\text { HTC at scale for simulation science. Flexible data methods at scale for messy data. } \\
\text { Machine learning and knowledge systems that integrate data from publications, } \\
\text { experiments, and simulations to advance goal-driven thinking in materials design. }\end{array}$} \\
\hline $\begin{array}{r}\text { Big Data Specific } \\
\text { Challenges in Mobility }\end{array}$ & \multicolumn{2}{|c|}{$\begin{array}{l}\text { Potential exists for widespread delivery of actionable knowledge in materials } \\
\text { science. Many materials genomics "apps" are amenable to a mobile platform. }\end{array}$} \\
\hline
\end{tabular}




\section{Commercial> Use Case 12: Simulation Driven Materials Genomics}

\begin{tabular}{|r|l|}
\hline $\begin{array}{r}\text { Security and Privacy } \\
\text { Requirements }\end{array}$ & $\begin{array}{l}\text { Ability to "sandbox" or create independent working areas between data } \\
\text { stakeholders. Policy-driven federation of datasets. }\end{array}$ \\
\hline $\begin{array}{r}\text { Highlight issues for } \\
\text { generalizing this use } \\
\text { case (e.g. for ref. } \\
\text { architecture) }\end{array}$ & $\begin{array}{l}\text { An OSTP blueprint toward broader materials genomics goals was made available in } \\
\text { Ma13. }\end{array}$ \\
\hline $\begin{array}{r}\text { More Information } \\
\text { (URLs) }\end{array}$ & http://www.materialsproject.org \\
\hline
\end{tabular}




\section{Defense> Use Case 13: Large Scale Geospatial Analysis and Visualization}

\begin{tabular}{|c|c|c|}
\hline Use Case Title & \multicolumn{2}{|c|}{ Large Scale Geospatial Analysis and Visualization } \\
\hline Vertical (area) & \multicolumn{2}{|c|}{ Defense - but applicable to many others } \\
\hline Author/Company/Email & \multicolumn{2}{|c|}{ David Boyd/Data Tactics/ dboyd@data-tactics.com } \\
\hline $\begin{array}{r}\text { Actors/Stakeholders } \\
\text { and their roles and } \\
\text { responsibilities }\end{array}$ & \multicolumn{2}{|c|}{$\begin{array}{l}\text { Geospatial Analysts } \\
\text { Decision Makers } \\
\text { Policy Makers }\end{array}$} \\
\hline Goals & \multicolumn{2}{|c|}{ Support large scale geospatial data analysis and visualization. } \\
\hline Use Case Description & \multicolumn{2}{|c|}{$\begin{array}{l}\text { As the number of geospatially aware sensors increase and the number of } \\
\text { geospatially tagged data sources increases the volume geospatial data requiring } \\
\text { complex analysis and visualization is growing exponentially. Traditional GIS systems } \\
\text { are generally capable of analyzing millions of objects and easily visualizing } \\
\text { thousands. Today's intelligence systems often contain trillions of geospatial objects } \\
\text { and need to be able to visualize and interact with millions of objects. }\end{array}$} \\
\hline \multirow[t]{4}{*}{$\begin{array}{r}\text { Current } \\
\text { Solutions }\end{array}$} & Compute(System) & $\begin{array}{l}\text { Compute and Storage systems - Laptops to Large } \\
\text { servers (see notes about clusters) } \\
\text { Visualization systems - handhelds to laptops }\end{array}$ \\
\hline & Storage & $\begin{array}{l}\text { Compute and Storage - local disk or SAN } \\
\text { Visualization - local disk, flash ram }\end{array}$ \\
\hline & Networking & $\begin{array}{l}\text { Compute and Storage - Gigabit or better LAN } \\
\text { connection } \\
\text { Visualization - Gigabit wired connections, Wireless } \\
\text { including WiFi (802.11), Cellular (3g/4g), or Radio Relay }\end{array}$ \\
\hline & Software & $\begin{array}{l}\text { Compute and Storage - generally Linux or Win Server } \\
\text { with Geospatially enabled RDBMS, Geospatial } \\
\text { server/analysis software - ESRI ArcServer, Geoserver } \\
\text { Visualization - Windows, Android, IOS - browser based } \\
\text { visualization. Some laptops may have local ArcMap. }\end{array}$ \\
\hline \multirow[t]{5}{*}{$\begin{array}{r}\text { Big Data } \\
\text { Characteristics }\end{array}$} & $\begin{array}{r}\text { Data Source } \\
\text { (distributed/centralized) } \\
\end{array}$ & Very distributed. \\
\hline & Volume (size) & $\begin{array}{l}\text { Imagery }-100 \text { s of Terabytes } \\
\text { Vector Data }-10 \text { s of GBs but billions of points }\end{array}$ \\
\hline & $\begin{array}{r}\text { Velocity } \\
\text { (e.g. real time) } \\
\end{array}$ & $\begin{array}{l}\text { Some sensors delivery vector data in NRT. Visualization } \\
\text { of changes should be NRT. }\end{array}$ \\
\hline & $\begin{array}{r}\text { Variety } \\
\text { (multiple datasets, } \\
\text { mashup) }\end{array}$ & $\begin{array}{l}\text { Imagery (various formats NITF, GeoTiff, CADRG) } \\
\text { Vector (various formats shape files, kml, text streams: } \\
\text { Object types include points, lines, areas, polylines, } \\
\text { circles, ellipses. }\end{array}$ \\
\hline & $\begin{array}{r}\text { Variability (rate of } \\
\text { change) }\end{array}$ & Moderate to high \\
\hline \multirow[t]{2}{*}{$\begin{array}{r}\text { Big Data Science } \\
\text { (collection, curation, } \\
\text { analysis, } \\
\text { action) }\end{array}$} & $\begin{array}{r}\text { Veracity (Robustness } \\
\text { Issues) }\end{array}$ & $\begin{array}{l}\text { Data accuracy is critical and is controlled generally by } \\
\text { three factors: } \\
\text { 1. Sensor accuracy is a big issue. } \\
\text { 2. datum/spheroid. } \\
\text { 3. Image registration accuracy }\end{array}$ \\
\hline & Visualization & $\begin{array}{l}\text { Displaying in a meaningful way large datasets (millions } \\
\text { of points) on small devices (handhelds) at the end of } \\
\text { low bandwidth networks. }\end{array}$ \\
\hline
\end{tabular}




\section{Defense> Use Case 13: Large Scale Geospatial Analysis and Visualization}

\begin{tabular}{|c|c|c|}
\hline & Data Quality & $\begin{array}{l}\text { The typical problem is visualization implying } \\
\text { quality/accuracy not available in the original data. All } \\
\text { data should include metadata for accuracy or circular } \\
\text { error probability. }\end{array}$ \\
\hline & Data Types & $\begin{array}{l}\text { Imagery (various formats NITF, GeoTiff, CADRG) } \\
\text { Vector (various formats shape files, kml, text streams: } \\
\text { Object types include points, lines, areas, polylines, } \\
\text { circles, ellipses. }\end{array}$ \\
\hline & Data Analytics & $\begin{array}{l}\text { Closest point of approach, deviation from route, point } \\
\text { density over time, PCA and ICA }\end{array}$ \\
\hline $\begin{array}{l}\text { Big Data Specific } \\
\text { Challenges (Gaps) }\end{array}$ & \multicolumn{2}{|c|}{$\begin{array}{l}\text { Indexing, retrieval and distributed analysis } \\
\text { Visualization generation and transmission }\end{array}$} \\
\hline $\begin{array}{r}\text { Big Data Specific } \\
\text { Challenges in Mobility }\end{array}$ & \multicolumn{2}{|c|}{ Visualization of data at the end of low bandwidth wireless connections. } \\
\hline $\begin{array}{r}\text { Security and Privacy } \\
\text { Requirements }\end{array}$ & \multicolumn{2}{|c|}{$\begin{array}{l}\text { Data is sensitive and must be completely secure in transit and at rest (particularly on } \\
\text { handhelds) }\end{array}$} \\
\hline $\begin{array}{r}\text { Highlight issues for } \\
\text { generalizing this use } \\
\text { case (e.g. for ref. } \\
\text { architecture) }\end{array}$ & \multicolumn{2}{|c|}{ Geospatial data requires unique approaches to indexing and distributed analysis. } \\
\hline $\begin{array}{r}\text { More Information } \\
\text { (URLs) }\end{array}$ & \multicolumn{2}{|c|}{$\begin{array}{l}\text { Applicable Standards: http://www.opengeospatial.org/standards } \\
\text { http://geojson.org/ } \\
\text { http://earth-info.nga.mil/publications/specs/printed/CADRG/cadrg.html } \\
\text { Geospatial Indexing: Quad Trees, Space Filling Curves (Hilbert Curves) - You can } \\
\text { google these for lots of references. }\end{array}$} \\
\hline \multicolumn{3}{|c|}{$\begin{array}{l}\text { Note: There has been some work with in DoD related to this problem set. Specifically, the DCGS-A standard } \\
\text { cloud (DSC) stores, indexes, and analyzes some Big Data sources. However, many issues remain with } \\
\text { visualization. }\end{array}$} \\
\hline
\end{tabular}




\section{Defense> Use Case 14: Object I dentification and Tracking - Persistent Surveillance}

\begin{tabular}{|c|c|c|}
\hline Use Case Title & \multicolumn{2}{|c|}{$\begin{array}{l}\text { Object identification and tracking from Wide Area Large Format Imagery (WALF) } \\
\text { Imagery or Full Motion Video (FMV) - Persistent Surveillance }\end{array}$} \\
\hline Vertical (area) & \multicolumn{2}{|l|}{ Defense (Intelligence) } \\
\hline Author/Company/Email & \multicolumn{2}{|c|}{ David Boyd/Data Tactics/dboyd@data-tactics.com } \\
\hline $\begin{array}{r}\text { Actors/Stakeholders } \\
\text { and their roles and } \\
\text { responsibilities }\end{array}$ & \multicolumn{2}{|c|}{$\begin{array}{l}\text { 1. Civilian Military decision makers } \\
\text { 2. Intelligence Analysts } \\
\text { 3. Warfighters }\end{array}$} \\
\hline Goals & \multicolumn{2}{|c|}{$\begin{array}{l}\text { To be able to process and extract/track entities (vehicles, people, packages) over time } \\
\text { from the raw image data. Specifically, the idea is to reduce the petabytes of data } \\
\text { generated by persistent surveillance down to a manageable size (e.g. vector tracks) }\end{array}$} \\
\hline Use Case Description & \multicolumn{2}{|c|}{$\begin{array}{l}\text { Persistent surveillance sensors can easily collect petabytes of imagery data in the space } \\
\text { of a few hours. It is unfeasible for this data to be processed by humans for either } \\
\text { alerting or tracking purposes. The data needs to be processed close to the sensor which } \\
\text { is likely forward deployed since it is too large to be easily transmitted. The data should } \\
\text { be reduced to a set of geospatial object (points, tracks, etc.) which can easily be } \\
\text { integrated with other data to form a common operational picture. }\end{array}$} \\
\hline \multirow[t]{4}{*}{$\begin{array}{r}\text { Current } \\
\text { Solutions }\end{array}$} & Compute(System) & $\begin{array}{l}\text { Various - they range from simple storage capabilities } \\
\text { mounted on the sensor, to simple display and storage, to } \\
\text { limited object extraction. Typical object extraction } \\
\text { systems are currently small (1-20 node) GPU enhanced } \\
\text { clusters. }\end{array}$ \\
\hline & Storage & $\begin{array}{l}\text { Currently flat files persisted on disk in most cases. } \\
\text { Sometimes RDBMS indexes pointing to files or portions of } \\
\text { files based on metadata/telemetry data. }\end{array}$ \\
\hline & Networking & Sensor comms tend to be Line of Sight or Satellite based. \\
\hline & Software & $\begin{array}{l}\text { A wide range custom software and tools including } \\
\text { traditional RDBMS and display tools. }\end{array}$ \\
\hline \multirow[t]{5}{*}{$\begin{array}{r}\text { Big Data } \\
\text { Characteristics }\end{array}$} & $\begin{array}{r}\text { Data Source } \\
\text { (distributed/centralized) }\end{array}$ & $\begin{array}{l}\text { Sensors include airframe mounted and fixed position } \\
\text { optical, IR, and SAR images. }\end{array}$ \\
\hline & Volume (size) & $\begin{array}{l}\text { FMV }-30 \text { to } 60 \text { frames per/sec at full color } 1080 \mathrm{P} \\
\text { resolution. } \\
\text { WALF }-1 \text { to } 10 \text { frames per } / \mathrm{sec} \text { at } 10 \mathrm{~K} \times 10 \mathrm{~K} \text { full color } \\
\text { resolution. }\end{array}$ \\
\hline & $\begin{array}{r}\text { Velocity } \\
\text { (e.g. real time) }\end{array}$ & Real Time \\
\hline & $\begin{array}{r}\text { Variety } \\
\text { (multiple datasets, } \\
\text { mashup) } \\
\end{array}$ & $\begin{array}{l}\text { Data Typically exists in one or more standard imagery or } \\
\text { video formats. }\end{array}$ \\
\hline & $\begin{array}{r}\text { Variability (rate of } \\
\text { change) }\end{array}$ & Little \\
\hline \multirow{3}{*}{$\begin{array}{r}\text { Big Data Science } \\
\text { (collection, curation, } \\
\text { analysis, } \\
\text { action) }\end{array}$} & $\begin{array}{r}\text { Veracity (Robustness } \\
\text { Issues) }\end{array}$ & $\begin{array}{l}\text { The veracity of extracted objects is critical. If the system } \\
\text { fails or generates false positives people are put at risk. }\end{array}$ \\
\hline & Visualization & $\begin{array}{l}\text { Visualization of extracted outputs will typically be as } \\
\text { overlays on a geospatial display. Overlay objects should } \\
\text { be links back to the originating image/video segment. }\end{array}$ \\
\hline & Data Quality & $\begin{array}{l}\text { Data quality is generally driven by a combination of sensor } \\
\text { characteristics and weather (both obscuring factors - } \\
\text { dust/moisture and stability factors - wind). }\end{array}$ \\
\hline
\end{tabular}




\section{Defense> Use Case 14: Object I dentification and Tracking - Persistent Surveillance}

\begin{tabular}{|c|c|c|}
\hline & Data Types & $\begin{array}{l}\text { Standard imagery and video formats are input. Output } \\
\text { should be in the form of OGC compliant web features or } \\
\text { standard geospatial files (shape files, KML). }\end{array}$ \\
\hline & Data Analytics & $\begin{array}{l}\text { 1. Object identification (type, size, color) and tracking. } \\
\text { 2. Pattern analysis of object (did the truck observed } \\
\text { every Weds. afternoon take a different route today or } \\
\text { is there a standard route this person takes every day). } \\
\text { 3. Crowd behavior/dynamics (is there a small group } \\
\text { attempting to incite a riot. Is this person out of place } \\
\text { in the crowd or behaving differently? } \\
\text { 4. Economic activity } \\
\text { a. is the line at the bread store, the butcher, or the } \\
\text { ice cream store, } \\
\text { b. are more trucks traveling north with goods than } \\
\text { c. Has activity at or the size of stores in this market } \\
\text { place increased or decreased over the past year. } \\
\text { F. Fusion of data with other data to improve quality and } \\
\text { confidence. }\end{array}$ \\
\hline $\begin{array}{l}\text { Big Data Specific } \\
\text { Challenges (Gaps) }\end{array}$ & \multicolumn{2}{|c|}{ Processing the volume of data in NRT to support alerting and situational awareness. } \\
\hline $\begin{array}{r}\text { Big Data Specific } \\
\text { Challenges in Mobility }\end{array}$ & \multicolumn{2}{|c|}{ Getting data from mobile sensor to processing } \\
\hline $\begin{array}{r}\text { Security and Privacy } \\
\text { Requirements }\end{array}$ & \multicolumn{2}{|c|}{$\begin{array}{l}\text { Significant - sources and methods cannot be compromised the enemy should not be } \\
\text { able to know what we see. }\end{array}$} \\
\hline $\begin{array}{r}\text { Highlight issues for } \\
\text { generalizing this use } \\
\text { case (e.g. for ref. } \\
\text { architecture) }\end{array}$ & \multicolumn{2}{|c|}{$\begin{array}{l}\text { Typically this type of processing fits well into massively parallel computing such as } \\
\text { provided by GPUs. Typical problem is integration of this processing into a larger cluster } \\
\text { capable of processing data from several sensors in parallel and in NRT. } \\
\text { Transmission of data from sensor to system is also a large challenge. }\end{array}$} \\
\hline $\begin{array}{r}\text { More Information } \\
\text { (URLs) }\end{array}$ & \multicolumn{2}{|c|}{$\begin{array}{l}\text { Motion Imagery Standards - http://www.gwg.nga.mil/misb/ } \\
\text { Some of many papers on object identity/tracking: } \\
\text { http://www.dabi.temple.edu/ hbling/publication/SPIE12 Dismount_Formatted_v2_B } \\
\text { W.pdf } \\
\text { http://csce.uark.edu/ jgauch/library/Tracking/Orten.2005.pdf } \\
\text { http://www.sciencedirect.com/science/article/pii/S0031320305004863 } \\
\text { General Articles on the need: } \\
\text { http://www.militaryaerospace.com/topics/m/video/79088650/persistent-surveillance- } \\
\underline{\text { relies-on-extracting-relevant-data-points-and-connecting-the-dots.htm }} \\
\text { http://www.defencetalk.com/wide-area-persistent-surveillance-revolutionizes-tactical- } \\
\text { isr-45745/ } \\
\text { istp://www.defencetalk.com/wide-area-persistent-surveillance-revolutionizes-tactical- }\end{array}$} \\
\hline
\end{tabular}




\section{Defense> Use Case 15: Intelligence Data Processing and Analysis}

\begin{tabular}{|c|c|c|}
\hline Use Case Title & \multicolumn{2}{|c|}{ Intelligence Data Processing and Analysis } \\
\hline Vertical (area) & \multicolumn{2}{|c|}{ Defense (Intelligence) } \\
\hline Author/Company/Email & \multicolumn{2}{|c|}{ David Boyd/Data Tactics/dboyd@data-tactics.com } \\
\hline $\begin{array}{r}\text { Actors/Stakeholders } \\
\text { and their roles and } \\
\text { responsibilities }\end{array}$ & \multicolumn{2}{|c|}{$\begin{array}{l}\text { Senior Civilian/Military Leadership } \\
\text { Field Commanders } \\
\text { Intelligence Analysts } \\
\text { Warfighters }\end{array}$} \\
\hline Goals & \multicolumn{2}{|c|}{$\begin{array}{l}\text { 1. Provide automated alerts to Analysts, Warfighters, Commanders, and Leadership } \\
\text { based on incoming intelligence data. } \\
\text { 2. Allow Intelligence Analysts to identify in Intelligence data } \\
\text { a. Relationships between entities (people, organizations, places, equipment) } \\
\text { b. Trends in sentiment or intent for either general population or leadership } \\
\text { group (state, non-state actors). } \\
\text { c. Location of and possibly timing of hostile actions (including implantation of } \\
\text { IEDs). } \\
\text { d. Track the location and actions of (potentially) hostile actors } \\
\text { 3. Ability to reason against and derive knowledge from diverse, disconnected, and } \\
\text { frequently unstructured (e.g. text) data sources. } \\
\text { 4. Ability to process data close to the point of collection and allow data to be } \\
\text { shared easily to/from individual soldiers, forward deployed units, and senior } \\
\text { leadership in garrison. }\end{array}$} \\
\hline Use Case Description & \multicolumn{2}{|c|}{$\begin{array}{l}\text { 1. Ingest/accept data from a wide range of sensors and sources across intelligence } \\
\text { disciplines (IMINT, MASINT, GEOINT, HUMINT, SIGINT, OSINT, etc.) } \\
\text { 2. Process, transform, or align date from disparate sources in disparate formats into } \\
\text { a unified data space to permit: } \\
\text { a. Search } \\
\text { b. Reasoning } \\
\text { c. Comparison } \\
\text { 3. Provide alerts to users of significant changes in the state of monitored entities or } \\
\text { significant activity within an area. } \\
\text { 4. Provide connectivity to the edge for the Warfighter (in this case the edge would } \\
\text { go as far as a single soldier on dismounted patrol) }\end{array}$} \\
\hline \multirow[t]{3}{*}{$\begin{array}{r}\text { Current } \\
\text { Solutions }\end{array}$} & Compute(System) & $\begin{array}{l}\text { Fixed and deployed computing clusters ranging from } \\
1000 \text { s of nodes to } 10 \text { s of nodes. }\end{array}$ \\
\hline & Storage & $\begin{array}{l}\text { 10s of Terabytes to } 100 \text { s of Petabytes for edge and fixed } \\
\text { site clusters. Dismounted soldiers would have at most 1- } \\
100 \text { s of GBs (mostly single digit handheld data storage } \\
\text { sizes). }\end{array}$ \\
\hline & Networking & $\begin{array}{l}\text { Networking with-in and between in garrison fixed sites is } \\
\text { robust. Connectivity to forward edge is limited and often } \\
\text { characterized by high latency and packet loss. Remote } \\
\text { comms might be Satellite based (high latency) or even } \\
\text { limited to RF Line of sight radio. }\end{array}$ \\
\hline
\end{tabular}




\section{Defense> Use Case 15: Intelligence Data Processing and Analysis}

\begin{tabular}{|c|c|c|}
\hline & Software & $\begin{array}{l}\text { Currently baseline leverages: } \\
\text { 1. Hadoop } \\
\text { 2. Accumulo (Big Table) } \\
\text { 3. Solr } \\
\text { 4. NLP (several variants) } \\
\text { 5. Puppet (for deployment and security) } \\
\text { 6. Storm } \\
\text { 7. } \\
\text { Custom applications and visualization tools }\end{array}$ \\
\hline \multirow[t]{5}{*}{$\begin{array}{r}\text { Big Data } \\
\text { Characteristics }\end{array}$} & $\begin{array}{r}\text { Data Source } \\
\text { (distributed/centralized) }\end{array}$ & Very distributed \\
\hline & Volume (size) & $\begin{array}{l}\text { Some IMINT sensors can produce over a petabyte of } \\
\text { data in the space of hours. Other data is as small as } \\
\text { infrequent sensor activations or text messages. }\end{array}$ \\
\hline & $\begin{array}{r}\text { Velocity } \\
\text { (e.g. real time) }\end{array}$ & $\begin{array}{l}\text { Much sensor data is real time (Full motion video, SIGINT) } \\
\text { other is less real time. The critical aspect is to be able } \\
\text { ingest, process, and disseminate alerts in NRT. }\end{array}$ \\
\hline & $\begin{array}{r}\text { Variety } \\
\text { (multiple datasets, } \\
\text { mashup) } \\
\end{array}$ & $\begin{array}{l}\text { Everything from text files, raw media, imagery, video, } \\
\text { audio, electronic data, human generated data. }\end{array}$ \\
\hline & $\begin{array}{r}\text { Variability (rate of } \\
\text { change) }\end{array}$ & $\begin{array}{l}\text { While sensor interface formats tend to be stable, most } \\
\text { other data is uncontrolled and may be in any format. } \\
\text { Much of the data is unstructured. }\end{array}$ \\
\hline \multirow[t]{5}{*}{$\begin{array}{r}\text { Big Data Science } \\
\text { (collection, curation, } \\
\text { analysis, } \\
\text { action) }\end{array}$} & $\begin{array}{l}\text { Veracity (Robustness } \\
\text { Issues, semantics) }\end{array}$ & $\begin{array}{l}\text { Data provenance (e.g. tracking of all transfers and } \\
\text { transformations) must be tracked over the life of the } \\
\text { data. } \\
\text { Determining the veracity of "soft" data sources } \\
\text { (generally human generated) is a critical requirement. }\end{array}$ \\
\hline & Visualization & $\begin{array}{l}\text { Primary visualizations will be Geospatial overlays and } \\
\text { network diagrams. Volume amounts might be millions of } \\
\text { points on the map and thousands of nodes in the } \\
\text { network diagram. }\end{array}$ \\
\hline & Data Quality (syntax) & $\begin{array}{l}\text { Data Quality for sensor generated data is generally } \\
\text { known (image quality, sig/noise) and good. } \\
\text { Unstructured or "captured" data quality varies } \\
\text { significantly and frequently cannot be controlled. }\end{array}$ \\
\hline & Data Types & $\begin{array}{l}\text { Imagery, Video, Text, Digital documents of all types, } \\
\text { Audio, Digital signal data. }\end{array}$ \\
\hline & Data Analytics & $\begin{array}{l}\text { 1. NRT Alerts based on patterns and baseline changes. } \\
\text { 2. Link Analysis } \\
\text { 3. Geospatial Analysis } \\
\text { 4. Text Analytics (sentiment, entity extraction, etc.) }\end{array}$ \\
\hline $\begin{array}{l}\text { Big Data Specific } \\
\text { Challenges (Gaps) }\end{array}$ & \multicolumn{2}{|c|}{$\begin{array}{l}\text { 1. Big (or even moderate size data) over tactical networks } \\
\text { 2. Data currently exists in disparate silos which must be accessible through a } \\
\text { semantically integrated data space. } \\
\text { 3. Most critical data is either unstructured or imagery/video which requires } \\
\text { significant processing to extract entities and information. }\end{array}$} \\
\hline $\begin{array}{r}\text { Big Data Specific } \\
\text { Challenges in Mobility }\end{array}$ & \multicolumn{2}{|c|}{$\begin{array}{l}\text { The outputs of this analysis and information must be transmitted to or accessed by } \\
\text { the dismounted forward soldier. }\end{array}$} \\
\hline
\end{tabular}




\section{Defense> Use Case 15: Intelligence Data Processing and Analysis}

\begin{tabular}{|c|c|}
\hline $\begin{array}{r}\text { Security and Privacy } \\
\text { Requirements }\end{array}$ & $\begin{array}{l}\text { Foremost. Data must be protected against: } \\
\text { 1. Unauthorized access or disclosure } \\
\text { 2. Tampering }\end{array}$ \\
\hline $\begin{array}{r}\text { Highlight issues for } \\
\text { generalizing this use } \\
\text { case (e.g. for ref. } \\
\text { architecture) }\end{array}$ & $\begin{array}{l}\text { Wide variety of data types, sources, structures, and quality which will span domains } \\
\text { and requires integrated search and reasoning. }\end{array}$ \\
\hline $\begin{array}{r}\text { More Information } \\
\text { (URLs) }\end{array}$ & $\begin{array}{l}\text { http://www.afcea- } \\
\text { aberdeen.org/files/presentations/AFCEAAberdeen DCGSA COLWells PS.pdf } \\
\text { http://stids.c4i.gmu.edu/papers/STIDSPapers/STIDS2012 T14 SmithEtAI Horizontall } \\
\text { ntegrationOfWarfighterIntel.pdf } \\
\text { http://stids.c4i.gmu.edu/STIDS2011/papers/STIDS2011 CR T1 SalmenEtAl.pdf } \\
\text { http://www.youtube.com/watch?v=|4Qii7T8zeg } \\
\text { http://dcgsa.apg.army.mil/ }\end{array}$ \\
\hline
\end{tabular}




\section{Healthcare and Life Sciences> Use Case 16: Electronic Medical Record Data}

\begin{tabular}{|c|c|c|}
\hline Use Case Title & \multicolumn{2}{|c|}{ Electronic Medical Record (EMR) Data } \\
\hline Vertical (area) & \multicolumn{2}{|c|}{ Healthcare } \\
\hline Author/Company/Email & \multicolumn{2}{|c|}{ Shaun Grannis/Indiana University/sgrannis@regenstrief.org } \\
\hline & \multicolumn{2}{|c|}{$\begin{array}{l}\text { Biomedical informatics research scientists (implement and evaluate enhanced } \\
\text { methods for seamlessly integrating, standardizing, analyzing, and operationalizing } \\
\text { highly heterogeneous, high-volume clinical data streams); Health services } \\
\text { researchers (leverage integrated and standardized EMR data to derive knowledge } \\
\text { that supports implementation and evaluation of translational, comparative } \\
\text { effectiveness, patient-centered outcomes research); Healthcare providers - } \\
\text { physicians, nurses, public health officials (leverage information and knowledge } \\
\text { derived from integrated and standardized EMR data to support direct patient care } \\
\text { and population health) }\end{array}$} \\
\hline Goals & \multicolumn{2}{|c|}{$\begin{array}{l}\text { Use advanced methods for normalizing patient, provider, facility and clinical concept } \\
\text { identification within and among separate health care organizations to enhance } \\
\text { models for defining and extracting clinical phenotypes from non-standard discrete } \\
\text { and free-text clinical data using feature selection, information retrieval and machine } \\
\text { learning decision-models. Leverage clinical phenotype data to support cohort } \\
\text { selection, clinical outcomes research, and clinical decision support. }\end{array}$} \\
\hline Use Case Description & \multicolumn{2}{|c|}{$\begin{array}{l}\text { As health care systems increasingly gather and consume EMR data, large national } \\
\text { initiatives aiming to leverage such data are emerging, and include developing a } \\
\text { digital learning health care system to support increasingly evidence-based clinical } \\
\text { decisions with timely accurate and up-to-date patient-centered clinical information; } \\
\text { using electronic observational clinical data to efficiently and rapidly translate } \\
\text { scientific discoveries into effective clinical treatments; and electronically sharing } \\
\text { integrated health data to improve healthcare process efficiency and outcomes. } \\
\text { These key initiatives all rely on high-quality, large-scale, standardized and aggregate } \\
\text { health data. Despite the promise that increasingly prevalent and ubiquitous EMR } \\
\text { data hold, enhanced methods for integrating and rationalizing these data are needed } \\
\text { for a variety of reasons. Data from clinical systems evolve over time. This is because } \\
\text { the concept space in healthcare is constantly evolving: new scientific discoveries lead } \\
\text { to new disease entities, new diagnostic modalities, and new disease management } \\
\text { approaches. These in turn lead to new clinical concepts, which drive the evolution of } \\
\text { health concept ontologies. Using heterogeneous data from the Indiana Network for } \\
\text { Patient Care (INPC), the nation's largest and longest-running health information } \\
\text { exchange, which includes more than } 4 \text { billion discrete coded clinical observations } \\
\text { from more than } 100 \text { hospitals for more than } 12 \text { million patients, we will use } \\
\text { information retrieval techniques to identify highly relevant clinical features from } \\
\text { electronic observational data. We will deploy information retrieval and natural } \\
\text { language processing techniques to extract clinical features. Validated features will be } \\
\text { used to parameterize clinical phenotype decision models based on maximum } \\
\text { likelihood estimators and Bayesian networks. Using these decision models we will } \\
\text { identify a variety of clinical phenotypes such as diabetes, congestive heart failure, } \\
\text { and pancreatic cancer. }\end{array}$} \\
\hline \multirow{4}{*}{$\begin{array}{r}\text { Current } \\
\text { Solutions }\end{array}$} & Compute(System) & Big Red II, a new Cray supercomputer at I.U. \\
\hline & Storage & Teradata, Po \\
\hline & & \\
\hline & Software & \\
\hline
\end{tabular}




\section{Healthcare and Life Sciences> Use Case 16: Electronic Medical Record Data}

\begin{tabular}{|c|c|c|}
\hline \multirow[t]{5}{*}{$\begin{array}{r}\text { Big Data } \\
\text { Characteristics }\end{array}$} & $\begin{array}{r}\text { Data Source } \\
\text { (distributed/centralized) }\end{array}$ & $\begin{array}{l}\text { Clinical data from more than } 1,100 \text { discrete logical, } \\
\text { operational healthcare sources in the Indiana Network } \\
\text { for Patient Care (INPC) the nation's largest and longest- } \\
\text { running health information exchange. }\end{array}$ \\
\hline & Volume (size) & $\begin{array}{l}\text { More than } 12 \text { million patients, more than } 4 \text { billion } \\
\text { discrete clinical observations. > } 20 \text { TB raw data. }\end{array}$ \\
\hline & $\begin{array}{r}\text { Velocity } \\
\text { (e.g. real time) }\end{array}$ & $\begin{array}{l}\text { Between } 500,000 \text { and } 1.5 \text { million new real-time clinical } \\
\text { transactions added per day. }\end{array}$ \\
\hline & $\begin{array}{r}\text { Variety } \\
\text { (multiple datasets, } \\
\text { mashup) }\end{array}$ & $\begin{array}{l}\text { We integrate a broad variety of clinical datasets from } \\
\text { multiple sources: free text provider notes; inpatient, } \\
\text { outpatient, laboratory, and emergency department } \\
\text { encounters; chromosome and molecular pathology; } \\
\text { chemistry studies; cardiology studies; hematology } \\
\text { studies; microbiology studies; neurology studies; } \\
\text { provider notes; referral labs; serology studies; surgical } \\
\text { pathology and cytology, blood bank, and toxicology } \\
\text { studies. }\end{array}$ \\
\hline & $\begin{array}{r}\text { Variability (rate of } \\
\text { change) }\end{array}$ & $\begin{array}{l}\text { Data from clinical systems evolve over time because } \\
\text { the clinical and biological concept space is constantly } \\
\text { evolving: new scientific discoveries lead to new disease } \\
\text { entities, new diagnostic modalities, and new disease } \\
\text { management approaches. These in turn lead to new } \\
\text { clinical concepts, which drive the evolution of health } \\
\text { concept ontologies, encoded in highly variable fashion. }\end{array}$ \\
\hline \multirow[t]{4}{*}{$\begin{array}{r}\text { Big Data Science } \\
\text { (collection, curation, } \\
\text { analysis, } \\
\text { action) }\end{array}$} & $\begin{array}{r}\text { Veracity (Robustness } \\
\text { Issues, semantics) }\end{array}$ & $\begin{array}{l}\text { Data from each clinical source are commonly gathered } \\
\text { using different methods and representations, yielding } \\
\text { substantial heterogeneity. This leads to systematic } \\
\text { errors and bias requiring robust methods for creating } \\
\text { semantic interoperability. }\end{array}$ \\
\hline & Visualization & $\begin{array}{l}\text { Inbound data volume, accuracy, and completeness } \\
\text { must be monitored on a routine basis using focus } \\
\text { visualization methods. Intrinsic informational } \\
\text { characteristics of data sources must be visualized to } \\
\text { identify unexpected trends. }\end{array}$ \\
\hline & Data Quality (syntax) & $\begin{array}{l}\text { A central barrier to leveraging EMR data is the highly } \\
\text { variable and unique local names and codes for the } \\
\text { same clinical test or measurement performed at } \\
\text { different institutions. When integrating many data } \\
\text { sources, mapping local terms to a common } \\
\text { standardized concept using a combination of } \\
\text { probabilistic and heuristic classification methods is } \\
\text { necessary. }\end{array}$ \\
\hline & Data Types & $\begin{array}{l}\text { Wide variety of clinical data types including numeric, } \\
\text { structured numeric, free-text, structured text, discrete } \\
\text { nominal, discrete ordinal, discrete structured, binary } \\
\text { large blobs (images and video). }\end{array}$ \\
\hline
\end{tabular}




\section{Healthcare and Life Sciences> Use Case 16: Electronic Medical Record Data}

\begin{tabular}{|c|c|c|}
\hline & Data Analytics & $\begin{array}{l}\text { Information retrieval methods to identify relevant } \\
\text { clinical features (tf-idf, latent semantic analysis, mutual } \\
\text { information). Natural Language Processing techniques } \\
\text { to extract relevant clinical features. Validated features } \\
\text { will be used to parameterize clinical phenotype } \\
\text { decision models based on maximum likelihood } \\
\text { estimators and Bayesian networks. Decision models will } \\
\text { be used to identify a variety of clinical phenotypes such } \\
\text { as diabetes, congestive heart failure, and pancreatic } \\
\text { cancer. }\end{array}$ \\
\hline $\begin{array}{r}\text { Big Data Specific } \\
\text { Challenges (Gaps) }\end{array}$ & \multicolumn{2}{|c|}{$\begin{array}{l}\text { Overcoming the systematic errors and bias in large-scale, heterogeneous clinical data } \\
\text { to support decision-making in research, patient care, and administrative use-cases } \\
\text { requires complex multistage processing and analytics that demands substantial } \\
\text { computing power. Further, the optimal techniques for accurately and effectively } \\
\text { deriving knowledge from observational clinical data are nascent. }\end{array}$} \\
\hline $\begin{array}{r}\text { Big Data Specific } \\
\text { Challenges in Mobility }\end{array}$ & \multicolumn{2}{|c|}{$\begin{array}{l}\text { Biological and clinical data are needed in a variety of contexts throughout the } \\
\text { healthcare ecosystem. Effectively delivering clinical data and knowledge across the } \\
\text { healthcare ecosystem will be facilitated by mobile platform such as mHealth. }\end{array}$} \\
\hline $\begin{array}{r}\text { Security and Privacy } \\
\text { Requirements }\end{array}$ & \multicolumn{2}{|c|}{$\begin{array}{l}\text { Privacy and confidentiality of individuals must be preserved in compliance with } \\
\text { federal and state requirements including HIPAA. Developing analytic models using } \\
\text { comprehensive, integrated clinical data requires aggregation and subsequent de- } \\
\text { identification prior to applying complex analytics. }\end{array}$} \\
\hline $\begin{array}{r}\text { Highlight issues for } \\
\text { generalizing this use } \\
\text { case (e.g. for ref. } \\
\text { architecture) }\end{array}$ & \multicolumn{2}{|c|}{$\begin{array}{l}\text { Patients increasingly receive health care in a variety of clinical settings. The } \\
\text { subsequent EMR data is fragmented and heterogeneous. In order to realize the } \\
\text { promise of a Learning Health Care system as advocated by the National Academy of } \\
\text { Science and the Institute of Medicine, EMR data must be rationalized and integrated. } \\
\text { The methods we propose in this use-case support integrating and rationalizing } \\
\text { clinical data to support decision-making at multiple levels. }\end{array}$} \\
\hline $\begin{array}{r}\text { More Information } \\
\text { (URLs) }\end{array}$ & \multicolumn{2}{|c|}{$\begin{array}{l}\text { Regenstrief Institute (http://www.regenstrief.org); Logical observation identifiers } \\
\text { names and codes (http://www.loinc.org); Indiana Health Information Exchange } \\
\text { (http://www.ihie.org); Institute of Medicine Learning Healthcare System } \\
\text { (http://www.iom.edu/Activities/Quality/LearningHealthcare.aspx) }\end{array}$} \\
\hline
\end{tabular}




\section{Healthcare and Life Sciences> Use Case 17: Pathology I maging/ Digital Pathology}

\begin{tabular}{|c|c|c|}
\hline Use Case Title & \multicolumn{2}{|c|}{ Pathology Imaging/digital pathology } \\
\hline Vertical (area) & \multicolumn{2}{|c|}{ Healthcare } \\
\hline Author/Company/Email & \multicolumn{2}{|c|}{ Fusheng Wang/Emory University/fusheng.wang@emory.edu } \\
\hline $\begin{array}{r}\text { Actors/Stakeholders } \\
\text { and their roles and } \\
\text { responsibilities }\end{array}$ & \multicolumn{2}{|c|}{$\begin{array}{l}\text { Biomedical researchers on translational research; hospital clinicians on imaging } \\
\text { guided diagnosis }\end{array}$} \\
\hline Goals & \multicolumn{2}{|c|}{$\begin{array}{l}\text { Develop high performance image analysis algorithms to extract spatial information } \\
\text { from images; provide efficient spatial queries and analytics, and feature clustering } \\
\text { and classification }\end{array}$} \\
\hline Use Case Description & \multicolumn{2}{|c|}{$\begin{array}{l}\text { Digital pathology imaging is an emerging field where examination of high resolution } \\
\text { images of tissue specimens enables novel and more effective ways for disease } \\
\text { diagnosis. Pathology image analysis segments massive (millions per image) spatial } \\
\text { objects such as nuclei and blood vessels, represented with their boundaries, along } \\
\text { with many extracted image features from these objects. The derived information is } \\
\text { used for many complex queries and analytics to support biomedical research and } \\
\text { clinical diagnosis. Recently, 3D pathology imaging is made possible through 3D laser } \\
\text { technologies or serially sectioning hundreds of tissue sections onto slides and } \\
\text { scanning them into digital images. Segmenting 3D microanatomic objects from } \\
\text { registered serial images could produce tens of millions of 3D objects from a single } \\
\text { image. This provides a deep "map" of human tissues for next generation diagnosis. }\end{array}$} \\
\hline \multirow{4}{*}{$\begin{array}{r}\text { Current } \\
\text { Solutions }\end{array}$} & Compute(System) & Supercomputers; Cloud \\
\hline & Storage & SAN or HDFS \\
\hline & Networking & Need excellent external network link \\
\hline & Software & $\begin{array}{l}\text { MPI for image analysis; Map/Reduce + Hive with spatial } \\
\text { extension }\end{array}$ \\
\hline \multirow[t]{5}{*}{$\begin{array}{r}\text { Big Data } \\
\text { Characteristics }\end{array}$} & $\begin{array}{r}\text { Data Source } \\
\text { (distributed/centralized) }\end{array}$ & Digitized pathology images from human tissues \\
\hline & Volume (size) & $\begin{array}{l}\text { 1GB raw image data }+1.5 \mathrm{~GB} \text { analytical results per } 2 \mathrm{D} \\
\text { image; } 1 \mathrm{~TB} \text { raw image data }+1 \mathrm{~TB} \text { analytical results per } \\
3 \mathrm{D} \text { image. } 1 \mathrm{~PB} \text { data per moderated hospital per year }\end{array}$ \\
\hline & $\begin{array}{r}\text { Velocity } \\
\text { (e.g. real time) }\end{array}$ & Once generated, data will not be changed \\
\hline & $\begin{array}{r}\text { Variety } \\
\text { (multiple datasets, } \\
\text { mashup) }\end{array}$ & $\begin{array}{l}\text { Image characteristics and analytics depend on disease } \\
\text { types }\end{array}$ \\
\hline & $\begin{array}{r}\text { Variability (rate of } \\
\text { change) }\end{array}$ & No change \\
\hline \multirow{5}{*}{$\begin{array}{r}\text { Big Data Science } \\
\text { (collection, curation, } \\
\text { analysis, } \\
\text { action) }\end{array}$} & $\begin{array}{r}\begin{array}{r}\text { Veracity (Robustness } \\
\text { Issues) }\end{array} \\
\end{array}$ & $\begin{array}{l}\text { High quality results validated with human annotations } \\
\text { are essential }\end{array}$ \\
\hline & Visualization & Needed for validation and training \\
\hline & Data Quality & $\begin{array}{l}\text { Depend on preprocessing of tissue slides such as } \\
\text { chemical staining and quality of image analysis } \\
\text { algorithms }\end{array}$ \\
\hline & Data Types & $\begin{array}{l}\text { Raw images are whole slide images (mostly based on } \\
\text { BIGTIFF), and analytical results are structured data } \\
\text { (spatial boundaries and features) }\end{array}$ \\
\hline & Data Analytics & $\begin{array}{l}\text { Image analysis, spatial queries and analytics, feature } \\
\text { clustering and classification }\end{array}$ \\
\hline
\end{tabular}




\section{Healthcare and Life Sciences> Use Case 17: Pathology I maging/ Digital Pathology}

\begin{tabular}{|r|l|}
\hline $\begin{array}{r}\text { Big Data Specific } \\
\text { Challenges (Gaps) }\end{array}$ & $\begin{array}{l}\text { Extreme large size; multi-dimensional; disease specific analytics; correlation with } \\
\text { other data types (clinical data, -omic data) }\end{array}$ \\
\hline $\begin{array}{r}\text { Big Data Specific } \\
\text { Challenges in Mobility }\end{array}$ & 3D visualization of 3D pathology images is not likely in mobile platforms \\
\hline $\begin{array}{r}\text { Security and Privacy } \\
\text { Requirements }\end{array}$ & $\begin{array}{l}\text { Protected health information has to be protected; public data have to be de- } \\
\text { identified }\end{array}$ \\
\hline $\begin{array}{r}\text { Highlight issues for } \\
\text { generalizing this use } \\
\text { case (e.g. for ref. } \\
\text { architecture) }\end{array}$ & \\
\hline $\begin{array}{r}\text { Imaging data; multi-dimensional spatial data analytics } \\
\text { More Information } \\
\text { (URLs) }\end{array}$ & $\underline{\text { https://web.cci.emory.edu/confluence/display/PAIS }}$ \\
\hline https://web.cci.emory.edu/confluence/display/HadoopGIS \\
\hline
\end{tabular}

See Figure 2: Pathology Imaging/Digital Pathology - Examples of 2-D and 3-D pathology images. See Figure 3: Pathology Imaging/Digital Pathology - Architecture of Hadoop-GIS, a spatial data warehousing system, over MapReduce to support spatial analytics for analytical pathology imaging. 


\section{Healthcare and Life Sciences> Use Case 18: Computational Bioimaging}

\begin{tabular}{|c|c|c|}
\hline Use Case Title & \multicolumn{2}{|l|}{ Computational Bioimaging } \\
\hline Vertical (area) & \multicolumn{2}{|c|}{ Scientific Research: Biological Science } \\
\hline Author/Company/Email & \multicolumn{2}{|c|}{$\begin{array}{l}\text { David Skinner }{ }^{1}, \underline{\text { deskinner@lbl.gov }} \\
\text { Joaquin Correa }{ }^{1}, \underline{\text { JoaquinCorrea@lbl.gov }} \\
\text { Daniela Ushizima }{ }^{2} \text {, dushizima@lbl.gov } \\
\text { Joerg Meyer }{ }^{2} \text {, joergmeyer@lbl.gov } \\
{ }^{1} \text { National Energy Scientific Computing Center (NERSC), Lawrence Berkeley National } \\
\text { Laboratory, USA } \\
{ }^{2} \text { Computational Research Division, Lawrence Berkeley National Laboratory, USA }\end{array}$} \\
\hline $\begin{array}{l}\text { Actors/Stakeholders } \\
\text { and their roles and } \\
\text { responsibilities }\end{array}$ & \multicolumn{2}{|c|}{$\begin{array}{l}\text { Capability providers: Bioimaging instrument operators, microscope developers, } \\
\text { imaging facilities, applied mathematicians, and data stewards. } \\
\text { User Community: DOE, industry and academic researchers seeking to collaboratively } \\
\text { build models from imaging data. }\end{array}$} \\
\hline Goals & \multicolumn{2}{|c|}{$\begin{array}{l}\text { Data delivered from bioimaging is increasingly automated, higher resolution, and } \\
\text { multi-modal. This has created a data analysis bottleneck that, if resolved, can } \\
\text { advance the biosciences discovery through Big Data techniques. Our goal is to solve } \\
\text { that bottleneck with extreme scale computing. } \\
\text { Meeting that goal will require more than computing. It will require building } \\
\text { communities around data resources and providing advanced algorithms for massive } \\
\text { image analysis. High-performance computational solutions can be harnessed by } \\
\text { community-focused science gateways to guide the application of massive data } \\
\text { analysis toward massive imaging datasets. Workflow components include data } \\
\text { acquisition, storage, enhancement, minimizing noise, segmentation of regions of } \\
\text { interest, crowd-based selection and extraction of features, and object classification, } \\
\text { and organization, and search. }\end{array}$} \\
\hline Use Case Description & \multicolumn{2}{|c|}{$\begin{array}{l}\text { Web-based one-stop-shop for high performance, high throughput image processing } \\
\text { for producers and consumers of models built on bio-imaging data. }\end{array}$} \\
\hline \multirow{4}{*}{$\begin{array}{r}\text { Current } \\
\text { Solutions }\end{array}$} & Compute(System) & Hopper.nersc.gov (150K cores) \\
\hline & Storage & Database and image collections \\
\hline & Networking & $10 \mathrm{~Gb}$, could use $100 \mathrm{~Gb}$ and advanced networking (SDN) \\
\hline & Software & $\begin{array}{l}\text { ImageJ, OMERO, VolRover, advanced segmentation and } \\
\text { feature detection methods from applied math } \\
\text { researchers }\end{array}$ \\
\hline \multirow[t]{5}{*}{$\begin{array}{r}\text { Big Data } \\
\text { Characteristics }\end{array}$} & $\begin{array}{r}\text { Data Source } \\
\text { (distributed/centralized) }\end{array}$ & $\begin{array}{l}\text { Distributed experimental sources of bioimages } \\
\text { (instruments). Scheduled high volume flows from } \\
\text { automated high-resolution optical and electron } \\
\text { microscopes. }\end{array}$ \\
\hline & Volume (size) & $\begin{array}{l}\text { Growing very fast. Scalable key-value and object store } \\
\text { databases needed. In-database processing and analytics. } \\
\text { 50TB here now, but currently over a petabyte overall. A } \\
\text { single scan on emerging machines is 32TB }\end{array}$ \\
\hline & $\begin{array}{r}\text { Velocity } \\
\text { (e.g. real time) }\end{array}$ & High throughput computing (HTC), responsive analysis \\
\hline & $\begin{array}{r}\text { Variety } \\
\text { (multiple datasets, } \\
\text { mashup) }\end{array}$ & $\begin{array}{l}\text { Multi-modal imaging essentially must mash-up disparate } \\
\text { channels of data with attention to registration and } \\
\text { dataset formats. }\end{array}$ \\
\hline & $\begin{array}{r}\text { Variability (rate of } \\
\text { change) }\end{array}$ & $\begin{array}{l}\text { Biological samples are highly variable and their analysis } \\
\text { workflows must cope with wide variation. }\end{array}$ \\
\hline
\end{tabular}




\section{Healthcare and Life Sciences> Use Case 18: Computational Bioimaging}

\begin{tabular}{|c|c|c|}
\hline \multirow{5}{*}{$\begin{array}{r}\text { Big Data Science } \\
\text { (collection, curation, } \\
\text { analysis, } \\
\text { action) }\end{array}$} & $\begin{array}{r}\text { Veracity (Robustness } \\
\text { Issues, semantics) }\end{array}$ & Data is messy overall as is training classifiers. \\
\hline & Visualization & Heavy use of 3D structural models. \\
\hline & Data Quality (syntax) & \\
\hline & Data Types & Imaging file formats \\
\hline & Data Analytics & $\begin{array}{l}\text { Machine learning (SVM and RF) for classification and } \\
\text { recommendation services. }\end{array}$ \\
\hline $\begin{array}{r}\text { Big Data Specific } \\
\text { Challenges (Gaps) }\end{array}$ & \multicolumn{2}{|c|}{$\begin{array}{l}\text { HTC at scale for simulation science. Flexible data methods at scale for messy data. } \\
\text { Machine learning and knowledge systems that drive pixel based data toward } \\
\text { biological objects and models. }\end{array}$} \\
\hline \multicolumn{3}{|l|}{$\begin{array}{r}\text { Big Data Specific } \\
\text { Challenges in Mobility }\end{array}$} \\
\hline \multicolumn{3}{|l|}{$\begin{array}{r}\text { Security and Privacy } \\
\text { Requirements }\end{array}$} \\
\hline $\begin{array}{r}\text { Highlight issues for } \\
\text { generalizing this use } \\
\text { case (e.g. for ref. } \\
\text { architecture) }\end{array}$ & \multicolumn{2}{|c|}{ There is potential in generalizing concepts of search in the context of bioimaging. } \\
\hline $\begin{array}{r}\text { More Information } \\
\text { (URLs) }\end{array}$ & & \\
\hline
\end{tabular}




\section{Healthcare and Life Sciences> Use Case 19: Genomic Measurements}

\begin{tabular}{|c|c|c|}
\hline Use Case Title & \multicolumn{2}{|l|}{ Genomic Measurements } \\
\hline Vertical (area) & \multicolumn{2}{|l|}{ Healthcare } \\
\hline Author/Company/Email & \multicolumn{2}{|c|}{ Justin Zook/NIST/izook@nist.gov } \\
\hline $\begin{array}{r}\text { Actors/Stakeholders } \\
\text { and their roles and } \\
\text { responsibilities }\end{array}$ & \multicolumn{2}{|c|}{ NIST/Genome in a Bottle Consortium - public/private/academic partnership } \\
\hline Goals & \multicolumn{2}{|c|}{$\begin{array}{l}\text { Develop well-characterized Reference Materials, Reference Data, and Reference } \\
\text { Methods needed to assess performance of genome sequencing }\end{array}$} \\
\hline Use Case Description & \multicolumn{2}{|c|}{$\begin{array}{l}\text { Integrate data from multiple sequencing technologies and methods to develop highly } \\
\text { confident characterization of whole human genomes as Reference Materials, and } \\
\text { develop methods to use these Reference Materials to assess performance of any } \\
\text { genome sequencing run }\end{array}$} \\
\hline \multirow[t]{4}{*}{$\begin{array}{r}\text { Current } \\
\text { Solutions }\end{array}$} & Compute(System) & $\begin{array}{l}\text { 72-core cluster for our NIST group, collaboration with } \\
>1000 \text { core clusters at FDA, some groups are using } \\
\text { cloud }\end{array}$ \\
\hline & Storage & $\approx 40 T B$ NFS at NIST, PBs of genomics data at NIH/NCBI \\
\hline & Networking & Varies. Significant I/O intensive processing needed \\
\hline & Software & $\begin{array}{l}\text { Open-source sequencing bioinformatics software from } \\
\text { academic groups (UNIX-based) }\end{array}$ \\
\hline \multirow[t]{5}{*}{$\begin{array}{r}\text { Big Data } \\
\text { Characteristics }\end{array}$} & $\begin{array}{r}\text { Data Source } \\
\text { (distributed/centralized) }\end{array}$ & $\begin{array}{l}\text { Sequencers are distributed across many laboratories, } \\
\text { though some core facilities exist. }\end{array}$ \\
\hline & Volume (size) & $\begin{array}{l}\text { 40TB NFS is full, will need }>100 \text { TB in } 1-2 \text { years at NIST; } \\
\text { Healthcare community will need many PBs of storage }\end{array}$ \\
\hline & $\begin{array}{r}\text { Velocity } \\
\text { (e.g. real time) }\end{array}$ & $\begin{array}{l}\text { DNA sequencers can generate } \approx 300 G B \text { compressed } \\
\text { data/day. Velocity has increased much faster than } \\
\text { Moore's Law }\end{array}$ \\
\hline & $\begin{array}{r}\text { Variety } \\
\text { (multiple datasets, } \\
\text { mashup) }\end{array}$ & $\begin{array}{l}\text { File formats not well-standardized, though some } \\
\text { standards exist. Generally structured data. }\end{array}$ \\
\hline & $\begin{array}{r}\text { Variability (rate of } \\
\text { change) }\end{array}$ & $\begin{array}{l}\text { Sequencing technologies have evolved very rapidly, and } \\
\text { new technologies are on the horizon. }\end{array}$ \\
\hline \multirow[t]{5}{*}{$\begin{array}{r}\text { Big Data Science } \\
\text { (collection, curation, } \\
\text { analysis, } \\
\text { action) }\end{array}$} & $\begin{array}{r}\text { Veracity (Robustness } \\
\text { Issues) }\end{array}$ & $\begin{array}{l}\text { All sequencing technologies have significant systematic } \\
\text { errors and biases, which require complex analysis } \\
\text { methods and combining multiple technologies to } \\
\text { understand, often with machine learning }\end{array}$ \\
\hline & Visualization & $\begin{array}{l}\text { "Genome browsers" have been developed to visualize } \\
\text { processed data }\end{array}$ \\
\hline & Data Quality & $\begin{array}{l}\text { Sequencing technologies and bioinformatics methods } \\
\text { have significant systematic errors and biases }\end{array}$ \\
\hline & Data Types & Mainly structured text \\
\hline & Data Analytics & $\begin{array}{l}\text { Processing of raw data to produce variant calls. Also, } \\
\text { clinical interpretation of variants, which is now very } \\
\text { challenging. }\end{array}$ \\
\hline $\begin{array}{l}\text { Big Data Specific } \\
\text { Challenges (Gaps) }\end{array}$ & \multicolumn{2}{|c|}{$\begin{array}{l}\text { Processing data requires significant computing power, which poses challenges } \\
\text { especially to clinical laboratories as they are starting to perform large-scale } \\
\text { sequencing. Long-term storage of clinical sequencing data could be expensive. } \\
\text { Analysis methods are quickly evolving. Many parts of the genome are challenging to } \\
\text { analyze, and systematic errors are difficult to characterize. }\end{array}$} \\
\hline $\begin{array}{r}\text { Big Data Specific } \\
\text { Challenges in Mobility }\end{array}$ & \multicolumn{2}{|c|}{ Physicians may need access to genomic data on mobile platforms } \\
\hline
\end{tabular}




\section{Healthcare and Life Sciences> Use Case 19: Genomic Measurements}

\begin{tabular}{|c|c|}
\hline $\begin{array}{r}\text { Security and Privacy } \\
\text { Requirements }\end{array}$ & $\begin{array}{l}\text { Sequencing data in health records or clinical research databases must be kept } \\
\text { secure/private, though our Consortium data is public. }\end{array}$ \\
\hline $\begin{array}{r}\text { Highlight issues for } \\
\text { generalizing this use } \\
\text { case (e.g. for ref. } \\
\text { architecture) }\end{array}$ & $\begin{array}{l}\text { I have some generalizations to medical genome sequencing above, but focus on } \\
\text { NIST/Genome in a Bottle Consortium work. Currently, labs doing sequencing range } \\
\text { from small to very large. Future data could include other 'omics' measurements, } \\
\text { which could be even larger than DNA sequencing }\end{array}$ \\
\hline $\begin{array}{r}\text { More Information } \\
\text { (URLs) }\end{array}$ & Genome in a Bottle Consortium: http://www.genomeinabottle.org \\
\hline
\end{tabular}

273

274

275

276 
Healthcare and Life Sciences> Use Case 20: Comparative Analysis for (meta) Genomes

\begin{tabular}{|c|c|c|}
\hline Use Case Title & \multicolumn{2}{|c|}{ Comparative analysis for metagenomes and genomes } \\
\hline Vertical (area) & \multicolumn{2}{|c|}{ Scientific Research: Genomics } \\
\hline Author/Company/Email & \multicolumn{2}{|c|}{ Ernest Szeto / LBNL / eszeto@lbl.gov } \\
\hline $\begin{array}{r}\text { Actors/Stakeholders } \\
\text { and their roles and } \\
\text { responsibilities }\end{array}$ & \multicolumn{2}{|c|}{$\begin{array}{l}\text { Joint Genome Institute (JGI) Integrated Microbial Genomes (IMG) project. Heads: } \\
\text { Victor M. Markowitz, and Nikos C. Kyrpides. User community: JGI, bioinformaticians } \\
\text { and biologists worldwide. }\end{array}$} \\
\hline Goals & \multicolumn{2}{|c|}{$\begin{array}{l}\text { Provide an integrated comparative analysis system for metagenomes and genomes. } \\
\text { This includes interactive Web UI with core data, backend precomputations, batch job } \\
\text { computation submission from the UI. }\end{array}$} \\
\hline Use Case Description & \multicolumn{2}{|c|}{$\begin{array}{l}\text { Given a metagenomic sample, (1) determine the community composition in terms of } \\
\text { other reference isolate genomes, (2) characterize the function of its genes, (3) begin } \\
\text { to infer possible functional pathways, (4) characterize similarity or dissimilarity with } \\
\text { other metagenomic samples, (5) begin to characterize changes in community } \\
\text { composition and function due to changes in environmental pressures, (6) isolate sub- } \\
\text { sections of data based on quality measures and community composition. }\end{array}$} \\
\hline \multirow[t]{4}{*}{$\begin{array}{r}\text { Current } \\
\text { Solutions }\end{array}$} & Compute(System) & $\begin{array}{l}\text { Linux cluster, Oracle RDBMS server, large memory } \\
\text { machines, standard Linux interactive hosts }\end{array}$ \\
\hline & Storage & $\begin{array}{l}\text { Oracle RDBMS, SQLite files, flat text files, Lucy (a } \\
\text { version of Lucene) for keyword searches, BLAST } \\
\text { databases, USEARCH databases }\end{array}$ \\
\hline & Networking & Provided by NERSC \\
\hline & Software & $\begin{array}{l}\text { Standard bioinformatics tools (BLAST, HMMER, multiple } \\
\text { alignment and phylogenetic tools, gene callers, } \\
\text { sequence feature predictors...), Perl/Python wrapper } \\
\text { scripts, Linux Cluster scheduling }\end{array}$ \\
\hline \multirow[t]{5}{*}{$\begin{array}{r}\text { Big Data } \\
\text { Characteristics }\end{array}$} & $\begin{array}{r}\text { Data Source } \\
\text { (distributed/centralized) }\end{array}$ & Centralized. \\
\hline & Volume (size) & $50 \mathrm{tb}$ \\
\hline & $\begin{array}{r}\text { Velocity } \\
\text { (e.g. real time) }\end{array}$ & $\begin{array}{l}\text { Front end web UI must be real time interactive. Back } \\
\text { end data loading processing must keep up with } \\
\text { exponential growth of sequence data due to the rapid } \\
\text { drop in cost of sequencing technology. }\end{array}$ \\
\hline & $\begin{array}{r}\text { Variety } \\
\text { (multiple datasets, } \\
\text { mashup) }\end{array}$ & $\begin{array}{l}\text { Biological data is inherently heterogeneous, complex, } \\
\text { structural, and hierarchical. One begins with sequences, } \\
\text { followed by features on sequences, such as genes, } \\
\text { motifs, regulatory regions, followed by organization of } \\
\text { genes in neighborhoods (operons), to proteins and } \\
\text { their structural features, to coordination and } \\
\text { expression of genes in pathways. Besides core genomic } \\
\text { data, new types of "Omics" data such as } \\
\text { transcriptomics, methylomics, and proteomics } \\
\text { describing gene expression under a variety of } \\
\text { conditions must be incorporated into the comparative } \\
\text { analysis system. }\end{array}$ \\
\hline & $\begin{array}{r}\text { Variability (rate of } \\
\text { change) }\end{array}$ & $\begin{array}{l}\text { The sizes of metagenomic samples can vary by several } \\
\text { orders of magnitude, such as several hundred thousand } \\
\text { genes to a billion genes (e.g., latter in a complex soil } \\
\text { sample). }\end{array}$ \\
\hline
\end{tabular}




\section{Healthcare and Life Sciences> Use Case 20: Comparative Analysis for (meta) Genomes}

\begin{tabular}{|c|c|c|}
\hline \multirow[t]{5}{*}{$\begin{array}{r}\text { Big Data Science } \\
\text { (collection, curation, } \\
\text { analysis, } \\
\text { action) }\end{array}$} & $\begin{array}{r}\text { Veracity (Robustness } \\
\text { Issues) }\end{array}$ & $\begin{array}{l}\text { Metagenomic sampling science is currently preliminary } \\
\text { and exploratory. Procedures for evaluating assembly of } \\
\text { highly fragmented data in raw reads are better defined, } \\
\text { but still an open research area. }\end{array}$ \\
\hline & Visualization & $\begin{array}{l}\text { Interactive speed of web UI on very large datasets is an } \\
\text { ongoing challenge. Web UI's still seem to be the } \\
\text { preferred interface for most biologists. It is use for } \\
\text { basic querying and browsing of data. More specialized } \\
\text { tools may be launched from them, e.g. for viewing } \\
\text { multiple alignments. Ability to download large amounts } \\
\text { of data for offline analysis is another requirement of } \\
\text { the system. }\end{array}$ \\
\hline & Data Quality & $\begin{array}{l}\text { Improving quality of metagenomic assembly is still a } \\
\text { fundamental challenge. Improving the quality of } \\
\text { reference isolate genomes, both in terms of the } \\
\text { coverage in the phylogenetic tree, improved gene } \\
\text { calling and functional annotation is a more mature } \\
\text { process, but an ongoing project. }\end{array}$ \\
\hline & Data Types & Cf. above on "Variety" \\
\hline & Data Analytics & $\begin{array}{l}\text { Descriptive statistics, statistical significance in } \\
\text { hypothesis testing, discovering new relationships, data } \\
\text { clustering and classification is a standard part of the } \\
\text { analytics. The less quantitative part includes the ability } \\
\text { to visualize structural details at different levels of } \\
\text { resolution. Data reduction, removing redundancies } \\
\text { through clustering, more abstract representations such } \\
\text { as representing a group of highly similar genomes in a } \\
\text { pangenome are all strategies for both data } \\
\text { management as well as analytics. }\end{array}$ \\
\hline $\begin{array}{r}\text { Big Data Specific } \\
\text { Challenges (Gaps) }\end{array}$ & \multicolumn{2}{|c|}{$\begin{array}{l}\text { The biggest friend for dealing with the heterogeneity of biological data is still the } \\
\text { RDBMS. Unfortunately, it does not scale for the current volume of data. NoSQL } \\
\text { solutions aim at providing an alternative. Unfortunately, NoSQL solutions do not } \\
\text { always lend themselves to real time interactive use, rapid and parallel bulk loading, } \\
\text { and sometimes have issues regarding robustness. Our current approach is currently } \\
\text { ad hoc, custom, relying mainly on the Linux cluster and the file system to supplement } \\
\text { the Oracle RDBMS. The custom solution oftentimes rely in knowledge of the } \\
\text { peculiarities of the data allowing us to devise horizontal partitioning schemes as well } \\
\text { as inversion of data organization when applicable. }\end{array}$} \\
\hline $\begin{array}{r}\text { Big Data Specific } \\
\text { Challenges in Mobility }\end{array}$ & \multicolumn{2}{|c|}{ No special challenges. Just world wide web access. } \\
\hline $\begin{array}{r}\text { Security and Privacy } \\
\text { Requirements }\end{array}$ & \multicolumn{2}{|c|}{ No special challenges. Data is either public or requires standard login with password. } \\
\hline $\begin{array}{r}\text { Highlight issues for } \\
\text { generalizing this use } \\
\text { case (e.g. for ref. } \\
\text { architecture) }\end{array}$ & \multicolumn{2}{|c|}{$\begin{array}{l}\text { A replacement for the RDBMS in Big Data would be of benefit to everyone. Many } \\
\text { NoSQL solutions attempt to fill this role, but have their limitations. }\end{array}$} \\
\hline $\begin{array}{r}\text { More Information } \\
\text { (URLs) }\end{array}$ & \multicolumn{2}{|l|}{ http://img.jgi.doe.gov } \\
\hline
\end{tabular}


NIST Big Data Interoperability Framework: Volume 3, Use CaSes and General ReQuiRements 


\section{Healthcare and Life Sciences> Use Case 21: I ndividualized Diabetes Management}

\begin{tabular}{|c|c|c|}
\hline Use Case Title & \multicolumn{2}{|c|}{ Individualized Diabetes Management } \\
\hline Vertical (area) & \multicolumn{2}{|c|}{ Healthcare } \\
\hline Author/Company/Email & \multicolumn{2}{|c|}{$\begin{array}{l}\text { Peter Li, Ying Ding, Philip Yu, Geoffrey Fox, David Wild at Mayo Clinic, Indiana } \\
\text { University, UIC; dingying@indiana.edu }\end{array}$} \\
\hline $\begin{array}{r}\text { Actors/Stakeholders } \\
\text { and their roles and } \\
\text { responsibilities }\end{array}$ & \multicolumn{2}{|c|}{$\begin{array}{l}\text { Mayo Clinic + IU/semantic integration of EHR data } \\
\text { UIC/semantic graph mining of EHR data } \\
\text { IU cloud and parallel computing }\end{array}$} \\
\hline Goals & \multicolumn{2}{|c|}{$\begin{array}{l}\text { Develop advanced graph-based data mining techniques applied to EHR to search for } \\
\text { these cohorts and extract their EHR data for outcome evaluation. These methods will } \\
\text { push the boundaries of scalability and data mining technologies and advance } \\
\text { knowledge and practice in these areas as well as clinical management of complex } \\
\text { diseases. }\end{array}$} \\
\hline Use Case Description & \multicolumn{2}{|c|}{$\begin{array}{l}\text { Diabetes is a growing illness in world population, affecting both developing and } \\
\text { developed countries. Current management strategies do not adequately take into } \\
\text { account of individual patient profiles, such as co-morbidities and medications, which } \\
\text { are common in patients with chronic illnesses. We propose to approach this } \\
\text { shortcoming by identifying similar patients from a large Electronic Health Record } \\
\text { (EHR) database, i.e., an individualized cohort, and evaluate their respective } \\
\text { management outcomes to formulate one best solution suited for a given patient } \\
\text { with diabetes. } \\
\text { Project under development as below } \\
\text { Stage 1: Use the Semantic Linking for Property Values method to convert an existing } \\
\text { data warehouse at Mayo Clinic, called the Enterprise Data Trust (EDT), into RDF } \\
\text { triples that enables us to find similar patients much more efficiently through linking } \\
\text { of both vocabulary-based and continuous values, } \\
\text { Stage 2: Needs efficient parallel retrieval algorithms, suitable for cloud or HPC, using } \\
\text { open source Hbase with both indexed and custom search to identify patients of } \\
\text { possible interest. } \\
\text { Stage 3: The EHR, as an RDF graph, provides a very rich environment for graph } \\
\text { pattern mining. Needs new distributed graph mining algorithms to perform pattern } \\
\text { analysis and graph indexing technique for pattern searching on RDF triple graphs. } \\
\text { Stage 4: Given the size and complexity of graphs, mining subgraph patterns could } \\
\text { generate numerous false positives and miss numerous false negatives. Needs robust } \\
\text { statistical analysis tools to manage false discovery rate and determine true subgraph } \\
\text { significance and validate these through several clinical use cases. }\end{array}$} \\
\hline \multirow{4}{*}{$\begin{array}{r}\text { Current } \\
\text { Solutions }\end{array}$} & Compute(System) & supercomputers; cloud \\
\hline & Storage & HDFS \\
\hline & Networking & Varies. Significant I/O intensive processing needed \\
\hline & Software & $\begin{array}{l}\text { Mayo internal data warehouse called Enterprise Data } \\
\text { Trust (EDT) }\end{array}$ \\
\hline \multirow[t]{3}{*}{$\begin{array}{r}\text { Big Data } \\
\text { Characteristics }\end{array}$} & $\begin{array}{r}\text { Data Source } \\
\text { (distributed/centralized) } \\
\end{array}$ & distributed EHR data \\
\hline & Volume (size) & $\begin{array}{l}\text { The Mayo Clinic EHR dataset is a very large dataset } \\
\text { containing over } 5 \text { million patients with thousands of } \\
\text { properties each and many more that are derived from } \\
\text { primary values. }\end{array}$ \\
\hline & $\begin{array}{r}\text { Velocity } \\
\text { (e.g. real time) }\end{array}$ & not real time but updated periodically \\
\hline
\end{tabular}




\section{Healthcare and Life Sciences> Use Case 21: I ndividualized Diabetes Management}

\begin{tabular}{|c|c|c|}
\hline & $\begin{array}{r}\text { Variety } \\
\text { (multiple datasets, } \\
\text { mashup) }\end{array}$ & $\begin{array}{l}\text { Structured data, a patient has controlled vocabulary } \\
\text { (CV) property values (demographics, diagnostic codes, } \\
\text { medications, procedures, etc.) and continuous property } \\
\text { values (lab tests, medication amounts, vitals, etc.). The } \\
\text { number of property values could range from less than } \\
100 \text { (new patient) to more than } 100,000 \text { (long term } \\
\text { patient) with typical patients composed of } 100 \mathrm{CV} \text { values } \\
\text { and } 1000 \text { continuous values. Most values are time } \\
\text { based, i.e., a timestamp is recorded with the value at } \\
\text { the time of observation. }\end{array}$ \\
\hline & $\begin{array}{r}\text { Variability (rate of } \\
\text { change) }\end{array}$ & Data will be updated or added during each patient visit. \\
\hline $\begin{array}{r}\text { Big Data Science } \\
\text { (collection, curation, } \\
\text { analysis, }\end{array}$ & $\begin{array}{r}\text { Veracity (Robustness } \\
\text { Issues) }\end{array}$ & $\begin{array}{l}\text { Data are annotated based on domain ontologies or } \\
\text { taxonomies. Semantics of data can vary from labs to } \\
\text { labs. }\end{array}$ \\
\hline action) & Visualization & no visualization \\
\hline & Data Quality & $\begin{array}{l}\text { Provenance is important to trace the origins of the data } \\
\text { and data quality }\end{array}$ \\
\hline & Data Types & text, and Continuous Numerical values \\
\hline & Data Analytics & $\begin{array}{l}\text { Integrating data into semantic graph, using graph } \\
\text { traverse to replace SQL join. Developing semantic graph } \\
\text { mining algorithms to identify graph patterns, index } \\
\text { graph, and search graph. Indexed Hbase. Custom code } \\
\text { to develop new patient properties from stored data. }\end{array}$ \\
\hline $\begin{array}{l}\text { Big Data Specific } \\
\text { Challenges (Gaps) }\end{array}$ & \multicolumn{2}{|c|}{$\begin{array}{l}\text { For individualized cohort, we will effectively be building a datamart for each patient } \\
\text { since the critical properties and indices will be specific to each patient. Due to the } \\
\text { number of patients, this becomes an impractical approach. Fundamentally, the } \\
\text { paradigm changes from relational row-column lookup to semantic graph traversal. }\end{array}$} \\
\hline $\begin{array}{r}\text { Big Data Specific } \\
\text { Challenges in Mobility }\end{array}$ & \multicolumn{2}{|c|}{ Physicians and patient may need access to this data on mobile platforms } \\
\hline $\begin{array}{r}\text { Security and Privacy } \\
\text { Requirements }\end{array}$ & \multicolumn{2}{|c|}{ Health records or clinical research databases must be kept secure/private. } \\
\hline $\begin{array}{r}\text { Highlight issues for } \\
\text { generalizing this use } \\
\text { case (e.g. for ref. } \\
\text { architecture) }\end{array}$ & \multicolumn{2}{|c|}{$\begin{array}{l}\text { Data integration: continuous values, ontological annotation, taxonomy } \\
\text { Graph Search: indexing and searching graph } \\
\text { Validation: Statistical validation }\end{array}$} \\
\hline $\begin{array}{r}\text { More Information } \\
\text { (URLs) }\end{array}$ & & \\
\hline
\end{tabular}




\section{Healthcare and Life Sciences> Use Case 22: Statistical Relational Artificial I ntelligence for Health Care}

\begin{tabular}{|c|c|c|}
\hline Use Case Title & \multicolumn{2}{|c|}{ Statistical Relational Artificial Intelligence for Health Care } \\
\hline Vertical (area) & \multicolumn{2}{|c|}{ Healthcare } \\
\hline Author/Company/Email & \multicolumn{2}{|c|}{ Sriraam Natarajan / Indiana University /natarasr@indiana.edu } \\
\hline $\begin{array}{r}\text { Actors/Stakeholders } \\
\text { and their roles and } \\
\text { responsibilities }\end{array}$ & \multicolumn{2}{|c|}{ Researchers in Informatics, medicine and practitioners in medicine. } \\
\hline Goals & \multicolumn{2}{|c|}{$\begin{array}{l}\text { The goal of the project is to analyze large, multi-modal, longitudinal data. Analyzing } \\
\text { different data types such as imaging, EHR, genetic and natural language data } \\
\text { requires a rich representation. This approach employs the relational probabilistic } \\
\text { models that have the capability of handling rich relational data and modeling } \\
\text { uncertainty using probability theory. The software learns models from multiple data } \\
\text { types and can possibly integrate the information and reason about complex queries. }\end{array}$} \\
\hline Use Case Description & \multicolumn{2}{|c|}{$\begin{array}{l}\text { Users can provide a set of descriptions - say for instance, MRI images and } \\
\text { demographic data about a particular subject. They can then query for the onset of a } \\
\text { particular disease (say Alzheimer's) and the system will then provide a probability } \\
\text { distribution over the possible occurrence of this disease. }\end{array}$} \\
\hline \multirow[t]{4}{*}{$\begin{array}{r}\text { Current } \\
\text { Solutions }\end{array}$} & Compute(System) & $\begin{array}{l}\text { A high performance computer ( } 48 \text { GB RAM) is needed to } \\
\text { run the code for a few hundred patients. Clusters for } \\
\text { large datasets }\end{array}$ \\
\hline & Storage & $\begin{array}{l}\text { A } 200 \text { GB to } 1 \text { TB hard drive typically stores the test } \\
\text { data. The relevant data is retrieved to main memory to } \\
\text { run the algorithms. Backend data in database or NoSQL } \\
\text { stores }\end{array}$ \\
\hline & Networking & Intranet. \\
\hline & Software & $\begin{array}{l}\text { Mainly Java based, in house tools are used to process } \\
\text { the data. }\end{array}$ \\
\hline \multirow[t]{5}{*}{$\begin{array}{r}\text { Big Data } \\
\text { Characteristics }\end{array}$} & $\begin{array}{r}\text { Data Source } \\
\text { (distributed/centralized) }\end{array}$ & $\begin{array}{l}\text { All the data about the users reside in a single disk file. } \\
\text { Sometimes, resources such as published text need to be } \\
\text { pulled from Ilnternet. }\end{array}$ \\
\hline & Volume (size) & $\begin{array}{l}\text { Variable due to the different amount of data collected. } \\
\text { Typically can be in } 100 \text { s of GBs for a single cohort of a } \\
\text { few hundred people. When dealing with millions of } \\
\text { patients, this can be in the order of } 1 \text { petabyte. }\end{array}$ \\
\hline & $\begin{array}{r}\text { Velocity } \\
\text { (e.g. real time) }\end{array}$ & $\begin{array}{l}\text { Varied. In some cases, EHRs are constantly being } \\
\text { updated. In other controlled studies, the data often } \\
\text { comes in batches in regular intervals. }\end{array}$ \\
\hline & $\begin{array}{r}\text { Variety } \\
\text { (multiple datasets, } \\
\text { mashup) }\end{array}$ & $\begin{array}{l}\text { This is the key property in medical datasets. That data is } \\
\text { typically in multiple tables and need to be merged in } \\
\text { order to perform the analysis. }\end{array}$ \\
\hline & $\begin{array}{r}\text { Variability (rate of } \\
\text { change) }\end{array}$ & $\begin{array}{l}\text { The arrival of data is unpredictable in many cases as } \\
\text { they arrive in real time. }\end{array}$ \\
\hline \multirow{3}{*}{$\begin{array}{r}\text { Big Data Science } \\
\text { (collection, curation, } \\
\text { analysis, } \\
\text { action) }\end{array}$} & $\begin{array}{r}\text { Veracity (Robustness } \\
\text { Issues, semantics) } \\
\end{array}$ & $\begin{array}{l}\text { Challenging due to different modalities of the data, } \\
\text { human errors in data collection and validation. }\end{array}$ \\
\hline & Visualization & $\begin{array}{l}\text { The visualization of the entire input data is nearly } \\
\text { impossible. But typically, partially visualizable. The } \\
\text { models built can be visualized under some reasonable } \\
\text { assumptions. }\end{array}$ \\
\hline & Data Quality (syntax) & \\
\hline
\end{tabular}




\section{Healthcare and Life Sciences> Use Case 22: Statistical Relational Artificial I ntelligence for Health Care}

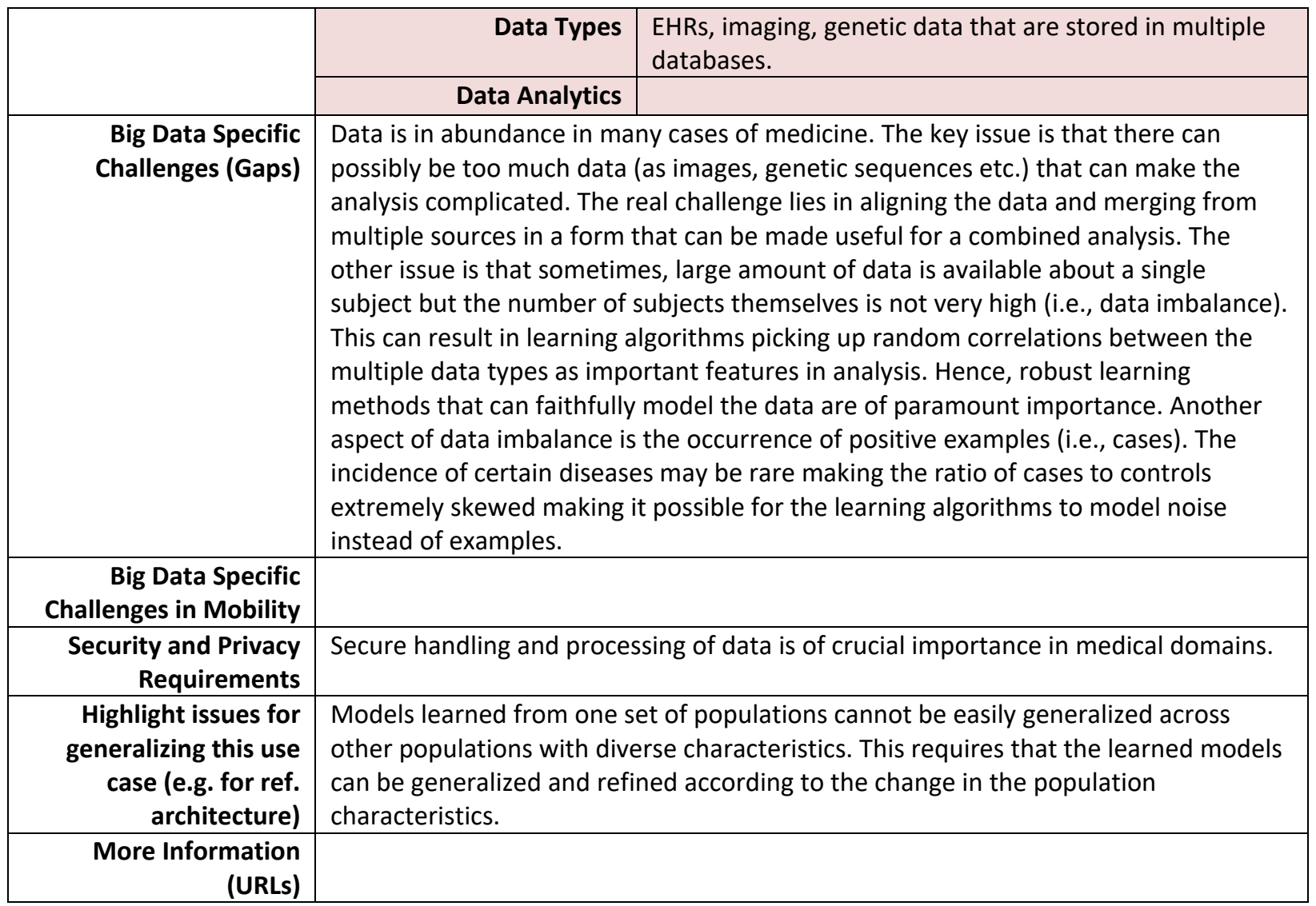




\section{Healthcare and Life Sciences> Use Case 23: World Population Scale Epidemiology}

\begin{tabular}{|c|c|c|}
\hline Use Case Title & \multicolumn{2}{|c|}{ World Population Scale Epidemiological Study } \\
\hline Vertical (area) & \multicolumn{2}{|c|}{ Epidemiology, Simulation Social Science, Computational Social Science } \\
\hline Author/Company/Email & \multicolumn{2}{|c|}{$\begin{array}{l}\text { Madhav Marathe Stephen Eubank or Chris Barrett/ Virginia Bioinformatics Institute, } \\
\text { Virginia Tech, mmarathe@vbi.vt.edu, seubank@vbi.vt.edu or cbarrett@vbi.vt.edu }\end{array}$} \\
\hline $\begin{array}{r}\text { Actors/Stakeholders } \\
\text { and their roles and } \\
\text { responsibilities }\end{array}$ & \multicolumn{2}{|c|}{$\begin{array}{l}\text { Government and non-profit institutions involved in health, public policy, and disaster } \\
\text { mitigation. Social Scientist who wants to study the interplay between behavior and } \\
\text { contagion. }\end{array}$} \\
\hline Goals & \multicolumn{2}{|c|}{$\begin{array}{l}\text { (a) Build a synthetic global population. (b) Run simulations over the global } \\
\text { population to reason about outbreaks and various intervention strategies. }\end{array}$} \\
\hline Use Case Description & \multicolumn{2}{|c|}{ Prediction and control of pandemic similar to the 2009 H1N1 influenza. } \\
\hline \multirow[t]{4}{*}{$\begin{array}{r}\text { Current } \\
\text { Solutions }\end{array}$} & Compute(System) & $\begin{array}{l}\text { Distributed (MPI) based simulation system written in } \\
\text { Charm++. Parallelism is achieved by exploiting the } \\
\text { disease residence time period. }\end{array}$ \\
\hline & Storage & $\begin{array}{l}\text { Network file system. Exploring database driven } \\
\text { techniques. }\end{array}$ \\
\hline & Networking & Infiniband. High bandwidth 3D Torus. \\
\hline & Software & Charm++, MPI \\
\hline \multirow[t]{5}{*}{$\begin{array}{r}\text { Big Data } \\
\text { Characteristics }\end{array}$} & $\begin{array}{r}\text { Data Source } \\
\text { (distributed/centralized) }\end{array}$ & $\begin{array}{l}\text { Generated from synthetic population generator. } \\
\text { Currently centralized. However, could be made } \\
\text { distributed as part of post-processing. }\end{array}$ \\
\hline & Volume (size) & 100TB \\
\hline & $\begin{array}{r}\text { Velocity } \\
\text { (e.g. real time) }\end{array}$ & $\begin{array}{l}\text { Interactions with experts and visualization routines } \\
\text { generate large amount of real time data. Data feeding } \\
\text { into the simulation is small but data generated by } \\
\text { simulation is massive. }\end{array}$ \\
\hline & $\begin{array}{r}\text { Variety } \\
\text { (multiple datasets, } \\
\text { mashup) }\end{array}$ & $\begin{array}{l}\text { Variety depends upon the complexity of the model } \\
\text { over which the simulation is being performed. Can be } \\
\text { very complex if other aspects of the world population } \\
\text { such as type of activity, geographical, socio-economic, } \\
\text { cultural variations are taken into account. }\end{array}$ \\
\hline & $\begin{array}{r}\text { Variability (rate of } \\
\text { change) }\end{array}$ & $\begin{array}{l}\text { Depends upon the evolution of the model and } \\
\text { corresponding changes in the code. This is complex and } \\
\text { time intensive. Hence low rate of change. }\end{array}$ \\
\hline \multirow{5}{*}{$\begin{array}{r}\text { Big Data Science } \\
\text { (collection, curation, } \\
\text { analysis, } \\
\text { action) }\end{array}$} & $\begin{array}{l}\text { Veracity (Robustness } \\
\text { Issues, semantics) }\end{array}$ & $\begin{array}{l}\text { Robustness of the simulation is dependent upon the } \\
\text { quality of the model. However, robustness of the } \\
\text { computation itself, although non-trivial, is tractable. }\end{array}$ \\
\hline & Visualization & $\begin{array}{l}\text { Would require very large amount of movement of data } \\
\text { to enable visualization. }\end{array}$ \\
\hline & Data Quality (syntax) & Consistent due to generation from a model \\
\hline & Data Types & Primarily network data. \\
\hline & Data Analytics & Summary of various runs and replicates of a simulation \\
\hline $\begin{array}{l}\text { Big Data Specific } \\
\text { Challenges (Gaps) }\end{array}$ & \multicolumn{2}{|c|}{$\begin{array}{l}\text { Computation of the simulation is both compute intensive and data intensive. } \\
\text { Moreover, due to unstructured and irregular nature of graph processing the problem } \\
\text { is not easily decomposable. Therefore it is also bandwidth intensive. Hence, a } \\
\text { supercomputer is applicable than cloud type clusters. }\end{array}$} \\
\hline $\begin{array}{r}\text { Big Data Specific } \\
\text { Challenges in Mobility }\end{array}$ & \multicolumn{2}{|c|}{ None } \\
\hline
\end{tabular}




\section{Healthcare and Life Sciences> Use Case 23: World Population Scale} Epidemiology

\begin{tabular}{|r|l|}
\hline $\begin{array}{r}\text { Security and Privacy } \\
\text { Requirements }\end{array}$ & $\begin{array}{l}\text { Several issues at the synthetic population-modeling phase (see social contagion } \\
\text { model). }\end{array}$ \\
\hline $\begin{array}{r}\text { Highlight issues for } \\
\text { generalizing this use } \\
\text { case (e.g. for ref. } \\
\text { architecture) }\end{array}$ & $\begin{array}{l}\text { In general contagion diffusion of various kinds: information, diseases, social unrest } \\
\text { underlying interaction network to study the evolution of the desired phenomena. }\end{array}$ \\
\hline $\begin{array}{r}\text { More Information } \\
\text { (URLs) }\end{array}$ & \\
\hline
\end{tabular}




\section{Healthcare and Life Sciences> Use Case 24: Social Contagion Modeling}

\begin{tabular}{|c|c|c|}
\hline Use Case Title & \multicolumn{2}{|l|}{ Social Contagion Modeling } \\
\hline Vertical (area) & \multicolumn{2}{|c|}{$\begin{array}{l}\text { Social behavior (including national security, public health, viral marketing, city } \\
\text { planning, disaster preparedness) }\end{array}$} \\
\hline Author/Company/Email & \multicolumn{2}{|c|}{$\begin{array}{l}\text { Madhav Marathe or Chris Kuhlman /Virginia Bioinformatics Institute, Virginia Tech } \\
\text { mmarathe@vbi.vt.edu or ckuhlman@vbi.vt.edu }\end{array}$} \\
\hline \multicolumn{3}{|l|}{$\begin{array}{l}\text { /Actors/Stakeholders } \\
\text { and their roles and } \\
\text { responsibilities }\end{array}$} \\
\hline Goals & \multicolumn{2}{|c|}{$\begin{array}{l}\text { Provide a computing infrastructure that models social contagion processes. } \\
\text { The infrastructure enables different types of human-to-human interactions (e.g., } \\
\text { face-to-face versus online media; mother-daughter relationships versus mother- } \\
\text { coworker relationships) to be simulated. It takes not only human-to-human } \\
\text { interactions into account, but also interactions among people, services (e.g., } \\
\text { transportation), and infrastructure (e.g., Internet, electric power). }\end{array}$} \\
\hline Use Case Description & \multicolumn{2}{|c|}{$\begin{array}{l}\text { Social unrest. People take to the streets to voice unhappiness with government } \\
\text { leadership. There are citizens that both support and oppose government. Quantify } \\
\text { the degrees to which normal business and activities are disrupted owing to fear and } \\
\text { anger. Quantify the possibility of peaceful demonstrations, violent protests. Quantify } \\
\text { the potential for government responses ranging from appeasement, to allowing } \\
\text { protests, to issuing threats against protestors, to actions to thwart protests. To } \\
\text { address these issues, must have fine-resolution models and datasets. }\end{array}$} \\
\hline \multirow[t]{4}{*}{$\begin{array}{r}\text { Current } \\
\text { Solutions }\end{array}$} & Compute(System) & $\begin{array}{l}\text { Distributed processing software running on commodity } \\
\text { clusters and newer architectures and systems (e.g., } \\
\text { clouds). }\end{array}$ \\
\hline & Storage & File servers (including archives), databases. \\
\hline & Networking & Ethernet, Infiniband, and similar. \\
\hline & Software & $\begin{array}{l}\text { Specialized simulators, open source software, and } \\
\text { proprietary modeling environments. Databases. }\end{array}$ \\
\hline \multirow[t]{6}{*}{$\begin{array}{r}\text { Big Data } \\
\text { Characteristics }\end{array}$} & $\begin{array}{r}\text { Data Source } \\
\text { (distributed/centralized) }\end{array}$ & $\begin{array}{l}\text { Many data sources: populations, work locations, travel } \\
\text { patterns, utilities (e.g., power grid) and other man- } \\
\text { made infrastructures, online (social) media. }\end{array}$ \\
\hline & Volume (size) & Easily 10 s of TB per year of new data. \\
\hline & $\begin{array}{r}\text { Velocity } \\
\text { (e.g. real time) }\end{array}$ & $\begin{array}{l}\text { During social unrest events, human interactions and } \\
\text { mobility key to understanding system dynamics. Rapid } \\
\text { changes in data; e.g., who follows whom in Twitter. }\end{array}$ \\
\hline & $\begin{array}{r}\text { Variety } \\
\text { (multiple datasets, } \\
\text { mashup) }\end{array}$ & $\begin{array}{l}\text { Variety of data seen in wide range of data sources. } \\
\text { Temporal data. Data fusion. }\end{array}$ \\
\hline & & $\begin{array}{l}\text { Data fusion a big issue. How to combine data from } \\
\text { different sources and how to deal with missing or } \\
\text { incomplete data? Multiple simultaneous contagion } \\
\text { processes. }\end{array}$ \\
\hline & $\begin{array}{r}\text { Variability (rate of } \\
\text { change) }\end{array}$ & $\begin{array}{l}\text { Because of stochastic nature of events, multiple } \\
\text { instances of models and inputs must be run to ranges } \\
\text { in outcomes. }\end{array}$ \\
\hline $\begin{array}{l}\text { Big Data Science } \\
\text { (collection, curation, }\end{array}$ & $\begin{array}{r}\text { Veracity (Robustness } \\
\text { Issues, semantics) }\end{array}$ & Failover of soft real-time analyses. \\
\hline
\end{tabular}




\section{Healthcare and Life Sciences> Use Case 24: Social Contagion Modeling}

\begin{tabular}{|c|c|c|}
\hline \multirow[t]{4}{*}{$\begin{array}{r}\text { analysis, } \\
\text { action) }\end{array}$} & Visualization & $\begin{array}{l}\text { Large datasets; time evolution; multiple contagion } \\
\text { processes over multiple network representations. } \\
\text { Levels of detail (e.g., individual, neighborhood, city, } \\
\text { state, country-level). }\end{array}$ \\
\hline & Data Quality (syntax) & $\begin{array}{l}\text { Checks for ensuring data consistency, corruption. } \\
\text { Preprocessing of raw data for use in models. }\end{array}$ \\
\hline & Data Types & $\begin{array}{l}\text { Wide-ranging data, from human characteristics to } \\
\text { utilities and transportation systems, and interactions } \\
\text { among them. }\end{array}$ \\
\hline & Data Analytics & $\begin{array}{l}\text { Models of behavior of humans and hard } \\
\text { infrastructures, and their interactions. Visualization of } \\
\text { results. }\end{array}$ \\
\hline $\begin{array}{l}\text { Big Data Specific } \\
\text { Challenges (Gaps) }\end{array}$ & \multicolumn{2}{|c|}{$\begin{array}{l}\text { How to take into account heterogeneous features of 100s of millions or billions of } \\
\text { individuals, models of cultural variations across countries that are assigned to } \\
\text { individual agents? How to validate these large models? Different types of models } \\
\text { (e.g., multiple contagions): disease, emotions, behaviors. Modeling of different } \\
\text { urban infrastructure systems in which humans act. With multiple replicates required } \\
\text { to assess stochasticity, large amounts of output data are produced; storage } \\
\text { requirements. }\end{array}$} \\
\hline $\begin{array}{r}\text { Big Data Specific } \\
\text { Challenges in Mobility }\end{array}$ & \multicolumn{2}{|c|}{$\begin{array}{l}\text { How and where to perform these computations? Combinations of cloud computing } \\
\text { and clusters. How to realize most efficient computations; move data to compute } \\
\text { resources? }\end{array}$} \\
\hline $\begin{array}{r}\text { Security and Privacy } \\
\text { Requirements }\end{array}$ & \multicolumn{2}{|c|}{$\begin{array}{l}\text { Two dimensions. First, privacy and anonymity issues for individuals used in modeling } \\
\text { (e.g., Twitter and Facebook users). Second, securing data and computing platforms } \\
\text { for computation. }\end{array}$} \\
\hline $\begin{array}{r}\text { Highlight issues for } \\
\text { generalizing this use } \\
\text { case (e.g. for ref. } \\
\text { architecture) }\end{array}$ & \multicolumn{2}{|c|}{$\begin{array}{l}\text { Fusion of different data types. Different datasets must be combined depending on } \\
\text { the particular problem. How to quickly develop, verify, and validate new models for } \\
\text { new applications. What is appropriate level of granularity to capture phenomena of } \\
\text { interest while generating results sufficiently quickly; i.e., how to achieve a scalable } \\
\text { solution. Data visualization and extraction at different levels of granularity. }\end{array}$} \\
\hline $\begin{array}{r}\text { More Information } \\
\text { (URLs) }\end{array}$ & & \\
\hline
\end{tabular}




\section{Healthcare and Life Sciences> Use Case 25: LifeWatch Biodiversity}

\begin{tabular}{|c|c|c|}
\hline Use Case Title & \multicolumn{2}{|c|}{$\begin{array}{l}\text { LifeWatch - E-Science European Infrastructure for Biodiversity and Ecosystem } \\
\text { Research }\end{array}$} \\
\hline Vertical (area) & \multicolumn{2}{|c|}{ Scientific Research: Life Science } \\
\hline Author/Company/Email & \multicolumn{2}{|c|}{ Wouter Los, Yuri Demchenko (y.demchenko@uva.nl), University of Amsterdam } \\
\hline $\begin{array}{r}\text { Actors/Stakeholders } \\
\text { and their roles and } \\
\text { responsibilities }\end{array}$ & \multicolumn{2}{|c|}{$\begin{array}{l}\text { End-users (biologists, ecologists, field researchers) } \\
\text { Data analysts, data archive managers, e-Science Infrastructure managers, EU states } \\
\text { national representatives }\end{array}$} \\
\hline Goals & \multicolumn{2}{|c|}{$\begin{array}{l}\text { Research and monitor different ecosystems, biological species, their dynamics and } \\
\text { migration. }\end{array}$} \\
\hline Use Case Description & \multicolumn{2}{|c|}{$\begin{array}{l}\text { LifeWatch project and initiative intends to provide integrated access to a variety of } \\
\text { data, analytical and modeling tools as served by a variety of collaborating initiatives. } \\
\text { Another service is offered with data and tools in selected workflows for specific } \\
\text { scientific communities. In addition, LifeWatch will provide opportunities to construct } \\
\text { personalized 'virtual labs', also allowing to enter new data and analytical tools. } \\
\text { New data will be shared with the data facilities cooperating with LifeWatch. } \\
\text { Particular case studies: Monitoring alien species, monitoring migrating birds, } \\
\text { wetlands } \\
\text { LifeWatch operates Global Biodiversity Information facility and Biodiversity } \\
\text { Catalogue that is Biodiversity Science Web Services Catalogue }\end{array}$} \\
\hline \multirow[t]{4}{*}{$\begin{array}{r}\text { Current } \\
\text { Solutions }\end{array}$} & Compute(System) & $\begin{array}{l}\text { Field facilities TBD } \\
\text { Data center: General Grid and cloud based resources } \\
\text { provided by national e-Science centers }\end{array}$ \\
\hline & Storage & Distributed, historical and trends data archiving \\
\hline & Networking & $\begin{array}{l}\text { May require special dedicated or overlay sensor } \\
\text { network. }\end{array}$ \\
\hline & Software & $\begin{array}{l}\text { Web Services based, Grid based services, relational } \\
\text { databases }\end{array}$ \\
\hline \multirow[t]{5}{*}{$\begin{array}{r}\text { Big Data } \\
\text { Characteristics }\end{array}$} & $\begin{array}{l}\text { Data Source } \\
\text { (distributed/centralized) }\end{array}$ & $\begin{array}{l}\text { Ecological information from numerous observation and } \\
\text { monitoring facilities and sensor network, satellite } \\
\text { images/information, climate and weather, all recorded } \\
\text { information. } \\
\text { Information from field researchers }\end{array}$ \\
\hline & Volume (size) & $\begin{array}{l}\text { Involves many existing datasets/sources } \\
\text { Collected amount of data TBD }\end{array}$ \\
\hline & $\begin{array}{l}\text { Velocity } \\
\text { (e.g. real time) }\end{array}$ & $\begin{array}{l}\text { Data analyzed incrementally, processes dynamics } \\
\text { corresponds to dynamics of biological and ecological } \\
\text { processes. } \\
\text { However may require real-time processing and analysis } \\
\text { in case of the natural or industrial disaster. } \\
\text { May require data streaming processing. }\end{array}$ \\
\hline & $\begin{array}{l}\text { Variety } \\
\text { (multiple datasets, } \\
\text { mashup) }\end{array}$ & $\begin{array}{l}\text { Variety and number of involved databases and } \\
\text { observation data is currently limited by available tools; } \\
\text { in principle, unlimited with the growing ability to } \\
\text { process data for identifying ecological changes, } \\
\text { factors/reasons, species evolution and trends. } \\
\text { See below in additional information. }\end{array}$ \\
\hline & $\begin{array}{l}\text { Variability (rate of } \\
\text { change) }\end{array}$ & $\begin{array}{l}\text { Structure of the datasets and models may change } \\
\text { depending on the data processing stage and tasks }\end{array}$ \\
\hline
\end{tabular}




\section{Healthcare and Life Sciences> Use Case 25: LifeWatch Biodiversity}

\begin{tabular}{|c|c|c|}
\hline \multirow[t]{5}{*}{$\begin{array}{r}\text { Big Data Science } \\
\text { (collection, curation, } \\
\text { analysis, } \\
\text { action) }\end{array}$} & $\begin{array}{l}\text { Veracity (Robustness } \\
\text { Issues) }\end{array}$ & $\begin{array}{l}\text { In normal monitoring mode are data are statistically } \\
\text { processed to achieve robustness. } \\
\text { Some biodiversity research is critical to data veracity } \\
\text { (reliability/trustworthiness). } \\
\text { In case of natural and technogenic disasters data } \\
\text { veracity is critical. }\end{array}$ \\
\hline & Visualization & $\begin{array}{l}\text { Requires advanced and rich visualization, high definition } \\
\text { visualization facilities, visualization data } \\
\text { - } \quad \text { 4D visualization } \\
\text { - } \quad \text { Visualizing effects of parameter change in } \\
\text { (computational) models } \\
\text { - Comparing model outcomes with actual } \\
\text { observations (multi-dimensional) }\end{array}$ \\
\hline & Data Quality & $\begin{array}{l}\text { Depends on and ensued by initial observation data. } \\
\text { Quality of analytical data depends on used mode and } \\
\text { algorithms that are constantly improved. } \\
\text { Repeating data analytics should be possible to re- } \\
\text { evaluate initial observation data. } \\
\text { Actionable data are human aided. }\end{array}$ \\
\hline & Data Types & $\begin{array}{l}\text { Multi-type. } \\
\text { Relational data, key-value, complex semantically rich } \\
\text { data }\end{array}$ \\
\hline & Data Analytics & Parallel data streams and streaming analytics \\
\hline $\begin{array}{l}\text { Big Data Specific } \\
\text { Challenges (Gaps) }\end{array}$ & \multicolumn{2}{|c|}{$\begin{array}{l}\text { Variety, multi-type data: SQL and no-SQL, distributed multi-source data. } \\
\text { Visualization, distributed sensor networks. } \\
\text { Data storage and archiving, data exchange and integration; data linkage: from the } \\
\text { initial observation data to processed data and reported/visualized data. } \\
\text { - Historical unique data } \\
\text { - } \quad \text { Curated (authorized) reference data (i.e., species names lists), algorithms, } \\
\text { - } \quad \text { Poftware code, workflows } \\
\text { - Processed (secondary) data serving as input for other researchers } \\
\text { workflows }\end{array}$} \\
\hline $\begin{array}{l}\text { Big Data Specific } \\
\text { Challenges in Mobility }\end{array}$ & \multicolumn{2}{|c|}{$\begin{array}{l}\text { Require supporting mobile sensors (e.g. birds migration) and mobile researchers } \\
\text { (both for information feed and catalogue search) } \\
\text { - Instrumented field vehicles, Ships, Planes, Submarines, floating buoys, sensor } \\
\text { tagging on organisms } \\
\text { - Photos, video, sound recording }\end{array}$} \\
\hline $\begin{array}{r}\text { Security and Privacy } \\
\text { Requirements }\end{array}$ & \multicolumn{2}{|c|}{$\begin{array}{l}\text { Data integrity, referral integrity of the datasets. } \\
\text { Federated identity management for mobile researchers and mobile sensors } \\
\text { Confidentiality, access control and accounting for information on protected species, } \\
\text { ecological information, space images, climate information. }\end{array}$} \\
\hline $\begin{array}{r}\text { Highlight issues for } \\
\text { generalizing this use } \\
\text { case (e.g. for ref. } \\
\text { architecture) }\end{array}$ & \multicolumn{2}{|c|}{$\begin{array}{l}\text { - } \\
\text { - } \\
\text { - } \\
\text { - } \\
\text { Dutati-type dife cycle management: data provenance, referral integrity and } \\
\text { identification } \\
\text { - }\end{array}$} \\
\hline $\begin{array}{r}\text { More Information } \\
\text { (URLs) }\end{array}$ & \multicolumn{2}{|c|}{$\begin{array}{l}\text { http://www.lifewatch.eu/web/guest/home } \\
\text { https://www.biodiversitycatalogue.org/ }\end{array}$} \\
\hline
\end{tabular}




\section{Healthcare and Life Sciences> Use Case 25: LifeWatch Biodiversity}

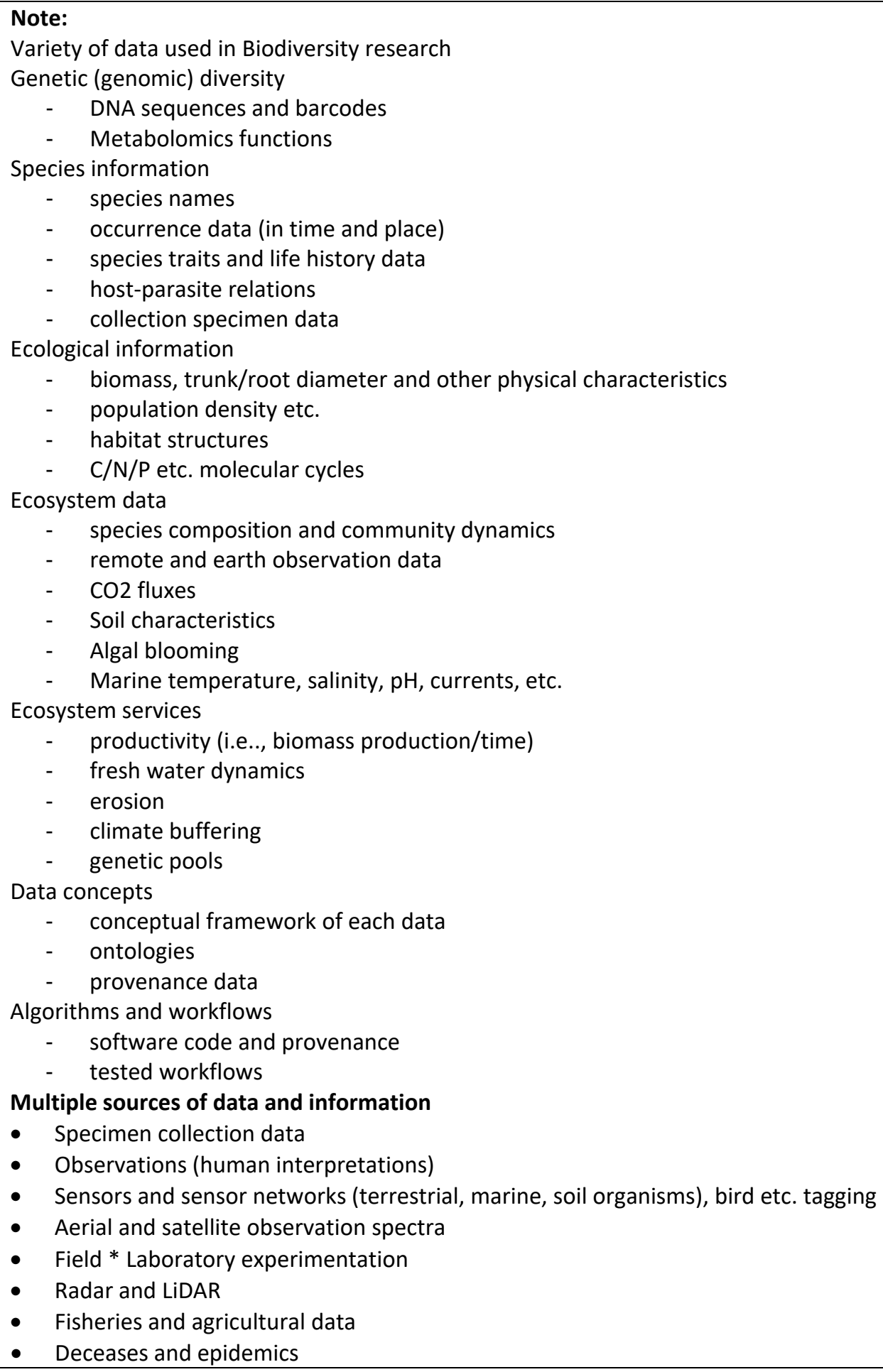

- Field * Laboratory experimentation

- Radar and LiDAR

- Fisheries and agricultural data

- Deceases and epidemics 


\section{Deep Learning and Social Media> Use Case 26: Large-scale Deep Learning}

\begin{tabular}{|c|c|c|}
\hline Use Case Title & \multicolumn{2}{|c|}{ Large-scale Deep Learning } \\
\hline Vertical (area) & \multicolumn{2}{|c|}{ Machine Learning/ artificial intelligence } \\
\hline Author/Company/Email & \multicolumn{2}{|c|}{ Adam Coates / Stanford University / acoates@cs.stanford.edu } \\
\hline $\begin{array}{l}\text { Actors/Stakeholders } \\
\text { and their roles and } \\
\text { responsibilities }\end{array}$ & \multicolumn{2}{|c|}{$\begin{array}{l}\text { Machine learning researchers and practitioners faced with large quantities of data } \\
\text { and complex prediction tasks. Supports state-of-the-art development in computer } \\
\text { vision as in automatic car driving, speech recognition, and natural language } \\
\text { processing in both academic and industry systems. }\end{array}$} \\
\hline Goals & \multicolumn{2}{|c|}{$\begin{array}{l}\text { Increase the size of datasets and models that can be tackled with deep learning } \\
\text { algorithms. Large models (e.g., neural networks with more neurons and connections) } \\
\text { combined with large datasets are increasingly the top performers in benchmark tasks } \\
\text { for vision, speech, and NLP. }\end{array}$} \\
\hline Use Case Description & \multicolumn{2}{|c|}{$\begin{array}{l}\text { A research scientist or machine learning practitioner wants to train a deep neural } \\
\text { network from a large (>>1TB) corpus of data (typically imagery, video, audio, or text). } \\
\text { Such training procedures often require customization of the neural network } \\
\text { architecture, learning criteria, and dataset preprocessing. In addition to the } \\
\text { computational expense demanded by the learning algorithms, the need for rapid } \\
\text { prototyping and ease of development is extremely high. }\end{array}$} \\
\hline \multirow[t]{4}{*}{$\begin{array}{r}\text { Current } \\
\text { Solutions }\end{array}$} & Compute(System) & $\begin{array}{l}\text { GPU cluster with high-speed interconnects (e.g., } \\
\text { Infiniband, 40gE) }\end{array}$ \\
\hline & Storage & 100TB Lustre filesystem \\
\hline & Networking & $\begin{array}{l}\text { Infiniband within HPC cluster; } 1 \mathrm{G} \text { ethernet to outside } \\
\text { infrastructure (e.g., Web, Lustre). }\end{array}$ \\
\hline & Software & $\begin{array}{l}\text { In-house GPU kernels and MPI-based communication } \\
\text { developed by Stanford CS. C++/Python source. }\end{array}$ \\
\hline \multirow[t]{5}{*}{$\begin{array}{r}\text { Big Data } \\
\text { Characteristics }\end{array}$} & $\begin{array}{r}\text { Data Source } \\
\text { (distributed/centralized) }\end{array}$ & $\begin{array}{l}\text { Centralized filesystem with a single large training } \\
\text { dataset. Dataset may be updated with new training } \\
\text { examples as they become available. }\end{array}$ \\
\hline & Volume (size) & $\begin{array}{l}\text { Current datasets typically } 1 \text { TB to } 10 \text { TB. With increases in } \\
\text { computation that enable much larger models, datasets of } \\
\text { 100TB or more may be necessary in order to exploit the } \\
\text { representational power of the larger models. Training a } \\
\text { self-driving car could take } 100 \text { million images. }\end{array}$ \\
\hline & $\begin{array}{r}\text { Velocity } \\
\text { (e.g. real time) }\end{array}$ & $\begin{array}{l}\text { Much faster than real-time processing is required. } \\
\text { Current computer vision applications involve processing } \\
\text { hundreds of image frames per second in order to ensure } \\
\text { reasonable training times. For demanding applications } \\
\text { (e.g., autonomous driving) we envision the need to } \\
\text { process many thousand high-resolution (6 megapixels or } \\
\text { more) images per second. }\end{array}$ \\
\hline & $\begin{array}{r}\text { Variety } \\
\text { (multiple datasets, } \\
\text { mashup) }\end{array}$ & $\begin{array}{l}\text { Individual applications may involve a wide variety of } \\
\text { data. Current research involves neural networks that } \\
\text { actively learn from heterogeneous tasks (e.g., learning to } \\
\text { perform tagging, chunking and parsing for text, or } \\
\text { learning to read lips from combinations of video and } \\
\text { audio). }\end{array}$ \\
\hline & $\begin{array}{r}\text { Variability (rate of } \\
\text { change) }\end{array}$ & $\begin{array}{l}\text { Low variability. Most data is streamed in at a consistent } \\
\text { pace from a shared source. Due to high computational } \\
\text { requirements, server loads can introduce burstiness into } \\
\text { data transfers. }\end{array}$ \\
\hline
\end{tabular}




\section{Deep Learning and Social Media> Use Case 26: Large-scale Deep Learning}

\begin{tabular}{|c|c|c|}
\hline \multirow[t]{5}{*}{$\begin{array}{r}\text { Big Data Science } \\
\text { (collection, curation, } \\
\text { analysis, } \\
\text { action) }\end{array}$} & $\begin{array}{r}\text { Veracity (Robustness } \\
\text { Issues, semantics) }\end{array}$ & $\begin{array}{l}\text { Datasets for ML applications are often hand-labeled and } \\
\text { verified. Extremely large datasets involve crowd-sourced } \\
\text { labeling and invite ambiguous situations where a label is } \\
\text { not clear. Automated labeling systems still require } \\
\text { human sanity-checks. Clever techniques for large dataset } \\
\text { construction is an active area of research. }\end{array}$ \\
\hline & Visualization & $\begin{array}{l}\text { Visualization of learned networks is an open area of } \\
\text { research, though partly as a debugging technique. Some } \\
\text { visual applications involve visualization predictions on } \\
\text { test imagery. }\end{array}$ \\
\hline & Data Quality (syntax) & $\begin{array}{l}\text { Some collected data (e.g., compressed video or audio) } \\
\text { may involve unknown formats, codecs, or may be } \\
\text { corrupted. Automatic filtering of original source data } \\
\text { removes these. }\end{array}$ \\
\hline & Data Types & Images, video, audio, text. (In practice: almost anything.) \\
\hline & Data Analytics & $\begin{array}{l}\text { Small degree of batch statistical preprocessing; all other } \\
\text { data analysis is performed by the learning algorithm } \\
\text { itself. }\end{array}$ \\
\hline $\begin{array}{r}\text { Big Data Specific } \\
\text { Challenges (Gaps) }\end{array}$ & \multicolumn{2}{|c|}{$\begin{array}{l}\text { Processing requirements for even modest quantities of data are extreme. Though the } \\
\text { trained representations can make use of many terabytes of data, the primary } \\
\text { challenge is in processing all of the data during training. Current state-of-the-art deep } \\
\text { learning systems are capable of using neural networks with more than } 10 \text { billion free } \\
\text { parameters (akin to synapses in the brain), and necessitate trillions of floating point } \\
\text { operations per training example. Distributing these computations over high- } \\
\text { performance infrastructure is a major challenge for which we currently use a largely } \\
\text { custom software system. }\end{array}$} \\
\hline $\begin{array}{r}\text { Big Data Specific } \\
\text { Challenges in Mobility }\end{array}$ & \multicolumn{2}{|c|}{$\begin{array}{l}\text { After training of large neural networks is completed, the learned network may be } \\
\text { copied to other devices with dramatically lower computational capabilities for use in } \\
\text { making predictions in real time. (E.g., in autonomous driving, the training procedure is } \\
\text { performed using a HPC cluster with } 64 \text { GPUs. The result of training, however, is a } \\
\text { neural network that encodes the necessary knowledge for making decisions about } \\
\text { steering and obstacle avoidance. This network can be copied to embedded hardware } \\
\text { in vehicles or sensors.) }\end{array}$} \\
\hline $\begin{array}{r}\text { Security and Privacy } \\
\text { Requirements }\end{array}$ & \multicolumn{2}{|c|}{ None. } \\
\hline
\end{tabular}




\section{Deep Learning and Social Media> Use Case 26: Large-scale Deep Learning}

\begin{tabular}{|r|l|}
$\begin{array}{r}\text { Highlight issues for } \\
\text { generalizing this use } \\
\text { case (e.g. for ref. } \\
\text { architecture) }\end{array}$ & $\begin{array}{l}\text { Deep Learning shares many characteristics with the broader field of machine learning. } \\
\text { The paramount requirements are high computational throughput for mostly dense } \\
\text { linear algebra operations, and extremely high productivity. Most deep learning } \\
\text { systems require a substantial degree of tuning on the target application for best } \\
\text { performance and thus necessitate a large number of experiments with designer } \\
\text { intervention in between. As a result, minimizing the turn-around time of experiments } \\
\text { and accelerating development is crucial. }\end{array}$ \\
& $\begin{array}{l}\text { These two requirements (high throughput and high productivity) are dramatically in } \\
\text { contention. HPC systems are available to accelerate experiments, but current HPC } \\
\text { software infrastructure is difficult to use which lengthens development and debugging } \\
\text { time and, in many cases, makes otherwise computationally tractable applications } \\
\text { infeasible. }\end{array}$ \\
& $\begin{array}{l}\text { The major components needed for these applications (which are currently in-house } \\
\text { custom software) involve dense linear algebra on distributed-memory HPC systems. } \\
\text { While libraries for single-machine or single-GPU computation are available (e.g., BLAS, } \\
\text { CuBLAS, MAGMA, etc.), distributed computation of dense BLAS-like or LAPACK-like } \\
\text { operations on GPUs remains poorly developed. Existing solutions (e.g., ScaLapack for } \\
\text { CPUs) are not well-integrated with higher level languages and require low-level } \\
\text { programming which lengthens experiment and development time. }\end{array}$ \\
\hline More Information \\
(URLs) & $\begin{array}{l}\text { Recent popular press coverage of deep learning technology: } \\
\text { http://www.nytimes.com/2012/11/24/science/scientists-see-advances-in-deep- }\end{array}$ \\
$\begin{array}{l}\text { learning-a-part-of-artificial-intelligence.html } \\
\text { http://www.nytimes.com/2012/06/26/technology/in-a-big-network-of-computers- } \\
\text { evidence-of-machine-learning.html } \\
\text { http://www.wired.com/wiredenterprise/2013/06/andrew ng/ }\end{array}$ \\
$\begin{array}{l}\text { A recent research paper on HPC for Deep Learning: } \\
\text { http://www.stanford.edu/ acoates/papers/CoatesHuvalWangWuNgCatanzaro icml2 } \\
\text { 013.pdf } \\
\text { Widely-used tutorials and references for Deep Learning: } \\
\text { http://ufldl.stanford.edu/wiki/index.php/Main Page } \\
\text { http://deeplearning.net/ }\end{array}$ \\
\hline
\end{tabular}




\section{Deep Learning and Social Media> Use Case 27: Large Scale Consumer Photos Organization}

\begin{tabular}{|c|c|c|}
\hline Use Case Title & \multicolumn{2}{|c|}{ Organizing large-scale, unstructured collections of consumer photos } \\
\hline Vertical (area) & \multicolumn{2}{|c|}{ (Scientific Research: Artificial Intelligence) } \\
\hline Author/Company/Email & \multicolumn{2}{|c|}{ David Crandall, Indiana University, djcran@indiana.edu } \\
\hline $\begin{array}{r}\text { Actors/Stakeholders } \\
\text { and their roles and } \\
\text { responsibilities }\end{array}$ & \multicolumn{2}{|c|}{$\begin{array}{l}\text { Computer vision researchers (to push forward state of art), media and social network } \\
\text { companies (to help organize large-scale photo collections), consumers (browsing } \\
\text { both personal and public photo collections), researchers and others interested in } \\
\text { producing cheap } 3 \mathrm{~d} \text { models (archaeologists, architects, urban planners, interior } \\
\text { designers...) }\end{array}$} \\
\hline Goals & \multicolumn{2}{|c|}{$\begin{array}{l}\text { Produce } 3 \mathrm{~d} \text { reconstructions of scenes using collections of millions to billions of } \\
\text { consumer images, where neither the scene structure nor the camera positions are } \\
\text { known a priori. Use resulting } 3 \mathrm{~d} \text { models to allow efficient and effective browsing of } \\
\text { large-scale photo collections by geographic position. Geolocate new images by } \\
\text { matching to 3d models. Perform object recognition on each image. }\end{array}$} \\
\hline Use Case Description & \multicolumn{2}{|c|}{$\begin{array}{l}\text { 3d reconstruction is typically posed as a robust non-linear least squares optimization } \\
\text { problem in which observed (noisy) correspondences between images are constraints } \\
\text { and unknowns are 6-d camera pose of each image and 3-d position of each point in } \\
\text { the scene. Sparsity and large degree of noise in constraints typically makes naïve } \\
\text { techniques fall into local minima that are not close to actual scene structure. Typical } \\
\text { specific steps are: (1) extracting features from images, (2) matching images to find } \\
\text { pairs with common scene structures, (3) estimating an initial solution that is close to } \\
\text { scene structure and/or camera parameters, (4) optimizing non-linear objective } \\
\text { function directly. Of these, (1) is embarrassingly parallel. (2) is an all-pairs matching } \\
\text { problem, usually with heuristics to reject unlikely matches early on. We solve (3) } \\
\text { using discrete optimization using probabilistic inference on a graph (Markov Random } \\
\text { Field) followed by robust Levenberg-Marquardt in continuous space. Others solve (3) } \\
\text { by solving (4) for a small number of images and then incrementally adding new } \\
\text { images, using output of last round as initialization for next round. (4) is typically } \\
\text { solved with Bundle Adjustment, which is a non-linear least squares solver that is } \\
\text { optimized for the particular constraint structure that occurs in 3d reconstruction } \\
\text { problems. Image recognition problems are typically embarrassingly parallel, although } \\
\text { learning object models involves learning a classifier (e.g. a Support Vector Machine), } \\
\text { a process that is often hard to parallelize. }\end{array}$} \\
\hline \multirow{4}{*}{$\begin{array}{r}\text { Current } \\
\text { Solutions }\end{array}$} & Compute(System) & Hadoop cluster (about 60 nodes, 480 core) \\
\hline & Storage & Hadoop DFS and flat files \\
\hline & Networking & Simple Unix \\
\hline & Software & $\begin{array}{l}\text { Hadoop Map-reduce, simple hand-written } \\
\text { multithreaded tools (ssh and sockets for } \\
\text { communication) }\end{array}$ \\
\hline \multirow[t]{4}{*}{$\begin{array}{r}\text { Big Data } \\
\text { Characteristics }\end{array}$} & $\begin{array}{r}\text { Data Source } \\
\text { (distributed/centralized) }\end{array}$ & $\begin{array}{l}\text { Publicly-available photo collections, e.g. on Flickr, } \\
\text { Panoramio, etc. }\end{array}$ \\
\hline & Volume (size) & $\begin{array}{l}500+\text { billion photos on Facebook, } 5+\text { billion photos on } \\
\text { Flickr. }\end{array}$ \\
\hline & $\begin{array}{r}\text { Velocity } \\
\text { (e.g. real time) }\end{array}$ & 100+ million new photos added to Facebook per day. \\
\hline & $\begin{array}{r}\text { Variety } \\
\text { (multiple datasets, } \\
\text { mashup) }\end{array}$ & $\begin{array}{l}\text { Images and metadata including EXIF tags (focal distance, } \\
\text { camera type, etc.), }\end{array}$ \\
\hline
\end{tabular}




\section{Deep Learning and Social Media> Use Case 27: Large Scale Consumer Photos Organization}

\begin{tabular}{|c|c|c|}
\hline & $\begin{array}{r}\text { Variability (rate of } \\
\text { change) }\end{array}$ & $\begin{array}{l}\text { Rate of photos varies significantly, e.g. roughly } 10 x \\
\text { photos to Facebook on New Year's versus other days. } \\
\text { Geographic distribution of photos follows long-tailed } \\
\text { distribution, with } 1000 \text { landmarks (totaling only about } \\
100 \text { square } \mathrm{km} \text { ) accounting for over } 20 \% \text { of photos on } \\
\text { Flickr. }\end{array}$ \\
\hline \multirow{5}{*}{$\begin{array}{r}\text { Big Data Science } \\
\text { (collection, curation, } \\
\text { analysis, } \\
\text { action) }\end{array}$} & $\begin{array}{r}\begin{array}{r}\text { Veracity (Robustness } \\
\text { Issues) }\end{array} \\
\end{array}$ & $\begin{array}{l}\text { Important to make as accurate as possible, subject to } \\
\text { limitations of computer vision technology. }\end{array}$ \\
\hline & Visualization & $\begin{array}{l}\text { Visualize large-scale } 3-d \text { reconstructions, and navigate } \\
\text { large-scale collections of images that have been aligned } \\
\text { to maps. }\end{array}$ \\
\hline & Data Quality & $\begin{array}{l}\text { Features observed in images are quite noisy due both to } \\
\text { imperfect feature extraction and to non-ideal properties } \\
\text { of specific images (lens distortions, sensor noise, image } \\
\text { effects added by user, etc.) }\end{array}$ \\
\hline & Data Types & Images, metadata \\
\hline & Data Analytics & \\
\hline $\begin{array}{r}\text { Big Data Specific } \\
\text { Challenges (Gaps) }\end{array}$ & \multicolumn{2}{|c|}{ Analytics needs continued monitoring and improvement. } \\
\hline $\begin{array}{r}\text { Big Data Specific } \\
\text { Challenges in Mobility }\end{array}$ & \multicolumn{2}{|c|}{$\begin{array}{l}\text { Many/most images are captured by mobile devices; eventual goal is to push } \\
\text { reconstruction and organization to phone to allow real-time interaction with the } \\
\text { user. }\end{array}$} \\
\hline $\begin{array}{r}\text { Security and Privacy } \\
\text { Requirements }\end{array}$ & \multicolumn{2}{|c|}{ Need to preserve privacy for users and digital rights for media. } \\
\hline $\begin{array}{r}\text { Highlight issues for } \\
\text { generalizing this use } \\
\text { case (e.g. for ref. } \\
\text { architecture) } \\
\end{array}$ & \multicolumn{2}{|c|}{$\begin{array}{l}\text { Components of this use case including feature extraction, feature matching, and } \\
\text { large-scale probabilistic inference appear in many or most computer vision and } \\
\text { image processing problems, including recognition, stereo resolution, image } \\
\text { denoising, etc. }\end{array}$} \\
\hline $\begin{array}{r}\text { More Information } \\
\text { (URLs) }\end{array}$ & \multicolumn{2}{|c|}{$\underline{\text { http://vision.soic.indiana.edu/disco }}$} \\
\hline
\end{tabular}




\section{Deep Learning and Social Media> Use Case 28: Truthy Twitter Data Analysis}

\begin{tabular}{|c|c|c|}
\hline Use Case Title & \multicolumn{2}{|c|}{ Truthy: Information diffusion research from Twitter Data } \\
\hline Vertical (area) & \multicolumn{2}{|c|}{ Scientific Research: Complex Networks and Systems research } \\
\hline Author/Company/Email & \multicolumn{2}{|c|}{$\begin{array}{l}\text { Filippo Menczer, Indiana University, fil@indiana.edu; } \\
\text { Alessandro Flammini, Indiana University, aflammin@indiana.edu; } \\
\text { Emilio Ferrara, Indiana University, ferrarae@indiana.edu; }\end{array}$} \\
\hline $\begin{array}{l}\text { Actors/Stakeholders } \\
\text { and their roles and } \\
\text { responsibilities }\end{array}$ & \multicolumn{2}{|c|}{ Research funded by NFS, DARPA, and McDonnel Foundation. } \\
\hline Goals & \multicolumn{2}{|c|}{$\begin{array}{l}\text { Understanding how communication spreads on socio-technical networks. Detecting } \\
\text { potentially harmful information spread at the early stage (e.g., deceiving messages, } \\
\text { orchestrated campaigns, untrustworthy information, etc.) }\end{array}$} \\
\hline Use Case Description & \multicolumn{2}{|c|}{$\begin{array}{l}\text { (1) Acquisition and storage of a large volume of continuous streaming data from } \\
\text { Twitter ( } \approx 100 \text { million messages per day, } \approx 500 G B \text { data/day increasing over time); } \\
\text { (2) near real-time analysis of such data, for anomaly detection, stream clustering, } \\
\text { signal classification and online-learning; (3) data retrieval, Big Data visualization, } \\
\text { data-interactive Web interfaces, public API for data querying. }\end{array}$} \\
\hline \multirow[t]{4}{*}{$\begin{array}{r}\text { Current } \\
\text { Solutions }\end{array}$} & Compute(System) & $\begin{array}{l}\text { Current: in-house cluster hosted by Indiana University. } \\
\text { Critical requirement: large cluster for data storage, } \\
\text { manipulation, querying and analysis. }\end{array}$ \\
\hline & Storage & $\begin{array}{l}\text { Current: Raw data stored in large compressed flat files, } \\
\text { since August 2010. Need to move towards } \\
\text { Hadoop/IndexedHBase and HDFS distributed storage. } \\
\text { Redis as an in-memory database as a buffer for real-time } \\
\text { analysis. }\end{array}$ \\
\hline & Networking & 10GB/Infiniband required. \\
\hline & Software & $\begin{array}{l}\text { Hadoop, Hive, Redis for data management. } \\
\text { Python/SciPy/NumPy/MPI for data analysis. }\end{array}$ \\
\hline \multirow[t]{5}{*}{$\begin{array}{r}\text { Big Data } \\
\text { Characteristics }\end{array}$} & $\begin{array}{r}\text { Data Source } \\
\text { (distributed/centralized) }\end{array}$ & Distributed - with replication/redundancy \\
\hline & Volume (size) & $\approx 30 \mathrm{~TB} /$ year compressed data \\
\hline & Velocity (e.g. real time) & Near real-time data storage, querying and analysis \\
\hline & $\begin{array}{r}\text { Variety (multiple } \\
\text { datasets, mashup) }\end{array}$ & $\begin{array}{l}\text { Data schema provided by social media data source. } \\
\text { Currently using Twitter only. We plan to expand } \\
\text { incorporating Google+, Facebook }\end{array}$ \\
\hline & $\begin{array}{r}\begin{array}{r}\text { Variability (rate of } \\
\text { change) }\end{array} \\
\end{array}$ & $\begin{array}{l}\text { Continuous real-time data stream incoming from each } \\
\text { source. }\end{array}$ \\
\hline \multirow{4}{*}{$\begin{array}{r}\text { Big Data Science } \\
\text { (collection, curation, } \\
\text { analysis, } \\
\text { action) }\end{array}$} & $\begin{array}{r}\text { Veracity (Robustness } \\
\text { Issues, semantics) }\end{array}$ & $\begin{array}{l}\text { 99.99\% uptime required for real-time data acquisition. } \\
\text { Service outages might corrupt data integrity and } \\
\text { significance. }\end{array}$ \\
\hline & Visualization & $\begin{array}{l}\text { Information diffusion, clustering, and dynamic network } \\
\text { visualization capabilities already exist. }\end{array}$ \\
\hline & Data Quality (syntax) & $\begin{array}{l}\text { Data structured in standardized formats, the overall } \\
\text { quality is extremely high. We generate aggregated } \\
\text { statistics; expand the features set, etc., generating high- } \\
\text { quality derived data. }\end{array}$ \\
\hline & Data Types & $\begin{array}{l}\text { Fully-structured data (JSON format) enriched with users' } \\
\text { meta-data, geo-locations, etc. }\end{array}$ \\
\hline
\end{tabular}




\section{Deep Learning and Social Media> Use Case 28: Truthy Twitter Data Analysis}

\begin{tabular}{|c|c|c|}
\hline & Data Analytics & $\begin{array}{l}\text { Stream clustering: data are aggregated according to } \\
\text { topics, meta-data and additional features, using ad hoc } \\
\text { online clustering algorithms. Classification: using multi- } \\
\text { dimensional time series to generate, network features, } \\
\text { users, geographical, content features, etc., we classify } \\
\text { information produced on the platform. Anomaly } \\
\text { detection: real-time identification of anomalous events } \\
\text { (e.g., induced by exogenous factors). Online learning: } \\
\text { applying machine learning/deep learning methods to } \\
\text { real-time information diffusion patterns analysis, users } \\
\text { profiling, etc. }\end{array}$ \\
\hline $\begin{array}{l}\text { Big Data Specific } \\
\text { Challenges (Gaps) }\end{array}$ & \multicolumn{2}{|c|}{$\begin{array}{l}\text { Dealing with real-time analysis of large volume of data. Providing a scalable } \\
\text { infrastructure to allocate resources, storage space, etc. on-demand if required by } \\
\text { increasing data volume over time. }\end{array}$} \\
\hline $\begin{array}{r}\text { Big Data Specific } \\
\text { Challenges in Mobility }\end{array}$ & \multicolumn{2}{|c|}{$\begin{array}{l}\text { Implementing low-level data storage infrastructure features to guarantee efficient, } \\
\text { mobile access to data. }\end{array}$} \\
\hline $\begin{array}{r}\text { Security and Privacy } \\
\text { Requirements }\end{array}$ & \multicolumn{2}{|c|}{$\begin{array}{l}\text { Twitter publicly releases data collected by our platform. Although, data-sources } \\
\text { incorporate user meta-data (in general, not sufficient to uniquely identify } \\
\text { individuals) therefore some policy for data storage security and privacy protection } \\
\text { must be implemented. }\end{array}$} \\
\hline $\begin{array}{r}\text { Highlight issues for } \\
\text { generalizing this use } \\
\text { case (e.g. for ref. } \\
\text { architecture) }\end{array}$ & \multicolumn{2}{|c|}{$\begin{array}{l}\text { Definition of high-level data schema to incorporate multiple data-sources providing } \\
\text { similarly structured data. }\end{array}$} \\
\hline $\begin{array}{r}\text { More Information } \\
\text { (URLs) }\end{array}$ & $\begin{array}{l}\text { http://truthy.indiana.edu/ } \\
\text { http://cnets.indiana.edu/g } \\
\text { http://cnets.indiana.edu/g }\end{array}$ & oups/nan/truthy \\
\hline
\end{tabular}




\section{Deep Learning and Social Media> Use Case 29: Crowd Sourcing in the Humanities}

\begin{tabular}{|c|c|c|}
\hline Use Case Title & \multicolumn{2}{|c|}{ Crowd Sourcing in the Humanities as Source for Big and Dynamic Data } \\
\hline Vertical (area) & \multicolumn{2}{|c|}{ Humanities, Social Sciences } \\
\hline Author/Company/Email & \multicolumn{2}{|c|}{$\begin{array}{l}\text { Sebastian Drude < Sebastian.Drude@mpi.nl }>\text {, Max Planck Institute for } \\
\text { Psycholinguistics }\end{array}$} \\
\hline $\begin{array}{r}\text { Actors/Stakeholders } \\
\text { and their roles and } \\
\text { responsibilities }\end{array}$ & \multicolumn{2}{|c|}{$\begin{array}{l}\text { Scientists (Sociologists, Psychologists, Linguists, Politic Scientists, Historians, etc.), } \\
\text { data managers and analysts, data archives } \\
\text { The general public as data providers and participants }\end{array}$} \\
\hline Goals & \multicolumn{2}{|c|}{$\begin{array}{l}\text { Capture information (manually entered, recorded multimedia, reaction times, } \\
\text { pictures, sensor information) from many individuals and their devices. } \\
\text { Thus capture wide ranging individual, social, cultural and linguistic variation among } \\
\text { several dimensions (space, social space, time). }\end{array}$} \\
\hline Use Case Description & \multicolumn{2}{|c|}{$\begin{array}{l}\text { Many different possible use cases: get recordings of language usage (words, } \\
\text { sentences, meaning descriptions, etc.), answers to surveys, info on cultural facts, } \\
\text { transcriptions of pictures and texts -- correlate these with other phenomena, detect } \\
\text { new cultural practices, behavior, values and believes, discover individual variation }\end{array}$} \\
\hline \multirow[t]{4}{*}{$\begin{array}{r}\text { Current } \\
\text { Solutions }\end{array}$} & Compute(System) & $\begin{array}{l}\text { Individual systems for manual data collection (mostly } \\
\text { Websites) }\end{array}$ \\
\hline & Storage & Traditional servers \\
\hline & Networking & barely used other than for data entry via web \\
\hline & Software & $\begin{array}{l}\text { XML technology, traditional relational databases for } \\
\text { storing pictures, not much multi-media yet. }\end{array}$ \\
\hline \multirow[t]{5}{*}{$\begin{array}{r}\text { Big Data } \\
\text { Characteristics }\end{array}$} & $\begin{array}{r}\text { Data Source } \\
\text { (distributed/centralized) }\end{array}$ & $\begin{array}{l}\text { Distributed, individual contributors via webpages and } \\
\text { mobile devices }\end{array}$ \\
\hline & Volume (size) & $\begin{array}{l}\text { Depends dramatically, from hundreds to millions of data } \\
\text { records. } \\
\text { Depending on data-type: from GBs (text, surveys, } \\
\text { experiment values) to hundreds of terabytes } \\
\text { (multimedia) }\end{array}$ \\
\hline & $\begin{array}{r}\text { Velocity } \\
\text { (e.g. real time) }\end{array}$ & $\begin{array}{l}\text { Depends very much on project: dozens to thousands of } \\
\text { new data records per day } \\
\text { Data has to be analyzed incrementally. }\end{array}$ \\
\hline & $\begin{array}{r}\text { Variety } \\
\text { (multiple datasets, } \\
\text { mashup) }\end{array}$ & $\begin{array}{l}\text { so far mostly homogeneous small datasets; expected } \\
\text { large distributed heterogeneous datasets which have to } \\
\text { be archived as primary data }\end{array}$ \\
\hline & $\begin{array}{r}\text { Variability (rate of } \\
\text { change) }\end{array}$ & $\begin{array}{l}\text { Data structure and content of collections are changing } \\
\text { during data life cycle. } \\
\text { There is no critical variation of data producing speed, or } \\
\text { runtime characteristics variations. }\end{array}$ \\
\hline \multirow{4}{*}{$\begin{array}{r}\text { Big Data Science } \\
\text { (collection, curation, } \\
\text { analysis, } \\
\text { action) }\end{array}$} & $\begin{array}{r}\text { Veracity (Robustness } \\
\text { Issues) }\end{array}$ & $\begin{array}{l}\text { Noisy data is possible, unreliable metadata, } \\
\text { identification and pre-selection of appropriate data }\end{array}$ \\
\hline & Visualization & $\begin{array}{l}\text { important for interpretation, no special visualization } \\
\text { techniques }\end{array}$ \\
\hline & Data Quality & $\begin{array}{l}\text { validation is necessary; quality of recordings, quality of } \\
\text { content, spam }\end{array}$ \\
\hline & Data Types & $\begin{array}{l}\text { individual data records (survey answers, reaction times); } \\
\text { text (e.g., comments, transcriptions,...); } \\
\text { multi-media (pictures, audio, video) }\end{array}$ \\
\hline
\end{tabular}




\section{Deep Learning and Social Media> Use Case 29: Crowd Sourcing in the Humanities}

\begin{tabular}{|r|l|l|}
\hline & Data Analytics & $\begin{array}{l}\text { pattern recognition of all kind (e.g., speech recognition, } \\
\text { automatic A\&V analysis, cultural patterns), identification } \\
\text { of structures (lexical units, linguistic rules, etc.) }\end{array}$ \\
\hline $\begin{array}{r}\text { Big Data Specific } \\
\text { Challenges (Gaps) }\end{array}$ & $\begin{array}{l}\text { Data management (metadata, provenance info, data identification with PIDs) } \\
\text { Data curation } \\
\text { Digitizing existing audio-video, photo and documents archives }\end{array}$ \\
\hline $\begin{array}{r}\text { Big Data Specific } \\
\text { Challenges in Mobility }\end{array}$ & $\begin{array}{l}\text { Include data from sensors of mobile devices (position, etc.); } \\
\text { Data collection from expeditions and field research. }\end{array}$ \\
\hline $\begin{array}{r}\text { Security and Privacy } \\
\text { Requirements }\end{array}$ & $\begin{array}{l}\text { Privacy issues may be involved (A/V from individuals), anonymization may be } \\
\text { necessary but not always possible (A/V analysis, small speech communities) }\end{array}$ \\
Archive and metadata integrity, long term preservation
\end{tabular}




\section{Deep Learning and Social Media> Use Case 30: CINET Network Science Cyberinfrastructure}

\begin{tabular}{|c|c|c|}
\hline Use Case Title & \multicolumn{2}{|c|}{ CINET: Cyberinfrastructure for Network (Graph) Science and Analytics } \\
\hline Vertical (area) & \multicolumn{2}{|c|}{ Network Science } \\
\hline Author/Company/Email & \multicolumn{2}{|c|}{$\begin{array}{l}\text { Team lead by Virginia Tech and comprising of researchers from Indiana University, } \\
\text { University at Albany, North Carolina AT, Jackson State University, University at } \\
\text { Houston Downtown, Argonne National Laboratory } \\
\text { Point of Contact: Madhav Marathe or Keith Bisset, Network Dynamics and Simulation } \\
\text { Science Laboratory, Virginia Bio-informatics Institute Virginia Tech, } \\
\text { mmarathe@vbi.vt.edu / kbisset@vbi.vt.edu }\end{array}$} \\
\hline $\begin{array}{l}\text { Actors/Stakeholders } \\
\text { and their roles and } \\
\text { responsibilities }\end{array}$ & \multicolumn{2}{|c|}{$\begin{array}{l}\text { Researchers, practitioners, educators and students interested in the study of } \\
\text { networks. }\end{array}$} \\
\hline Goals & \multicolumn{2}{|c|}{$\begin{array}{l}\text { CINET cyberinfrastructure middleware to support network science. This middleware } \\
\text { will give researchers, practitioners, teachers and students access to a computational } \\
\text { and analytic environment for research, education and training. The user interface } \\
\text { provides lists of available networks and network analysis modules (implemented } \\
\text { algorithms for network analysis). A user, who can be a researcher in network science } \\
\text { area, can select one or more networks and analysis them with the available network } \\
\text { analysis tools and modules. A user can also generate random networks following } \\
\text { various random graph models. Teachers and students can use CINET for classroom } \\
\text { use to demonstrate various graph theoretic properties and behaviors of various } \\
\text { algorithms. A user is also able to add a network or network analysis module to the } \\
\text { system. This feature of CINET allows it to grow easily and remain up-to-date with the } \\
\text { latest algorithms. } \\
\text { The goal is to provide a common web-based platform for accessing various } \\
\text { (i) network and graph analysis tools such as SNAP, NetworkX, Galib, etc. (ii) real- } \\
\text { world and synthetic networks, (iii) computing resources and (iv) data management } \\
\text { systems to the end-user in a seamless manner. }\end{array}$} \\
\hline Use Case Description & \multicolumn{2}{|c|}{$\begin{array}{l}\text { Users can run one or more structural or dynamic analysis on a set of selected } \\
\text { networks. The domain specific language allows users to develop flexible high level } \\
\text { workflows to define more complex network analysis. }\end{array}$} \\
\hline \multirow[t]{4}{*}{$\begin{array}{r}\text { Current } \\
\text { Solutions }\end{array}$} & Compute(System) & $\begin{array}{l}\text { A high performance computing cluster (DELL C6100), } \\
\text { named Shadowfax, of } 60 \text { compute nodes and } 12 \\
\text { processors (Intel Xeon X5670 2.93GHz) per compute } \\
\text { node with a total of } 720 \text { processors and 4GB main } \\
\text { memory per processor. } \\
\text { Shared memory systems; EC2 based clouds are also } \\
\text { used } \\
\text { Some of the codes and networks can utilize single node } \\
\text { systems and thus are being currently mapped to Open } \\
\text { Science Grid }\end{array}$ \\
\hline & Storage & 628 TB GPFS \\
\hline & Networking & $\begin{array}{l}\text { Internet, infiniband. A loose collection of } \\
\text { supercomputing resources. }\end{array}$ \\
\hline & Software & $\begin{array}{l}\text { Graph libraries: Galib, NetworkX. } \\
\text { Distributed Workflow Management: Simfrastructure, } \\
\text { databases, semantic web tools }\end{array}$ \\
\hline
\end{tabular}




\section{Deep Learning and Social Media> Use Case 30: CINET Network Science Cyberinfrastructure}

\begin{tabular}{|c|c|c|}
\hline \multirow[t]{5}{*}{$\begin{array}{r}\text { Big Data } \\
\text { Characteristics }\end{array}$} & $\begin{array}{r}\text { Data Source } \\
\text { (distributed/centralized) }\end{array}$ & $\begin{array}{l}\text { A single network remains in a single disk file accessible } \\
\text { by multiple processors. However, during the execution } \\
\text { of a parallel algorithm, the network can be partitioned } \\
\text { and the partitions are loaded in the main memory of } \\
\text { multiple processors. }\end{array}$ \\
\hline & Volume (size) & Can be hundreds of GB for a single network. \\
\hline & $\begin{array}{r}\text { Velocity } \\
\text { (e.g. real time) }\end{array}$ & $\begin{array}{l}\text { Two types of changes: (i) the networks are very } \\
\text { dynamic and (ii) as the repository grows, we expect at } \\
\text { least a rapid growth to lead to over } 1000-5000 \\
\text { networks and methods in about a year }\end{array}$ \\
\hline & $\begin{array}{r}\text { Variety } \\
\text { (multiple datasets, } \\
\text { mashup) }\end{array}$ & $\begin{array}{l}\text { Datasets are varied: (i) directed as well as undirected } \\
\text { networks, (ii) static and dynamic networks, (iii) labeled, } \\
\text { (iv) can have dynamics over these networks, }\end{array}$ \\
\hline & $\begin{array}{r}\text { Variability (rate of } \\
\text { change) }\end{array}$ & $\begin{array}{l}\text { The rate of graph-based data is growing at increasing } \\
\text { rate. Moreover, increasingly other life sciences } \\
\text { domains are using graph-based techniques to address } \\
\text { problems. Hence, we expect the data and the } \\
\text { computation to grow at a significant pace. }\end{array}$ \\
\hline \multirow{5}{*}{$\begin{array}{r}\text { Big Data Science } \\
\text { (collection, curation, } \\
\text { analysis, } \\
\text { action) }\end{array}$} & $\begin{array}{r}\text { Veracity (Robustness } \\
\text { Issues, semantics) }\end{array}$ & $\begin{array}{l}\text { Challenging due to asynchronous distributed } \\
\text { computation. Current systems are designed for real- } \\
\text { time synchronous response. }\end{array}$ \\
\hline & Visualization & $\begin{array}{l}\text { As the input graph size grows the visualization system } \\
\text { on client side is stressed heavily both in terms of data } \\
\text { and compute. }\end{array}$ \\
\hline & Data Quality (syntax) & \\
\hline & Data Types & \\
\hline & Data Analytics & \\
\hline $\begin{array}{l}\text { Big Data Specific } \\
\text { Challenges (Gaps) }\end{array}$ & \multicolumn{2}{|c|}{$\begin{array}{l}\text { Parallel algorithms are necessary to analyze massive networks. Unlike many } \\
\text { structured data, network data is difficult to partition. The main difficulty in } \\
\text { partitioning a network is that different algorithms require different partitioning } \\
\text { schemes for efficient operation. Moreover, most of the network measures are global } \\
\text { in nature and require either i) huge duplicate data in the partitions or ii) very large } \\
\text { communication overhead resulted from the required movement of data. These } \\
\text { issues become significant challenges for big networks. } \\
\text { Computing dynamics over networks is harder since the network structure often } \\
\text { interacts with the dynamical process being studied. } \\
\text { CINET enables large class of operations across wide variety, both in terms of } \\
\text { structure and size, of graphs. Unlike other compute + data intensive systems, such as } \\
\text { parallel databases or CFD, performance on graph computation is sensitive to } \\
\text { underlying architecture. Hence, a unique challenge in CINET is manage the mapping } \\
\text { between workload (graph type + operation) to a machine whose architecture and } \\
\text { runtime is conducive to the system. } \\
\text { Data manipulation and bookkeeping of the derived for users is another big challenge } \\
\text { since unlike enterprise data there is no well-defined and effective models and tools } \\
\text { for management of various graph data in a unified fashion. }\end{array}$} \\
\hline \multicolumn{3}{|l|}{$\begin{array}{r}\text { Big Data Specific } \\
\text { Challenges in Mobility }\end{array}$} \\
\hline $\begin{array}{r}\text { Security and Privacy } \\
\text { Requirements }\end{array}$ & & \\
\hline
\end{tabular}




\section{Deep Learning and Social Media> Use Case 30: CINET Network Science Cyberinfrastructure}

Highlight issues for generalizing this use case (e.g. for ref. architecture)

More Information http://cinet.vbi.vt.edu/cinet new/
HPC as a service. As data volume grows increasingly large number of applications such as biological sciences need to use HPC systems. CINET can be used to deliver the compute resource necessary for such domains.

(URLs) 


\section{Deep Learning and Social Media> Use Case 31: NIST Analytic Technology Measurement and Evaluations}

\begin{tabular}{|c|c|c|}
\hline Use Case Title & \multicolumn{2}{|c|}{$\begin{array}{l}\text { NIST Information Access Division analytic technology performance measurement, } \\
\text { evaluations, and standards }\end{array}$} \\
\hline Vertical (area) & \multicolumn{2}{|c|}{$\begin{array}{l}\text { Analytic technology performance measurement and standards for government, } \\
\text { industry, and academic stakeholders }\end{array}$} \\
\hline Author/Company/Email & \multicolumn{2}{|c|}{ John Garofolo (john.garofolo@nist.gov) } \\
\hline $\begin{array}{r}\text { Actors/Stakeholders } \\
\text { and their roles and } \\
\text { responsibilities }\end{array}$ & \multicolumn{2}{|c|}{$\begin{array}{l}\text { NIST developers of measurement methods, data contributors, analytic algorithm } \\
\text { developers, users of analytic technologies for unstructured, semi-structured data, } \\
\text { and heterogeneous data across all sectors. }\end{array}$} \\
\hline Goals & \multicolumn{2}{|c|}{$\begin{array}{l}\text { Accelerate the development of advanced analytic technologies for unstructured, } \\
\text { semi-structured, and heterogeneous data through performance measurement and } \\
\text { standards. Focus communities of interest on analytic technology challenges of } \\
\text { importance, create consensus-driven measurement metrics and methods for } \\
\text { performance evaluation, evaluate the performance of the performance metrics and } \\
\text { methods via community-wide evaluations which foster knowledge exchange and } \\
\text { accelerate progress, and build consensus towards widely-accepted standards for } \\
\text { performance measurement. }\end{array}$} \\
\hline Use Case Description & \multicolumn{2}{|c|}{$\begin{array}{l}\text { Develop performance metrics, measurement methods, and community evaluations } \\
\text { to ground and accelerate the development of advanced analytic technologies in the } \\
\text { areas of speech and language processing, video and multimedia processing, } \\
\text { biometric image processing, and heterogeneous data processing as well as the } \\
\text { interaction of analytics with users. Typically employ one of two processing models: 1) } \\
\text { Push test data out to test participants and analyze the output of participant systems, } \\
\text { 2) Push algorithm test harness interfaces out to participants and bring in their } \\
\text { algorithms and test them on internal computing clusters. Developing approaches to } \\
\text { support scalable Cloud-based developmental testing. Also perform usability and } \\
\text { utility testing on systems with users in the loop. }\end{array}$} \\
\hline \multirow[t]{4}{*}{$\begin{array}{r}\text { Current } \\
\text { Solutions }\end{array}$} & Compute (System) & $\begin{array}{l}\text { Linux and OS-10 clusters; distributed computing with } \\
\text { stakeholder collaborations; specialized image processing } \\
\text { architectures. }\end{array}$ \\
\hline & Storage & $\begin{array}{l}\text { RAID arrays, and distribute data on 1-2TB drives, and } \\
\text { occasionally FTP. Distributed data distribution with } \\
\text { stakeholder collaborations. }\end{array}$ \\
\hline & Networking & $\begin{array}{l}\text { Fiber channel disk storage, Gigabit Ethernet for system- } \\
\text { system communication, general intra- and Internet } \\
\text { resources within NIST and shared networking resources } \\
\text { with its stakeholders. }\end{array}$ \\
\hline & Software & $\begin{array}{l}\text { PERL, Python, } \mathrm{C} / \mathrm{C}++ \text {, Matlab, } \mathrm{R} \text { development tools. } \\
\text { Create ground-up test and measurement applications. }\end{array}$ \\
\hline \multirow[t]{2}{*}{$\begin{array}{r}\text { Big Data } \\
\text { Characteristics }\end{array}$} & $\begin{array}{r}\text { Data Source } \\
\text { (distributed/centralized) }\end{array}$ & $\begin{array}{l}\text { Large annotated corpora of unstructured/semi- } \\
\text { structured text, audio, video, images, multimedia, and } \\
\text { heterogeneous collections of the above including } \\
\text { ground truth annotations for training, developmental } \\
\text { testing, and summative evaluations. }\end{array}$ \\
\hline & Volume (size) & $\begin{array}{l}\text { The test corpora exceed 900M Web pages occupying } 30 \\
\text { TB of storage, } 100 \mathrm{M} \text { tweets, } 100 \mathrm{M} \text { ground-truthed } \\
\text { biometric images, several hundred thousand partially } \\
\text { ground-truthed video clips, and terabytes of smaller } \\
\text { fully ground-truthed test collections. Even larger data } \\
\text { collections are being planned for future evaluations of }\end{array}$ \\
\hline
\end{tabular}




\section{Deep Learning and Social Media> Use Case 31: NIST Analytic Technology Measurement and Evaluations}

\begin{tabular}{|c|c|c|}
\hline & & $\begin{array}{l}\text { analytics involving multiple data streams and very } \\
\text { heterogeneous data. }\end{array}$ \\
\hline & $\begin{array}{r}\text { Velocity } \\
\text { (e.g. real time) }\end{array}$ & $\begin{array}{l}\text { Most legacy evaluations are focused on retrospective } \\
\text { analytics. Newer evaluations are focusing on simulations } \\
\text { of real-time analytic challenges from multiple data } \\
\text { streams. }\end{array}$ \\
\hline & $\begin{array}{r}\text { Variety } \\
\text { (multiple datasets, } \\
\text { mashup) }\end{array}$ & $\begin{array}{l}\text { The test collections span a wide variety of analytic } \\
\text { application types including textual search/extraction, } \\
\text { machine translation, speech recognition, image and } \\
\text { voice biometrics, object and person recognition and } \\
\text { tracking, document analysis, human-computer dialogue, } \\
\text { and multimedia search/extraction. Future test } \\
\text { collections will include mixed type data and applications. }\end{array}$ \\
\hline & $\begin{array}{r}\text { Variability (rate of } \\
\text { change) }\end{array}$ & $\begin{array}{l}\text { Evaluation of tradeoffs between accuracy and data rates } \\
\text { as well as variable numbers of data streams and variable } \\
\text { stream quality. }\end{array}$ \\
\hline $\begin{array}{r}\text { Big Data Science } \\
\text { (collection, curation, } \\
\text { analysis, } \\
\text { action) }\end{array}$ & $\begin{array}{r}\text { Veracity (Robustness } \\
\text { Issues, semantics) }\end{array}$ & $\begin{array}{l}\text { The creation and measurement of the uncertainty } \\
\text { associated with the ground-truthing process - especially } \\
\text { when humans are involved - is challenging. The manual } \\
\text { ground-truthing processes that have been used in the } \\
\text { past are not scalable. Performance measurement of } \\
\text { complex analytics must include measurement of } \\
\text { intrinsic uncertainty as well as ground truthing error to } \\
\text { be useful. }\end{array}$ \\
\hline & Visualization & $\begin{array}{l}\text { Visualization of analytic technology performance results } \\
\text { and diagnostics including significance and various forms } \\
\text { of uncertainty. Evaluation of analytic presentation } \\
\text { methods to users for usability, utility, efficiency, and } \\
\text { accuracy. }\end{array}$ \\
\hline & Data Quality (syntax) & $\begin{array}{l}\text { The performance of analytic technologies is highly } \\
\text { impacted by the quality of the data they are employed } \\
\text { against with regard to a variety of domain- and } \\
\text { application-specific variables. Quantifying these } \\
\text { variables is a challenging research task in itself. Mixed } \\
\text { sources of data and performance measurement of } \\
\text { analytic flows pose even greater challenges with regard } \\
\text { to data quality. }\end{array}$ \\
\hline & Data Types & $\begin{array}{l}\text { Unstructured and semi-structured text, still images, } \\
\text { video, audio, multimedia (audio+video). }\end{array}$ \\
\hline & Data Analytics & $\begin{array}{l}\text { Information extraction, filtering, search, and } \\
\text { summarization; image and voice biometrics; speech } \\
\text { recognition and understanding; machine translation; } \\
\text { video person/object detection and tracking; event } \\
\text { detection; imagery/document matching; novelty } \\
\text { detection; a variety of structural/semantic/temporal } \\
\text { analytics and many subtypes of the above. }\end{array}$ \\
\hline $\begin{array}{r}\text { Big Data Specific } \\
\text { Challenges (Gaps) }\end{array}$ & \multicolumn{2}{|c|}{$\begin{array}{l}\text { Scaling ground-truthing to larger data, intrinsic and annotation uncertainty } \\
\text { measurement, performance measurement for incompletely annotated data, } \\
\text { measuring analytic performance for heterogeneous data and analytic flows involving }\end{array}$} \\
\hline
\end{tabular}




\section{Deep Learning and Social Media> Use Case 31: NIST Analytic Technology Measurement and Evaluations}

\begin{tabular}{|r|l|}
\hline $\begin{array}{r}\text { Big Data Specific } \\
\text { Challenges in Mobility }\end{array}$ & $\begin{array}{l}\text { Moving training, development, and test data to evaluation participants or moving } \\
\text { evaluation participants' analytic algorithms to computational testbeds for } \\
\text { performance assessment. Providing developmental tools and data. Supporting agile } \\
\text { developmental testing approaches. }\end{array}$ \\
\hline $\begin{array}{r}\text { Security and Privacy } \\
\text { Requirements }\end{array}$ & $\begin{array}{l}\text { Analytic algorithms working with written language, speech, human imagery, etc. } \\
\text { must generally be tested against real or realistic data. It's extremely challenging to } \\
\text { engineer artificial data that sufficiently captures the variability of real data involving } \\
\text { humans. Engineered data may provide artificial challenges that may be directly or } \\
\text { indirectly modeled by analytic algorithms and result in overstated performance. The } \\
\text { advancement of analytic technologies themselves is increasing privacy sensitivities. } \\
\text { Future performance testing methods will need to isolate analytic technology } \\
\text { algorithms from the data the algorithms are tested against. Advanced architectures } \\
\text { are needed to support security requirements for protecting sensitive data while } \\
\text { enabling meaningful developmental performance evaluation. Shared evaluation } \\
\text { testbeds must protect the intellectual property of analytic algorithm developers. }\end{array}$ \\
\hline $\begin{array}{r}\text { Highlight issues for } \\
\text { generalizing this use }\end{array}$ & $\begin{array}{l}\text { Scalability of analytic technology performance testing methods, source data } \\
\text { creation, and ground truthing; approaches and architectures supporting } \\
\text { case (e.g. for ref. } \\
\text { architecture) } \\
\text { and other personal information in test data; measurement of uncertainty using } \\
\text { partially-annotated data; composing test data with regard to qualities impacting } \\
\text { performance and estimating test set difficulty; evaluating complex analytic flows } \\
\text { involving multiple analytics, data types, and user interactions; multiple } \\
\text { heterogeneous data streams and massive numbers of streams; mixtures of } \\
\text { structured, semi-structured, and unstructured data sources; agile scalable } \\
\text { developmental testing approaches and mechanisms. }\end{array}$ \\
\hline http://www.nist.gov/it//iad/ \\
\hline More Information
\end{tabular}




\section{The Ecosystem for Research> Use Case 32: DataNet Federation Consortium (DFC)}

\begin{tabular}{|c|c|c|}
\hline Use Case Title & \multicolumn{2}{|c|}{ DataNet Federation Consortium (DFC) } \\
\hline Vertical (area) & \multicolumn{2}{|c|}{ Collaboration Environments } \\
\hline Author/Company/Email & \multicolumn{2}{|c|}{ Reagan Moore / University of North Carolina at Chapel Hill / rwmoore@renci.org } \\
\hline $\begin{array}{r}\text { Actors/Stakeholders } \\
\text { and their roles and } \\
\text { responsibilities }\end{array}$ & \multicolumn{2}{|c|}{$\begin{array}{l}\text { National Science Foundation research projects: Ocean Observatories Initiative } \\
\text { (sensor archiving); Temporal Dynamics of Learning Center (Cognitive science data } \\
\text { grid); the iPlant Collaborative (plant genomics); Drexel engineering digital library; } \\
\text { Odum Institute for social science research (data grid federation with Dataverse). }\end{array}$} \\
\hline Goals & \multicolumn{2}{|c|}{$\begin{array}{l}\text { Provide national infrastructure (collaboration environments) that enables } \\
\text { researchers to collaborate through shared collections and shared workflows. Provide } \\
\text { policy-based data management systems that enable the formation of collections, } \\
\text { data grid, digital libraries, archives, and processing pipelines. Provide interoperability } \\
\text { mechanisms that federate existing data repositories, information catalogs, and web } \\
\text { services with collaboration environments. }\end{array}$} \\
\hline Use Case Description & \multicolumn{2}{|c|}{$\begin{array}{l}\text { Promote collaborative and interdisciplinary research through federation of data } \\
\text { management systems across federal repositories, national academic research } \\
\text { initiatives, institutional repositories, and international collaborations. The } \\
\text { collaboration environment runs at scale: petabytes of data, hundreds of millions of } \\
\text { files, hundreds of millions of metadata attributes, tens of thousands of users, and a } \\
\text { thousand storage resources. }\end{array}$} \\
\hline \multirow[t]{4}{*}{$\begin{array}{r}\text { Current } \\
\text { Solutions }\end{array}$} & Compute(System) & $\begin{array}{l}\text { Interoperability with workflow systems (NCSA } \\
\text { Cyberintegrator, Kepler, Taverna) }\end{array}$ \\
\hline & Storage & $\begin{array}{l}\text { Interoperability across file systems, tape archives, cloud } \\
\text { storage, object-based storage }\end{array}$ \\
\hline & Networking & $\begin{array}{l}\text { Interoperability across TCP/IP, parallel TCP/IP, RBUDP, } \\
\text { HTTP }\end{array}$ \\
\hline & Software & Integrated Rule Oriented Data System (iRODS) \\
\hline \multirow[t]{5}{*}{$\begin{array}{r}\text { Big Data } \\
\text { Characteristics }\end{array}$} & $\begin{array}{r}\text { Data Source } \\
\text { (distributed/centralized) } \\
\end{array}$ & Manage internationally distributed data \\
\hline & Volume (size) & Petabytes, hundreds of millions of files \\
\hline & $\begin{array}{r}\text { Velocity } \\
\text { (e.g. real time) }\end{array}$ & $\begin{array}{l}\text { Support sensor data streams, satellite imagery, } \\
\text { simulation output, observational data, experimental } \\
\text { data }\end{array}$ \\
\hline & $\begin{array}{r}\text { Variety } \\
\text { (multiple datasets, } \\
\text { mashup) } \\
\end{array}$ & $\begin{array}{l}\text { Support logical collections that span administrative } \\
\text { domains, data aggregation in containers, metadata, and } \\
\text { workflows as objects }\end{array}$ \\
\hline & $\begin{array}{r}\text { Variability (rate of } \\
\text { change) }\end{array}$ & $\begin{array}{l}\text { Support active collections (mutable data), versioning of } \\
\text { data, and persistent identifiers }\end{array}$ \\
\hline \multirow{4}{*}{$\begin{array}{r}\text { Big Data Science } \\
\text { (collection, curation, } \\
\text { analysis, } \\
\text { action) }\end{array}$} & $\begin{array}{r}\text { Veracity (Robustness } \\
\text { Issues) }\end{array}$ & $\begin{array}{l}\text { Provide reliable data transfer, audit trails, event } \\
\text { tracking, periodic validation of assessment criteria } \\
\text { (integrity, authenticity), distributed debugging }\end{array}$ \\
\hline & Visualization & $\begin{array}{l}\text { Support execution of external visualization systems } \\
\text { through automated workflows (GRASS) }\end{array}$ \\
\hline & Data Quality & $\begin{array}{l}\text { Provide mechanisms to verify quality through } \\
\text { automated workflow procedures }\end{array}$ \\
\hline & Data Types & $\begin{array}{l}\text { Support parsing of selected formats (NetCDF, HDF5, } \\
\text { Dicom), and provide mechanisms to invoke other data } \\
\text { manipulation methods }\end{array}$ \\
\hline
\end{tabular}




\section{The Ecosystem for Research> Use Case 32: DataNet Federation Consortium (DFC)}

\begin{tabular}{|c|c|c|}
\hline & Data Analytics & $\begin{array}{l}\text { Provide support for invoking analysis workflows, } \\
\text { tracking workflow provenance, sharing of workflows, } \\
\text { and re-execution of workflows }\end{array}$ \\
\hline $\begin{array}{l}\text { Big Data Specific } \\
\text { Challenges (Gaps) }\end{array}$ & \multicolumn{2}{|c|}{$\begin{array}{l}\text { Provide standard policy sets that enable a new community to build upon data } \\
\text { management plans that address federal agency requirements }\end{array}$} \\
\hline $\begin{array}{r}\text { Big Data Specific } \\
\text { Challenges in Mobility }\end{array}$ & \multicolumn{2}{|c|}{$\begin{array}{l}\text { Capture knowledge required for data manipulation, and apply resulting procedures } \\
\text { at either the storage location, or a computer server. }\end{array}$} \\
\hline $\begin{array}{r}\text { Security and Privacy } \\
\text { Requirements }\end{array}$ & \multicolumn{2}{|c|}{$\begin{array}{l}\text { Federate across existing authentication environments through Generic Security } \\
\text { Service API and Pluggable Authentication Modules (GSI, Kerberos, InCommon, } \\
\text { Shibboleth). Manage access controls on files independently of the storage location. }\end{array}$} \\
\hline $\begin{array}{r}\text { Highlight issues for } \\
\text { generalizing this use } \\
\text { case (e.g. for ref. } \\
\text { architecture) }\end{array}$ & $\begin{array}{l}\text { Currently } 25 \text { science and en } \\
\text { policy-based data managem } \\
\text { Astrophysics } \\
\text { Atmospheric science } \\
\text { Biology } \\
\text { Climate } \\
\text { Cognitive Science } \\
\text { Computer Science } \\
\text { Cosmic Ray } \\
\text { Dark Matter Physics } \\
\text { Earth Science } \\
\text { Ecology } \\
\text { Engineering } \\
\text { High Energy Physics } \\
\text { Hydrology } \\
\text { Genomics } \\
\text { Medicine } \\
\text { Neuroscience } \\
\text { Neutrino Physics } \\
\text { Oceanography } \\
\text { Optical Astronomy } \\
\text { Particle Physics } \\
\text { Plant genetics } \\
\text { Quantum Chromodynamics } \\
\text { Radio Astronomy } \\
\text { Seismology } \\
\text { Social Science }\end{array}$ & $\begin{array}{l}\text { gineering domains have projects that rely on the iRODS } \\
\text { Ient system: } \\
\text { Auger supernova search } \\
\text { NASA Langley Atmospheric Sciences Center } \\
\text { Phylogenetics at CC IN2P3 } \\
\text { NOAA National Climatic Data Center } \\
\text { Temporal Dynamics of Learning Center } \\
\text { GENI experimental network } \\
\text { AMS experiment on the International Space Station } \\
\text { Edelweiss II } \\
\text { NASA Center for Climate Simulations } \\
\text { CEED Caveat Emptor Ecological Data } \\
\text { CIBER-U } \\
\text { BaBar } \\
\text { Institute for the Environment, UNC-CH; Hydroshare } \\
\text { Broad Institute, Wellcome Trust Sanger Institute } \\
\text { Sick Kids Hospital } \\
\text { International Neuroinformatics Coordinating Facility } \\
\text { T2K and dChooz neutrino experiments } \\
\text { Ocean Observatories Initiative } \\
\text { National Optical Astronomy Observatory } \\
\text { Indra } \\
\text { the iPlant Collaborative } \\
\text { IN2P3 } \\
\text { Cyber Square Kilometer Array, TREND, BAOradio } \\
\text { Southern California Earthquake Center } \\
\text { Odum Institute for Social Science Research, TerraPop }\end{array}$ \\
\hline $\begin{array}{r}\text { More Information } \\
\text { (URLs) }\end{array}$ & \multicolumn{2}{|c|}{$\begin{array}{l}\text { The DataNet Federation Consortium: http://www.datafed.org } \\
\text { iRODS: http://www.irods.org }\end{array}$} \\
\hline \multicolumn{3}{|c|}{$\begin{array}{l}\text { Note: A major challenge is the ability to capture knowledge needed to interact with the data products of a } \\
\text { research domain. In policy-based data management systems, this is done by encapsulating the knowledge in } \\
\text { procedures that are controlled through policies. The procedures can automate retrieval of data from external } \\
\text { repositories, or execute processing workflows, or enforce management policies on the resulting data products. } \\
\text { A standard application is the enforcement of data management plans and the verification that the plan has been } \\
\text { successfully applied. }\end{array}$} \\
\hline
\end{tabular}

301 See Figure 4: DataNet Federation Consortium DFC - iRODS architecture. 


\section{The Ecosystem for Research> Use Case 33: The 'Discinnet Process'}

\begin{tabular}{|c|c|c|}
\hline Use Case Title & \multicolumn{2}{|c|}{ The 'Discinnet process', metadata <-> Big Data global experiment } \\
\hline Vertical (area) & \multicolumn{2}{|c|}{ Scientific Research: Interdisciplinary Collaboration } \\
\hline Author/Company/Email & \multicolumn{2}{|c|}{ P. Journeau / Discinnet Labs / phjourneau@discinnet.org } \\
\hline $\begin{array}{r}\text { Actors/Stakeholders } \\
\text { and their roles and } \\
\text { responsibilities }\end{array}$ & \multicolumn{2}{|c|}{$\begin{array}{l}\text { Actors Richeact, Discinnet Labs and I4OpenResearch fund France/Europe. American } \\
\text { equivalent pending. Richeact is fundamental research and development } \\
\text { epistemology, Discinnet Labs applied in web } 2.0 \mathrm{http} / / \text { www.discinnet.org, } 14 \text { non- } \\
\text { profit warrant. }\end{array}$} \\
\hline Goals & \multicolumn{2}{|c|}{$\begin{array}{l}\text { Richeact scientific goal is to reach predictive interdisciplinary model of research } \\
\text { fields' behavior (with related meta-grammar). Experimentation through global } \\
\text { sharing of now multidisciplinary, later interdisciplinary Discinnet process/web } \\
\text { mapping and new scientific collaborative communication and publication system. } \\
\text { Expected sharp impact to reducing uncertainty and time between theoretical, } \\
\text { applied, technology research and development steps. }\end{array}$} \\
\hline Use Case Description & \multicolumn{2}{|c|}{$\begin{array}{l}\text { Currently } 35 \text { clusters started, close to } 100 \text { awaiting more resources and potentially } \\
\text { much more open for creation, administration and animation by research } \\
\text { communities. Examples range from optics, cosmology, materials, microalgae, health } \\
\text { to applied maths, computation, rubber and other chemical products/issues. } \\
\text { How does a typical case currently work: } \\
\text { - A researcher or group wants to see how a research field is faring and in a } \\
\text { minute defines the field on Discinnet as a 'cluster' } \\
\text { - Then it takes another } 5 \text { to } 10 \text { mn to parameter the first/main dimensions, } \\
\text { mainly measurement units and categories, but possibly later on some } \\
\text { variable limited time for more dimensions } \\
\text { Cluster then may be filled either by doctoral students or reviewing } \\
\text { researchers and/or communities/researchers for projects/progress } \\
\text { Already significant value but now needs to be disseminated and advertised although } \\
\text { maximal value to come from interdisciplinary/projective next version. Value is to } \\
\text { detect quickly a paper/project of interest for its results and next step is trajectory of } \\
\text { the field under types of interactions from diverse levels of oracles (subjects/objects) } \\
+ \text { from interdisciplinary context. }\end{array}$} \\
\hline \multirow[t]{4}{*}{$\begin{array}{r}\text { Current } \\
\text { Solutions }\end{array}$} & Compute(System) & $\begin{array}{l}\text { Currently on OVH (Hosting company } \\
\text { http://www.ovh.co.uk/) servers (mix shared + } \\
\text { dedicated) }\end{array}$ \\
\hline & Storage & $\mathrm{OVH}$ \\
\hline & Networking & To be implemented with desired integration with others \\
\hline & Software & Current version with Symfony-PHP, Linux, MySQL \\
\hline \multirow[t]{5}{*}{$\begin{array}{r}\text { Big Data } \\
\text { Characteristics }\end{array}$} & $\begin{array}{r}\text { Data Source } \\
\text { (distributed/centralized) }\end{array}$ & $\begin{array}{l}\text { Currently centralized, soon distributed per country and } \\
\text { even per hosting institution interested by own platform }\end{array}$ \\
\hline & Volume (size) & Not significant : this is a metadata base, not Big Data \\
\hline & $\begin{array}{r}\text { Velocity } \\
\text { (e.g. real time) } \\
\end{array}$ & Real time \\
\hline & $\begin{array}{r}\text { Variety } \\
\text { (multiple datasets, } \\
\text { mashup) }\end{array}$ & $\begin{array}{l}\text { Link to Big data still to be established in a Meta<->Big } \\
\text { relationship not yet implemented (with experimental } \\
\text { databases and already } 1^{\text {st }} \text { level related metadata) }\end{array}$ \\
\hline & $\begin{array}{r}\text { Variability (rate of } \\
\text { change) }\end{array}$ & $\begin{array}{l}\text { Currently real time, for further multiple locations and } \\
\text { distributed architectures, periodic (such as nightly) }\end{array}$ \\
\hline \multirow{2}{*}{$\begin{array}{r}\text { Big Data Science } \\
\text { (collection, curation, } \\
\text { analysis, }\end{array}$} & $\begin{array}{r}\text { Veracity (Robustness } \\
\text { Issues, semantics) }\end{array}$ & $\begin{array}{l}\text { Methods to detect overall consistency, holes, errors, } \\
\text { misstatements, known but mostly to be implemented }\end{array}$ \\
\hline & Visualization & Multidimensional (hypercube) \\
\hline
\end{tabular}




\section{The Ecosystem for Research> Use Case 33: The 'Discinnet Process'}

\begin{tabular}{|c|c|c|}
\hline \multirow[t]{3}{*}{ action) } & Data Quality (syntax) & $\begin{array}{l}\text { A priori correct (directly human captured) with sets of } \\
\text { checking + evaluation processes partly implemented }\end{array}$ \\
\hline & Data Types & 'cluster displays' (image), vectors, categories, PDFs \\
\hline & Data Analytics & \\
\hline $\begin{array}{l}\text { Big Data Specific } \\
\text { Challenges (Gaps) }\end{array}$ & \multicolumn{2}{|c|}{$\begin{array}{l}\text { Our goal is to contribute to Big } 2 \text { Metadata challenge by systematic reconciling } \\
\text { between metadata from many complexity levels with ongoing input from } \\
\text { researchers from ongoing research process. } \\
\text { Current relationship with Richeact is to reach the interdisciplinary model, using } \\
\text { meta-grammar itself to be experimented and its extent fully proven to bridge } \\
\text { efficiently the gap between as remote complexity levels as semantic and most } \\
\text { elementary (big) signals. Example with cosmological models versus many levels of } \\
\text { intermediary models (particles, gases, galactic, nuclear, geometries). Others with } \\
\text { computational versus semantic levels. }\end{array}$} \\
\hline $\begin{array}{r}\text { Big Data Specific } \\
\text { Challenges in Mobility }\end{array}$ & \multicolumn{2}{|c|}{ Appropriate graphic interface power } \\
\hline $\begin{array}{r}\text { Security and Privacy } \\
\text { Requirements }\end{array}$ & \multicolumn{2}{|c|}{$\begin{array}{l}\text { Several levels already available and others planned, up to physical access keys and } \\
\text { isolated servers. Optional anonymity, usual protected exchanges }\end{array}$} \\
\hline $\begin{array}{r}\text { Highlight issues for } \\
\text { generalizing this use } \\
\text { case (e.g. for ref. } \\
\text { architecture) }\end{array}$ & \multicolumn{2}{|c|}{$\begin{array}{l}\text { Through 2011-2013, we have shown on http://www.discinnet.org that all kinds of } \\
\text { research fields could easily get into Discinnet type of mapping, yet developing and } \\
\text { filling a cluster requires time and/or dedicated workers. }\end{array}$} \\
\hline $\begin{array}{r}\text { More Information } \\
\text { (URLs) }\end{array}$ & \multicolumn{2}{|c|}{$\begin{array}{l}\text { On http://www.discinnet.org the already started or starting clusters can be watched } \\
\text { in one click on 'cluster' (field) title and even more detail is available through free } \\
\text { registration (more resource available when registering as researcher (publications) or } \\
\text { pending (doctoral student) } \\
\text { Maximum level of detail is free for contributing researchers in order to protect } \\
\text { communities but available to external observers for symbolic fee: all suggestions for } \\
\text { improvements and better sharing welcome. } \\
\text { We are particularly open to provide and support experimental appropriation by } \\
\text { doctoral schools to build and study the past and future behavior of clusters in Earth } \\
\text { sciences, Cosmology, Water, Health, Computation, Energy/Batteries, Climate models, } \\
\text { Space, etc.. }\end{array}$} \\
\hline & & \\
\hline
\end{tabular}




\section{The Ecosystem for Research> Use Case 34: Graph Search on Scientific Data}

\begin{tabular}{|c|c|c|}
\hline Use Case Title & \multicolumn{2}{|c|}{$\begin{array}{l}\text { Enabling Face-Book like Semantic Graph-search on Scientific Chemical and Text- } \\
\text { based Data }\end{array}$} \\
\hline Vertical (area) & \multicolumn{2}{|c|}{ Management of Information from Research Articles } \\
\hline Author/Company/Email & \multicolumn{2}{|c|}{ Talapady Bhat, bhat@nist.gov } \\
\hline $\begin{array}{r}\text { Actors/Stakeholders } \\
\text { and their roles and } \\
\text { responsibilities }\end{array}$ & \multicolumn{2}{|c|}{$\begin{array}{l}\text { Chemical structures, Protein Data Bank, Material Genome Project, Open-GOV } \\
\text { initiative, Semantic Web, Integrated Data-graphs, Scientific social media }\end{array}$} \\
\hline Goals & \multicolumn{2}{|c|}{$\begin{array}{l}\text { Establish infrastructure, terminology and semantic data-graphs to annotate and } \\
\text { present technology information using 'root' and rule-based methods used primarily } \\
\text { by some Indo-European languages like Sanskrit and Latin. }\end{array}$} \\
\hline Use Case Description & \multicolumn{2}{|c|}{$\begin{array}{l}\text { - Social media hype } \\
\text { Internet and social media play a significant role in modern information } \\
\text { exchange. Every day most of us use social-media both to distribute and } \\
\text { receive information. Two of the special features of many social media like } \\
\text { Face-Book are } \\
\text { - the community is both data-providers and data-users } \\
\text { - They store information in a pre-defined 'data-shelf' of a data-graph } \\
\text { language free } \\
\text { What this has to do with managing scientific information? } \\
\text { activity involving every country and almost every household. We routinely 'tune- } \\
\text { in' to Internet resources to share and seek scientific information. } \\
\text { What are the challenges in creating social media for science } \\
\text { Creating a social media of scientific information needs an infrastructure } \\
\text { where many scientists from various parts of the world can participate and } \\
\text { deposit results of their experiment. Some of the issues that one has to } \\
\text { resolve prior to establishing a scientific social media are: } \\
\text { - How to minimize challenges related to local language and its grammar? } \\
\text { - How to determining the 'data-graph' to place an information in an } \\
\quad \text { intuitive way without knowing too much about the data management? } \\
\text { - How to find relevant scientific data without spending too much time on } \\
\text { the Internet? } \\
\text { Approach: Most languages and more so Sanskrit and Latin use a novel 'root'-based } \\
\text { method to facilitate the creation of on-demand, discriminating words to define } \\
\text { concepts. Some such examples from English are Bio-logy, Bio-chemistry. Youga, Yogi, } \\
\text { Yogendra, Yogesh are examples from Sanskrit. Genocide is an example from Latin. } \\
\text { These words are created on-demand based on best-practice terms and their } \\
\text { capability to serve as node in a discriminating data-graph with self-explained } \\
\text { meaning. }\end{array}$} \\
\hline \multirow{4}{*}{$\begin{array}{r}\text { Current } \\
\text { Solutions }\end{array}$} & Compute(System) & Cloud for the participation of community \\
\hline & Storage & $\begin{array}{l}\text { Requires expandable on-demand based resource that is } \\
\text { suitable for global users location and requirements }\end{array}$ \\
\hline & Networking & Needs good network for the community participation \\
\hline & Software & $\begin{array}{l}\text { Good database tools and servers for data-graph } \\
\text { manipulation are needed }\end{array}$ \\
\hline \multirow[t]{2}{*}{$\begin{array}{r}\text { Big Data } \\
\text { Characteristics }\end{array}$} & $\begin{array}{r}\text { Data Source } \\
\text { (distributed/centralized) }\end{array}$ & $\begin{array}{l}\text { Distributed resource with a limited centralized } \\
\text { capability }\end{array}$ \\
\hline & Volume (size) & Undetermined. May be few terabytes at the beginning \\
\hline
\end{tabular}




\section{The Ecosystem for Research> Use Case 34: Graph Search on Scientific Data}

\begin{tabular}{|c|c|c|}
\hline & $\begin{array}{r}\text { Velocity } \\
\text { (e.g. real time) }\end{array}$ & Evolving with time to accommodate new best-practices \\
\hline & $\begin{array}{r}\text { Variety } \\
\text { (multiple datasets, } \\
\text { mashup) }\end{array}$ & $\begin{array}{l}\text { Wildly varying depending on the types available } \\
\text { technological information }\end{array}$ \\
\hline & $\begin{array}{r}\text { Variability (rate of } \\
\text { change) }\end{array}$ & $\begin{array}{l}\text { Data-graphs are likely to change in time based on } \\
\text { customer preferences and best-practices }\end{array}$ \\
\hline \multirow{5}{*}{$\begin{array}{r}\text { Big Data Science } \\
\text { (collection, curation, } \\
\text { analysis, } \\
\text { action) }\end{array}$} & $\begin{array}{r}\text { Veracity (Robustness } \\
\text { Issues) }\end{array}$ & $\begin{array}{l}\text { Technological information is likely to be stable and } \\
\text { robust }\end{array}$ \\
\hline & Visualization & Efficient data-graph based visualization is needed \\
\hline & Data Quality & Expected to be good \\
\hline & Data Types & $\begin{array}{l}\text { All data types, image to text, structures to protein } \\
\text { sequence }\end{array}$ \\
\hline & Data Analytics & \\
\hline & \multicolumn{2}{|c|}{$\begin{array}{l}\text { This is a community effort similar to many social media. Providing a robust, scalable, } \\
\text { on-demand infrastructures in a manner that is use-case and user-friendly is a real } \\
\text { challenge by any existing conventional methods }\end{array}$} \\
\hline & \multicolumn{2}{|c|}{$\begin{array}{l}\text { A community access is required for the data and thus it has to be media and location } \\
\text { independent and thus requires high mobility too. }\end{array}$} \\
\hline & \multicolumn{2}{|c|}{$\begin{array}{l}\text { None since the effort is initially focused on publicly accessible data provided by } \\
\text { open-platform projects like open-gov, MGI and protein data bank. }\end{array}$} \\
\hline $\begin{array}{r}\text { Highlight issues for } \\
\text { generalizing this use } \\
\text { case (e.g. for ref. } \\
\text { architecture) }\end{array}$ & \multicolumn{2}{|c|}{$\begin{array}{l}\text { This effort includes many local and networked resources. Developing an } \\
\text { infrastructure to automatically integrate information from all these resources using } \\
\text { data-graphs is a challenge that we are trying to solve. }\end{array}$} \\
\hline $\begin{array}{r}\text { More Information } \\
\text { (URLs) }\end{array}$ & $\begin{array}{l}\text { http://www.eurekalert.org/ } \\
\text { http://xpdb.nist.gov/chemb } \\
\text { http://xpdb.nist.gov/chemb }\end{array}$ & $\begin{array}{l}\text { pub releases/2013-07/aiop-ffm071813.php } \\
\text { last/pdb.pl } \\
\text { last/pdb.pl }\end{array}$ \\
\hline \multicolumn{3}{|c|}{$\begin{array}{l}\text { Note: Many reports, including a recent one on Material Genome Project finds that exclusive top-down solutions } \\
\text { to facilitate data sharing and integration are not desirable for federated multi-disciplinary efforts. However, a } \\
\text { bottom-up approach can be chaotic. For this reason, there is need for a balanced blend of the two approaches } \\
\text { to support easy-to-use techniques to metadata creation, integration and sharing. This challenge is very similar } \\
\text { to the challenge faced by language developer at the beginning. One of the successful effort used by many } \\
\text { prominent languages is that of 'roots' and rules that form the framework for creating on-demand words for } \\
\text { communication. In this approach a top-down method is used to establish a limited number of highly re-usable } \\
\text { words called 'roots' by surveying the existing best practices in building terminology. These 'roots' are combined } \\
\text { using few 'rules' to create terms on-demand by a bottom-up step. } \\
\text { Y(uj) (join), O (creator, God, brain), Ga (motion, initiation) -leads to 'Yoga' in Sanskrit, English } \\
\text { Geno (genos)-cide-race based killing - Latin, English } \\
\text { Bio-technology -English, Latin } \\
\text { Red-light, red-laser-light -English. } \\
\text { A press release by the American Institute of Physics on this approach is at } \\
\text { http://www.eurekalert.org/pub releases/2013-07/aiop-ffm071813.php }\end{array}$} \\
\hline \multicolumn{3}{|c|}{$\begin{array}{l}\text { Our efforts to develop automated and rule and root-based methods (Chem-BLAST -. } \\
\text { http://xpdb.nist.gov/chemblast/pdb.pl) to identify and use best-practice, discriminating terms in generating } \\
\text { semantic data-graphs for science started almost a decade back with a chemical structure database. This } \\
\text { database has millions of structures obtained from the Protein Data Bank and the PubChem used world-wide. } \\
\text { Subsequently we extended our efforts to build root-based terms to text-based data of cell-images. In this work }\end{array}$} \\
\hline
\end{tabular}




\section{The Ecosystem for Research> Use Case 34: Graph Search on Scientific Data}

we use few simple rules to define and extend terms based on best-practice as decided by weaning through millions of popular use-cases chosen from over hundred biological ontologies.

Currently we are working on extending this method to publications of interest to Material Genome, Open-Gov and NIST-wide publication archive - NIKE. - http://xpdb.nist.gov/nike/term.pl. These efforts are a component of Research Data Alliance Working Group on Metadata https://www.rd-alliance.org/filedepot download/694/160 and https://rd-alliance.org/poster-session-rda-2nd-plenary-meeting.html 


\section{The Ecosystem for Research> Use Case 35: Light Source Beamlines}

\begin{tabular}{|c|c|c|}
\hline Use Case Title & \multicolumn{2}{|l|}{ Light source beamlines } \\
\hline Vertical (area) & \multicolumn{2}{|c|}{ Research (Biology, Chemistry, Geophysics, Materials Science, others) } \\
\hline Author/Company/Email & \multicolumn{2}{|c|}{ Eli Dart, LBNL (eddart@|bl.gov) } \\
\hline $\begin{array}{r}\text { Actors/Stakeholders } \\
\text { and their roles and } \\
\text { responsibilities }\end{array}$ & \multicolumn{2}{|c|}{ Research groups from a variety of scientific disciplines (see above) } \\
\hline Goals & \multicolumn{2}{|c|}{$\begin{array}{l}\text { Use of a variety of experimental techniques to determine structure, composition, } \\
\text { behavior, or other attributes of a sample relevant to scientific enquiry. }\end{array}$} \\
\hline Use Case Description & \multicolumn{2}{|c|}{$\begin{array}{l}\text { Samples are exposed to X-rays in a variety of configurations depending on the } \\
\text { experiment. Detectors (essentially high-speed digital cameras) collect the data. The data } \\
\text { are then analyzed to reconstruct a view of the sample or process being studied. The } \\
\text { reconstructed images are used by scientist's analyses. }\end{array}$} \\
\hline \multirow[t]{4}{*}{$\begin{array}{r}\text { Current } \\
\text { Solutions }\end{array}$} & Compute(System) & $\begin{array}{l}\text { Computation ranges from single analysis hosts to high- } \\
\text { throughput computing systems at computational facilities }\end{array}$ \\
\hline & Storage & $\begin{array}{l}\text { Local storage on the order of 1-40TB on Windows or Linux } \\
\text { data servers at facility for temporary storage, over 60TB on } \\
\text { disk at NERSC, over 300TB on tape at NERSC }\end{array}$ \\
\hline & Networking & 10Gbps Ethernet at facility, 100Gbps to NERSC \\
\hline & Software & $\begin{array}{l}\text { A variety of commercial and open source software is used } \\
\text { for data analysis - examples include: } \\
\text { - Octopus ( }(\mathrm{http}: / / \text { www.inct.be/en/software/octopus) } \\
\text { for Tomographic Reconstruction } \\
\text { - Avizo (http://vsg3d.com) and FIJI (a distribution of } \\
\text { ImageJ; http://fiji.sc) for Visualization and Analysis } \\
\text { Data transfer is accomplished using physical transport of } \\
\text { portable media (severely limits performance) or using high- } \\
\text { performance GridFTP, managed by Globus Online or } \\
\text { workflow systems such as SPADE. }\end{array}$ \\
\hline \multirow[t]{5}{*}{$\begin{array}{r}\text { Big Data } \\
\text { Characteristics }\end{array}$} & $\begin{array}{r}\text { Data Source } \\
\text { (distributed/centralized) }\end{array}$ & $\begin{array}{l}\text { Centralized (high resolution camera at facility). Multiple } \\
\text { beamlines per facility with high-speed detectors. }\end{array}$ \\
\hline & Volume (size) & 3GB to $30 \mathrm{~GB}$ per sample - up to 15 samples/day \\
\hline & $\begin{array}{r}\text { Velocity } \\
\text { (e.g. real time) }\end{array}$ & $\begin{array}{l}\text { Near real-time analysis needed for verifying experimental } \\
\text { parameters (lower resolution OK). Automation of analysis } \\
\text { would dramatically improve scientific productivity. }\end{array}$ \\
\hline & $\begin{array}{r}\text { Variety } \\
\text { (multiple datasets, } \\
\text { mashup) }\end{array}$ & $\begin{array}{l}\text { Many detectors produce similar types of data (e.g. TIFF } \\
\text { files), but experimental context varies widely }\end{array}$ \\
\hline & $\begin{array}{r}\text { Variability (rate of } \\
\text { change) }\end{array}$ & $\begin{array}{l}\text { Detector capabilities are increasing rapidly. Growth is } \\
\text { essentially Moore's Law. Detector area is increasing } \\
\text { exponentially }(1 \mathrm{k} \times 1 \mathrm{k}, 2 \mathrm{k} \times 2 \mathrm{k}, 4 \mathrm{k} \times 4 \mathrm{k}, \ldots) \text { and readout is } \\
\text { increasing exponentially }(1 \mathrm{~Hz}, 10 \mathrm{~Hz}, 100 \mathrm{~Hz}, 1 \mathrm{kHz}, \ldots) \text {. } \\
\text { Single detector data rates are expected to reach } 1 \mathrm{~GB} \text { per } \\
\text { second within } 2 \text { years. }\end{array}$ \\
\hline $\begin{array}{r}\text { Big Data Science } \\
\text { (collection, curation, } \\
\text { analysis, } \\
\text { action) }\end{array}$ & $\begin{array}{r}\text { Veracity (Robustness } \\
\text { Issues) }\end{array}$ & $\begin{array}{l}\text { Near real-time analysis required to verify experimental } \\
\text { parameters. In many cases, early analysis can dramatically } \\
\text { improve experiment productivity by providing early } \\
\text { feedback. This implies high-throughput computing, high- } \\
\text { performance data transfer, and high-speed storage are } \\
\text { routinely available. }\end{array}$ \\
\hline
\end{tabular}




\section{The Ecosystem for Research> Use Case 35: Light Source Beamlines}

\begin{tabular}{|c|c|c|}
\hline & Visualization & $\begin{array}{l}\text { Visualization is key to a wide variety of experiments at all } \\
\text { light source facilities }\end{array}$ \\
\hline & Data Quality & $\begin{array}{l}\text { Data quality and precision are critical (especially since } \\
\text { beam time is scarce, and re-running an experiment is often } \\
\text { impossible). }\end{array}$ \\
\hline & Data Types & Many beamlines generate image data (e.g., TIFF files) \\
\hline & Data Analytics & Volume reconstruction, feature identification, others \\
\hline $\begin{array}{r}\text { Big Data Specific } \\
\text { Challenges (Gaps) }\end{array}$ & \multicolumn{2}{|c|}{$\begin{array}{l}\text { Rapid increase in camera capabilities, need for automation of data transfer and near- } \\
\text { real-time analysis. }\end{array}$} \\
\hline $\begin{array}{r}\text { Big Data Specific } \\
\text { Challenges in Mobility }\end{array}$ & \multicolumn{2}{|c|}{$\begin{array}{l}\text { Data transfer to large-scale computing facilities is becoming necessary because of the } \\
\text { computational power required to conduct the analysis on time scales useful to the } \\
\text { experiment. Large number of beamlines (e.g., } 39 \text { at LBNL ALS) means that aggregate data } \\
\text { load is likely to increase significantly over the coming years. }\end{array}$} \\
\hline $\begin{array}{r}\text { Security and Privacy } \\
\text { Requirements }\end{array}$ & \multicolumn{2}{|c|}{ Varies with project. } \\
\hline $\begin{array}{r}\text { Highlight issues for } \\
\text { generalizing this use } \\
\text { case (e.g. for ref. } \\
\text { architecture) }\end{array}$ & \multicolumn{2}{|c|}{$\begin{array}{l}\text { There will be significant need for a generalized infrastructure for analyzing GBs per } \\
\text { second of data from many beamline detectors at multiple facilities. Prototypes exist now, } \\
\text { but routine deployment will require additional resources. }\end{array}$} \\
\hline $\begin{array}{r}\text { More Information } \\
\text { (URLs) }\end{array}$ & \multicolumn{2}{|c|}{$\begin{array}{l}\text { http://www-als.lbl.gov/ } \\
\text { http://www.aps.anl.gov/ } \\
\text { https://portal.slac.stanford.edu/sites/Icls public/Pages/Default.aspx }\end{array}$} \\
\hline
\end{tabular}




\section{Astronomy and Physics> Use Case 36: Catalina Digital Sky Survey for Transients}

\begin{tabular}{|c|c|c|}
\hline Use Case Title & \multicolumn{2}{|c|}{ Catalina Real-Time Transient Survey (CRTS): a digital, panoramic, synoptic sky survey } \\
\hline Vertical (area) & \multicolumn{2}{|c|}{ Scientific Research: Astronomy } \\
\hline Author/Company/Email & \multicolumn{2}{|c|}{ S. G. Djorgovski / Caltech / george@astro.caltech.edu } \\
\hline & \multicolumn{2}{|c|}{$\begin{array}{l}\text { The survey team: data processing, quality control, analysis and interpretation, } \\
\text { publishing, and archiving. } \\
\text { Collaborators: a number of research groups world-wide: further work on data } \\
\text { analysis and interpretation, follow-up observations, and publishing. } \\
\text { User community: all of the above, plus the astronomical community world-wide: } \\
\text { further work on data analysis and interpretation, follow-up observations, and } \\
\text { publishing. }\end{array}$} \\
\hline Goals & \multicolumn{2}{|c|}{$\begin{array}{l}\text { The survey explores the variable universe in the visible light regime, on time scales } \\
\text { ranging from minutes to years, by searching for variable and transient sources. It } \\
\text { discovers a broad variety of astrophysical objects and phenomena, including various } \\
\text { types of cosmic explosions (e.g., Supernovae), variable stars, phenomena associated } \\
\text { with accretion to massive black holes (active galactic nuclei) and their relativistic jets, } \\
\text { high proper motion stars, etc. }\end{array}$} \\
\hline Use Case Description & \multicolumn{2}{|c|}{$\begin{array}{l}\text { The data are collected from } 3 \text { telescopes ( } 2 \text { in Arizona and } 1 \text { in Australia), with } \\
\text { additional ones expected in the near future (in Chile). The original motivation is a } \\
\text { search for near-Earth (NEO) and potential planetary hazard (PHO) asteroids, funded } \\
\text { by NASA, and conducted by a group at the Lunar and Planetary Laboratory (LPL) at } \\
\text { the Univ. of Arizona (UA); that is the Catalina Sky Survey proper (CSS). The data } \\
\text { stream is shared by the CRTS for the purposes for exploration of the variable } \\
\text { universe, beyond the Solar system, led by the Caltech group. Approximately } 83 \% \text { of } \\
\text { the entire sky is being surveyed through multiple passes (crowded regions near the } \\
\text { Galactic plane, and small areas near the celestial poles are excluded). } \\
\text { The data are preprocessed at the telescope, and transferred to LPL/UA, and hence to } \\
\text { Caltech, for further analysis, distribution, and archiving. The data are processed in } \\
\text { real time, and detected transient events are published electronically through a } \\
\text { variety of dissemination mechanisms, with no proprietary period (CRTS has a } \\
\text { completely open data policy). } \\
\text { Further data analysis includes automated and semi-automated classification of the } \\
\text { detected transient events, additional observations using other telescopes, scientific } \\
\text { interpretation, and publishing. In this process, it makes a heavy use of the archival } \\
\text { data from a wide variety of geographically distributed resources connected through } \\
\text { the Virtual Observatory (VO) framework. } \\
\text { Light curves (flux histories) are accumulated for } \approx 500 \text { million sources detected in the } \\
\text { survey, each with a few hundred data points on average, spanning up to } 8 \text { years, and } \\
\text { growing. These are served to the community from the archives at Caltech, and } \\
\text { shortly from IUCAA, India. This is an unprecedented dataset for the exploration of } \\
\text { time domain in astronomy, in terms of the temporal and area coverage and depth. } \\
\text { CRTS is a scientific and methodological testbed and precursor of the grander surveys } \\
\text { to come, notably the Large Synoptic Survey Telescope (LSST), expected to operate in } \\
\text { 2020's. }\end{array}$} \\
\hline & Compute(System) & $\begin{array}{l}\text { Instrument and data processing computers: a number of } \\
\text { desktop and small server class machines, although more } \\
\text { powerful machinery is needed for some data analysis } \\
\text { tasks. } \\
\text { This is not so much a computationally-intensive project, } \\
\text { but rather a data-handling-intensive one. }\end{array}$ \\
\hline
\end{tabular}




\section{Astronomy and Physics> Use Case 36: Catalina Digital Sky Survey for Transients}

\begin{tabular}{|c|c|c|}
\hline & Storage & Several multi-TB / tens of TB servers. \\
\hline & Networking & Standard inter-university Internet connections. \\
\hline & Software & $\begin{array}{l}\text { Custom data processing pipeline and data analysis } \\
\text { software, operating under Linux. Some archives on } \\
\text { Windows machines, running a MS SQL server databases. }\end{array}$ \\
\hline $\begin{array}{r}\text { Big Data } \\
\text { Characteristics }\end{array}$ & $\begin{array}{r}\text { Data Source } \\
\text { (distributed/centralized) }\end{array}$ & 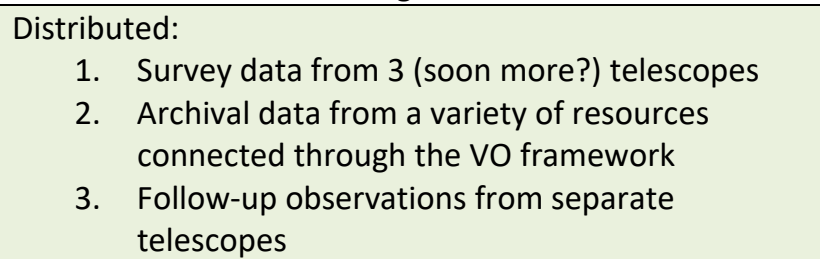 \\
\hline & Volume (size) & $\begin{array}{l}\text { The survey generates up to } \approx 0.1 \text { TB per clear night; } \approx \\
100 \text { TB in current data holdings. Follow-up observational } \\
\text { data amount to no more than a few \% of that. } \\
\text { Archival data in external (VO-connected) archives are in } \\
\text { PBs, but only a minor fraction is used. }\end{array}$ \\
\hline & $\begin{array}{r}\text { Velocity } \\
\text { (e.g. real time) }\end{array}$ & Up to $\approx 0.1$ TB / night of the raw survey data. \\
\hline & $\begin{array}{r}\text { Variety } \\
\text { (multiple datasets, } \\
\text { mashup) }\end{array}$ & $\begin{array}{l}\text { The primary survey data in the form of images, } \\
\text { processed to catalogs of sources (db tables), and time } \\
\text { series for individual objects (light curves). } \\
\text { Follow-up observations consist of images and spectra. } \\
\text { Archival data from the VO data grid include all of the } \\
\text { above, from a wide variety of sources and different } \\
\text { wavelengths. }\end{array}$ \\
\hline & $\begin{array}{r}\text { Variability (rate of } \\
\text { change) }\end{array}$ & $\begin{array}{l}\text { Daily data traffic fluctuates from } \approx 0.01 \text { to } \approx 0.1 \mathrm{~TB} / \text { day, } \\
\text { not including major data transfers between the principal } \\
\text { archives (Caltech, UA, and IUCAA). }\end{array}$ \\
\hline $\begin{array}{r}\text { Big Data Science } \\
\text { (collection, curation, } \\
\text { analysis, }\end{array}$ & $\begin{array}{r}\text { Veracity (Robustness } \\
\text { Issues, semantics) }\end{array}$ & $\begin{array}{l}\text { A variety of automated and human inspection quality } \\
\text { control mechanisms is implemented at all stages of the } \\
\text { process. }\end{array}$ \\
\hline action) & Visualization & $\begin{array}{l}\text { Standard image display and data plotting packages are } \\
\text { used. We are exploring visualization mechanisms for } \\
\text { highly dimensional data parameter spaces. }\end{array}$ \\
\hline & Data Quality (syntax) & $\begin{array}{l}\text { It varies, depending on the observing conditions, and it } \\
\text { is evaluated automatically: error bars are estimated for } \\
\text { all relevant quantities. }\end{array}$ \\
\hline & Data Types & Images, spectra, time series, catalogs. \\
\hline & Data Analytics & $\begin{array}{l}\text { A wide variety of the existing astronomical data analysis } \\
\text { tools, plus a large amount of custom developed tools } \\
\text { and software, some of it a research project in itself. }\end{array}$ \\
\hline $\begin{array}{r}\text { Big Data Specific } \\
\text { Challenges (Gaps) }\end{array}$ & \multicolumn{2}{|c|}{$\begin{array}{l}\text { Development of machine learning tools for data exploration, and in particular for an } \\
\text { automated, real-time classification of transient events, given the data sparsity and } \\
\text { heterogeneity. } \\
\text { Effective visualization of hyper-dimensional parameter spaces is a major challenge } \\
\text { for all of us. }\end{array}$} \\
\hline $\begin{array}{r}\text { Big Data Specific } \\
\text { Challenges in Mobility }\end{array}$ & \multicolumn{2}{|c|}{ Not a significant limitation at this time. } \\
\hline
\end{tabular}




\section{Astronomy and Physics> Use Case 36: Catalina Digital Sky Survey for Transients}

\begin{tabular}{|c|c|}
\hline $\begin{array}{r}\text { Security and Privacy } \\
\text { Requirements }\end{array}$ & None. \\
\hline $\begin{array}{r}\text { Highlight issues for } \\
\text { generalizing this use } \\
\text { case (e.g. for ref. } \\
\text { architecture) }\end{array}$ & $\begin{array}{l}\text { - Real-time processing and analysis of massive data streams from a distributed } \\
\text { sensor network (in this case telescopes), with a need to identify, characterize, } \\
\text { and respond to the transient events of interest in (near) real time. } \\
\text { Use of highly distributed archival data resources (in this case VO-connected } \\
\text { archives) for data analysis and interpretation. } \\
\text { - Automated classification given the very sparse and heterogeneous data, } \\
\text { dynamically evolving in time as more data come in, and follow-up decision } \\
\text { making given limited and sparse resources (in this case follow-up observations } \\
\text { with other telescopes). }\end{array}$ \\
\hline $\begin{array}{r}\text { More Information } \\
\text { (URLs) }\end{array}$ & $\begin{array}{l}\text { CRTS survey: } \underline{\text { http://crts.caltech.edu }} \\
\text { CSS survey: } \underline{\text { http://www.Ipl.arizona.edu/css }} \\
\text { For an overview of the classification challenges, see, e.g., } \\
\text { http://arxiv.org/abs/1209.1681 } \\
\text { For a broader context of sky surveys, past, present, and future, see, e.g., the review } \\
\text { http://arxiv.org/abs/1209.1681 }\end{array}$ \\
\hline \multicolumn{2}{|c|}{$\begin{array}{l}\text { Note: CRTS can be seen as a good precursor to the astronomy's flagship project, the Large Synoptic Sky Survey } \\
\text { (LSST; http://www.Isst.org), now under development. Their anticipated data rates ( } \approx 20 \text { TB to } 30 \text { TB per clear } \\
\text { night, tens of PB over the duration of the survey) are directly on the Moore's law scaling from the current CRTS } \\
\text { data rates and volumes, and many technical and methodological issues are very similar. } \\
\text { It is also a good case for real-time data mining and knowledge discovery in massive data streams, with } \\
\text { distributed data sources and computational resources. }\end{array}$} \\
\hline
\end{tabular}

See Figure 5: Catalina CRTS: A Digital, Panoramic, Synoptic Sky Survey

The figure shows one possible schematic architecture for a cyber-infrastructure for time domain astronomy. Transient event data streams are produced by survey pipelines from the telescopes on the ground or in space, and the events with their observational descriptions are ingested by one or more depositories, from which they can be disseminated electronically to human astronomers or robotic telescopes. Each event is assigned an evolving portfolio of information, which would include all of the available data on that celestial position, from a wide variety of data archives unified under the Virtual Observatory framework, expert annotations, etc. Representations of such federated information can be both human-readable and machine-readable. They are fed into one or more automated event characterization, classification, and prioritization engines that deploy a variety of machine learning tools for these tasks. Their output, which evolves dynamically as new information arrives and is processed, informs the follow-up observations of the selected events, and the resulting data are communicated back to the event portfolios, for the next iteration. Users (human or robotic) can tap into the system at multiple points, both for an information retrieval, and to contribute new information, through a standardized set of formats and protocols. This could be done in a (near) real time, or in an archival (not time critical) modes. 


\section{Astronomy and Physics> Use Case 37: Cosmological Sky Survey and Simulations}

\begin{tabular}{|c|c|c|}
\hline Use Case Title & \multicolumn{2}{|c|}{ DOE Extreme Data from Cosmological Sky Survey and Simulations } \\
\hline Vertical (area) & \multicolumn{2}{|c|}{ Scientific Research: Astrophysics } \\
\hline Author/Company/Email & \multicolumn{2}{|c|}{$\begin{array}{l}\text { Pls: Salman Habib, Argonne National Laboratory; Andrew Connolly, University of } \\
\text { Washington }\end{array}$} \\
\hline $\begin{array}{r}\text { Actors/Stakeholders } \\
\text { and their roles and } \\
\text { responsibilities }\end{array}$ & \multicolumn{2}{|c|}{$\begin{array}{l}\text { Researchers studying dark matter, dark energy, and the structure of the early } \\
\text { universe. }\end{array}$} \\
\hline Goals & \multicolumn{2}{|c|}{$\begin{array}{l}\text { Clarify the nature of dark matter, dark energy, and inflation, some of the most exciting, } \\
\text { perplexing, and challenging questions facing modern physics. Emerging, unanticipated } \\
\text { measurements are pointing toward a need for physics beyond the successful Standard } \\
\text { Model of particle physics. }\end{array}$} \\
\hline Use Case Description & \multicolumn{2}{|c|}{$\begin{array}{l}\text { This investigation requires an intimate interplay between Big Data from experiment } \\
\text { and simulation as well as massive computation. The melding of all will } \\
\text { 1) Provide the direct means for cosmological discoveries that require a strong } \\
\text { connection between theory and observations ('precision cosmology'); } \\
\text { 2) Create an essential 'tool of discovery' in dealing with large datasets generated by } \\
\text { complex instruments; and, } \\
\text { 3) Generate and share results from high-fidelity simulations that are necessary to } \\
\text { understand and control systematics, especially astrophysical systematics. }\end{array}$} \\
\hline \multirow[t]{4}{*}{$\begin{array}{r}\text { Current } \\
\text { Solutions }\end{array}$} & Compute(System) & $\begin{array}{l}\text { Hours: 24M (NERSC / Berkeley Lab), 190M (ALCF / } \\
\text { Argonne), 10M (OLCF / Oak Ridge) } \\
\end{array}$ \\
\hline & Storage & 180 TB (NERSC / Berkeley Lab) \\
\hline & Networking & $\begin{array}{l}\text { ESNet connectivity to the national labs is adequate } \\
\text { today. }\end{array}$ \\
\hline & Software & $\begin{array}{l}\text { MPI, OpenMP, C, C++, F90, FFTW, viz packages, python, } \\
\text { FFTW, numpy, Boost, OpenMP, ScaLAPCK, PSQL and } \\
\text { MySQL databases, Eigen, cfitsio, astrometry.net, and } \\
\text { Minuit2 }\end{array}$ \\
\hline \multirow[t]{5}{*}{$\begin{array}{r}\text { Big Data } \\
\text { Characteristics }\end{array}$} & $\begin{array}{r}\text { Data Source } \\
\text { (distributed/centralized) }\end{array}$ & $\begin{array}{l}\text { Observational data will be generated by the Dark Energy } \\
\text { Survey (DES) and the Zwicky Transient Factory in } 2015 \\
\text { and by the Large Synoptic Sky Survey starting in } 2019 . \\
\text { Simulated data will generated at DOE supercomputing } \\
\text { centers. }\end{array}$ \\
\hline & Volume (size) & $\begin{array}{l}\text { DES: } 4 \text { PB, ZTF } 1 \text { PB/year, LSST } 7 \text { PB/year, Simulations > } \\
10 \text { PB in } 2017\end{array}$ \\
\hline & $\begin{array}{r}\text { Velocity } \\
\text { (e.g. real time) }\end{array}$ & LSST: 20 TB/day \\
\hline & $\begin{array}{r}\text { Variety } \\
\text { (multiple datasets, } \\
\text { mashup) } \\
\end{array}$ & $\begin{array}{l}\text { 1) Raw Data from sky surveys 2) Processed Image data } \\
\text { 3) Simulation data }\end{array}$ \\
\hline & $\begin{array}{r}\text { Variability (rate of } \\
\text { change) }\end{array}$ & $\begin{array}{l}\text { Observations are taken nightly; supporting simulations } \\
\text { are run throughout the year, but data can be produced } \\
\text { sporadically depending on access to resources }\end{array}$ \\
\hline \multirow[t]{2}{*}{$\begin{array}{r}\text { Big Data Science } \\
\text { (collection, curation, } \\
\text { analysis, } \\
\text { action) } \\
\end{array}$} & $\begin{array}{r}\text { Veracity (Robustness } \\
\text { Issues) }\end{array}$ & \\
\hline & Visualization & $\begin{array}{l}\text { Interpretation of results from detailed simulations } \\
\text { requires advanced analysis and visualization techniques }\end{array}$ \\
\hline
\end{tabular}




\section{Astronomy and Physics> Use Case 37: Cosmological Sky Survey and Simulations}

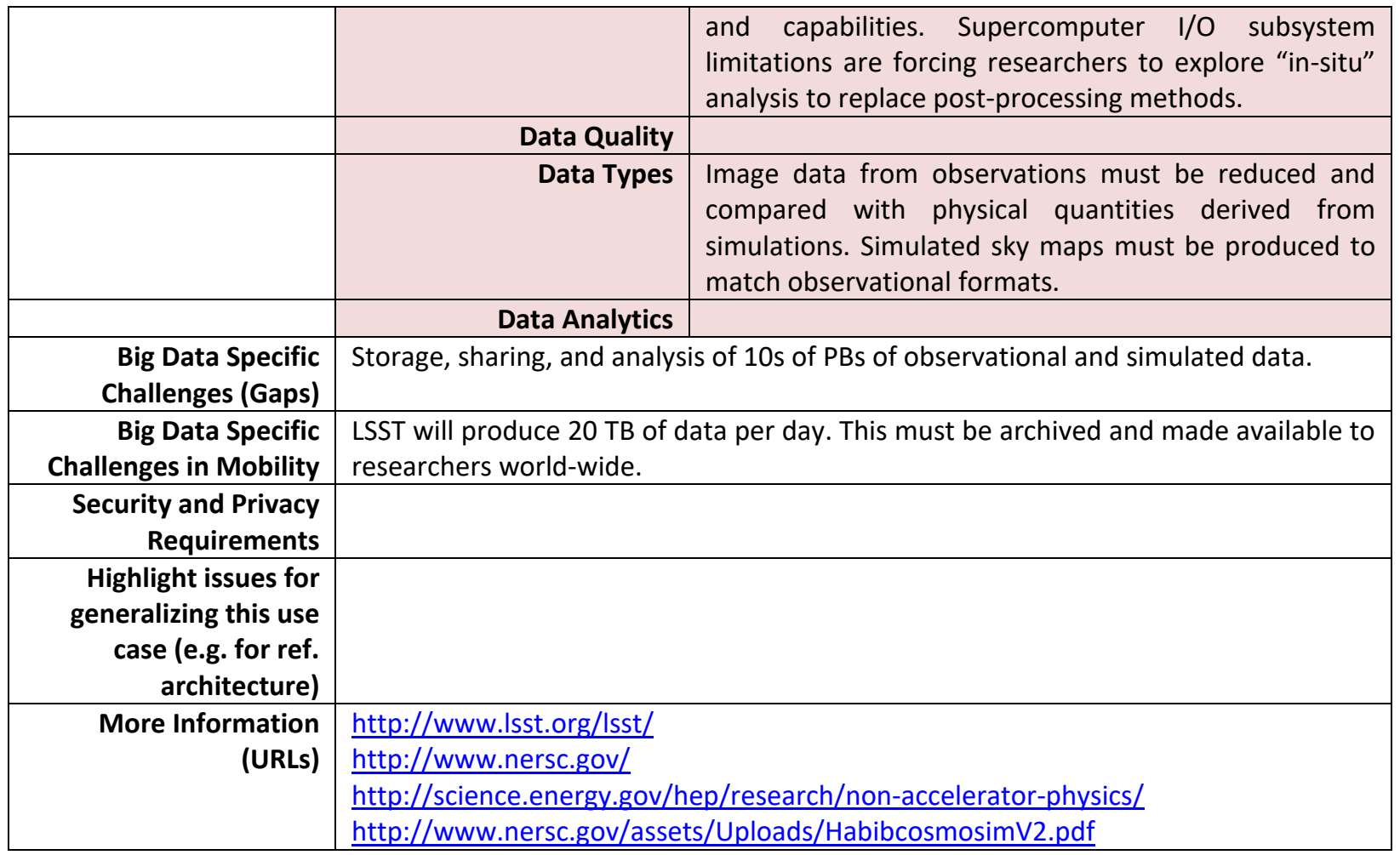




\section{Astronomy and Physics> Use Case 38: Large Survey Data for Cosmology}

\begin{tabular}{|c|c|c|}
\hline Use Case Title & \multicolumn{2}{|c|}{ Large Survey Data for Cosmology } \\
\hline Vertical (area) & \multicolumn{2}{|c|}{ Scientific Research: Cosmic Frontier } \\
\hline Author/Company/Email & \multicolumn{2}{|c|}{ Peter Nugent / LBNL / penugent@lbl.gov } \\
\hline $\begin{array}{r}\text { Actors/Stakeholders } \\
\text { and their roles and } \\
\text { responsibilities }\end{array}$ & \multicolumn{2}{|c|}{$\begin{array}{l}\text { Dark Energy Survey, Dark Energy Spectroscopic Instrument, Large Synoptic Survey } \\
\text { Telescope. ANL, BNL, FNAL, LBNL and SLAC: Create the instruments/telescopes, run } \\
\text { the survey and perform the cosmological analysis. }\end{array}$} \\
\hline Goals & \multicolumn{2}{|c|}{$\begin{array}{l}\text { Provide a way to reduce photometric data in real time for supernova discovery and } \\
\text { follow-up and to handle the large volume of observational data (in conjunction with } \\
\text { simulation data) to reduce systematic uncertainties in the measurement of the } \\
\text { cosmological parameters via baryon acoustic oscillations, galaxy cluster counting and } \\
\text { weak lensing measurements. }\end{array}$} \\
\hline Use Case Description & \multicolumn{2}{|c|}{$\begin{array}{l}\text { For DES the data are sent from the mountaintop via a microwave link to La Serena, } \\
\text { Chile. From there, an optical link forwards them to the NCSA as well as NERSC for } \\
\text { storage and "reduction". Subtraction pipelines are run using extant imaging data to } \\
\text { find new optical transients through machine learning algorithms. Then galaxies and } \\
\text { stars in both the individual and stacked images are identified, catalogued, and finally } \\
\text { their properties measured and stored in a database. }\end{array}$} \\
\hline \multirow[t]{4}{*}{$\begin{array}{r}\text { Current } \\
\text { Solutions }\end{array}$} & Compute(System) & $\begin{array}{l}\text { Linux cluster, Oracle RDBMS server, large memory } \\
\text { machines, standard Linux interactive hosts. For } \\
\text { simulations, HPC resources. }\end{array}$ \\
\hline & Storage & $\begin{array}{l}\text { Oracle RDBMS, Postgres psql, as well as GPFS and Lustre } \\
\text { file systems and tape archives. }\end{array}$ \\
\hline & Networking & Provided by NERSC \\
\hline & Software & $\begin{array}{l}\text { Standard astrophysics reduction software as well as } \\
\text { Perl/Python wrapper scripts, Linux Cluster scheduling } \\
\text { and comparison to large amounts of simulation data via } \\
\text { techniques like Cholesky decomposition. }\end{array}$ \\
\hline \multirow[t]{5}{*}{$\begin{array}{r}\text { Big Data } \\
\text { Characteristics }\end{array}$} & $\begin{array}{r}\text { Data Source } \\
\text { (distributed/centralized) }\end{array}$ & $\begin{array}{l}\text { Distributed. Typically between observation and } \\
\text { simulation data. }\end{array}$ \\
\hline & Volume (size) & $\begin{array}{l}\text { LSST will generate } 60 \text { PB of imaging data and } 15 \text { PB of } \\
\text { catalog data and a correspondingly large (or larger) } \\
\text { amount of simulation data. Over } 20 \text { TB of data per night. }\end{array}$ \\
\hline & $\begin{array}{r}\text { Velocity } \\
\text { (e.g. real time) }\end{array}$ & $\begin{array}{l}\text { 20TB of data will have to be subtracted each night in as } \\
\text { near real time as possible in order to maximize the } \\
\text { science for supernovae. }\end{array}$ \\
\hline & $\begin{array}{r}\text { Variety } \\
\text { (multiple datasets, } \\
\text { mashup) }\end{array}$ & $\begin{array}{l}\text { While the imaging data is similar, the analysis for the } 4 \\
\text { different types of cosmological measurements and } \\
\text { comparisons to simulation data is quite different. }\end{array}$ \\
\hline & $\begin{array}{r}\text { Variability (rate of } \\
\text { change) }\end{array}$ & $\begin{array}{l}\text { Weather and sky conditions can radically change both } \\
\text { the quality and quantity of data. }\end{array}$ \\
\hline $\begin{array}{r}\text { Big Data Science } \\
\text { (collection, curation, } \\
\text { analysis, } \\
\text { action) }\end{array}$ & $\begin{array}{r}\text { Veracity (Robustness } \\
\text { Issues) }\end{array}$ & $\begin{array}{l}\text { Astrophysical data is a statistician's nightmare as the } \\
\text { both the uncertainties in a given measurement change } \\
\text { from night-to-night in addition to the cadence being } \\
\text { highly unpredictable. Also, most all of the cosmological } \\
\text { measurements are systematically limited, and thus } \\
\text { understanding these as best possible is the highest } \\
\text { priority for a given survey. }\end{array}$ \\
\hline
\end{tabular}




\section{Astronomy and Physics> Use Case 38: Large Survey Data for Cosmology}

\begin{tabular}{|c|c|c|}
\hline & Visualization & $\begin{array}{l}\text { Interactive speed of web UI on very large datasets is an } \\
\text { ongoing challenge. Basic querying and browsing of data } \\
\text { to find new transients as well as monitoring the quality } \\
\text { of the survey is a must. Ability to download large } \\
\text { amounts of data for offline analysis is another } \\
\text { requirement of the system. Ability to combine both } \\
\text { simulation and observational data is also necessary. }\end{array}$ \\
\hline & Data Quality & $\begin{array}{l}\text { Understanding the systematic uncertainties in the } \\
\text { observational data is a prerequisite to a successful } \\
\text { cosmological measurement. Beating down the } \\
\text { uncertainties in the simulation data to under this level is } \\
\text { a huge challenge for future surveys. }\end{array}$ \\
\hline & Data Types & Cf. above on "Variety" \\
\hline & Data Analytics & \\
\hline $\begin{array}{l}\text { Big Data Specific } \\
\text { Challenges (Gaps) }\end{array}$ & \multicolumn{2}{|c|}{$\begin{array}{l}\text { New statistical techniques for understanding the limitations in simulation data would } \\
\text { be beneficial. Often it is the case where there is not enough computing time to } \\
\text { generate all the simulations one wants and thus there is a reliance on emulators to } \\
\text { bridge the gaps. Techniques for handling Cholesky decomposition for thousands of } \\
\text { simulations with matrices of order } 1 \mathrm{M} \text { on a side. }\end{array}$} \\
\hline $\begin{array}{l}\text { Big Data Specific } \\
\text { Challenges in Mobility }\end{array}$ & \multicolumn{2}{|c|}{ Performing analysis on both the simulation and observational data simultaneously. } \\
\hline $\begin{array}{r}\text { Security and Privacy } \\
\text { Requirements }\end{array}$ & \multicolumn{2}{|c|}{ No special challenges. Data is either public or requires standard login with password. } \\
\hline $\begin{array}{r}\text { Highlight issues for } \\
\text { generalizing this use } \\
\text { case (e.g. for ref. } \\
\text { architecture) }\end{array}$ & \multicolumn{2}{|c|}{$\begin{array}{l}\text { Parallel databases which could handle imaging data would be an interesting avenue } \\
\text { for future research. }\end{array}$} \\
\hline $\begin{array}{r}\text { More Information } \\
\text { (URLs) }\end{array}$ & \multicolumn{2}{|c|}{ http://www.Isst.org/lsst, http://desi.Ibl.gov, and http://www.darkenergysurvey.org } \\
\hline
\end{tabular}




\section{Astronomy and Physics> Use Case 39: Analysis of LHC (Large Hadron Collider) Data}

\begin{tabular}{|c|c|c|}
\hline Use Case Title & \multicolumn{2}{|c|}{$\begin{array}{l}\text { Particle Physics: Analysis of LHC (Large Hadron Collider) Data (Discovery of Higgs } \\
\text { particle) }\end{array}$} \\
\hline Vertical (area) & \multicolumn{2}{|c|}{ Scientific Research: Physics } \\
\hline Author/Company/Emai & \multicolumn{2}{|c|}{$\begin{array}{l}\text { Michael Ernst mernst@bnl.gov, Lothar Bauerdick bauerdick@fnal.gov based on an } \\
\text { initial version written by Geoffrey Fox, Indiana University gcf@indiana.edu, Eli Dart, } \\
\text { LBNL eddart@|bl.gov, }\end{array}$} \\
\hline $\begin{array}{l}\text { Actors/Stakeholders } \\
\text { and their roles and } \\
\text { responsibilities }\end{array}$ & \multicolumn{2}{|c|}{$\begin{array}{l}\text { Physicists(Design and Identify need for Experiment, Analyze Data) Systems Staff } \\
\text { (Design, Build and Support distributed Computing Grid), Accelerator Physicists } \\
\text { (Design, Build and Run Accelerator), Government (funding based on long term } \\
\text { importance of discoveries in field)) }\end{array}$} \\
\hline Goals & \multicolumn{2}{|c|}{ Understanding properties of fundamental particles } \\
\hline Use Case Description & \multicolumn{2}{|c|}{$\begin{array}{l}\text { CERN LHC Detectors and Monte Carlo producing events describing particle-apparatus } \\
\text { interaction. Processed information defines physics properties of events (lists of } \\
\text { particles with type and momenta). These events are analyzed to find new effects; } \\
\text { both new particles (Higgs) and present evidence that conjectured particles } \\
\text { (Supersymmetry) not seen. }\end{array}$} \\
\hline \multirow[t]{3}{*}{$\begin{array}{r}\text { Current } \\
\text { Solutions }\end{array}$} & Compute(System) & $\begin{array}{l}\text { WLCG and Open Science Grid in the US integrate } \\
\text { computer centers worldwide that provide computing } \\
\text { and storage resources into a single infrastructure } \\
\text { accessible by all LHC physicists. } \\
\text { 350,000 cores running "continuously" arranged in } 3 \text { tiers } \\
\text { (CERN, "Continents/Countries". "Universities"). Uses } \\
\text { "Distributed High Throughput Computing (DHTC)"; } \\
\text { 200PB storage, >2million jobs/day. }\end{array}$ \\
\hline & Storage & $\begin{array}{ll}\text { ATLAS: } & \\
\text { - } & \text { Brookhaven National Laboratory Tier1 tape: } \\
& \text { 10PB ATLAS data on tape managed by HPSS } \\
\text { (incl. RHIC/NP the total data volume is 35PB) } \\
\text { - } \text { Brookhaven National Laboratory Tier1 disk: } \\
\text { 11PB; using dCache to virtualize a set of } \approx 60 \\
\text { heterogeneous storage servers with high- } \\
\text { density disk backend systems } \\
\text { - US Tier2 centers, disk cache: } 16 \mathrm{~PB} \\
\text { CMS: } \\
\text { - Fermilab US Tier1, reconstructed, tape/cache: } \\
\text { - } 20.4 \mathrm{~PB} \\
\text { - US Tier2 centers, disk cache: } 7 \mathrm{~PB} \\
\text { US Tier3 sites, disk cache: } 1.04 \mathrm{~PB}\end{array}$ \\
\hline & Networking & $\begin{array}{l}\text { - As experiments have global participants (CMS } \\
\text { has } 3600 \text { participants from } 183 \text { institutions in } \\
38 \text { countries), the data at all levels is } \\
\text { transported and accessed across continents. } \\
\text { - Large scale automated data transfers occur } \\
\text { over science networks across the globe. } \\
\text { LHCOPN and LHCONE network overlay provide } \\
\text { dedicated network allocations and traffic } \\
\text { isolation for LHC data traffic }\end{array}$ \\
\hline
\end{tabular}




\section{Astronomy and Physics> Use Case 39: Analysis of LHC (Large Hadron Collider) Data}

\begin{tabular}{|c|c|c|}
\hline & & $\begin{array}{l}\text { - ATLAS Tier1 data center at BNL has 160Gbps } \\
\text { internal paths (often fully loaded). 70Gbps WAN } \\
\text { connectivity provided by ESnet. } \\
\text { CMS Tier1 data center at FNAL has 90Gbps } \\
\text { WAN connectivity provided by ESnet } \\
\text { - Aggregate wide area network traffic for LHC } \\
\text { experiments is about 25Gbps steady state } \\
\text { worldwide }\end{array}$ \\
\hline & Software & $\begin{array}{l}\text { The scalable ATLAS workload/workflow management } \\
\text { system PanDA manages } \approx 1 \text { million production and user } \\
\text { analysis jobs on globally distributed computing resources } \\
\text { ( } \approx 100 \text { sites) per day. } \\
\text { The new ATLAS distributed data management system } \\
\text { Rucio is the core component keeping track of an } \\
\text { inventory of currently } \approx 130 P B \text { of data distributed across } \\
\text { grid resources and to orchestrate data movement } \\
\text { between sites. The data volume is expected to grow to } \\
\text { exascale size in the next few years. Based on the xrootd } \\
\text { system ATLAS has developed FAX, a federated storage } \\
\text { system that allows remote data access. } \\
\text { Similarly, CMS is using the OSG glideinWMS } \\
\text { infrastructure to manage its workflows for production } \\
\text { and data analysis the PhEDEx system to orchestrate data } \\
\text { movements, and the AAA/xrootd system to allow } \\
\text { remote data access. } \\
\text { Experiment-specific physics software including } \\
\text { simulation packages, data processing, advanced statistic } \\
\text { packages, etc. }\end{array}$ \\
\hline \multirow[t]{4}{*}{$\begin{array}{r}\text { Big Data } \\
\text { Characteristics }\end{array}$} & $\begin{array}{r}\text { Data Source } \\
\text { (distributed/centralized) }\end{array}$ & $\begin{array}{l}\text { High speed detectors produce large data volumes: } \\
\text { - ATLAS detector at CERN: Originally } 1 \mathrm{~PB} / \mathrm{sec} \text { raw } \\
\text { data rate, reduced to } 300 \mathrm{MB} / \mathrm{sec} \text { by multi-stage } \\
\text { trigger. } \\
\text { CMS detector at CERN: similar } \\
\text { Data distributed to Tier1 centers globally, which serve as } \\
\text { data sources for Tier2 and Tier3 analysis centers }\end{array}$ \\
\hline & Volume (size) & 15 Petabytes per year from Detectors and Analysis \\
\hline & $\begin{array}{r}\text { Velocity } \\
\text { (e.g. real time) }\end{array}$ & $\begin{array}{l}\text { - Real time with some long LHC "shut downs" (to } \\
\text { improve accelerator and detectors) with no } \\
\text { data except Monte Carlo. } \\
\text { - Besides using programmatically and } \\
\text { dynamically replicated datasets, real-time } \\
\text { remote I/O (using XrootD) is increasingly used } \\
\text { by analysis which requires reliable high- } \\
\text { performance networking capabilities to reduce } \\
\text { file copy and storage system overhead }\end{array}$ \\
\hline & Variety & $\begin{array}{l}\text { Lots of types of events with from 2- few hundred final } \\
\text { particle but all data is collection of particles after initial }\end{array}$ \\
\hline
\end{tabular}




\section{Astronomy and Physics> Use Case 39: Analysis of LHC (Large Hadron Collider) Data}

\begin{tabular}{|c|c|c|}
\hline & $\begin{array}{r}\text { (multiple datasets, } \\
\text { mashup) }\end{array}$ & $\begin{array}{l}\text { analysis. Events are grouped into datasets; real detector } \\
\text { data is segmented into } \approx 20 \text { datasets (with partial } \\
\text { overlap) on the basis of event characteristics determined } \\
\text { through real-time trigger system, while different } \\
\text { simulated datasets are characterized by the physics } \\
\text { process being simulated. }\end{array}$ \\
\hline & $\begin{array}{r}\text { Variability (rate of } \\
\text { change) }\end{array}$ & $\begin{array}{l}\text { Data accumulates and does not change character. What } \\
\text { you look for may change based on physics insight. As } \\
\text { understanding of detectors increases, large scale data } \\
\text { reprocessing tasks are undertaken. }\end{array}$ \\
\hline \multirow[t]{5}{*}{$\begin{array}{r}\text { Big Data Science } \\
\text { (collection, curation, } \\
\text { analysis, } \\
\text { action) }\end{array}$} & $\begin{array}{r}\text { Veracity (Robustness } \\
\text { Issues) }\end{array}$ & $\begin{array}{l}\text { One can lose modest amount of data without much pain } \\
\text { as errors proportional to } 1 / \text { SquareRoot(Events } \\
\text { gathered), but such data loss must be carefully } \\
\text { accounted. Importance that accelerator and } \\
\text { experimental apparatus work both well and in } \\
\text { understood fashion. Otherwise data too "dirty" / } \\
\text { "uncorrectable". }\end{array}$ \\
\hline & Visualization & $\begin{array}{l}\text { Modest use of visualization outside histograms and } \\
\text { model fits. Nice event displays but discovery requires } \\
\text { lots of events so this type of visualization of secondary } \\
\text { importance }\end{array}$ \\
\hline & Data Quality & $\begin{array}{l}\text { Huge effort to make certain complex apparatus well } \\
\text { understood (proper calibrations) and "corrections" } \\
\text { properly applied to data. Often requires data to be re- } \\
\text { analyzed }\end{array}$ \\
\hline & Data Types & $\begin{array}{l}\text { Raw experimental data in various binary forms with } \\
\text { conceptually a name: value syntax for name spanning } \\
\text { "chamber readout" to "particle momentum". } \\
\text { Reconstructed data is processed to produce dense data } \\
\text { formats optimized for analysis }\end{array}$ \\
\hline & Data Analytics & $\begin{array}{l}\text { Initial analysis is processing of experimental data specific } \\
\text { to each experiment (ALICE, ATLAS, CMS, LHCb) } \\
\text { producing summary information. Second step in analysis } \\
\text { uses "exploration" (histograms, scatter-plots) with } \\
\text { model fits. Substantial Monte-Carlo computations are } \\
\text { necessary to estimate analysis quality. } \\
\text { A large fraction ( } \approx 60 \% \text { ) of the available CPU resources } \\
\text { available to the ATLAS collaboration at the Tier-1 and } \\
\text { the Tier- } 2 \text { centers is used for simulated event } \\
\text { production. The ATLAS simulation requirements are } \\
\text { completely driven by the physics community in terms of } \\
\text { analysis needs and corresponding physics goals. The } \\
\text { current physics analyses are looking at real data samples } \\
\text { of roughly } 2 \text { billion (B) events taken in } 2011 \text { and } 3 B \\
\text { events taken in } 2012 \text { (this represents } \approx 5 \text { PB of } \\
\text { experimental data), and ATLAS has roughly } 3.5 B \text { MC } \\
\text { events for } 2011 \text { data, and } 2.5 B \text { MC events for } 2012 \text { (this } \\
\text { represents } \approx 6 \text { PB of simulated data). Given the resource } \\
\text { requirements to fully simulate an event using the GEANT }\end{array}$ \\
\hline
\end{tabular}




\section{Astronomy and Physics> Use Case 39: Analysis of LHC (Large Hadron Collider) Data}

\begin{tabular}{|c|c|}
\hline & $\begin{array}{l}4 \text { package, ATLAS can currently produce about } 4 \text { million } \\
\text { events per day using the entire processing capacity } \\
\text { available to production worldwide. } \\
\text { Due to its high CPU cost, the outputs of full Geant } 4 \\
\text { simulation (HITS) are stored in one custodial tape copy } \\
\text { on Tier1 tapes to be re-used in several Monte-Carlo re- } \\
\text { processings. The HITS from faster simulation flavors will } \\
\text { be only of transient nature in LHC Run } 2 \text {. }\end{array}$ \\
\hline $\begin{array}{r}\text { Big Data Specific } \\
\text { Challenges (Gaps) }\end{array}$ & 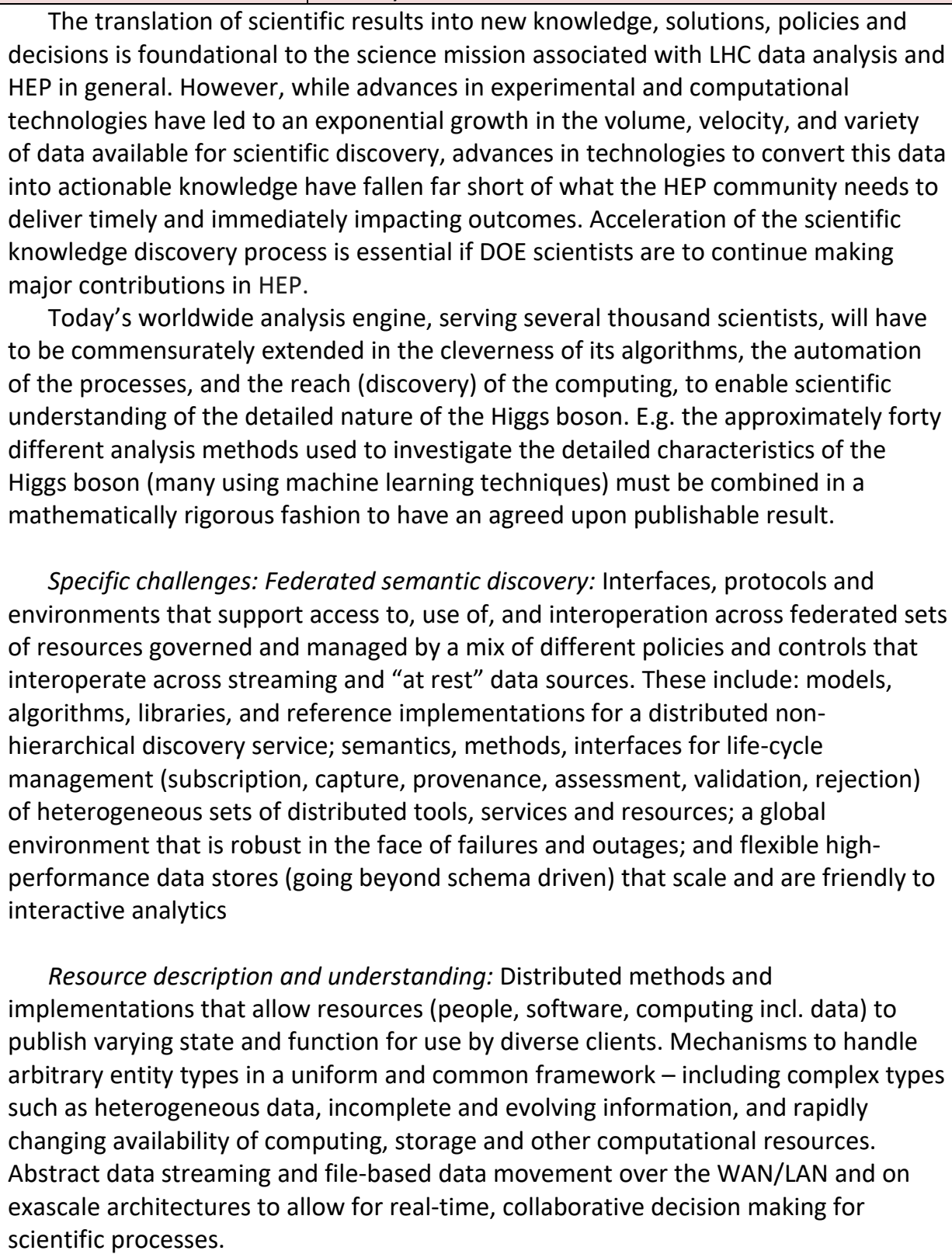 \\
\hline $\begin{array}{r}\text { Big Data Specific } \\
\text { Challenges in Mobility }\end{array}$ & $\begin{array}{l}\text { The agility to use any appropriate available resources and to ensure that all data } \\
\text { needed is dynamically available at that resource is fundamental to future discoveries } \\
\text { in HEP. In this context "resource" has a broad meaning and includes data and people } \\
\text { as well as computing and other non-computer based entities: thus, any kind of data- }\end{array}$ \\
\hline
\end{tabular}




\section{Astronomy and Physics> Use Case 39: Analysis of LHC (Large Hadron Collider) Data}

\begin{tabular}{|c|c|}
\hline & $\begin{array}{l}\text { raw data, information, knowledge, etc., and any type of resource-people, } \\
\text { computers, storage systems, scientific instruments, software, resource, service, etc. } \\
\text { In order to make effective use of such resources, a wide range of management } \\
\text { capabilities must be provided in an efficient, secure, and reliable manner, } \\
\text { encompassing for example collection, discovery, allocation, movement, access, use, } \\
\text { release, and reassignment. These capabilities must span and control large ensembles } \\
\text { of data and other resources that are constantly changing and evolving, and will often } \\
\text { be in-deterministic and fuzzy in many aspects. } \\
\text { Specific Challenges: Globally optimized dynamic allocation of resources: These } \\
\text { need to take account of the lack of strong consistency in knowledge across the entire } \\
\text { system. } \\
\text { Minimization of time-to-delivery of data and services: Not only to reduce the time } \\
\text { to delivery of the data or service but also allow for a predictive capability, so } \\
\text { physicists working on data analysis can deal with uncertainties in the real-time } \\
\text { decision making processes. }\end{array}$ \\
\hline $\begin{array}{r}\text { Security and Privacy } \\
\text { Requirements }\end{array}$ & $\begin{array}{l}\text { While HEP data itself is not proprietary unintended alteration and/or cyber- } \\
\text { security related facility service compromises could potentially be very disruptive to } \\
\text { the analysis process. Besides the need of having personal credentials and the related } \\
\text { virtual organization credential management systems to maintain access rights to a } \\
\text { certain set of resources, a fair amount of attention needs to be devoted to the } \\
\text { development and operation of the many software components the community needs } \\
\text { to conduct computing in this vastly distributed environment. } \\
\text { The majority of software and systems development for LHC data analysis is } \\
\text { carried out inside the HEP community or by adopting software components from } \\
\text { other parties which involves numerous assumptions and design decisions from the } \\
\text { early design stages throughout its life cycle. Software systems make a number of } \\
\text { assumptions about their environment - how they are deployed, configured, who runs } \\
\text { it, what sort of network is it on, is its input or output sensitive, can it trust its input, } \\
\text { does it preserve privacy, etc.? When multiple software components are } \\
\text { interconnected, for example in the deep software stacks used in DHTC, without clear } \\
\text { understanding of their security assumptions, the security of the resulting system } \\
\text { becomes an unknown. } \\
\text { A trust framework is a possible way of addressing this problem. A DHTC trust } \\
\text { framework, by describing what software, systems and organizations provide and } \\
\text { expect of their environment regarding policy enforcement, security and privacy, } \\
\text { allows for a system to be analyzed for gaps in trust, fragility and fault tolerance. }\end{array}$ \\
\hline $\begin{array}{l}\text { Highlight issues for } \\
\text { generalizing this use } \\
\text { case (e.g. for ref. } \\
\text { architecture) }\end{array}$ & $\begin{array}{l}\text { Large scale example of an event based analysis with core statistics needed. Also } \\
\text { highlights importance of virtual organizations as seen in global collaboration. } \\
\text { The LHC experiments are pioneers of distributed Big Data science infrastructure, } \\
\text { and several aspects of the LHC experiments' workflow highlight issues that other } \\
\text { disciplines will need to solve. These include automation of data distribution, high } \\
\text { performance data transfer, and large-scale high-throughput computing. }\end{array}$ \\
\hline $\begin{array}{r}\text { More Information } \\
\text { (URLs) }\end{array}$ & $\begin{array}{l}\text { http://grids.ucs.indiana.edu/ptliupages/publications/Where\%20does\%20all\%20the\% } \\
\text { 20data\%20come\%20from\%20v7.pdf } \\
\text { http://www.es.net/assets/pubs presos/High-throughput-lessons-from-the-LHC- } \\
\text { experience.Johnston.TNC2013.pdf }\end{array}$ \\
\hline
\end{tabular}


NIST Big DATA INTEROPERABILITY FRAMEWORK: Volume 3, USE CASES AND GENERAL REQUIREMENTS

\begin{tabular}{|c|c|c|c|c|c|}
\hline Use Case Stages & Data Sources & Data Usage & $\begin{array}{l}\text { Transformations } \\
\text { (Data Analytics) }\end{array}$ & Infrastructure & Security and Privacy \\
\hline \multicolumn{6}{|c|}{ Particle Physics: Analysis of LHC Large Hadron Collider Data, Discovery of Higgs particle (Scientific Research: Physics) } \\
\hline Record Raw Data & \begin{tabular}{|l|} 
CERN LHC \\
Accelerator
\end{tabular} & \begin{tabular}{|l|} 
This data is staged at \\
CERN and then \\
distributed across the \\
globe for next stage in \\
processing
\end{tabular} & $\begin{array}{l}\text { LHC has } 10^{9} \text { collisions } \\
\text { per second; the } \\
\text { hardware + software } \\
\text { trigger selects } \\
\text { "interesting events". } \\
\text { Other utilities distribute } \\
\text { data across the globe } \\
\text { with fast transport } \\
\end{array}$ & \begin{tabular}{|l|} 
Accelerator and \\
sophisticated data \\
selection (trigger \\
process) that uses \\
$\approx 7000$ cores at CERN \\
to record $\approx 100-500$ \\
events each second \\
$(\approx 1$ megabyte each) \\
\end{tabular} & $\mathrm{N} / \mathrm{A}$ \\
\hline $\begin{array}{l}\text { Process Raw Data to } \\
\text { Information }\end{array}$ & Disk Files of Raw Data & \begin{tabular}{|l|} 
Iterative calibration \\
and checking of \\
analysis which has for \\
example "heuristic" \\
track finding \\
algorithms. \\
Produce "large" full \\
physics files and \\
stripped down Analysis \\
Object Data $(A O D)$ \\
files that are $\approx 10 \%$ \\
original size \\
\end{tabular} & $\begin{array}{l}\text { Full analysis code that } \\
\text { builds in complete } \\
\text { understanding of } \\
\text { complex experimental } \\
\text { detector. } \\
\text { Also Monte Carlo } \\
\text { codes to produce } \\
\text { simulated data to } \\
\text { evaluate efficiency of } \\
\text { experimental } \\
\text { detection. }\end{array}$ & $\begin{array}{l}\approx 300,000 \text { cores } \\
\text { arranged in } 3 \text { tiers. } \\
\text { Tier 0: CERN } \\
\text { Tier 1: "Major } \\
\text { Countries" } \\
\text { Tier 2: Universities and } \\
\text { laboratories. } \\
\text { Note processing is } \\
\text { compute and data } \\
\text { intensive }\end{array}$ & N/A \\
\hline $\begin{array}{l}\text { Physics Analysis } \\
\text { Information to } \\
\text { Knowledge/Discovery }\end{array}$ & $\begin{array}{l}\text { Disk Files of } \\
\text { Information including } \\
\text { accelerator and Monte } \\
\text { Carlo data. } \\
\text { Include wisdom from } \\
\text { lots of physicists } \\
\text { (papers) in analysis } \\
\text { choices }\end{array}$ & \begin{tabular}{|l|} 
Use simple statistical \\
techniques (like \\
histogramming, \\
multi-variate analysis \\
methods and other \\
data analysis \\
techniques and model \\
fits to discover new \\
effects (particles) and \\
put limits on effects not \\
seen
\end{tabular} & $\begin{array}{l}\text { Data reduction and } \\
\text { processing steps with } \\
\text { advanced physics } \\
\text { algorithms to identify } \\
\text { event properties, } \\
\text { particle hypothesis etc. } \\
\text { For interactive data } \\
\text { analysis of those } \\
\text { reduced and selected } \\
\text { datasets the classic } \\
\text { program is Root from } \\
\text { CERN that reads } \\
\text { multiple event (AOD, } \\
\text { NTUP) files from } \\
\text { selected datasets and } \\
\text { use physicist } \\
\text { generated C++ code to } \\
\text { calculate new } \\
\text { quantities such as } \\
\text { implied mass of an } \\
\text { unstable (new) particle }\end{array}$ & \begin{tabular}{|l|} 
While the bulk of data \\
processing is done at \\
Tier 1 and Tier 2 \\
resources, the end \\
stage analysis is \\
usually done by users \\
at a local Tier 3 facility. \\
The scale of \\
computing resources \\
at Tier 3 sites range \\
from workstations to \\
small clusters. ROOT \\
is the most common \\
software stack used to \\
analyze compact data \\
formats generated on \\
distributed computing \\
resources. Data \\
transfer is done using \\
ATLAS and CMS DDM \\
tools, which mostly rely \\
on gridFTP \\
middleware. XROOTD \\
based direct data \\
access is also gaining \\
importance wherever \\
high network \\
bandwidth is available.
\end{tabular} & $\begin{array}{l}\text { Physics discoveries } \\
\text { and results are } \\
\text { confidential until } \\
\text { certified by group and } \\
\text { presented at } \\
\text { meeting/journal. Data } \\
\text { preserved so results } \\
\text { reproducible }\end{array}$ \\
\hline
\end{tabular}

See Figure 6: Particle Physics: Analysis of LHC Data: Discovery of Higgs Particle - CERN LHC 329 location.

330 See Figure 7: Particle Physics: Analysis of LHC Data: Discovery of Higgs Particle - The multi-tier LHC 331 computing infrastructure. 


\section{Astronomy and Physics> Use Case 40: Belle II Experiment}

\begin{tabular}{|c|c|c|}
\hline Use Case Title & \multicolumn{2}{|l|}{ Belle II Experiment } \\
\hline Vertical (area) & \multicolumn{2}{|c|}{ Scientific Research: High Energy Physics } \\
\hline Author/Company/Email & \multicolumn{2}{|c|}{$\begin{array}{l}\text { David Asner and Malachi Schram, PNNL, david.asner@pnnl.gov and } \\
\text { malachi.schram@pnnl.gov }\end{array}$} \\
\hline $\begin{array}{r}\text { Actors/Stakeholders } \\
\text { and their roles and } \\
\text { responsibilities }\end{array}$ & \multicolumn{2}{|c|}{$\begin{array}{l}\text { David Asner is the Chief Scientist for the US Belle II Project } \\
\text { Malachi Schram is Belle II network and data transfer coordinator and the PNNL Belle } \\
\text { II computing center manager }\end{array}$} \\
\hline Goals & \multicolumn{2}{|c|}{$\begin{array}{l}\text { Perform precision measurements to search for new phenomena beyond the } \\
\text { Standard Model of Particle Physics }\end{array}$} \\
\hline Use Case Description & \multicolumn{2}{|c|}{$\begin{array}{l}\text { Study numerous decay modes at the Upsilon(4S) resonance to search for new } \\
\text { phenomena beyond the Standard Model of Particle Physics }\end{array}$} \\
\hline \multirow{4}{*}{$\begin{array}{r}\text { Current } \\
\text { Solutions }\end{array}$} & Compute(System) & Distributed (Grid computing using DIRAC) \\
\hline & Storage & Distributed (various technologies) \\
\hline & Networking & $\begin{array}{l}\text { Continuous RAW data transfer of } \approx 20 \text { Gbps at designed } \\
\text { luminosity between Japan and US } \\
\text { Additional transfer rates are currently being investigated }\end{array}$ \\
\hline & Software & $\begin{array}{l}\text { Open Science Grid, Geant4, DIRAC, FTS, Belle II } \\
\text { framework }\end{array}$ \\
\hline \multirow[t]{5}{*}{$\begin{array}{r}\text { Big Data } \\
\text { Characteristics }\end{array}$} & $\begin{array}{r}\text { Data Source } \\
\text { (distributed/centralized) }\end{array}$ & $\begin{array}{l}\text { Distributed data centers } \\
\text { Primary data centers are in Japan (KEK) and US (PNNL) }\end{array}$ \\
\hline & Volume (size) & $\begin{array}{l}\text { Total integrated RAW data } \approx 120 \mathrm{~PB} \text { and physics data } \\
\approx 15 \mathrm{~PB} \text { and } \approx 100 \mathrm{~PB} \mathrm{MC} \text { samples }\end{array}$ \\
\hline & $\begin{array}{r}\text { Velocity } \\
\text { (e.g. real time) }\end{array}$ & $\begin{array}{l}\text { Data will be re-calibrated and analyzed incrementally } \\
\text { Data rates will increase based on the accelerator } \\
\text { luminosity }\end{array}$ \\
\hline & $\begin{array}{r}\text { Variety } \\
\text { (multiple datasets, } \\
\text { mashup) }\end{array}$ & Data will be re-calibrated and distributed incrementally. \\
\hline & $\begin{array}{r}\text { Variability (rate of } \\
\text { change) }\end{array}$ & $\begin{array}{l}\text { Collisions will progressively increase until the designed } \\
\text { luminosity is reached ( } 3000 \mathrm{BB} \text { pairs per sec). } \\
\text { Expected event size is } \approx 300 \mathrm{kB} \text { per events. }\end{array}$ \\
\hline \multirow{5}{*}{$\begin{array}{r}\text { Big Data Science } \\
\text { (collection, curation, } \\
\text { analysis, } \\
\text { action) }\end{array}$} & $\begin{array}{r}\text { Veracity (Robustness } \\
\text { Issues) }\end{array}$ & $\begin{array}{l}\text { Validation will be performed using known reference } \\
\text { physics processes }\end{array}$ \\
\hline & Visualization & $\mathrm{N} / \mathrm{A}$ \\
\hline & Data Quality & $\begin{array}{l}\text { Output data will be re-calibrated and validated } \\
\text { incrementally }\end{array}$ \\
\hline & Data Types & Tuple based output \\
\hline & Data Analytics & $\begin{array}{l}\text { Data clustering and classification is an integral part of } \\
\text { the computing model. Individual scientists define event } \\
\text { level analytics. }\end{array}$ \\
\hline $\begin{array}{r}\text { Big Data Specific } \\
\text { Challenges (Gaps) }\end{array}$ & \multicolumn{2}{|c|}{ Data movement and bookkeeping (file and event level meta-data). } \\
\hline $\begin{array}{r}\text { Big Data Specific } \\
\text { Challenges in Mobility }\end{array}$ & \multicolumn{2}{|c|}{$\begin{array}{l}\text { Network infrastructure required for continuous data transfer between Japan (KEK) } \\
\text { and US (PNNL). }\end{array}$} \\
\hline $\begin{array}{r}\text { Security and Privacy } \\
\text { Requirements }\end{array}$ & \multicolumn{2}{|c|}{ No special challenges. Data is accessed using grid authentication. } \\
\hline $\begin{array}{r}\text { Highlight issues for } \\
\text { generalizing this use } \\
\text { case (e.g. for ref. } \\
\text { architecture) }\end{array}$ & & \\
\hline
\end{tabular}


NIST Big Data Interoperability Framework: Volume 3, Use CaSes and General ReQuiRements

\section{Astronomy and Physics> Use Case 40: Belle II Experiment}

333

More Information http://belle2.kek.jp (URLS)

334 


\section{Earth, Environmental and Polar Science> Use Case 41: EISCAT 3D I ncoherent Scatter Radar System}

\begin{tabular}{|c|c|c|}
\hline Use Case Title & \multicolumn{2}{|c|}{ EISCAT 3D incoherent scatter radar system } \\
\hline Vertical (area) & \multicolumn{2}{|c|}{ Environmental Science } \\
\hline Author/Company/Email & \multicolumn{2}{|c|}{$\begin{array}{l}\text { Yin Chen /Cardiff University/ chenY58@ cardiff.ac.uk } \\
\text { Ingemar Häggström, Ingrid Mann, Craig Heinselman/ } \\
\text { EISCAT Science Association/\{Ingemar.Haggstrom, Ingrid.mann, } \\
\text { Craig.Heinselman\}@eiscat.se }\end{array}$} \\
\hline $\begin{array}{r}\text { Actors/Stakeholders } \\
\text { and their roles and } \\
\text { responsibilities }\end{array}$ & \multicolumn{2}{|c|}{$\begin{array}{l}\text { The EISCAT Scientific Association is an international research organization operating } \\
\text { incoherent scatter radar systems in Northern Europe. It is funded and operated by } \\
\text { research councils of Norway, Sweden, Finland, Japan, China and the United Kingdom } \\
\text { (collectively, the EISCAT Associates). In addition to the incoherent scatter radars, } \\
\text { EISCAT also operates an lonospheric Heater facility, as well as two Dynasondes. }\end{array}$} \\
\hline Goals & \multicolumn{2}{|c|}{$\begin{array}{l}\text { EISCAT, the European Incoherent Scatter Scientific Association, is established to } \\
\text { conduct research on the lower, middle and upper atmosphere and ionosphere using } \\
\text { the incoherent scatter radar technique. This technique is the most powerful ground- } \\
\text { based tool for these research applications. EISCAT is also being used as a coherent } \\
\text { scatter radar for studying instabilities in the ionosphere, as well as for investigating } \\
\text { the structure and dynamics of the middle atmosphere and as a diagnostic instrument } \\
\text { in ionospheric modification experiments with the Heating facility. }\end{array}$} \\
\hline Use Case Description & \multicolumn{2}{|c|}{$\begin{array}{l}\text { The design of the next generation incoherent scatter radar system, EISCAT_3D, } \\
\text { opens up opportunities for physicists to explore many new research fields. On the } \\
\text { other hand, it also introduces significant challenges in handling large-scale } \\
\text { experimental data which will be massively generated at great speeds and volumes. } \\
\text { This challenge is typically referred to as a Big Data problem and requires solutions } \\
\text { from beyond the capabilities of conventional database technologies. }\end{array}$} \\
\hline \multirow[t]{4}{*}{$\begin{array}{r}\text { Current } \\
\text { Solutions }\end{array}$} & Compute(System) & $\begin{array}{l}\text { EISCAT 3D data e-Infrastructure plans to use the high } \\
\text { performance computers for central site data processing } \\
\text { and high throughput computers for mirror sites data } \\
\text { processing }\end{array}$ \\
\hline & Storage & 32 TB \\
\hline & Networking & $\begin{array}{l}\text { The estimated data rates in local networks at the active } \\
\text { site run from } 1 \mathrm{~GB} / \mathrm{s} \text { to } 10 \mathrm{~GB} / \mathrm{s} \text {. Similar capacity is } \\
\text { needed to connect the sites through dedicated high- } \\
\text { speed network links. Downloading the full data is not } \\
\text { time critical, but operations require real-time } \\
\text { information about certain pre-defined events to be sent } \\
\text { from the sites to the operation centre and a real-time } \\
\text { link from the operation centre to the sites to set the } \\
\text { mode of radar operation on with immediate action. }\end{array}$ \\
\hline & Software & $\begin{array}{l}\text { - Mainstream operating systems, e.g., Windows, } \\
\text { Linux, Solaris, HP/UX, or FreeBSD } \\
\text { - Simple, flat file storage with required capabilities } \\
\text { e.g., compression, file striping and file journaling } \\
\text { - Self-developed software } \\
\text { o Control and monitoring tools including, system } \\
\text { configuration, quick-look, fault reporting, etc. } \\
\text { o Data dissemination utilities } \\
\text { o User software e.g., for cyclic buffer, data } \\
\text { cleaning, RFI detection and excision, auto- } \\
\text { correlation, data integration, data analysis, }\end{array}$ \\
\hline
\end{tabular}




\section{Earth, Environmental and Polar Science> Use Case 41: EISCAT 3D I ncoherent Scatter Radar System}

\begin{tabular}{|c|c|c|}
\hline & & $\begin{array}{ll} & \text { event identification, discovery and retrieval, } \\
\text { calculation of value-added data products, } \\
\text { ingestion/extraction, plot } \\
\text { o User-oriented computing } \\
\text { o APIs into standard software environments } \\
\text { o } & \text { Data processing chains and workflow }\end{array}$ \\
\hline \multirow[t]{5}{*}{$\begin{array}{r}\text { Big Data } \\
\text { Characteristics }\end{array}$} & $\begin{array}{r}\text { Data Source } \\
\text { (distributed/centralized) }\end{array}$ & $\begin{array}{l}\text { EISCAT_3D will consist of a core site with a transmitting } \\
\text { and receiving radar arrays and four sites with receiving } \\
\text { antenna arrays at some } 100 \mathrm{~km} \text { from the core. }\end{array}$ \\
\hline & Volume (size) & $\begin{array}{l}\text { - The fully operational 5-site system will generate } 40 \\
\text { PB/year in } 2022 \text {. } \\
\text { It is expected to operate for } 30 \text { years, and data } \\
\text { products to be stored at less } 10 \text { years }\end{array}$ \\
\hline & $\begin{array}{r}\text { Velocity } \\
\text { (e.g. real time) }\end{array}$ & $\begin{array}{l}\text { At each of 5-receiver-site: } \\
\text { - each antenna generates } 30 \mathrm{Msamples} / \mathrm{s}(120 \mathrm{MB} / \mathrm{s}) \text {; } \\
\text { - each antenna group (consists of } 100 \text { antennas) to } \\
\text { form beams at speed of } 2 \mathrm{Gbit} / \mathrm{s} / \text { group; } \\
\text { these data are temporary stored in a ringbuffer: } 160 \\
\text { groups }->125 \mathrm{~TB} / \mathrm{h} \text {. }\end{array}$ \\
\hline & $\begin{array}{r}\text { Variety } \\
\text { (multiple datasets, } \\
\text { mashup) }\end{array}$ & $\begin{array}{l}\text { - Measurements: different versions, formats, } \\
\text { - } \quad \text { System information: configuration, monitoring, } \\
\text { logs/provenance ... } \\
\text { - Users' metadata/data: experiments, analysis, } \\
\text { sharing, communications ... }\end{array}$ \\
\hline & $\begin{array}{r}\text { Variability (rate of } \\
\text { change) }\end{array}$ & $\begin{array}{l}\text { In time, instantly, a few ms. } \\
\text { Along the radar beams, } 100 \mathrm{~ns} \text {. }\end{array}$ \\
\hline \multirow[t]{2}{*}{$\begin{array}{r}\text { Big Data Science } \\
\text { (collection, curation, } \\
\text { analysis, } \\
\text { action) }\end{array}$} & $\begin{array}{r}\text { Veracity (Robustness } \\
\text { Issues) }\end{array}$ & $\begin{array}{l}\text { - } \text { Running 24/7, EISCAT_3D have very high demands } \\
\text { on robustness. } \\
\text { Data and performance assurance is vital for the } \\
\text { ring-buffer and archive systems. These systems } \\
\text { must be able to guarantee to meet minimum data } \\
\text { rate acceptance at all times or scientific data will be } \\
\text { lost. } \\
\text { Similarly, the systems must guarantee that data } \\
\text { held is not volatile or corrupt. This latter } \\
\text { requirement is particularly vital at the permanent } \\
\text { archive where data is most likely to be accessed by } \\
\text { scientific users and least easy to check; data } \\
\text { corruption here has a significant possibility of being } \\
\text { non-recoverable and of poisoning the scientific } \\
\text { literature. }\end{array}$ \\
\hline & Visualization & $\begin{array}{l}\text { Real-time visualization of analyzed data, e.g., with a } \\
\text { figure of updating panels showing electron density, } \\
\text { temperatures and ion velocity to those data for } \\
\text { each beam. } \\
\text { Non-real-time (post-experiment) visualization of the } \\
\text { physical parameters of interest, e.g., } \\
\text { o by standard plots, }\end{array}$ \\
\hline
\end{tabular}




\section{Earth, Environmental and Polar Science> Use Case 41: EISCAT 3D I ncoherent Scatter Radar System}

\begin{tabular}{|c|c|c|}
\hline & & $\begin{array}{l}\text { o } \begin{array}{l}\text { using three-dimensional block to show to } \\
\text { spatial variation (in the user selected cuts), } \\
\text { using animations to show the temporal } \\
\text { variation, } \\
\text { allow the visualization of } 5 \text { or higher } \\
\text { dimensional data, e.g., using the 'cut up } \\
\text { and stack' technique to reduce the } \\
\text { dimensionality, that is take one or more } \\
\text { independent coordinates as discrete; or } \\
\text { volume rendering technique to display a } \\
\text { 2D projection of a 3D discretely sampled } \\
\text { dataset. }\end{array} \\
\text { (Interactive) Visualization. E.g., to allow users to } \\
\text { combine the information on several spectral } \\
\text { features, e.g., by using color coding, and to provide } \\
\text { real-time visualization facility to allow the users to } \\
\text { link or plug in tailor-made data visualization } \\
\text { functions, and more importantly functions to signal } \\
\text { for special observational conditions. }\end{array}$ \\
\hline & Data Quality & $\begin{array}{l}\text { Monitoring software will be provided which allows } \\
\text { The Operator to see incoming data via the } \\
\text { Visualization system in real-time and react } \\
\text { appropriately to scientifically interesting events. } \\
\text { Control software will be developed to time- } \\
\text { integrate the signals and reduce the noise variance } \\
\text { and the total data throughput of the system that } \\
\text { reached the data archive. }\end{array}$ \\
\hline & Data Types & HDF-5 \\
\hline & Data Analytics & $\begin{array}{l}\text { Pattern recognition, demanding correlation routines, } \\
\text { high level parameter extraction }\end{array}$ \\
\hline $\begin{array}{l}\text { Big Data Specific } \\
\text { Challenges (Gaps) }\end{array}$ & \multicolumn{2}{|c|}{$\begin{array}{l}\text { - High throughput of data for reduction into higher levels. } \\
\text { - Discovery of meaningful insights from low-value-density data needs new } \\
\text { approaches to the deep, complex analysis e.g., using machine learning, } \\
\text { statistical modelling, graph algorithms etc. which go beyond traditional } \\
\text { approaches to the space physics. }\end{array}$} \\
\hline $\begin{array}{r}\text { Big Data Specific } \\
\text { Challenges in Mobility }\end{array}$ & \multicolumn{2}{|c|}{ Is not likely in mobile platforms } \\
\hline $\begin{array}{r}\text { Security and Privacy } \\
\text { Requirements }\end{array}$ & \multicolumn{2}{|c|}{$\begin{array}{l}\text { Lower level of data has restrictions for } 1 \text { year within the associate countries. All data } \\
\text { open after } 3 \text { years. }\end{array}$} \\
\hline $\begin{array}{r}\text { Highlight issues for } \\
\text { generalizing this use } \\
\text { case (e.g. for ref. } \\
\text { architecture) }\end{array}$ & \multicolumn{2}{|c|}{$\begin{array}{l}\text { EISCAT 3D data e-Infrastructure shares similar architectural characteristics with other } \\
\text { ISR radars, and many existing Big Data systems, such as LOFAR, LHC, and SKA }\end{array}$} \\
\hline $\begin{array}{r}\text { More Information } \\
\text { (URLs) }\end{array}$ & \multicolumn{2}{|l|}{ https://www.eiscat3d.se/ } \\
\hline
\end{tabular}

See Figure 8: EISCAT 3D Incoherent Scatter Radar System - System architecture. 


\section{Earth, Environmental and Polar Science> Use Case 42: Common Environmental Research I nfrastructure}

\begin{tabular}{|c|c|}
\hline Use Case Title & ENVRI (Common Operations of Environmental Research Infrastructure) \\
\hline Vertical (area) & Environmental Science \\
\hline Author/Company/Email & Yin Chen/ Cardiff University / ChenY58@cardiff.ac.uk \\
\hline $\begin{array}{r}\text { Actors/Stakeholders } \\
\text { and their roles and } \\
\text { responsibilities }\end{array}$ & $\begin{array}{l}\text { The ENVRI project is a collaboration conducted within the European Strategy Forum } \\
\text { on Research Infrastructures (ESFRI) Environmental Cluster. The ESFRI Environmental } \\
\text { research infrastructures involved in ENVRI including: } \\
\text { - ICOS is a European distributed infrastructure dedicated to the monitoring of } \\
\text { greenhouse gases (GHG) through its atmospheric, ecosystem and ocean } \\
\text { networks. } \\
\text { - EURO-Argo is the European contribution to Argo, which is a global ocean } \\
\text { observing system. } \\
\text { - EISCAT-3D is a European new-generation incoherent-scatter research radar for } \\
\text { upper atmospheric science. } \\
\text { - LifeWatch is an e-science Infrastructure for biodiversity and ecosystem research. } \\
\text { EPOS is a European Research Infrastructure on earthquakes, volcanoes, surface } \\
\text { dynamics and tectonics. } \\
\text { EMSO is a European network of seafloor observatories for the long-term } \\
\text { monitoring of environmental processes related to ecosystems, climate change } \\
\text { and geo-hazards. } \\
\text { ENVRI also maintains close contact with the other not-directly involved ESFRI } \\
\text { Environmental research infrastructures by inviting them for joint meetings. These } \\
\text { projects are: } \\
\text { - } \quad \text { IAGOS } \quad \text { Aircraft for global observing system } \\
\text { ENOS } \quad \text { Svalbard arctic Earth observing system } \\
\text { ENVRI IT community provides common policies and technical solutions for the } \\
\text { Cardiff University, CNR-ISTI, CNRS (Centre National de la Recherche Scientifique), } \\
\text { CSC, EAA (Umweltbundesamt Gmbh), EGI, ESA-ESRIN, University of Amsterdam, and } \\
\text { University of Edinburgh. }\end{array}$ \\
\hline Goals & $\begin{array}{l}\text { The ENVRI project gathers } 6 \text { EU ESFRI environmental science infra-structures } \\
\text { (ICOS, EURO-Argo, EISCAT-3D, LifeWatch, EPOS, and EMSO) in order to develop } \\
\text { common data and software services. The results will accelerate the construction of } \\
\text { these infrastructures and improve interoperability among them. } \\
\text { The primary goal of ENVRI is to agree on a reference model for joint operations. } \\
\text { The ENVRI RM is a common ontological framework and standard for the description } \\
\text { and characterisation of computational and storage infrastructures in order to } \\
\text { achieve seamless interoperability between the heterogeneous resources of different } \\
\text { infrastructures. The ENVRI RM serves as a common language for community } \\
\text { communication, providing a uniform framework into which the infrastructure's } \\
\text { components can be classified and compared, also serving to identify common } \\
\text { solutions to common problems. This may enable reuse, share of resources and } \\
\text { experiences, and avoid duplication of efforts. }\end{array}$ \\
\hline Use Case Description & $\begin{array}{l}\text { ENVRI project implements harmonized solutions and draws up guidelines for the } \\
\text { common needs of the environmental ESFRI projects, with a special focus on issues as } \\
\text { architectures, metadata frameworks, data discovery in scattered repositories, } \\
\text { visualization and data curation. This will empower the users of the collaborating } \\
\text { environmental research infrastructures and enable multidisciplinary scientists to } \\
\text { access, study and correlate data from multiple domains for "system level" research. } \\
\text { ENVRI investigates a collection of representative research infrastructures for }\end{array}$ \\
\hline
\end{tabular}




\section{Earth, Environmental and Polar Science> Use Case 42: Common Environmental Research I nfrastructure}

\begin{tabular}{|c|c|c|}
\hline & \multicolumn{2}{|c|}{$\begin{array}{l}\text { environmental sciences, and provides a projection of Europe-wide requirements } \\
\text { they have; identifying in particular, requirements they have in common. Based on } \\
\text { the analysis evidence, the ENVRI Reference Model (http://www.envri.eu/rm) is } \\
\text { developed using ISO standard Open Distributed Processing. Fundamentally the } \\
\text { model serves to provide a universal reference framework for discussing many } \\
\text { common technical challenges facing all of the ESFRI-environmental research } \\
\text { infrastructures. By drawing analogies between the reference components of the } \\
\text { model and the actual elements of the infrastructures (or their proposed designs) as } \\
\text { they exist now, various gaps and points of overlap can be identified. }\end{array}$} \\
\hline \multirow{4}{*}{$\begin{array}{r}\text { Current } \\
\text { Solutions }\end{array}$} & Compute(System) & \\
\hline & Storage & File systems and relational databases \\
\hline & Networking & \\
\hline & Software & Own \\
\hline $\begin{array}{r}\text { Big Data } \\
\text { Characteristics }\end{array}$ & $\begin{array}{r}\text { Data Source } \\
\text { (distributed/centralized) }\end{array}$ & $\begin{array}{l}\text { Most of the ENVRI Research Infrastructures (ENV RIs) } \\
\text { are distributed, long-term, remote controlled } \\
\text { observational networks focused on understanding } \\
\text { processes, trends, thresholds, interactions and } \\
\text { feedbacks and increasing the predictive power to } \\
\text { address future environmental challenges. They are } \\
\text { spanning from the Arctic areas to the European } \\
\text { Southernmost areas and from Atlantic on west to the } \\
\text { Black Sea on east. More precisely: } \\
\text { - EMSO, network of fixed-point, deep-seafloor and } \\
\text { water column observatories, is geographically } \\
\text { distributed in key sites of European waters, } \\
\text { presently consisting of thirteen sites. } \\
\text { EPOS aims at integrating the existing European } \\
\text { facilities in solid Earth science into one coherent } \\
\text { multidisciplinary RI, and to increase the accessibility } \\
\text { and usability of multidisciplinary data from seismic } \\
\text { and geodetic monitoring networks, volcano } \\
\text { observatories, laboratory experiments and } \\
\text { computational simulations enhancing worldwide } \\
\text { interoperability in Earth Science. } \\
\text { ICOS dedicates to the monitoring of greenhouse } \\
\text { gases (GHG) through its atmospheric, ecosystem } \\
\text { and ocean networks. The ICOS network includes } \\
\text { more than } 30 \text { atmospheric and more than } 30 \\
\text { ecosystem primary long term sites located across } \\
\text { Europe, and additional secondary sites. It also } \\
\text { includes three Thematic Centres to process the data } \\
\text { from all the stations from each network, and } \\
\text { provide access to these data. } \\
\text { LifeWatch is a "virtual" infrastructure for } \\
\text { biodiversity and ecosystem research with services } \\
\text { mainly provided through the Internet. Its Common } \\
\text { Facilities is coordinated and managed at a central } \\
\text { European level; and the LifeWatch Centres serve as } \\
\text { specialized facilities from member countries }\end{array}$ \\
\hline
\end{tabular}




\section{Earth, Environmental and Polar Science> Use Case 42: Common Environmental Research I nfrastructure}

\begin{tabular}{|c|c|c|}
\hline & & $\begin{array}{l}\text { (regional partner facilities) or research } \\
\text { communities. } \\
\text { Euro-Argo provides, deploys and operates an array } \\
\text { of around } 800 \text { floats contributing to the global array } \\
\text { (3,000 floats) and thus provide enhanced coverage } \\
\text { in the European regional seas. } \\
\text { EISCAT- } 3 D \text {, makes continuous measurements of } \\
\text { the geospace environment and its coupling to the } \\
\text { Earth's atmosphere from its location in the auroral } \\
\text { zone at the southern edge of the northern polar } \\
\text { vortex, and is a distributed infrastructure. }\end{array}$ \\
\hline & Volume (size) & $\begin{array}{l}\text { Variable data size. e.g., } \\
\text { - The amount of data within the EMSO is depending } \\
\text { on the instrumentation and configuration of the } \\
\text { observatory between several MBs to several GB per } \\
\text { dataset. } \\
\text { - Within EPOS, the EIDA network is currently } \\
\text { providing access to continuous raw data coming } \\
\text { from approximately more than } 1000 \text { stations } \\
\text { recording about } 40 G B \text { per day, so over } 15 \text { TB per } \\
\text { year. EMSC stores a Database of } 1.85 \text { GB of } \\
\text { earthquake parameters, which is constantly } \\
\text { growing and updated with refined information. } \\
\text { - } 222705 \text { - events } \\
\text { - } 632327 \text { - origins } \\
\text { - } 642555 \text { - magnitudes } \\
\text { Within EISCAT 3D raw voltage data will reach } \\
\text { 40PB/year in 2023. }\end{array}$ \\
\hline & $\begin{array}{r}\text { Velocity } \\
\text { (e.g. real time) } \\
\end{array}$ & $\begin{array}{l}\text { Real-time data handling is a common request of the } \\
\text { environmental research infrastructures }\end{array}$ \\
\hline & $\begin{array}{r}\text { Variety } \\
\text { (multiple datasets, } \\
\text { mashup) }\end{array}$ & Highly complex and heterogeneous \\
\hline & $\begin{array}{r}\text { Variability (rate of } \\
\text { change) }\end{array}$ & Relative low rate of change \\
\hline \multirow{2}{*}{$\begin{array}{r}\text { Big Data Science } \\
\text { (collection, curation, } \\
\text { analysis, } \\
\text { action) }\end{array}$} & $\begin{array}{r}\text { Veracity (Robustness } \\
\text { Issues, semantics) }\end{array}$ & Normal \\
\hline & Visualization & $\begin{array}{l}\text { Most of the projects have not yet developed the } \\
\text { visualization technique to be fully operational. } \\
\text { - EMSO is not yet fully operational, currently only } \\
\text { simple graph plotting tools. } \\
\text { - Visualization techniques are not yet defined for } \\
\text { EPOS. } \\
\text { Within ICOS Level-1.b data products such as near } \\
\text { real time GHG measurements are available to users } \\
\text { via ATC web portal. Based on Google Chart Tools, an } \\
\text { interactive time series line chart with optional } \\
\text { annotations allows user to scroll and zoom inside a } \\
\text { time series of } \mathrm{CO} 2 \text { or } \mathrm{CH} 4 \text { measurement at an ICOS }\end{array}$ \\
\hline
\end{tabular}




\section{Earth, Environmental and Polar Science> Use Case 42: Common Environmental Research I nfrastructure}

\begin{tabular}{|c|c|c|}
\hline & & $\begin{array}{l}\text { Atmospheric station. The chart is rendered within } \\
\text { the browser using Flash. Some Level-2 products are } \\
\text { also available to ensure instrument monitoring to } \\
\text { PIs. It is mainly instrumental and comparison data } \\
\text { plots automatically generated (R language and } \\
\text { Python Matplotlib 2D plotting library) and daily } \\
\text { pushed on ICOS web server. Level-3 data products } \\
\text { such as gridded GHG fluxes derived from ICOS } \\
\text { observations increase the scientific impact of ICOS. } \\
\text { For this purpose ICOS supports its community of } \\
\text { users. The Carbon portal is expected to act as a } \\
\text { platform that will offer visualization of the flux } \\
\text { products that incorporate ICOS data. Example of } \\
\text { candidate Level-3 products from future ICOS GHG } \\
\text { concentration data are for instance maps of } \\
\text { European high-resolution CO2 or CH4 fluxes } \\
\text { obtained by atmospheric inversion modellers in } \\
\text { Europe. Visual tools for comparisons between } \\
\text { products will be developed by the Carbon Portal. } \\
\text { Contributions will be open to any product of high } \\
\text { scientific quality. } \\
\text { LifeWatch will provide common visualization } \\
\text { techniques, such as the plotting of species on maps. } \\
\text { New techniques will allow visualizing the effect of } \\
\text { changing data and/or parameters in models. }\end{array}$ \\
\hline & Data Quality (syntax) & Highly important \\
\hline & Data Types & $\begin{array}{l}\text { - Measurements (often in file formats), } \\
\text { - } \quad \text { Metadata, } \\
\text { - } \text { Ontology, } \\
\text { - Annotations }\end{array}$ \\
\hline & Data Analytics & $\begin{array}{l}\text { - Data assimilation, } \\
\text { - (Statistical) analysis, } \\
\text { - Data mining, } \\
\text { - } \text { Data extraction, } \\
\text { - Scientific modeling and simulation, } \\
\text { - Scientific workflow }\end{array}$ \\
\hline $\begin{array}{r}\text { Big Data Specific } \\
\text { Challenges (Gaps) }\end{array}$ & \multicolumn{2}{|c|}{$\begin{array}{l}\text { - } \text { Real-time handling of extreme high volume of data } \\
\text { - } \quad \text { Data staging to mirror archives } \\
\text { - Integrated Data access and discovery } \\
\text { - } \quad \text { Data processing and analysis }\end{array}$} \\
\hline $\begin{array}{r}\text { Big Data Specific } \\
\text { Challenges in Mobility }\end{array}$ & \multicolumn{2}{|c|}{$\begin{array}{l}\text { The need for efficient and high performance mobile detectors and instrumentation is } \\
\text { common: } \\
\text { - In ICOS, various mobile instruments are used to collect data from marine } \\
\text { observations, atmospheric observations, and ecosystem monitoring. } \\
\text { - In Euro-Argo, thousands of submersible robots to obtain observations of all of } \\
\text { the oceans } \\
\text { - In Lifewatch, biologists use mobile instruments for observations and } \\
\text { measurements. }\end{array}$} \\
\hline Security and Privacy & \multicolumn{2}{|c|}{ Most of the projects follow the open data sharing policy. E.g., } \\
\hline
\end{tabular}




\section{Earth, Environmental and Polar Science> Use Case 42: Common Environmental Research I nfrastructure}

\begin{tabular}{|c|c|}
\hline Requirements & $\begin{array}{l}\text { - The vision of EMSO is to allow scientists all over the world to access } \\
\text { observatories data following an open access model. } \\
\text { Within EPOS, EIDA data and Earthquake parameters are generally open and free } \\
\text { to use. Few restrictions are applied on few seismic networks and the access is } \\
\text { regulated depending on email based authentication/authorization. } \\
\text { The ICOS data will be accessible through a license with full and open access. No } \\
\text { particular restriction in the access and eventual use of the data is anticipated, } \\
\text { expected the inability to redistribute the data. Acknowledgement of ICOS and } \\
\text { traceability of the data will be sought in a specific, way (e.g. DOI of dataset). A } \\
\text { large part of relevant data and resources are generated using public funding } \\
\text { from national and international sources. } \\
\text { LifeWatch is following the appropriate European policies, such as: the European } \\
\text { Research Council (ERC) requirement; the European Commission's open access } \\
\text { pilot mandate in } 2008 \text {. For publications, initiatives such as Dryad instigated by } \\
\text { publishers and the Open Access Infrastructure for Research in Europe } \\
\text { (OpenAIRE). The private sector may deploy their data in the LifeWatch } \\
\text { infrastructure. A special company will be established to manage such } \\
\text { commercial contracts. } \\
\text { In EISCAT 3D, lower level of data has restrictions for } 1 \text { year within the associate } \\
\text { countries. All data open after } 3 \text { years. }\end{array}$ \\
\hline $\begin{array}{r}\text { Highlight issues for } \\
\text { generalizing this use } \\
\text { case (e.g. for ref. } \\
\text { architecture) }\end{array}$ & $\begin{array}{l}\text { Different research infrastructures are designed for different purposes and evolve } \\
\text { over time. The designers describe their approaches from different points of view, in } \\
\text { different levels of detail and using different typologies. The documentation provided } \\
\text { is often incomplete and inconsistent. What is needed is a uniform platform for } \\
\text { interpretation and discussion, which helps to unify understanding. } \\
\text { In ENVRI, we choose to use a standard model, Open Distributed Processing (ODP), to } \\
\text { interpret the design of the research infrastructures, and place their requirements } \\
\text { into the ODP framework for further analysis and comparison. }\end{array}$ \\
\hline $\begin{array}{r}\text { More Information } \\
\text { (URLs) }\end{array}$ & 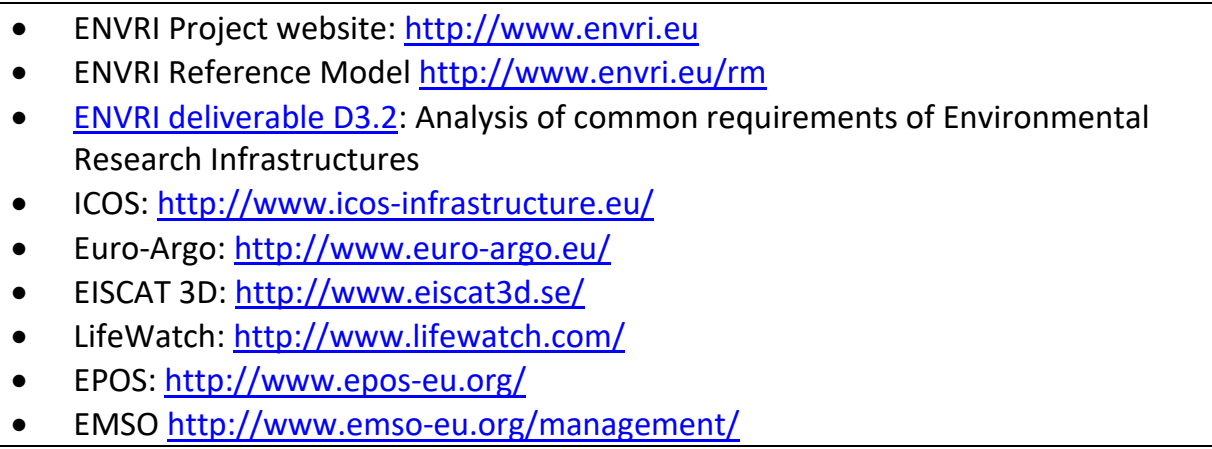 \\
\hline
\end{tabular}

See Figure 9: ENVRI, Common Operations of Environmental Research Infrastructure - ENVRI common architecture.

340 See Figure 10(a): ICOS architecture

341 See Figure 10(b): LifeWatch architecture

342 See Figure 10(c): EMSO architecture

343 See Figure 10(d): EURO-Argo architecture

344 See Figure 10(e): EISCAT 3D architecture 
NIST Big Data Interoperability Framework: Volume 3, Use CaSes and General ReQuiRements 


\section{Earth, Environmental and Polar Science> Use Case 43: Radar Data Analysis for CReSIS}

\begin{tabular}{|c|c|c|}
\hline Use Case Title & \multicolumn{2}{|l|}{ Radar Data Analysis for CReSIS } \\
\hline Vertical (area) & \multicolumn{2}{|c|}{ Scientific Research: Polar Science and Remote Sensing of Ice Sheets } \\
\hline Author/Company/Email & \multicolumn{2}{|c|}{ Geoffrey Fox, Indiana University gcf@indiana.edu } \\
\hline $\begin{array}{r}\text { Actors/Stakeholders } \\
\text { and their roles and } \\
\text { responsibilities }\end{array}$ & \multicolumn{2}{|c|}{$\begin{array}{l}\text { Research funded by NSF and NASA with relevance to near and long term climate } \\
\text { change. Engineers designing novel radar with "field expeditions" for } 1-2 \text { months to } \\
\text { remote sites. Results used by scientists building models and theories involving Ice } \\
\text { Sheets }\end{array}$} \\
\hline Goals & \multicolumn{2}{|c|}{$\begin{array}{l}\text { Determine the depths of glaciers and snow layers to be fed into higher level scientific } \\
\text { analyses }\end{array}$} \\
\hline Use Case Description & \multicolumn{2}{|c|}{$\begin{array}{l}\text { Build radar; build UAV or use piloted aircraft; overfly remote sites (Arctic, Antarctic, } \\
\text { Himalayas). Check in field that experiments configured correctly with detailed } \\
\text { analysis later. Transport data by air-shipping disk as poor Internet connection. Use } \\
\text { image processing to find ice/snow sheet depths. Use depths in scientific discovery of } \\
\text { melting ice caps etc. }\end{array}$} \\
\hline \multirow[t]{4}{*}{$\begin{array}{r}\text { Current } \\
\text { Solutions }\end{array}$} & Compute(System) & $\begin{array}{l}\text { Field is a low power cluster of rugged laptops plus } \\
\text { classic } 2-4 \text { CPU servers with } \approx 40 \text { TB removable disk } \\
\text { array. Off line is about } 2500 \text { cores }\end{array}$ \\
\hline & Storage & $\begin{array}{l}\text { Removable disk in field. (Disks suffer in field so } 2 \text { copies } \\
\text { made) Lustre or equivalent for offline }\end{array}$ \\
\hline & Networking & Terrible Internet linking field sites to continental USA. \\
\hline & Software & $\begin{array}{l}\text { Radar signal processing in Matlab. Image analysis is } \\
\text { Map/Reduce or MPI plus C/Java. User Interface is a } \\
\text { Geographical Information System }\end{array}$ \\
\hline \multirow[t]{5}{*}{$\begin{array}{r}\text { Big Data } \\
\text { Characteristics }\end{array}$} & $\begin{array}{r}\text { Data Source } \\
\text { (distributed/centralized) } \\
\end{array}$ & $\begin{array}{l}\text { Aircraft flying over ice sheets in carefully planned paths } \\
\text { with data downloaded to disks. }\end{array}$ \\
\hline & Volume (size) & $\approx 0.5$ Petabytes per year raw data \\
\hline & $\begin{array}{r}\text { Velocity } \\
\text { (e.g. real time) }\end{array}$ & $\begin{array}{l}\text { All data gathered in real time but analyzed } \\
\text { incrementally and stored with a GIS interface }\end{array}$ \\
\hline & $\begin{array}{r}\text { Variety } \\
\text { (multiple datasets, } \\
\text { mashup) }\end{array}$ & $\begin{array}{l}\text { Lots of different datasets - each needing custom signal } \\
\text { processing but all similar in structure. This data needs } \\
\text { to be used with wide variety of other polar data. }\end{array}$ \\
\hline & $\begin{array}{r}\text { Variability (rate of } \\
\text { change) }\end{array}$ & $\begin{array}{l}\text { Data accumulated in } \approx 100 \text { TB chunks for each } \\
\text { expedition }\end{array}$ \\
\hline \multirow{5}{*}{$\begin{array}{r}\text { Big Data Science } \\
\text { (collection, curation, } \\
\text { analysis, } \\
\text { action) }\end{array}$} & $\begin{array}{r}\text { Veracity (Robustness } \\
\text { Issues) }\end{array}$ & $\begin{array}{l}\text { Essential to monitor field data and correct instrumental } \\
\text { problems. Implies must analyze fully portion of data in } \\
\text { field }\end{array}$ \\
\hline & Visualization & Rich user interface for layers and glacier simulations \\
\hline & Data Quality & $\begin{array}{l}\text { Main engineering issue is to ensure instrument gives } \\
\text { quality data }\end{array}$ \\
\hline & Data Types & Radar Images \\
\hline & Data Analytics & $\begin{array}{l}\text { Sophisticated signal processing; novel new image } \\
\text { processing to find layers (can be 100's one per year) }\end{array}$ \\
\hline $\begin{array}{l}\text { Big Data Specific } \\
\text { Challenges (Gaps) }\end{array}$ & \multicolumn{2}{|c|}{$\begin{array}{l}\text { Data volumes increasing. Shipping disks clumsy but no other obvious solution. Image } \\
\text { processing algorithms still very active research }\end{array}$} \\
\hline $\begin{array}{r}\text { Big Data Specific } \\
\text { Challenges in Mobility }\end{array}$ & \multicolumn{2}{|c|}{ Smart phone interfaces not essential but LOW power technology essential in field } \\
\hline $\begin{array}{r}\text { Security and Privacy } \\
\text { Requirements }\end{array}$ & \multicolumn{2}{|c|}{$\begin{array}{l}\text { Himalaya studies fraught with political issues and require UAV. Data itself open after } \\
\text { initial study }\end{array}$} \\
\hline
\end{tabular}




\section{Earth, Environmental and Polar Science> Use Case 43: Radar Data Analysis for CReSIS}

\begin{tabular}{|r|l|}
\hline $\begin{array}{r}\text { Highlight issues for } \\
\text { generalizing this use } \\
\text { case (e.g. for ref. } \\
\text { architecture) }\end{array}$ & \\
\hline $\begin{array}{r}\text { More Information } \\
\text { (URLs) }\end{array}$ & $\begin{array}{l}\text { http://polargrid.org/polargrid } \\
\text { https://www.cresis.ku.edu/ }\end{array}$ \\
\hline See movie at http://polargrid.org/polargrid/gallery \\
\hline Note:
\end{tabular}

\begin{tabular}{|l|l|l|l|l|l|}
\hline \multicolumn{1}{|c|}{ Use Case Stages } & \multicolumn{1}{|c|}{ Data Sources } & \multicolumn{1}{|c|}{ Data Usage } & \multicolumn{1}{|c|}{$\begin{array}{l}\text { Transformations } \\
\text { (Data Analytics) }\end{array}$} & \multicolumn{1}{|c|}{$\begin{array}{l}\text { Security } \\
\text { Ind Prastructure }\end{array}$} \\
\hline Radar Data Analysis for CReSIS (Scientific Research: Polar Science and Remote Sensing of Ice Sheets)
\end{tabular}

See Figure 11: Radar Data Analysis for CReSIS Remote Sensing of Ice Sheets- Typical CReSIS radar data after analysis.

See Figure 12: Radar Data Analysis for CReSIS Remote Sensing of Ice Sheets- Typical flight paths of data gathering in survey region.

351 See Figure 13: Radar Data Analysis for CReSIS Remote Sensing of Ice Sheets - Typical echogram with 352 detected boundaries. The upper (green) boundary is between air and ice layers, while the lower (red) 353 boundary is between ice and terrain. 


\section{Earth, Environmental and Polar Science> Use Case 44: UAVSAR Data Processing}

\begin{tabular}{|c|c|c|}
\hline Use Case Title & \multicolumn{2}{|c|}{ UAVSAR Data Processing, Data Product Delivery, and Data Services } \\
\hline Vertical (area) & \multicolumn{2}{|c|}{ Scientific Research: Earth Science } \\
\hline Author/Company/Email & \multicolumn{2}{|c|}{$\begin{array}{l}\text { Andrea Donnellan, NASA JPL, andrea.donnellan@jpl.nasa.gov; Jay Parker, NASA JPL, } \\
\text { jay.w.parker@jpl.nasa.gov }\end{array}$} \\
\hline $\begin{array}{l}\text { Actors/Stakeholders } \\
\text { and their roles and } \\
\text { responsibilities }\end{array}$ & \multicolumn{2}{|c|}{$\begin{array}{l}\text { NASA UAVSAR team, NASA QuakeSim team, ASF (NASA SAR DAAC), USGS, CA } \\
\text { Geological Survey }\end{array}$} \\
\hline Goals & \multicolumn{2}{|c|}{$\begin{array}{l}\text { Use of Synthetic Aperture Radar (SAR) to identify landscape changes caused by } \\
\text { seismic activity, landslides, deforestation, vegetation changes, flooding, etc.; } \\
\text { increase its usability and accessibility by scientists. }\end{array}$} \\
\hline Use Case Description & \multicolumn{2}{|c|}{$\begin{array}{l}\text { A scientist who wants to study the after effects of an earthquake examines multiple } \\
\text { standard SAR products made available by NASA. The scientist may find it useful to } \\
\text { interact with services provided by intermediate projects that add value to the official } \\
\text { data product archive. }\end{array}$} \\
\hline \multirow[t]{4}{*}{$\begin{array}{r}\text { Current } \\
\text { Solutions }\end{array}$} & Compute(System) & $\begin{array}{l}\text { Raw data processing at NASA Ames Pleiades, Endeavour. } \\
\text { Commercial clouds for storage and service front ends } \\
\text { have been explored. }\end{array}$ \\
\hline & Storage & File based. \\
\hline & Networking & $\begin{array}{l}\text { Data require one time transfers between instrument and } \\
\text { JPL, JPL and other NASA computing centers (NASA Ames), } \\
\text { and JPL and ASF. } \\
\text { Individual data files are not too large for individual users } \\
\text { to download, but entire dataset is unwieldy to transfer. } \\
\text { This is a problem to downstream groups like QuakeSim } \\
\text { who want to reformat and add value to datasets. }\end{array}$ \\
\hline & Software & ROI_PAC, GeoServer, GDAL, GeoTIFF-supporting tools. \\
\hline \multirow[t]{5}{*}{$\begin{array}{r}\text { Big Data } \\
\text { Characteristics }\end{array}$} & $\begin{array}{r}\text { Data Source } \\
\text { (distributed/centralized) }\end{array}$ & $\begin{array}{l}\text { Data initially acquired by unmanned aircraft. Initially } \\
\text { processed at NASA JPL. Archive is centralized at ASF } \\
\text { (NASA DAAC). QuakeSim team maintains separate } \\
\text { downstream products (GeoTIFF conversions). }\end{array}$ \\
\hline & Volume (size) & $\begin{array}{l}\text { Repeat Pass Interferometry (RPI) Data: } \approx 3 \text { TB. Increasing } \\
\text { about 1-2 TB/year. } \\
\text { Polarimetric Data: } \approx 40 \text { TB (processed) } \\
\text { Raw Data: } 110 \text { TB } \\
\text { Proposed satellite missions (Earth Radar Mission, } \\
\text { formerly DESDynl) could dramatically increase data } \\
\text { volumes (TBs per day). }\end{array}$ \\
\hline & $\begin{array}{r}\text { Velocity } \\
\text { (e.g. real time) }\end{array}$ & RPI Data: 1-2 TB/year. Polarimetric data is faster. \\
\hline & $\begin{array}{r}\text { Variety } \\
\text { (multiple datasets, } \\
\text { mashup) }\end{array}$ & $\begin{array}{l}\text { Two main types: Polarimetric and RPI. Each RPI product } \\
\text { is a collection of files (annotation file, unwrapped, etc.). } \\
\text { Polarimetric products also consist of several files each. }\end{array}$ \\
\hline & $\begin{array}{r}\text { Variability (rate of } \\
\text { change) }\end{array}$ & $\begin{array}{l}\text { Data products change slowly. Data occasionally get } \\
\text { reprocessed: new processing methods or parameters. } \\
\text { There may be additional quality assurance and quality } \\
\text { control issues. }\end{array}$ \\
\hline
\end{tabular}




\section{Earth, Environmental and Polar Science> Use Case 44: UAVSAR Data Processing}

\begin{tabular}{|c|c|c|}
\hline \multirow[t]{5}{*}{$\begin{array}{r}\text { Big Data Science } \\
\text { (collection, curation, } \\
\text { analysis, } \\
\text { action) }\end{array}$} & $\begin{array}{l}\text { Veracity (Robustness } \\
\text { Issues, semantics) }\end{array}$ & $\begin{array}{l}\text { Provenance issues need to be considered. This } \\
\text { provenance has not been transparent to downstream } \\
\text { consumers in the past. Versioning used now; versions } \\
\text { described in the UAVSAR web page in notes. }\end{array}$ \\
\hline & Visualization & $\begin{array}{l}\text { Uses Geospatial Information System tools, services, } \\
\text { standards. }\end{array}$ \\
\hline & Data Quality (syntax) & $\begin{array}{l}\text { Many frames and collections are found to be unusable } \\
\text { due to unforeseen flight conditions. }\end{array}$ \\
\hline & Data Types & GeoTIFF and related imagery data \\
\hline & Data Analytics & $\begin{array}{l}\text { Done by downstream consumers (such as edge } \\
\text { detections): research issues. }\end{array}$ \\
\hline $\begin{array}{r}\text { Big Data Specific } \\
\text { Challenges (Gaps) }\end{array}$ & \multicolumn{2}{|c|}{$\begin{array}{l}\text { Data processing pipeline requires human inspection and intervention. Limited } \\
\text { downstream data pipelines for custom users. } \\
\text { Cloud architectures for distributing entire data product collections to downstream } \\
\text { consumers should be investigated, adopted. }\end{array}$} \\
\hline $\begin{array}{r}\text { Big Data Specific } \\
\text { Challenges in Mobility }\end{array}$ & \multicolumn{2}{|c|}{$\begin{array}{l}\text { Some users examine data in the field on mobile devices, requiring interactive } \\
\text { reduction of large datasets to understandable images or statistics. }\end{array}$} \\
\hline $\begin{array}{r}\text { Security and Privacy } \\
\text { Requirements }\end{array}$ & \multicolumn{2}{|c|}{ Data is made immediately public after processing (no embargo period). } \\
\hline $\begin{array}{r}\text { Highlight issues for } \\
\text { generalizing this use } \\
\text { case (e.g. for ref. } \\
\text { architecture) }\end{array}$ & \multicolumn{2}{|c|}{$\begin{array}{l}\text { Data is geolocated, and may be angularly specified. Categories: GIS; standard } \\
\text { instrument data processing pipeline to produce standard data products. }\end{array}$} \\
\hline $\begin{array}{r}\text { More Information } \\
\text { (URLs) }\end{array}$ & \multicolumn{2}{|c|}{$\begin{array}{l}\text { http://uavsar.jpl.nasa.gov/, http://www.asf.alaska.edu/program/sdc, } \\
\text { http://quakesim.org }\end{array}$} \\
\hline
\end{tabular}

See Figure 14: UAVSAR Data Processing, Data Product Delivery, and Data Services - Combined unwrapped coseismic interferograms for flight lines 26501, 26505, and 08508 for the October 2009-April 2010 time period. End points where slip can be seen on the Imperial, Superstition Hills, and Elmore Ranch faults are noted. GPS stations are marked by dots and are labeled. 


\section{Earth, Environmental and Polar Science> Use Case 45: NASA LaRC/ GSFC iRODS Federation Testbed}

\begin{tabular}{|c|c|}
\hline Use Case Title & NASA LaRC/GSFC iRODS Federation Testbed \\
\hline Vertical (area) & Earth Science Research and Applications \\
\hline Author/Company/Email & $\begin{array}{l}\text { Michael Little, Roger Dubois, Brandi Quam, Tiffany Mathews, Andrei Vakhnin, Beth } \\
\text { Huffer, Christian Johnson / NASA Langley Research Center (LaRC) / } \\
\text { M.M.Little@NASA.gov, Roger.A.Dubois@nasa.gov, Brandi.M.Quam@NASA.gov, } \\
\text { Tiffany.J.Mathews@NASA.gov, and Andrei.A.Vakhnin@NASA.gov } \\
\text { John Schnase, Daniel Duffy, Glenn Tamkin, Scott Sinno, John Thompson, and Mark } \\
\text { McInerney / NASA Goddard Space Flight Center (GSFC) / John.L.Schnase@NASA.gov, } \\
\text { Daniel.Q.Duffy@NASA.gov, Glenn.S.Tamkin@nasa.gov. Scott.S.Sinno@nasa.gov, } \\
\text { John.H.Thompson@nasa.gov, and Mark.Mcinerney@nasa.gov }\end{array}$ \\
\hline $\begin{array}{r}\text { Actors/Stakeholders } \\
\text { and their roles and } \\
\text { responsibilities }\end{array}$ & $\begin{array}{l}\text { NASA's Atmospheric Science Data Center (ASDC) at Langley Research Center (LaRC) } \\
\text { in Hampton, Virginia, and the Center for Climate Simulation (NCCS) at Goddard Space } \\
\text { Flight Center (GSFC) both ingest, archive, and distribute data that is essential to } \\
\text { stakeholders including the climate research community, science applications } \\
\text { community, and a growing community of government and private-sector customers } \\
\text { who have a need for atmospheric and climatic data. }\end{array}$ \\
\hline Goals & $\begin{array}{l}\text { To implement a data federation ability to improve and automate the discovery of } \\
\text { heterogeneous data, decrease data transfer latency, and meet customizable criteria } \\
\text { based on data content, data quality, metadata, and production. } \\
\text { To support/enable applications and customers that require the integration of } \\
\text { multiple heterogeneous data collections. }\end{array}$ \\
\hline Use Case Description & $\begin{array}{l}\text { ASDC and NCCS have complementary datasets, each containing vast amounts of data } \\
\text { that is not easily shared and queried. Climate researchers, weather forecasters, } \\
\text { instrument teams, and other scientists need to access data from across multiple } \\
\text { datasets in order to compare sensor measurements from various instruments, } \\
\text { compare sensor measurements to model outputs, calibrate instruments, look for } \\
\text { correlations across multiple parameters, etc. To analyze, visualize and otherwise } \\
\text { process data from heterogeneous datasets is currently a time consuming effort that } \\
\text { requires scientists to separately access, search for, and download data from multiple } \\
\text { servers and often the data is duplicated without an understanding of the } \\
\text { authoritative source. Many scientists report spending more time in accessing data } \\
\text { than in conducting research. Data consumers need mechanisms for retrieving } \\
\text { heterogeneous data from a single point-of-access. This can be enabled through the } \\
\text { use of iRODS, a Data grid software system that enables parallel downloads of } \\
\text { datasets from selected replica servers that can be geographically dispersed, but still } \\
\text { accessible by users worldwide. Using iRODS in conjunction with semantically } \\
\text { enhanced metadata, managed via a highly precise Earth Science ontology, the } \\
\text { ASDC's Data Products Online (DPO) will be federated with the data at the NASA } \\
\text { Center for Climate Simulation (NCCS) at Goddard Space Flight Center (GSFC). The } \\
\text { heterogeneous data products at these two NASA facilities are being semantically } \\
\text { annotated using common concepts from the NASA Earth Science ontology. The } \\
\text { semantic annotations will enable the iRODS system to identify complementary } \\
\text { datasets and aggregate data from these disparate sources, facilitating data sharing } \\
\text { between climate modelers, forecasters, Earth scientists, and scientists from other } \\
\text { disciplines that need Earth science data. The iRODS data federation system will also } \\
\text { support cloud-based data processing services in the Amazon Web Services (AWS) } \\
\text { cloud. }\end{array}$ \\
\hline Current & \begin{tabular}{c|l} 
Compute (System) & NASA Center for Climate Simulation (NCCS) and
\end{tabular} \\
\hline
\end{tabular}




\section{Earth, Environmental and Polar Science> Use Case 45: NASA LaRC/ GSFC iRODS Federation Testbed}

\begin{tabular}{|c|c|c|}
\hline \multirow[t]{4}{*}{ Solutions } & & $\begin{array}{l}\text { NASA Atmospheric Science Data Center (ASDC): Two } \\
\text { GPFS systems }\end{array}$ \\
\hline & Storage & $\begin{array}{l}\text { The ASDC's Data Products Online (DPO) GPFS File } \\
\text { system consists of } 12 \times \text { IBM DC } 4800 \text { and } 6 \text { x IBM } \\
\text { DCS3700 Storage subsystems, } 144 \text { Intel } 2.4 \mathrm{GHz} \text { cores, } \\
1,400 \text { TB usable storage. NCCS data is stored in the } \\
\text { NCCS MERRA cluster, which is a } 36 \text { node Dell cluster, } \\
576 \text { Intel } 2.6 \mathrm{GHz} \text { SandyBridge cores, } 1,300 \text { TB raw } \\
\text { storage, } 1,250 \mathrm{~GB} \text { RAM, } 11.7 \text { TF theoretical peak } \\
\text { compute capacity. }\end{array}$ \\
\hline & Networking & $\begin{array}{l}\text { A combination of Fibre Channel SAN and 10GB LAN. } \\
\text { The NCCS cluster nodes are connected by an FDR } \\
\text { Infiniband network with peak TCP/IP speeds }>20 \text { Gbps. }\end{array}$ \\
\hline & Software & $\begin{array}{l}\text { SGE Univa Grid Engine Version 8.1, iRODS version } 3.2 \\
\text { and/or 3.3, IBM General Parallel File System (GPFS) } \\
\text { version 3.4, Cloudera version 4.5.2-1. }\end{array}$ \\
\hline $\begin{array}{r}\text { Big Data } \\
\text { Characteristics }\end{array}$ & $\begin{array}{r}\text { Data Source } \\
\text { (distributed/centralized) }\end{array}$ & $\begin{array}{l}\text { iRODS will be leveraged to share data collected from } \\
\text { CERES Level 3B data products including: CERES EBAF- } \\
\text { TOA and CERES-Surface products. } \\
\text { Surface fluxes in EBAF-Surface are derived from two } \\
\text { CERES data products: } 1 \text { ) CERES SYN1deg-Month Ed3 - } \\
\text { which provides computed surface fluxes to be adjusted } \\
\text { and } 2 \text { ) CERES EBAF-TOA Ed2.7 - which uses } \\
\text { observations to provide CERES-derived TOA flux } \\
\text { constraints. Access to these products will enable the } \\
\text { NCCS at GSFC to run data from the products in a } \\
\text { simulation model in order to produce an assimilated } \\
\text { flux. } \\
\text { The NCCS will introduce Modern-Era Retrospective } \\
\text { Analysis for Research and Applications (MERRA) data to } \\
\text { the iRODS federation. MERRA integrates observational } \\
\text { data with numerical models to produce a global } \\
\text { temporally and spatially consistent synthesis of } 26 \text { key } \\
\text { climate variables. MERRA data files are created from } \\
\text { the Goddard Earth Observing System version } 5 \text { (GEOS- } \\
5 \text { ) model and are stored in HDF-EOS and (Network } \\
\text { Common Data Form) NetCDF formats. } \\
\text { Spatial resolution is } 1 / 2^{\circ} \text { latitude } \times 2 / 3^{\circ} \text { longitude } \times \\
72 \text { vertical levels extending through the stratosphere. } \\
\text { Temporal resolution is } 6 \text {-hours for three-dimensional, } \\
\text { full spatial resolution, extending from } 1979-\text { present, } \\
\text { nearly the entire satellite era. } \\
\text { Each file contains a single grid with multiple } 2 D \text { and } \\
3 D \text { variables. All data are stored on a longitude-latitude } \\
\text { grid with a vertical dimension applicable for all } 3 D \\
\text { variables. The GEOS- } 5 \text { MERRA products are divided into } \\
25 \text { collections: } 18 \text { standard products, chemistry } \\
\text { products. The collections comprise monthly means files } \\
\text { and daily files at six-hour intervals running from } 1979-\end{array}$ \\
\hline
\end{tabular}




\section{Earth, Environmental and Polar Science> Use Case 45: NASA LaRC/ GSFC iRODS Federation Testbed}

\begin{tabular}{|c|c|c|}
\hline & & $\begin{array}{l}\text { 2012. MERRA data are typically packaged as multi- } \\
\text { dimensional binary data within a self-describing NetCDF } \\
\text { file format. Hierarchical metadata in the NetCDF } \\
\text { header contain the representation information that } \\
\text { allows NetCDF- aware software to work with the data. } \\
\text { It also contains arbitrary preservation description and } \\
\text { policy information that can be used to bring the data } \\
\text { into use-specific compliance. }\end{array}$ \\
\hline & Volume (size) & $\begin{array}{l}\text { Currently, Data from the EBAF-TOA Product is about } \\
420 \mathrm{MB} \text { and Data from the EBAF-Surface Product is } \\
\text { about } 690 \mathrm{MB} \text {. Data grows with each version update } \\
\text { (about every six months). The MERRA collection } \\
\text { represents about } 160 \text { TB of total data (uncompressed); } \\
\text { compressed is } \approx 80 \text { TB. }\end{array}$ \\
\hline & $\begin{array}{r}\text { Velocity } \\
\text { (e.g. real time) }\end{array}$ & $\begin{array}{l}\text { Periodic since updates are performed with each new } \\
\text { version update. }\end{array}$ \\
\hline & $\begin{array}{r}\text { Variety } \\
\text { (multiple datasets, } \\
\text { mashup) }\end{array}$ & $\begin{array}{l}\text { There is a need in many types of applications to } \\
\text { combine MERRA reanalysis data with other reanalyses } \\
\text { and observational data such as CERES. The NCCS is } \\
\text { using the Climate Model Intercomparison Project } \\
\text { (CMIP5) Reference standard for ontological alignment } \\
\text { across multiple, disparate datasets. }\end{array}$ \\
\hline & $\begin{array}{r}\text { Variability (rate of } \\
\text { change) }\end{array}$ & $\begin{array}{l}\text { The MERRA reanalysis grows by approximately one TB } \\
\text { per month. }\end{array}$ \\
\hline \multirow[t]{5}{*}{$\begin{array}{r}\text { Big Data Science } \\
\text { (collection, curation, } \\
\text { analysis, } \\
\text { action) }\end{array}$} & $\begin{array}{r}\text { Veracity (Robustness } \\
\text { Issues) }\end{array}$ & $\begin{array}{l}\text { Validation and testing of semantic metadata, and of } \\
\text { federated data products will be provided by data } \\
\text { producers at NASA Langley Research Center and at } \\
\text { Goddard through regular testing. Regression testing } \\
\text { will be implemented to ensure that updates and } \\
\text { changes to the iRODS system, newly added data } \\
\text { sources, or newly added metadata do not introduce } \\
\text { errors to federated data products. MERRA validation is } \\
\text { provided by the data producers, NASA Goddard's } \\
\text { Global Modeling and Assimilation Office (GMAO). }\end{array}$ \\
\hline & Visualization & $\begin{array}{l}\text { There is a growing need in the scientific community for } \\
\text { data management and visualization services that can } \\
\text { aggregate data from multiple sources and display it in a } \\
\text { single graphical display. Currently, such capabilities are } \\
\text { hindered by the challenge of finding and downloading } \\
\text { comparable data from multiple servers, and then } \\
\text { transforming each heterogeneous dataset to make it } \\
\text { usable by the visualization software. Federation of } \\
\text { NASA datasets using iRODS will enable scientists to } \\
\text { quickly find and aggregate comparable datasets for use } \\
\text { with visualization software. }\end{array}$ \\
\hline & Data Quality & $\begin{array}{l}\text { For MERRA, quality controls are applied by the data } \\
\text { producers, GMAO. }\end{array}$ \\
\hline & Data Types & See above. \\
\hline & Data Analytics & Pursuant to the first goal of increasing accessibility and \\
\hline
\end{tabular}




\section{Earth, Environmental and Polar Science> Use Case 45: NASA LaRC/ GSFC iRODS Federation Testbed}

\begin{tabular}{|c|c|}
\hline & $\begin{array}{l}\text { discoverability through innovative technologies, the } \\
\text { ASDC and NCCS are exploring a capability to improve } \\
\text { data access capabilities. Using iRODS, the ASDC's Data } \\
\text { Products Online (DPO) can be federated with data at } \\
\text { GSFC's NCCS creating a data access system that can } \\
\text { serve a much broader customer base than is currently } \\
\text { being served. Federating and sharing information will } \\
\text { enable the ASDC and NCCS to fully utilize multi-year } \\
\text { and multi-instrument data and will improve and } \\
\text { automate the discovery of heterogeneous data, } \\
\text { increase data transfer latency, and meet customizable } \\
\text { criteria based on data content, data quality, metadata, } \\
\text { and production. }\end{array}$ \\
\hline \multicolumn{2}{|l|}{$\begin{array}{l}\text { Big Data Specific } \\
\text { Challenges (Gaps) }\end{array}$} \\
\hline $\begin{array}{r}\text { Big Data Specific } \\
\text { Challenges in Mobility }\end{array}$ & $\begin{array}{l}\text { A major challenge includes defining an enterprise architecture that can deliver real- } \\
\text { time analytics via communication with multiple APIs and cloud computing systems. } \\
\text { By keeping the computation resources on cloud systems, the challenge with mobility } \\
\text { resides in not overpowering mobile devices with displaying CPU intensive } \\
\text { visualizations that may hinder the performance or usability of the data being } \\
\text { presented to the user. }\end{array}$ \\
\hline \multicolumn{2}{|l|}{$\begin{array}{r}\text { Security and Privacy } \\
\text { Requirements }\end{array}$} \\
\hline $\begin{array}{r}\text { Highlight issues for } \\
\text { generalizing this use } \\
\text { case (e.g. for ref. } \\
\text { architecture) }\end{array}$ & $\begin{array}{l}\text { This federation builds on several years of iRODS research and development } \\
\text { performed at the NCCS. During this time, the NCCS vetted the iRODS features while } \\
\text { extending its core functions with domain-specific extensions. For example, the NCCS } \\
\text { created and installed Python-based scientific kits within iRODS that automatically } \\
\text { harvest metadata when the associated data collection is registered. One of these } \\
\text { scientific kits was developed for the MERRA collection. This kit in conjunction with } \\
\text { iRODS bolsters the strength of the LaRC/GSFC federation by providing advanced } \\
\text { search capabilities. LaRC is working through the establishment of an advanced } \\
\text { architecture that leverages multiple technology pilots and tools (access, discovery, } \\
\text { and analysis) designed to integrate capabilities across the earth science community - } \\
\text { the research and development completed by both data centers is complementary } \\
\text { and only further enhances this use case. } \\
\text { Other scientific kits that have been developed include: NetCDF, Intergovernmental } \\
\text { Panel on Climate Change (IPCC), and Ocean Modeling and Data Assimilation (ODAS). } \\
\text { The combination of iRODS and these scientific kits has culminated in a configurable } \\
\text { technology stack called the virtual Climate Data Server (vCDS), meaning that this } \\
\text { runtime environment can be deployed to multiple destinations (e.g., bare metal, } \\
\text { virtual servers, cloud) to support various scientific needs. The vCDS, which can be } \\
\text { viewed as a reference architecture for easing the federation of disparate data } \\
\text { repositories, is leveraged by but not limited to LaRC and GSFC. }\end{array}$ \\
\hline $\begin{array}{r}\text { More Information } \\
\text { (URLs) }\end{array}$ & Please contact the authors for additional information. \\
\hline
\end{tabular}




\section{Earth, Environmental and Polar Science> Use Case 46: MERRA Analytic Services}

\begin{tabular}{|c|c|c|}
\hline Use Case Title & \multicolumn{2}{|c|}{ MERRA Analytic Services (MERRA/AS) } \\
\hline Vertical (area) & \multicolumn{2}{|c|}{ Scientific Research: Earth Science } \\
\hline Author/Company/Email & \multicolumn{2}{|c|}{$\begin{array}{l}\text { John L. Schnase and Daniel Q. Duffy / NASA Goddard Space Flight Center } \\
\text { John.L.Schnase@NASA.gov, Daniel.Q.Duffy@NASA.gov }\end{array}$} \\
\hline $\begin{array}{r}\text { Actors/Stakeholders } \\
\text { and their roles and } \\
\text { responsibilities }\end{array}$ & \multicolumn{2}{|c|}{$\begin{array}{l}\text { NASA's Modern-Era Retrospective Analysis for Research and Applications (MERRA) } \\
\text { integrates observational data with numerical models to produce a global temporally } \\
\text { and spatially consistent synthesis of } 26 \text { key climate variables. Actors and } \\
\text { stakeholders who have an interest in MERRA include the climate research } \\
\text { community, science applications community, and a growing number of government } \\
\text { and private-sector customers who have a need for the MERRA data in their decision } \\
\text { support systems. }\end{array}$} \\
\hline Goals & \multicolumn{2}{|c|}{$\begin{array}{l}\text { Increase the usability and use of large-scale scientific data collections, such as } \\
\text { MERRA. }\end{array}$} \\
\hline Use Case Description & \multicolumn{2}{|c|}{$\begin{array}{l}\text { MERRA Analytic Services enables Map/Reduce analytics over the MERRA collection. } \\
\text { MERRA/AS is an example of cloud-enabled climate analytics as a service (CAaS), } \\
\text { which is an approach to meeting the Big Data challenges of climate science through } \\
\text { the combined use of 1) high performance, data proximal analytics, (2) scalable data } \\
\text { management, (3) software appliance virtualization, (4) adaptive analytics, and (5) a } \\
\text { domain-harmonized API. The effectiveness of MERRA/AS is being demonstrated in } \\
\text { several applications, including data publication to the Earth System Grid Federation } \\
\text { (ESGF) in support of Intergovernmental Panel on Climate Change (IPCC) research, the } \\
\text { NASA/Department of Interior RECOVER wild land fire decision support system, and } \\
\text { data interoperability testbed evaluations between NASA Goddard Space Flight } \\
\text { Center and the NASA Langley Atmospheric Data Center. }\end{array}$} \\
\hline \multirow{4}{*}{$\begin{array}{r}\text { Current } \\
\text { Solutions }\end{array}$} & Compute(System) & NASA Center for Climate Simulation (NCCS) \\
\hline & Storage & $\begin{array}{l}\text { The MERRA Analytic Services Hadoop Filesystem (HDFS) } \\
\text { is a } 36 \text { node Dell cluster, } 576 \text { Intel } 2.6 \mathrm{GHz} \text { SandyBridge } \\
\text { cores, } 1300 \text { TB raw storage, } 1250 \mathrm{~GB} \text { RAM, } 11.7 \text { TF } \\
\text { theoretical peak compute capacity. }\end{array}$ \\
\hline & Networking & $\begin{array}{l}\text { Cluster nodes are connected by an FDR Infiniband } \\
\text { network with peak TCP/IP speeds }>20 \text { Gbps. }\end{array}$ \\
\hline & Software & Cloudera, iRODS, Amazon AWS \\
\hline $\begin{array}{r}\text { Big Data } \\
\text { Characteristics }\end{array}$ & $\begin{array}{r}\text { Data Source } \\
\text { (distributed/centralized) }\end{array}$ & $\begin{array}{l}\text { MERRA data files are created from the GEOS- } 5 \text { model } \\
\text { and are stored in HDF-EOS and NetCDF formats. Spatial } \\
\text { resolution is } 1 / 2{ }^{\circ} \text { latitude } \times 2 / 3 \text { longitude } \times 72 \text { vertical } \\
\text { levels extending through the stratosphere. Temporal } \\
\text { resolution is } 6 \text {-hours for three-dimensional, full spatial } \\
\text { resolution, extending from } 1979 \text {-present, nearly the } \\
\text { entire satellite era. Each file contains a single grid with } \\
\text { multiple } 2 D \text { and 3D variables. All data are stored on a } \\
\text { longitude latitude grid with a vertical dimension } \\
\text { applicable for all 3D variables. The GEOS- } 5 \text { MERRA } \\
\text { products are divided into } 25 \text { collections: } 18 \text { standard } \\
\text { products, } 7 \text { chemistry products. The collections } \\
\text { comprise monthly means files and daily files at six-hour } \\
\text { intervals running from } 1979-2012 \text {. MERRA data are } \\
\text { typically packaged as multi-dimensional binary data } \\
\text { within a self-describing NetCDF file format. Hierarchical } \\
\text { metadata in the NetCDF header contain the }\end{array}$ \\
\hline
\end{tabular}




\section{Earth, Environmental and Polar Science> Use Case 46: MERRA Analytic Services}

\begin{tabular}{|c|c|c|}
\hline & & $\begin{array}{l}\text { representation information that allows NetCDF aware } \\
\text { software to work with the data. It also contains arbitrary } \\
\text { preservation description and policy information that can } \\
\text { be used to bring the data into use-specific compliance. }\end{array}$ \\
\hline & Volume (size) & 480TB \\
\hline & $\begin{array}{r}\text { Velocity } \\
\text { (e.g. real time) }\end{array}$ & $\begin{array}{l}\text { Real-time or batch, depending on the analysis. We're } \\
\text { developing a set of "canonical ops" -early stage, near- } \\
\text { data operations common to many analytic workflows. } \\
\text { The goal is for the canonical ops to run in near real-time. }\end{array}$ \\
\hline & $\begin{array}{r}\text { Variety } \\
\text { (multiple datasets, } \\
\text { mashup) }\end{array}$ & $\begin{array}{l}\text { There is a need in many types of applications to } \\
\text { combine MERRA reanalysis data with other re-analyses } \\
\text { and observational data. We are using the Climate Model } \\
\text { Inter-comparison Project (CMIP5) Reference standard } \\
\text { for ontological alignment across multiple, disparate } \\
\text { datasets. }\end{array}$ \\
\hline & $\begin{array}{r}\text { Variability (rate of } \\
\text { change) }\end{array}$ & $\begin{array}{l}\text { The MERRA reanalysis grows by approximately one TB } \\
\text { per month. }\end{array}$ \\
\hline $\begin{array}{l}\text { Big Data Science } \\
\text { (collection, curation, }\end{array}$ & $\begin{array}{r}\text { Veracity (Robustness } \\
\text { Issues, semantics) }\end{array}$ & $\begin{array}{l}\text { Validation provided by data producers, NASA Goddard's } \\
\text { Global Modeling and Assimilation Office (GMAO). }\end{array}$ \\
\hline $\begin{array}{r}\text { analysis, } \\
\text { action) }\end{array}$ & Visualization & $\begin{array}{l}\text { There is a growing need for distributed visualization of } \\
\text { analytic outputs. }\end{array}$ \\
\hline & Data Quality (syntax) & Quality controls applied by data producers, GMAO. \\
\hline & Data Types & See above. \\
\hline & Data Analytics & $\begin{array}{l}\text { In our efforts to address the Big Data challenges of } \\
\text { climate science, we are moving toward a notion of } \\
\text { climate analytics-as-a-service. We focus on analytics, } \\
\text { because it is the knowledge gained from our } \\
\text { interactions with Big Data that ultimately produce } \\
\text { societal benefits. We focus on CAaaS because we } \\
\text { believe it provides a useful way of thinking about the } \\
\text { problem: a specialization of the concept of business } \\
\text { process-as-a-service, which is an evolving extension of } \\
\text { laaS, PaaS, and SaaS enabled by Cloud Computing. }\end{array}$ \\
\hline $\begin{array}{l}\text { Big Data Specific } \\
\text { Challenges (Gaps) }\end{array}$ & \multicolumn{2}{|c|}{$\begin{array}{l}\text { A big question is how to use cloud computing to enable better use of climate } \\
\text { science's earthbound compute and data resources. Cloud Computing is providing for } \\
\text { us a new tier in the data services stack - a cloud-based layer where agile } \\
\text { customization occurs and enterprise-level products are transformed to meet the } \\
\text { specialized requirements of applications and consumers. It helps us close the gap } \\
\text { between the world of traditional, high-performance computing, which, at least for } \\
\text { now, resides in a finely-tuned climate modeling environment at the enterprise level } \\
\text { and our new customers, whose expectations and manner of work are increasingly } \\
\text { influenced by the smart mobility megatrend. }\end{array}$} \\
\hline $\begin{array}{r}\text { Big Data Specific } \\
\text { Challenges in Mobility }\end{array}$ & \multicolumn{2}{|c|}{$\begin{array}{l}\text { Most modern smartphones, tablets, etc. actually consist of just the display and user } \\
\text { interface components of sophisticated applications that run in cloud data centers. } \\
\text { This is a mode of work that CAaaS is intended to accommodate. }\end{array}$} \\
\hline $\begin{array}{r}\text { Security and Privacy } \\
\text { Requirements }\end{array}$ & \multicolumn{2}{|c|}{ No critical issues identified at this time. } \\
\hline $\begin{array}{l}\text { Highlight issues for } \\
\text { generalizing this use }\end{array}$ & \multicolumn{2}{|c|}{$\begin{array}{l}\text { Map/Reduce and iRODS fundamentally make analytics and data aggregation easier; } \\
\text { our approach to software appliance virtualization in makes it easier to transfer }\end{array}$} \\
\hline
\end{tabular}




\section{Earth, Environmental and Polar Science> Use Case 46: MERRA Analytic Services}

\begin{tabular}{|r|l|}
\hline $\begin{array}{r}\text { case (e.g. for ref. } \\
\text { architecture) }\end{array}$ & $\begin{array}{l}\text { capabilities to new users and simplifies their ability to build new applications; the } \\
\text { social construction of extended capabilities facilitated by the notion of canonical } \\
\text { operations enable adaptability; and the Climate Data Services API that we're } \\
\text { developing enables ease of mastery. Taken together, we believe that these core } \\
\text { technologies behind CAaaS creates a generative context where inputs from diverse } \\
\text { people and groups, who may or may not be working in concert, can contribute } \\
\text { capabilities that help address the Big Data challenges of climate science. }\end{array}$ \\
\hline $\begin{array}{r}\text { More Information } \\
\text { (URLs) }\end{array}$ & Please contact the authors for additional information. \\
\hline
\end{tabular}




\section{Earth, Environmental and Polar Science> Use Case 47: Atmospheric Turbulence-Event Discovery}

\begin{tabular}{|c|c|c|}
\hline Use Case Title & \multicolumn{2}{|c|}{ Atmospheric Turbulence - Event Discovery and Predictive Analytics } \\
\hline Vertical (area) & \multicolumn{2}{|c|}{ Scientific Research: Earth Science } \\
\hline Author/Company/Email & \multicolumn{2}{|c|}{ Michael Seablom, NASA Headquarters, michael.s.seablom@nasa.gov } \\
\hline $\begin{array}{r}\text { Actors/Stakeholders } \\
\text { and their roles and } \\
\text { responsibilities }\end{array}$ & \multicolumn{2}{|c|}{$\begin{array}{l}\text { Researchers with NASA or NSF grants, weather forecasters, aviation interests (for the } \\
\text { generalized case, any researcher who has a role in studying phenomena-based } \\
\text { events). }\end{array}$} \\
\hline Goals & \multicolumn{2}{|c|}{$\begin{array}{l}\text { Enable the discovery of high-impact phenomena contained within voluminous Earth } \\
\text { Science data stores and which are difficult to characterize using traditional numerical } \\
\text { methods (e.g., turbulence). Correlate such phenomena with global atmospheric re- } \\
\text { analysis products to enhance predictive capabilities. }\end{array}$} \\
\hline Use Case Description & \multicolumn{2}{|c|}{$\begin{array}{l}\text { Correlate aircraft reports of turbulence (either from pilot reports or from automated } \\
\text { aircraft measurements of eddy dissipation rates) with recently completed } \\
\text { atmospheric re-analyses of the entire satellite-observing era. Reanalysis products } \\
\text { include the North American Regional Reanalysis (NARR) and the Modern-Era } \\
\text { Retrospective-Analysis for Research (MERRA) from NASA. }\end{array}$} \\
\hline \multirow{4}{*}{$\begin{array}{r}\text { Current } \\
\text { Solutions }\end{array}$} & Compute(System) & NASA Earth Exchange (NEX) - Pleiades supercomputer. \\
\hline & Storage & $\begin{array}{l}\text { Re-analysis products are on the order of 100TB each; } \\
\text { turbulence data are negligible in size. }\end{array}$ \\
\hline & Networking & $\begin{array}{l}\text { Re-analysis datasets are likely to be too large to } \\
\text { relocate to the supercomputer of choice (in this case } \\
\text { NEX), therefore the fastest networking possible would } \\
\text { be needed. }\end{array}$ \\
\hline & Software & $\begin{array}{l}\text { Map/Reduce or the like; SciDB or other scientific } \\
\text { database. }\end{array}$ \\
\hline \multirow[t]{5}{*}{$\begin{array}{r}\text { Big Data } \\
\text { Characteristics }\end{array}$} & $\begin{array}{r}\text { Data Source } \\
\text { (distributed/centralized) }\end{array}$ & Distributed \\
\hline & Volume (size) & 200TB (current), 500TB within 5 years \\
\hline & $\begin{array}{r}\text { Velocity } \\
\text { (e.g. real time) }\end{array}$ & Data analyzed incrementally \\
\hline & $\begin{array}{r}\text { Variety } \\
\text { (multiple datasets, } \\
\text { mashup) }\end{array}$ & $\begin{array}{l}\text { Re-analysis datasets are inconsistent in format, } \\
\text { resolution, semantics, and metadata. Likely each of } \\
\text { these input streams will have to be } \\
\text { interpreted/analyzed into a common product. }\end{array}$ \\
\hline & $\begin{array}{r}\text { Variability (rate of } \\
\text { change) }\end{array}$ & $\begin{array}{l}\text { Turbulence observations would be updated } \\
\text { continuously; re-analysis products are released about } \\
\text { once every five years. }\end{array}$ \\
\hline \multirow{5}{*}{$\begin{array}{r}\text { Big Data Science } \\
\text { (collection, curation, } \\
\text { analysis, } \\
\text { action) }\end{array}$} & $\begin{array}{r}\text { Veracity (Robustness } \\
\text { Issues) }\end{array}$ & $\begin{array}{l}\text { Validation would be necessary for the output product } \\
\text { (correlations). }\end{array}$ \\
\hline & Visualization & Useful for interpretation of results. \\
\hline & Data Quality & $\begin{array}{l}\text { Input streams would have already been subject to } \\
\text { quality control. }\end{array}$ \\
\hline & Data Types & $\begin{array}{l}\text { Gridded output from atmospheric data assimilation } \\
\text { systems and textual data from turbulence } \\
\text { observations. }\end{array}$ \\
\hline & Data Analytics & $\begin{array}{l}\text { Event-specification language needed to perform data } \\
\text { mining / event searches. }\end{array}$ \\
\hline $\begin{array}{r}\text { Big Data Specific } \\
\text { Challenges (Gaps) }\end{array}$ & \multicolumn{2}{|c|}{$\begin{array}{l}\text { Semantics (interpretation of multiple reanalysis products); data movement; } \\
\text { database(s) with optimal structuring for 4-dimensional data mining. }\end{array}$} \\
\hline
\end{tabular}




\section{Earth, Environmental and Polar Science> Use Case 47: Atmospheric Turbulence-Event Discovery}

\begin{tabular}{|r|l|}
\hline $\begin{array}{r}\text { Big Data Specific } \\
\text { Challenges in Mobility }\end{array}$ & Development for mobile platforms not essential at this time. \\
\hline $\begin{array}{r}\text { Security and Privacy } \\
\text { Requirements }\end{array}$ & No critical issues identified. \\
\hline $\begin{array}{r}\text { Highlight issues for } \\
\text { generalizing this use } \\
\text { case (e.g. for ref. } \\
\text { architecture) }\end{array}$ & $\begin{array}{l}\text { Atmospheric turbulence is only one of many phenomena-based events that could be } \\
\text { useful for understanding anomalies in the atmosphere or the ocean that are } \\
\text { connected over long distances in space and time. However the process has limits to } \\
\text { extensibility, i.e., each phenomena may require very different processes for data } \\
\text { mining and predictive analysis. }\end{array}$ \\
\hline $\begin{array}{r}\text { More Information } \\
\text { (URLs) }\end{array}$ & $\begin{array}{l}\text { http://oceanworld.tamu.edu/resources/oceanography-book/teleconnections.htm } \\
\text { http://www.forbes.com/sites/toddwoody/2012/03/21/meet-the-scientists-mining- } \\
\text { big-data-to-predict-the-weather/ }\end{array}$ \\
\hline
\end{tabular}

See Figure 16: Atmospheric Turbulence - Event Discovery and Predictive Analytics (Section 2.9.7) 367 Typical NASA image of turbulent waves 


\section{Earth, Environmental and Polar Science> Use Case 48: Climate Studies using the Community Earth System Model}

\begin{tabular}{|c|c|c|}
\hline Use Case Title & \multicolumn{2}{|c|}{ Climate Studies using the Community Earth System Model at DOE's NERSC center } \\
\hline Vertical (area) & \multicolumn{2}{|c|}{ Research: Climate } \\
\hline Author/Company/Email & \multicolumn{2}{|c|}{ PI: Warren Washington, NCAR } \\
\hline $\begin{array}{r}\text { Actors/Stakeholders } \\
\text { and their roles and } \\
\text { responsibilities }\end{array}$ & \multicolumn{2}{|c|}{ Climate scientists, U.S. policy makers } \\
\hline Goals & \multicolumn{2}{|c|}{$\begin{array}{l}\text { The goals of the Climate Change Prediction (CCP) group at NCAR are to understand } \\
\text { and quantify contributions of natural and anthropogenic-induced patterns of climate } \\
\text { variability and change in the 20th and 21st centuries by means of simulations with } \\
\text { the Community Earth System Model (CESM). }\end{array}$} \\
\hline Use Case Description & \multicolumn{2}{|c|}{$\begin{array}{l}\text { With these model simulations, researchers are able to investigate mechanisms of } \\
\text { climate variability and change, as well as to detect and attribute past climate } \\
\text { changes, and to project and predict future changes. The simulations are motivated } \\
\text { by broad community interest and are widely used by the national and international } \\
\text { research communities. }\end{array}$} \\
\hline \multirow{4}{*}{$\begin{array}{r}\text { Current } \\
\text { Solutions }\end{array}$} & Compute(System) & NERSC (24M Hours), DOE LCF (41M), NCAR CSL (17M) \\
\hline & Storage & 1.5 PB at NERSC \\
\hline & Networking & ESNet \\
\hline & Software & $\begin{array}{l}\text { NCAR PIO library and utilities NCL and NCO, parallel } \\
\text { NetCDF }\end{array}$ \\
\hline \multirow[t]{4}{*}{$\begin{array}{r}\text { Big Data } \\
\text { Characteristics }\end{array}$} & $\begin{array}{r}\text { Data Source } \\
\text { (distributed/centralized) }\end{array}$ & $\begin{array}{l}\text { Data is produced at computing centers. The Earth } \\
\text { Systems Grid is an open source effort providing a robust, } \\
\text { distributed data and computation platform, enabling } \\
\text { world wide access to Peta/Exa-scale scientific data. ESGF } \\
\text { manages the first-ever decentralized database for } \\
\text { handling climate science data, with multiple petabytes } \\
\text { of data at dozens of federated sites worldwide. It is } \\
\text { recognized as the leading infrastructure for the } \\
\text { management and access of large distributed data } \\
\text { volumes for climate change research. It supports the } \\
\text { Coupled Model Intercomparison Project (CMIP), whose } \\
\text { protocols enable the periodic assessments carried out } \\
\text { by the Intergovernmental Panel on Climate Change } \\
\text { (IPCC). }\end{array}$ \\
\hline & Volume (size) & $\begin{array}{l}30 \text { PB at NERSC (assuming } 15 \text { end-to-end climate change } \\
\text { experiments) in 2017; many times more worldwide }\end{array}$ \\
\hline & $\begin{array}{r}\text { Velocity } \\
\text { (e.g. real time) } \\
\end{array}$ & $42 \mathrm{~GB} / \mathrm{s}$ are produced by the simulations \\
\hline & $\begin{array}{r}\text { Variety } \\
\text { (multiple datasets, } \\
\text { mashup) }\end{array}$ & $\begin{array}{l}\text { Data must be compared among those from } \\
\text { observations, historical reanalysis, and a number of } \\
\text { independently produced simulations. The Program for } \\
\text { Climate Model Diagnosis and Intercomparison develops } \\
\text { methods and tools for the diagnosis and inter- } \\
\text { comparison of general circulation models (GCMs) that } \\
\text { simulate the global climate. The need for innovative } \\
\text { analysis of GCM climate simulations is apparent, as } \\
\text { increasingly more complex models are developed, while } \\
\text { the disagreements among these simulations and relative } \\
\text { to climate observations remain significant and poorly }\end{array}$ \\
\hline
\end{tabular}




\section{Earth, Environmental and Polar Science> Use Case 48: Climate Studies using the Community Earth System Model}

\begin{tabular}{|c|c|c|}
\hline & & $\begin{array}{l}\text { understood. The nature and causes of these } \\
\text { disagreements must be accounted for in a systematic } \\
\text { fashion in order to confidently use GCMs for simulation } \\
\text { of putative global climate change. }\end{array}$ \\
\hline & $\begin{array}{r}\text { Variability (rate of } \\
\text { change) }\end{array}$ & $\begin{array}{l}\text { Data is produced by codes running at supercomputer } \\
\text { centers. During runtime, intense periods of data i/O } \\
\text { occur regularly, but typically consume only a few } \\
\text { percent of the total run time. Runs are carried out } \\
\text { routinely, but spike as deadlines for reports approach. }\end{array}$ \\
\hline $\begin{array}{r}\text { Big Data Science } \\
\text { (collection, curation, } \\
\text { analysis, } \\
\text { action) }\end{array}$ & $\begin{array}{l}\text { Veracity (Robustness } \\
\text { Issues) and Quality }\end{array}$ & $\begin{array}{l}\text { Data produced by climate simulations is plays a large } \\
\text { role in informing discussion of climate change } \\
\text { simulations. Therefore, it must be robust, both from the } \\
\text { standpoint of providing a scientifically valid } \\
\text { representation of processes that influence climate, but } \\
\text { also as that data is stored long term and transferred } \\
\text { world-wide to collaborators and other scientists. }\end{array}$ \\
\hline & Visualization & $\begin{array}{l}\text { Visualization is crucial to understanding a system as } \\
\text { complex as the Earth ecosystem. }\end{array}$ \\
\hline & Data Types & $\begin{array}{l}\text { Earth system scientists are being inundated by an } \\
\text { explosion of data generated by ever-increasing } \\
\text { resolution in both global models and remote sensors. }\end{array}$ \\
\hline & Data Analytics & $\begin{array}{l}\text { There is a need to provide data reduction and analysis } \\
\text { web services through the Earth System Grid (ESG). A } \\
\text { pressing need is emerging for data analysis capabilities } \\
\text { closely linked to data archives. }\end{array}$ \\
\hline $\begin{array}{r}\text { Big Data Specific } \\
\text { Challenges (Gaps) }\end{array}$ & \multicolumn{2}{|c|}{$\begin{array}{l}\text { The rapidly growing size of datasets makes scientific analysis a challenge. The need } \\
\text { to write data from simulations is outpacing supercomputers' ability to accommodate } \\
\text { this need. }\end{array}$} \\
\hline $\begin{array}{r}\text { Big Data Specific } \\
\text { Challenges in Mobility }\end{array}$ & \multicolumn{2}{|c|}{$\begin{array}{l}\text { Data from simulations and observations must be shared among a large widely } \\
\text { distributed community. }\end{array}$} \\
\hline \multicolumn{3}{|l|}{$\begin{array}{r}\text { Security and Privacy } \\
\text { Requirements }\end{array}$} \\
\hline $\begin{array}{r}\text { Highlight issues for } \\
\text { generalizing this use } \\
\text { case (e.g. for ref. } \\
\text { architecture) }\end{array}$ & \multicolumn{2}{|c|}{$\begin{array}{l}\text { ESGF is in the early stages of being adapted for use in two additional domains: } \\
\text { biology (to accelerate drug design and development) and energy (infrastructure for } \\
\text { California Energy Systems for the 21st Century (CES21)). }\end{array}$} \\
\hline $\begin{array}{r}\text { More Information } \\
\text { (URLs) }\end{array}$ & \multicolumn{2}{|c|}{$\begin{array}{l}\text { http://esgf.org/ } \\
\text { http://www-pcmdi.IInl.gov/ } \\
\text { http://www.nersc.gov/ } \\
\text { http://science.energy.gov/ber/research/cesd/ } \\
\text { http://www2.cisl.ucar.edu/ }\end{array}$} \\
\hline
\end{tabular}




\section{Earth, Environmental and Polar Science> Use Case 49: Subsurface Biogeochemistry}

\begin{tabular}{|c|c|c|}
\hline Use Case Title & \multicolumn{2}{|c|}{ DOE-BER Subsurface Biogeochemistry Scientific Focus Area } \\
\hline Vertical (area) & \multicolumn{2}{|c|}{ Research: Earth Science } \\
\hline Author/Company/Email & \multicolumn{2}{|c|}{ Deb Agarwal, Lawrence Berkeley Lab. daagarwal@lbl.gov } \\
\hline $\begin{array}{r}\text { Actors/Stakeholders } \\
\text { and their roles and } \\
\text { responsibilities }\end{array}$ & \multicolumn{2}{|c|}{$\begin{array}{l}\text { LBNL Sustainable Systems SFA 2.0, Subsurface Scientists, Hydrologists, Geophysicists, } \\
\text { Genomics Experts, JGI, Climate scientists, and DOE SBR. }\end{array}$} \\
\hline Goals & \multicolumn{2}{|c|}{$\begin{array}{l}\text { The Sustainable Systems Scientific Focus Area } 2.0 \text { Science Plan ("SFA 2.0") has been } \\
\text { developed to advance predictive understanding of complex and multiscale terrestrial } \\
\text { environments relevant to the DOE mission through specifically considering the } \\
\text { scientific gaps defined above. }\end{array}$} \\
\hline Use Case Description & \multicolumn{2}{|c|}{$\begin{array}{l}\text { Development of a Genome-Enabled Watershed Simulation Capability (GEWaSC) that } \\
\text { will provide a predictive framework for understanding how genomic information } \\
\text { stored in a subsurface microbiome affects biogeochemical watershed functioning, } \\
\text { how watershed-scale processes affect microbial functioning, and how these } \\
\text { interactions co-evolve. While modeling capabilities developed by our team and } \\
\text { others in the community have represented processes occurring over an impressive } \\
\text { range of scales (ranging from a single bacterial cell to that of a contaminant plume), } \\
\text { to date little effort has been devoted to developing a framework for systematically } \\
\text { connecting scales, as is needed to identify key controls and to simulate important } \\
\text { feedbacks. A simulation framework that formally scales from genomes to watersheds } \\
\text { is the primary focus of this GEWaSC deliverable. }\end{array}$} \\
\hline \multirow{4}{*}{$\begin{array}{l}\text { Current } \\
\text { Solutions }\end{array}$} & Compute(System) & NERSC \\
\hline & Storage & NERSC \\
\hline & Networking & ESNet \\
\hline & Software & PFLOWTran, postgres, HDF5, Akuna, NEWT, etc. \\
\hline \multirow[t]{5}{*}{$\begin{array}{r}\text { Big Data } \\
\text { Characteristics }\end{array}$} & $\begin{array}{r}\text { Data Source } \\
\text { (distributed/centralized) }\end{array}$ & $\begin{array}{l}\text { Terabase-scale sequencing data from JGI, subsurface } \\
\text { and surface hydrological and biogeochemical data from } \\
\text { a variety of sensors (including dense geophysical } \\
\text { datasets) experimental data from field and lab analysis }\end{array}$ \\
\hline & Volume (size) & \\
\hline & $\begin{array}{r}\text { Velocity } \\
\text { (e.g. real time) }\end{array}$ & \\
\hline & $\begin{array}{r}\text { Variety } \\
\text { (multiple datasets, } \\
\text { mashup) }\end{array}$ & $\begin{array}{l}\text { Data crosses all scales from genomics of the microbes in } \\
\text { the soil to watershed hydro-biogeochemistry. The SFA } \\
\text { requires the synthesis of diverse and disparate field, } \\
\text { laboratory, and simulation datasets across different } \\
\text { semantic, spatial, and temporal scales through GEWaSC. } \\
\text { Such datasets will be generated by the different } \\
\text { research areas and include simulation data, field data } \\
\text { (hydrological, geochemical, geophysical), 'omics data, } \\
\text { and data from laboratory experiments. }\end{array}$ \\
\hline & $\begin{array}{r}\text { Variability (rate of } \\
\text { change) }\end{array}$ & Simulations and experiments \\
\hline $\begin{array}{r}\text { Big Data Science } \\
\text { (collection, curation, } \\
\text { analysis, } \\
\text { action) }\end{array}$ & $\begin{array}{l}\text { Veracity (Robustness } \\
\text { Issues) and Quality }\end{array}$ & $\begin{array}{l}\text { Each of the sources samples different properties with } \\
\text { different footprints - extremely heterogeneous. Each of } \\
\text { the sources has different levels of uncertainty and } \\
\text { precision associated with it. In addition, the translation } \\
\text { across scales and domains introduces uncertainty as } \\
\text { does the data mining. Data quality is critical. }\end{array}$ \\
\hline
\end{tabular}




\section{Earth, Environmental and Polar Science> Use Case 49: Subsurface Biogeochemistry}

\begin{tabular}{|c|c|c|}
\hline & Visualization & Visualization is crucial to understanding the data. \\
\hline & Data Types & Described in "Variety" above. \\
\hline & Data Analytics & $\begin{array}{l}\text { Data mining, data quality assessment, cross-correlation } \\
\text { across datasets, reduced model development, statistics, } \\
\text { quality assessment, data fusion, etc. }\end{array}$ \\
\hline $\begin{array}{l}\text { Big Data Specific } \\
\text { Challenges (Gaps) }\end{array}$ & \multicolumn{2}{|c|}{ Translation across diverse and large datasets that cross domains and scales. } \\
\hline $\begin{array}{r}\text { Big Data Specific } \\
\text { Challenges in Mobility }\end{array}$ & \multicolumn{2}{|c|}{$\begin{array}{l}\text { Field experiment data taking would be improved by access to existing data and } \\
\text { automated entry of new data via mobile devices. }\end{array}$} \\
\hline $\begin{array}{r}\text { Security and Privacy } \\
\text { Requirements }\end{array}$ & & \\
\hline $\begin{array}{r}\text { Highlight issues for } \\
\text { generalizing this use } \\
\text { case (e.g. for ref. } \\
\text { architecture) }\end{array}$ & \multicolumn{2}{|c|}{$\begin{array}{l}\text { A wide array of programs in the earth sciences are working on challenges that cross } \\
\text { the same domains as this project. }\end{array}$} \\
\hline $\begin{array}{r}\text { More Information } \\
\text { (URLs) }\end{array}$ & \multicolumn{2}{|l|}{ Under development } \\
\hline
\end{tabular}




\section{Earth, Environmental and Polar Science> Use Case 50: Ameriflux and FLUXNET}

\begin{tabular}{|c|c|c|}
\hline Use Case Title & \multicolumn{2}{|c|}{ DOE-BER AmeriFlux and FLUXNET Networks } \\
\hline Vertical (area) & \multicolumn{2}{|c|}{ Research: Earth Science } \\
\hline Author/Company/Email & \multicolumn{2}{|c|}{ Deb Agarwal, Lawrence Berkeley Lab. daagarwal@|bl.gov } \\
\hline $\begin{array}{r}\text { Actors/Stakeholders } \\
\text { and their roles and } \\
\text { responsibilities }\end{array}$ & \multicolumn{2}{|c|}{$\begin{array}{l}\text { AmeriFlux scientists, Data Management Team, ICOS, DOE TES, USDA, NSF, and } \\
\text { Climate modelers. }\end{array}$} \\
\hline Goals & \multicolumn{2}{|c|}{$\begin{array}{l}\text { AmeriFlux Network and FLUXNET measurements provide the crucial linkage between } \\
\text { organisms, ecosystems, and process-scale studies at climate-relevant scales of } \\
\text { landscapes, regions, and continents, which can be incorporated into biogeochemical } \\
\text { and climate models. Results from individual flux sites provide the foundation for a } \\
\text { growing body of synthesis and modeling analyses. }\end{array}$} \\
\hline Use Case Description & \multicolumn{2}{|c|}{$\begin{array}{l}\text { AmeriFlux network observations enable scaling of trace gas fluxes (CO2, water vapor) } \\
\text { across a broad spectrum of times (hours, days, seasons, years, and decades) and } \\
\text { space. Moreover, Ameriflux and FLUXNET datasets provide the crucial linkages } \\
\text { among organisms, ecosystems, and process-scale studies-at climate-relevant scales } \\
\text { of landscapes, regions, and continents-for incorporation into biogeochemical and } \\
\text { climate models }\end{array}$} \\
\hline \multirow{4}{*}{$\begin{array}{r}\text { Current } \\
\text { Solutions }\end{array}$} & Compute(System) & NERSC \\
\hline & Storage & NERSC \\
\hline & Networking & ESNet \\
\hline & Software & $\begin{array}{l}\text { EddyPro, Custom analysis software, R, python, neural } \\
\text { networks, Matlab. }\end{array}$ \\
\hline \multirow[t]{5}{*}{$\begin{array}{r}\text { Big Data } \\
\text { Characteristics }\end{array}$} & $\begin{array}{r}\text { Data Source } \\
\text { (distributed/centralized) }\end{array}$ & $\begin{array}{l}\approx 150 \text { towers in AmeriFlux and over } 500 \text { towers } \\
\text { distributed globally collecting flux measurements. }\end{array}$ \\
\hline & Volume (size) & \\
\hline & $\begin{array}{r}\text { Velocity } \\
\text { (e.g. real time) }\end{array}$ & \\
\hline & $\begin{array}{r}\text { Variety } \\
\text { (multiple datasets, } \\
\text { mashup) }\end{array}$ & $\begin{array}{l}\text { The flux data is relatively uniform, however, the } \\
\text { biological, disturbance, and other ancillary data needed } \\
\text { to process and to interpret the data is extensive and } \\
\text { varies widely. Merging this data with the flux data is } \\
\text { challenging in today's systems. }\end{array}$ \\
\hline & $\begin{array}{r}\text { Variability (rate of } \\
\text { change) }\end{array}$ & \\
\hline \multirow[t]{4}{*}{$\begin{array}{r}\text { Big Data Science } \\
\text { (collection, curation, } \\
\text { analysis, } \\
\text { action) }\end{array}$} & $\begin{array}{l}\text { Veracity (Robustness } \\
\text { Issues) and Quality }\end{array}$ & $\begin{array}{l}\text { Each site has unique measurement and data processing } \\
\text { techniques. The network brings this data together and } \\
\text { performs a common processing, gap-filling, and quality } \\
\text { assessment. Thousands of users }\end{array}$ \\
\hline & Visualization & Graphs and 3D surfaces are used to visualize the data. \\
\hline & Data Types & Described in "Variety" above. \\
\hline & Data Analytics & $\begin{array}{l}\text { Data mining, data quality assessment, cross-correlation } \\
\text { across datasets, data assimilation, data interpolation, } \\
\text { statistics, quality assessment, data fusion, etc. }\end{array}$ \\
\hline $\begin{array}{l}\text { Big Data Specific } \\
\text { Challenges (Gaps) }\end{array}$ & \multicolumn{2}{|c|}{ Translation across diverse datasets that cross domains and scales. } \\
\hline $\begin{array}{r}\text { Big Data Specific } \\
\text { Challenges in Mobility }\end{array}$ & \multicolumn{2}{|c|}{$\begin{array}{l}\text { Field experiment data taking would be improved by access to existing data and } \\
\text { automated entry of new data via mobile devices. }\end{array}$} \\
\hline $\begin{array}{r}\text { Security and Privacy } \\
\text { Requirements }\end{array}$ & & \\
\hline
\end{tabular}


NIST Big Data Interoperability Framework: Volume 3, Use CaSes and General ReQuiRements

\section{Earth, Environmental and Polar Science> Use Case 50: Ameriflux and FLUXNET}

\begin{tabular}{|r|l|}
\hline $\begin{array}{r}\text { Highlight issues for } \\
\text { generalizing this use } \\
\text { case (e.g. for ref. } \\
\text { architecture) }\end{array}$ & \\
\hline $\begin{array}{r}\text { More Information } \\
\text { (URLs) }\end{array}$ & $\begin{array}{l}\text { http://Ameriflux.lbl.gov } \\
\text { http://www.fluxdata.org }\end{array}$ \\
\hline
\end{tabular}




\section{Energy> Use Case 51: Consumption Forecasting in Smart Grids}

\begin{tabular}{|c|c|c|}
\hline Use Case Title & \multicolumn{2}{|c|}{ Consumption forecasting in Smart Grids } \\
\hline Vertical (area) & \multicolumn{2}{|c|}{ Energy Informatics } \\
\hline Author/Company/Email & \multicolumn{2}{|c|}{ Yogesh Simmhan, University of Southern California, simmhan@usc.edu } \\
\hline $\begin{array}{r}\text { Actors/Stakeholders } \\
\text { and their roles and } \\
\text { responsibilities }\end{array}$ & \multicolumn{2}{|c|}{$\begin{array}{l}\text { Electric Utilities, Campus MicroGrids, Building Managers, Power Consumers, Energy } \\
\text { Markets }\end{array}$} \\
\hline Goals & \multicolumn{2}{|c|}{$\begin{array}{l}\text { Develop scalable and accurate forecasting models to predict the energy consumption } \\
\text { (kWh) within the utility service area under different spatial and temporal } \\
\text { granularities to help improve grid reliability and efficiency. }\end{array}$} \\
\hline Use Case Description & \multicolumn{2}{|c|}{$\begin{array}{l}\text { Deployment of smart meters are making available near-realtime energy usage } \\
\text { data (kWh) every 15-mins at the granularity individual consumers within the service } \\
\text { area of smart power utilities. This unprecedented and growing access to fine-grained } \\
\text { energy consumption information allows novel analytics capabilities to be developed } \\
\text { for predicting energy consumption for customers, transformers, sub-stations and the } \\
\text { utility service area. Near-term forecast can be used by utilities and microgrid } \\
\text { managers to take preventive action before consumption spikes cause } \\
\text { brown/blackouts through demand-response optimization by engaging consumers, } \\
\text { bringing peaker units online, or purchasing power from the energy markets. These } \\
\text { form an OODA feedback loop. Customers can also use them for energy use planning } \\
\text { and budgeting. Medium- to long-term predictions can help utilities and building } \\
\text { managers plan generation capacity, renewable portfolio, energy purchasing } \\
\text { contracts and sustainable building improvements. } \\
\text { Steps involved include 1) Data Collection and Storage: time-series data from } \\
\text { (potentially) millions of smart meters in near real time, features on consumers, } \\
\text { facilities and regions, weather forecasts, archival of data for training, testing and } \\
\text { validating models; 2) Data Cleaning and Normalization: Spatio-temporal } \\
\text { normalization, gap filling/Interpolation, outlier detection, semantic annotation; 3) } \\
\text { Training Forecast Models: Using univariate timeseries models like ARIMA, and data- } \\
\text { driven machine learning models like regression tree, ANN, for different spatial } \\
\text { (consumer, transformer) and temporal (15-min, 24-hour) granularities; 4) Prediction: } \\
\text { Predict consumption for different spatio-temporal granularities and prediction } \\
\text { horizons using near-realtime and historic data fed to the forecast model with } \\
\text { thresholds on prediction latencies. }\end{array}$} \\
\hline \multirow{4}{*}{$\begin{array}{r}\text { Current } \\
\text { Solutions }\end{array}$} & Compute(System) & Many-core servers, Commodity Cluster, Workstations \\
\hline & Storage & $\begin{array}{l}\text { SQL Databases, CSV Files, HDFS, Meter Data } \\
\text { Management }\end{array}$ \\
\hline & Networking & Gigabit Ethernet \\
\hline & Software & R/Matlab, Weka, Hadoop \\
\hline \multirow[t]{3}{*}{$\begin{array}{r}\text { Big Data } \\
\text { Characteristics }\end{array}$} & $\begin{array}{r}\text { Data Source } \\
\text { (distributed/centralized) }\end{array}$ & $\begin{array}{l}\text { Head-end of smart meters (distributed), Utility } \\
\text { databases (Customer Information, Network topology; } \\
\text { centralized), US Census data (distributed), NOAA } \\
\text { weather data (distributed), Microgrid building } \\
\text { information system (centralized), Microgrid sensor } \\
\text { network (distributed) }\end{array}$ \\
\hline & Volume (size) & $10 \mathrm{~GB} /$ day; $4 \mathrm{~TB} /$ year (City scale) \\
\hline & $\begin{array}{r}\text { Velocity } \\
\text { (e.g. real time) }\end{array}$ & $\begin{array}{l}\text { Los Angeles: Once every } 15 \text {-mins ( } \approx 100 \mathrm{k} \text { streams); } \\
\text { Once every } 8 \text {-hours ( } \approx 1.4 \mathrm{M} \text { streams) with finer grain } \\
\text { data aggregated to } 8 \text {-hour interval }\end{array}$ \\
\hline
\end{tabular}




\section{Energy> Use Case 51: Consumption Forecasting in Smart Grids}

\begin{tabular}{|c|c|c|}
\hline & $\begin{array}{r}\text { Variety } \\
\text { (multiple datasets, } \\
\text { mashup) }\end{array}$ & $\begin{array}{l}\text { Tuple-based: Timeseries, database rows; Graph-based: } \\
\text { Network topology, customer connectivity; Some } \\
\text { semantic data for normalization. }\end{array}$ \\
\hline & $\begin{array}{r}\text { Variability (rate of } \\
\text { change) }\end{array}$ & $\begin{array}{l}\text { Meter and weather data change, and are } \\
\text { collected/used, on hourly basis. Customer/building/grid } \\
\text { topology information is slow changing on a weekly } \\
\text { basis }\end{array}$ \\
\hline $\begin{array}{r}\text { Big Data Science } \\
\text { (collection, curation, } \\
\text { analysis, } \\
\text { action) }\end{array}$ & $\begin{array}{r}\text { Veracity (Robustness } \\
\text { Issues, semantics) }\end{array}$ & $\begin{array}{l}\text { Versioning and reproducibility is necessary to } \\
\text { validate/compare past and current models. Resilience } \\
\text { of storage and analytics is important for operational } \\
\text { needs. Semantic normalization can help with inter- } \\
\text { disciplinary analysis (e.g. utility operators, building } \\
\text { managers, power engineers, behavioral scientists) }\end{array}$ \\
\hline & Visualization & $\begin{array}{l}\text { Map-based visualization of grid service topology, stress; } \\
\text { Energy heat-maps; Plots of demand forecasts vs. } \\
\text { capacity, what-if analysis; Realtime information display; } \\
\text { Apps with push notification of alerts }\end{array}$ \\
\hline & Data Quality (syntax) & $\begin{array}{l}\text { Gaps in smart meters and weather data; Quality issues } \\
\text { in sensor data; Rigorous checks done for "billing } \\
\text { quality" meter data; }\end{array}$ \\
\hline & Data Types & $\begin{array}{l}\text { Timeseries (CSV, SQL tuples), Static information (RDF, } \\
\mathrm{XML} \text { ), topology (shape files) }\end{array}$ \\
\hline & Data Analytics & $\begin{array}{l}\text { Forecasting models, machine learning models, time } \\
\text { series analysis, clustering, motif detection, complex } \\
\text { event processing, visual network analysis, }\end{array}$ \\
\hline $\begin{array}{l}\text { Big Data Specific } \\
\text { Challenges (Gaps) }\end{array}$ & \multicolumn{2}{|c|}{$\begin{array}{l}\text { Scalable realtime analytics over large data streams } \\
\text { Low-latency analytics for operational needs } \\
\text { Federated analytics at utility and microgrid levels } \\
\text { Robust time series analytics over millions of customer consumption data } \\
\text { Customer behavior modeling, targeted curtailment requests }\end{array}$} \\
\hline $\begin{array}{r}\text { Big Data Specific } \\
\text { Challenges in Mobility }\end{array}$ & \multicolumn{2}{|c|}{$\begin{array}{l}\text { Apps for engaging with customers: Data collection from customers/premises for } \\
\text { behavior modeling, feature extraction; Notification of curtailment requests by } \\
\text { utility/building managers; Suggestions on energy efficiency; Geo-localized display of } \\
\text { energy footprint. }\end{array}$} \\
\hline $\begin{array}{r}\text { Security and Privacy } \\
\text { Requirements }\end{array}$ & \multicolumn{2}{|c|}{$\begin{array}{l}\text { Personally identifiable customer data requires careful handling. Customer energy } \\
\text { usage data can reveal behavior patterns. Anonymization of information. Data } \\
\text { aggregation to avoid customer identification. Data sharing restrictions by federal and } \\
\text { state energy regulators. Surveys by behavioral scientists may have IRB (Institutional } \\
\text { Review Board) restrictions. }\end{array}$} \\
\hline $\begin{array}{r}\text { Highlight issues for } \\
\text { generalizing this use } \\
\text { case (e.g. for ref. } \\
\text { architecture) }\end{array}$ & \multicolumn{2}{|c|}{ Realtime data-driven analytics for cyber-physical systems } \\
\hline $\begin{array}{r}\text { More Information } \\
\text { (URLs) }\end{array}$ & \multicolumn{2}{|c|}{$\begin{array}{l}\text { http://smartgrid.usc.edu } \\
\text { http://ganges.usc.edu/wiki/Smart Grid } \\
\text { https://www.ladwp.com/ladwp/faces/ladwp/aboutus/a-power/a-p-smartgridla } \\
\text { http://ieeexplore.ieee.org/xpl/articleDetails.jsp?arnumber=6475927 }\end{array}$} \\
\hline
\end{tabular}




\section{Appendix B: Summary of Key Properties}

Information related to five key properties was extracted from each use case. The five key properties were three Big Data characteristics (volume, velocity, and variety), software related information, and associated analytics. The extracted information is presented in Table B-1. The use case number listed in the first column corresponds to the use case number used in this report. The use case number in the second column (e.g., M0147) corresponds to the document number on the NIST Big Data Public Working Group Document Repository

(https://bigdatawg.nist.gov/show_InputDoc.php).

Table B-1: Use Case Specific Information by Key Properties

\begin{tabular}{|c|c|c|c|c|c|c|}
\hline No. & Use Case & Volume & Velocity & Variety & Software & Analytics \\
\hline 1 & $\begin{array}{l}\frac{\text { M0147 }}{\text { Census } 2000 \text { and }} \\
2010\end{array}$ & 380 TB & Static for 75 years & Scanned documents & $\begin{array}{l}\text { Robust archival } \\
\text { storage }\end{array}$ & None for 75 years \\
\hline 2 & $\begin{array}{l}\text { M0148 } \\
\text { NARA: Search, } \\
\text { Retrieve, } \\
\text { Preservation }\end{array}$ & $\begin{array}{l}\text { Hundreds of } \\
\text { terabytes, and } \\
\text { growing }\end{array}$ & $\begin{array}{l}\text { Data loaded in } \\
\text { batches, so bursty }\end{array}$ & $\begin{array}{l}\text { Unstructured and } \\
\text { structured data: textual } \\
\text { documents, emails, } \\
\text { photos, scanned } \\
\text { documents, multimedia, } \\
\text { social networks, web sites, } \\
\text { databases, etc. }\end{array}$ & $\begin{array}{l}\text { Custom software, } \\
\text { commercial search } \\
\text { products, commercial } \\
\text { databases }\end{array}$ & $\begin{array}{l}\text { Crawl/index, search, ranking, } \\
\text { predictive search; data } \\
\text { categorization (sensitive, } \\
\text { confidential, etc.); personally } \\
\text { identifiable information (PII) } \\
\text { detection and flagging }\end{array}$ \\
\hline 3 & $\begin{array}{l}\text { M0219 } \\
\text { Statistical } \\
\text { Survey } \\
\text { Response } \\
\text { Improvement }\end{array}$ & Approximately 1 PB & $\begin{array}{l}\text { Variable, field data } \\
\text { streamed } \\
\text { continuously, } \\
\text { Census was } \approx 150 \\
\text { million records } \\
\text { transmitted }\end{array}$ & Strings and numerical data & $\begin{array}{l}\text { Hadoop, Spark, Hive, } \\
\text { R, SAS, Mahout, } \\
\text { Allegrograph, MySQL, } \\
\text { Oracle, Storm, } \\
\text { BigMemory, } \\
\text { Cassandra, Pig }\end{array}$ & $\begin{array}{l}\text { Recommendation systems, } \\
\text { continued monitoring }\end{array}$ \\
\hline
\end{tabular}




\begin{tabular}{|c|c|c|c|c|c|c|}
\hline No. & Use Case & Volume & Velocity & Variety & Software & Analytics \\
\hline 4 & $\begin{array}{l}\text { M0222 } \\
\text { Non-Traditional } \\
\text { Data in } \\
\text { Statistical } \\
\text { Survey } \\
\text { Response } \\
\text { Improvement }\end{array}$ & - & - & $\begin{array}{l}\text { Survey data, other } \\
\text { government } \\
\text { administrative data, web- } \\
\text { scraped data, wireless } \\
\text { data, e-transaction data, } \\
\text { (potentially) social media } \\
\text { data and positioning data } \\
\text { from various sources }\end{array}$ & $\begin{array}{l}\text { Hadoop, Spark, Hive, } \\
\text { R, SAS, Mahout, } \\
\text { Allegrograph, MySQL, } \\
\text { Oracle, Storm, } \\
\text { BigMemory, } \\
\text { Cassandra, Pig }\end{array}$ & $\begin{array}{l}\text { New analytics to create } \\
\text { reliable information from } \\
\text { non-traditional disparate } \\
\text { sources }\end{array}$ \\
\hline 5 & $\begin{array}{l}\text { M0175 } \\
\text { Cloud Eco- } \\
\text { System for } \\
\text { Finance }\end{array}$ & - & Real time & - & Hadoop RDBMS XBRL & Fraud detection \\
\hline 6 & $\frac{\text { M0161 }}{\text { Mendeley }}$ & $\begin{array}{l}15 \text { TB presently, } \\
\text { growing about } 1 \text { TB } \\
\text { per month }\end{array}$ & $\begin{array}{l}\text { Currently Hadoop } \\
\text { batch jobs } \\
\text { scheduled daily, } \\
\text { real-time } \\
\text { recommended in } \\
\text { future }\end{array}$ & $\begin{array}{l}\text { PDF documents and log } \\
\text { files of social network and } \\
\text { client activities }\end{array}$ & $\begin{array}{l}\text { Hadoop, Scribe, Hive, } \\
\text { Mahout, Python }\end{array}$ & $\begin{array}{l}\text { Standard libraries for } \\
\text { machine learning and } \\
\text { analytics, LDA, custom-built } \\
\text { reporting tools for } \\
\text { aggregating readership and } \\
\text { social activities per } \\
\text { document }\end{array}$ \\
\hline 7 & $\begin{array}{l}\text { M0164 } \\
\text { Netflix Movie } \\
\text { Service }\end{array}$ & $\begin{array}{l}\text { Summer } 2012-25 \\
\text { million subscribers, } \\
4 \text { million ratings per } \\
\text { day, } 3 \text { million } \\
\text { searches per day, } 1 \\
\text { billion hours } \\
\text { streamed in June } \\
2012 \text {; Cloud storage } \\
-2 \text { petabytes in } \\
\text { June } 2013\end{array}$ & $\begin{array}{l}\text { Media (video and } \\
\text { properties) and } \\
\text { rankings } \\
\text { continually } \\
\text { updated }\end{array}$ & $\begin{array}{l}\text { Data vary from digital } \\
\text { media to user rankings, } \\
\text { user profiles, and media } \\
\text { properties for content- } \\
\text { based recommendations }\end{array}$ & $\begin{array}{l}\text { Hadoop and Pig; } \\
\text { Cassandra; Teradata }\end{array}$ & $\begin{array}{l}\text { Personalized recommender } \\
\text { systems using logistic/linear } \\
\text { regression, elastic nets, } \\
\text { matrix factorization, } \\
\text { clustering, LDA, association } \\
\text { rules, gradient-boosted } \\
\text { decision trees, and others; } \\
\text { streaming video delivery }\end{array}$ \\
\hline 8 & $\frac{\text { M0165 }}{\text { Web Search }}$ & $\begin{array}{l}45 \text { billion web pages } \\
\text { total, } 500 \text { million } \\
\text { photos uploaded } \\
\text { each day, } 100 \text { hours } \\
\text { of video uploaded to } \\
\text { YouTube each } \\
\text { minute }\end{array}$ & $\begin{array}{l}\text { Real-time updating } \\
\text { and real-time } \\
\text { responses to } \\
\text { queries }\end{array}$ & Multiple media & $\begin{array}{l}\text { Map/Reduce + } \\
\text { Bigtable; Dryad + } \\
\text { Cosmos; PageRank; } \\
\text { final step essentially a } \\
\text { recommender engine }\end{array}$ & $\begin{array}{l}\text { Crawling; searching, } \\
\text { including topic-based } \\
\text { searches; ranking; } \\
\text { recommending }\end{array}$ \\
\hline
\end{tabular}




\begin{tabular}{|c|c|c|c|c|c|c|}
\hline No. & Use Case & Volume & Velocity & Variety & Software & Analytics \\
\hline 9 & $\begin{array}{l}\text { M0137 } \\
\text { Business } \\
\text { Continuity and } \\
\text { Disaster } \\
\text { Recovery Within } \\
\text { a Cloud Eco- } \\
\text { System }\end{array}$ & $\begin{array}{l}\text { Terabytes up to } \\
\text { petabytes }\end{array}$ & $\begin{array}{l}\text { Can be real time } \\
\text { for recent changes }\end{array}$ & Must work for all data & $\begin{array}{l}\text { Hadoop, Map/Reduce, } \\
\text { open source, and/or } \\
\text { vendor proprietary } \\
\text { such as AWS, Google } \\
\text { Cloud Services, and } \\
\text { Microsoft }\end{array}$ & Robust backup \\
\hline 10 & $\frac{\text { M0103 }}{\text { Cargo Shipping }}$ & - & $\begin{array}{l}\text { Needs to become } \\
\text { real time, currently } \\
\text { updated at events }\end{array}$ & Event-based & - & $\begin{array}{l}\text { Distributed event analysis } \\
\text { identifying problems }\end{array}$ \\
\hline 11 & $\begin{array}{l}\text { M0162 } \\
\text { Materials Data } \\
\text { for } \\
\text { Manufacturing }\end{array}$ & $\begin{array}{l}500,000 \text { material } \\
\text { types in } 1980 \text { s, } \\
\text { much growth since } \\
\text { then }\end{array}$ & $\begin{array}{l}\text { Ongoing increase } \\
\text { in new materials }\end{array}$ & $\begin{array}{l}\text { Many datasets with no } \\
\text { standards }\end{array}$ & $\begin{array}{l}\text { National programs } \\
\text { (Japan, Korea, and } \\
\text { China), application } \\
\text { areas (EU nuclear } \\
\text { program), proprietary } \\
\text { systems (Granta, etc.) }\end{array}$ & $\begin{array}{l}\text { No broadly applicable } \\
\text { analytics }\end{array}$ \\
\hline 12 & $\begin{array}{l}\text { M0176 } \\
\text { Simulation- } \\
\text { Driven Materials } \\
\text { Genomics }\end{array}$ & $\begin{array}{l}100 \text { TB (current), } \\
500 \text { TB within five } \\
\text { years, scalable key- } \\
\text { value and object } \\
\text { store databases } \\
\text { needed }\end{array}$ & $\begin{array}{l}\text { Regular data } \\
\text { added from } \\
\text { simulations }\end{array}$ & $\begin{array}{l}\text { Varied data and simulation } \\
\text { results }\end{array}$ & $\begin{array}{l}\text { MongoDB, GPFS, } \\
\text { PyMatGen, FireWorks, } \\
\text { VASP, ABINIT, } \\
\text { NWChem, } \\
\text { BerkeleyGW, varied } \\
\text { community codes }\end{array}$ & $\begin{array}{l}\text { Map/Reduce and search that } \\
\text { join simulation and } \\
\text { experimental data }\end{array}$ \\
\hline 13 & $\begin{array}{l}\text { M0213 } \\
\text { Large-Scale } \\
\text { Geospatial } \\
\text { Analysis and } \\
\text { Visualization }\end{array}$ & $\begin{array}{l}\text { Imagery - hundreds } \\
\text { of terabytes; vector } \\
\text { data - tens of GBs } \\
\text { but billions of points }\end{array}$ & $\begin{array}{l}\text { Vectors } \\
\text { transmitted in } \\
\text { near real time }\end{array}$ & $\begin{array}{l}\text { Imagery, vector (various } \\
\text { formats such as shape } \\
\text { files, KML, text streams) } \\
\text { and many object } \\
\text { structures }\end{array}$ & $\begin{array}{l}\text { Geospatially enabled } \\
\text { RDBMS, Esri } \\
\text { ArcServer, Geoserver }\end{array}$ & $\begin{array}{l}\text { Closest point of approach, } \\
\text { deviation from route, point } \\
\text { density over time, PCA and } \\
\text { ICA }\end{array}$ \\
\hline 14 & $\begin{array}{l}\text { M0214 } \\
\text { Object } \\
\text { Identification } \\
\text { and Tracking }\end{array}$ & $\begin{array}{l}\text { FMV - } 30 \text { to } 60 \\
\text { frames per second } \\
\text { at full-color } 1080 \mathrm{P} \\
\text { resolution; WALF - } 1 \\
\text { to } 10 \text { frames per } \\
\text { second at } 10,000 \mathrm{x} \\
10,000 \text { full-color } \\
\text { resolution }\end{array}$ & Real time & $\begin{array}{l}\text { A few standard imagery or } \\
\text { video formats }\end{array}$ & $\begin{array}{l}\text { Custom software and } \\
\text { tools including } \\
\text { traditional RDBMS and } \\
\text { display tools }\end{array}$ & $\begin{array}{l}\text { Visualization as overlays on a } \\
\text { GIS, basic object detection } \\
\text { analytics and integration } \\
\text { with sophisticated situation } \\
\text { awareness tools with data } \\
\text { fusion }\end{array}$ \\
\hline
\end{tabular}




\begin{tabular}{|c|c|c|c|c|c|c|}
\hline No. & Use Case & Volume & Velocity & Variety & Software & Analytics \\
\hline 15 & $\begin{array}{l}\text { M0215 } \\
\text { Intelligence } \\
\text { Data Processing } \\
\text { and Analysis }\end{array}$ & $\begin{array}{l}\text { Tens of terabytes to } \\
\text { hundreds of } \\
\text { petabytes, individual } \\
\text { warfighters (first } \\
\text { responders) would } \\
\text { have at most one to } \\
\text { hundreds of GBs }\end{array}$ & $\begin{array}{l}\text { Much real-time, } \\
\text { imagery } \\
\text { intelligence } \\
\text { devices that gather } \\
\text { a petabyte of data } \\
\text { in a few hours }\end{array}$ & $\begin{array}{l}\text { Text files, raw media, } \\
\text { imagery, video, audio, } \\
\text { electronic data, human- } \\
\text { generated data }\end{array}$ & $\begin{array}{l}\text { Hadoop, Accumulo } \\
\text { (BigTable), Solr, NLP, } \\
\text { Puppet (for } \\
\text { deployment and } \\
\text { security) and Storm; } \\
\text { GIS }\end{array}$ & $\begin{array}{l}\text { Near real-time alerts based } \\
\text { on patterns and baseline } \\
\text { changes, link analysis, } \\
\text { geospatial analysis, text } \\
\text { analytics (sentiment, entity } \\
\text { extraction, etc.) }\end{array}$ \\
\hline 16 & $\frac{\text { M0177 }}{\text { EMR Data }}$ & $\begin{array}{l}12 \text { million patients, } \\
\text { more than } 4 \text { billion } \\
\text { discrete clinical } \\
\text { observations, }>20 \\
\text { TB raw data }\end{array}$ & $\begin{array}{l}0.5 \text { to } 1.5 \text { million } \\
\text { new real-time } \\
\text { clinical } \\
\text { transactions added } \\
\text { per day }\end{array}$ & $\begin{array}{l}\text { Broad variety of data from } \\
\text { doctors, nurses, } \\
\text { laboratories and } \\
\text { instruments }\end{array}$ & $\begin{array}{l}\text { Teradata, PostgreSQL, } \\
\text { MongoDB, Hadoop, } \\
\text { Hive, R }\end{array}$ & $\begin{array}{l}\text { Information retrieval } \\
\text { methods (tf-idf), NLP, } \\
\text { maximum likelihood } \\
\text { estimators, Bayesian } \\
\text { networks }\end{array}$ \\
\hline 17 & $\begin{array}{l}\text { M0089 } \\
\text { Pathology } \\
\text { Imaging }\end{array}$ & $\begin{array}{l}1 \mathrm{~GB} \text { raw image data } \\
+1.5 \mathrm{~GB} \text { analytical } \\
\text { results per } 2 \mathrm{D} \\
\text { image, } 1 \mathrm{~TB} \text { raw } \\
\text { image data }+1 \mathrm{~TB} \\
\text { analytical results per } \\
3 \mathrm{D} \text { image, } 1 \mathrm{~PB} \text { data } \\
\text { per moderated } \\
\text { hospital per year }\end{array}$ & $\begin{array}{l}\text { Once generated, } \\
\text { data will not be } \\
\text { changed }\end{array}$ & Images & $\begin{array}{l}\text { MPI for image } \\
\text { analysis, Map/Reduce } \\
+ \text { Hive with spatial } \\
\text { extension }\end{array}$ & $\begin{array}{l}\text { Image analysis, spatial } \\
\text { queries and analytics, } \\
\text { feature clustering and } \\
\text { classification }\end{array}$ \\
\hline 18 & $\begin{array}{l}\text { M0191 } \\
\text { Computational } \\
\text { Bioimaging }\end{array}$ & $\begin{array}{l}\text { Medical diagnostic } \\
\text { imaging around } 70 \\
\text { PB annually, } 32 \text { TB } \\
\text { on emerging } \\
\text { machines for a single } \\
\text { scan }\end{array}$ & $\begin{array}{l}\text { Volume of data } \\
\text { acquisition } \\
\text { requires HPC back } \\
\text { end }\end{array}$ & $\begin{array}{l}\text { Multi-modal imaging with } \\
\text { disparate channels of data }\end{array}$ & $\begin{array}{l}\text { Scalable key-value and } \\
\text { object store } \\
\text { databases; ImageJ, } \\
\text { OMERO, VolRover, } \\
\text { advanced } \\
\text { segmentation and } \\
\text { feature detection } \\
\text { methods }\end{array}$ & $\begin{array}{l}\text { Machine learning (support } \\
\text { vector machine }[\mathrm{SVM}] \text { and } \\
\text { random forest }[\mathrm{RF}] \text { ) for } \\
\text { classification and } \\
\text { recommendation services }\end{array}$ \\
\hline 19 & $\begin{array}{l}\text { M0078 } \\
\text { Genomic } \\
\text { Measurements }\end{array}$ & $\begin{array}{l}>100 \text { TB in } 1 \text { to } 2 \\
\text { years at NIST, many } \\
\text { PBs in healthcare } \\
\text { community }\end{array}$ & $\begin{array}{l}\approx 300 \mathrm{~GB} \text { of } \\
\text { compressed } \\
\text { data/day } \\
\text { generated by DNA } \\
\text { sequencers }\end{array}$ & $\begin{array}{l}\text { File formats not well- } \\
\text { standardized, though } \\
\text { some standards exist; } \\
\text { generally structured data }\end{array}$ & $\begin{array}{l}\text { Open-source } \\
\text { sequencing } \\
\text { bioinformatics } \\
\text { software from } \\
\text { academic groups }\end{array}$ & $\begin{array}{l}\text { Processing of raw data to } \\
\text { produce variant calls, clinical } \\
\text { interpretation of variants }\end{array}$ \\
\hline
\end{tabular}




\begin{tabular}{|c|c|c|c|c|c|c|}
\hline No. & Use Case & Volume & Velocity & Variety & Software & Analytics \\
\hline 20 & $\begin{array}{l}\text { M0188 } \\
\text { Comparative } \\
\text { Analysis for } \\
\text { Metagenomes } \\
\text { and Genomes }\end{array}$ & $50 \mathrm{~TB}$ & $\begin{array}{l}\text { New sequencers } \\
\text { stream in data at } \\
\text { growing rate }\end{array}$ & $\begin{array}{l}\text { Biological data that are } \\
\text { inherently heterogeneous, } \\
\text { complex, structural, and } \\
\text { hierarchical; besides core } \\
\text { genomic data, new types } \\
\text { of omics data such as } \\
\text { transcriptomics, } \\
\text { methylomics, and } \\
\text { proteomics }\end{array}$ & $\begin{array}{l}\text { Standard } \\
\text { bioinformatics tools } \\
\text { (BLAST, HMMER, } \\
\text { multiple alignment } \\
\text { and phylogenetic } \\
\text { tools, gene callers, } \\
\text { sequence feature } \\
\text { predictors), } \\
\text { Perl/Python wrapper } \\
\text { scripts }\end{array}$ & $\begin{array}{l}\text { Descriptive statistics, } \\
\text { statistical significance in } \\
\text { hypothesis testing, data } \\
\text { clustering and classification }\end{array}$ \\
\hline 21 & $\begin{array}{l}\frac{\text { M0140 }}{\text { Individualized }} \\
\text { Diabetes } \\
\text { Management }\end{array}$ & 5 million patients & $\begin{array}{l}\text { Not real time but } \\
\text { updated } \\
\text { periodically }\end{array}$ & $\begin{array}{l}100 \text { controlled vocabulary } \\
\text { values and } 1,000 \\
\text { continuous values per } \\
\text { patient, mostly time- } \\
\text { stamped values }\end{array}$ & $\begin{array}{l}\text { HDFS supplementing } \\
\text { Mayo internal data } \\
\text { warehouse (EDT) }\end{array}$ & $\begin{array}{l}\text { Integration of data into } \\
\text { semantic graphs, using graph } \\
\text { traverse to replace SQL join; } \\
\text { development of semantic } \\
\text { graph-mining algorithms to } \\
\text { identify graph patterns, } \\
\text { index graph, and search } \\
\text { graph; indexed Hbase; } \\
\text { custom code to develop new } \\
\text { patient properties from } \\
\text { stored data }\end{array}$ \\
\hline 22 & $\begin{array}{l}\text { M0174 } \\
\text { Statistical } \\
\text { Relational } \\
\text { Artificial } \\
\text { Intelligence for } \\
\text { Health Care }\end{array}$ & $\begin{array}{l}\text { Hundreds of GBs for } \\
\text { a single cohort of a } \\
\text { few hundred people; } \\
\text { possibly on the } \\
\text { order of } 1 \text { PB when } \\
\text { dealing with millions } \\
\text { of patients }\end{array}$ & $\begin{array}{l}\text { Constant updates } \\
\text { to EHRs; in other } \\
\text { controlled studies, } \\
\text { data often in } \\
\text { batches at regular } \\
\text { intervals }\end{array}$ & $\begin{array}{l}\text { Critical feature - data } \\
\text { typically in multiple tables, } \\
\text { need to be merged to } \\
\text { perform analysis }\end{array}$ & $\begin{array}{l}\text { Mainly Java-based, in- } \\
\text { house tools to process } \\
\text { the data }\end{array}$ & $\begin{array}{l}\text { Relational probabilistic } \\
\text { models (Statistical Relational } \\
\text { Artificial Intelligence) learned } \\
\text { from multiple data types }\end{array}$ \\
\hline 23 & $\begin{array}{l}\text { M0172 } \\
\text { World } \\
\text { Population- } \\
\text { Scale } \\
\text { Epidemiological } \\
\text { Study }\end{array}$ & $100 \mathrm{~TB}$ & $\begin{array}{l}\text { Low number of } \\
\text { data feeding into } \\
\text { the simulation, } \\
\text { massive amounts } \\
\text { of real-time data } \\
\text { generated by } \\
\text { simulation }\end{array}$ & $\begin{array}{l}\text { Can be rich with various } \\
\text { population activities, } \\
\text { geographical, socio- } \\
\text { economic, cultural } \\
\text { variations }\end{array}$ & Charm++, MPI & $\begin{array}{l}\text { Simulations on a synthetic } \\
\text { population }\end{array}$ \\
\hline
\end{tabular}




\begin{tabular}{|c|c|c|c|c|c|c|}
\hline No. & Use Case & Volume & Velocity & Variety & Software & Analytics \\
\hline 24 & $\begin{array}{l}\frac{\text { M0173 }}{\text { Social Contagion }} \\
\text { Modeling for } \\
\text { Planning }\end{array}$ & $\begin{array}{l}\text { Tens of terabytes } \\
\text { per year }\end{array}$ & $\begin{array}{l}\text { During social } \\
\text { unrest events, } \\
\text { human } \\
\text { interactions and } \\
\text { mobility leads to } \\
\text { rapid changes in } \\
\text { data; e.g., who } \\
\text { follows whom in } \\
\text { Twitter }\end{array}$ & $\begin{array}{l}\text { Big issues - data fusion, } \\
\text { combining data from } \\
\text { different sources, dealing } \\
\text { with missing or incomplete } \\
\text { data }\end{array}$ & $\begin{array}{l}\text { Specialized simulators, } \\
\text { open source software, } \\
\text { proprietary modeling } \\
\text { environments; } \\
\text { databases }\end{array}$ & $\begin{array}{l}\text { Models of behavior of } \\
\text { humans and hard } \\
\text { infrastructures, models of } \\
\text { their interactions, } \\
\text { visualization of results }\end{array}$ \\
\hline 25 & $\begin{array}{l}\frac{\text { M0141 }}{\text { Biodiversity and }} \\
\text { LifeWatch }\end{array}$ & N/A & $\begin{array}{l}\text { Real-time } \\
\text { processing and } \\
\text { analysis in case of } \\
\text { natural or } \\
\text { industrial disaster }\end{array}$ & $\begin{array}{l}\text { Rich variety and number } \\
\text { of involved databases and } \\
\text { observation data }\end{array}$ & RDBMS & $\begin{array}{l}\text { Requires advanced and rich } \\
\text { visualization }\end{array}$ \\
\hline 26 & $\begin{array}{l}\text { M0136 } \\
\text { Large-Scale } \\
\text { Deep Learning }\end{array}$ & $\begin{array}{l}\text { Current datasets } \\
\text { typically } 1 \text { TB to } 10 \\
\text { TB, possibly } 100 \\
\text { million images to } \\
\text { train a self-driving } \\
\text { car }\end{array}$ & $\begin{array}{l}\text { Much faster than } \\
\text { real-time } \\
\text { processing; for } \\
\text { autonomous } \\
\text { driving, need to } \\
\text { process thousands } \\
\text { of high-resolution } \\
\text { (six megapixels or } \\
\text { more) images per } \\
\text { second }\end{array}$ & $\begin{array}{l}\text { Neural net very } \\
\text { heterogeneous as it learns } \\
\text { many different features }\end{array}$ & $\begin{array}{l}\text { In-house GPU kernels } \\
\text { and MPI-based } \\
\text { communication } \\
\text { developed by } \\
\text { Stanford, C++/Python } \\
\text { source }\end{array}$ & $\begin{array}{l}\text { Small degree of batch } \\
\text { statistical preprocessing, all } \\
\text { other data analysis } \\
\text { performed by the learning } \\
\text { algorithm itself }\end{array}$ \\
\hline 27 & $\begin{array}{l}\text { M0171 } \\
\text { Organizing } \\
\text { Large-Scale } \\
\text { Unstructured } \\
\text { Collections of } \\
\text { Consumer } \\
\text { Photos }\end{array}$ & $\begin{array}{l}500+\text { billion photos } \\
\text { on Facebook, } 5+ \\
\text { billion photos on } \\
\text { Flickr }\end{array}$ & $\begin{array}{l}\text { Over } 500 \text { million } \\
\text { images uploaded } \\
\text { to Facebook each } \\
\text { day }\end{array}$ & $\begin{array}{l}\text { Images and metadata } \\
\text { including EXIF } \\
\text { (Exchangeable Image File) } \\
\text { tags (focal distance, } \\
\text { camera type, etc.) }\end{array}$ & $\begin{array}{l}\text { Hadoop Map/Reduce, } \\
\text { simple hand-written } \\
\text { multi-threaded tools } \\
\text { (Secure Shell [SSH] } \\
\text { and sockets for } \\
\text { communication) }\end{array}$ & $\begin{array}{l}\text { Robust non-linear least } \\
\text { squares optimization } \\
\text { problem, SVM }\end{array}$ \\
\hline
\end{tabular}




\begin{tabular}{|c|c|c|c|c|c|c|}
\hline No. & Use Case & Volume & Velocity & Variety & Software & Analytics \\
\hline 28 & $\begin{array}{l}\frac{\text { M0160 }}{\text { Truthy Twitter }} \\
\text { Data }\end{array}$ & $\begin{array}{l}30 \text { TB/year } \\
\text { compressed data }\end{array}$ & $\begin{array}{l}\text { Near real-time } \\
\text { data storage, } \\
\text { querying and } \\
\text { analysis }\end{array}$ & $\begin{array}{l}\text { Schema provided by social } \\
\text { media data source; } \\
\text { currently using Twitter } \\
\text { only; plans to expand, } \\
\text { incorporating Google+ and } \\
\text { Facebook }\end{array}$ & $\begin{array}{l}\text { Hadoop IndexedHBase } \\
\text { and HDFS; Hadoop, } \\
\text { Hive, Redis for data } \\
\text { management; Python: } \\
\text { SciPy NumPy and MPI } \\
\text { for data analysis }\end{array}$ & $\begin{array}{l}\text { Anomaly detection, stream } \\
\text { clustering, signal } \\
\text { classification, online } \\
\text { learning; information } \\
\text { diffusion, clustering, dynamic } \\
\text { network visualization }\end{array}$ \\
\hline 29 & $\begin{array}{l}\text { M0211 } \\
\text { Crowd Sourcing } \\
\text { in Humanities }\end{array}$ & $\begin{array}{l}\text { GBs (text, surveys, } \\
\text { experiment values) } \\
\text { to hundreds of } \\
\text { terabytes } \\
\text { (multimedia) }\end{array}$ & $\begin{array}{l}\text { Data continuously } \\
\text { updated and } \\
\text { analyzed } \\
\text { incrementally }\end{array}$ & $\begin{array}{l}\text { So far mostly } \\
\text { homogeneous small } \\
\text { datasets; expected large } \\
\text { distributed heterogeneous } \\
\text { datasets }\end{array}$ & $\begin{array}{l}\text { XML technology, } \\
\text { traditional relational } \\
\text { databases }\end{array}$ & $\begin{array}{l}\text { Pattern recognition (e.g., } \\
\text { speech recognition, } \\
\text { automatic audio-visual } \\
\text { analysis, cultural patterns), } \\
\text { identification of structures } \\
\text { (lexical units, linguistic rules, } \\
\text { etc.) }\end{array}$ \\
\hline 30 & $\begin{array}{l}\text { M0158 } \\
\text { CINET for } \\
\text { Network Science }\end{array}$ & $\begin{array}{l}\text { Can be hundreds of } \\
\text { GBs for a single } \\
\text { network, } 1,000 \text { to } \\
5,000 \text { networks and } \\
\text { methods }\end{array}$ & $\begin{array}{l}\text { Dynamic networks, } \\
\text { network collection } \\
\text { growing }\end{array}$ & Many types of networks & $\begin{array}{l}\text { Graph libraries (Galib, } \\
\text { NetworkX); } \\
\text { distributed workflow } \\
\text { management } \\
\text { (Simfrastructure, } \\
\text { databases, semantic } \\
\text { web tools) }\end{array}$ & Network visualization \\
\hline 31 & $\begin{array}{l}\text { M0190 } \\
\text { NIST } \\
\text { Information } \\
\text { Access Division }\end{array}$ & $\begin{array}{l}>900 \text { million web } \\
\text { pages occupying } 30 \\
\text { TB of storage, } 100 \\
\text { million tweets, } 100 \\
\text { million ground- } \\
\text { truthed biometric } \\
\text { images, hundreds of } \\
\text { thousands of } \\
\text { partially ground- } \\
\text { truthed video clips, } \\
\text { terabytes of smaller } \\
\text { fully ground-truthed } \\
\text { test collections }\end{array}$ & $\begin{array}{l}\text { Legacy evaluations } \\
\text { mostly focused on } \\
\text { retrospective } \\
\text { analytics, newer } \\
\text { evaluations } \\
\text { focused on } \\
\text { simulations of real- } \\
\text { time analytic } \\
\text { challenges from } \\
\text { multiple data } \\
\text { streams }\end{array}$ & $\begin{array}{l}\text { Wide variety of data types } \\
\text { including textual } \\
\text { search/extraction, } \\
\text { machine translation, } \\
\text { speech recognition, image } \\
\text { and voice biometrics, } \\
\text { object and person } \\
\text { recognition and tracking, } \\
\text { document analysis, } \\
\text { human-computer } \\
\text { dialogue, multimedia } \\
\text { search/extraction }\end{array}$ & $\begin{array}{l}\text { PERL, Python, C/C++, } \\
\text { Matlab, R } \\
\text { development tools; } \\
\text { create ground-up test } \\
\text { and measurement } \\
\text { applications }\end{array}$ & $\begin{array}{l}\text { Information extraction, } \\
\text { filtering, search, and } \\
\text { summarization; image and } \\
\text { voice biometrics; speech } \\
\text { recognition and } \\
\text { understanding; machine } \\
\text { translation; video } \\
\text { person/object detection and } \\
\text { tracking; event detection; } \\
\text { imagery/document } \\
\text { matching; novelty detection; } \\
\text { structural semantic temporal } \\
\text { analytics }\end{array}$ \\
\hline 32 & $\frac{\text { M0130 }}{\text { DataNet (iRODS) }}$ & $\begin{array}{l}\text { Petabytes, hundreds } \\
\text { of millions of files }\end{array}$ & $\begin{array}{l}\text { Real time and } \\
\text { batch }\end{array}$ & Rich & iRODS & $\begin{array}{l}\text { Supports general analysis } \\
\text { workflows }\end{array}$ \\
\hline
\end{tabular}




\begin{tabular}{|c|c|c|c|c|c|c|}
\hline No. & Use Case & Volume & Velocity & Variety & Software & Analytics \\
\hline 33 & $\begin{array}{l}\text { M0163 } \\
\text { The Discinnet } \\
\text { Process }\end{array}$ & $\begin{array}{l}\text { Small as metadata to } \\
\text { Big Data }\end{array}$ & Real time & $\begin{array}{l}\text { Can tackle arbitrary Big } \\
\text { Data }\end{array}$ & $\begin{array}{l}\text { Symfony-PHP, Linux, } \\
\text { MySQL }\end{array}$ & -- \\
\hline 34 & $\begin{array}{l}\text { M0131 } \\
\text { Semantic Graph- } \\
\text { Search }\end{array}$ & A few terabytes & Evolving in time & Rich & Database & Data graph processing \\
\hline 35 & $\begin{array}{l}\text { M0189 } \\
\text { Light Source } \\
\text { Beamlines }\end{array}$ & $\begin{array}{l}50 \text { to } 400 \mathrm{~GB} \text { per } \\
\text { day, total } \approx 400 \mathrm{~TB}\end{array}$ & $\begin{array}{l}\text { Continuous stream } \\
\text { of data, but } \\
\text { analysis need not } \\
\text { be real time }\end{array}$ & Images & $\begin{array}{l}\text { Octopus for } \\
\text { Tomographic } \\
\text { Reconstruction, Avizo } \\
\text { (http://vsg3d.com) } \\
\text { and FIJI (a distribution } \\
\text { of ImageJ) }\end{array}$ & $\begin{array}{l}\text { Volume reconstruction, } \\
\text { feature identification, etc. }\end{array}$ \\
\hline 36 & $\begin{array}{l}\text { M0170 } \\
\text { Catalina Real- } \\
\text { Time Transient } \\
\text { Survey }\end{array}$ & $\begin{array}{l}\approx 100 \text { TB total } \\
\text { increasing by } 0.1 \text { TB } \\
\text { a night accessing PBs } \\
\text { of base astronomy } \\
\text { data, } 30 \text { TB a night } \\
\text { from successor LSST } \\
\text { in } 2020 \text { s }\end{array}$ & $\begin{array}{l}\text { Nightly update } \\
\text { runs processes in } \\
\text { real time }\end{array}$ & $\begin{array}{l}\text { Images, spectra, time } \\
\text { series, catalogs }\end{array}$ & $\begin{array}{l}\text { Custom data } \\
\text { processing pipeline } \\
\text { and data analysis } \\
\text { software }\end{array}$ & $\begin{array}{l}\text { Detection of rare events and } \\
\text { relation to existing diverse } \\
\text { data }\end{array}$ \\
\hline 37 & $\begin{array}{l}\text { M0185 } \\
\text { DOE Extreme } \\
\text { Data from } \\
\text { Cosmological } \\
\text { Sky Survey }\end{array}$ & $\begin{array}{l}\text { Several petabytes } \\
\text { from Dark Energy } \\
\text { Survey and Zwicky } \\
\text { Transient Factory, } \\
\text { simulations > } 10 \text { PB }\end{array}$ & $\begin{array}{l}\text { Analysis done in } \\
\text { batch mode with } \\
\text { data from } \\
\text { observations and } \\
\text { simulations } \\
\text { updated daily }\end{array}$ & Image and simulation data & $\begin{array}{l}\text { MPI, FFTW, viz } \\
\text { packages, numpy, } \\
\text { Boost, OpenMP, } \\
\text { ScaLAPCK, PSQL and } \\
\text { MySQL databases, } \\
\text { Eigen, cfitsio, } \\
\text { astrometry.net, and } \\
\text { Minuit2 }\end{array}$ & $\begin{array}{l}\text { New analytics needed to } \\
\text { analyze simulation results }\end{array}$ \\
\hline
\end{tabular}




\begin{tabular}{|c|c|c|c|c|c|c|}
\hline No. & Use Case & Volume & Velocity & Variety & Software & Analytics \\
\hline 38 & $\begin{array}{l}\text { M0209 } \\
\text { Large Survey } \\
\text { Data for } \\
\text { Cosmology }\end{array}$ & $\begin{array}{l}\text { Petabytes of data } \\
\text { from Dark Energy } \\
\text { Survey }\end{array}$ & $\begin{array}{l}400 \text { images of } 1 \mathrm{~GB} \\
\text { in size per night }\end{array}$ & Images & $\begin{array}{l}\text { Linux cluster, Oracle } \\
\text { RDBMS server, } \\
\text { Postgres PSQL, large } \\
\text { memory machines, } \\
\text { standard Linux } \\
\text { interactive hosts, } \\
\text { GPFS; for simulations, } \\
\text { HPC resources; } \\
\text { standard astrophysics } \\
\text { reduction software as } \\
\text { well as Perl/Python } \\
\text { wrapper scripts }\end{array}$ & $\begin{array}{l}\text { Machine learning to find } \\
\text { optical transients, Cholesky } \\
\text { decomposition for thousands } \\
\text { of simulations with matrices } \\
\text { of order } 1 \text { million on a side } \\
\text { and parallel image storage }\end{array}$ \\
\hline 39 & $\begin{array}{l}\text { M0166 } \\
\text { Particle Physics } \\
\text { at LHC }\end{array}$ & $\begin{array}{l}15 \text { PB of data } \\
\text { (experiment and } \\
\text { Monte Carlo } \\
\text { combined) per year }\end{array}$ & $\begin{array}{l}\text { Data updated } \\
\text { continuously with } \\
\text { sophisticated real- } \\
\text { time selection and } \\
\text { test analysis but all } \\
\text { analyzed } \\
\text { "properly" offline }\end{array}$ & $\begin{array}{l}\text { Different format for each } \\
\text { stage in analysis but data } \\
\text { uniform within each stage }\end{array}$ & $\begin{array}{l}\text { Grid-based } \\
\text { environment with } \\
\text { over } 350,000 \text { cores } \\
\text { running } \\
\text { simultaneously }\end{array}$ & $\begin{array}{l}\text { Sophisticated specialized } \\
\text { data analysis code followed } \\
\text { by basic exploratory statistics } \\
\text { (histogram) with complex } \\
\text { detector efficiency } \\
\text { corrections }\end{array}$ \\
\hline 40 & $\begin{array}{l}\text { M0210 } \\
\text { Belle II High } \\
\text { Energy Physics } \\
\text { Experiment }\end{array}$ & $\begin{array}{l}\text { Eventually } 120 \text { PB of } \\
\text { Monte Carlo and } \\
\text { observational data }\end{array}$ & $\begin{array}{l}\text { Data updated } \\
\text { continuously with } \\
\text { sophisticated real- } \\
\text { time selection and } \\
\text { test analysis but all } \\
\text { analyzed } \\
\text { "properly" offline }\end{array}$ & $\begin{array}{l}\text { Different format for each } \\
\text { stage in analysis but data } \\
\text { uniform within each stage }\end{array}$ & DIRAC Grid software & $\begin{array}{l}\text { Sophisticated specialized } \\
\text { data analysis code followed } \\
\text { by basic exploratory statistics } \\
\text { (histogram) with complex } \\
\text { detector efficiency } \\
\text { corrections }\end{array}$ \\
\hline 41 & $\begin{array}{l}\text { M0155 } \\
\text { EISCAT 3D } \\
\text { incoherent } \\
\text { scatter radar } \\
\text { system }\end{array}$ & $\begin{array}{l}\text { Terabytes/year } \\
\text { (current), } 40 \text { PB/year } \\
\text { starting } \approx 2022\end{array}$ & $\begin{array}{l}\text { Data updated } \\
\text { continuously with } \\
\text { real-time test } \\
\text { analysis and batch } \\
\text { full analysis }\end{array}$ & Big data uniform & $\begin{array}{l}\text { Custom analysis based } \\
\text { on flat file data } \\
\text { storage }\end{array}$ & $\begin{array}{l}\text { Pattern recognition, } \\
\text { demanding correlation } \\
\text { routines, high-level } \\
\text { parameter extraction }\end{array}$ \\
\hline 42 & $\begin{array}{l}\text { M0157 } \\
\text { ENVRI } \\
\text { Environmental } \\
\text { Research } \\
\text { Infrastructure }\end{array}$ & $\begin{array}{l}\text { Low volume (apart } \\
\text { from EISCAT 3D } \\
\text { given above), one } \\
\text { system EPOS } \approx 15 \\
\text { TB/year }\end{array}$ & $\begin{array}{l}\text { Mainly real-time } \\
\text { data streams }\end{array}$ & $\begin{array}{l}\text { Six separate projects with } \\
\text { common architecture for } \\
\text { infrastructure, data very } \\
\text { diverse across projects }\end{array}$ & $\begin{array}{l}\text { R and Python } \\
\text { (Matplotlib) for } \\
\text { visualization, custom } \\
\text { software for } \\
\text { processing }\end{array}$ & $\begin{array}{l}\text { Data assimilation, (statistical) } \\
\text { analysis, data mining, data } \\
\text { extraction, scientific } \\
\text { modeling and simulation, } \\
\text { scientific workflow }\end{array}$ \\
\hline
\end{tabular}




\begin{tabular}{|c|c|c|c|c|c|c|}
\hline No. & Use Case & Volume & Velocity & Variety & Software & Analytics \\
\hline 43 & $\begin{array}{l}\frac{\text { M0167 }}{\text { CReSIS Remote }} \\
\text { Sensing }\end{array}$ & $\begin{array}{l}\text { Around } 1 \text { PB } \\
\text { (current) increasing } \\
\text { by } 50 \text { to } 100 \text { TB per } \\
\text { mission, future } \\
\text { expedition } \approx 1 \text { PB } \\
\text { each }\end{array}$ & $\begin{array}{l}\text { Data taken in } \\
\text { ztwo-month } \\
\text { missions including } \\
\text { test analysis and } \\
\text { then later batch } \\
\text { processing }\end{array}$ & $\begin{array}{l}\text { Raw data, images with } \\
\text { final layer data used for } \\
\text { science }\end{array}$ & $\begin{array}{l}\text { Matlab for custom } \\
\text { raw data processing, } \\
\text { custom image } \\
\text { processing software, } \\
\text { GIS as user interface }\end{array}$ & $\begin{array}{l}\text { Custom signal processing to } \\
\text { produce radar images that } \\
\text { are analyzed by image } \\
\text { processing to find layers }\end{array}$ \\
\hline 44 & $\begin{array}{l}\frac{\text { M0127 }}{\text { UAVSAR Data }} \\
\text { Processing }\end{array}$ & $\begin{array}{l}110 \text { TB raw data and } \\
40 \text { TB processed, } \\
\text { plus smaller samples }\end{array}$ & $\begin{array}{l}\text { Data come from } \\
\text { aircraft and so } \\
\text { incrementally } \\
\text { added, data } \\
\text { occasionally get } \\
\text { reprocessed: new } \\
\text { processing } \\
\text { methods or } \\
\text { parameters }\end{array}$ & Image and annotation files & $\begin{array}{l}\text { ROI_PAC, GeoServer, } \\
\text { GDAL, GeoTIFF- } \\
\text { supporting tools; } \\
\text { moving to clouds }\end{array}$ & $\begin{array}{l}\text { Process raw data to get } \\
\text { images that are run through } \\
\text { image processing tools and } \\
\text { accessed from GIS }\end{array}$ \\
\hline 45 & $\begin{array}{l}\text { M0182 } \\
\text { NASA } \\
\text { LaRC/GSFC } \\
\text { iRODS }\end{array}$ & $\begin{array}{l}\text { MERRA collection } \\
\text { (below) represents } \\
\text { most of total data, } \\
\text { other smaller } \\
\text { collections }\end{array}$ & $\begin{array}{l}\text { Periodic updates } \\
\text { every six months }\end{array}$ & $\begin{array}{l}\text { Many applications to } \\
\text { combine MERRA } \\
\text { reanalysis data with other } \\
\text { reanalyses and } \\
\text { observational data such as } \\
\text { CERES }\end{array}$ & $\begin{array}{l}\text { SGE Univa Grid Engine } \\
\text { Version } 8.1 \text {, iRODS } \\
\text { Version } 3.2 \text { and/or } \\
\text { 3.3, IBM GPFS Version } \\
\text { 3.4, Cloudera Version } \\
\text { 4.5.2-1 }\end{array}$ & Federation software \\
\hline 46 & $\begin{array}{l}\frac{\text { M0129 }}{\text { MERRA Analytic }} \\
\text { Services }\end{array}$ & 480 TB from MERRA & $\begin{array}{l}\text { Increases at } \approx 1 \\
\text { TB/month }\end{array}$ & $\begin{array}{l}\text { Applications to combine } \\
\text { MERRA reanalysis data } \\
\text { with other re-analyses and } \\
\text { observational data }\end{array}$ & $\begin{array}{l}\text { Cloudera, iRODS, } \\
\text { Amazon AWS }\end{array}$ & CAaaS \\
\hline 47 & $\begin{array}{l}\text { M0090 } \\
\text { Atmospheric } \\
\text { Turbulence }\end{array}$ & $\begin{array}{l}200 \text { TB (current), } \\
500 \text { TB within } 5 \\
\text { years }\end{array}$ & $\begin{array}{l}\text { Data analyzed } \\
\text { incrementally }\end{array}$ & $\begin{array}{l}\text { Re-analysis datasets are } \\
\text { inconsistent in format, } \\
\text { resolution, semantics, and } \\
\text { metadata; } \\
\text { interpretation/analysis of } \\
\text { each of these input } \\
\text { streams into a common } \\
\text { product }\end{array}$ & $\begin{array}{l}\text { Map/Reduce or the } \\
\text { like, SciDB or other } \\
\text { scientific database }\end{array}$ & $\begin{array}{l}\text { Data mining customized for } \\
\text { specific event types }\end{array}$ \\
\hline
\end{tabular}




\begin{tabular}{|c|c|c|c|c|c|c|}
\hline No. & Use Case & Volume & Velocity & Variety & Software & Analytics \\
\hline 48 & $\frac{\text { M0186 }}{\text { Climate Studies }}$ & $\begin{array}{l}\text { Up to } 30 \text { PB/year } \\
\text { from } 15 \text { end-to-end } \\
\text { simulations at } \\
\text { NERSC, more at } \\
\text { other HPC centers }\end{array}$ & $\begin{array}{l}42 \mathrm{~GB} / \text { second } \\
\text { from simulations }\end{array}$ & $\begin{array}{l}\text { Variety across simulation } \\
\text { groups and between } \\
\text { observation and } \\
\text { simulation }\end{array}$ & $\begin{array}{l}\text { National Center for } \\
\text { Atmospheric Research } \\
\text { (NCAR) PIO library and } \\
\text { utilities NCL and NCO, } \\
\text { parallel NetCDF }\end{array}$ & $\begin{array}{l}\text { Need analytics next to data } \\
\text { storage }\end{array}$ \\
\hline 49 & $\begin{array}{l}\text { M0183 } \\
\text { DOE-BER } \\
\text { Subsurface } \\
\text { Biogeochemistry }\end{array}$ & - & - & $\begin{array}{l}\text { From omics of the } \\
\text { microbes in the soil to } \\
\text { watershed hydro- } \\
\text { biogeochemistry, from } \\
\text { observation to simulation }\end{array}$ & $\begin{array}{l}\text { PFLOWTran, postgres, } \\
\text { HDF5, Akuna, NEWT, } \\
\text { etc. }\end{array}$ & $\begin{array}{l}\text { Data mining, data quality } \\
\text { assessment, cross- } \\
\text { correlation across datasets, } \\
\text { reduced model } \\
\text { development, statistics, } \\
\text { quality assessment, data } \\
\text { fusion }\end{array}$ \\
\hline 50 & $\begin{array}{l}\text { M0184 } \\
\text { DOE-BER } \\
\text { AmeriFlux and } \\
\text { FLUXNET } \\
\text { Networks }\end{array}$ & - & $\begin{array}{l}\text { Streaming data } \\
\text { from } \approx 150 \text { towers } \\
\text { in AmeriFlux and } \\
\text { over } 500 \text { towers } \\
\text { distributed } \\
\text { globally collecting } \\
\text { flux measurements }\end{array}$ & $\begin{array}{l}\text { Flux data merged with } \\
\text { biological, disturbance, } \\
\text { and other ancillary data }\end{array}$ & $\begin{array}{l}\text { EddyPro, custom } \\
\text { analysis software, R, } \\
\text { Python, neural } \\
\text { networks, Matlab }\end{array}$ & $\begin{array}{l}\text { Data mining, data quality } \\
\text { assessment, cross- } \\
\text { correlation across datasets, } \\
\text { data assimilation, data } \\
\text { interpolation, statistics, } \\
\text { quality assessment, data } \\
\text { fusion }\end{array}$ \\
\hline 51 & $\begin{array}{l}\text { M0223 } \\
\text { Consumption } \\
\text { forecasting in } \\
\text { Smart Grids }\end{array}$ & $\begin{array}{l}4 \mathrm{~TB} / \text { year for a city } \\
\text { with } 1.4 \text { million } \\
\text { sensors, such as Los } \\
\text { Angeles }\end{array}$ & $\begin{array}{l}\text { Streaming data } \\
\text { from millions of } \\
\text { sensors }\end{array}$ & $\begin{array}{l}\text { Tuple-based: timeseries, } \\
\text { database rows; graph- } \\
\text { based: network topology, } \\
\text { customer connectivity; } \\
\text { some semantic data for } \\
\text { normalization }\end{array}$ & $\begin{array}{l}\text { R/Matlab, Weka, } \\
\text { Hadoop; GIS-based } \\
\text { visualization }\end{array}$ & $\begin{array}{l}\text { Forecasting models, machine } \\
\text { learning models, time series } \\
\text { analysis, clustering, motif } \\
\text { detection, complex event } \\
\text { processing, visual network } \\
\text { analysis }\end{array}$ \\
\hline
\end{tabular}




\begin{tabular}{|c|c|c|c|c|c|c|}
\hline No. & Use Case & Volume & Velocity & Variety & Software & Analytics \\
\hline $2-1$ & $\begin{array}{l}\frac{\text { M0633 }}{\text { NASA Earth }} \\
\text { Observing } \\
\text { System Data } \\
\text { and Information } \\
\text { System (EOSDIS) }\end{array}$ & $\begin{array}{l}\text { Data size is 22PB } \\
\text { corresponding to } \\
\text { Total Earth } \\
\text { Observation Data } \\
\text { managed by NASA } \\
\text { EOSDIS accumulated } \\
\text { since } 1994 \text {. Higher } \\
\text { resolution } \\
\text { spaceborne } \\
\text { instruments are } \\
\text { expected to increase } \\
\text { that volume by two } \\
\text { orders of magnitude } \\
\text { ( 200 PB) over the } \\
\text { next } 7 \text { years. In a } \\
\text { given year, EOSDIS } \\
\text { distributes a volume } \\
\text { that is comparable } \\
\text { to the overall } \\
\text { cumulative archive } \\
\text { volume. }\end{array}$ & $\begin{array}{l}\text { This is now an } \\
\text { archive of } 23 \text { years } \\
\text { data but is } \\
\text { continually } \\
\text { increasing in both } \\
\text { gathered and } \\
\text { distributed data. } \\
\text { In a given year, } \\
\text { EOSDIS distributes } \\
\text { a volume that is } \\
\text { comparable to the } \\
\text { overall cumulative } \\
\text { archive volume. }\end{array}$ & $\begin{array}{l}\text { EOSDIS's Common } \\
\text { Metadata Repository } \\
\text { includes over } 6400 \text { EOSDIS } \\
\text { data collections as of June } \\
2017 \text {, providing significant } \\
\text { challenges in data } \\
\text { discovery. CMR and other } \\
\text { interoperability } \\
\text { frameworks (metrics, } \\
\text { browse imagery, } \\
\text { governance) knit together } \\
12 \text { different archives, each } \\
\text { with a different } \\
\text { implementation. Nearly all } \\
\text { Earth science disciplines } \\
\text { are represented in EOSDIS. }\end{array}$ & $\begin{array}{l}\text { EOSDIS uses high- } \\
\text { performance } \\
\text { software, such as the } \\
\text { netCDF Command } \\
\text { Operators. However, } \\
\text { current prototypes are } \\
\text { using cloud computing } \\
\text { and data-parallel } \\
\text { algorithms (e.g., } \\
\text { Spark) to achieve an } \\
\text { order of magnitude } \\
\text { speed-up. Cloud } \\
\text { storage and database } \\
\text { schemes are being } \\
\text { investigated. Python, } \\
\text { Fortran, C languages. } \\
\text { Visualization through } \\
\text { tools such as } \\
\text { Giovanni. }\end{array}$ & $\begin{array}{l}\text { Analytics used includes: } \\
\text { (1) computing statistical } \\
\text { measures of Earth } \\
\text { Observation data across a } \\
\text { variety of dimensions } \\
\text { (2) examining covariance and } \\
\text { correlation of a variety of } \\
\text { Earth observations } \\
\text { (3) assimilating multiple data } \\
\text { variables into a model using } \\
\text { Kalman filtering } \\
\text { (4) analyzing time series. }\end{array}$ \\
\hline
\end{tabular}




\begin{tabular}{|c|c|c|c|c|c|c|}
\hline No. & Use Case & Volume & Velocity & Variety & Software & Analytics \\
\hline $2-2$ & $\begin{array}{l}\text { M0634 } \\
\text { Web-Enabled } \\
\text { Landsat Data } \\
\text { (WELD) } \\
\text { Processing }\end{array}$ & $\begin{array}{l}\text { The data represent } \\
\text { the operational time } \\
\text { period of } 1984 \text { to } \\
2011 \text { for the Landsat } \\
4,5 \text {, and } 7 \text { satellites } \\
\text { and corresponds to } \\
\text { 30PB of processed } \\
\text { data through the } \\
\text { pipeline (1PB inputs, } \\
\text { 10PB intermediate, } \\
6 \text { PB outputs) }\end{array}$ & $\begin{array}{l}\text { Data was collected } \\
\text { over a period of } 27 \\
\text { years and is being } \\
\text { processed over a } \\
\text { period of } 5 \text { years. } \\
\text { Based on } \\
\text { programmatic } \\
\text { goals of processing } \\
\text { several iterations } \\
\text { of the final } \\
\text { product over the } \\
\text { span of the } \\
\text { project, } 150 T B / \text { day } \\
\text { is processed per } \\
\text { day during } \\
\text { processing time } \\
\text { periods. }\end{array}$ & $\begin{array}{l}\text { None. This use case } \\
\text { basically deals with a } \\
\text { single dataset. }\end{array}$ & $\begin{array}{l}\text { NEX science platform } \\
\text { - data management, } \\
\text { workflow processing, } \\
\text { provenance capture; } \\
\text { WELD science } \\
\text { processing algorithms } \\
\text { from South Dakota } \\
\text { State University } \\
\text { (SDSU), browse } \\
\text { visualization, and } \\
\text { time-series code; } \\
\text { Global Imagery } \\
\text { Browse Service (GIBS) } \\
\text { data visualization } \\
\text { platform; USGS data } \\
\text { distribution platform. } \\
\text { Custom-built } \\
\text { application and } \\
\text { libraries built on top } \\
\text { of open-source } \\
\text { libraries. }\end{array}$ & $\begin{array}{l}\text { There are number of } \\
\text { analytics processes } \\
\text { throughout the processing } \\
\text { pipeline. The key analytics is } \\
\text { identifying best available } \\
\text { pixels for spatio-temporal } \\
\text { composition and spatial } \\
\text { aggregation processes as a } \\
\text { part of the overall QA. The } \\
\text { analytics algorithms are } \\
\text { custom developed for this } \\
\text { use case. }\end{array}$ \\
\hline
\end{tabular}




\begin{tabular}{|c|c|c|c|c|c|c|}
\hline No. & Use Case & Volume & Velocity & Variety & Software & Analytics \\
\hline $2-3$ & $\begin{array}{l}\text { M0676 } \\
\text { Urban context- } \\
\text { aware event } \\
\text { management for } \\
\text { Smart Cities - } \\
\text { Public safety }\end{array}$ & $\begin{array}{l}\text { Depending on the } \\
\text { sensor type and data } \\
\text { type, some sensors } \\
\text { can produce over a } \\
\text { gigabyte of data in } \\
\text { the space of hours. } \\
\text { Other data is as } \\
\text { small as infrequent } \\
\text { sensor activations or } \\
\text { text messages. }\end{array}$ & $\begin{array}{l}\text { New records were } \\
\text { gathered per week } \\
\text { or when available, } \\
\text { except for city } \\
\text { events when the } \\
\text { data was gathered } \\
\text { once per month } \\
\text { and social media } \\
\text { when data was } \\
\text { gathered every } \\
\text { day. }\end{array}$ & $\begin{array}{l}\text { Everything from text files, } \\
\text { raw media, imagery, } \\
\text { electronic data, human- } \\
\text { generated data all in } \\
\text { various formats. } \\
\text { Heterogeneous datasets } \\
\text { are fused together for } \\
\text { analytical use. }\end{array}$ & $\begin{array}{l}\text { Currently, baseline } \\
\text { leverages } 1 . \text { NLP } \\
\text { (several variants); } 2 \text {. } \\
\text { R/R } \\
\text { Studio/Python/Java; } 3 . \\
\text { Spark/Kafka; } 4 . \\
\text { Custom applications } \\
\text { and visualization } \\
\text { tools. }\end{array}$ & $\begin{array}{l}\text { - Pattern detection, Link } \\
\text { analysis, Sentiment } \\
\text { analysis, Time-series } \\
\text { forecasting } \\
\text { - Pattern recognition of all } \\
\text { kind (e.g., event behavior } \\
\text { automatic analysis, cultural } \\
\text { patterns). } \\
\text { - Classification: event type, } \\
\text { classification, using } \\
\text { multivariate time series to } \\
\text { generate network, content, } \\
\text { geographical features and } \\
\text { so forth. } \\
\text { - Clustering: per topic, } \\
\text { similarity, spatial-temporal, } \\
\text { and additional features. } \\
\text { - Text Analytics (sentiment, } \\
\text { entity similarity) } \\
\text { - Link Analysis: using } \\
\text { similarity and statistical } \\
\text { techniques } \\
\text { - Online learning: real-time } \\
\text { information analysis. } \\
\text { - Multiview learning: data } \\
\text { fusion feature learning } \\
\text { - Anomaly detection: } \\
\text { unexpected event behavior } \\
\text { - Visualizations based on } \\
\text { patterns, spatial-temporal } \\
\text { changes. }\end{array}$ \\
\hline
\end{tabular}




\section{Appendix C: Use Case Requirements Summary}

Requirements were extracted from each Version 1 use case (the Version 2 use cases were not included) within seven characteristic categories introduced in Section 3.1. The number of requirements within each category varied for each use case. Table C-1 contains the use case specific requirements.

Table C-1: Use Case Specific Requirements

\begin{tabular}{|c|c|c|c|c|c|c|c|c|}
\hline No. & Use Case & $\begin{array}{c}\text { Data } \\
\text { Sources }\end{array}$ & $\begin{array}{c}\text { Data } \\
\text { Transformation }\end{array}$ & Capabilities & $\begin{array}{c}\text { Data } \\
\text { Consumer }\end{array}$ & $\begin{array}{l}\text { Security and } \\
\text { Privacy }\end{array}$ & $\begin{array}{c}\text { Life Cycle } \\
\text { Management }\end{array}$ & Other \\
\hline 1 & $\begin{array}{l}\frac{\text { M0147 }}{\text { Census }} \\
2010 \text { and } \\
2000\end{array}$ & $\begin{array}{l}\text { 1. Large } \\
\text { document format } \\
\text { from centralized } \\
\text { storage }\end{array}$ & -- & $\begin{array}{l}\text { 1. Large } \\
\text { centralized } \\
\text { storage (storage) }\end{array}$ & -- & 1. Title 13 data & $\begin{array}{l}\text { 1. Long-term } \\
\text { preservation of data } \\
\text { as-is for } 75 \text { years } \\
\text { 2. Long-term } \\
\text { preservation at the } \\
\text { bit level } \\
\text { 3. Curation process } \\
\text { including format } \\
\text { transformation } \\
\text { 4. Access and } \\
\text { analytics processing } \\
\text { after } 75 \text { years } \\
5 . \text { No data loss }\end{array}$ & -- \\
\hline 2 & $\begin{array}{l}\frac{\text { M0148 }}{\text { NARA: }} \\
\text { Search, } \\
\text { Retrieve, } \\
\text { Preservatio } \\
n\end{array}$ & $\begin{array}{l}\text { 1. Distributed } \\
\text { data sources } \\
\text { 2. Large data } \\
\text { storage } \\
\text { 3. Bursty data } \\
\text { ranging from GBs } \\
\text { to hundreds of } \\
\text { terabytes } \\
\text { 4. Wide variety of } \\
\text { data formats } \\
\text { including } \\
\text { unstructured and }\end{array}$ & $\begin{array}{l}\text { 1. Crawl and index } \\
\text { from distributed } \\
\text { data sources } \\
\text { 2. Various analytics } \\
\text { processing } \\
\text { including ranking, } \\
\text { data } \\
\text { categorization, } \\
\text { detection of PII } \\
\text { data } \\
\text { 3. Data } \\
\text { preprocessing }\end{array}$ & $\begin{array}{l}\text { 1. Large data } \\
\text { storage } \\
\text { 2. Various storage } \\
\text { systems such as } \\
\text { NetApps, Hitachi, } \\
\text { magnetic tapes }\end{array}$ & $\begin{array}{l}\text { 1. High } \\
\text { relevancy and } \\
\text { high recall } \\
\text { from search } \\
\text { 2. High } \\
\text { accuracy from } \\
\text { categorization } \\
\text { of records } \\
\text { 3. Various } \\
\text { storage } \\
\text { systems such } \\
\text { as NetApps, }\end{array}$ & $\begin{array}{l}\text { 1. Security } \\
\text { policy }\end{array}$ & $\begin{array}{l}\text { 1. Pre-process for } \\
\text { virus scan } \\
\text { 2. File format } \\
\text { identification } \\
\text { 3. Indexing } \\
\text { 4. Records } \\
\text { categorization }\end{array}$ & $\begin{array}{l}\text { 1. Mobile } \\
\text { search with } \\
\text { similar } \\
\text { interfaces/ } \\
\text { results from } \\
\text { desktop }\end{array}$ \\
\hline
\end{tabular}




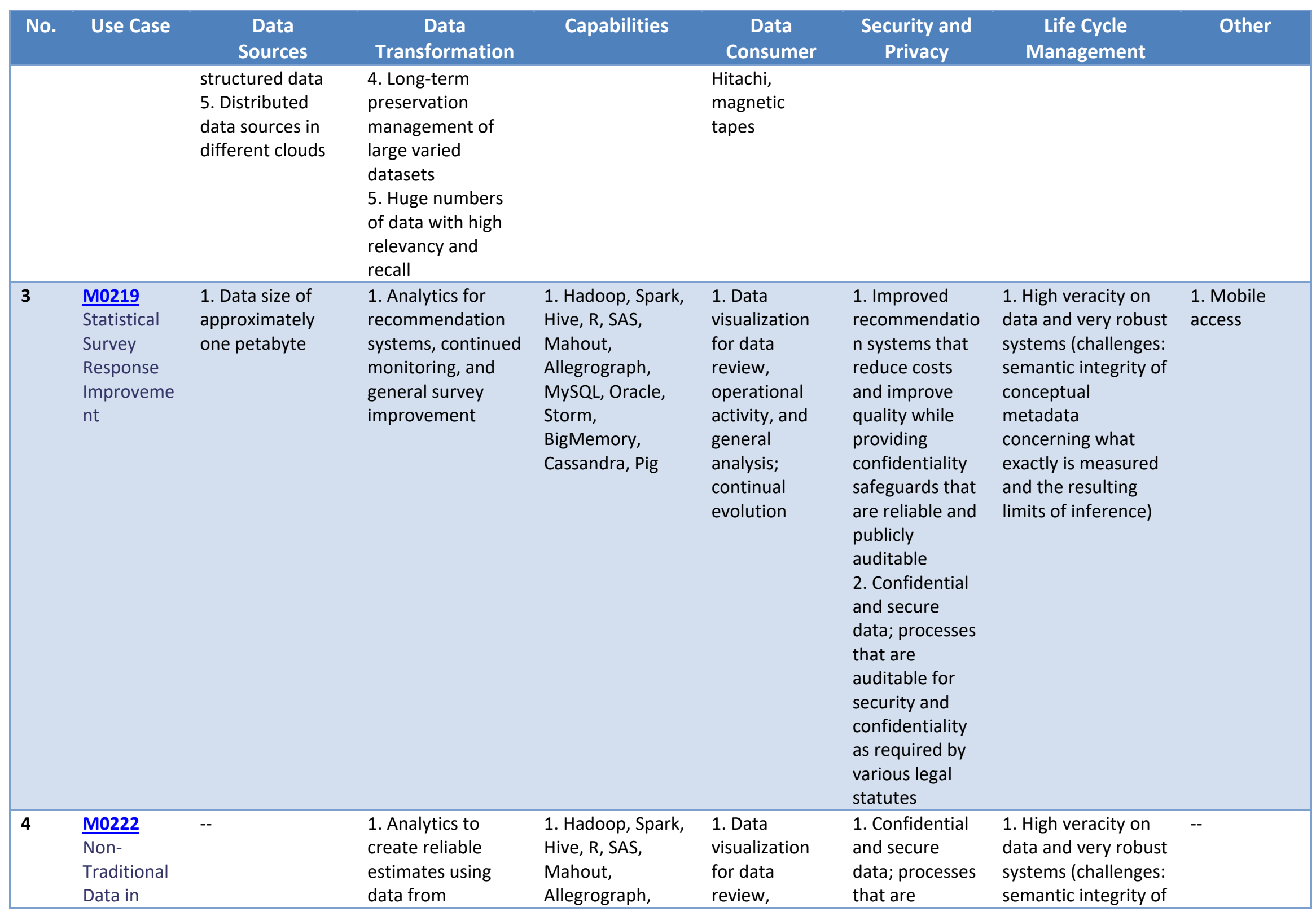




\begin{tabular}{|c|c|c|c|c|c|c|c|c|}
\hline No. & Use Case & $\begin{array}{l}\text { Data } \\
\text { Sources }\end{array}$ & $\begin{array}{c}\text { Data } \\
\text { Transformation }\end{array}$ & Capabilities & $\begin{array}{c}\text { Data } \\
\text { Consumer }\end{array}$ & $\begin{array}{l}\text { Security and } \\
\text { Privacy }\end{array}$ & $\begin{array}{c}\text { Life Cycle } \\
\text { Management }\end{array}$ & Other \\
\hline & $\begin{array}{l}\text { Statistical } \\
\text { Survey } \\
\text { Response } \\
\text { Improveme } \\
\text { nt }\end{array}$ & & $\begin{array}{l}\text { traditional survey } \\
\text { sources, } \\
\text { government } \\
\text { administrative data } \\
\text { sources, and non- } \\
\text { traditional sources } \\
\text { from the digital } \\
\text { economy }\end{array}$ & $\begin{array}{l}\text { MySQL, Oracle, } \\
\text { Storm, } \\
\text { BigMemory, } \\
\text { Cassandra, Pig }\end{array}$ & $\begin{array}{l}\text { operational } \\
\text { activity, and } \\
\text { general } \\
\text { analysis; } \\
\text { continual } \\
\text { evolution }\end{array}$ & $\begin{array}{l}\text { auditable for } \\
\text { security and } \\
\text { confidentiality } \\
\text { as required by } \\
\text { various legal } \\
\text { statutes }\end{array}$ & $\begin{array}{l}\text { conceptual } \\
\text { metadata } \\
\text { concerning what } \\
\text { exactly is measured } \\
\text { and the resulting } \\
\text { limits of inference) }\end{array}$ & \\
\hline 6 & $\frac{\text { M0161 }}{\text { Mendeley }}$ & $\begin{array}{l}\text { 1. File-based } \\
\text { documents with } \\
\text { constant new } \\
\text { uploads } \\
\text { 2. Variety of file } \\
\text { types such as } \\
\text { PDFs, social } \\
\text { network log files, } \\
\text { client activities } \\
\text { images, } \\
\text { spreadsheet, } \\
\text { presentation files }\end{array}$ & $\begin{array}{l}\text { 1. Standard } \\
\text { machine learning } \\
\text { and analytics } \\
\text { libraries } \\
\text { 2. Efficient scalable } \\
\text { and parallelized } \\
\text { way to match } \\
\text { between } \\
\text { documents } \\
\text { 3. Third-party } \\
\text { annotation tools or } \\
\text { publisher } \\
\text { watermarks and } \\
\text { cover pages }\end{array}$ & $\begin{array}{l}\text { 1. Amazon Elastic } \\
\text { Compute Cloud } \\
\text { (EC2) with HDFS } \\
\text { (infrastructure) } \\
\text { 2. S3 (storage) } \\
\text { 3. Hadoop } \\
\text { (platform) } \\
\text { 4. Scribe, Hive, } \\
\text { Mahout, Python } \\
\text { (language) } \\
\text { 5. Moderate } \\
\text { storage (15 TB } \\
\text { with } 1 \mathrm{~TB} / \text { month) } \\
\text { 6. Batch and real- } \\
\text { time processing }\end{array}$ & $\begin{array}{l}\text { 1. Custom- } \\
\text { built reporting } \\
\text { tools } \\
2 . \\
\text { Visualization } \\
\text { tools such as } \\
\text { networking } \\
\text { graph, } \\
\text { scatterplots, } \\
\text { etc. }\end{array}$ & $\begin{array}{l}\text { 1. Access } \\
\text { controls for } \\
\text { who reads what } \\
\text { content }\end{array}$ & $\begin{array}{l}\text { 1. Metadata } \\
\text { management from } \\
\text { PDF extraction } \\
\text { 2. Identification of } \\
\text { document } \\
\text { duplication } \\
\text { 3. Persistent } \\
\text { identifier } \\
\text { 4. Metadata } \\
\text { correlation between } \\
\text { data repositories } \\
\text { such as CrossRef, } \\
\text { PubMed, and Arxiv }\end{array}$ & $\begin{array}{l}\text { 1. Windows } \\
\text { Android and } \\
\text { iOS mobile } \\
\text { devices for } \\
\text { content } \\
\text { deliverables } \\
\text { from } \\
\text { Windows } \\
\text { desktops }\end{array}$ \\
\hline 7 & $\begin{array}{l}\text { M0164 } \\
\text { Netflix } \\
\text { Movie } \\
\text { Service }\end{array}$ & $\begin{array}{l}\text { 1. User profiles } \\
\text { and ranking } \\
\text { information }\end{array}$ & $\begin{array}{l}\text { 1. Streaming video } \\
\text { contents to } \\
\text { multiple clients } \\
\text { 2. Analytic } \\
\text { processing for } \\
\text { matching client } \\
\text { interest in movie } \\
\text { selection }\end{array}$ & $\begin{array}{l}\text { 1. Hadoop } \\
\text { (platform) } \\
\text { 2. Pig (language) } \\
\text { 3. Cassandra and } \\
\text { Hive } \\
\text { 4. Huge numbers } \\
\text { of subscribers, } \\
\text { ratings, and }\end{array}$ & $\begin{array}{l}\text { 1. Streaming } \\
\text { and rendering } \\
\text { media }\end{array}$ & $\begin{array}{l}\text { 1. Preservation } \\
\text { of users, } \\
\text { privacy and } \\
\text { digital rights for } \\
\text { media }\end{array}$ & $\begin{array}{l}\text { 1. Continued ranking } \\
\text { and updating based } \\
\text { on user profile and } \\
\text { analytic results }\end{array}$ & $\begin{array}{l}\text { 1. Smart } \\
\text { interface } \\
\text { accessing } \\
\text { movie } \\
\text { content on } \\
\text { mobile } \\
\text { platforms }\end{array}$ \\
\hline
\end{tabular}




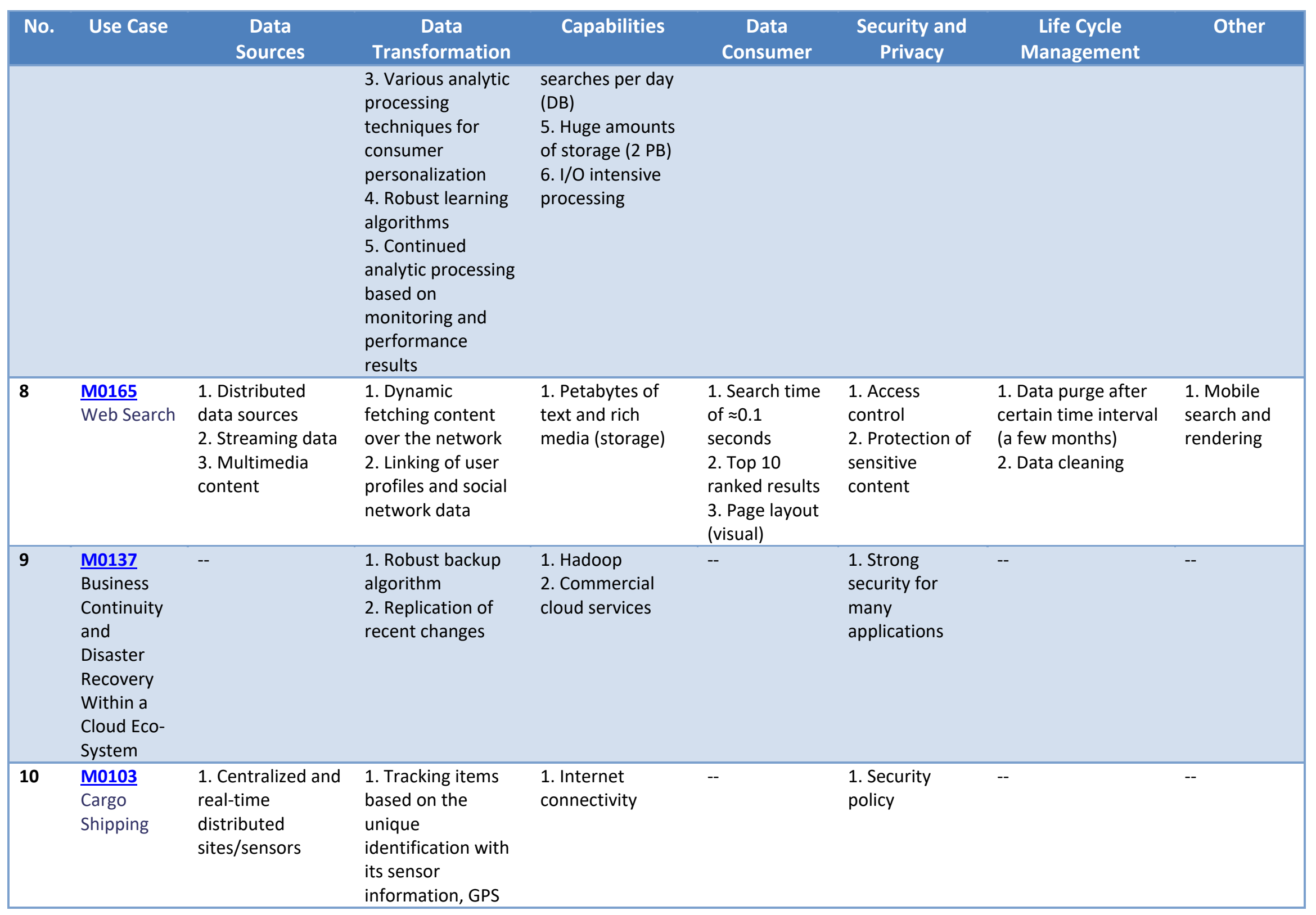




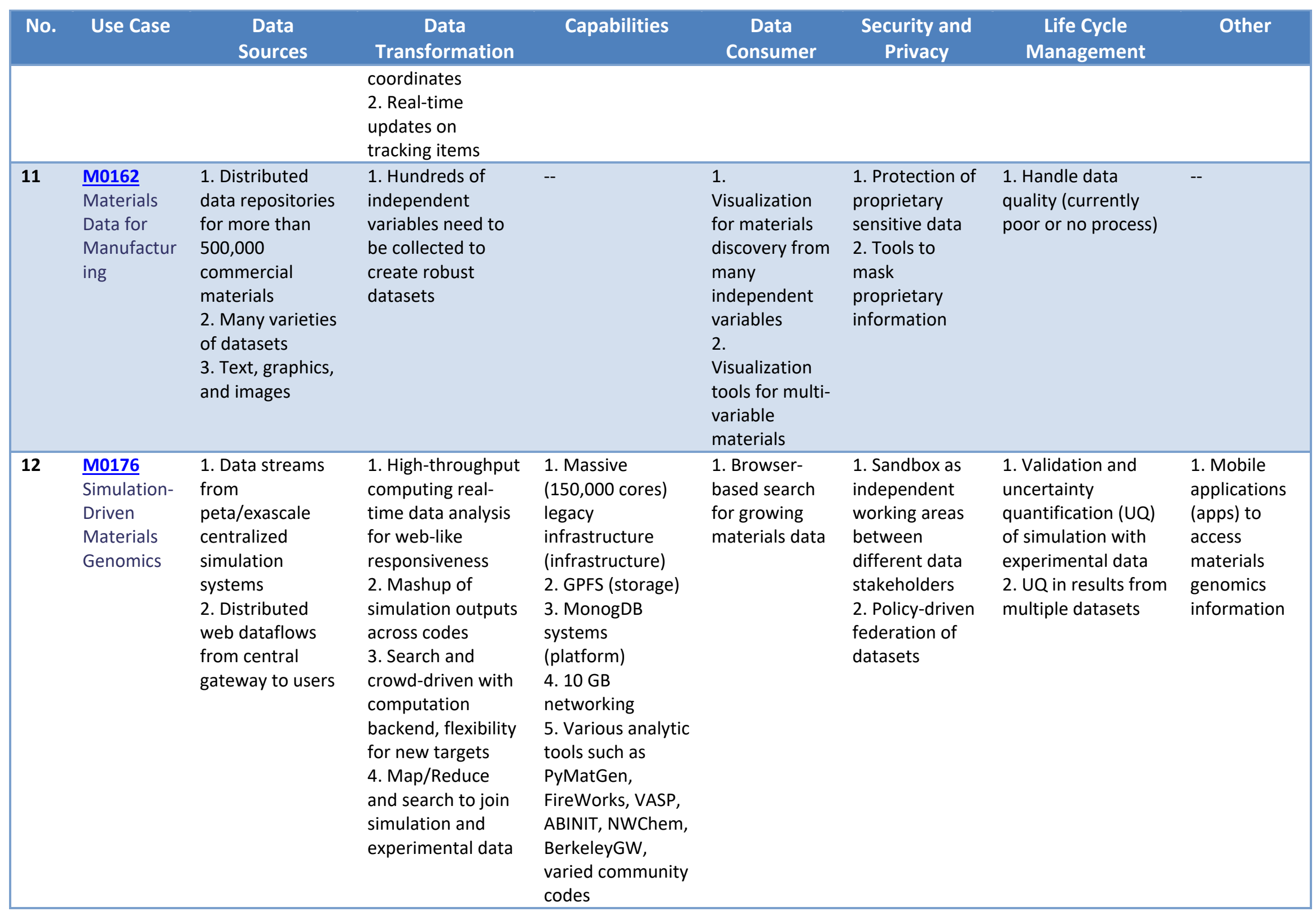




\begin{tabular}{|c|c|c|c|c|c|c|c|c|}
\hline No. & Use Case & $\begin{array}{l}\text { Data } \\
\text { Sources }\end{array}$ & $\begin{array}{c}\text { Data } \\
\text { Transformation }\end{array}$ & Capabilities & $\begin{array}{l}\text { Data } \\
\text { Consumer }\end{array}$ & $\begin{array}{l}\text { Security and } \\
\text { Privacy }\end{array}$ & $\begin{array}{c}\text { Life Cycle } \\
\text { Management }\end{array}$ & Other \\
\hline 13 & $\begin{array}{l}\text { M0213 } \\
\text { Large-Scale } \\
\text { Geospatial } \\
\text { Analysis } \\
\text { and } \\
\text { Visualizatio } \\
\mathrm{n}\end{array}$ & $\begin{array}{l}\text { 1. Unique } \\
\text { approaches to } \\
\text { indexing and } \\
\text { distributed } \\
\text { analysis required } \\
\text { for geospatial } \\
\text { data }\end{array}$ & $\begin{array}{l}\text { 1. Analytics: closest } \\
\text { point of approach, } \\
\text { deviation from } \\
\text { route, point } \\
\text { density over time, } \\
\text { PCA and ICA } \\
\text { 2. Unique } \\
\text { approaches to } \\
\text { indexing and } \\
\text { distributed analysis } \\
\text { required for } \\
\text { geospatial data }\end{array}$ & $\begin{array}{l}\text { 1. Geospatially } \\
\text { enabled RDBMS, } \\
\text { geospatial } \\
\text { server/analysis } \\
\text { software, e.g., } \\
\text { ESRI ArcServer, } \\
\text { Geoserver }\end{array}$ & $\begin{array}{l}1 . \\
\text { Visualization } \\
\text { with GIS at } \\
\text { high and low } \\
\text { network } \\
\text { bandwidths } \\
\text { and on } \\
\text { dedicated } \\
\text { facilities and } \\
\text { handhelds }\end{array}$ & $\begin{array}{l}\text { 1. Complete } \\
\text { security of } \\
\text { sensitive data } \\
\text { in transit and at } \\
\text { rest } \\
\text { (particularly on } \\
\text { handhelds) }\end{array}$ & -- & -- \\
\hline 14 & $\begin{array}{l}\text { M0214 } \\
\text { Object } \\
\text { Identificatio } \\
\mathrm{n} \text { and } \\
\text { Tracking }\end{array}$ & $\begin{array}{l}\text { 1. Real-time data } \\
\text { FMV ( } 30 \text { to } 60 \\
\text { frames/ second at } \\
\text { full-color } 1080 \mathrm{P} \\
\text { resolution) and } \\
\text { WALF ( } 1 \text { to } 10 \\
\text { frames/ second at } \\
10,000 \times 10,000 \\
\text { full-color } \\
\text { resolution) }\end{array}$ & $\begin{array}{l}\text { 1. Rich analytics } \\
\text { with object } \\
\text { identification, } \\
\text { pattern } \\
\text { recognition, crowd } \\
\text { behavior, } \\
\text { economic activity, } \\
\text { and data fusion }\end{array}$ & $\begin{array}{l}\text { 1. Wide range of } \\
\text { custom software } \\
\text { and tools } \\
\text { including } \\
\text { traditional } \\
\text { RDBMSs and } \\
\text { display tools } \\
\text { 2. Several } \\
\text { network } \\
\text { requirements } \\
\text { 3. GPU usage } \\
\text { important }\end{array}$ & $\begin{array}{l}1 . \\
\text { Visualization } \\
\text { of extracted } \\
\text { outputs as } \\
\text { overlays on a } \\
\text { geospatial } \\
\text { display; links } \\
\text { back to the } \\
\text { originating } \\
\text { image/video } \\
\text { segment as } \\
\text { overlay }\end{array}$ & $\begin{array}{l}\text { 1. Significant } \\
\text { security and } \\
\text { privacy issues; } \\
\text { sources and } \\
\text { methods never } \\
\text { compromised }\end{array}$ & $\begin{array}{l}\text { 1. Veracity of } \\
\text { extracted objects }\end{array}$ & -- \\
\hline
\end{tabular}


NIST BIg DATA INTEROPERABILITY FRAMEWORK: Volume 3, USE CASES AND GENERAL REQUIREMENTS

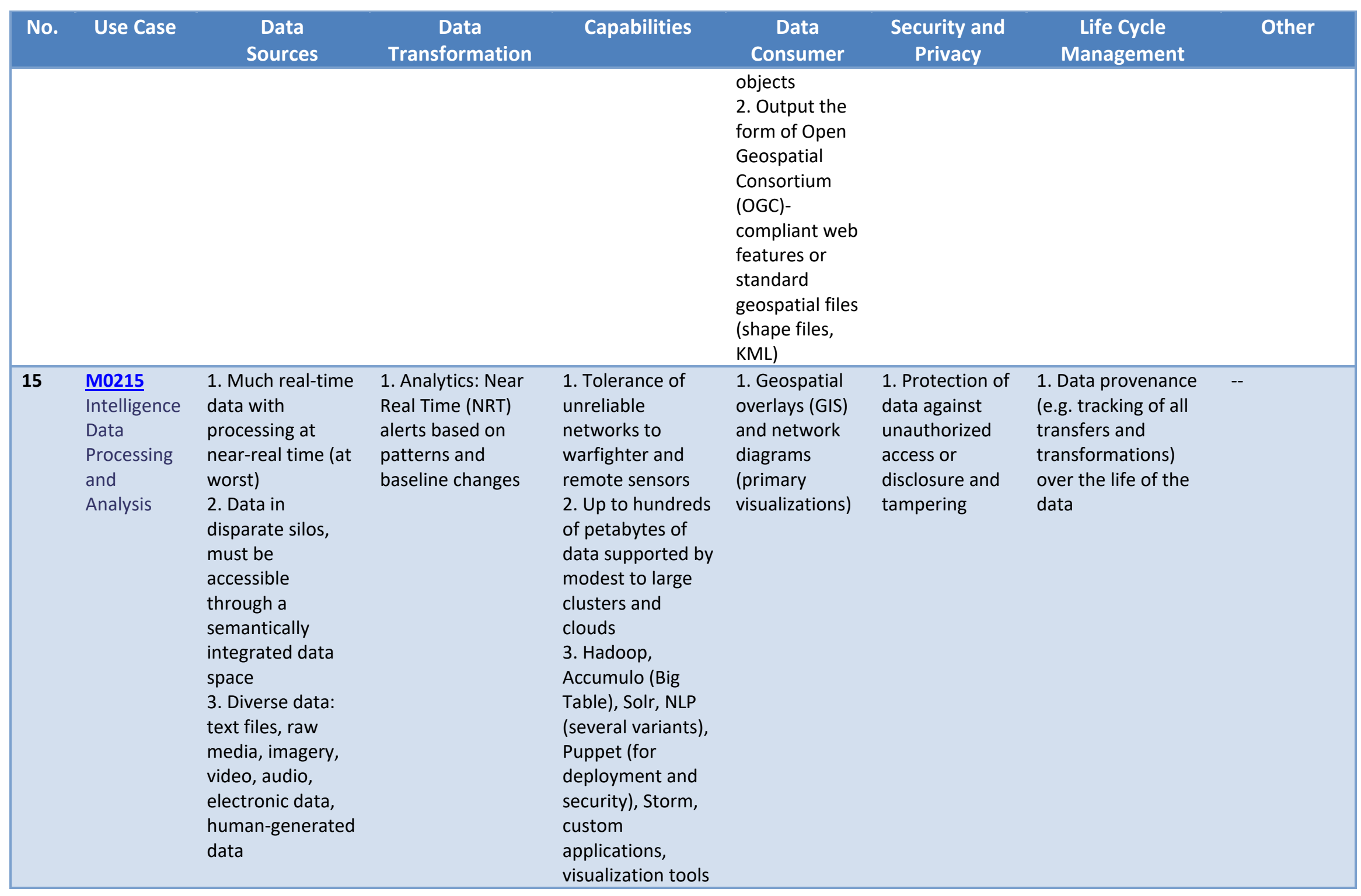




\begin{tabular}{|c|c|c|c|c|c|c|c|c|}
\hline No. & Use Case & $\begin{array}{l}\text { Data } \\
\text { Sources }\end{array}$ & $\begin{array}{c}\text { Data } \\
\text { Transformation }\end{array}$ & Capabilities & $\begin{array}{l}\text { Data } \\
\text { Consumer }\end{array}$ & $\begin{array}{l}\text { Security and } \\
\text { Privacy }\end{array}$ & $\begin{array}{l}\text { Life Cycle } \\
\text { Management }\end{array}$ & Other \\
\hline
\end{tabular}




\begin{tabular}{|c|c|c|c|c|c|c|c|c|}
\hline No. & Use Case & $\begin{array}{l}\text { Data } \\
\text { Sources }\end{array}$ & $\begin{array}{c}\text { Data } \\
\text { Transformation }\end{array}$ & Capabilities & $\begin{array}{l}\text { Data } \\
\text { Consumer }\end{array}$ & $\begin{array}{l}\text { Security and } \\
\text { Privacy }\end{array}$ & $\begin{array}{c}\text { Life Cycle } \\
\text { Management }\end{array}$ & Other \\
\hline & & $\begin{array}{l}\text { highly variable } \\
\text { fashion }\end{array}$ & & & & & & \\
\hline 18 & $\begin{array}{l}\text { M0191 } \\
\begin{array}{l}\text { Computatio } \\
\text { nal } \\
\text { Bioimaging }\end{array}\end{array}$ & $\begin{array}{l}\text { 1. Distributed } \\
\text { multi-modal high- } \\
\text { resolution } \\
\text { experimental } \\
\text { sources of } \\
\text { bioimages } \\
\text { (instruments) } \\
2.50 \text { TB of data in } \\
\text { formats that } \\
\text { include images }\end{array}$ & $\begin{array}{l}\text { 1. High-throughput } \\
\text { computing with } \\
\text { responsive analysis } \\
\text { 2. Segmentation of } \\
\text { regions of interest; } \\
\text { crowd-based } \\
\text { selection and } \\
\text { extraction of } \\
\text { features; object } \\
\text { classification, and } \\
\text { organization; and } \\
\text { search }\end{array}$ & $\begin{array}{l}\text { 1. ImageJ, } \\
\text { OMERO, } \\
\text { VolRover, } \\
\text { advanced } \\
\text { segmentation and } \\
\text { feature detection } \\
\text { methods from } \\
\text { applied math } \\
\text { researchers; } \\
\text { scalable key-value } \\
\text { and object store } \\
\text { databases needed }\end{array}$ & $\begin{array}{l}\text { 1. 3D } \\
\text { structural } \\
\text { modeling }\end{array}$ & $\begin{array}{l}\text { 1. Significant } \\
\text { but optional } \\
\text { security and } \\
\text { privacy } \\
\text { including } \\
\text { secure servers } \\
\text { and } \\
\text { anonymization }\end{array}$ & $\begin{array}{l}\text { 1. Workflow } \\
\text { components } \\
\text { including data } \\
\text { acquisition, storage, } \\
\text { enhancement, } \\
\text { minimizing noise }\end{array}$ & -- \\
\hline
\end{tabular}




\begin{tabular}{|c|c|c|c|c|c|c|c|c|}
\hline No. & Use Case & $\begin{array}{l}\text { Data } \\
\text { Sources }\end{array}$ & $\begin{array}{c}\text { Data } \\
\text { Transformation }\end{array}$ & Capabilities & $\begin{array}{l}\text { Data } \\
\text { Consumer }\end{array}$ & $\begin{array}{l}\text { Security and } \\
\text { Privacy }\end{array}$ & $\begin{array}{c}\text { Life Cycle } \\
\text { Management }\end{array}$ & Other \\
\hline & & & $\begin{array}{l}\text { 3. Advanced } \\
\text { biosciences } \\
\text { discovery through } \\
\text { Big Data } \\
\text { techniques / } \\
\text { extreme-scale } \\
\text { computing; in- } \\
\text { database } \\
\text { processing and } \\
\text { analytics; machine } \\
\text { learning (SVM and } \\
\text { RF) for } \\
\text { classification and } \\
\text { recommendation } \\
\text { services; advanced } \\
\text { algorithms for } \\
\text { massive image } \\
\text { analysis; high- } \\
\text { performance } \\
\text { computational } \\
\text { solutions } \\
\text { 4. Massive data } \\
\text { analysis toward } \\
\text { massive imaging } \\
\text { datasets. }\end{array}$ & $\begin{array}{l}\text { 2. NERSC's } \\
\text { Hopper } \\
\text { infrastructure } \\
\text { 3. database and } \\
\text { image collections } \\
\text { 4. } 10 \text { GB and } \\
\text { future } 100 \mathrm{~GB} \text { and } \\
\text { advanced } \\
\text { networking } \\
\text { (software defined } \\
\text { networking } \\
\text { [SDN]) }\end{array}$ & & & & \\
\hline 19 & $\begin{array}{l}\text { M0078 } \\
\text { Genomic } \\
\text { Measureme } \\
\text { nts }\end{array}$ & $\begin{array}{l}\text { 1. High- } \\
\text { throughput } \\
\text { compressed data } \\
\text { ( } 300 \mathrm{~GB} / \text { day) } \\
\text { from various DNA } \\
\text { sequencers } \\
\text { 2. Distributed } \\
\text { data source } \\
\text { (sequencers) } \\
\text { 3. Various file }\end{array}$ & $\begin{array}{l}\text { 1. Processing raw } \\
\text { data in variant calls } \\
\text { 2. Challenge: } \\
\text { characterizing } \\
\text { machine learning } \\
\text { for complex } \\
\text { analysis on } \\
\text { systematic errors } \\
\text { from sequencing } \\
\text { technologies }\end{array}$ & $\begin{array}{l}\text { 1. Legacy } \\
\text { computing cluster } \\
\text { and other PaaS } \\
\text { and laaS } \\
\text { (computing } \\
\text { cluster) } \\
\text { 2. Huge data } \\
\text { storage in PB } \\
\text { range (storage) } \\
\text { 3. Unix-based }\end{array}$ & $\begin{array}{l}\text { 1. Data format } \\
\text { for genome } \\
\text { browsers }\end{array}$ & $\begin{array}{l}\text { 1. Security and } \\
\text { privacy } \\
\text { protection of } \\
\text { health records } \\
\text { and clinical } \\
\text { research } \\
\text { databases }\end{array}$ & -- & $\begin{array}{l}\text { 1. Mobile } \\
\text { platforms for } \\
\text { physicians } \\
\text { accessing } \\
\text { genomic data } \\
\text { (mobile } \\
\text { device) }\end{array}$ \\
\hline
\end{tabular}




\begin{tabular}{|c|c|c|c|c|c|c|c|c|}
\hline No. & Use Case & $\begin{array}{l}\text { Data } \\
\text { Sources }\end{array}$ & $\begin{array}{c}\text { Data } \\
\text { Transformation }\end{array}$ & Capabilities & $\begin{array}{c}\text { Data } \\
\text { Consumer }\end{array}$ & $\begin{array}{l}\text { Security and } \\
\text { Privacy }\end{array}$ & $\begin{array}{c}\text { Life Cycle } \\
\text { Management }\end{array}$ & Other \\
\hline 20 & $\begin{array}{l}\text { M0188 } \\
\text { Comparativ } \\
\text { e Analysis } \\
\text { for } \\
\text { Metageno } \\
\text { mes and } \\
\text { Genomes }\end{array}$ & $\begin{array}{l}\text { 1. Multiple } \\
\text { centralized data } \\
\text { sources } \\
\text { 2. Proteins and } \\
\text { their structural } \\
\text { features, core } \\
\text { genomic data, } \\
\text { new types of } \\
\text { omics data such } \\
\text { as } \\
\text { transcriptomics, } \\
\text { methylomics, and } \\
\text { proteomics } \\
\text { describing gene } \\
\text { expression } \\
\text { 3. Front real-time } \\
\text { web UI } \\
\text { interactive; } \\
\text { backend data } \\
\text { loading } \\
\text { processing that } \\
\text { keeps up with } \\
\text { exponential } \\
\text { growth of } \\
\text { sequence data } \\
\text { due to the rapid } \\
\text { drop in cost of } \\
\text { sequencing } \\
\text { technology } \\
4 .\end{array}$ & $\begin{array}{l}\text { 2. Scalable RDBMS } \\
\text { for heterogeneous } \\
\text { biological data } \\
\text { 2. Real-time rapid } \\
\text { and parallel bulk } \\
\text { loading } \\
\text { 3. Oracle RDBMS, } \\
\text { SQLite files, flat } \\
\text { text files, Lucy (a } \\
\text { version of Lucene) } \\
\text { for keyword } \\
\text { searches, BLAST } \\
\text { databases, } \\
\text { USEARCH } \\
\text { databases } \\
\text { 4. Linux cluster, } \\
\text { Oracle RDBMS } \\
\text { server, large } \\
\text { memory machines, } \\
\text { standard Linux } \\
\text { interactive hosts } \\
\text { 5. Sequencing and } \\
\text { comparative } \\
\text { analysis techniques } \\
\text { for highly complex } \\
\text { data } \\
\text { 6. Descriptive } \\
\text { statistics }\end{array}$ & $\begin{array}{l}\text { 1. Huge data } \\
\text { storage }\end{array}$ & $\begin{array}{l}\text { 1. Real-time } \\
\text { interactive } \\
\text { parallel bulk } \\
\text { loading } \\
\text { capability } \\
\text { 2. Interactive } \\
\text { Web UI, } \\
\text { backend pre- } \\
\text { computations, } \\
\text { batch job } \\
\text { computation } \\
\text { submission } \\
\text { from the UI. } \\
\text { 3. Download } \\
\text { of assembled } \\
\text { and annotated } \\
\text { datasets for } \\
\text { offline analysis } \\
\text { 4. Ability to } \\
\text { query and } \\
\text { browse data } \\
\text { via interactive } \\
\text { web UI } \\
\text { 5. Visualize } \\
\text { data structure } \\
\text { at different } \\
\text { levels of } \\
\text { resolution; } \\
\text { ability to view } \\
\text { abstract }\end{array}$ & $\begin{array}{l}\text { 1. Login } \\
\text { security: } \\
\text { username and } \\
\text { password } \\
\text { 2. Creation of } \\
\text { user account to } \\
\text { submit and } \\
\text { access dataset } \\
\text { to system via } \\
\text { web interface } \\
\text { 3. Single sign- } \\
\text { on capability } \\
\text { (SSO) }\end{array}$ & $\begin{array}{l}\text { 1. Methods to } \\
\text { improve data quality } \\
\text { 2. Data clustering, } \\
\text { classification, } \\
\text { reduction } \\
\text { 3. Integration of } \\
\text { new data/content } \\
\text { into the system's } \\
\text { data store and data } \\
\text { annotation }\end{array}$ & -- \\
\hline
\end{tabular}




\begin{tabular}{|c|c|c|c|c|c|c|c|c|}
\hline No. & Use Case & $\begin{array}{c}\text { Data } \\
\text { Sources }\end{array}$ & $\begin{array}{c}\text { Data } \\
\text { Transformation }\end{array}$ & Capabilities & $\begin{array}{l}\text { Data } \\
\text { Consumer }\end{array}$ & $\begin{array}{l}\text { Security and } \\
\text { Privacy }\end{array}$ & $\begin{array}{c}\text { Life Cycle } \\
\text { Management }\end{array}$ & Other \\
\hline & & $\begin{array}{l}\text { Heterogeneous, } \\
\text { complex, } \\
\text { structural, and } \\
\text { hierarchical } \\
\text { biological data } \\
5 . \text { Metagenomic } \\
\text { samples that can } \\
\text { vary by several } \\
\text { orders of } \\
\text { magnitude, such } \\
\text { as several } \\
\text { hundred } \\
\text { thousand genes } \\
\text { to a billion genes }\end{array}$ & & & $\begin{array}{l}\text { representation } \\
\text { s of highly } \\
\text { similar data }\end{array}$ & & & \\
\hline 21 & $\begin{array}{l}\text { M0140 } \\
\text { Individualiz } \\
\text { ed Diabetes } \\
\text { Manageme } \\
\text { nt }\end{array}$ & $\begin{array}{l}\text { 1. Distributed EHR } \\
\text { data } \\
\text { 2. Over } 5 \text { million } \\
\text { patients with } \\
\text { thousands of } \\
\text { properties each } \\
\text { and many more } \\
\text { derived from } \\
\text { primary values } \\
3 . \text { Each record: a } \\
\text { range of } 100 \text { to } \\
100,000 \text { data } \\
\text { property values, } \\
\text { average of } 100 \\
\text { controlled } \\
\text { vocabulary } \\
\text { values, and } \\
\text { average of } 1,000 \\
\text { continuous values } \\
4 \text {. No real-time, } \\
\text { but data updated }\end{array}$ & $\begin{array}{l}\text { 1. Data integration } \\
\text { using ontological } \\
\text { annotation and } \\
\text { taxonomies } \\
\text { 2. Parallel retrieval } \\
\text { algorithms for both } \\
\text { indexed and } \\
\text { custom searches; } \\
\text { identification of } \\
\text { data of interest; } \\
\text { patient cohorts, } \\
\text { patients' meeting } \\
\text { certain criteria, } \\
\text { patients sharing } \\
\text { similar } \\
\text { characteristics } \\
\text { 3. Distributed } \\
\text { graph mining } \\
\text { algorithms, pattern } \\
\text { analysis and graph } \\
\text { indexing, pattern }\end{array}$ & $\begin{array}{l}\text { 1. data } \\
\text { warehouse, open } \\
\text { source indexed } \\
\text { Hbase } \\
\text { 2. } \\
\text { supercomputers, } \\
\text { cloud and parallel } \\
\text { computing } \\
\text { 3. I/O intensive } \\
\text { processing } \\
\text { 4. HDFS storage } \\
\text { 5. custom code to } \\
\text { develop new } \\
\text { properties from } \\
\text { stored data. }\end{array}$ & $\begin{array}{l}\text { 1. Efficient } \\
\text { data graph- } \\
\text { based } \\
\text { visualization } \\
\text { needed }\end{array}$ & $\begin{array}{l}\text { 1. Protection of } \\
\text { health data in } \\
\text { accordance } \\
\text { with privacy } \\
\text { policies and } \\
\text { legal } \\
\text { requirements, } \\
\text { e.g., HIPAA. } \\
\text { 2. Security } \\
\text { policies for } \\
\text { different user } \\
\text { roles }\end{array}$ & $\begin{array}{l}\text { 1. Data annotated } \\
\text { based on domain } \\
\text { ontologies or } \\
\text { taxonomies } \\
\text { 2. Traceability of } \\
\text { data from origin } \\
\text { (initial point of } \\
\text { collection) through } \\
\text { use } \\
\text { 3. Data conversion } \\
\text { from existing data } \\
\text { warehouse into RDF } \\
\text { triples }\end{array}$ & $\begin{array}{l}\text { 1. Mobile } \\
\text { access }\end{array}$ \\
\hline
\end{tabular}




\begin{tabular}{|c|c|c|c|c|c|c|c|c|}
\hline No. & Use Case & $\begin{array}{l}\text { Data } \\
\text { Sources }\end{array}$ & $\begin{array}{c}\text { Data } \\
\text { Transformation }\end{array}$ & Capabilities & $\begin{array}{c}\text { Data } \\
\text { Consumer }\end{array}$ & $\begin{array}{l}\text { Security and } \\
\text { Privacy }\end{array}$ & $\begin{array}{c}\text { Life Cycle } \\
\text { Management }\end{array}$ & Other \\
\hline & & $\begin{array}{l}\text { periodically; data } \\
\text { timestamped with } \\
\text { the time of } \\
\text { observation (time } \\
\text { the value is } \\
\text { recorded) } \\
5 \text {. Two main } \\
\text { categories of } \\
\text { structured data } \\
\text { about a patient: } \\
\text { data with } \\
\text { controlled } \\
\text { vocabulary (CV) } \\
\text { property values } \\
\text { and data with } \\
\text { continuous } \\
\text { property values } \\
\text { (recorded/ } \\
\text { captured more } \\
\text { frequently) } \\
6 \text {. Data consist of } \\
\text { text and } \\
\text { continuous } \\
\text { numerical values }\end{array}$ & $\begin{array}{l}\text { searching on RDF } \\
\text { triple graphs } \\
\text { 4. Robust statistical } \\
\text { analysis tools to } \\
\text { manage false } \\
\text { discovery rates, } \\
\text { determine true } \\
\text { sub-graph } \\
\text { significance, } \\
\text { validate results, } \\
\text { eliminate false } \\
\text { positive/false } \\
\text { negative results } \\
\text { 5. Semantic graph } \\
\text { mining algorithms } \\
\text { to identify graph } \\
\text { patterns, index and } \\
\text { search graph } \\
6 \text {. Semantic graph } \\
\text { traversal }\end{array}$ & & & & & \\
\hline 22 & $\begin{array}{l}\text { M0174 } \\
\text { Statistical } \\
\text { Relational } \\
\text { Artificial } \\
\text { Intelligence } \\
\text { for Health } \\
\text { Care }\end{array}$ & $\begin{array}{l}\text { 1. Centralized } \\
\text { data, with some } \\
\text { data retrieved } \\
\text { from Internet } \\
\text { sources } \\
\text { 2. Range from } \\
\text { hundreds of GBs } \\
\text { for a sample size } \\
\text { to } 1 \text { PB for very } \\
\text { large studies } \\
\text { 3. Both constant }\end{array}$ & $\begin{array}{l}\text { 1. Relational } \\
\text { probabilistic } \\
\text { models/ } \\
\text { probability theory; } \\
\text { software that } \\
\text { learns models from } \\
\text { multiple data types } \\
\text { and can possibly } \\
\text { integrate the } \\
\text { information and } \\
\text { reason about }\end{array}$ & $\begin{array}{l}\text { 1. Java, some in } \\
\text { house tools, } \\
\text { [relational] } \\
\text { database and } \\
\text { NoSQL stores } \\
\text { 2. Cloud and } \\
\text { parallel } \\
\text { computing } \\
\text { 3. High- } \\
\text { performance } \\
\text { computer, } 48 \mathrm{~GB}\end{array}$ & $\begin{array}{l}1 . \\
\text { Visualization } \\
\text { of very large } \\
\text { data subsets }\end{array}$ & $\begin{array}{l}\text { 1. Secure } \\
\text { handling and } \\
\text { processing of } \\
\text { data }\end{array}$ & $\begin{array}{l}\text { 1. Merging multiple } \\
\text { tables before } \\
\text { analysis } \\
\text { 2. Methods to } \\
\text { validate data to } \\
\text { minimize errors }\end{array}$ & \\
\hline
\end{tabular}




\begin{tabular}{|c|c|c|c|c|c|c|c|c|}
\hline No. & Use Case & $\begin{array}{l}\text { Data } \\
\text { Sources }\end{array}$ & $\begin{array}{c}\text { Data } \\
\text { Transformation }\end{array}$ & Capabilities & $\begin{array}{l}\text { Data } \\
\text { Consumer }\end{array}$ & $\begin{array}{l}\text { Security and } \\
\text { Privacy }\end{array}$ & $\begin{array}{c}\text { Life Cycle } \\
\text { Management }\end{array}$ & Other \\
\hline & & $\begin{array}{l}\text { updates/additions } \\
\text { (to data subsets) } \\
\text { and scheduled } \\
\text { batch inputs } \\
\text { 4. Large, multi- } \\
\text { modal, } \\
\text { longitudinal data } \\
\text { 5. Rich relational } \\
\text { data comprising } \\
\text { multiple tables, } \\
\text { different data } \\
\text { types such as } \\
\text { imaging, EHR, } \\
\text { demographic, } \\
\text { genetic, and } \\
\text { natural language } \\
\text { data requiring } \\
\text { rich } \\
\text { representation } \\
6 . \text { Unpredictable } \\
\text { arrival rates, } \\
\text { often real time }\end{array}$ & $\begin{array}{l}\text { complex queries } \\
\text { 2. Robust and } \\
\text { accurate learning } \\
\text { methods to } \\
\text { account for data } \\
\text { imbalance (where } \\
\text { large numbers of } \\
\text { data are available } \\
\text { for a small number } \\
\text { of subjects) } \\
\text { 3. Learning } \\
\text { algorithms to } \\
\text { identify skews in } \\
\text { data, so as to not } \\
\text { to (incorrectly) } \\
\text { model noise } \\
\text { 4. Generalized and } \\
\text { refined learned } \\
\text { models for } \\
\text { application to } \\
\text { diverse sets of data } \\
\text { 5. Challenge: } \\
\text { acceptance of data } \\
\text { in different } \\
\text { modalities (and } \\
\text { from disparate } \\
\text { sources) }\end{array}$ & $\begin{array}{l}\text { RAM (to perform } \\
\text { analysis for a } \\
\text { moderate sample } \\
\text { size) } \\
\text { 4. Dlusters for } \\
\text { large datasets } \\
5.200 \text { GB- } 1 \text { TB } \\
\text { hard drive for test } \\
\text { data }\end{array}$ & & & & \\
\hline 23 & $\begin{array}{l}\frac{\text { M0172 }}{\text { World }} \\
\text { Population } \\
\text { Scale } \\
\text { Epidemiolo } \\
\text { gical Study }\end{array}$ & $\begin{array}{l}\text { 1. File-based } \\
\text { synthetic } \\
\text { population, either } \\
\text { centralized or } \\
\text { distributed sites } \\
\text { 2. Large volume } \\
\text { of real-time } \\
\text { output data }\end{array}$ & $\begin{array}{l}\text { 1. Compute- } \\
\text { intensive and data- } \\
\text { intensive } \\
\text { computation, like } \\
\text { supercomputer } \\
\text { performance } \\
2 \text {. Unstructured } \\
\text { and irregular }\end{array}$ & $\begin{array}{l}\text { 1. Movement of } \\
\text { very large volume } \\
\text { of data for } \\
\text { visualization } \\
\text { (networking) } \\
\text { 2. Distributed } \\
\text { MPI-based } \\
\text { simulation system }\end{array}$ & $\begin{array}{l}1 . \\
\text { Visualization }\end{array}$ & $\begin{array}{l}\text { 1. Protection of } \\
\text { PII on } \\
\text { individuals used } \\
\text { in modeling } \\
\text { 2. Data } \\
\text { protection and } \\
\text { secure platform }\end{array}$ & $\begin{array}{l}\text { 1. Data quality, } \\
\text { ability to capture the } \\
\text { traceability of } \\
\text { quality from } \\
\text { computation }\end{array}$ & \\
\hline
\end{tabular}




\begin{tabular}{|c|c|c|c|c|c|c|c|c|}
\hline No. & Use Case & $\begin{array}{l}\text { Data } \\
\text { Sources }\end{array}$ & $\begin{array}{c}\text { Data } \\
\text { Transformation }\end{array}$ & Capabilities & $\begin{array}{l}\text { Data } \\
\text { Consumer }\end{array}$ & $\begin{array}{c}\text { Security and } \\
\text { Privacy }\end{array}$ & $\begin{array}{c}\text { Life Cycle } \\
\text { Management }\end{array}$ & Other \\
\hline & & $\begin{array}{l}\text { 3. Variety of } \\
\text { output datasets } \\
\text { depending on the } \\
\text { model's } \\
\text { complexity }\end{array}$ & $\begin{array}{l}\text { nature of graph } \\
\text { processing } \\
\text { 3. Summary of } \\
\text { various runs of } \\
\text { simulation }\end{array}$ & $\begin{array}{l}\text { (platform) } \\
\text { 3. Charm++ on } \\
\text { multi-nodes } \\
\text { (software) } \\
\text { 4. Network file } \\
\text { system (storage) } \\
\text { 5. Infiniband } \\
\text { network } \\
\text { (networking) }\end{array}$ & & $\begin{array}{l}\text { for } \\
\text { computation }\end{array}$ & & \\
\hline 24 & $\begin{array}{l}\text { M0173 } \\
\text { Social } \\
\text { Contagion } \\
\text { Modeling } \\
\text { for Planning }\end{array}$ & $\begin{array}{l}\text { 1. Traditional and } \\
\text { new architecture } \\
\text { for dynamic } \\
\text { distributed } \\
\text { processing on } \\
\text { commodity } \\
\text { clusters } \\
\text { 2. Fine-resolution } \\
\text { models and } \\
\text { datasets to } \\
\text { support Twitter } \\
\text { network traffic } \\
\text { 3. Huge data } \\
\text { storage } \\
\text { supporting annual } \\
\text { data growth }\end{array}$ & $\begin{array}{l}\text { 1. Large-scale } \\
\text { modeling for } \\
\text { various events } \\
\text { (disease, emotions, } \\
\text { behaviors, etc.) } \\
\text { 2. Scalable fusion } \\
\text { between combined } \\
\text { datasets } \\
\text { 3. Multilevel } \\
\text { analysis while } \\
\text { generating } \\
\text { sufficient results } \\
\text { quickly }\end{array}$ & $\begin{array}{l}\text { 1. Computing } \\
\text { infrastructure } \\
\text { that can capture } \\
\text { human-to-human } \\
\text { interactions on } \\
\text { various social } \\
\text { events via the } \\
\text { Internet } \\
\text { (infrastructure) } \\
\text { 2. File servers and } \\
\text { databases } \\
\text { (platform) } \\
\text { 3. Ethernet and } \\
\text { Infiniband } \\
\text { networking } \\
\text { (networking) } \\
\text { 4. Specialized } \\
\text { simulators, open } \\
\text { source software, } \\
\text { and proprietary } \\
\text { modeling } \\
\text { (application) } \\
\text { 5. Huge user } \\
\text { accounts across } \\
\text { country }\end{array}$ & $\begin{array}{l}\text { 1. Multilevel } \\
\text { detailed } \\
\text { network } \\
\text { representation } \\
\mathrm{s} \\
2 . \\
\text { Visualization } \\
\text { with } \\
\text { interactions }\end{array}$ & $\begin{array}{l}\text { 1. Protection of } \\
\text { PII of } \\
\text { individuals used } \\
\text { in modeling } \\
\text { 2. Data } \\
\text { protection and } \\
\text { secure platform } \\
\text { for } \\
\text { computation }\end{array}$ & $\begin{array}{l}\text { 1. Data fusion from } \\
\text { variety of data } \\
\text { sources (i.e., Stata } \\
\text { data files) } \\
\text { 2. Data consistency } \\
\text { and no corruption } \\
\text { 3. Preprocessing of } \\
\text { raw data }\end{array}$ & $\begin{array}{l}\text { 1. Efficient } \\
\text { method of } \\
\text { moving data }\end{array}$ \\
\hline
\end{tabular}




\begin{tabular}{|c|c|c|c|c|c|c|c|c|}
\hline No. & Use Case & $\begin{array}{l}\text { Data } \\
\text { Sources }\end{array}$ & $\begin{array}{c}\text { Data } \\
\text { Transformation }\end{array}$ & Capabilities & $\begin{array}{l}\text { Data } \\
\text { Consumer }\end{array}$ & $\begin{array}{l}\text { Security and } \\
\text { Privacy }\end{array}$ & $\begin{array}{c}\text { Life Cycle } \\
\text { Management }\end{array}$ & Other \\
\hline & & & & $\begin{array}{l}\text { boundaries } \\
\text { (networking) }\end{array}$ & & & & \\
\hline
\end{tabular}


NIST BIg DATA INTERoperability FRAmEWORK: Volume 3, USE CASES AND GENERAL REQUIREMENTS

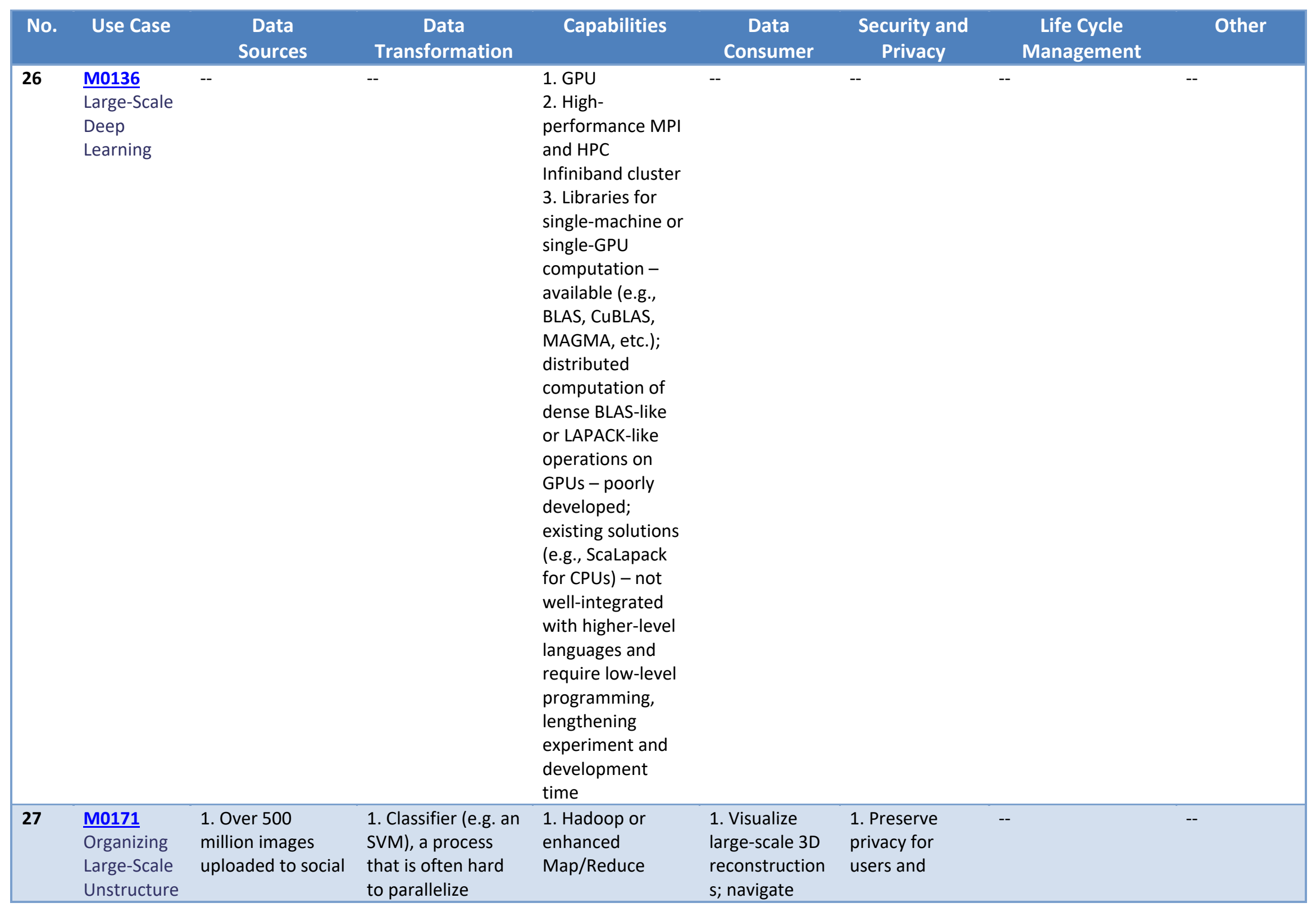




\begin{tabular}{|c|c|c|c|c|c|c|c|c|}
\hline No. & Use Case & $\begin{array}{l}\text { Data } \\
\text { Sources }\end{array}$ & $\begin{array}{c}\text { Data } \\
\text { Transformation }\end{array}$ & Capabilities & $\begin{array}{l}\text { Data } \\
\text { Consumer }\end{array}$ & $\begin{array}{c}\text { Security and } \\
\text { Privacy }\end{array}$ & $\begin{array}{c}\text { Life Cycle } \\
\text { Management }\end{array}$ & Other \\
\hline & $\begin{array}{l}\text { d } \\
\text { Collections } \\
\text { of } \\
\text { Consumer } \\
\text { Photos }\end{array}$ & $\begin{array}{l}\text { media sites each } \\
\text { day }\end{array}$ & $\begin{array}{l}\text { 2. Features seen in } \\
\text { many large-scale } \\
\text { image processing } \\
\text { problems }\end{array}$ & & $\begin{array}{l}\text { large-scale } \\
\text { collections of } \\
\text { images that } \\
\text { have been } \\
\text { aligned to } \\
\text { maps }\end{array}$ & $\begin{array}{l}\text { digital rights for } \\
\text { media }\end{array}$ & & \\
\hline 28 & $\begin{array}{l}\text { M0160 } \\
\text { Truthy } \\
\text { Twitter } \\
\text { Data }\end{array}$ & $\begin{array}{l}\text { 1. Distributed } \\
\text { data sources } \\
\text { 2. Large volume } \\
\text { of real-time } \\
\text { streaming data } \\
\text { 3. Raw data in } \\
\text { compressed } \\
\text { formats } \\
\text { 4. Fully structured } \\
\text { data in JSON, user } \\
\text { metadata, geo- } \\
\text { location data } \\
\text { 5. Multiple data } \\
\text { schemas }\end{array}$ & $\begin{array}{l}\text { 1. Various real- } \\
\text { time data analysis } \\
\text { for anomaly } \\
\text { detection, stream } \\
\text { clustering, signal } \\
\text { classification on } \\
\text { multi-dimensional } \\
\text { time series, online } \\
\text { learning }\end{array}$ & $\begin{array}{l}\text { 1. Hadoop and } \\
\text { HDFS (platform) } \\
\text { 2. IndexedHBase, } \\
\text { Hive, SciPy, } \\
\text { NumPy (software) } \\
\text { 3. In-memory } \\
\text { database, MPI } \\
\text { (platform) } \\
\text { 4. High-speed } \\
\text { Infiniband } \\
\text { network } \\
\text { (networking) }\end{array}$ & $\begin{array}{l}\text { 1. Data } \\
\text { retrieval and } \\
\text { dynamic } \\
\text { visualization } \\
\text { 2. Data-driven } \\
\text { interactive } \\
\text { web interfaces } \\
\text { 3. API for data } \\
\text { query }\end{array}$ & $\begin{array}{l}\text { 1. Security and } \\
\text { privacy policy }\end{array}$ & $\begin{array}{l}\text { 1. Standardized data } \\
\text { structures/ formats } \\
\text { with extremely high } \\
\text { data quality }\end{array}$ & $\begin{array}{l}\text { 1. Low-level } \\
\text { data storage } \\
\text { infrastructur } \\
\text { e for efficient } \\
\text { mobile } \\
\text { access to } \\
\text { data }\end{array}$ \\
\hline 29 & $\begin{array}{l}\text { M0211 } \\
\text { Crowd } \\
\text { Sourcing in } \\
\text { Humanities }\end{array}$ & -- & $\begin{array}{l}\text { 1. Digitize existing } \\
\text { audio-video, } \\
\text { photo, and } \\
\text { documents } \\
\text { archives } \\
\text { 2. Analytics: } \\
\text { pattern recognition } \\
\text { of all kinds (e.g., } \\
\text { speech } \\
\text { recognition, } \\
\text { automatic A\&V } \\
\text { analysis, cultural } \\
\text { patterns), } \\
\text { identification of } \\
\text { structures (lexical }\end{array}$ & -- & -- & $\begin{array}{l}\text { 1. Privacy issues } \\
\text { in preserving } \\
\text { anonymity of } \\
\text { responses in } \\
\text { spite of } \\
\text { computer } \\
\text { recording of } \\
\text { access ID and } \\
\text { reverse } \\
\text { engineering of } \\
\text { unusual user } \\
\text { responses }\end{array}$ & -- & -- \\
\hline
\end{tabular}




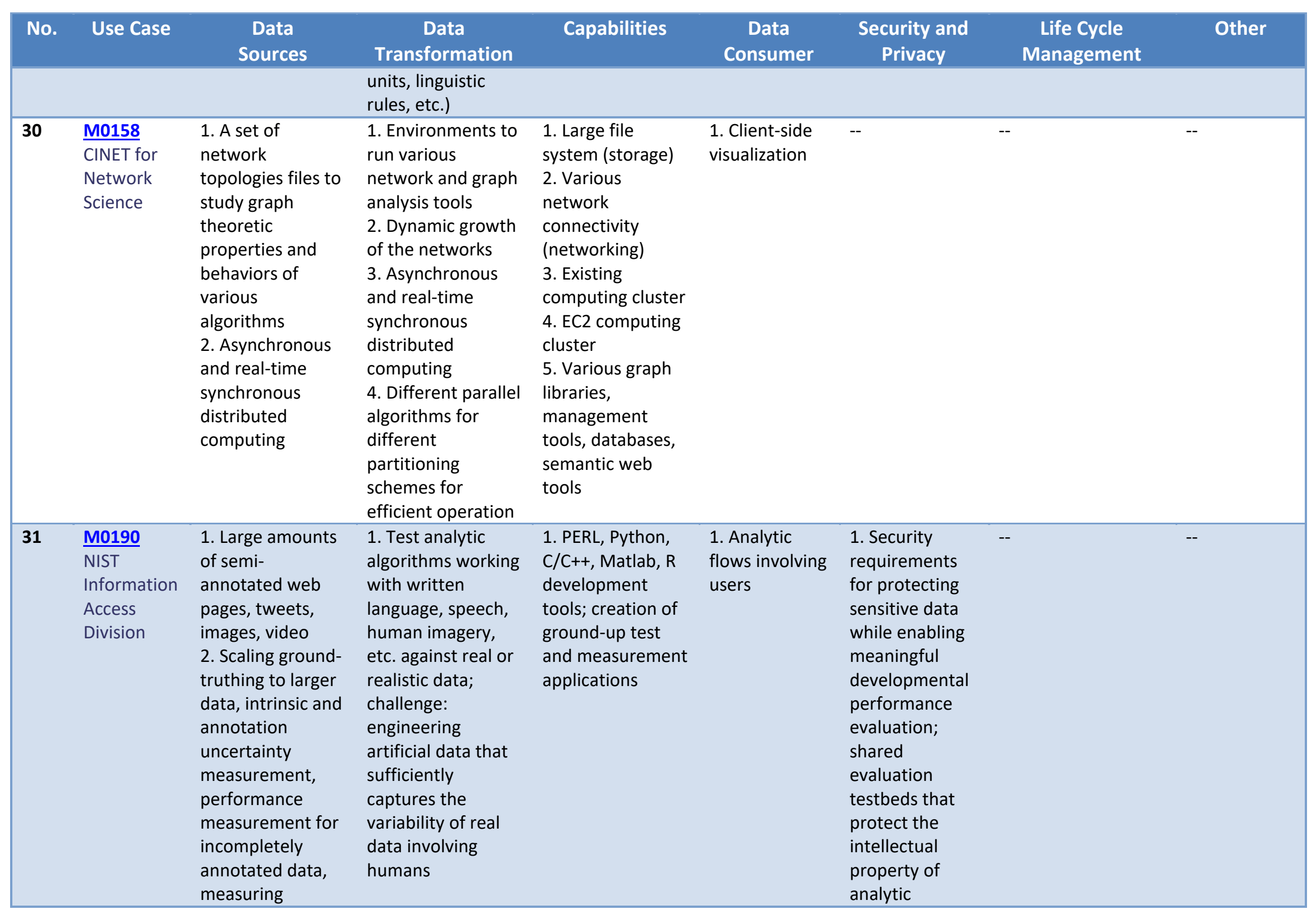


NIST Big Data INTEROPERABILITY FRAmEWORK: VolUme 3, Use CASES AND GENERAL ReQUIREMENTS

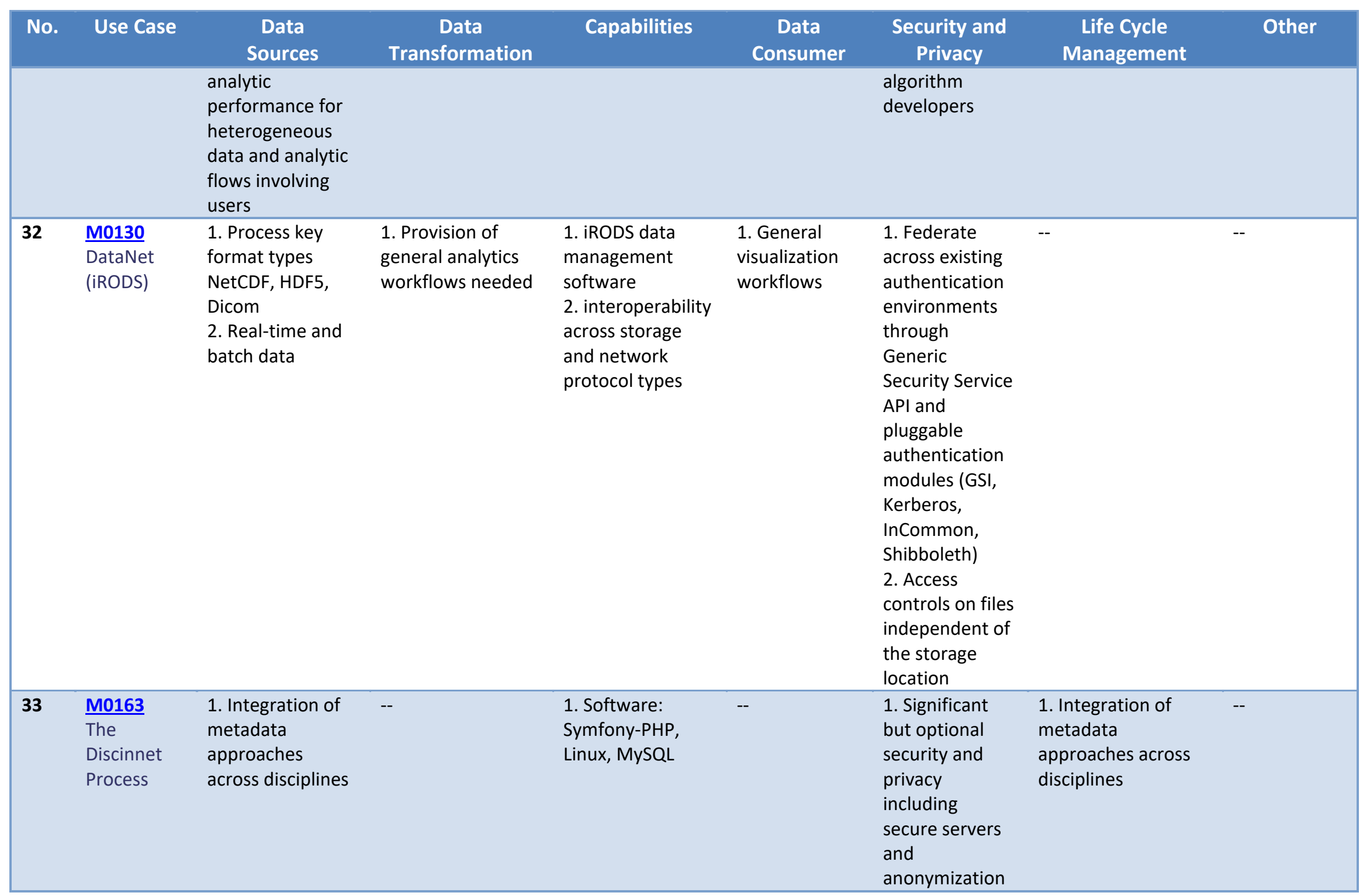




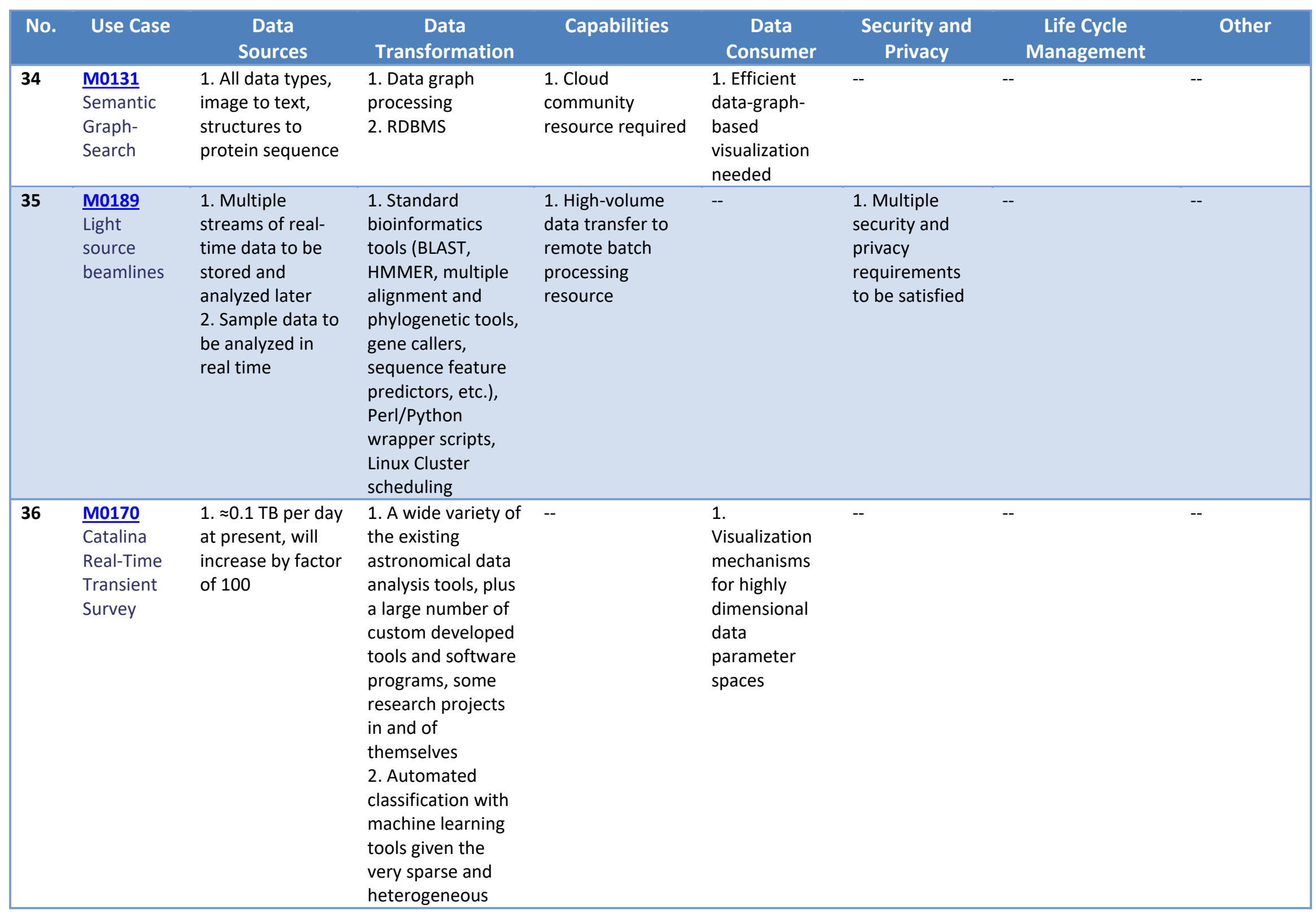




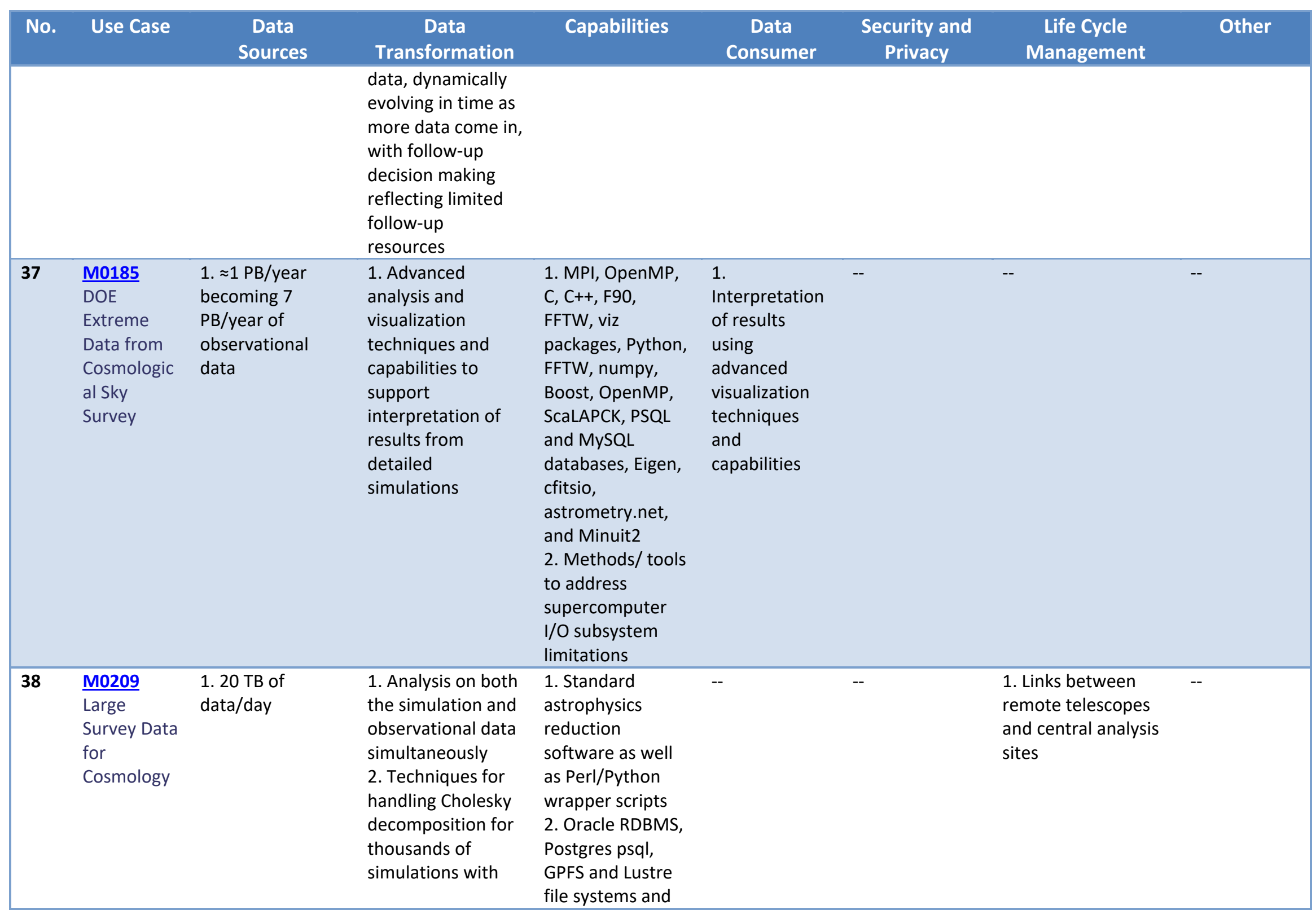




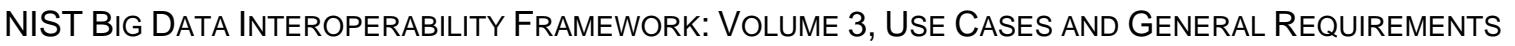

\begin{tabular}{|c|c|c|c|c|c|c|c|c|}
\hline No. & Use Case & $\begin{array}{l}\text { Data } \\
\text { Sources }\end{array}$ & $\begin{array}{c}\text { Data } \\
\text { Transformation }\end{array}$ & Capabilities & $\begin{array}{l}\text { Data } \\
\text { Consumer }\end{array}$ & $\begin{array}{l}\text { Security and } \\
\text { Privacy }\end{array}$ & $\begin{array}{c}\text { Life Cycle } \\
\text { Management }\end{array}$ & Other \\
\hline 39 & $\begin{array}{l}\frac{\text { M0166 }}{\text { Particle }} \\
\text { Physics at } \\
\text { LHC }\end{array}$ & $\begin{array}{l}\text { 1. Real-time data } \\
\text { from accelerator } \\
\text { and analysis } \\
\text { instruments } \\
2 \text {. } \\
\text { Asynchronization } \\
\text { data collection } \\
\text { 3. Calibration of } \\
\text { instruments }\end{array}$ & $\begin{array}{l}\text { 1. Experimental } \\
\text { data from ALICE, } \\
\text { ATLAS, CMS, LHB } \\
\text { 2. Histograms, } \\
\text { scatter-plots with } \\
\text { model fits } \\
\text { 3. Monte-Carlo } \\
\text { computations }\end{array}$ & $\begin{array}{l}\text { 1. Legacy } \\
\text { computing } \\
\text { infrastructure } \\
\text { (computing } \\
\text { nodes) } \\
\text { 2. Distributed } \\
\text { cached files } \\
\text { (storage) } \\
\text { 3. Object } \\
\text { databases } \\
\text { (software } \\
\text { package) }\end{array}$ & $\begin{array}{l}\text { 1. Histograms } \\
\text { and model fits } \\
\text { (visual) }\end{array}$ & $\begin{array}{l}\text { 1. Data } \\
\text { protection }\end{array}$ & $\begin{array}{l}\text { 1. Data quality on } \\
\text { complex apparatus }\end{array}$ & -- \\
\hline
\end{tabular}




\begin{tabular}{|c|c|c|c|c|c|c|c|c|}
\hline No. & Use Case & $\begin{array}{l}\text { Data } \\
\text { Sources }\end{array}$ & $\begin{array}{c}\text { Data } \\
\text { Transformation }\end{array}$ & Capabilities & $\begin{array}{l}\text { Data } \\
\text { Consumer }\end{array}$ & $\begin{array}{l}\text { Security and } \\
\text { Privacy }\end{array}$ & $\begin{array}{c}\text { Life Cycle } \\
\text { Management }\end{array}$ & Other \\
\hline 42 & $\begin{array}{l}\text { M0157 } \\
\text { ENVRI } \\
\text { Environme } \\
\text { ntal } \\
\text { Research } \\
\text { Infrastructu } \\
\text { re }\end{array}$ & $\begin{array}{l}\text { 1. Huge volume of } \\
\text { data from real- } \\
\text { time distributed } \\
\text { data sources } \\
\text { 2. Variety of } \\
\text { instrumentation } \\
\text { datasets and } \\
\text { metadata }\end{array}$ & $\begin{array}{l}\text { 1. Diversified } \\
\text { analytics tools }\end{array}$ & $\begin{array}{l}\text { 1. Variety of } \\
\text { computing } \\
\text { infrastructures } \\
\text { and architectures } \\
\text { (infrastructure) } \\
\text { 2. Scattered } \\
\text { repositories } \\
\text { (storage) }\end{array}$ & $\begin{array}{l}\text { 1. Graph } \\
\text { plotting tools } \\
\text { 2. Time series } \\
\text { interactive } \\
\text { tools } \\
\text { 3. Brower- } \\
\text { based flash } \\
\text { playback } \\
\text { 4. Earth high- } \\
\text { resolution } \\
\text { map display } \\
\text { 5. Visual tools } \\
\text { for quality } \\
\text { comparisons }\end{array}$ & $\begin{array}{l}\text { 1. Open data } \\
\text { policy with } \\
\text { minor } \\
\text { restrictions }\end{array}$ & $\begin{array}{l}\text { 1. High data quality } \\
\text { 2. Mirror archives } \\
\text { 3. Various metadata } \\
\text { frameworks } \\
\text { 4. Scattered } \\
\text { repositories and } \\
\text { data curation }\end{array}$ & $\begin{array}{l}\text { 1. Various } \\
\text { kinds of } \\
\text { mobile } \\
\text { sensor } \\
\text { devices for } \\
\text { data } \\
\text { acquisition }\end{array}$ \\
\hline
\end{tabular}




\begin{tabular}{|c|c|c|c|c|c|c|c|c|}
\hline No. & Use Case & $\begin{array}{c}\text { Data } \\
\text { Sources }\end{array}$ & $\begin{array}{c}\text { Data } \\
\text { Transformation }\end{array}$ & Capabilities & $\begin{array}{c}\text { Data } \\
\text { Consumer }\end{array}$ & $\begin{array}{l}\text { Security and } \\
\text { Privacy }\end{array}$ & $\begin{array}{c}\text { Life Cycle } \\
\text { Management }\end{array}$ & Other \\
\hline 43 & $\begin{array}{l}\text { M0167 } \\
\text { CReSIS } \\
\text { Remote } \\
\text { Sensing }\end{array}$ & $\begin{array}{l}\text { 1. Provision of } \\
\text { reliable data } \\
\text { transmission from } \\
\text { aircraft sensors/ } \\
\text { instruments or } \\
\text { removable disks } \\
\text { from remote sites } \\
\text { 2. Data gathering } \\
\text { in real time } \\
\text { 3. Varieties of } \\
\text { datasets }\end{array}$ & $\begin{array}{l}\text { 1. Legacy software } \\
\text { (Matlab) and } \\
\text { language (C/Java) } \\
\text { binding for } \\
\text { processing } \\
\text { 2. Signal } \\
\text { processing and } \\
\text { advanced image } \\
\text { processing to find } \\
\text { layers needed }\end{array}$ & $\begin{array}{l}\text { 1. } \approx 0.5 \mathrm{~PB} / \text { year of } \\
\text { raw data } \\
\text { 2. Transfer } \\
\text { content from } \\
\text { removable disk to } \\
\text { computing cluster } \\
\text { for parallel } \\
\text { processing } \\
\text { 3. Map/Reduce or } \\
\text { MPI plus language } \\
\text { binding for } \mathrm{C} / \text { Java }\end{array}$ & $\begin{array}{l}\text { 1. GIS user } \\
\text { interface } \\
\text { 2. Rich user } \\
\text { interface for } \\
\text { simulations }\end{array}$ & $\begin{array}{l}\text { 1. Security and } \\
\text { privacy on } \\
\text { sensitive } \\
\text { political issues } \\
\text { 2. Dynamic } \\
\text { security and } \\
\text { privacy policy } \\
\text { mechanisms }\end{array}$ & $\begin{array}{l}\text { 1. Data quality } \\
\text { assurance }\end{array}$ & $\begin{array}{l}\text { 1. Monitoring } \\
\text { data } \\
\text { collection } \\
\text { instruments/ } \\
\text { sensors }\end{array}$ \\
\hline 44 & $\begin{array}{l}\text { M0127 } \\
\text { UAVSAR } \\
\text { Data } \\
\text { Processing }\end{array}$ & $\begin{array}{l}\text { 1. Angular and } \\
\text { spatial data } \\
\text { 2. Compatibility } \\
\text { with other NASA } \\
\text { radar systems and } \\
\text { repositories } \\
\text { (Alaska Satellite } \\
\text { Facility) }\end{array}$ & $\begin{array}{l}\text { 1. Geolocated data } \\
\text { that require GIS } \\
\text { integration of data } \\
\text { as custom overlays } \\
\text { 2. Significant } \\
\text { human } \\
\text { intervention in } \\
\text { data processing } \\
\text { pipeline } \\
\text { 3. Hosting of rich } \\
\text { set of radar image } \\
\text { processing services } \\
\text { 4. ROI_PAC, } \\
\text { GeoServer, GDAL, } \\
\text { GeoTIFF- } \\
\text { supporting tools }\end{array}$ & $\begin{array}{l}\text { 1. Support for } \\
\text { interoperable } \\
\text { Cloud-HPC } \\
\text { architecture } \\
\text { 2. Hosting of rich } \\
\text { set of radar image } \\
\text { processing } \\
\text { services } \\
\text { 3. ROI_PAC, } \\
\text { GeoServer, GDAL, } \\
\text { GeoTIFF- } \\
\text { supporting tools } \\
\text { 4. Compatibility } \\
\text { with other NASA } \\
\text { radar systems and } \\
\text { repositories } \\
\text { (Alaska Satellite } \\
\text { Facility) }\end{array}$ & $\begin{array}{l}\text { 1. Support for } \\
\text { field } \\
\text { expedition } \\
\text { users with } \\
\text { phone/tablet } \\
\text { interface and } \\
\text { low-resolution } \\
\text { downloads }\end{array}$ & -- & $\begin{array}{l}\text { 1. Significant human } \\
\text { intervention in data } \\
\text { processing pipeline } \\
2 \text {. Rich robust } \\
\text { provenance defining } \\
\text { complex } \\
\text { machine/human } \\
\text { processing }\end{array}$ & $\begin{array}{l}\text { 1. Support } \\
\text { for field } \\
\text { expedition } \\
\text { users with } \\
\text { phone/tablet } \\
\text { interface and } \\
\text { low- } \\
\text { resolution } \\
\text { downloads }\end{array}$ \\
\hline 45 & $\begin{array}{l}\text { M0182 } \\
\text { NASA LaRC/ } \\
\text { GSFC iRODS }\end{array}$ & $\begin{array}{l}\text { 1. Federate } \\
\text { distributed } \\
\text { heterogeneous } \\
\text { datasets }\end{array}$ & 1. CAaaS on clouds & $\begin{array}{l}\text { 1. Support virtual } \\
\text { climate data } \\
\text { server (vCDS) } \\
\text { 2. GPFS parallel } \\
\text { file system } \\
\text { integrated with }\end{array}$ & $\begin{array}{l}\text { 1. Support } \\
\text { needed to } \\
\text { visualize } \\
\text { distributed } \\
\text { heterogeneou } \\
\text { s data }\end{array}$ & -- & -- & -- \\
\hline
\end{tabular}




\begin{tabular}{|c|c|c|c|c|c|c|c|c|}
\hline No. & Use Case & $\begin{array}{l}\text { Data } \\
\text { Sources }\end{array}$ & $\begin{array}{c}\text { Data } \\
\text { Transformation }\end{array}$ & Capabilities & $\begin{array}{l}\text { Data } \\
\text { Consumer }\end{array}$ & $\begin{array}{l}\text { Security and } \\
\text { Privacy }\end{array}$ & $\begin{array}{c}\text { Life Cycle } \\
\text { Management }\end{array}$ & Other \\
\hline & & & & $\begin{array}{l}\text { Hadoop } \\
\text { 3. iRODS }\end{array}$ & & & & \\
\hline 47 & $\begin{array}{l}\text { M0090 } \\
\text { Atmospheri } \\
\text { c } \\
\text { Turbulence }\end{array}$ & $\begin{array}{l}\text { 1. Real-time } \\
\text { distributed } \\
\text { datasets } \\
\text { 2. Various } \\
\text { formats, } \\
\text { resolution, } \\
\text { semantics, and } \\
\text { metadata }\end{array}$ & $\begin{array}{l}\text { 1. Map/Reduce, } \\
\text { SciDB, and other } \\
\text { scientific databases } \\
\text { 2. Continuous } \\
\text { computing for } \\
\text { updates } \\
\text { 3. Event } \\
\text { specification } \\
\text { language for data } \\
\text { mining and event } \\
\text { searching } \\
\text { 4. Semantics } \\
\text { interpretation and } \\
\text { optimal structuring } \\
\text { for } 4 D \text { data mining } \\
\text { and predictive } \\
\text { analysis }\end{array}$ & $\begin{array}{l}\text { 1. Other legacy } \\
\text { computing } \\
\text { systems (e.g. } \\
\text { supercomputer) } \\
\text { 2. high } \\
\text { throughput data } \\
\text { transmission over } \\
\text { the network }\end{array}$ & $\begin{array}{l}1 . \\
\text { Visualization } \\
\text { to interpret } \\
\text { results }\end{array}$ & -- & $\begin{array}{l}\text { 1. Validation for } \\
\text { output products } \\
\text { (correlations) }\end{array}$ & -- \\
\hline 48 & $\begin{array}{l}\text { M0186 } \\
\text { Climate } \\
\text { Studies }\end{array}$ & $\begin{array}{l}\text { 1. } \approx 100 \text { PB data in } \\
2017 \text { streaming at } \\
\text { high data rates } \\
\text { from large }\end{array}$ & $\begin{array}{l}\text { 1. Data analytics } \\
\text { close to data } \\
\text { storage }\end{array}$ & $\begin{array}{l}\text { 1. Extension of } \\
\text { architecture to } \\
\text { several other } \\
\text { fields }\end{array}$ & $\begin{array}{l}\text { 1. Worldwide } \\
\text { climate data } \\
\text { sharing }\end{array}$ & -- & -- & $\begin{array}{l}\text { 1. Phone- } \\
\text { based input } \\
\text { and access }\end{array}$ \\
\hline
\end{tabular}


NIST Big Data INTEROPERABILITY FRAmEWORK: VolUme 3, Use CASES AND GENERAL ReQUIREMENTS

\begin{tabular}{|c|c|c|c|c|c|c|c|c|}
\hline No. & Use Case & $\begin{array}{l}\text { Data } \\
\text { Sources }\end{array}$ & $\begin{array}{c}\text { Data } \\
\text { Transformation }\end{array}$ & Capabilities & $\begin{array}{c}\text { Data } \\
\text { Consumer }\end{array}$ & $\begin{array}{c}\text { Security and } \\
\text { Privacy }\end{array}$ & $\begin{array}{c}\text { Life Cycle } \\
\text { Management }\end{array}$ & Other \\
\hline & & $\begin{array}{l}\text { supercomputers } \\
\text { across the world } \\
2 \text {. Integration of } \\
\text { large-scale } \\
\text { distributed data } \\
\text { from simulations } \\
\text { with diverse } \\
\text { observations } \\
\text { 3. Linking of } \\
\text { diverse data to } \\
\text { novel HPC } \\
\text { simulation }\end{array}$ & & & $\begin{array}{l}\text { 2. High-end } \\
\text { distributed } \\
\text { visualization }\end{array}$ & & & \\
\hline 49 & $\begin{array}{l}\text { M0183 } \\
\text { DOE-BER } \\
\text { Subsurface } \\
\text { Biogeoche } \\
\text { mistry }\end{array}$ & $\begin{array}{l}\text { 1. Heterogeneous } \\
\text { diverse data with } \\
\text { different domains } \\
\text { and scales, } \\
\text { translation across } \\
\text { diverse datasets } \\
\text { that cross } \\
\text { domains and } \\
\text { scales } \\
\text { 2. Synthesis of } \\
\text { diverse and } \\
\text { disparate field, } \\
\text { laboratory, omic, } \\
\text { and simulation } \\
\text { datasets across } \\
\text { different } \\
\text { semantic, spatial, } \\
\text { and temporal } \\
\text { scales } \\
\text { 3. Linking of } \\
\text { diverse data to } \\
\text { novel HPC } \\
\text { simulation }\end{array}$ & -- & $\begin{array}{l}\text { 1. Postgres, HDF5 } \\
\text { data } \\
\text { technologies, and } \\
\text { many custom } \\
\text { software systems }\end{array}$ & $\begin{array}{l}\text { 1. Phone- } \\
\text { based input } \\
\text { and access }\end{array}$ & -- & -- & $\begin{array}{l}\text { 1. Phone- } \\
\text { based input } \\
\text { and access }\end{array}$ \\
\hline
\end{tabular}


NIST BIg DATA INTERoperability FRAmEWORK: Volume 3, USE CASES AND GENERAL REQUIREMENTS

\begin{tabular}{|c|c|c|c|c|c|c|c|c|}
\hline No. & Use Case & $\begin{array}{l}\text { Data } \\
\text { Sources }\end{array}$ & $\begin{array}{c}\text { Data } \\
\text { Transformation }\end{array}$ & Capabilities & $\begin{array}{l}\text { Data } \\
\text { Consumer }\end{array}$ & $\begin{array}{l}\text { Security and } \\
\text { Privacy }\end{array}$ & $\begin{array}{c}\text { Life Cycle } \\
\text { Management }\end{array}$ & Other \\
\hline 50 & $\begin{array}{l}\text { M0184 } \\
\text { DOE-BER } \\
\text { AmeriFlux } \\
\text { and } \\
\text { FLUXNET } \\
\text { Networks }\end{array}$ & $\begin{array}{l}\text { 1. Heterogeneous } \\
\text { diverse data with } \\
\text { different domains } \\
\text { and scales, } \\
\text { translation across } \\
\text { diverse datasets } \\
\text { that cross } \\
\text { domains and } \\
\text { scales } \\
\text { 2. Link to many } \\
\text { other } \\
\text { environment and } \\
\text { biology datasets } \\
\text { 3. Link to HPC } \\
\text { climate and other } \\
\text { simulations } \\
\text { 4. Link to } \\
\text { European data } \\
\text { sources and } \\
\text { projects } \\
5 . \text { Access to data } \\
\text { from } 500 \\
\text { distributed } \\
\text { sources }\end{array}$ & $\begin{array}{l}\text { 1. Custom } \\
\text { software such as } \\
\text { EddyPro, and } \\
\text { custom analysis } \\
\text { software, such as } \\
\text { R, Python, neural } \\
\text { networks, Matlab }\end{array}$ & $\begin{array}{l}\text { 1. Custom } \\
\text { software, such as } \\
\text { EddyPro, and } \\
\text { custom analysis } \\
\text { software, such as } \\
\text { R, Python, neural } \\
\text { networks, Matlab } \\
\text { 2. Analytics } \\
\text { including data } \\
\text { mining, data } \\
\text { quality } \\
\text { assessment, } \\
\text { cross-correlation } \\
\text { across datasets, } \\
\text { data assimilation, } \\
\text { data } \\
\text { interpolation, } \\
\text { statistics, quality } \\
\text { assessment, data } \\
\text { fusion, etc. }\end{array}$ & $\begin{array}{l}\text { 1. Phone- } \\
\text { based input } \\
\text { and access }\end{array}$ & -- & -- & $\begin{array}{l}\text { 1. Phone- } \\
\text { based input } \\
\text { and access }\end{array}$ \\
\hline 51 & $\begin{array}{l}\frac{\text { M0223 }}{\text { Consumpti }} \\
\text { on } \\
\text { Forecasting } \\
\text { in Smart } \\
\text { Grids }\end{array}$ & $\begin{array}{l}\text { 1. Diverse data } \\
\text { from smart grid } \\
\text { sensors, city } \\
\text { planning, } \\
\text { weather, utilities } \\
\text { 2. Data updated } \\
\text { every } 15 \text { minutes }\end{array}$ & $\begin{array}{l}\text { 1. New machine } \\
\text { learning analytics } \\
\text { to predict } \\
\text { consumption }\end{array}$ & $\begin{array}{l}\text { 1. SQL databases, } \\
\text { CVS files, HDFS } \\
\text { (platform) } \\
\text { 2. R/Matlab, } \\
\text { Weka, Hadoop } \\
\text { (platform) }\end{array}$ & -- & $\begin{array}{l}\text { 1. Privacy and } \\
\text { anonymization } \\
\text { by aggregation }\end{array}$ & -- & $\begin{array}{l}\text { 1. Mobile } \\
\text { access for } \\
\text { clients }\end{array}$ \\
\hline
\end{tabular}




\section{Appendix D: Use Case Detail Requirements}

This appendix contains the Version 1 use case specific requirements and the aggregated general requirements within each of the following seven characteristic categories:

- Data sources

- Data transformation

- Capabilities

- Data consumer

- Security and privacy

- Life cycle management

- Other

Within each characteristic category, the general requirements are listed with the use cases to which that requirement applies. The use case IDs, in the form of MNNNN, contain links to the use case documents in the NIST document library (http://bigdatawg.nist.gov/usecases.php).

After the general requirements, the use case specific requirements for the characterization category are listed by use case. If requirements were not extracted from a use case for a particular characterization category, the use case will not be in this section of the table.

\section{TABLE D-1: DATA SOURCES REQUIREMENTS}

\section{GENERAL REQUIREMENTS}

Needs to support reliable real time, Applies to 28 use cases: $\mathrm{M} 0078, \mathrm{M} 0090, \mathrm{M} 0103$, asynchronous, streaming, and batch processing to collect data from centralized, distributed, and cloud data sources, sensors, or instruments.

Needs to support slow, bursty, and highthroughput data transmission between data sources and computing clusters.

Needs to support diversified data content: structured and unstructured text, document, graph, web, geospatial, compressed, timed, spatial, multimedia, simulation, instrumental data.

$\mathrm{M} 0127, \mathrm{M0129}, \mathrm{M} 0140, \mathrm{M0141}, \mathrm{M0147}, \mathrm{M0148}$, $\mathrm{M} 0157, \mathrm{M} 0160, \mathrm{M} 0160, \mathrm{M} 0162, \mathrm{M} 0165, \mathrm{M} 0166$, $\mathrm{M} 0166, \mathrm{M} 0167, \overline{\mathrm{M} 0172}, \mathrm{M} 0173, \mathrm{M} 0174, \mathrm{M} 0176$, $\underline{\mathrm{M} 0177}, \underline{\mathrm{M} 0183}, \underline{\mathrm{M} 0184}, \underline{\mathrm{M} 0186}, \underline{\mathrm{M} 0188}, \underline{\mathrm{M} 0191}, \underline{\mathrm{M} 0215}$ Applies to 22 use cases: $\mathrm{M0078}, \mathrm{M} 0148, \mathrm{M} 0155$, M0157, M0162, M0165, M0167, M0170, M0171, $\mathrm{M} 0172, \mathrm{M} 0174, \mathrm{M} 0176, \mathrm{M} 0177, \mathrm{M} 0184, \mathrm{M} 0185$, $\mathrm{M} 0186, \underline{\mathrm{M} 0188}, \underline{\mathrm{M} 0191}, \underline{\mathrm{M} 0209}, \mathrm{M} 0210, \underline{\mathrm{M} 0219}, \mathrm{M} 0223$ Applies to 28 use cases: $\mathrm{M0089}, \mathrm{M0090}, \mathrm{M0140}$, $\mathrm{M} 0141, \mathrm{M} 0147, \mathrm{M} 0148, \mathrm{M0155}, \mathrm{M0158}, \mathrm{M0160}$, $\mathrm{M} 0161, \mathrm{M} 0162, \mathrm{M} 0165, \mathrm{M} 0166, \mathrm{M} 0167, \mathrm{M} 0171$, $\mathrm{M} 0172, \mathrm{M} 0173, \mathrm{M} 0177, \mathrm{M0183}, \mathrm{M0184}, \mathrm{M0186}$, M0188, M0190, M0191, M0213, M0214, M0215, M0223

\section{USE CASE SPECIFIC REQUIREMENTS FOR DATA SOURCES}

$1 \quad$ M0147 Census 2010 and 2000

- $\quad$ Needs to support large document format from a centralized storage. 


\section{TABLE D-1: DATA SOURCES REQUIREMENTS}

2 M0148 NARA: Search, Retrieve, Preservation

- $\quad$ Needs to support distributed data sources.

- Needs to support large data storage.

- Needs to support bursty data ranging from a GB to hundreds of terabytes.

- Needs to support a wide variety of data formats including unstructured and structured data.

- Needs to support distributed data sources in different clouds.

$3 \quad$ M0219 Statistical Survey Response Improvement

- Needs to support data size of approximately one petabyte.

$5 \quad$ M0175 Cloud Eco-System for Finance

- Needs to support real-time ingestion of data.

$6 \quad$ M0161 Mendeley

- $\quad$ Needs to support file-based documents with constant new uploads.

- Needs to support a variety of file types such as PDFs, social network log files, client activities images, spreadsheets, presentation files.

$7 \quad$ M0164 Netflix Movie Service

- Needs to support user profiles and ranking information.

$8 \quad$ M0165 Web Search

- Needs to support distributed data sources

- $\quad$ Needs to support streaming data.

- $\quad$ Needs to support multimedia content.

$10 \underline{\mathrm{M} 0103}$ Cargo Shipping

- Needs to support centralized and real-time distributed sites/sensors.

11 M0162 Materials Data for Manufacturing

- Needs to support distributed data repositories for more than 500,000 commercial materials.

- Needs to support many varieties of datasets.

- Needs to support text, graphics, and images.

12 M0176 Simulation-Driven Materials Genomics

- $\quad$ Needs to support data streams from peta/exascale centralized simulation systems.

- Needs to support distributed web dataflows from central gateway to users.

13 M0213 Large-Scale Geospatial Analysis and Visualization

- Needs to support geospatial data that require unique approaches to indexing and distributed analysis.

$14 \underline{\mathrm{M} 0214}$ Object identification and tracking

- Needs to support real-time data FMV (30 to 60 frames per second at full-color 1080P resolution) and WALF ( 1 to 10 frames per second at 10,000 x 10,000 full-color resolution).

15 M0215 Intelligence Data Processing and Analysis

- $\quad$ Needs to support real-time data with processing at (at worst) near-real time.

- Needs to support data that currently exist in disparate silos that must be accessible through a semantically integrated data space.

- Needs to support diverse data: text files, raw media, imagery, video, audio, electronic data, humangenerated data. 


\section{TABLE D-1: DATA SOURCES REQUIREMENTS}

\section{M0177 EMR Data}

- $\quad$ Needs to support heterogeneous, high-volume, diverse data sources.

- Needs to support volume of $>12$ million entities (patients), $>4$ billion records or data points (discrete clinical observations), aggregate of $>20 \mathrm{~TB}$ of raw data.

- Needs to support velocity: 500,000 to 1.5 million new transactions per day.

- Needs to support variety: formats include numeric, structured numeric, free-text, structured text, discrete nominal, discrete ordinal, discrete structured, binary large blobs (images and video).

- Needs to support data that evolve in a highly variable fashion.

- Needs to support a comprehensive and consistent view of data across sources and over time.

17 M0089 Pathology Imaging

- Needs to support high-resolution spatial digitized pathology images.

- Needs to support various image quality analysis algorithms.

- Needs to support various image data formats, especially BigTIFF, with structured data for analytical results.

- Needs to support image analysis, spatial queries and analytics, feature clustering, and classification.

18 M0191 Computational Bioimaging

- Needs to support distributed multi-modal high-resolution experimental sources of bioimages (instruments).

- Needs to support $50 \mathrm{~TB}$ of data in formats that include images.

19 M0078 Genomic Measurements

- $\quad$ Needs to support high-throughput compressed data (300 GB per day) from various DNA sequencers.

- Needs to support distributed data source (sequencers).

- Needs to support various file formats for both structured and unstructured data.

\section{M0188 Comparative Analysis for Metagenomes and Genomes}

- Needs to support multiple centralized data sources.

- Needs to support proteins and their structural features, core genomic data, and new types of omics data such as transcriptomics, methylomics, and proteomics describing gene expression.

- Needs to support front real-time web UI interactive. Backend data loading processing must keep up with the exponential growth of sequence data due to the rapid drop in cost of sequencing technology.

- Needs to support heterogeneous, complex, structural, and hierarchical biological data.

- Needs to support metagenomic samples that can vary by several orders of magnitude, such as several hundred thousand genes to a billion genes.

21 M0140 Individualized Diabetes Management

- $\quad$ Needs to support distributed EHR data.

- Needs to support over 5 million patients with thousands of properties each and many more that are derived from primary values.

- Needs to support each record, a range of 100 to 100,000 data property values, an average of 100 controlled vocabulary values, and an average of 1,000 continuous values.

- Needs to support data that are updated periodically (not real time). Data are timestamped with the time of observation (the time that the value is recorded).

- Needs to support structured data about patients. The data fall into two main categories: data with controlled vocabulary (CV) property values and data with continuous property values (which are recorded/captured more frequently).

- Needs to support data that consist of text and continuous numerical values. 


\section{TABLE D-1: DATA SOURCES REQUIREMENTS}

22 M0174 Statistical Relational Artificial Intelligence for Health Care

- $\quad$ Needs to support centralized data, with some data retrieved from Internet sources.

- Needs to support data ranging from hundreds of GBs for a sample size to one petabyte for very large studies.

- Needs to support both constant updates/additions (to data subsets) and scheduled batch inputs.

- Needs to support large, multi-modal, longitudinal data.

- Needs to support rich relational data comprising multiple tables, as well as different data types such as imaging, EHR, demographic, genetic and natural language data requiring rich representation.

- Needs to support unpredictable arrival rates; in many cases, data arrive in real-time.

23 M0172 World Population-Scale Epidemiological Study

- Needs to support file-based synthetic populations on either centralized or distributed sites.

- Needs to support a large volume of real-time output data.

- Needs to support a variety of output datasets, depending on the complexity of the model.

24 M0173 Social Contagion Modeling for Planning

- Needs to support traditional and new architecture for dynamic distributed processing on commodity clusters.

- Needs to support fine-resolution models and datasets to support Twitter network traffic.

- Needs to support huge data storage per year.

25 M0141 Biodiversity and LifeWatch

- $\quad$ Needs to support special dedicated or overlay sensor network.

- $\quad$ Needs to support storage for distributed, historical, and trends data archiving.

- Needs to support distributed data sources and include observation and monitoring facilities, sensor network, and satellites.

- Needs to support a wide variety of data, including satellite images/information, climate and weather data, photos, video, sound recordings, etc.

- Needs to support multi-type data combinations and linkages with potentially unlimited data variety.

- Needs to support data streaming.

27 M0171 Organizing Large-Scale Unstructured Collections of Consumer Photos

- $\quad$ Needs to support over 500 million images uploaded to social media sites each day.

28 M0160 Truthy Twitter Data

- $\quad$ Needs to support distributed data sources.

- Needs to support large data volumes and real-time streaming.

- Needs to support raw data in compressed formats.

- Needs to support fully structured data in JSON, user metadata, and geo-location data.

- Needs to support multiple data schemas.

30 M0158 CINET for Network Science

- Needs to support a set of network topologies files to study graph theoretic properties and behaviors of various algorithms.

- Needs to support asynchronous and real-time synchronous distributed computing.

31 M0190 NIST Information Access Division

- Needs to support large amounts of semi-annotated web pages, tweets, images, and video.

- Needs to support scaling of ground-truthing to larger data, intrinsic and annotation uncertainty measurement, performance measurement for incompletely annotated data, measurement of analytic performance for heterogeneous data, and analytic flows involving users.

$32 \underline{\text { M0130 DataNet (iRODS) }}$

- Needs to support process key format types: NetCDF, HDF5, Dicom.

- $\quad$ Needs to support real-time and batch data.

33 M0163 The Discinnet Process

- Needs to support integration of metadata approaches across disciplines. 


\section{TABLE D-1: DATA SOURCES REQUIREMENTS}

34 M0131 Semantic Graph-Search

- Needs to support all data types, image to text, structures to protein sequence.

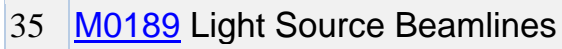

- Needs to support multiple streams of real-time data to be stored and analyzed later.

- $\quad$ Needs to support sample data to be analyzed in real time.

36 M0170 Catalina Real-Time Transient Survey

- Needs to support $\approx 0.1$ TB per day at present; the volume will increase by a factor of 100 .

$37 \underline{\text { M0185 }}$ DOE Extreme Data from Cosmological Sky Survey

- $\quad$ Needs to support $\approx 1$ PB per year, becoming 7 PB per year, of observational data.

38 M0209 Large Survey Data for Cosmology

- Needs to support $20 \mathrm{~TB}$ of data per day.

39 M0166 Particle Physics at LHC

- Needs to support real-time data from accelerator and analysis instruments.

- Needs to support asynchronization data collection.

- Needs to support calibration of instruments.

$40 \quad$ M0210 Belle II High Energy Physics Experiment

- $\quad$ Needs to support $120 \mathrm{~PB}$ of raw data.

41 M0155 EISCAT 3D Incoherent Scatter Radar System

- $\quad$ Needs to support remote sites generating 40 PB of data per year by 2022.

- Needs to support HDF5 data format.

- $\quad$ Needs to support visualization of high-dimensional $(\geq 5)$ data.

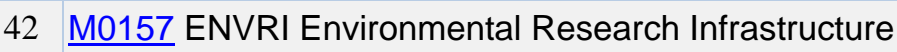

- Needs to support a huge volume of data from real-time distributed data sources.

- Needs to support a variety of instrumentation datasets and metadata.

43 M0167 CReSIS Remote Sensing

- Needs to provide reliable data transmission from aircraft sensors/instruments or removable disks from remote sites.

- Needs to support data gathering in real time.

- $\quad$ Needs to support varieties of datasets.

44 M0127 UAVSAR Data Processing

- $\quad$ Needs to support angular and spatial data.

- Needs to support compatibility with other NASA radar systems and repositories (Alaska Satellite Facility).

45 M0182 NASA LaRC/GSFC iRODS

- $\quad$ Needs to support federated distributed heterogeneous datasets.

$46 \underline{\text { M0129 MERRA Analytic Services }}$

- Needs to support integration of simulation output and observational data, NetCDF files.

- Needs to support real-time and batch mode.

- Needs to support interoperable use of AWS and local clusters.

- Needs to support iRODS data management.

47 M0090 Atmospheric Turbulence

- Needs to support real-time distributed datasets.

- Needs to support various formats, resolution, semantics, and metadata. 


\section{TABLE D-1: DATA SOURCES REQUIREMENTS}

\section{$48 \quad$ M0186 Climate Studies}

- Needs to support $\approx 100 \mathrm{~PB}$ of data (in 2017) streaming at high data rates from large supercomputers across the world.

- Needs to support integration of large-scale distributed data from simulations with diverse observations.

- Needs to link diverse data to novel HPC simulation.

49 M0183 DOE-BER Subsurface Biogeochemistry

- Needs to support heterogeneous diverse data with different domains and scales, and translation across diverse datasets that cross domains and scales.

- $\quad$ Needs to support synthesis of diverse and disparate field, laboratory, omic, and simulation datasets across different semantic, spatial, and temporal scales.

- Needs to link diverse data to novel HPC simulation.

50 M0184 DOE-BER AmeriFlux and FLUXNET Networks

- Needs to support heterogeneous diverse data with different domains and scales, and translation across diverse datasets that cross domains and scales.

- Needs to support links to many other environment and biology datasets.

- Needs to support links to HPC for climate and other simulations.

- Needs to support links to European data sources and projects.

- Needs to support access to data from 500 distributed sources.

$51 \mathrm{M} 0223$ Consumption Forecasting in Smart Grids

- Needs to support diverse data from smart grid sensors, city planning, weather, and utilities.

- Needs to support data from updates every 15 minutes.

\section{TABLE D-2: DATA TRANSFORMATION}

\section{GENERAL REQUIREMENTS}

1. Needs to support diversified computeintensive, analytic processing, and machine learning techniques.
Applies to 38 use cases: $\mathrm{M0078}, \mathrm{M0089}, \mathrm{M0103}, \mathrm{M0127}$, $\mathrm{M} 0129, \mathrm{M0140}, \mathrm{M0141}, \mathrm{M0148}, \mathrm{M0155}, \mathrm{M0157}, \mathrm{M0158}$, $\mathrm{M} 0160, \mathrm{M} 0161, \mathrm{M} 0164, \mathrm{M} 0164, \mathrm{M} 0166, \mathrm{M} 0166, \mathrm{M} 0167$, $\mathrm{M} 0170, \mathrm{M} 0171, \mathrm{M} 0172, \mathrm{M} 0173, \mathrm{M} 0174, \mathrm{M} 0176, \mathrm{M} 0177$, $\mathrm{M} 0182, \mathrm{M0185}, \underline{\mathrm{M} 0186}, \mathrm{M0190}, \mathrm{M0191}, \mathrm{M0209}, \mathrm{M0211}$, $\mathrm{M0213}, \mathrm{M0214}, \mathrm{M0215}, \mathrm{M0219}, \mathrm{M0222}, \underline{\mathrm{M} 0223}$

2. Needs to support batch and real-time analytic Applies to 7 use cases: $\underline{M 0090}, \underline{M 0103}, \underline{M 0141}, \underline{M 0155}$, processing.

3. Needs to support processing of large diversified data content and modeling.

Applies to 15 use cases: $\underline{\mathrm{M} 0078}, \underline{\mathrm{M} 0089}, \underline{\mathrm{M} 0127}, \underline{\mathrm{M} 0140}$, $\underline{M 0158}, \underline{M 0162}, \underline{M 0165}, \underline{M 0166}, \underline{M 0166}, \underline{M 0167}, \underline{M 0171}$, $\mathrm{M} 0172, \underline{\mathrm{M} 0173}, \underline{\mathrm{M} 0176}, \underline{\mathrm{M} 0213}$

4, Needs to support processing of data in motion Applies to 6 use cases: M0078, M0090, M0103, M0164, (streaming, fetching new content, tracking, etc.) M0165, M0166

\section{Use CASE SPECIFIC REQUIREMENTS For DATA TRANSFORMATION}

1. M0148 NARA: Search, Retrieve, Preservation Transformation Requirements:

- Needs to support crawl and index from distributed data sources.

- Needs to support various analytics processing including ranking, data categorization, and PII data detection.

- Needs to support preprocessing of data.

- Needs to support long-term preservation management of large varied datasets. Needs to support a huge amount of data with high relevancy and recall.

2. M0219 Statistical Survey Response Improvement Transformation Requirements: 


\section{TABLE D-2: DATA TRANSFORMATION}

- Needs to support analytics that are required for recommendation systems, continued monitoring, and general survey improvement.

3. M0222 Non-Traditional Data in Statistical Survey Response Improvement Transformation Requirements:

- Needs to support analytics to create reliable estimates using data from traditional survey sources, government administrative data sources, and non-traditional sources from the digital economy.

4. M0175 Cloud Eco-System for Finance Transformation Requirements:

- Needs to support real-time analytics.

5. M0161 Mendeley Transformation Requirements:

- Needs to support standard machine learning and analytics libraries.

- Needs to support efficient scalable and parallelized ways of matching between documents.

- Needs to support third-party annotation tools or publisher watermarks and cover pages.

6. M0164 Netflix Movie Service Transformation Requirements:

- $\quad$ Needs to support streaming video contents to multiple clients.

- Needs to support analytic processing for matching client interest in movie selection.

- Needs to support various analytic processing techniques for consumer personalization.

- Needs to support robust learning algorithms.

- Needs to support continued analytic processing based on the monitoring and performance results.

7. M0165 Web Search Transformation Requirements:

- Needs to support dynamic fetching content over the network.

- $\quad$ Needs to link user profiles and social network data.

8. M0137 Business Continuity and Disaster Recovery within a Cloud Eco-System Transformation Requirements:

- Needs to support a robust backup algorithm.

- Needs to replicate recent changes.

9. M0103 Cargo Shipping Transformation Requirements:

- Needs to support item tracking based on unique identification using an item's sensor information and GPS coordinates.

- $\quad$ Needs to support real-time updates on tracking items.

10. M0162 Materials Data for Manufacturing Transformation Requirements:

- Needs to support hundreds of independent variables by collecting these variables to create robust datasets.

11. M0176 Simulation-Driven Materials Genomics Transformation Requirements:

- Needs to support high-throughput computing real-time data analysis for web-like responsiveness.

- Needs to support mashup of simulation outputs across codes.

- Needs to support search and crowd-driven functions with computation backend flexibility for new targets.

- Needs to support Map/Reduce and search functions to join simulation and experimental data.

12. M0213 Large-Scale Geospatial Analysis and Visualization Transformation Requirements:

- Needs to support analytics including closest point of approach, deviation from route, point density over time, PCA, and ICA.

- Needs to support geospatial data that require unique approaches to indexing and distributed analysis.

13. M0214 Object Identification and Tracking Transformation Requirements:

- Needs to support rich analytics with object identification, pattern recognition, crowd behavior, economic activity, and data fusion.

14. M0215 Intelligence Data Processing and Analysis Transformation Requirements:

- Needs to support analytics including NRT alerts based on patterns and baseline changes.

15. M0177 EMR Data Transformation Requirements: 


\section{TABLE D-2: DATA TRANSFORMATION}

- Needs to support a comprehensive and consistent view of data across sources and over time.

- Needs to support analytic techniques: information retrieval, natural language processing, machine learning decision models, maximum likelihood estimators, and Bayesian networks.

16. M0089 Pathology Imaging Transformation Requirements:

- Needs to support high-performance image analysis to extract spatial information.

- Needs to support spatial queries and analytics, and feature clustering and classification.

- Needs to support analytic processing on a huge multi-dimensional dataset and be able to correlate with other data types such as clinical data and omic data.

17. M0191 Computational Bioimaging Transformation Requirements:

- Needs to support high-throughput computing with responsive analysis.

- Needs to support segmentation of regions of interest; crowd-based selection and extraction of features; and object classification, organization, and search.

- Needs to support advanced biosciences discovery through Big Data techniques/extreme-scale computing, in-database processing and analytics, machine learning (SVM and RF) for classification and recommendation services, advanced algorithms for massive image analysis, and high-performance computational solutions.

- Needs to support massive data analysis toward massive imaging datasets.

18. M0078 Genomic Measurements Transformation Requirements:

- $\quad$ Needs to support processing of raw data in variant calls.

- Needs to support machine learning for complex analysis on systematic errors from sequencing technologies, which are hard to characterize.

19. M0188 Comparative Analysis for Metagenomes and Genomes Transformation Requirements:

- Needs to support sequencing and comparative analysis techniques for highly complex data.

- Needs to support descriptive statistics.

20. M0140 Individualized Diabetes Management Transformation Requirements:

- Needs to support data integration using ontological annotation and taxonomies.

- Needs to support parallel retrieval algorithms for both indexed and custom searches and the ability to identify data of interest. Potential results include patient cohorts, patients meeting certain criteria, and patients sharing similar characteristics.

- Needs to support distributed graph mining algorithms, pattern analysis and graph indexing, and pattern searching on RDF triple graphs.

- Needs to support robust statistical analysis tools to manage false discovery rates, determine true subgraph significance, validate results, and eliminate false positive/false negative results.

- Needs to support semantic graph mining algorithms to identify graph patterns, index, and search graphs.

- Needs to support semantic graph traversal.

21. M0174 Statistical Relational Artificial Intelligence for Health Care Transformation Requirements:

- $\quad$ Needs to support relational probabilistic models/probability theory. The software learns models from multiple data types and can possibly integrate the information and reason about complex queries.

- Needs to support robust and accurate learning methods to account for data imbalance, i.e., situations in which large amounts of data are available for a small number of subjects.

- Needs to support learning algorithms to identify skews in data, so as to not-incorrectly-model noise.

- Needs to support learned models that can be generalized and refined to be applied to diverse sets of data.

- $\quad$ Needs to support acceptance of data in different modalities and from disparate sources.

22. M0172 World Population-Scale Epidemiological Study Transformation Requirements:

- Needs to support compute-intensive and data-intensive computation, like a supercomputer's performance.

- $\quad$ Needs to support the unstructured and irregular nature of graph processing.

- Needs to support summaries of various runs of simulation. 


\section{TABLE D-2: DATA TRANSFORMATION}

23. $\mathrm{M} 0173$ Social Contagion Modeling for Planning Transformation Requirements:

- $\quad$ Needs to support large-scale modeling for various events (disease, emotions, behaviors, etc.).

- $\quad$ Needs to support scalable fusion between combined datasets.

- Needs to support multilevels analysis while generating sufficient results quickly.

24. M0141 Biodiversity and LifeWatch Transformation Requirements:

- Needs to support incremental and/or real-time data analysis; rates vary because of variations in source processes.

- Needs to support a variety of data, analytical, and modeling tools to support analytics for diverse scientific communities.

- Needs to support parallel data streams and streaming analytics.

- Needs to support access and integration of multiple distributed databases.

25. M0171 Large-Scale Deep Learning Transformation Requirements:

- Needs to support classifier (e.g., an SVM), a process that is often hard to parallelize.

- Needs to support features seen in many large-scale image processing problems.

26. M0160 Truthy Twitter Data Transformation Requirements:

- Needs to support various real-time data analyses for anomaly detection, stream clustering, signal classification on multi-dimensional time series, and online learning.

27. M0211 Crowd Sourcing in Humanities Transformation Requirements:

- $\quad$ Needs to support digitization of existing audio-video, photo, and document archives.

- Needs to support analytics including pattern recognition of all kinds (e.g., speech recognition, automatic $A \& V$ analysis, cultural patterns) and identification of structures (lexical units, linguistics rules, etc.).

28. M0158 CINET for Network Science Transformation Requirements:

- Needs to support environments to run various network and graph analysis tools.

- Needs to support dynamic growth of the networks.

- $\quad$ Needs to support asynchronous and real-time synchronous distributed computing.

- Needs to support different parallel algorithms for different partitioning schemes for efficient operation.

29. M0190 NIST Information Access Division Transformation Requirements:

- Needs to support analytic algorithms working with written language, speech, human imagery, etc. The algorithms generally need to be tested against real or realistic data. It is extremely challenging to engineer artificial data that sufficiently capture the variability of real data involving humans.

30. M0130 DataNet (iRODS) Transformation Requirements:

- Needs to provide general analytics workflows.

31. M0131 Semantic Graph-Search Transformation Requirements:

- Needs to support data graph processing.

- $\quad$ Needs to support RDBMS.

32. M0189 Light Source Beamlines Transformation Requirements:

- $\quad$ Needs to support standard bioinformatics tools (BLAST, HMMER, multiple alignment and phylogenetic tools, gene callers, sequence feature predictors, etc.), Perl/Python wrapper scripts, and Linux Cluster scheduling.

33. M0170 Catalina Real-Time Transient Survey Transformation Requirements:

- Needs to support a wide variety of the existing astronomical data analysis tools, plus a large number of custom-developed tools and software programs, some of which are research projects in and of themselves.

- Needs to support automated classification with machine learning tools given very sparse and heterogeneous data, dynamically evolving as more data are generated, with follow-up decision making reflecting limited follow up resources.

34. M0185 DOE Extreme Data from Cosmological Sky Survey Transformation Requirements: 


\section{TABLE D-2: DATA TRANSFORMATION}

- Needs to support interpretation of results from detailed simulations. Interpretation requires advanced analysis and visualization techniques and capabilities.

35. M0209 Large Survey Data for Cosmology Transformation Requirements:

- Needs to support analysis on both the simulation and observational data simultaneously.

- Needs to support techniques for handling Cholesky decomposition for thousands of simulations with matrices of order 1 million on a side.

36. M0166 Particle Physics at LHC Transformation Requirements:

- Needs to support experimental data from ALICE, ATLAS, CMS, and LHb.

- Needs to support histograms and scatter-plots with model fits.

- Needs to support Monte Carlo computations.

37. M0155 EISCAT 3D Incoherent Scatter Radar System Transformation Requirements:

- Needs to support Queen Bea architecture with mix of distributed on-sensor and central processing for 5 distributed sites.

- $\quad$ Needs to support real-time monitoring of equipment by partial streaming analysis.

- Needs to host rich set of radar image processing services using machine learning, statistical modelling, and graph algorithms.

38. M0157 ENVRI Environmental Research Infrastructure Transformation Requirements:

- Needs to support diversified analytics tools.

39. M0167 CReSIS Remote Sensing Transformation Requirements:

- Needs to support legacy software (Matlab) and language (C/Java) binding for processing.

- Needs signal processing and advanced image processing to find layers.

40. M0127 UAVSAR Data Processing Transformation Requirements:

- Needs to support geolocated data that require GIS integration of data as custom overlays.

- Needs to support significant human intervention in data-processing pipeline.

- Needs to host rich sets of radar image processing services.

- Needs to support ROI_PAC, GeoServer, GDAL, and GeoTIFF-supporting tools.

41. M0182 NASA LaRC/GSFC iRODS Transformation Requirements:

- Needs to support CAaaS on clouds.

42. M0129 MERRA Analytic Services Transformation Requirements:

- Needs to support CAaaS on clouds.

43. M0090 Atmospheric Turbulence Transformation Requirements:

- $\quad$ Needs to support Map/Reduce, SciDB, and other scientific databases.

- $\quad$ Needs to support continuous computing for updates.

- Needs to support event specification language for data mining and event searching.

- Needs to support semantics interpretation and optimal structuring for 4D data mining and predictive analysis.

44. M0186 Climate Studies Transformation Requirements:

- Needs to support data analytics close to data storage.

45. M0184 DOE-BER AmeriFlux and FLUXNET Networks Transformation Requirements:

- Needs to support custom software, such as EddyPro, and custom analysis software, such as R, python, neural networks, Matlab.

46. M0223 Consumption Forecasting in Smart Grids Transformation Requirements:

- Needs to support new machine learning analytics to predict consumption.

\section{TABLE D-3: CAPABILITIES}

GENERAL REQUIREMENTS 


\section{TABLE D-3: CAPABILITIES}

1. Needs to support legacy and advanced software packages (subcomponent: SaaS).

2. Needs to support legacy and advanced computing platforms (subcomponent: PaaS).

3. Needs to support legacy and advanced distributed computing clusters, co-processors, and I/O processing (subcomponent: laaS).

4. Needs to support elastic data transmission (subcomponent: networking).
Applies to 30 use cases: M0078, M0089, M0127, M0136, M0140, M0141, M0158, M0160, M0161, M0164, M0164, $\mathrm{M} 0166, \underline{\mathrm{M} 0167}, \underline{\mathrm{M} 0172}, \mathrm{M0173}, \mathrm{M0174}, \mathrm{M0176}, \underline{\mathrm{M} 0177}$ $\mathrm{M} 0183, \underline{\mathrm{M} 0188}, \underline{\mathrm{M} 0191}, \mathrm{M0209}, \mathrm{M0210}, \mathrm{M0212}, \underline{\mathrm{M} 0213}$, $\mathrm{M} 0214, \mathrm{M} 0215, \mathrm{M} 0219, \mathrm{M} 0219, \mathrm{M} 0223$

Applies to 17 use cases: $\underline{\mathrm{M} 0078}, \underline{\mathrm{M} 0089}, \underline{\mathrm{M} 0127}, \underline{\mathrm{M} 0158}$, $\mathrm{M} 0160, \mathrm{M0161}, \mathrm{M0164}, \mathrm{M0164}, \mathrm{M0171}, \mathrm{M0172}, \mathrm{M0173}$, $\mathrm{M} 0177, \mathrm{M0182}, \mathrm{M0188}, \mathrm{M0191}, \mathrm{M0209}, \mathrm{M0223}$

Applies to 24 use cases: M0015, M0078, M0089, M0090, $\mathrm{M} 0129, \mathrm{M0136}, \mathrm{M0140}, \mathrm{M0141}, \mathrm{M0155}, \mathrm{M0158}, \mathrm{M0161}$, $\mathrm{M} 0164, \mathrm{M} 0164, \mathrm{M0166}, \mathrm{M0167}, \mathrm{M0173}, \mathrm{M0174}, \mathrm{M0176}$, $\mathrm{M} 0177, \mathrm{M} 0185, \underline{\mathrm{M} 0186}, \mathrm{M0191}, \mathrm{M0214}, \mathrm{M0215}$

Applies to 4 use cases: $\underline{\mathrm{M} 0089}, \mathrm{M0090}, \mathrm{M0103}, \mathrm{M0136}$, M0141, M0158, M0160, M0172, M0173, M0176, M0191, $\underline{\mathrm{M} 0210}, \underline{\mathrm{M} 0214}, \underline{\mathrm{M} 0215}$

5. Needs to support legacy, large, and advanced Applies to 35 use cases: M0078, M0089, M0127, M0140, distributed data storage (subcomponent: storage).

6. Needs to support legacy and advanced executable programming: applications, tools, utilities, and libraries. $\mathrm{M} 0147, \mathrm{M0147}, \mathrm{M0148}, \mathrm{M0148}, \mathrm{M0155}, \mathrm{M0157}, \mathrm{M0157}$ $\mathrm{M} 0158, \mathrm{M} 0160, \mathrm{M} 0161, \mathrm{M} 0164, \mathrm{M} 0164, \mathrm{M} 0165, \mathrm{M} 0166$, M0167, M0c170, M0171, M0172, M0173, M0174, M0176, $\mathrm{M} 0176, \mathrm{M0182}, \mathrm{M0185}, \mathrm{M0188}, \mathrm{M0209}, \mathrm{M0209}, \mathrm{M0210}$, $\mathrm{M} 0210, \underline{\mathrm{M} 0215}, \underline{\mathrm{M} 0219}$

Applies to 13 use cases: $\mathrm{M0078}, \underline{\mathrm{M} 0089}, \underline{\mathrm{M} 0140}, \underline{\mathrm{M} 0164}$, M0c166, M0167, M0174, M0176, M0184, M0185, M0190, $\underline{\mathrm{M} 0214}, \underline{\mathrm{M} 0215}$

\section{USE CASE SPeCIFIC ReQUiRements for CAPABILITIES}

1. M0147 Census 2010 and 2000 Capability Requirements:

- Needs to support large centralized storage.

2. M0148 NARA: Search, Retrieve, Preservation Capability Requirements:

- Needs to support large data storage.

- Needs to support various storages such as NetApps, Hitachi, and magnetic tapes.

3. M0219 Statistical Survey Response Improvement Capability Requirements:

- Needs to support the following software: Hadoop, Spark, Hive, R, SAS, Mahout, Allegrograph, MySQL, Oracle, Storm, BigMemory, Cassandra, and Pig.

4. M0222 Non-Traditional Data in Statistical Survey Response Improvement Capability Requirements:

- Needs to support the following software: Hadoop, Spark, Hive, R, SAS, Mahout, Allegrograph, MySQL, Oracle, Storm, BigMemory, Cassandra, and Pig.

5. M0161 Mendeley Capability Requirements:

- $\quad$ Needs to support EC2 with HDFS (infrastructure).

- $\quad$ Needs to support S3 (storage).

- $\quad$ Needs to support Hadoop (platform).

- Needs to support Scribe, Hive, Mahout, and Python (language).

- Needs to support moderate storage (15 TB with $1 \mathrm{~TB} /$ month).

- Needs to support batch and real-time processing. 


\section{TABLE D-3: CAPABILITIES}

6. M0164 Netflix Movie Service Capability Requirements:

- $\quad$ Needs to support Hadoop (platform).

- Needs to support Pig (language).

- Needs to support Cassandra and Hive.

- Needs to support a huge volume of subscribers, ratings, and searches per day (DB).

- Needs to support huge storage (2 PB).

- $\quad$ Needs to support I/O-intensive processing.

7. M0165 Web Search Capability Requirements:

- $\quad$ Needs to support petabytes of text and rich media (storage).

8. M0137 Business Continuity and Disaster Recovery within a Cloud Eco-System Capability

\section{Requirements:}

- $\quad$ Needs to support Hadoop.

- Needs to support commercial cloud services.

9. M0103 Cargo Shipping Capability Requirements:

- Needs to support Internet connectivity.

10. M0176 Simulation-Driven Materials Genomics Capability Requirements:

- $\quad$ Needs to support massive (150,000 cores) of legacy infrastructure (infrastructure).

- $\quad$ Needs to support GPFS (storage).

- Needs to support MonogDB systems (platform).

- Needs to support 10 GB of networking data.

- Needs to support various analytic tools such as PyMatGen, FireWorks, VASP, ABINIT, NWChem, BerkeleyGW, and varied community codes.

- Needs to support large storage (storage).

- Needs to support scalable key-value and object store (platform).

- Needs to support data streams from peta/exascale centralized simulation systems.

11. M0213 Large-Scale Geospatial Analysis and Visualization Capability Requirements:

- Needs to support geospatially enabled RDBMS and geospatial server/analysis software (ESRI ArcServer, Geoserver).

12. M0214 Object Identification and Tracking Capability Requirements:

- Needs to support a wide range of custom software and tools including traditional RDBMS and display tools.

- Needs to support several network capability requirements.

- $\quad$ Needs to support GPU usage.

13. M0215 Intelligence Data Processing and Analysis Capability Requirements:

- Needs to support tolerance of unreliable networks to warfighter and remote sensors.

- Needs to support up to hundreds of petabytes of data supported by modest to large clusters and clouds.

- Needs to support the following software: Hadoop, Accumulo (Big Table), Solr, NLP (several variants), Puppet (for deployment and security), Storm, and custom applications and visualization tools.

14. M0177 EMR Data Capability Requirements:

- $\quad$ Needs to support Hadoop, Hive, and R Unix-based.

- Needs to support a Cray supercomputer.

- $\quad$ Needs to support teradata, PostgreSQL, MongoDB.

- Needs to support various capabilities with significant I/O-intensive processing.

15. M0089 Pathology Imaging Capability Requirements:

- Needs to support legacy systems and clouds (computing cluster).

- Needs to support huge legacy and new storage such as SAN or HDFS (storage).

- Needs to support high-throughput network links (networking).

- Needs to support MPI image analysis, Map/Reduce, and Hive with spatial extension (software packages). 


\section{TABLE D-3: CAPABILITIES}

16. M0191 Computational Bioimaging Capability Requirements:

- Needs to support ImageJ, OMERO, VolRover, advanced segmentation, and feature detection methods from applied math researchers. Scalable key-value and object store databases are needed.

- Needs to support NERSC's Hopper infrastructure

- Needs to support database and image collections.

- Needs to support $10 \mathrm{~GB}$ and future $100 \mathrm{~GB}$ and advanced networking (SDN).

17. M0078 Genomic Measurements Capability Requirements:

- Needs to support legacy computing cluster and other PaaS and IaaS (computing cluster).

- $\quad$ Needs to support huge data storage in the petabyte range (storage).

- Needs to support Unix-based legacy sequencing bioinformatics software (software package).

18. M0188 Comparative Analysis for Metagenomes and Genomes Capability Requirements:

- Needs to support huge data storage.

- Needs to support scalable RDBMS for heterogeneous biological data.

- Needs to support real-time rapid and parallel bulk loading.

- Needs to support Oracle RDBMS, SQLite files, flat text files, Lucy (a version of Lucene) for keyword searches, BLAST databases, and USEARCH databases.

- Needs to support Linux cluster, Oracle RDBMS server, large memory machines, and standard Linux interactive hosts.

19. M0140 Individualized Diabetes Management Capability Requirements:

- $\quad$ Needs to support a data warehouse, specifically open source indexed Hbase.

- Needs to support supercomputers with cloud and parallel computing.

- Needs to support I/O-intensive processing.

- $\quad$ Needs to support HDFS storage.

- Needs to support custom code to develop new properties from stored data.

20. M0174 Statistical Relational Artificial Intelligence for Health Care Capability Requirements:

- $\quad$ Needs to support Java, some in-house tools, a relational database, and NoSQL stores.

- Needs to support cloud and parallel computing.

- Needs to support a high-performance computer with 48 GB RAM (to perform analysis for a moderate sample size).

- $\quad$ Needs to support clusters for large datasets.

- $\quad$ Needs to support 200 GB to 1 TB hard drive for test data.

21. M0172 World Population-Scale Epidemiological Study Capability Requirements:

- $\quad$ Needs to support movement of very large numbers of data for visualization (networking).

- Needs to support distributed an MPI-based simulation system (platform).

- Needs to support Charm++ on multi-nodes (software).

- Needs to support a network file system (storage).

- Needs to support an Infiniband network (networking).

22. $\underline{\mathrm{M} 0173}$ Social Contagion Modeling for Planning Capability Requirements:

- Needs to support a computing infrastructure that can capture human-to-human interactions on various social events via the Internet (infrastructure).

- $\quad$ Needs to support file servers and databases (platform).

- $\quad$ Needs to support Ethernet and Infiniband networking (networking).

- Needs to support specialized simulators, open source software, and proprietary modeling (application).

- Needs to support huge user accounts across country boundaries (networking).

23. M0141 Biodiversity and LifeWatch Capability Requirements:

- $\quad$ Needs to support expandable on-demand-based storage resources for global users.

- Needs to support cloud community resources. 


\section{TABLE D-3: CAPABILITIES}

\section{M0136 Large-scale Deep Learning Capability Requirements:}

- $\quad$ Needs to support GPU usage.

- Needs to support a high-performance MPI and HPC Infiniband cluster.

- Needs to support libraries for single-machine or single-GPU computation (e.g., BLAS, CuBLAS, MAGMA, etc.).

- Needs to support distributed computation of dense BLAS-like or LAPACK-like operations on GPUs, which remains poorly developed. Existing solutions (e.g., ScaLapack for CPUs) are not well integrated with higher-level languages and require low-level programming, which lengthens experiment and development time.

25. M0171 Organizing Large-Scale Unstructured Collections of Consumer Photos Capability Requirements:

- Needs to support Hadoop or enhanced Map/Reduce.

26. M0160 Truthy Twitter Data Capability Requirements:

- Needs to support Hadoop and HDFS (platform).

- $\quad$ Needs to support IndexedHBase, Hive, SciPy, and NumPy (software).

- $\quad$ Needs to support in-memory database and MPI (platform).

- Needs to support high-speed Infiniband network (networking).

27. M0158 CINET for Network Science Capability Requirements:

- $\quad$ Needs to support a large file system (storage).

- Needs to support various network connectivity (networking).

- Needs to support an existing computing cluster.

- Needs to support an EC2 computing cluster.

- Needs to support various graph libraries, management tools, databases, and semantic web tools.

28. M0190 NIST Information Access Division Capability Requirements:

- $\quad$ Needs to support PERL, Python, C/C++, Matlab, and R development tools.

- Needs to support creation of a ground-up test and measurement applications.

29. M0130 DataNet (iRODS) Capability Requirements:

- Needs to support iRODS data management software.

- Needs to support interoperability across storage and network protocol types.

30. M0163 The Discinnet Process Capability Requirements:

- Needs to support the following software: Symfony-PHP, Linux, and MySQL.

31. M0131 Semantic Graph-Search Capability Requirements:

- $\quad$ Needs to support a cloud community resource.

32. M0189 Light Source Beamlines Capability Requirements:

- Needs to support high-volume data transfer to a remote batch processing resource.

33. M0185 DOE Extreme Data from Cosmological Sky Survey Capability Requirements:

- Needs to support MPI, OpenMP, C, C++, F90, FFTW, viz packages, Python, FFTW, numpy, Boost, OpenMP, ScaLAPCK, PSQL and MySQL databases, Eigen, cfitsio, astrometry.net, and Minuit2.

- $\quad$ Needs to address limitations of supercomputer I/O subsystem.

34. M0209 Large Survey Data for Cosmology Capability Requirements:

- Needs to support standard astrophysics reduction software as well as Perl/Python wrapper scripts.

- Needs to support Oracle RDBMS and Postgres psql, as well as GPFS and Lustre file systems and tape archives.

- $\quad$ Needs to support parallel image storage.

35. M0166 Particle Physics at LHC Capability Requirements:

- Needs to support legacy computing infrastructure (computing nodes).

- Needs to support distributed cached files (storage).

- $\quad$ Needs to support object databases (software package). 


\section{TABLE D-3: CAPABILITIES}

36. $\underline{\mathrm{M} 0210}$ Belle II High Energy Physics Experiment Capability Requirements:

- $\quad$ Needs to support $120 \mathrm{~PB}$ of raw data.

- Needs to support an international distributed computing model to augment that at the accelerator in Japan.

- Needs to support data transfer of $\approx 20$ BG per second at designed luminosity between Japan and the United States.

- Needs to support software from Open Science Grid, Geant4, DIRAC, FTS, and the Belle II framework.

37. M0155 EISCAT 3D Incoherent Scatter Radar System Capability Requirements:

- Needs to support architecture compatible with the ENVRI collaboration.

38. M0157 ENVRI Environmental Research Infrastructure Capability Requirements:

- Needs to support a variety of computing infrastructures and architectures (infrastructure).

- Needs to support scattered repositories (storage).

39. M0167 CReSIS Remote Sensing Capability Requirements:

- $\quad$ Needs to support $\approx 0.5$ PB per year of raw data.

- Needs to support transfer of content from removable disk to computing cluster for parallel processing.

- Needs to support Map/Reduce or MPI plus language binding for C/Java.

40. M0127 UAVSAR Data Processing Capability Requirements:

- Needs to support an interoperable cloud-HPC architecture.

- Needs to host rich sets of radar image processing services.

- Needs to support ROI_PAC, GeoServer, GDAL, and GeoTIFF-supporting tools.

- Needs to support compatibility with other NASA radar systems and repositories (Alaska Satellite Facility).

41. M0182 NASA LaRC/GSFC iRODS Capability Requirements:

- $\quad$ Needs to support vCDS.

- Needs to support a GPFS integrated with Hadoop.

- Needs to support iRODS.

42. M0129 MERRA Analytic Services Capability Requirements:

- $\quad$ Needs to support NetCDF aware software.

- Needs to support Map/Reduce.

- Needs to support interoperable use of AWS and local clusters.

43. M0090 Atmospheric Turbulence Capability Requirements:

- Needs to support other legacy computing systems (e.g., a supercomputer).

- Needs to support high-throughput data transmission over the network.

44. M0186 Climate Studies Capability Requirements:

- Needs to support extension of architecture to several other fields.

45. M0183 DOE-BER Subsurface Biogeochemistry Capability Requirements:

- Needs to support Postgres, HDF5 data technologies, and many custom software systems.

46. M0184 DOE-BER AmeriFlux and FLUXNET Networks Capability Requirements:

- Needs to support custom software, such as EddyPro, and analysis software, such as R, Python, neural networks, and Matlab.

- Needs to support analytics: data mining, data quality assessment, cross-correlation across datasets, data assimilation, data interpolation, statistics, quality assessment, data fusion, etc.

47. M0223 Consumption Forecasting in Smart Grids Capability Requirements:

- $\quad$ Needs to support SQL databases, CVS files, and HDFS (platform).

- Needs to support R/Matlab, Weka, and Hadoop (platform). 


\section{TABLE D-4: DATA CONSUMER}

\section{GENERAL REQUIREMENTS}

1. Needs to support fast searches from processed Applies to 4 use cases: $\underline{M 0148}, \underline{M 0160}, \underline{M 0165}$, data with high relevancy, accuracy, and high recall. M0176

2. Needs to support diversified output file formats for visualization, rendering, and reporting.

3. Needs to support visual layouts for results presentation.

4. Needs to support rich user interfaces for access using browsers, visualization tools.

5. Needs to support a high-resolution multidimension layer of data visualization.

6. Needs to support streaming results to clients.
Applies to 16 use cases: $\underline{\mathrm{M} 0078}, \underline{\mathrm{M} 0089}, \underline{\mathrm{M} 0090}$ M0157, M0c161, M0164, M0164, M0165, M0166, $\underline{\mathrm{M} 0166}, \underline{\mathrm{M} 0167}, \underline{\mathrm{M} 0167}, \underline{\mathrm{M} 0174}, \underline{\mathrm{M} 0177}, \underline{\mathrm{M} 0213}$, $\underline{\mathrm{M} 0214}$

Applies to 2 use cases: $\underline{\mathrm{M} 0165}$, M0167

Applies to 1 use cases: $\underline{\mathrm{M} 0089}, \mathrm{M0127}, \mathrm{M0157}$ $\mathrm{M} 0160, \underline{\mathrm{M} 0162}, \underline{\mathrm{M} 0167}, \underline{\mathrm{M} 0167}, \underline{\mathrm{M} 0183}, \underline{\mathrm{M} 0184}$, M0188, M0190

Applies to 21 use cases: $\underline{\mathrm{M} 0129}, \underline{\mathrm{M} 0155}, \underline{\mathrm{M} 0155}$ $\underline{\mathrm{M} 0158}, \mathrm{M0161}, \mathrm{M0162}, \mathrm{M0171}, \mathrm{M0172}, \mathrm{M0173}$ $\mathrm{M} 0177, \mathrm{M0179}, \mathrm{M0182}, \mathrm{M0185}, \mathrm{M018 \textrm {c } 6}, \mathrm{M0188}$ $\underline{\mathrm{M} 0191}, \underline{\mathrm{M} 0213}, \underline{\mathrm{M} 0214}, \underline{\mathrm{M} 02 \mathrm{c} 15}, \underline{\mathrm{M} 0219}, \underline{\mathrm{M} 0222}$

Applies to 1 use case: $\underline{\mathrm{M} 0164}$

\section{USE CASE SPECIFIC REQUIREMENTS FOR DATA CONSUMERS}

1. M0148 NARA: Search, Retrieve, Preservation Data Consumer Requirements:

- $\quad$ Needs to support high relevancy and high recall from search.

- Needs to support high accuracy from categorization of records.

- Needs to support various storages such as NetApps, Hitachi, and magnetic tapes.

2. M0219 Statistical Survey Response Improvement Data Consumer Requirements:

- Needs to support evolving data visualization for data review, operational activity, and general analysis.

3. M0222 Non-Traditional Data in Statistical Survey Response Improvement Data Consumer Requirements:

- Needs to support evolving data visualization for data review, operational activity, and general analysis.

4. M0161 Mendeley Data Consumer Requirements:

- $\quad$ Needs to support custom-built reporting tools.

- $\quad$ Needs to support visualization tools such as networking graphs, scatterplots, etc.

5. M0164 Netflix Movie Service Data Consumer Requirements:

- Needs to support streaming and rendering media

6. M0165 Web Search Data Consumer Requirements:

- $\quad$ Needs to support search times of $\approx 0.1$ seconds.

- Needs to support top 10 ranked results.

- Needs to support appropriate page layout (visual).

7. M0162 Materials Data for Manufacturing Data Consumer Requirements:

- Needs to support visualization for materials discovery from many independent variables.

- Needs to support visualization tools for multi-variable materials.

8. M0176 Simulation-Driven Materials Genomics Data Consumer Requirements:

- Needs to support browser-based searches for growing material data.

9. M0213 Large-Scale Geospatial Analysis and Visualization Data Consumer Requirements:

- Needs to support visualization with GIS at high and low network bandwidths and on dedicated facilities and handhelds. 


\section{TABLE D-4: DATA CONSUMER}

10. M0214 Object Identification and Tracking Data Consumer Requirements:

- Needs to support visualization of extracted outputs. These will typically be overlays on a geospatial display. Overlay objects should be links back to the originating image/video segment.

- Needs to output the form of OGC-compliant web features or standard geospatial files (shape files, KML).

11. M0215 Intelligence Data Processing and Analysis Data Consumer Requirements:

- Needs to support primary visualizations, i.e., geospatial overlays (GIS) and network diagrams.

12. M0177 EMR Data Data Consumer Requirements:

- Needs to provide results of analytics for use by data consumers/stakeholders, i.e., those who did not actually perform the analysis.

- Needs to support specific visualization techniques.

13. M0089 Pathology Imaging Data Consumer Requirements:

- $\quad$ Needs to support visualization for validation and training.

14. M0191 Computational Bioimaging Data Consumer Requirements:

- $\quad$ Needs to support 3D structural modeling.

15. M0078 Genomic Measurements Data Consumer Requirements:

- Needs to support data format for genome browsers.

16. M0188 Comparative Analysis for Metagenomes and Genomes Data Consumer Requirements:

- $\quad$ Needs to support real-time interactive parallel bulk loading capability.

- Needs to support interactive web UI, backend pre-computations, and batch job computation submission from the UI.

- Needs to support download assembled and annotated datasets for offline analysis.

- Needs to support ability to query and browse data via interactive web UI.

- Needs to support visualized data structure at different levels of resolution, as well as the ability to view abstract representations of highly similar data.

17. M0174 Statistical Relational Artificial Intelligence for Health Care Data Consumer Requirements:

- Needs to support visualization of subsets of very large data.

18. M0172 World Population-Scale Epidemiological Study Data Consumer Requirements:

- Needs to support visualization.

19. M0173 Social Contagion Modeling for Planning Data Consumer Requirements:

- $\quad 1$. Needs to support multilevel detail network representations.

- Needs to support visualization with interactions.

20. M0141 Biodiversity and LifeWatch Data Consumer Requirements:

- $\quad$ Needs to support advanced/rich/high-definition visualization.

- Needs to support 4D visualization.

21. M0171 Organizing Large-Scale Unstructured Collections of Consumer Photos Data Consumer Requirements:

- Needs to support visualization of large-scale 3D reconstructions and navigation of large-scale collections of images that have been aligned to maps.

22. M0160 Truthy Twitter Data Data Consumer Requirements:

- Needs to support data retrieval and dynamic visualization.

- Needs to support data-driven interactive web interfaces.

- $\quad$ Needs to support API for data query.

23. M0158 CINET for Network Science Data Consumer Requirements:

- Needs to support client-side visualization.

24. M0190 NIST Information Access Division Data Consumer Requirements:

- Needs to support analytic flows involving users. 


\section{TABLE D-4: DATA CONSUMER}

\section{M0130 DataNet (iRODS) Data Consumer Requirements:}

- Needs to support general visualization workflows.

26. M0131 Semantic Graph-Search Data Consumer Requirements:

- $\quad$ Needs to support efficient data-graph-based visualization.

27. M0170 Catalina Real-Time Transient Survey Data Consumer Requirements:

- Needs to support visualization mechanisms for highly dimensional data parameter spaces.

28. M0185 DOE Extreme Data from Cosmological Sky Survey Data Consumer Requirements:

- Needs to support interpretation of results using advanced visualization techniques and capabilities.

29. M0166 Particle Physics at LHC Data Consumer Requirements:

- $\quad$ Needs to support histograms and model fits (visual).

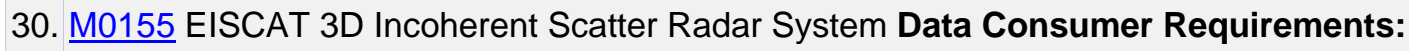

- $\quad$ Needs to support visualization of high-dimensional $(\geq 5)$ data.

31. M0157 ENVRI Environmental Research Infrastructure Data Consumer Requirements:

- Needs to support graph-plotting tools.

- Needs to support time series interactive tools.

- Needs to support browser-based flash playback.

- Needs to support earth high-resolution map displays.

- Needs to support visual tools for quality comparisons.

32. M0167 CReSIS Remote Sensing Data Consumer Requirements:

- $\quad$ Needs to support GIS user interface.

- Needs to support rich user interface for simulations.

33. M0127 UAVSAR Data Processing Data Consumer Requirements:

- Needs to support field expedition users with phone/tablet interface and low-resolution downloads.

34. M0182 NASA LaRC/GSFC iRODS Data Consumer Requirements:

- Needs to support visualization of distributed heterogeneous data.

35. M0129 MERRA Analytic Services Data Consumer Requirements:

- Needs to support high-end distributed visualization.

36. M0090 Atmospheric Turbulence Data Consumer Requirements:

- $\quad$ Needs to support visualization to interpret results.

37. M0186 Climate Studies Data Consumer Requirements:

- Needs to support worldwide climate data sharing.

- Needs to support high-end distributed visualization.

38. M0183 DOE-BER Subsurface Biogeochemistry Data Consumer Requirements:

- Needs to support phone-based input and access.

39. M0184 DOE-BER AmeriFlux and FLUXNET Networks Data Consumer Requirements:

- $\quad$ Needs to support phone-based input and access.

\section{TABLE D-5: SECURITY AND PRIVACY}

\section{GENERAL REQUIREMENTS}

1. Needs to protect and preserve security and privacy for sensitive data.
Applies to 32 use cases: $\underline{\mathrm{M} 0078}, \mathrm{M0089}, \mathrm{M0103}$, $\mathrm{M} 0140, \mathrm{M0141}, \mathrm{M0147}, \overline{\mathrm{M} 0148}, \overline{\mathrm{M} 0157}, \overline{\mathrm{M} 0160}$, $\mathrm{M} 0162, \underline{\mathrm{M} 0164}, \mathrm{M0165}, \mathrm{M0166}, \underline{\mathrm{M} 0166}, \underline{\mathrm{M} 0167}$, $\mathrm{M} 0167, \mathrm{M0171}, \mathrm{M0172}, \mathrm{M0173}, \mathrm{M0174}, \mathrm{M0176}$, $\mathrm{M} 0177, \mathrm{M} 0190, \mathrm{M} 0191, \mathrm{M0210}, \mathrm{M0211}, \mathrm{M0213}$, $\mathrm{M} 0214, \underline{\mathrm{M} 0215}, \underline{\mathrm{M} 0219}, \underline{\mathrm{M} 0222}, \underline{\mathrm{M} 0223}$ 


\section{TABLE D-5: SeCURITY AND PRIVACy}

2. Needs to support sandbox, access control, and multilevel policy-driven authentication on protected data.
Applies to 13 use cases: $\underline{\mathrm{M} 0006}, \underline{\mathrm{M} 0078}, \underline{\mathrm{M} 0089}$, $\mathrm{M} 0103, \mathrm{M} 0140, \mathrm{M0161}, \mathrm{M0165}, \underline{\mathrm{M} 0167}, \underline{\mathrm{M} 0176}$, $\underline{\mathrm{M} 0177}, \underline{\mathrm{M} 0188}, \underline{\mathrm{M} 0210}, \underline{\mathrm{M} 0211}$

\section{Use CASe SPECIFIC RequiRements for SeCURITY ANd PRIVACY}

1. M0147 Census 2010 and 2000 Security and Privacy Requirements:

- Needs to support Title 13 data.

2. M0148 NARA: Search, Retrieve, Preservation Security and Privacy Requirements:

- $\quad$ Needs to support security policy.

3. M0219 Statistical Survey Response Improvement Security and Privacy Requirements:

- Needs to support improved recommendation systems that reduce costs and improve quality while providing confidentiality safeguards that are reliable and publicly auditable.

- Needs to support confidential and secure data. All processes must be auditable for security and confidentiality as required by various legal statutes.

4. M0222 Non-Traditional Data in Statistical Survey Response Improvement Security and Privacy Requirements:

- Needs to support confidential and secure data. All processes must be auditable for security and confidentiality as required by various legal statutes.

5. M0175 Cloud Eco-System for Finance Security and Privacy Requirements:

- Needs to support strong security and privacy constraints.

6. M0161 Mendeley Security and Privacy Requirements:

- Needs to support access controls for who is reading what content.

7. M0164 Netflix Movie Service Security and Privacy Requirements:

- Needs to support preservation of users' privacy and digital rights for media.

8. M0165 Web Search Security and Privacy Requirements:

- $\quad$ Needs to support access control.

- Needs to protect sensitive content.

9. M0137 Business Continuity and Disaster Recovery within a Cloud Eco-System Security and Privacy Requirements:

- Needs to support strong security for many applications.

10. M0103 Cargo Shipping Security and Privacy Requirements:

- Needs to support security policy.

11. M0162 Materials Data for Manufacturing Security and Privacy Requirements:

- Needs to support protection of proprietary sensitive data.

- Needs to support tools to mask proprietary information.

12. M0176 Simulation-Driven Materials Genomics Security and Privacy Requirements:

- Needs to support sandbox as independent working areas between different data stakeholders. 2. Needs to support policy-driven federation of datasets.

13. $\underline{\mathrm{M} 0213}$ Large-Scale Geospatial Analysis and Visualization Security and Privacy Requirements:

- Needs to support complete security of sensitive data in transit and at rest (particularly on handhelds).

14. M0214 Object Identification and Tracking Security and Privacy Requirements:

- Needs to support significant security and privacy; sources and methods cannot be compromised. The enemy should not be able to know what the user sees.

15. M0215 Intelligence Data Processing and Analysis Security and Privacy Requirements:

- Needs to support protection of data against unauthorized access or disclosure and tampering. 


\section{TABLE D-5: SeCURITY AND PRIVACy}

\section{M0177 EMR Data Security and Privacy Requirements:}

- Needs to support direct consumer access to data, as well as referral to results of analytics performed by informatics research scientists and health service researchers.

- Needs to support protection of all health data in compliance with government regulations.

- Needs to support protection of data in accordance with data providers' policies.

- Needs to support security and privacy policies, which may be unique to a subset of the data.

- Needs to support robust security to prevent data breaches.

17. M0089 Pathology Imaging Security and Privacy Requirements:

- Needs to support security and privacy protection for protected health information.

18. M0191 Computational Bioimaging Security and Privacy Requirements:

- Needs to support significant but optional security and privacy, including secure servers and anonymization.

19. M0078 Genomic Measurements Security and Privacy Requirements:

- Needs to support security and privacy protection of health records and clinical research databases.

20. M0188 Comparative Analysis for Metagenomes and Genomes Security and Privacy Requirements:

- Needs to support login security, i.e., usernames and passwords.

- Needs to support creation of user accounts to access datasets, and submit datasets to systems, via a web interface.

- $\quad$ Needs to support single sign-on (SSO) capability.

21. M0140 Individualized Diabetes Management Security and Privacy Requirements:

- Needs to support protection of health data in accordance with privacy policies and legal security and privacy requirements, e.g., HIPAA.

- Needs to support security policies for different user roles.

22. M0174 Statistical Relational Artificial Intelligence for Health Care Security and Privacy Requirements:

- Needs to support secure handling and processing of data.

23. M0172 World Population-Scale Epidemiological Study Security and Privacy Requirements:

- $\quad$ Needs to support protection of PII on individuals used in modeling.

- Needs to support data protection and a secure platform for computation.

24. M0173 Social Contagion Modeling for Planning Security and Privacy Requirements:

- $\quad$ Needs to support protection of PII on individuals used in modeling.

- Needs to support data protection and a secure platform for computation.

25. M0141 Biodiversity and LifeWatch Security and Privacy Requirements:

- $\quad$ Needs to support federated identity management for mobile researchers and mobile sensors.

- Needs to support access control and accounting.

26. M0171 Organizing Large-Scale Unstructured Collections of Consumer Photos Security and Privacy Requirements:

- Needs to preserve privacy for users and digital rights for media.

27. M0160 Truthy Twitter Data Security and Privacy Requirements:

- Needs to support security and privacy policy.

28. M0211 Crowd Sourcing in Humanities Security and Privacy Requirements:

- Needs to support privacy issues in preserving anonymity of responses in spite of computer recording of access ID and reverse engineering of unusual user responses.

29. M0190 NIST Information Access Division Security and Privacy Requirements:

- Needs to support security and privacy requirements for protecting sensitive data while enabling meaningful developmental performance evaluation. Shared evaluation testbeds must protect the intellectual property of analytic algorithm developers. 


\section{TABLE D-5: SeCURITY AND PRIVACy}

30. M0130 DataNet (iRODS) Security and Privacy Requirements:

- $\quad$ Needs to support federation across existing authentication environments through Generic Security Service API and pluggable authentication modules (GSI, Kerberos, InCommon, Shibboleth).

- Needs to support access controls on files independent of the storage location.

31. M0163 The Discinnet Process Security and Privacy Requirements:

- Needs to support significant but optional security and privacy, including secure servers and anonymization.

32. M0189 Light Source Beamlines Security and Privacy Requirements:

- Needs to support multiple security and privacy requirements.

33. M0166 Particle Physics at LHC Security and Privacy Requirements:

- Needs to support data protection.

34. M0210 Belle II High Energy Physics Experiment Security and Privacy Requirements:

- $\quad$ Needs to support standard grid authentication.

35. M0157 ENVRI Environmental Research Infrastructure Security and Privacy Requirements:

- Needs to support an open data policy with minor restrictions.

36. M0167 CReSIS Remote Sensing Security and Privacy Requirements:

- $\quad$ Needs to support security and privacy on sensitive political issues.

- Needs to support dynamic security and privacy policy mechanisms.

37. M0223 Consumption Forecasting in Smart Grids Security and Privacy Requirements:

- $\quad$ Needs to support privacy and anonymization by aggregation.

\section{TABLE D-6: LIFE CYCLE MANAGEMENT}

\section{GENERAL REQUIREMENTS}

1. Needs to support data quality curation including preprocessing, data clustering, classification, reduction, and format transformation.
Applies to 20 use cases: M0141, M0147, M0148, M0157, M0160, M0161, M0162, M0165, M0166, M0167, M0172, $\underline{\mathrm{M} 0173}, \mathrm{M0174}, \underline{\mathrm{M} 0177}, \underline{\mathrm{M} 0188}, \mathrm{M0191}, \underline{\mathrm{M} 0214}, \underline{\mathrm{M} 0215}$, $\underline{\mathrm{M} 0219}, \underline{\mathrm{M} 0222}$

2. Needs to support dynamic updates on data, Applies to 2 use cases: $\underline{\mathrm{M} 0164}, \underline{\mathrm{M} 0209})$ user profiles, and links.

3. Needs to support data life cycle and longterm preservation policy, including data provenance.

4. Needs to support data validation.

Applies to 6 use cases: $\underline{\mathrm{M} 0141}, \underline{\mathrm{M} 0 \mathrm{c} 147}, \underline{\mathrm{M} 0155}, \underline{\mathrm{M} 0163}$, $\underline{\mathrm{M} 0164}, \underline{\mathrm{M} 0165}$

5. Needs to support human annotation for data Applies to 4 use cases: $\underline{\mathrm{M} 0089}, \underline{\mathrm{M} 01 \mathrm{c} 27}, \underline{\mathrm{M} 0140}, \underline{\mathrm{M} 0188}$ validation.

6. Needs to support prevention of data loss or Applies to 3 use cases: $\underline{M 0147}, \underline{M 0155}, \underline{M 0173})$ corruption.

7. Needs to support multisites archival.

Applies to 1 use case: $\underline{\mathrm{M} 0157}$

8. Needs to support persistent identifier and data traceability.

9. Needs to standardize, aggregate, and Applies to 2 use cases: $\underline{\text { M0140, }}$ M0161) normalize data from disparate sources.

Applies to 1 use case: $\underline{\mathrm{M} 0177)}$

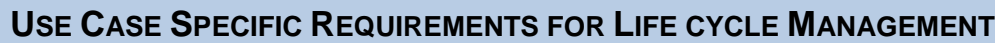




\section{TABLE D-6: LIFE CYCLE MANAGEMENT}

1. M0147 Census 2010 and 2000 Life Cycle Requirements:

- Needs to support long-term preservation of data as-is for 75 years.

- Needs to support long-term preservation at the bit level.

- Needs to support the curation process, including format transformation.

- Needs to support access and analytics processing after 75 years.

- $\quad$ Needs to ensure there is no data loss.

2. M0148 NARA: Search, Retrieve, Preservation Life Cycle Requirements:

- Needs to support pre-process for virus scans.

- Needs to support file format identification.

- $\quad$ Needs to support indexing.

- Needs to support record categorization.

3. M0219 Statistical Survey Response Improvement Life Cycle Requirements:

- Needs to support high veracity of data, and systems must be very robust. The semantic integrity of conceptual metadata concerning what exactly is measured and the resulting limits of inference remain a challenge.

4. M0222 Non-Traditional Data in Statistical Survey Response Improvement Life Cycle Requirements:

- Needs to support high veracity of data, and systems must be very robust. The semantic integrity of conceptual metadata concerning what exactly is measured and the resulting limits of inference remain a challenge.

5. M0161 Mendeley Life Cycle Requirements:

- Needs to support metadata management from PDF extraction.

- Needs to support identify of document duplication.

- Needs to support persistent identifiers.

- Needs to support metadata correlation between data repositories such as CrossRef, PubMed and Arxiv.

6. M0164 Netflix Movie Service Life Cycle Requirements:

- Needs to support continued ranking and updating based on user profiles and analytic results.

7. M0165 Web Search Life Cycle Requirements:

- $\quad$ Needs to support purge data after a certain time interval (a few months).

- Needs to support data cleaning.

8. M0162 Materials Data for Manufacturing Life Cycle Requirements:

- Needs to support data quality handling; current process is poor or unknown.

9. M0176 Simulation-Driven Materials Genomics Life Cycle Requirements:

- Needs to support validation and UQ of simulation with experimental data.

- Needs to support UQ in results from multiple datasets.

10. M0214 Object Identification and Tracking Life Cycle Requirements:

- Needs to support veracity of extracted objects.

11. M0215 Intelligence Data Processing and Analysis Life Cycle Requirements:

- Needs to support data provenance (e.g., tracking of all transfers and transformations) over the life of the data.

12. M0177 EMR Data Life Cycle Requirements:

- $\quad$ Needs to standardize, aggregate, and normalize data from disparate sources.

- Needs to reduce errors and bias.

- Needs to support common nomenclature and classification of content across disparate sources.

13. M0089 Pathology Imaging Life Cycle Requirements:

- Needs to support human annotations for validation. 


\section{TABLE D-6: LIFE CYCLE MANAGEMENT}

14. M0191 Computational Bioimaging Life Cycle Requirements:

- Needs to support workflow components include data acquisition, storage, enhancement, and noise minimization.

15. M0188 Comparative Analysis for Metagenomes and Genomes Life Cycle Requirements:

- Needs to support methods to improve data quality.

- Needs to support data clustering, classification, and reduction.

- Needs to support integration of new data/content into the system's data store and annotate data.

16. M0140 Individualized Diabetes Management Life Cycle Requirements:

- Needs to support data annotation based on domain ontologies or taxonomies.

- $\quad$ Needs to ensure traceability of data from origin (initial point of collection) through use.

- Needs to support data conversion from existing data warehouse into RDF triples.

17. M0174 Statistical Relational Artificial Intelligence for Health Care Life Cycle Requirements:

- Needs to support merging multiple tables before analysis.

- Needs to support methods to validate data to minimize errors.

18. M0172 World Population-Scale Epidemiological Study Life Cycle Requirements:

- Needs to support data quality and capture traceability of quality from computation.

19. M0173 Social Contagion Modeling for Planning Life Cycle Requirements:

- $\quad$ Needs to support data fusion from variety of data sources.

- Needs to support data consistency and prevent corruption.

- Needs to support preprocessing of raw data.

20. M0141 Biodiversity and LifeWatch Life Cycle Requirements:

- $\quad$ Needs to support data storage and archiving, data exchange, and integration.

- Needs to support data life cycle management: data provenance, referral integrity, and identification traceability back to initial observational data.

- Needs to support processed (secondary) data (in addition to original source data) that may be stored for future uses.

- $\quad$ Needs to support provenance (and PID) control of data, algorithms, and workflows.

- Needs to support curated (authorized) reference data (i.e., species name lists), algorithms, software code, and workflows.

21. M0160 Truthy Twitter Data Life Cycle Requirements:

- Needs to support standardized data structures/formats with extremely high data quality.

22. M0163 The Discinnet Process Life Cycle Requirements:

- Needs to support integration of metadata approaches across disciplines.

23. M0209 Large Survey Data for Cosmology Life Cycle Requirements:

- Needs to support links between remote telescopes and central analysis sites.

24. M0166 Particle Physics at LHC Life Cycle Requirements:

- Needs to support data quality on complex apparatus.

25. M0155 EISCAT 3D Incoherent Scatter Radar System Life Cycle Requirements:

- Needs to support preservation of data and avoid data loss due to instrument malfunction.

26. M0157 ENVRI Environmental Research Infrastructure Life Cycle Requirements:

- Needs to support high data quality.

- Needs to support mirror archives.

- Needs to support various metadata frameworks.

- Needs to support scattered repositories and data curation.

27. M0167 CReSIS Remote Sensing Life Cycle Requirements:

- Needs to support data quality assurance. 


\section{TABLE D-6: LIFE CYCLE MANAGEMENT}

28. M0127 UAVSAR Data Processing Life Cycle Requirements:

- Needs to support significant human intervention in data processing pipeline.

- Needs to support rich robust provenance defining complex machine/human processing.

29. M0090 Atmospheric Turbulence Life Cycle Requirements:

- Needs to support validation for output products (correlations).

\section{TABLE D-7: OTHERS}

\section{General ReQuirements}

1. Needs to support rich user interfaces from mobile platforms to access processed results.

2. Needs to support performance monitoring on analytic processing from mobile platforms.

3. Needs to support rich visual content search and rendering from mobile platforms.

4. Needs to support mobile device data acquisition.

5. Needs to support security across mobile devices.
Applies to 6 use cases: $\underline{\mathrm{M} 0078}, \underline{\mathrm{M} 0127}, \underline{\mathrm{M} 0129}, \underline{\mathrm{M} 0148}$ $\underline{\mathrm{M} 0160}, \underline{\mathrm{M} 0164}$

Applies to 2 use cases: $\underline{\mathrm{M} 0155}, \underline{\mathrm{M} 0167}$

Applies to 13 use cases: $\mathrm{M0078}, \mathrm{M0089}, \underline{\mathrm{M} 0161}$, $\underline{\mathrm{M} 0164}, \underline{\mathrm{M} 0165}, \underline{\mathrm{M} 0166}, \underline{\mathrm{M} 0176}, \underline{\mathrm{M} 0177}, \underline{\mathrm{M} 0183}, \underline{\mathrm{M} 0184}$ $\mathrm{M} 0186, \underline{\mathrm{M} 0219}, \underline{\mathrm{M} 0223}$

Applies to 1 use case: $\underline{\mathrm{M} 0157}$

\section{Use CASe SPECIFIC Requirements for Others}

1. M0148 NARA: Search, Retrieve, Preservation Other Requirements:

- Needs to support mobile search with similar interfaces/results from a desktop.

2. M0219 Statistical Survey Response Improvement Other Requirements:

- $\quad$ Needs to support mobile access.

3. M0175 Cloud Eco-System for Finance Other Requirements:

- $\quad$ Needs to support mobile access.

4. M0161 Mendeley Other Requirements:

- Needs to support Windows Android and iOS mobile devices for content deliverables from Windows desktops.

5. M0164 Netflix Movie Service Other Requirements:

- Needs to support smart interfaces for accessing movie content on mobile platforms.

6. M0165 Web Search Other Requirements:

- Needs to support mobile search and rendering.

7. M0176 Simulation-Driven Materials Genomics Other Requirements:

- Needs to support mobile apps to access materials genomics information.

8. M0177 EMR Data Other Requirements:

- Needs to support security across mobile devices.

9. M0089 Pathology Imaging Other Requirements:

- Needs to support 3D visualization and rendering on mobile platforms.

10. M0078 Genomic Measurements Other Requirements:

- Needs to support mobile platforms for physicians accessing genomic data (mobile device).

11. M0140 Individualized Diabetes Management Other Requirements:

- Needs to support mobile access.

12. M0173 Social Contagion Modeling for Planning Other Requirements:

- $\quad$ Needs to support an efficient method of moving data. 


\section{TABLE D-7: OTHERS}

13. M0141 Biodiversity and LifeWatch Other Requirements:

- $\quad$ Needs to support access by mobile users.

14. M0160 Truthy Twitter Data Other Requirements:

- Needs to support a low-level data storage infrastructure for efficient mobile access to data.

15. M0155 EISCAT 3D Incoherent Scatter Radar System Other Requirements:

- Needs to support real-time monitoring of equipment by partial streaming analysis.

16. M0157 ENVRI Environmental Research Infrastructure Other Requirements:

- Needs to support various kinds of mobile sensor devices for data acquisition.

17. M0167 CReSIS Remote Sensing Other Requirements:

- Needs to support monitoring of data collection instruments/sensors.

18. M0127 UAVSAR Data Processing Other Requirements:

- Needs to support field expedition users with phone/tablet interface and low-resolution downloads.

19. M0129 MERRA Analytic Services Other Requirements:

- Needs to support smart phone and tablet access.

- Needs to support iRODS data management.

20. M0186 Climate Studies Other Requirements:

- Needs to support phone-based input and access.

21. M0183 DOE-BER Subsurface Biogeochemistry Other Requirements:

- Needs to support phone-based input and access.

22. M0184 DOE-BER AmeriFlux and FLUXNET Networks Other Requirements:

- Needs to support phone-based input and access.

23. $\mathrm{M} 0223$ Consumption Forecasting in Smart Grids Other Requirements:

- Needs to support mobile access for clients. 


\section{Appendix E: Use Case Template 2}

418 Use Case Template 2 was used to gather information on additional use cases, which were incorporated 419 into the work of the NBDIF. Appendix E contains an outline of the questions in the Use Case Template 2 420 and is provided for the readers' reference. The fillable PDF form of Use Case template 2can be 421 downloaded from the NBD-PWG website at 422 https://bigdatawg.nist.gov/_uploadfiles/M0621_v2_7345181325.pdf. 
BIG DATA USE CASE TEMPLATE 2

428 This template was designed by the NIST Big Data Public Working Group (NBD-PWG) to gather Big Data use cases. The use case information you provide in this template will greatly help the NBD-PWG in the next phase of developing the NIST Big Data Interoperability Framework. We sincerely appreciate your effort and realize it is nontrivial.

432 The template can also be completed in the Google Form for Use Case Template 2: http://bit.ly/1ff7iM9.

\section{TEMPLATE OUTLI NE}

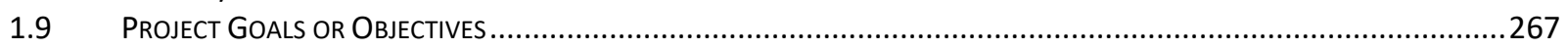

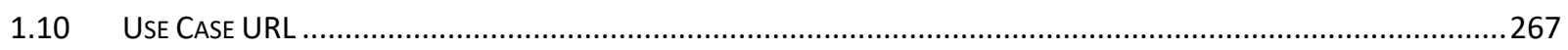

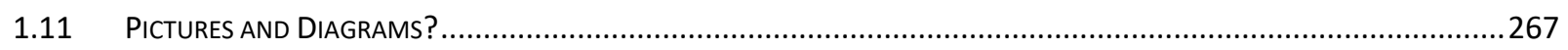

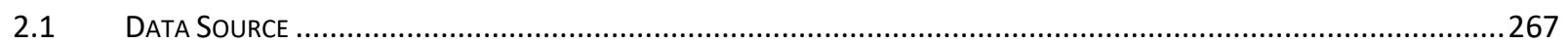

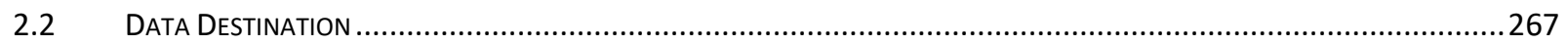

2.3 VOLUME

2.4 VELOCITY

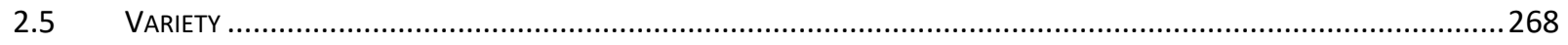

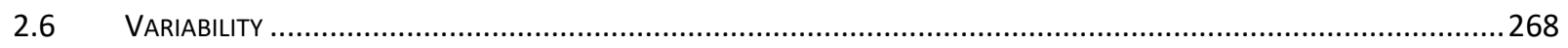

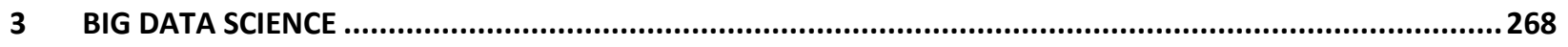

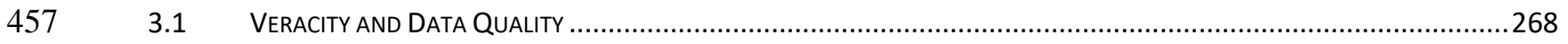

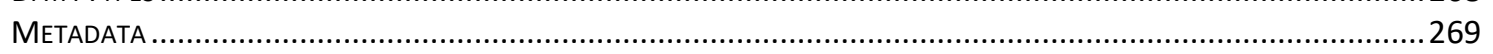

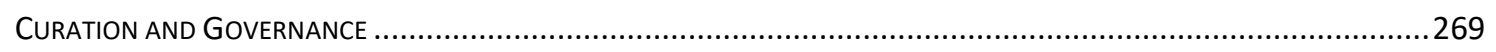

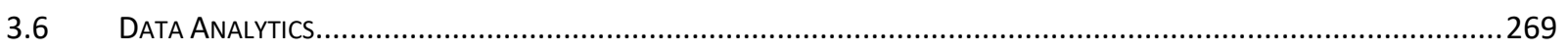

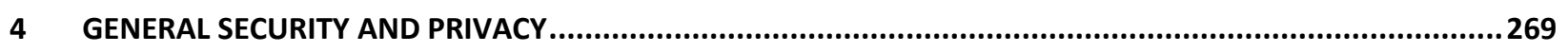

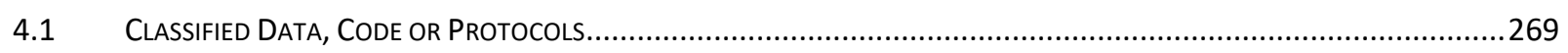

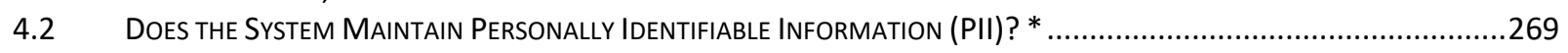

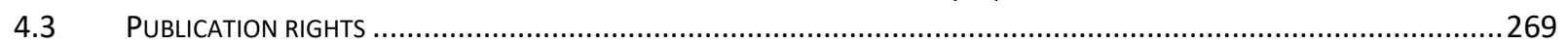




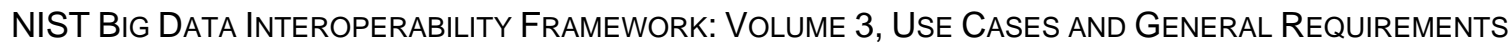

467

468

469

470

471

472

473

474

475

476

477

478

479

480

481

482

483

484

485

486

487

488

489

490

491

492

493

494

495

496

497

498

499

500

501

502

503

504

505

506

507

508

509

510

511

512

513

514

515

4.4 IS THERE AN EXPLICIT DATA GOVERNANCE PLAN OR FRAMEWORK FOR THE EFFORT?

4.5 DO YOU FORESEE ANY POTENTIAL RISKS FROM PUBLIC OR PRIVATE OPEN DATA PROJECTS? .........................................270

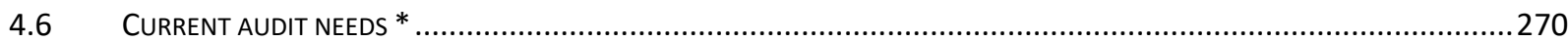

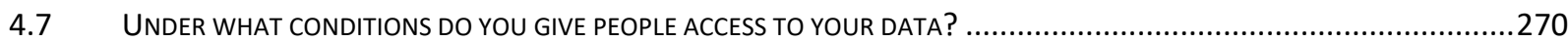

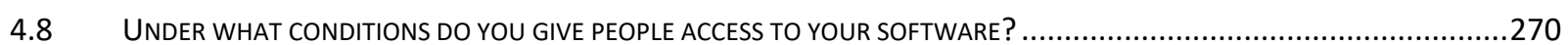

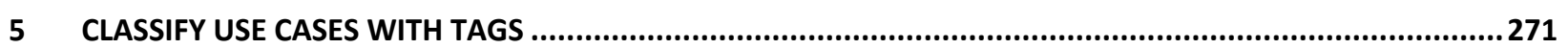

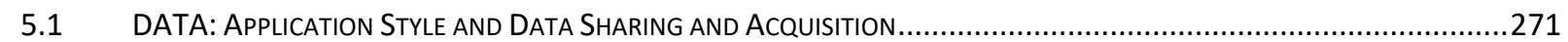

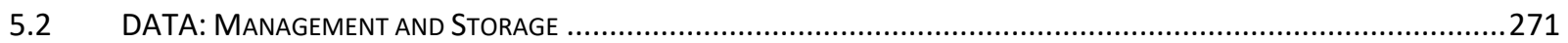

5.3 DATA: Describe Other DATA ACQUisition/ AcCESS/ Sharing/ Management/ Storage ISSUES..........................271

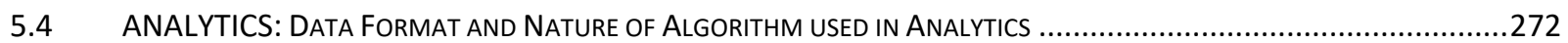

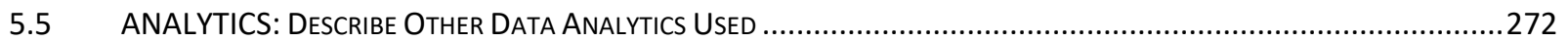

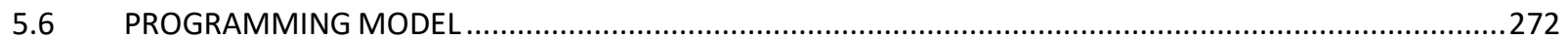

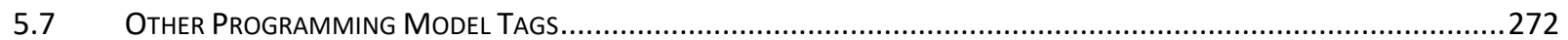

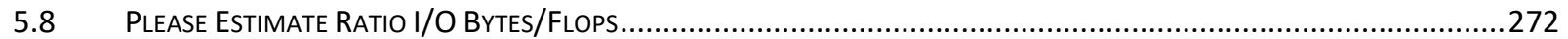

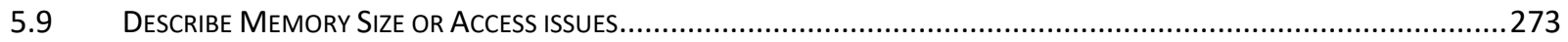

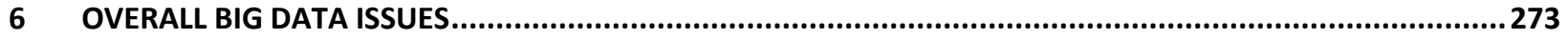

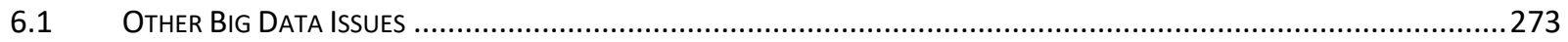

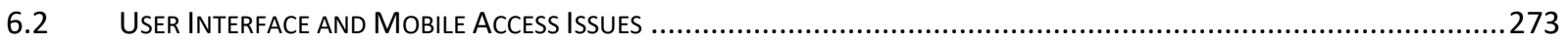

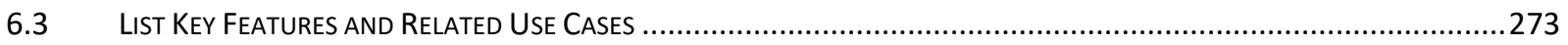

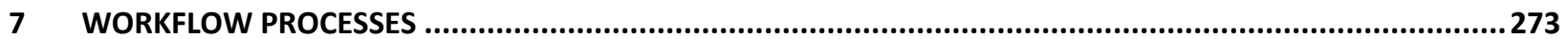

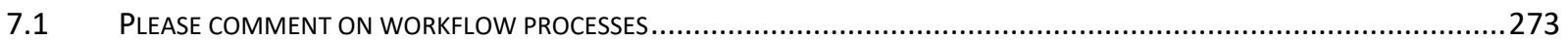

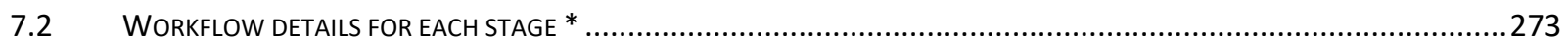

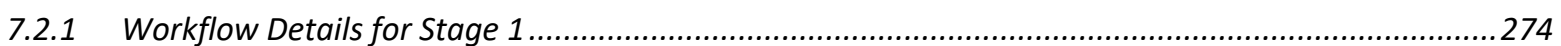

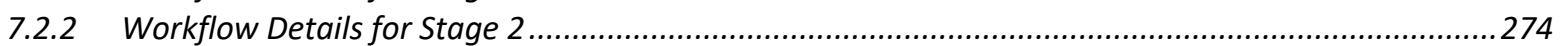

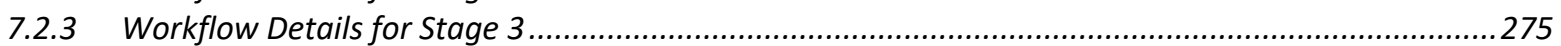

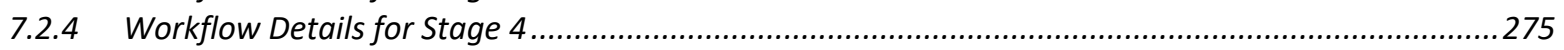

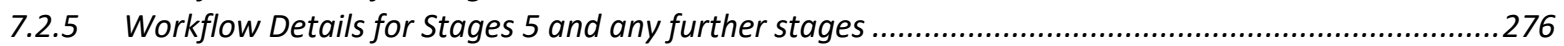

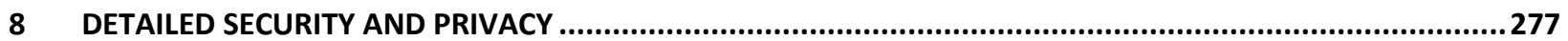

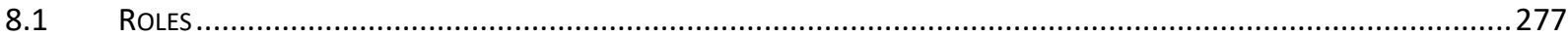

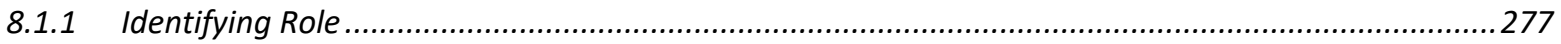

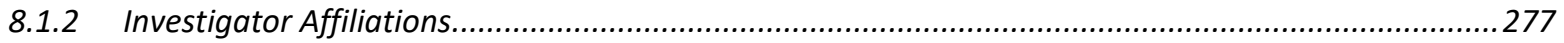

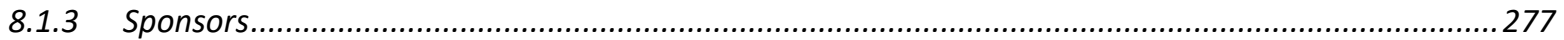

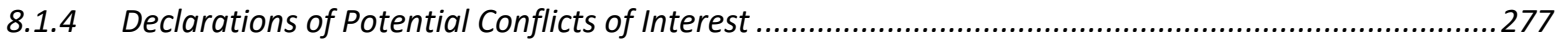

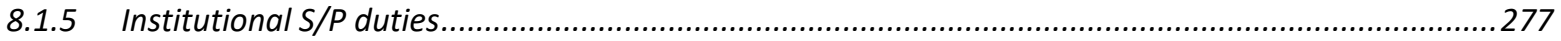

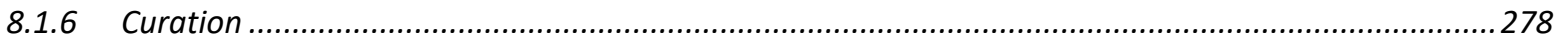

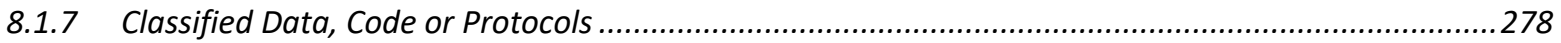

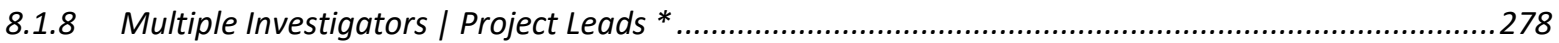

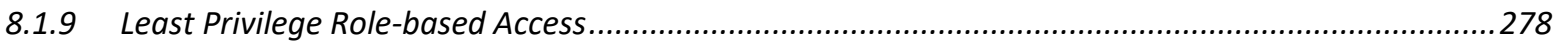

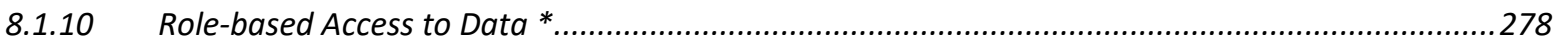

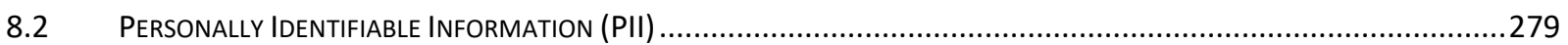

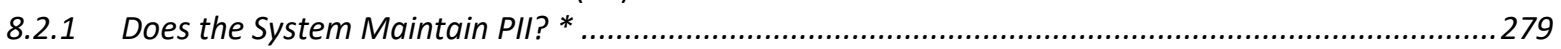

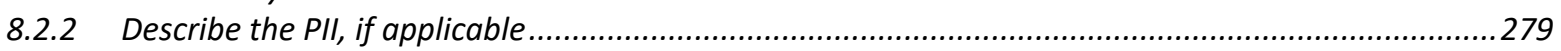

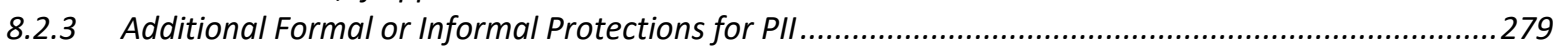

8.2.4 Algorithmic / Statistical Segmentation of Human Populations .......................................................2.279

8.2.5 Protections afforded statistical / deep learning discrimination ........................................................2.279

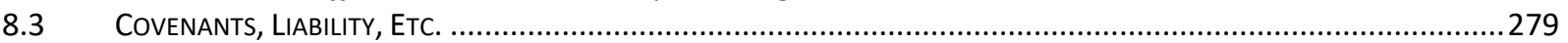

8.3.1 Identify any Additional Security, Compliance, Regulatory Requirements * ....................................279

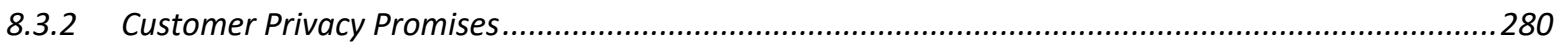

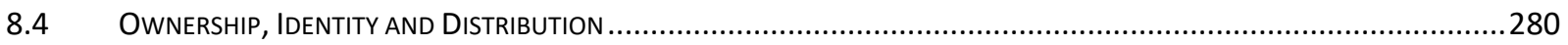


8.4.1 Publication rights

8.4.2 Chain of Trust............

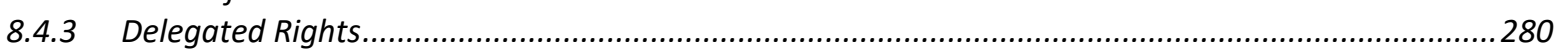

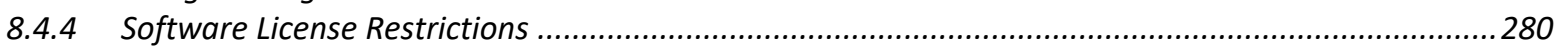

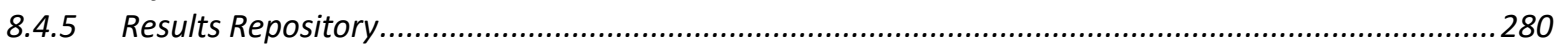

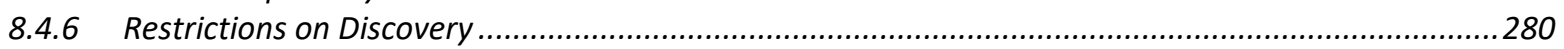

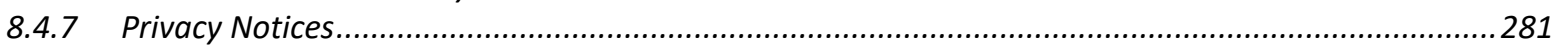

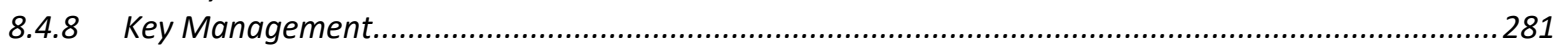

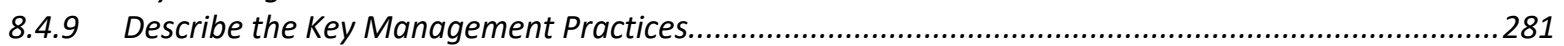

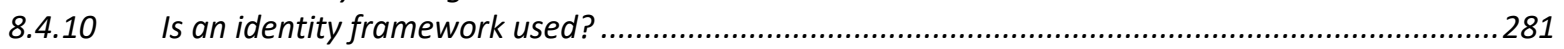

8.4.11 CAC / ECA Cards or Other Enterprise-wide Framework ...........................................................281

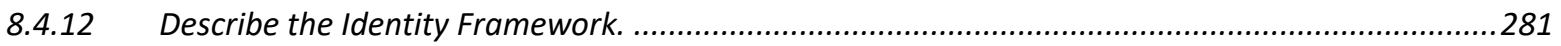

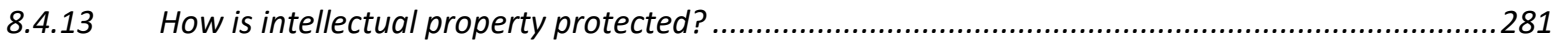

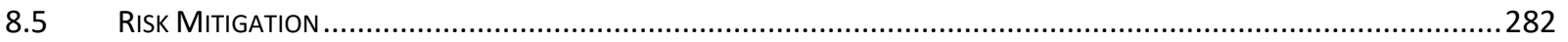

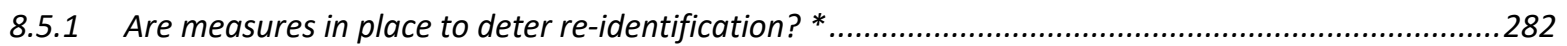

8.5.2 Please describe any re-identification deterrents in place ............................................................282

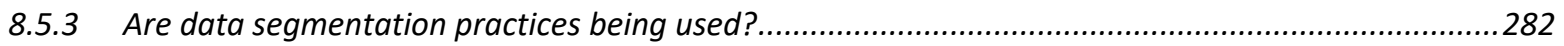

8.5.4 Is there an explicit data governance plan or framework for the effort?...........................................282

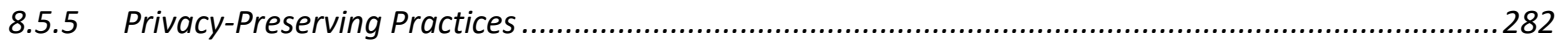

8.5.6 Do you foresee any potential risks from public or private open data projects? ................................282

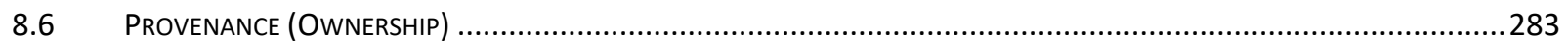

8.6.1 Describe your metadata management practices..............................................................................283

8.6.2 If a metadata management system is present, what measures are in place to verify and protect its

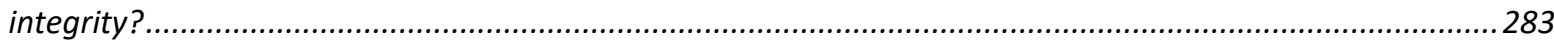

8.6.3 Describe provenance as related to instrumentation, sensors or other devices. .................................283

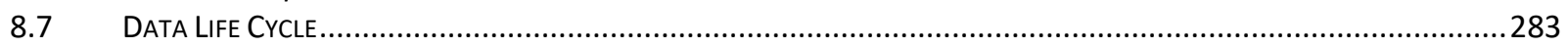

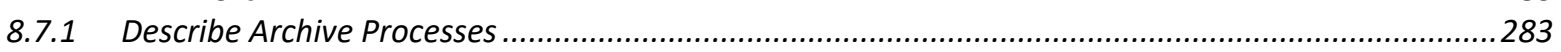

8.7.2 Describe Point in Time and Other Dependency Issues ....................................................................284

8.7.3 Compliance with Secure Data Disposal Requirements....................................................................284

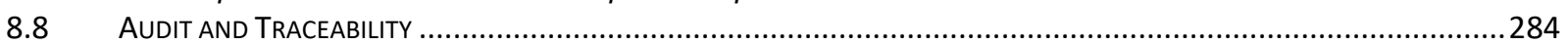

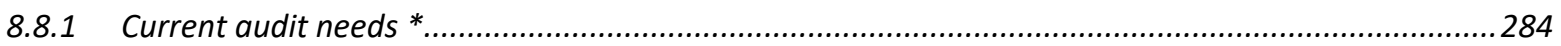

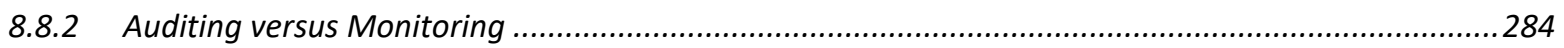

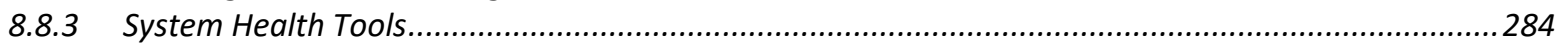

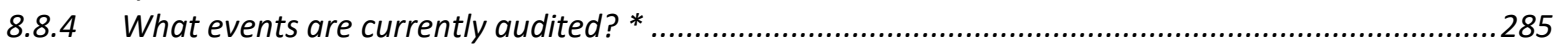

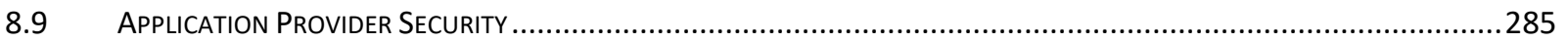

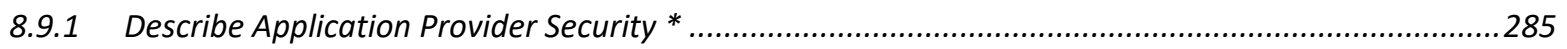

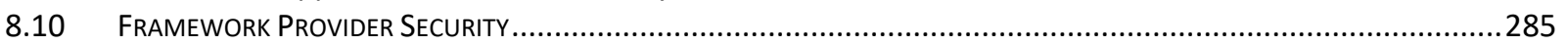

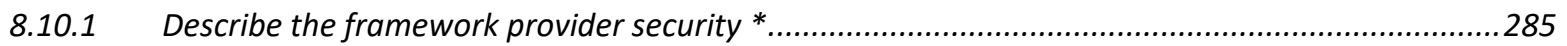

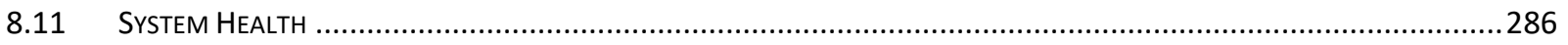

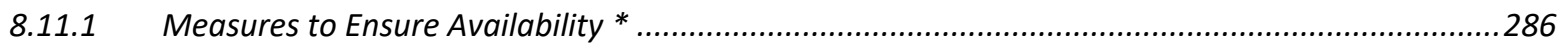

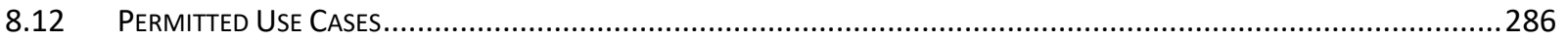

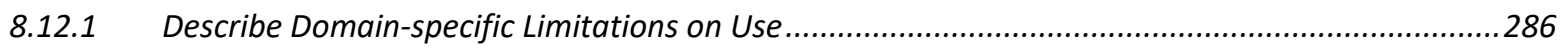

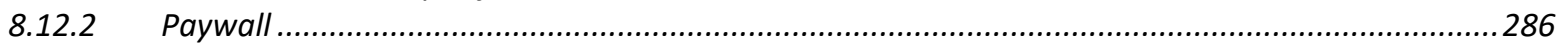

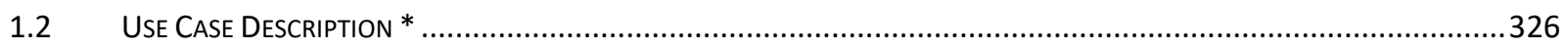

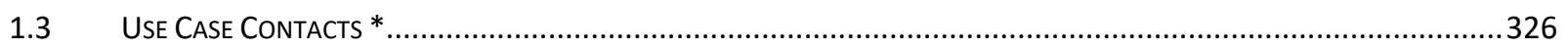

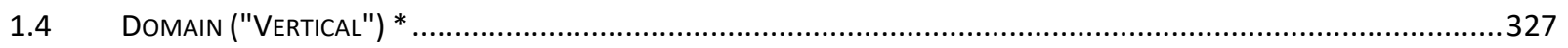

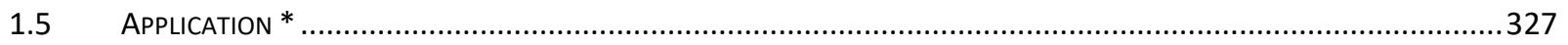

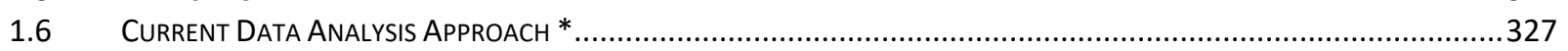

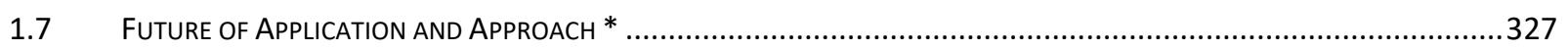

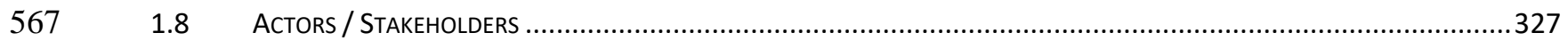


NIST Big Data Interoperability Framework: Volume 3, Use CaSes and General ReQuiRements

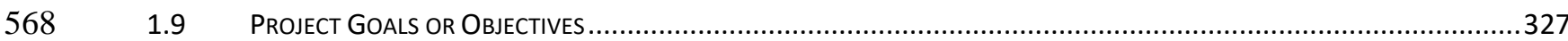

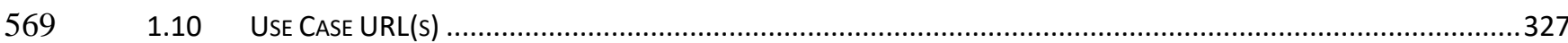

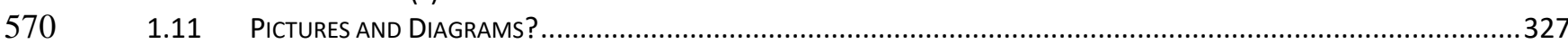

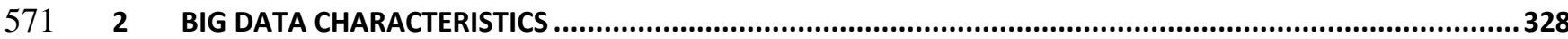

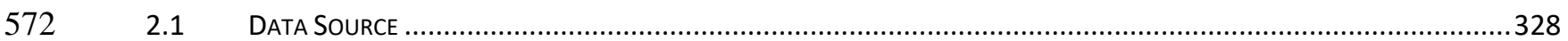

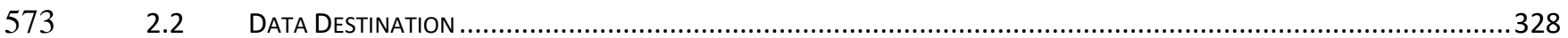

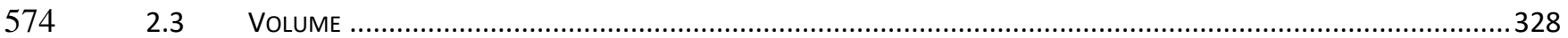

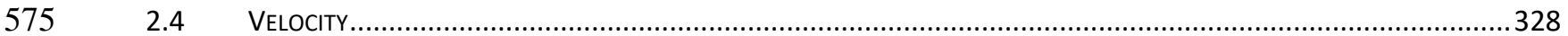

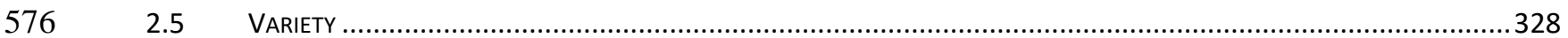

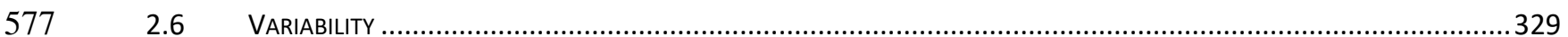

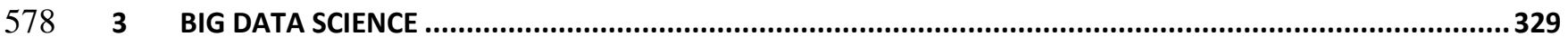

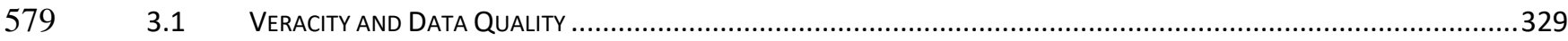

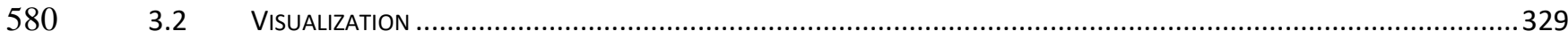

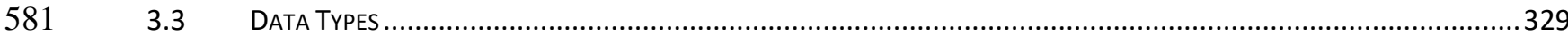

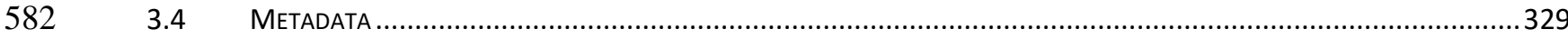

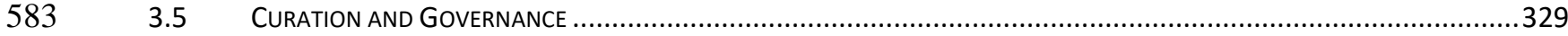

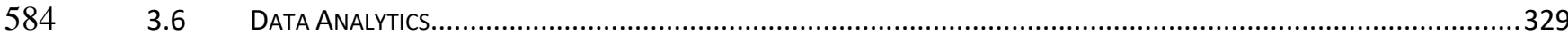

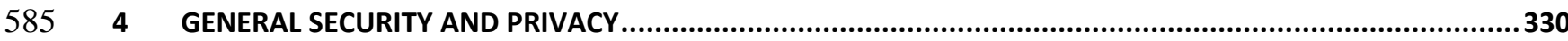

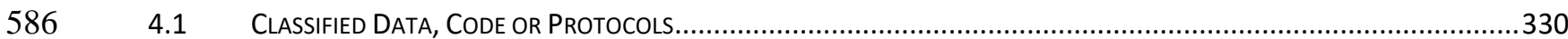

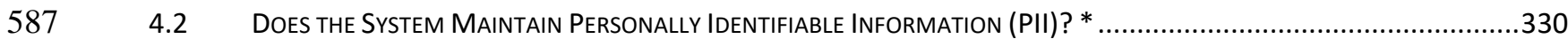

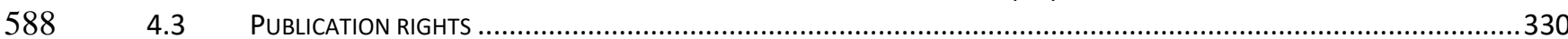

$589 \quad 4.4 \quad$ IS THERE AN EXPLICIT DATA GOVERNANCE PLAN OR FRAMEWORK FOR THE EFFORT? ..................................................330

$590 \quad 4.5 \quad$ DO YOU FORESEE ANY POTENTIAL RISKS FROM PUBLIC OR PRIVATE OPEN DATA PROJECTS? .......................................3

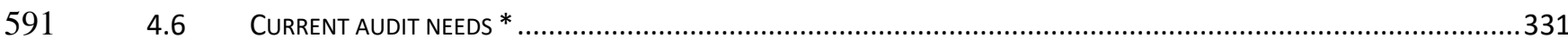

$592 \quad 4.7 \quad$ UNDER WHAT CONDITIONS DO YOU GIVE PEOPLE ACCESS TO YOUR DATA? ............................................................3

$593 \quad 4.8 \quad$ UNDER WHAT CONDITIONS DO YOU GIVE PEOPLE ACCESS TO YOUR SOFTWARE? .........................................................331

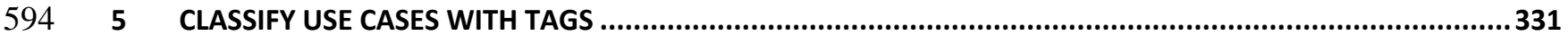

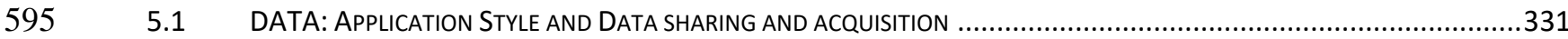

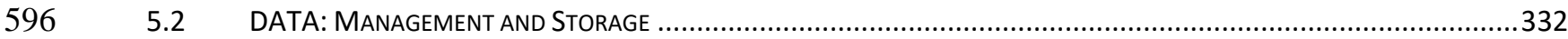

$597 \quad 5.3 \quad$ DATA: Describe Other DatA ACQUISITION/ ACCESS/ SHARING/ MANAGEMENT/ StORAGE ISSUES...........................332

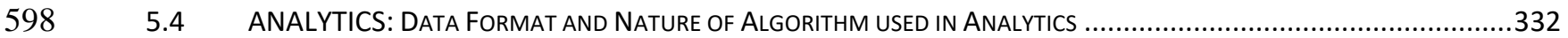

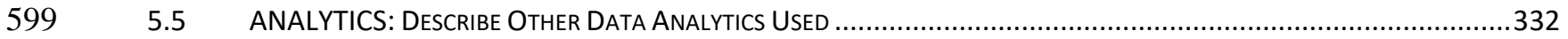

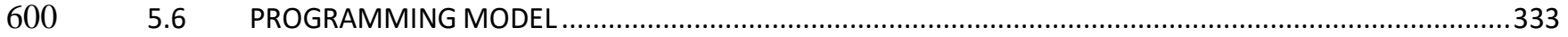

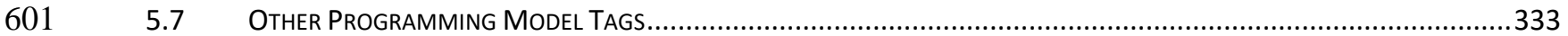

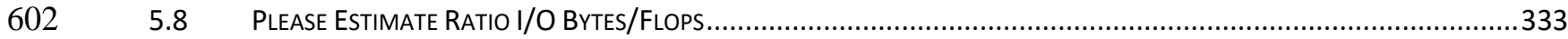

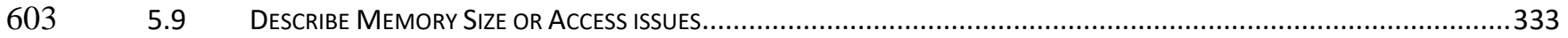

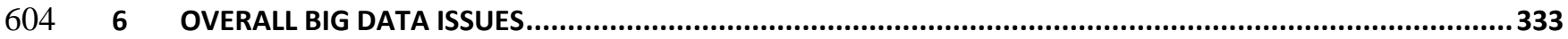

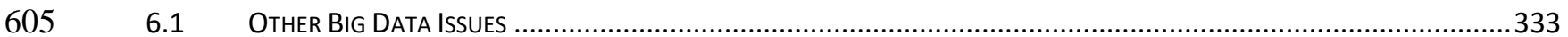

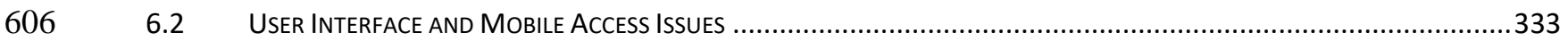

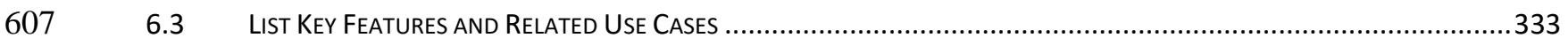

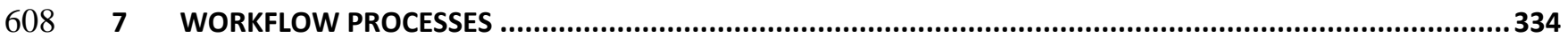

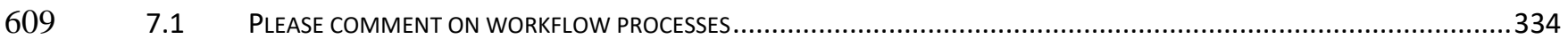

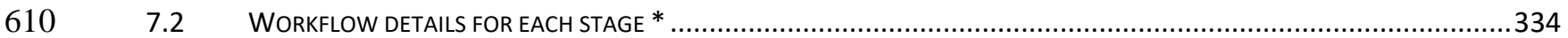

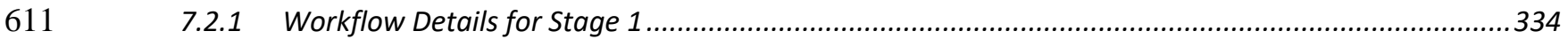

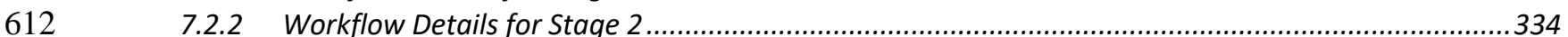

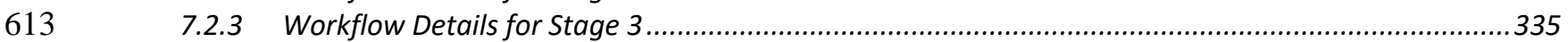

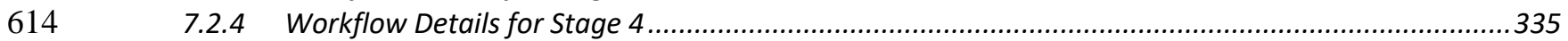


8.1 ROLES

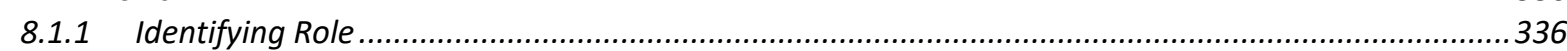

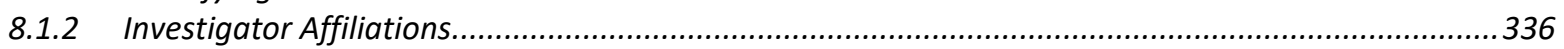

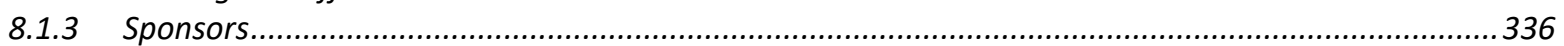

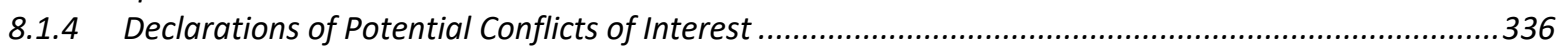

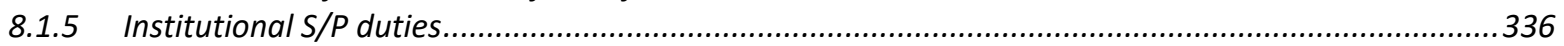

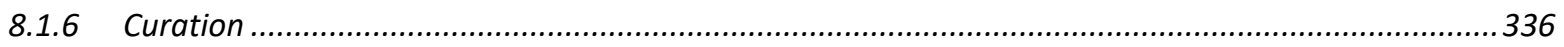

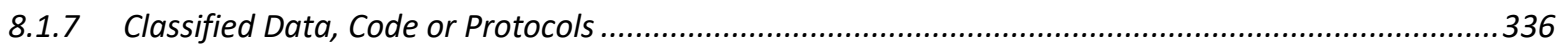

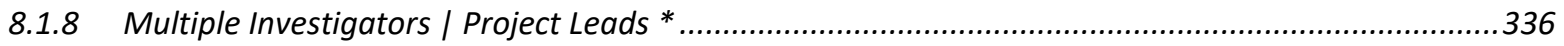

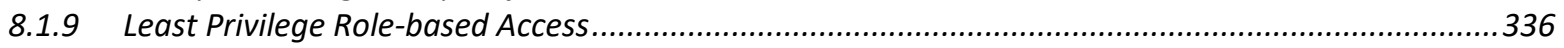

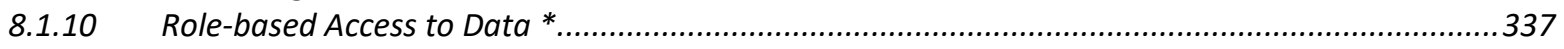

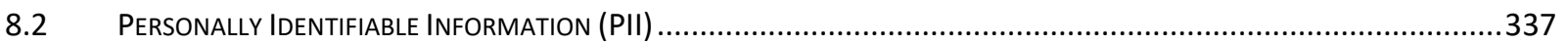

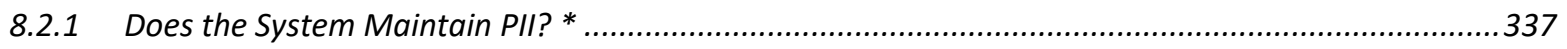

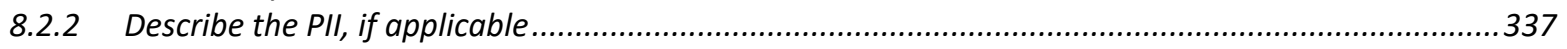

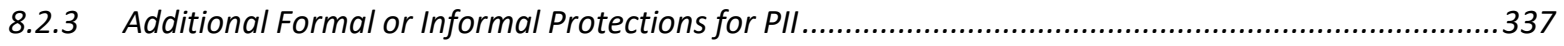

8.2.4 Algorithmic / Statistical Segmentation of Human Populations .......................................................337

8.2.5 Protections afforded statistical / deep learning discrimination.........................................................337

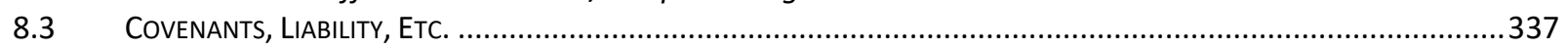

8.3.1 Identify any Additional Security, Compliance, Regulatory Requirements *.........................................337

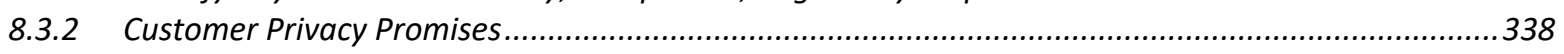

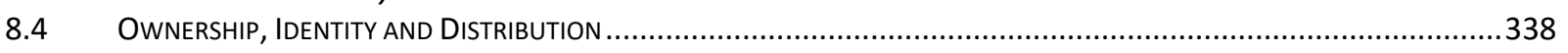

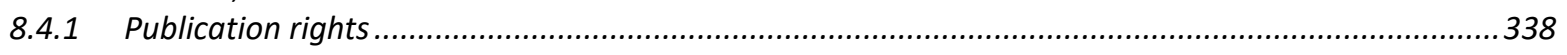

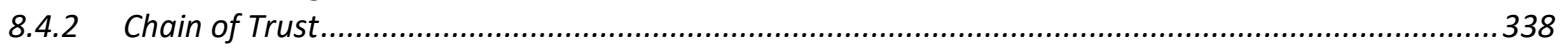

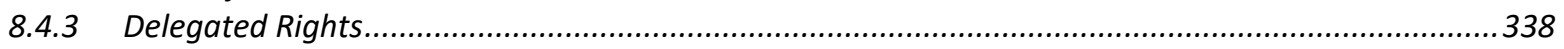

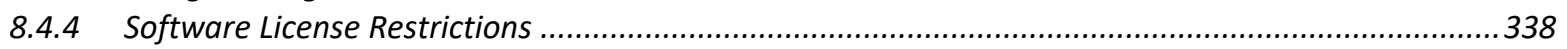

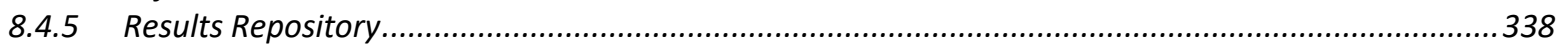

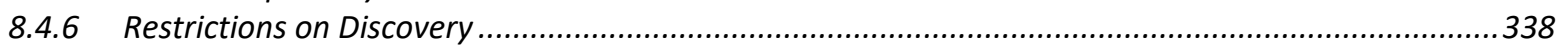

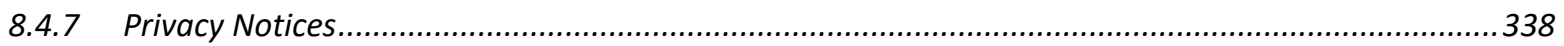

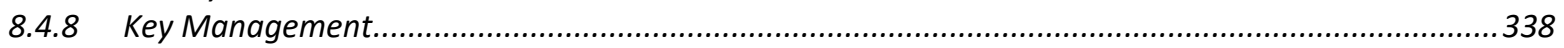

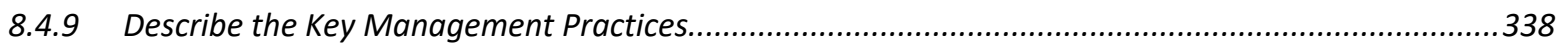

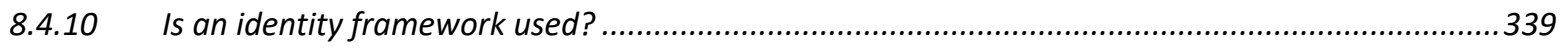

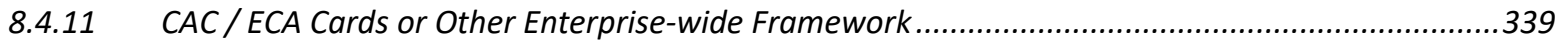

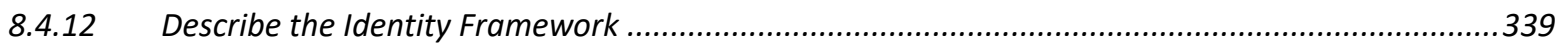

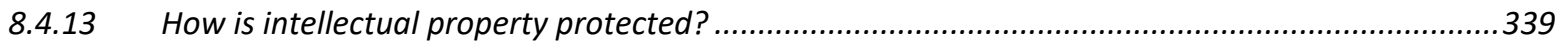

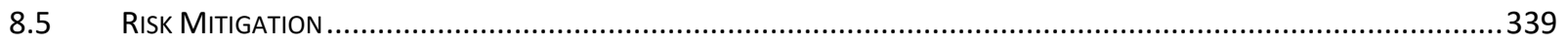

8.5.1 Are measures in place to deter re-identification? * ..................................................................... 339

8.5.2 Please describe any re-identification deterrents in place ...............................................................339

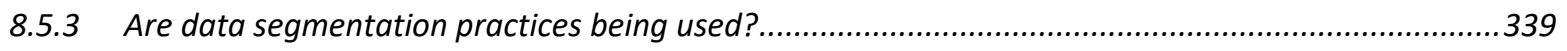

8.5.4 Is there an explicit data governance plan or framework for the effort?............................................339

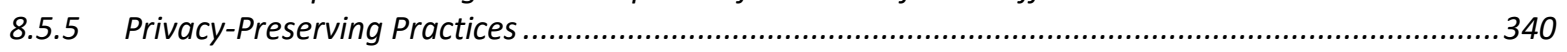

8.5.6 Do you foresee any potential risks from public or private open data projects? ..................................340

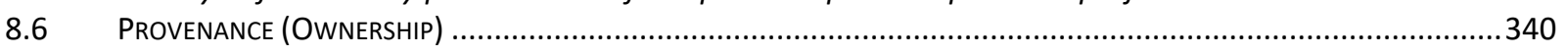

8.6.1 Describe your metadata management practices............................................................................ 340

8.6.2 If a metadata management system is present, what measures are in place to verify and protect its

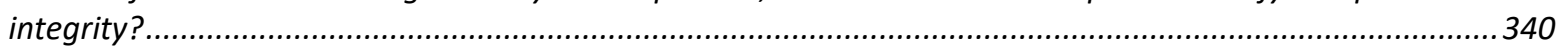

8.6.3 Describe provenance as related to instrumentation, sensors or other devices. .................................340

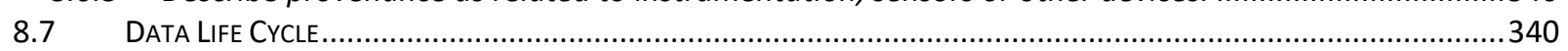

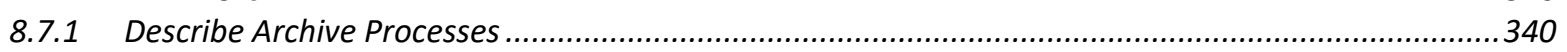

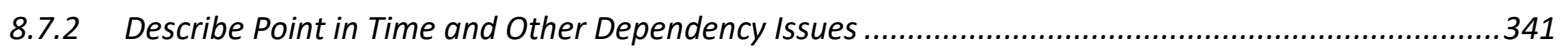

8.7.3 Compliance with Secure Data Disposal Requirements......................................................................341 
667

668

669

670

671

672

673

674

675

676

677

678

679

680

681

682

683 General Instructions:

684 Brief instructions are provided with each question requesting an answer in a text field. For the questions 685

686

687

688

689

690

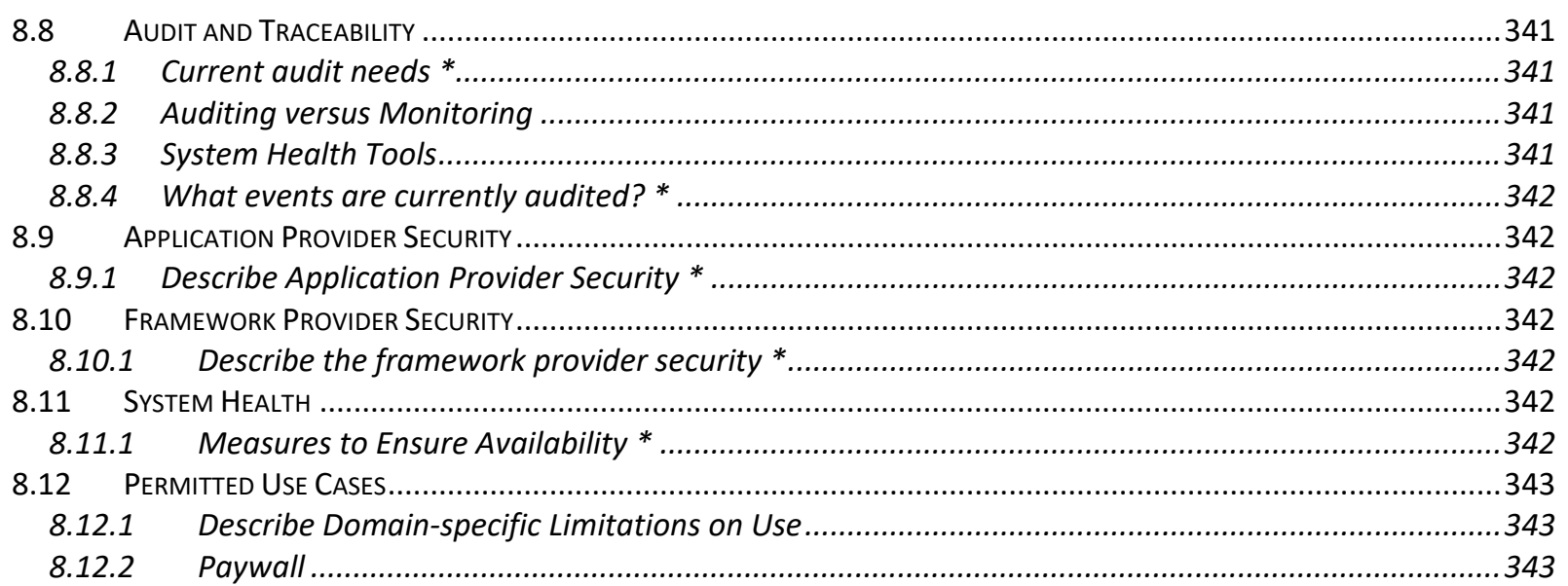
offering check boxes, please check any that apply to the use case.

No fields are required to be filled in. Please fill in the fields that you are comfortable answering. The fields that are particularly important to the work of the NBD-PWG are marked with *

Please email the completed template to Wo Chang at wchang@nist.gov.

NOTE: No proprietary or confidential information should be included. 


\section{OVERALL PROJECT DESCRIPTION}

Please limit to one line. A description field is provided below for a longer description.

\subsection{Use Case Description *}

Summarize all aspects of use case focusing on application issues (later questions will highlight technology).

\subsection{Use Case Contacts *}

700 Add names, phone number, and email of key people associated with this use case. Please designate who is authorized to edit this use case.

$$
\text { Name }
$$

Phone

Email

PI / Author

Edit rights?

Primary

\subsection{Domain ("Vertical") *}

\subsection{Application *}

709 Summarize the use case applications.

\subsection{Current Data Analysis Approach *}

\subsection{Actors / Stakeholders}

$720 \quad$ Please describe the players and their roles in the use case. Identify relevant stakeholder roles and 721 responsibilities. Note: Security and privacy roles are discussed in a separate part of this template. 


\subsection{Project Goals or Objectives}

724 Please describe the objectives of the use case.

\subsection{Use Case URL}

\subsection{Pictures and Diagrams?}

$730 \quad$ Please email any pictures or diagrams with this template.

\section{BIG DATA CHARACTERISTICS}

Big Data Characteristics describe the properties of the (raw) data including the four major ' $\mathrm{V}$ 's' of Big Data described in NIST Big Data Interoperability Framework: Volume 1, Big Data Definition.

\subsection{Data Source}

\subsection{Data Destination}

If the data is transformed in the use case, describe where the final results end up. This has similar characteristics to data source.

\subsection{Volume}

\begin{tabular}{|l|l|}
\hline Size & \\
\hline Units & \\
\hline Time Period & \\
\hline Proviso & \\
\hline
\end{tabular}

Proviso: The criterion (e.g. data gathered by a particular organization) used to get size with units in time period in three fields above 


\subsection{Velocity}

Enter if real-time or streaming data is important. Be quantitative: this number qualified by 3 fields below: units, time period, proviso. Refers to the rate of flow at which the data is created, stored, analyzed, and visualized. For example, big velocity means that a large quantity of data is being processed in a short amount of time.

\begin{tabular}{|l|l|}
\hline Unit of measure & \\
\hline Time Period & \\
\hline Proviso & \\
& \\
\hline
\end{tabular}

Unit of Measure: Units of Velocity size given above. What is measured such as "New Tweets gathered per second", etc.?

Time Period: Time described and interval such as September 2015; items per minute

Proviso: The criterion (e.g., data gathered by a particular organization) used to get Velocity measure with units in time period in three fields above

\subsection{Variety}

Variety refers to data from multiple repositories, domains, or types. Please indicate if the data is from multiple datasets, mashups, etc.

\subsection{Variability}

764

765

766 of changes than Velocity which is just change in size. Please describe the use case data variability.

\section{BIG DATA SCIENCE}

\subsection{Veracity and Data Quality}

769 This covers the completeness and accuracy of the data with respect to semantic content as well as 770 syntactical quality of data (e.g., presence of missing fields or incorrect values).

\subsection{Visualization}

\subsection{Data Types}


Note that we have a separate section for security and privacy. Comment on process to ensure good data quality and who is responsible.

\subsection{Data Analytics}

In the context of these use cases, analytics refers broadly to tools and algorithms used in processing the data at any stage including the data to information or knowledge to wisdom stages, as well as the information to knowledge stage. This section should be reasonably precise so quantitative comparisons

\section{GENERAL SECURITY AND PRIVACY}

794 The following questions are intended to cover general security and privacy topics. Security and privacy

795 topics are explored in more detail in Section 8. For the questions with checkboxes, please select the 796 item(s) that apply to the use case.

\subsection{Classified Data, Code or Protocols}

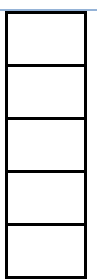

Intellectual property protections

Military classifications, e.g., FOUO, or Controlled Classified

Not applicable

Creative commons/ open source

Other:

\subsection{Does the System Maintain Personally Identifiable Information (PII)? *}

No, but it is possible that individuals could be identified via third party databases

Other:

\subsection{Publication rights}

801 Open publisher; traditional publisher; white paper; working paper

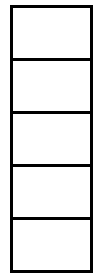

Open publication

Proprietary

Traditional publisher rights (e.g., Springer, Elsevier, IEEE)

"Big Science" tools in use

Other: 
802

803

804

805

\subsection{Is there an explicit data governance plan or framework for the effort?}

Data governance refers to the overall management of the availability, usability, integrity, and security of the data employed in an enterprise.

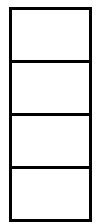

Explicit data governance plan

No data governance plan, but could use one

Data governance does not appear to be necessary

Other:

\subsection{Do you foresee any potential risks from public or private open data projects?}

Transparency and data sharing initiatives can release into public use datasets that can be used to undermine privacy (and, indirectly, security.)

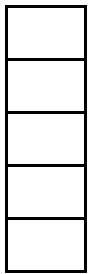

Risks are known.

Currently no known risks, but it is conceivable.

Not sure

Unlikely that this will ever be an issue (e.g., no PII, human-agent related data or subsystems.) Other:

\subsection{Current audit needs *}

\begin{tabular}{|l|l}
\hline We have third party registrar or other audits, such as for ISO 9001 \\
We have internal enterprise audit requirements \\
Audit is only for system health or other management requirements \\
No audit, not needed or does not apply \\
Other:
\end{tabular}

\subsection{Under what conditions do you give people access to your data?}

\subsection{Under what conditions do you give people access to your software?}




\section{CLASSIFY USE CASES WITH TAGS}

The questions below will generate tags that can be used to classify submitted use cases. See

\subsection{DATA: Application Style and Data Sharing and} Acquisition

Uses Geographical Information Systems?

Use case involves Internet of Things?

Data comes from HPC or other simulations?

Data Fusion important?

Data is Real time Streaming?

Data is Batched Streaming (e.g. collected remotely and uploaded every so often)?

\begin{tabular}{ll}
\hline & Important Data is in a Permanent Repository (Not streamed)? \\
\hline & Transient Data important?
\end{tabular}

Permanent Data Important?

Data shared between different applications/users?

Data largely dedicated to only this use case?

\subsection{DATA: Management and Storage}

\begin{tabular}{|l|l|}
\hline & Application data system based on Files? \\
& Application data system based on Objects? \\
& Uses HDFS style File System? \\
& Uses Wide area File System like Lustre? \\
& Uses HPC parallel file system like GPFS? \\
& Uses SQL? \\
\cline { 1 - 1 } Uses NoSQL? \\
Uses NewSQL? \\
Uses Graph Database?
\end{tabular}

\subsection{DATA: Describe Other Data Acquisition/ Access/ Sharing/ Management/ Storage Issues}



in Analytics

Data regular?

Data dynamic?

Algorithm $\mathrm{O}\left(\mathrm{N}^{\wedge} 2\right)$ ?

Basic statistics (regression, moments) used?

Search/Query/Index of application data Important?

Classification of data Important?

Recommender Engine Used?

Clustering algorithms used?

Alignment algorithms used?

(Deep) Learning algorithms used?

Graph Analytics Used?

\subsection{ANALYTICS: Describe Other Data Analytics Used}

\subsection{PROGRAMMING MODEL}

\begin{tabular}{|c|}
\hline $\begin{array}{l}\text { Pleasingly parallel Structure? Parallel execution over independent data. Called Many Task } \\
\text { or high throughput computing. MapReduce with only Map and no Reduce of this type }\end{array}$ \\
\hline $\begin{array}{l}\text { Use case NOT Pleasingly Parallel -- Parallelism involves linkage between tasks. MapReduce } \\
\text { (with Map and Reduce) of this type }\end{array}$ \\
\hline Uses Classic MapReduce? such as Hadoop \\
\hline Uses Apache Spark or similar Iterative MapReduce? \\
\hline Uses Graph processing as in Apache Giraph? \\
\hline Uses MPI (HPC Communication) and/or Bulk Synchronous Processing BSP? \\
\hline Dataflow Programming Model used? \\
\hline Workflow or Orchestration software used? \\
\hline Python or Scripting front ends used? Maybe used for orchestration \\
\hline Shared memory architectures important? \\
\hline Event-based Programming Model used? \\
\hline Agent-based Programming Model used? \\
\hline Use case $1 / O$ dominated? $\mathrm{I} / \mathrm{O}$ time $>$ or $>>$ Compute time \\
\hline Use case involves little I/O? Compute >> I/O \\
\hline
\end{tabular}




\section{OVERALL BIG DATA ISSUES}

\subsection{Other Big Data Issues}

\subsection{User Interface and Mobile Access Issues}

\section{WORKFLOW PROCESSES}

Please answer this question if the use case contains multiple steps where Big Data characteristics, recorded in this template, vary across steps. If possible, flesh out workflow in the separate set of questions. Only use this section if your use case has multiple stages where Big Data issues differ significantly between stages.

\subsection{Please comment on workflow processes}

\subsection{Workflow details for each stage *}

\section{Description of table fields below:}

Data Source(s): The origin of data, which could be from instruments, Internet of Things, Web, Surveys, Commercial activity, or from simulations. The source(s) can be distributed, centralized, local, or remote. Often data source at one stage is destination of previous stage with raw data driving first stage.

Nature of Data: What items are in the data?

Software Used: List software packages used

Data Analytics: List algorithms and analytics libraries/packages used

Infrastructure: Compute, Network and Storage used. Note sizes infrastructure -- especially if "big".

Percentage of Use Case Effort: Explain units. Could be clock time elapsed or fraction of compute cycles

Other Comments: Include comments here on items like veracity and variety present in upper level but omitted in summary. 
NIST Big Data INTEROperability Framework: Volume 3, Use CaSes and General ReQUiRements

876

\subsubsection{WORKFLOW DETAILS FOR STAGE 1}

\begin{tabular}{|l|l|}
\hline Stage 1 Name & \\
\hline Data Source(s) & \\
\hline Nature of Data & \\
\hline Software Used & \\
\hline Data Analytics & \\
\hline Infrastructure & \\
\hline $\begin{array}{l}\text { Percentage of Use } \\
\text { Case Effort }\end{array}$ & \\
\hline Other Comments & \\
\hline
\end{tabular}

\subsubsection{WORKFLOW DETAILS FOR STAGE 2}

\begin{tabular}{|l|l|}
\hline Stage 2 Name & \\
\hline Data Source(s) & \\
\hline Nature of Data & \\
\hline Software Used & \\
\hline Data Analytics & \\
\hline Infrastructure & \\
\hline $\begin{array}{l}\text { Percentage of Use } \\
\text { Case Effort }\end{array}$ & \\
\hline Other Comments & \\
\hline
\end{tabular}


NIST Big Data Interoperability Framework: Volume 3, Use CaSes and General ReQuiRements

878

\subsubsection{WORKFLOW DETAILS FOR STAGE 3}

\begin{tabular}{|l|l|}
\hline Stage 3 Name & \\
\hline Data Source(s) & \\
\hline Nature of Data & \\
\hline Software Used & \\
\hline Data Analytics & \\
\hline Infrastructure & \\
\hline $\begin{array}{l}\text { Percentage of Use } \\
\text { Case Effort }\end{array}$ & \\
\hline Other Comments & \\
\hline
\end{tabular}

1.45.4 WORKFLOW DETAILS FOR STAGE 4

\begin{tabular}{|l|l|}
\hline Stage 4 Name & \\
\hline Data Source(s) & \\
\hline Nature of Data & \\
\hline Software Used & \\
\hline Data Analytics & \\
\hline Infrastructure & \\
\hline $\begin{array}{l}\text { Percentage of Use } \\
\text { Case Effort }\end{array}$ & \\
\hline Other Comments & \\
\hline
\end{tabular}


NIST Big Data Interoperability Framework: Volume 3, Use CaSes and General ReQuiRements

881

882

\subsection{5 .5}

WORKFLOW DETAILS FOR STAGES 5 AND ANY FURTHER STAGES
If you have more than five stages, please put stages 5 and higher here.

\begin{tabular}{|l|l|}
\hline Stage 5 Name & \\
\hline Data Source(s) & \\
\hline Nature of Data & \\
\hline Software Used & \\
\hline Data Analytics & \\
\hline Infrastructure & \\
\hline $\begin{array}{l}\text { Percentage of Use } \\
\text { Case Effort }\end{array}$ & \\
\hline Other Comments & \\
\hline
\end{tabular}




\section{DETAILED SECURITY AND PRIVACY}

885

886

887

888

889

890

891

892

893

894

895

896

897

898

899

900

901

902

903

904

905

906

907

908

909

910

911

912

913

914

915

916

917

918

919

920

921

922

Questions in this section are designed to gather a comprehensive image of security and privacy aspects (e.g., security, privacy, provenance, governance, curation, and system health) of the use case. Other sections contain aspects of curation, provenance, and governance that are not strictly speaking only security and privacy considerations. The answers will be very beneficial to the NBD-PWG in understanding your use case. However, if you are unable to answer the questions in this section, the NBDPWG would still be interested in the information gathered in the rest of the template. The security and privacy questions are grouped as follows:

- Roles

- Personally Identifiable Information

- Covenants and Liability

- Ownership, Distribution, Publication

- Risk Mitigation

- Audit and Traceability

- Data Life Cycle

- Dependencies

- Framework provider S\&P

- Application Provider S\&P

- Information Assurance | System Health

- $\quad$ Permitted Use Cases

\subsection{Roles}

Roles may be associated with multiple functions within a big data ecosystem.

\subsubsection{IDENTIFYING ROLE}

Identify the role (e.g., Investigator, Lead Analyst, Lead Scientists, Project Leader, Manager of Product Development, VP Engineering) associated with identifying the use case need, requirements, and deployment.

\subsubsection{INVESTIGATOR AFFILIATIONS}

This can be time-dependent and can include past affiliations in some domains.

\subsubsection{SPONSORS}

Include disclosure requirements mandated by sponsors, funders, etc.

\subsubsection{Declarations of Potential Conflicts of Interest}

\subsubsection{INSTITUTIONAL S/P DUTIES}

List and describe roles assigned by the institution, such as via an IRB (Institutional Review Board). 
923

924

925

926

927

928

\subsection{6 .8}

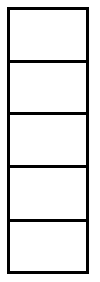
duties.

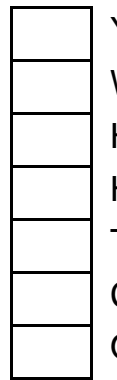

\section{CURATION}

List and describe roles associated with data quality and curation, independent of any specific Big Data component. Example: Role responsible for identifying U.S. government data as FOUO or Controlled Unclassified Information, etc.

\subsubsection{Classified Data, Code or Protocols}

\begin{tabular}{|l|l}
\hline & Intellectual property protections \\
& Military classifications, e.g., FOUO, or Controlled Classified \\
& Not applicable \\
Creative commons/ open source \\
Other:
\end{tabular}

\subsubsection{LEAST PRIVILEge Role-based Access}

Least privilege requires that a user receives no more permissions than necessary to perform the user's

Yes, roles are segregated and least privilege is enforced

We do have least privilege and role separation but the admin role(s) may be too all-inclusion Handled at application provider level Handled at framework provider level There is no need for this feature in our application Could be applicable in production or future versions of our work Other:

\subsubsection{Role-based Access to Data *}

Please describe the level at which access to data is limited in your system.

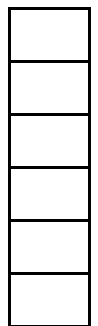

Dataset

Data record / row

Data element / field

Handled at application provider level

Handled at framework provider level

Other: 


\subsection{Personally Identifiable Information (PII)}

\subsubsection{DOES THE SySteM MAINTAIN PII? *}

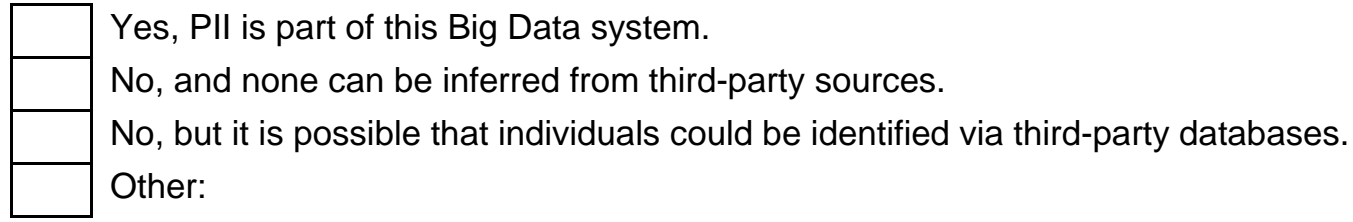

\subsubsection{DESCRIBE THE PII, IF APPLICABLE}

Describe how PII is collected, anonymized, etc. Also list disclosures to human subjects, interviewees, or web visitors.

\subsubsection{AdDitional Formal or Informal Protections for PII}

\subsubsection{Algorithmic / Statistical Segmentation of Human Populations}

$\square$ Yes, doing segmentation, possible discrimination issues if abused. Please also answer the next question.

Yes, doing segmentation, but no foreseeable discrimination issues.

Does not apply to this use case at all (e.g., no human subject data). Other:

\subsubsection{PROTECTIONS AFFORDED STATISTICAL / DEEP LEARNING DISCRIMINATION}

Identify what measures are in place to address this concern regarding human populations, if it applies. Refer to the previous question.

\subsection{Covenants, Liability, Etc.}

\subsubsection{IDENTIFY ANY AdDITIONAL SECURITY, COMPLIANCE, REgULATORY REQUIREMENTS * \\ Refer to 45 CFR 46: http://1.usa.gov/1bg6JQ2}

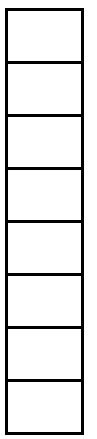

FTC regulations apply

HHS 45 CFR 46

HIPAA

EU General Data Protection (Reference: http://bit.ly/1Ta8S1C )

COPPA

Other Transborder issues

Fair Credit Reporting Act (Reference: http://bit.ly/1Ta8XSN )

Family Educational Rights and Protection (FERPA) 
NIST Big Data Interoperability Framework: Volume 3, Use CaSes and General ReQuiRements

\begin{tabular}{ll}
\hline & None apply \\
& Other:
\end{tabular}

$954 \quad \mathbf{1 . 4 8 . 2}$ CUSTOMER PRIVACy PROMISES

955 Select all that apply,e.g., RadioShack promise that is subject of this DOJ ruling: http://bit.ly/1fOMW9t

\begin{tabular}{|l|l|}
\hline & Yes, we're making privacy promises to customers or subjects. \\
We are using a notice-and-consent model. \\
Not applicable \\
Other:
\end{tabular}

\subsection{Ownership, Identity and Distribution}

\subsubsection{PUBLICATION RIGHTS}

958 Open publisher; traditional publisher; white paper; working paper

\begin{tabular}{|l|l}
\hline & Open publication \\
& Proprietary \\
& Traditional publisher rights (e.g., Springer, Elsevier, IEEE) \\
& "Big Science" tools in use \\
Other:
\end{tabular}

959

960

961

962

\section{3}

964

965

966

967

968

969

970

971

972

973

974

975

976

\subsubsection{ChAIN OF TRUST}

Identify any chain-of-trust mechanisms in place (e.g., ONC Data Provenance Initiative). Potentially very domain-dependent; see the ONC event grid, for instance. Reference: http://bit.ly/1fOPGDL

\subsubsection{DELEGATED RIGHTS}

Example of one approach: "Delegation Logic: A Logic-based Approach to Distributed Authorization", Li, N., Grosof, B.N., Feigenbaum, J.(2003) https://www.cs.purdue.edu/homes/ninghui/papers/thesis.pdf

\subsubsection{SOFTWARE LICENSE RESTRICTIONS}

Identify proprietary software used in the use case Big Data system which could restrict use, reproducibility, results, or distribution.

\subsubsection{RESULTS REPOSITORY}

Identify any public or private / federated consortia maintaining a shared repository.

\subsubsection{RESTRICTIONS ON DISCOVERY}

Describe restrictions or protocols imposed on discoverable end points. 
$977 \quad \mathbf{1 . 4 9 . 7} \quad$ PRIVACY NOTICES

978 Indicate any privacy notices required / associated with data collected for redistribution to others,

\begin{tabular}{|l|l}
\hline & Privacy notices apply \\
\cline { 1 - 1 } Privacy notices do not apply \\
Other:
\end{tabular}

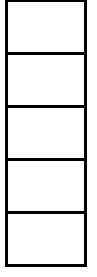

A key management scheme is part of our system.

We are using public key infrastructure.

We do not use key management, but it could have been useful.

No readily identifiable use for key management.

Other:

Describe the Key Management Practices

\subsubsection{0}

IS AN IDENTITY FRAMEWORK USED?

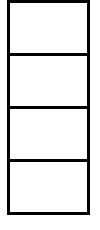

A framework is in place. (See next question.)

Not currently using a framework.

There is no perceived need for an identity framework.

Other:

\subsubsection{1}

CAC I ECA CARDS OR OTHER ENTERPRISE-WIDE FRAMEWORK

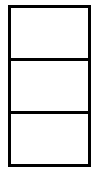

Using an externally maintained enterprise-wide identity framework.

Could be used, but none are available.

Not applicable

\subsubsection{DESCRIBE THE IDENTITY FRAMEWORK.}

HOW IS INTELLECTUAL PROPERTY PROTECTED?

Login screens advising of IP issues

Employee or team training

Official guidelines limiting access or distribution

Required to track all access to, distribution of digital assets

Does not apply to this effort (e.g., public effort)

Other: 


\subsection{Risk Mitigation}

1.50 .1

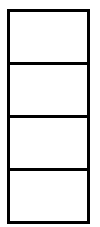

ARE MEASURES IN PLACE TO DETER RE-IDENTIFICATION? *

\subsubsection{PLEASE DESCRIBE ANY RE-IDENTIFICATION DETERRENTS IN PLACE}

\subsubsection{ARE DATA SEGMENTATION PRACTICES BEING USED?}

Data segmentation for privacy has been suggested as one strategy to enhance privacy protections. Reference: http://bit.ly/1P3h12Y

\begin{tabular}{|l|l}
\hline & Yes, being used \\
& Not in use, but does apply \\
& Not applicable \\
& Other:
\end{tabular}

\subsubsection{IS THERE AN EXPLICIT DATA GOVERNANCE PLAN OR FRAMEWORK FOR} THE EFFORT?

Data governance refers to the overall management of the availability, usability, integrity, and security of the data employed in an enterprise.

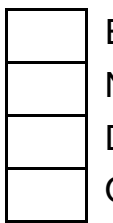

Explicit data governance plan

No data governance plan, but could use one

Data governance does not appear to be necessary

Other:

\subsubsection{PRIVACY-PRESERVING PRACTICES}

\subsubsection{DO YOU FORESEE ANY POTENTIAL RISKS FROM PUBLIC OR PRIVATE} OPEN DATA PROJECTS?

Transparency and data sharing initiatives can release into public use datasets that can be used to undermine privacy (and, indirectly, security).

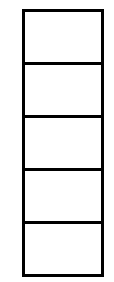

Risks are known.

Currently no known risks, but it is conceivable.

Not sure

Unlikely that this will ever be an issue (e.g., no PII, human-agent related data or subsystems). Other: 


\subsection{Provenance (Ownership)}

1006 Provenance viewed from a security or privacy perspective. The primary meaning for some domains is

1007 digital reproducibility, but it could apply in simulation scenarios as well.

DESCRIBE YOUR METADATA MANAGEMENT PRACTICES

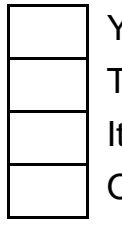
Yes, we have a metadata management system. There is no need for a metadata management system in this use case. It is applicable but we do not currently have one. Other:

\subsubsection{IF A METADATA MANAGEMENT SYSTEM IS PRESENT, WHAT MEASURES ARE IN PLACE TO VERIFY AND PROTECT ITS INTEGRITY?}

\subsubsection{DESCRIBE PROVENANCE AS RELATED TO INSTRUMENTATION,} SENSORS OR OTHER DEVICES.
\begin{tabular}{|l|}
\hline \\
\hline \\
\hline \\
\hline \\
\hline \\
\hline
\end{tabular}
We have potential machine-to-machine traffic provenance concerns.
Endpoint sensors or instruments have signatures periodically updated.
Using hardware or software methods, we detect and remediate outlier signatures.
Endpoint signature detection and upstream flow are built into system processing.
We rely on third-party vendors to manage endpoint integrity.
We use a sampling method to verify endpoint integrity.
Not a concern at this time.
Other:

\subsection{Data Life Cycle}

\section{1.52.1 DESCRIBE ARCHIVE PROCESSES}

\footnotetext{
\begin{tabular}{|l|}
\hline \\
\hline \\
\hline \\
\hline \\
\hline \\
\hline \\
\hline
\end{tabular}

Our application has no separate "archive" process.

We offload data using certain criteria to removable media which are taken offline.

We use a multi-stage, tiered archive process.

We allow for "forgetting" of individual PII on request.

Have ability to track individual data elements across all stages of processing, including archive.

Additional protections, such as separate encryption, are applied to archival data.

Archived data is saved for potential later use by applications or analytics yet to be built.

Does not apply to our application.

Other:
} 


\subsubsection{Describe Point In Time AND Other Dependency IsSues}

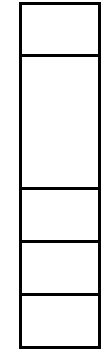

Some data is valid only within a point in time,

Some data is only valid with other, related data is available or applicable, such as the existence of a building, the presence of a weather event, or the active use of a vehicle.

There are specific events in the application that render certain data obsolete or unusable.

Point and Time and related dependencies do not apply.

Other:

\subsection{2 .3}

Complance With Secure Data Disposal ReQuirements

Per NCSL: "at least 29 states have enacted laws that require entities to destroy, dispose. . ." http://www.ncsl.org/research/telecommunications-and-information-technology/privacy-and-security.aspx

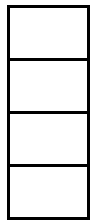

We are required to destroy or otherwise dispose of data.

Does not apply to us.

Not sure

Other:

\subsection{Audit and Traceability}

1021 Big Data use case: SEC Rule 613 initiative

1.53.1

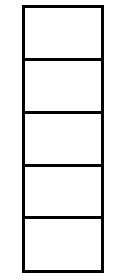

\section{CURRENT AUDIT NEEDS *}

We have third-party registrar or other audits, such as for ISO 9001.

We have internal enterprise audit requirements.

Audit is only for system health or other management requirements.

No audit, not needed or does not apply.

Other:

\subsection{2}

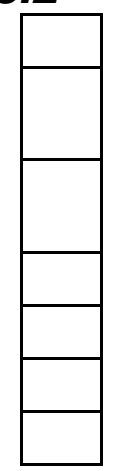

\section{AUDITING VERSUS MONITORING}

We rely on third-party or O.S. tools to audit, e.g., Windows or Linux auditing.

There are built-in tools for monitoring or logging that are only used for system or application health monitoring.

Monitoring services include logging of role-based access to assets such as PII or other resources.

The same individual(s) in the enterprise are responsible for auditing as for monitoring.

This aspect of our application is still in flux.

Does not apply to our setting.

Other:

\section{SYSTEM HEALTH TOOLS}

We rely on system-wide tools for health monitoring.

We built application health tools specifically to address integrity, performance monitoring, and related concerns.

There is no need in our setting.

Other: 


\subsubsection{WHAT EVENTS ARE CURRENTLY AUDITED? *}

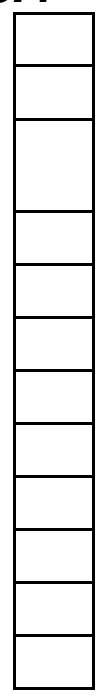

All data access must be audited.

Only selected / protected data must be audited.

Maintenance on user roles must be audited (new users, disabled user, updated roles or permissions).

Purge and archive events.

Domain-dependent events (e.g., adding a new sensor).

REST or SOAP events

Changes in system configuration

Organizational changes

External project ownership / management changes

Requirements are externally set, e.g., by PCl compliance.

Domain-specific events (patient death in a drug trial)

Other:

\subsection{Application Provider Security}

\subsubsection{DESCRIBE APPLICATION PROVIDER SECURITY *}

1028 One example of application layer security is the SAP ERP application.

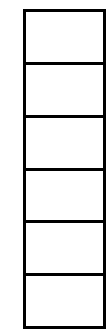

There is a security mechanism implemented at the application level.

The app provider level is aware of PII or privacy data elements.

The app provider implements audit and logging.

The app provider security relies on framework-level security for its operation.

Does not apply to our application.

Other:

\subsection{Framework Provider Security}

1030 One example is Microsoft Active Directory as applied across LANs to Azure, or LDAP mapped to Hadoop. 1031 Reference: http://bit.ly/1fOVDR3

\section{2 \\ 1.55 .1 \\ DESCRIBE THE FRAMEWORK PROVIDER SECURITY *}

Security is implemented at the framework level.

Roles can be defined at the framework level.

The framework level is aware of PII or related sensitive data.

Does not apply in our setting.

Is provided by the Big Data tool.

Other: 


\subsection{System Health}

1034 Also included in this grouping: Availability, Resilience, Information Assurance

1035

\subsubsection{MEASURES TO ENSURE AVAILABILITY *}

\begin{tabular}{|l|l}
\hline & Deterrents to man-in-the-middle attacks \\
\hline & Deterrents to denial of service attacks \\
& Replication, redundancy or other resilience measures \\
\hline & Deterrents to data corruption, drops or other critical big data components \\
Other:
\end{tabular}

\section{$1036 \quad 1.57$ Permitted Use Cases}

1037 Beyond the scope of S\&P considerations presented thus far, please identify particular domain-specific 1038 limitations

\subsubsection{DESCRIBE DOMAIN-SPECIFIC LIMITATIONS ON USE}

\subsubsection{PAYWALL}

A paywall is in use at some stage in the workflow. 


\section{Description of NIST Public Working Group on Big Data}

1044 NIST is leading the development of a Big Data Technology Roadmap. This roadmap will define and prioritize 1045 requirements for interoperability, portability, reusability, and extendibility for Big Data analytic techniques and 1046 technology infrastructure in order to support secure and effective adoption of Big Data. To help develop the 1047 ideas in the Big Data Technology Roadmap, NIST created the Public Working Group for Big Data.

1048 Scope: The focus of the NBD-PWG is to form a community of interest from industry, academia, and 1049 government, with the goal of developing consensus definitions, taxonomies, secure reference architectures, and 1050 a technology roadmap. The aim is to create vendor-neutral, technology- and infrastructure-agnostic 1051 deliverables to enable Big Data stakeholders to pick and choose best analytics tools for their processing and visualization requirements on the most suitable computing platforms and clusters while allowing value-added from Big Data service providers and flow of data between the stakeholders in a cohesive and secure manner.

1054 For more, refer to the website at http://bigdatawg.nist.gov. 


\section{Appendix F: Version 2 Raw Use Case Data}

This appendix contains the raw data from the three Template 2 use cases that have been submitted to date. Summaries of these use cases are included in Section 2. The first two use cases were submitted in an earlier version of Template 2. The third use case (Use Case 2-3) was submitted with a later version of Template 2. The difference between the two Template 2 versions are the location of the Detailed Security and Privacy section (Section 8 in the later version) and the addition of a General Security and Privacy Section in the later version. The later Template 2 version is the current version and should be used for submitted use cases from this point forward.

\section{Overall Project Description}

Use Case 2-1

\begin{tabular}{|ll}
\hline 1.1 & Use Case Title * \\
\hline 1.2 & Use Case Description \\
$*$ &
\end{tabular}

NASA Earth Observing System Data and Information System (EOSDIS)

The Earth Observing System Data and Information System archive and dissemination of Earth Observation data. The system comprises 12 discipline-oriented data systems spread across the United States. This network is linked together using interoperability frameworks such as the Common Metadata Repository, a file-level database that supports one-stop searching across EOSDIS. The data consist of satellite, aircraft, field campaign and in situ data over a variety of disciplines related to Earth science, covering the Atmosphere, Hydrosphere, Cryosphere, Lithosphere, Biosphere, and Anthroposphere. Data are distributed to a diverse community ranging from Earth science researchers to applications to citizen science and educational users. EOSDIS faces major challenges in both Volume and Variety. As of early 2017, the cumulative archive data volume is over 20 Petabytes. Higher resolution spaceborne instruments are expected to increase that volume by two orders of magnitude ( 200 PB) over the next 7 years. More importantly, the data distribution to users is equally high. In a given year, EOSDIS distributes a volume that is comparable to the overall cumulative archive volume. 


\section{Overall Project Description Use Case 2-1}

\subsection{Use Case Contacts *}

\begin{tabular}{|c|c|}
\hline Name & Christopher Lynnes \\
\hline PI or Author & Author \\
\hline Edit Privileges? & Yes \\
\hline Primary author? & Yes \\
\hline 1.4 Domain ("Vertical") * & Earth Science \\
\hline 1.5 Application * & $\begin{array}{l}\text { Data Archiving: storing NASA's Earth Observation dataData } \\
\text { Distribution: disseminating data to end users in Research, } \\
\text { Applications (e.g., water resource management) and } \\
\text { EducationData Discovery: search and access to Earth } \\
\text { Observation dataData Visualization: static browse images and } \\
\text { dynamically constructed visualizationsData } \\
\text { Customization: subsetting, reformatting, regridding, } \\
\text { mosaicking, and quality screening on behalf of end usersData } \\
\text { Processing: routine production of standard scientific datasets, } \\
\text { converting raw data to geophysical variables.Data } \\
\text { Analytics: end-user analysis of large datasets, such as time- } \\
\text { averaged maps and area-averaged time series }\end{array}$ \\
\hline $\begin{array}{l}1.6 \text { Current Data } \\
\text { Analysis Approach * }\end{array}$ & $\begin{array}{l}\text { Standard data processing converts raw data to geophysical } \\
\text { parameters. Though much of this is heritage custom Fortran or } \\
\text { C code running, current prototypes are using cloud computing } \\
\text { to scale up to rapid reprocessing campaigns.EOSDIS support of } \\
\text { end-user analysis currently uses high-performance software, } \\
\text { such as the netCDF Command Operators. However, current } \\
\text { prototypes are using cloud computing and data-parallel } \\
\text { algorithms (e.g., Spark) to achieve an order of magnitude } \\
\text { speed-up. }\end{array}$ \\
\hline $\begin{array}{l}1.7 \text { Future of Application } \\
\text { and Approach * }\end{array}$ & $\begin{array}{l}\text { EOSDIS is beginning to migrate data archiving to the cloud in } \\
\text { order to enable end users to bring algorithms to the data. We } \\
\text { also expect to reorganize certain high-value datasets into forms } \\
\text { that lend themselves to cloud data-parallel computing. } \\
\text { Prototypes are underway to prove out storage schemes that } \\
\text { are optimized for cloud analytics, such as space-time tiles } \\
\text { stored in cloud databases and cloud filesystems. }\end{array}$ \\
\hline $\begin{array}{l}1.8 \text { Actors / } \\
\text { Stakeholders }\end{array}$ & $\begin{array}{l}\text { Science Research Users consume the data and apply their } \\
\text { analysis techniques to derive knowledge of Earth System } \\
\text { Science.Applications users consume the data for real-world } \\
\text { practical use, such as hazard mitigation or resource } \\
\text { management.Educational users and citizen scientists consume } \\
\text { the data in order to understand more about the world in which } \\
\text { they live.Satellite project and science teams use EOSDIS as a } \\
\text { data archive and dissemination agent. }\end{array}$ \\
\hline
\end{tabular}




\section{Overall Project Description \\ Use Case 2-1 \\ 1.9 Project Goals or The objectives are to distribute useful and usable science data Objectives and information relating to Earth system science to a diverse community. \\ 1.10 Use Case URL(s) https://earthdata.nasa.gov}

\section{Big Data Characteristics}

Use Case 2-1

2.1 Data Source

2.2 Data Destination
The two most voluminous sources are:1. high spatial resolution satellite-borne instruments; and 2. long-time-series models assimilating data from satellites and instruments. Most of the Variety comes from the many field campaigns that are run to validate satellite data and explore questions that cannot be answered by spaceborne instruments alone.

Final results most often end up in science research papers.

Data consumed by Applications users may end up in Decision Support Systems, systems that Applications users employ to properly digest and infer information from the data.

\subsection{Volume}

Size $22 \mathrm{~PB}$

Units Total Earth Observation Data managed by NASA EOSDIS

Time Period Accumulated since 1994

Proviso

\subsection{Velocity}

Unit of measure

Time Period

\section{Proviso}

\subsection{Variety}

EOSDIS's Common Metadata Repository includes over 6400 EOSDIS data collections as of June 2017, providing significant challenges in data discovery. CMR and other interoperability frameworks (metrics, browse imagery, governance) knit together 12 different archives, each with a different implementation. Nearly all Earth science disciplines are represented in EOSDIS.

2.6 Variability Data latency varies from Near Real Time (within 3-5 hours) to research-scale times (days to weeks time lag). Datasets also vary widely in size from small to multi-terabyte size. (Future radar data will be petabyte-scale.) 


\section{Big Data Science Use Case 2-1}

\subsection{Veracity and Data Quality}

\subsection{Visualization}

\section{Security and Privacy}

\section{Use Case 2-1}

\subsection{Roles}

\subsubsection{Identifying Role System Architect}

4.1.2 Investigator NASA Affiliations 


\section{Security and Privacy Use Case 2-1}

\begin{tabular}{|c|c|}
\hline 4.1.3 Sponsors & NASA Program Executive for Earth Science Data Systems \\
\hline \multicolumn{2}{|l|}{$\begin{array}{l}\text { 4.1.4 Declarations of } \\
\text { Potential Conflicts of } \\
\text { Interest }\end{array}$} \\
\hline \multicolumn{2}{|l|}{$\begin{array}{l}\text { 4.1.5 Institutional S/P } \\
\text { duties }\end{array}$} \\
\hline 4.1.6 Curation & Distributed Active Archive Center Manager \\
\hline \multicolumn{2}{|l|}{$\begin{array}{l}\text { 4.1.7 Classified Data, Code } \\
\text { or Protocols }\end{array}$} \\
\hline Intellectual property protections & Yes \\
\hline $\begin{array}{l}\text { Military classifications, e.g., } \\
\text { FOUO, or Controlled Classified }\end{array}$ & Yes \\
\hline \multicolumn{2}{|l|}{ Not applicable } \\
\hline \multicolumn{2}{|l|}{ Other: } \\
\hline \multicolumn{2}{|l|}{ Other text } \\
\hline \multicolumn{2}{|l|}{$\begin{array}{l}\text { 4.1.8 Multiple Investigators } \\
\text { / Project Leads * }\end{array}$} \\
\hline \multicolumn{2}{|l|}{$\begin{array}{l}\text { Only one investigator | project } \\
\text { lead | developer }\end{array}$} \\
\hline \multicolumn{2}{|l|}{$\begin{array}{l}\text { Multiple team members, but in } \\
\text { the same organization }\end{array}$} \\
\hline $\begin{array}{l}\text { Multiple leads across legal } \\
\text { organizational boundaries }\end{array}$ & Yes \\
\hline \multicolumn{2}{|l|}{$\begin{array}{l}\text { Multinational investigators | } \\
\text { project leads }\end{array}$} \\
\hline \multicolumn{2}{|l|}{ Other: } \\
\hline \multicolumn{2}{|l|}{ Other text } \\
\hline \multicolumn{2}{|l|}{$\begin{array}{l}\text { 4.1.9 Least Privilege Role- } \\
\text { based Access }\end{array}$} \\
\hline $\begin{array}{l}\text { Yes, roles are segregated and } \\
\text { least privilege is enforced }\end{array}$ & Yes \\
\hline \multicolumn{2}{|l|}{$\begin{array}{l}\text { We do have least privilege and } \\
\text { role separation but the admin } \\
\text { role(s) may be too all-inclusion }\end{array}$} \\
\hline \multicolumn{2}{|l|}{$\begin{array}{l}\text { Handled at application provider } \\
\text { level }\end{array}$} \\
\hline \multicolumn{2}{|l|}{$\begin{array}{l}\text { Handled at framework provider } \\
\text { level }\end{array}$} \\
\hline \multicolumn{2}{|l|}{$\begin{array}{l}\text { There is no need for this feature } \\
\text { in our application }\end{array}$} \\
\hline \multicolumn{2}{|l|}{$\begin{array}{l}\text { Could be applicable in production } \\
\text { or future versions of our work }\end{array}$} \\
\hline \multicolumn{2}{|l|}{ Other: } \\
\hline Other text & \\
\hline
\end{tabular}




\section{Security and Privacy \\ Use Case 2-1}

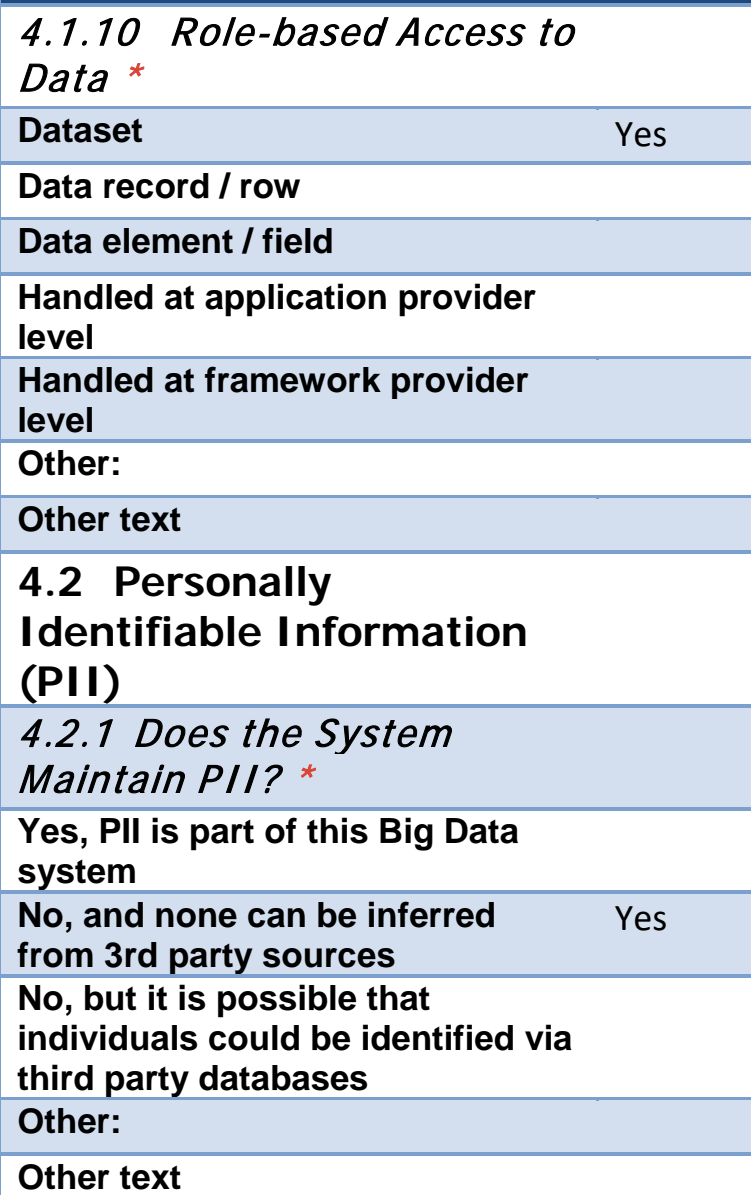

4.2.2 Describe the PII, if applicable

4.2.3 Additional Formal or

Informal Protections for PII

4.2.4 Algorithmic/

Statistical Segmentation of

Human Populations

Yes, doing segmentation, possible discrimination issues if abused. Please also answer the next question.

Yes, doing segmentation, but no foreseeable discrimination

issues.

Does not apply to this use case at Yes

all (e.g., no human subject data)

Other:

Other text 


\section{Security and Privacy}

Use Case 2-1

\subsubsection{Protections afforded statistical / deep learning}

discrimination

\subsection{Covenants, Liability,}

Etc.

4.3.1 Identify any Additional

Security, Compliance,

Regulatory Requirements *

FTC regulations apply

HHS 45 CFR 46

HIPAA

EU General Data Protection

(Reference: http://bit.ly/1Ta8S1C)

COPPA

Other Transborder issues

Fair Credit Reporting Act

(Reference: http://bit.ly/1Ta8XSN

1

Family Educational Rights and

Protection (FERPA)

None apply

$\begin{array}{ll}\text { Other: } & \text { Yes } \\ \text { Other text } & \text { HSPD-12 }\end{array}$

4.3.2 Customer Privacy

Promises

Yes, we're making privacy

promises to customers or

subjects

We are using a notice-and- Yes

consent model

Not applicable

Other:

Other text

\subsection{Ownership, I dentity} and Distribution

\subsubsection{Publication rights}

Open publication Yes

Proprietary

Traditional publisher rights (e.g.,

Springer, Elsevier, IEEE)

"Big Science" tools in use 


\section{Security and Privacy \\ Use Case 2-1}

Other:

Other text

4.4.2 Chain of Trust

4.4.3 Delegated Rights

4.4.4 Software License

Restrictions

Patents are applicable in some cases. Off-the-shelf commercial analysis packages are also used. Software which has not passed through NASA Software Release process is not eligible for public distribution.

\subsubsection{Results Repository PubMed Central (PMC)}

\subsubsection{Restrictions on} Discovery

\subsubsection{Privacy Notices}

Privacy notices apply

Privacy notices do not apply Yes

Other:

Other text

\subsubsection{Key Management}

A key management scheme is part of our system

We are using public key Yes

infrastructure.

We do not use key management,

but it could have been useful

No readily identifiable use for key

management

Other:

Other text

\subsubsection{Describe and Key}

Management Practices

4.4.10 Is an identity

framework used?

A framework is in place. (See Yes next question.)

Not currently using a framework.

There is no perceived need for an

identity framework.

Other:

Other text

\subsubsection{CAC/ ECA Cards or}

Other Enterprise-wide

Framework 


\section{Security and Privacy}

\section{Use Case 2-1}

Using an externally maintained Yes

enterprise-wide identity

framework

Could be used, but none are

available

Not applicable

4.4.12 Describe the

I dentity Framework.

4.4.13 How is intellectual

property protected?

Login screens advising of IP

issues

Employee or team training

Official guidelines limiting access

or distribution

Required to track all access to, distribution of digital assets

Does not apply to this effort (e.g., Yes

public effort)

Other:

Other text

\subsection{Risk Mitigation}

\subsubsection{Are measures in place} to deter re-identification? *

Yes, in place

Not in place, but such measures

do apply

Not applicable

Yes

Other:

Other text

4.5.2 Please describe any re-identification deterrents in place

4.5.3 Are data segmentation

practices being used?

Yes, being used

Not in use, but does apply

Not applicable Yes

Other:

Other text

4.5.4 Is there an explicit governance plan or

framework for the effort? 


\section{Security and Privacy}

\section{Use Case 2-1}

Explicit governance plan Yes

No governance plan, but could

use one

I don't think governance

contributes anything to this

project

Other:

Other text

4.5.5 Privacy-Preserving Practices
A privacy assessment is performed for each new publicly accessible NASA system and tracked in a NASA-wide database.

4.5.6 Do you foresee any potential risks from public or private open data projects?

Risks are known.

Currently no known risks, but it is conceivable.

Not sure

Unlikely that this will ever be an issue (e.g., no PII, human-agent related data or subsystems.)

Other:

Other text

\subsection{Provenance}

(Ownership)

\subsubsection{Describe your} metadata management

practices

Yes, we have a metadata management system.

There is no need for a metadata management system in this use

case

It is applicable but we do not

currently have one.

Other:

Other text

\subsubsection{If a metadata}

management system is

present, what measures are

in place to verify and protect

its integrity?

4.6.3 Describe provenance as related to 


\section{Security and Privacy \\ Use Case 2-1 \\ instrumentation, sensors or \\ other devices.}

We have potential machine-tomachine traffic provenance

concerns.

Endpoint sensors or instruments

have signatures periodically

updated

Using hardware or software

methods, we detect and

remediate outlier signatures

Endpoint signature detection and

upstream flow are built into

system processing

We rely on third party vendors to

manage endpoint integrity

We use a sampling method to

verify endpoint integrity

Not a concern at this time Yes

Other:

Other text

\subsection{Data Life Cycle}

\subsubsection{Describe Archive} Processes

Our application has no separate

"archive" process

We offload data using certain

criteria to removable media which

are taken offline

we use a multi-stage, tiered Yes

archive process

We allow for "forgetting" of

individual PII on request

Have ability to track individual Yes

data elements across all stages

of processing, including archive

Additional protections, such as

separate encryption, are applied

to archival data

Archived data is saved for

potential later use by applications

or analytics yet to be built

Does not apply to our application

Other:

Other text 


\section{Security and Privacy \\ Use Case 2-1}

\subsubsection{Describe Point in Time and Other Dependency}

Issues

Some data is valid only within a point in time,

Some data is only valid with

other, related data is available or

applicable, such as the existence

of a building, the presence of a

weather event, or the active use

of a vehicle

There are specific events in the

application that render certain

data obsolete or unusable

Point and Time and related

dependencies do not apply

Other:

Other text

\subsubsection{Compliance with}

Secure Data Disposal

Requirements

We are required to destroy or

otherwise dispose of data

Does not apply to us Yes

Not sure

Other:

Other text

\subsection{Audit and}

Traceability

4.8.1 Current audit needs *

We have third party registrar or Yes

other audits, such as for ISO 9001

We have internal enterprise audit Yes

requirements

Audit is only for system health or other management requirements

No audit, not needed or does not

apply

Other:

Other text

\subsubsection{Auditing versus} Monitoring

We rely on third-party or O.S. Yes

tools to audit, e.g., Windows or

Linux auditing 


\section{Security and Privacy}

\section{Use Case 2-1}

There are built-in tools for Yes monitoring or logging that are only used for system or application health monitoring Monitoring services include logging of role-based access to assets such as PII or other resources

The same individual(s) in the enterprise are responsible for auditing as for monitoring

This aspect of our application is still in flux

Does not apply to our setting

Other:

Other text

\subsubsection{System Health Tools}

We rely on system-wide tools for Yes health monitoring

We built application health tools Yes specifically to address integrity, performance monitoring and related concerns

There is no need in our setting

Other:

Other text

\subsubsection{What events are} currently audited? *

All data access must be audited

Only selected $/$ protected data Yes must be audited

Maintenance on user roles must Yes be audited (new users, disabled user, updated roles or permissions)

Purge and archive events

Domain-dependent events (e.g., adding a new sensor)

REST or SOAP events

Changes in system configuration Yes

Organizational changes

External project ownership I management changes

Requirements are externally set, e.g., by $\mathrm{PCl}$ compliance 


\section{Security and Privacy}

Use Case 2-1

Domain-specific events (patient

death in a drug trial)

Other:

Other text

\subsection{Application Provider}

Security

4.9.1 Describe Application

Provider Security*

There is a security mechanism

implemented at the application

level

The app provider level is aware of

PII or privacy data elements

The app provider implements

audit and logging

The app provider security relies

on framework-level security for

its operation

Does not apply to our application Yes

Other:

Other text

\subsection{Framework Provider}

Security

4.10.1 Describe the

framework provider security

*

Security is implemented at the

framework level

Roles can be defined at the

framework level

The framework level is aware of

PII or related sensitive data

Does not apply in our setting Yes

Is provided by the Big Data tool

Other:

Other text

\subsection{System Health}

\subsubsection{Measures to}

Ensure Availability *

Deterrents to man-in-the-middle

attacks

Deterrents to denial of service

attacks 


\section{Security and Privacy \\ Use Case 2-1}

Replication, redundancy or other

resilience measures

Deterrents to data corruption, drops or other critical big data components

Other:

Other text

4.12 Permitted Use Cases

4.12.1 Describe

Domain-specific Limitations

on Use

4.12.2 Paywall

A paywall is in use at some stage

in the workflow

Not applicable

\section{Classify Use Cases with Tags}

Use Case 2-1

\subsection{DATA: Application}

Style and Data sharing

and acquisition

Uses Geographical Information Yes

Systems?

Use case involves Internet of

Things?

Data comes from HPC or other Yes

simulations?

Data Fusion important? Yes

Data is Real time Streaming?

Data is Batched Streaming (e.g. Yes collected remotely and uploaded every so often)?

Important Data is in a Permanent Yes

Repository (Not streamed)?

Transient Data important? Yes

Permanent Data Important? Yes

Data shared between different Yes

applications/users?

Data largely dedicated to only

this use case?

5.2 DATA: Management and Storage 


\section{Classify Use Cases with Tags}

\section{Use Case 2-1}

\begin{tabular}{|c|c|}
\hline $\begin{array}{l}\text { Application data system based } \\
\text { on Files? }\end{array}$ & Yes \\
\hline $\begin{array}{l}\text { Application data system based } \\
\text { on Objects? }\end{array}$ & \\
\hline Uses HDFS style File System? & \\
\hline $\begin{array}{l}\text { Uses Wide area File System like } \\
\text { Lustre? }\end{array}$ & \\
\hline $\begin{array}{l}\text { Uses HPC parallel file system like } \\
\text { GPFS? }\end{array}$ & \\
\hline Uses SQL? & Yes \\
\hline Uses NoSQL? & Yes \\
\hline Uses NewSQL? & \\
\hline
\end{tabular}

\subsection{DATA: Describe} Other Data Acquisition/ Access/ Sharing/ Management/ Storage I ssues

\subsection{ANALYTI CS: Data}

Format and Nature of Algorithm used in Analytics

\begin{tabular}{l}
\hline Data regular? \\
\hline Data dynamic? \\
\hline Algorithm O(N^2) ? \\
\hline $\begin{array}{l}\text { Basic statistics (regression, } \\
\text { moments) used? }\end{array}$ \\
\hline $\begin{array}{l}\text { Search/Query/Index of } \\
\text { application data Important? }\end{array}$ \\
\hline Classification of data Important? \\
\hline Recommender Engine Used? \\
\hline Clustering algorithms used? \\
\hline Alignment algorithms used? \\
\hline $\begin{array}{l}\text { (Deep) Learning algorithms } \\
\text { used? }\end{array}$ \\
\hline Graph Analytics Used? \\
\hline
\end{tabular}

\subsection{ANALYTICS:} Describe Other Data Analytics Used

\subsection{PROGRAMMI NG MODEL}




\section{Classify Use Cases with Tags}

\section{Use Case 2-1}

Pleasingly parallel Structure? Yes

Parallel execution over

independent data. Called Many

Task or high throughput

computing. MapReduce with only

Map and no Reduce of this type

Use case NOT Pleasingly Parallel

-- Parallelism involves linkage

between tasks. MapReduce (with

Map and Reduce) of this type

Uses Classic MapReduce? such

as Hadoop

Uses Apache Spark or similar Yes

Iterative MapReduce?

Uses Graph processing as in

Apache Giraph?

Uses MPI (HPC Communication)

and/or Bulk Synchronous

Processing BSP?

Dataflow Programming Model

used?

Workflow or Orchestration Yes

software used?

Python or Scripting front ends Yes

used? Maybe used for

orchestration

Shared memory architectures

important?

Event-based Programming Model

used?

Agent-based Programming Model

used?

Use case I/O dominated? I/O time Yes

$>$ or $>$ Compute time

Use case involves little $/ / O$ ?

Compute $\gg 1 / 0$

5.7 Other Programming

Model Tags

5.8 Please Estimate

Ratio I/ O Bytes/ Flops

5.9 Describe Memory

Size or Access issues 


\begin{tabular}{|ll|}
$\begin{array}{l}\text { 6.1 Other Big Data } \\
\text { I ssues }\end{array}$ & $\begin{array}{l}\text { Currently, the Variety in Big Data is producing a set of data } \\
\text { discovery issues for the end users. Searching for datasets turns } \\
\text { out to be different from searching for documents in a variety of } \\
\text { subtle, but important, ways. }\end{array}$ \\
\hline $\begin{array}{l}\text { 6.2 User I nterface and } \\
\text { Mobile Access I ssues }\end{array}$ & \\
\hline $\begin{array}{l}\text { 6.3 List Key Features } \\
\text { and Related Use Cases }\end{array}$ & $\begin{array}{l}\text { More data will be stored in the cloud, likely with copies in } \\
\text { some cases of reorganized data in order to make them more } \\
\text { tractable to data-parallel algorithms. More analysis support will } \\
\text { also be offered to users that want to run analyses of data n the } \\
\text { cloud. }\end{array}$ \\
\hline
\end{tabular}

\section{Workflow Processes}

Use Case 2-1

7.1 Please comment on workflow processes

\subsection{Workflow details for each stage *}

\subsubsection{Workflow Details for}

Stage 1

Stage 1 Name

Data Source(s)

Nature of Data

Software Used

Data Analytics

Infrastructure

Percentage of Use Case Effort

Other Comments

\subsubsection{Workflow Details for}

\section{Stage 2}

Stage 2 Name

Data Source(s)

Nature of Data

Software Used

Level 0 Processing

Satellite Data Processing commonly goes through the following processing steps: Level 0 - raw data in files, de-duplicatedLevel 1 - calibrated data with geolocation Level 2 - inferred geophysical measurements, in sensor coordinates Level 3 geophysical measurements Level 4 - model output (usually done outside EOSDIS)The characteristics of the data, especially their geolocations vary significantly from $L 0$ to $L 1$, and from $L 2$ to $L 3$. The usability to various audiences crosses a significant border between $\mathrm{L} 1$ and $\mathrm{L} 2$. 


\begin{tabular}{|c|c|}
\hline Data Analytics & Geolocation and calibration of raw data \\
\hline Infrastructure & Multiple local servers \\
\hline \multicolumn{2}{|l|}{ Percentage of Use Case Effort } \\
\hline \multicolumn{2}{|l|}{ Other Comments } \\
\hline \multicolumn{2}{|l|}{$\begin{array}{l}\text { 7.2.3 Workflow Details for } \\
\text { Stage } 3\end{array}$} \\
\hline Stage 3 Name & Level 2 Processing \\
\hline Data Source(s) & Level 1B processing system \\
\hline Nature of Data & Level 1B geolocated, calibrated data \\
\hline Software Used & Scientist-authored physical retrieval code \\
\hline Data Analytics & $\begin{array}{l}\text { Transform calibrated data (radiances, waveforms, ...) into } \\
\text { geophysical measurements }\end{array}$ \\
\hline Infrastructure & Large compute clusters \\
\hline \multicolumn{2}{|l|}{ Percentage of Use Case Effort } \\
\hline \multicolumn{2}{|l|}{ Other Comments } \\
\hline \multicolumn{2}{|l|}{$\begin{array}{l}\text { 7.2.4 Workflow Details for } \\
\text { Stage } 4\end{array}$} \\
\hline Stage 4 Name & Level 3 Processing \\
\hline Data Source(s) & Level 2 Processor \\
\hline Nature of Data & Geophysical variables in sensor coordinates \\
\hline Software Used & Scientist-authored gridding code \\
\hline Data Analytics & Data projection and aggregation over space and/or time \\
\hline Infrastructure & Compute clusters with large amounts of disk space \\
\hline \multicolumn{2}{|l|}{ Percentage of Use Case Effort } \\
\hline \multicolumn{2}{|l|}{ Other Comments } \\
\hline \multicolumn{2}{|l|}{$\begin{array}{l}\text { 7.2.5 Workflow Details for } \\
\text { Stages } 5 \text { and any further } \\
\text { stages }\end{array}$} \\
\hline \multicolumn{2}{|l|}{ Stage 5 Name } \\
\hline \multicolumn{2}{|l|}{ Data Source(s) } \\
\hline \multicolumn{2}{|l|}{ Nature of Data } \\
\hline \multicolumn{2}{|l|}{ Software Used } \\
\hline \multicolumn{2}{|l|}{ Data Analytics } \\
\hline \multicolumn{2}{|l|}{ Infrastructure } \\
\hline \multicolumn{2}{|l|}{ Percentage of Use Case Effort } \\
\hline Other Comments & \\
\hline
\end{tabular}




\section{F.2 Use Case 2-2: Web-Enabled Landsat Data (WELD) Processing}

\section{Overall Project Description}

\section{Use Case 2-2}

\subsection{Use Case Title * Web-Enabled Landsat Data (WELD) Processing \\ 1.2 Use Case The use case is specific to the part of the project where data is Description * available on the HPC platform and processed through the science workflow. It is a 32-stage processing pipeline that includes two separate science products (Top-of-the-Atmosphere (TOA) reflectances and surface reflectances) as well as QA and visualization components.}

\subsection{Use Case Contacts *}

\begin{tabular}{|c|c|}
\hline & Andrew Michaelis \\
\hline & Author \\
\hline & Yes \\
\hline & Yes \\
\hline $\begin{array}{l}1.4 \text { Domain } \\
\text { ("Vertical") * }\end{array}$ & Land use science: image processing \\
\hline 1.5 Application * & $\begin{array}{l}\text { The product of this use case is a dataset of science products of use to } \\
\text { the land surface science community that is made freely available by } \\
\text { NASA. The dataset is produced through processing of images from } \\
\text { the Landsat } 4,5 \text {, and } 7 \text { satellites. }\end{array}$ \\
\hline $\begin{array}{l}1.6 \text { Current Data } \\
\text { Analysis Approach * }\end{array}$ & $\begin{array}{l}\text { > Compute System: Shared High Performance Computing (HPC) } \\
\text { system at NASA Ames Research Center (Pleiades) } \\
\text { > Storage: NASA Earth Exchange (NEX) NFS storage system for read- } \\
\text { only data storage (2.5PB), Lustre for read-write access during } \\
\text { processing (1PB), tape for near-line storage (5OPB) } \\
\text { >> Networking: InfiniBand partial hypercube internal interconnect } \\
\text { within the HPC system; } 1 \mathrm{G} \text { to } 10 \mathrm{G} \text { connection to external data } \\
\text { providers } \\
\text { >> Software: NEX science platform - data management, workflow } \\
\text { processing, provenance capture; WELD science processing algorithms } \\
\text { from South Dakota State University (SDSU), browse visualization, and } \\
\text { time-series code; Global Imagery Browse Service (GIBS) data } \\
\text { visualization platform; USGS data distribution platform. Custom-built } \\
\text { application and libraries built on top of open-source libraries. }\end{array}$ \\
\hline $\begin{array}{l}1.7 \text { Future of } \\
\text { Application and } \\
\text { Approach * }\end{array}$ & $\begin{array}{l}\text { Processing will be improved with newer and updated algorithms. } \\
\text { This process may also be applied to future datasets and processing } \\
\text { systems (Landsat } 8 \text { and Sentinel-2 satellites, for example) }\end{array}$ \\
\hline
\end{tabular}




\section{Overall Project Description Use Case 2-2}

\subsection{Actors / Stakeholders}

\subsection{Project Goals or Objectives}

South Dakota State University - science, algorithm development, QA, data browse visualization and distribution framework; NASA Advanced Supercomputing Division at NASA Ames Research Center data processing at scale; USGS - data source and data distribution; NASA GIBS - native resolution data visualization; NASA HQ and NASA EOSDIS - sponsor.

The WELD products are developed specifically to provide consistent data that can be used to derive land cover as well as geophysical and biophysical products for assessment of surface dynamics and to study Earth system functioning. The WELD products are free and are available via the Internet. The WELD products are processed so that users do not need to apply the equations and spectral calibration coefficients and solar information to convert the Landsat digital numbers to reflectance and brightness temperature, and successive products are defined in the same coordinate system and align precisely, making them simple to use for multi-temporal applications.

1.10 Use Case URL(s) http://globalmonitoring.sdstate.edu/projects/weldglobal/gweld.html http://globalweld.cr.usgs.gov/ https://nex.nasa.gov http://www.nas.nasa.gov/hecc/resources/pleiades.html https://earthdata.nasa.gov/about/science-systemdescription/eosdis-components/global-imagery-browse-services-gibs https://worldview.earthdata.nasa.gov/

\section{Big Data Characteristics}

Use Case 2-2

2.1 Data Source Satellite Earth observation data from Landsat 4, 5, and 7 missions. The data source is remote and centralized - distributed from USGS EROS Center.

2.2 Data Destination centralized data system. It is also available on the NEX platform for further analysis and product development.

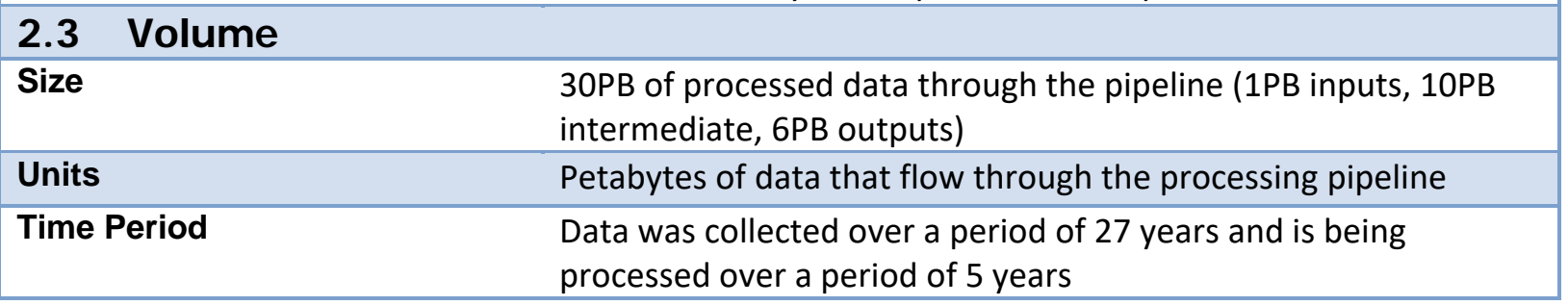




\begin{tabular}{|c|c|}
\hline Proviso & $\begin{array}{l}\text { The data represent the operational time period of } 1984 \text { to } 2011 \\
\text { for the Landsat 4, 5, and } 7 \text { satellites }\end{array}$ \\
\hline 2.4 Velocity & \\
\hline Unit of measure & $\begin{array}{l}\text { Terabytes processed per day during processing time periods: } 150 \\
\text { TB/day }\end{array}$ \\
\hline Time Period & 24 hours \\
\hline Proviso & $\begin{array}{l}\text { Based on programmatic goals of processing several iterations of } \\
\text { the final product over the span of the project. Observed run-time } \\
\text { and volumes during processing }\end{array}$ \\
\hline Variety & This use case basically deals with a single dataset. \\
\hline 2.6 Variability & $\begin{array}{l}\text { Not clear what the difference is between variability and variety. } \\
\text { This use case basically deals with a single dataset. }\end{array}$ \\
\hline
\end{tabular}

\section{Big Data Science} Use Case 2-2

3.1 Veracity and Data Quality

3.2 Visualization
This data dealt with in this use case are a high-quality, curated dataset.

Visualization is not used in this use case per se, but visualization is important in QA processes conducted outside of the use case as well as in the ultimate use by scientists of the product datasets that result from this use case

\subsection{Data Types structured image data}

3.4 Metadata Metadata adhere to accepted metadata standards widely used in the earth science imagery field.

3.5 Curation and Governance

Data is governed by NASA data release policy; data is referred to by the DOI and the algorithms have been peer-reviewed. The data distribution center and the $\mathrm{PI}$ are responsible for science data support.

\subsection{Data Analytics}

There are number of analytics processes throughout the processing pipeline. The key analytics is identifying best available pixels for spatio-temporal composition and spatial aggregation processes as a part of the overall QA. The analytics algorithms are custom developed for this use case. 


\section{Security and Privacy Use Case 2-2}

\subsection{Roles}

\begin{tabular}{|c|c|}
\hline 4.1.1 Identifying Role & PI; Project sponsor (NASA EOSDIS program) \\
\hline $\begin{array}{l}\text { 4.1.2 Investigator } \\
\text { Affiliations }\end{array}$ & $\begin{array}{l}\text { Andrew Michaelis, NASA, NEX Processing Pipeline Development } \\
\text { and Operations } \\
\text { David Roy, South Dakota State University, Project PI } \\
\text { Hankui Zhang, South Dakota State University, Science Algorithm } \\
\text { Development } \\
\text { Adam Dosch, South Dakota State University, SDSU } \\
\text { operations/data management } \\
\text { Lisa Johnson, USGS, Data Distribution } \\
\text { Matthew Cechini, Ryan Boller, Kevin Murphy, NASA, GIBS } \\
\text { project }\end{array}$ \\
\hline 4.1.3 Sponsors & NASA EOSDIS project \\
\hline $\begin{array}{l}\text { 4.1.4 Declarations of } \\
\text { Potential Conflicts of } \\
\text { Interest }\end{array}$ & None \\
\hline $\begin{array}{l}\text { 4.1.5 Institutional S/P } \\
\text { duties }\end{array}$ & None \\
\hline 4.1.6 Curation & Joint responsibility of NASA, USGS, and Principal Investigator \\
\hline \multicolumn{2}{|l|}{$\begin{array}{l}\text { 4.1.7 Classified Data, Code } \\
\text { or Protocols }\end{array}$} \\
\hline Intellectual property protections & Off \\
\hline $\begin{array}{l}\text { Military classifications, e.g., } \\
\text { FOUO, or Controlled Classified }\end{array}$ & Off \\
\hline Not applicable & Yes \\
\hline Other: & Off \\
\hline \multicolumn{2}{|l|}{ Other text } \\
\hline \multicolumn{2}{|l|}{$\begin{array}{l}\text { 4.1.8 Multiple Investigators } \\
\text { / Project Leads * }\end{array}$} \\
\hline $\begin{array}{l}\text { Only one investigator | project } \\
\text { lead | developer }\end{array}$ & Off \\
\hline $\begin{array}{l}\text { Multiple team members, but in } \\
\text { the same organization }\end{array}$ & Off \\
\hline $\begin{array}{l}\text { Multiple leads across legal } \\
\text { organizational boundaries }\end{array}$ & Yes \\
\hline $\begin{array}{l}\text { Multinational investigators I } \\
\text { project leads }\end{array}$ & Off \\
\hline Other: & Off \\
\hline \multicolumn{2}{|l|}{ Other text } \\
\hline $\begin{array}{l}\text { 4.1.9 Least Privilege Role- } \\
\text { based Access }\end{array}$ & \\
\hline
\end{tabular}




\section{Security and Privacy Use Case 2-2}

\begin{tabular}{|c|c|}
\hline $\begin{array}{l}\text { Yes, roles are segregated and } \\
\text { least privilege is enforced }\end{array}$ & Off \\
\hline $\begin{array}{l}\text { We do have least privilege and } \\
\text { role separation but the admin } \\
\text { role(s) may be too all-inclusion }\end{array}$ & Off \\
\hline $\begin{array}{l}\text { Handled at application provider } \\
\text { level }\end{array}$ & Off \\
\hline $\begin{array}{l}\text { Handled at framework provider } \\
\text { level }\end{array}$ & Off \\
\hline $\begin{array}{l}\text { There is no need for this feature } \\
\text { in our application }\end{array}$ & Off \\
\hline $\begin{array}{l}\text { Could be applicable in } \\
\text { production or future versions of } \\
\text { our work }\end{array}$ & Off \\
\hline Other: & Yes \\
\hline Other text & Not used \\
\hline $\begin{array}{l}\text { 4.1.10 Role-based } \\
\text { Access to Data * }\end{array}$ & \\
\hline Dataset & Yes \\
\hline Data record / row & Off \\
\hline Data element / field & Off \\
\hline $\begin{array}{l}\text { Handled at application provider } \\
\text { level }\end{array}$ & Off \\
\hline $\begin{array}{l}\text { Handled at framework provider } \\
\text { level }\end{array}$ & Off \\
\hline Other: & Off \\
\hline \multicolumn{2}{|l|}{ Other text } \\
\hline \multicolumn{2}{|l|}{$\begin{array}{l}4.2 \text { Personally } \\
\text { I dentifiable I nformation } \\
\text { (PI I ) }\end{array}$} \\
\hline \multicolumn{2}{|l|}{$\begin{array}{l}\text { 4.2.1 Does the System } \\
\text { Maintain PII? * }\end{array}$} \\
\hline $\begin{array}{l}\text { Yes, PII is part of this Big Data } \\
\text { system }\end{array}$ & Off \\
\hline $\begin{array}{l}\text { No, and none can be inferred } \\
\text { from 3rd party sources }\end{array}$ & Yes \\
\hline $\begin{array}{l}\text { No, but it is possible that } \\
\text { individuals could be identified } \\
\text { via third party databases }\end{array}$ & Off \\
\hline Other: & Off \\
\hline \multicolumn{2}{|l|}{ Other text } \\
\hline $\begin{array}{l}\text { 4.2.2 Describe the PII, if } \\
\text { applicable }\end{array}$ & \\
\hline
\end{tabular}




\section{Security and Privacy \\ Use Case 2-2}

\subsubsection{Additional Formal or \\ Informal Protections for PII \\ 4.2.4 Algorithmic / \\ Statistical Segmentation of \\ Human Populations}

Yes, doing segmentation, possible discrimination issues if

Off

abused. Please also answer the

next question.

Yes, doing segmentation, but no Off

foreseeable discrimination

issues.

Does not apply to this use case Yes

at all (e.g., no human subject

data)

Other:

Off

Other text

4.2.5 Protections afforded Not applicable to this use case. statistical / deep learning

discrimination

\subsection{Covenants, Liability,}

Etc.

4.3.1 Identify any

Additional Security,

Compliance, Regulatory

Requirements *

FTC regulations apply Off

HHS 45 CFR 46

HIPAA Off

EU General Data Protection Off

(Reference: http://bit.ly/1Ta8S1C

1

COPPA Off

Other Transborder issues Off

Fair Credit Reporting Act Off

(Reference: http://bit.ly/1Ta8XSN

1

Family Educational Rights and Off

Protection (FERPA)

None apply Yes

Other: Off

Other text 


\section{Security and Privacy Use Case 2-2}

\begin{tabular}{|c|c|}
\hline $\begin{array}{l}\text { Yes, we're making privacy } \\
\text { promises to customers or } \\
\text { subjects }\end{array}$ & Off \\
\hline $\begin{array}{l}\text { We are using a notice-and- } \\
\text { consent model }\end{array}$ & Off \\
\hline Not applicable & Yes \\
\hline Other: & Off \\
\hline
\end{tabular}

\subsection{Ownership, I dentity} and Distribution

\subsubsection{Publication rights}

Open publication Off

Proprietary Off

Traditional publisher rights (e.g., Off

Springer, Elsevier, IEEE)

"Big Science" tools in use Off

Other: Yes

Other text Datasets produced are available to the public with a requirement for appropriate citation when used.

\begin{tabular}{|ll|}
\hline 4.4.2 Chain of Trust & None \\
\hline 4.4.3 Delegated Rights & None \\
\hline $\begin{array}{l}\text { 4.4.4 Software License } \\
\text { Restrictions }\end{array}$ & None \\
\hline
\end{tabular}

4.4.5 Results Repository The datasets produced from this dataset are distributed to the public from repositories at the USGS EROS Center and the NASA EOSDIS program.

\subsubsection{Restrictions on None} Discovery

\subsubsection{Privacy Notices}

\begin{tabular}{|ll}
\hline Privacy notices apply & Off \\
\hline Privacy notices do not apply & Yes \\
\hline Other: & Off \\
\hline
\end{tabular}

Other text

4.4.8 Key Management

A key management scheme is Off part of our system

We are using public key Off
infrastructure. 


\section{Security and Privacy Use Case 2-2}

\begin{tabular}{l}
$\begin{array}{l}\text { We do not use key management, Off } \\
\text { but it could have been useful }\end{array}$ \\
$\begin{array}{l}\text { No readily identifiable use for } \\
\text { key management }\end{array}$ \\
\hline Other:
\end{tabular}

\subsubsection{Describe and Key Management Practices}

4.4.10 Is an identity framework used?

A framework is in place. (See Off next question.)

Not currently using a Off

framework.

There is no perceived need for Yes an identity framework.

Other: Off

Other text

\subsubsection{CAC/ ECA Cards} or Other Enterprise-wide

Framework

Using an externally maintained Off enterprise-wide identity

framework

Could be used, but none are Off

available

Not applicable Yes

\subsubsection{Describe the} Identity Framework.

\subsubsection{How is} intellectual property

protected?

\begin{tabular}{|ll|}
\hline $\begin{array}{l}\text { Login screens advising of IP } \\
\text { issues }\end{array}$ & Off \\
\hline Employee or team training & Off \\
\hline $\begin{array}{l}\text { Official guidelines limiting } \\
\text { access or distribution }\end{array}$ & Off \\
\hline $\begin{array}{l}\text { Required to track all access to, } \\
\text { distribution of digital assets }\end{array}$ & Off \\
\hline $\begin{array}{l}\text { Does not apply to this effort } \\
\text { (e.g., public effort) }\end{array}$ & Off \\
\hline Other: & Yes \\
\hline
\end{tabular}




\section{Security and Privacy} Use Case 2-2

Other text

Believe there are standards for citation of datasets that apply to use of the datasets from the USGS or NASA repositories.

\subsection{Risk Mitigation}

\begin{tabular}{ll}
$\begin{array}{l}\text { 4.5.1 Are measures in place } \\
\text { to deter re-identification? }\end{array}$ & \\
\hline Yes, in place & Off \\
\hline $\begin{array}{l}\text { Not in place, but such measures } \\
\text { do apply }\end{array}$ & Off \\
\hline Not applicable & Yes \\
\hline Other: & Off \\
\hline Other text & \\
\hline
\end{tabular}

4.5.2 Please describe any re-identification deterrents in place

\begin{tabular}{|c|c|}
\hline $\begin{array}{l}\text { 4.5.3 Are data } \\
\text { segmentation practices } \\
\text { being used? }\end{array}$ & \\
\hline Yes, being used & Off \\
\hline Not in use, but does apply & Off \\
\hline Not applicable & Yes \\
\hline Other: & Off \\
\hline
\end{tabular}

4.5.4 Is there an explicit governance plan or framework for the effort?

\begin{tabular}{ll|}
\hline $\begin{array}{l}\text { Explicit governance plan } \\
\text { No governance plan, but could } \\
\text { use one }\end{array}$ & Off \\
\hline $\begin{array}{l}\text { I don't think governance } \\
\text { contributes anything to this } \\
\text { project }\end{array}$ & Off \\
\hline Other: & Yes \\
\hline Other text & $\begin{array}{l}\text { Resulting datasets are governed by the data access policies of } \\
\text { the USGS and NASA. }\end{array}$ \\
\hline $\begin{array}{l}\text { 4.5.5 Privacy-Preserving } \\
\text { Practices }\end{array}$ & None \\
\hline $\begin{array}{l}\text { 4.5.6 Do you foresee any } \\
\text { potential risks from public } \\
\text { or private open data } \\
\text { projects? }\end{array}$ & \\
\hline \begin{tabular}{l} 
Risks are known. \\
\hline
\end{tabular} & Off \\
\hline
\end{tabular}




\section{Security and Privacy} Use Case 2-2

\begin{tabular}{|l|l|}
\hline $\begin{array}{l}\text { Currently no known risks, but it } \\
\text { is conceivable. }\end{array}$ & Off \\
\hline Not sure & Yes \\
\hline $\begin{array}{l}\text { Unlikely that this will ever be an } \\
\text { issue (e.g., no PII, human-agent } \\
\text { related data or subsystems.) }\end{array}$ \\
\hline Other: & Off \\
\hline Other text & \\
\hline
\end{tabular}

\subsection{Provenance (Ownership)}

\subsubsection{Describe your} metadata management practices

Yes, we have a metadata Off management system.

\begin{tabular}{ll}
$\begin{array}{l}\text { There is no need for a metadata } \\
\text { management system in this use } \\
\text { case }\end{array}$ & Off \\
\hline $\begin{array}{l}\text { It is applicable but we do not } \\
\text { currently have one. }\end{array}$ & Off \\
\hline Other: & Yes \\
\hline Other text & $\begin{array}{l}\text { There is no metadata management system within this use case, } \\
\text { but the resultant datasets' metadata is managed as NASA } \\
\text { EOSDIS datasets. }\end{array}$ \\
\end{tabular}

\subsubsection{If a metadata}

management system is

present, what measures are

in place to verify and

protect its integrity?

\subsubsection{Describe provenance \\ as related to \\ instrumentation, sensors or \\ other devices.}

We have potential machine-to- Off

machine traffic provenance

concerns.

Endpoint sensors or

Off

instruments have signatures

periodically updated

Using hardware or software Off

methods, we detect and

remediate outlier signatures

Endpoint signature detection and upstream flow are built into system processing 


\section{Security and Privacy Use Case 2-2}

\begin{tabular}{|ll|}
$\begin{array}{l}\text { We rely on third party vendors } \\
\text { to manage endpoint integrity }\end{array}$ & Off \\
\hline $\begin{array}{l}\text { We use a sampling method to } \\
\text { verify endpoint integrity }\end{array}$ & Off \\
\hline Not a concern at this time & Off \\
\hline Other: & Off \\
\hline Other text & \\
\hline
\end{tabular}

\subsection{Data Life Cycle}

\subsubsection{Describe Archive Processes}

Our application has no separate Off "archive" process

\begin{tabular}{ll|}
$\begin{array}{l}\text { We offload data using certain } \\
\text { criteria to removable media } \\
\text { which are taken offline }\end{array}$ & Off \\
\hline $\begin{array}{l}\text { we use a multi-stage, tiered } \\
\text { archive process }\end{array}$ & Off \\
\hline $\begin{array}{l}\text { We allow for "forgetting" of } \\
\text { individual PII on request }\end{array}$ & Off \\
\hline $\begin{array}{l}\text { Have ability to track individual } \\
\text { data elements across all stages } \\
\text { of processing, including archive }\end{array}$ & Off \\
\hline $\begin{array}{l}\text { Additional protections, such as } \\
\text { separate encryption, are applied } \\
\text { to archival data }\end{array}$ & Off \\
\hline $\begin{array}{l}\text { Archived data is saved for } \\
\text { potential later use by } \\
\text { applications or analytics yet to } \\
\text { be built }\end{array}$ & Off \\
\hline $\begin{array}{l}\text { Does not apply to our } \\
\text { application }\end{array}$ & Off \\
\hline $\begin{array}{ll}\text { Other: } \\
\text { Other text }\end{array}$ & Yes \\
\hline
\end{tabular}

\subsubsection{Describe Point in}

Time and Other

Dependency / ssues

Some data is valid only within a Off point in time,

Some data is only valid with Off other, related data is available or applicable, such as the existence of a building, the presence of a weather event, or the active use of a vehicle 


\section{Security and Privacy \\ Use Case 2-2}

There are specific events in the application that render certain data obsolete or unusable

Point and Time and related Off dependencies do not apply

Other:

Off

Other text

Yes

Data are relevant and valid independent of when

accessed/used, but all data have a specific date/time/location

reference that is part of the metadata.

4.7.3 Compliance with

Secure Data Disposal

Requirements

We are required to destroy or Off

otherwise dispose of data

Does not apply to us Yes

Not sure Off

Other: Off

Other text

4.8 Audit and

Traceability

4.8.1 Current audit needs *

We have third party registrar or Off

other audits, such as for ISO

9001

We have internal enterprise Off

audit requirements

Audit is only for system health Off or other management

requirements

No audit, not needed or does Yes

not apply

Other:

Off

Other text

4.8.2 Auditing versus

Monitoring

We rely on third party or O.S. Off

tools to audit, e.g., Windows or

Linux auditing

There are built-in tools for

Off

monitoring or logging that are

only used for system or

application health monitoring 


\section{Security and Privacy} Use Case 2-2

\begin{tabular}{|c|c|}
\hline $\begin{array}{l}\text { Monitoring services include } \\
\text { logging of role-based access to } \\
\text { assets such as PII or other } \\
\text { resources }\end{array}$ & Off \\
\hline $\begin{array}{l}\text { The same individual(s) in the } \\
\text { enterprise are responsible for } \\
\text { auditing as for monitoring }\end{array}$ & Off \\
\hline $\begin{array}{l}\text { This aspect of our application is } \\
\text { still in flux }\end{array}$ & Off \\
\hline Does not apply to our setting & Yes \\
\hline Other: & Off \\
\hline
\end{tabular}

\subsubsection{System Health Tools}

We rely on system-wide tools Off for health monitoring

We built application health tools Off specifically to address integrity, performance monitoring and related concerns

\begin{tabular}{ll} 
There is no need in our setting & Off \\
\hline Other: & Yes \\
\hline Other text & $\begin{array}{l}\text { Systems employed in the use case are operated and maintained } \\
\text { by the NASA Advanced Supercomputing Division and the use } \\
\text { case staff do not have to deal with system health. Repositories } \\
\text { for the resultant data are operated and maintained under the } \\
\text { auspices of NASA and the USGS. }\end{array}$
\end{tabular}

\subsubsection{What events are} currently audited? *

\begin{tabular}{|c|c|}
\hline All data access must be audited & Off \\
\hline $\begin{array}{l}\text { Only selected / protected data } \\
\text { must be audited }\end{array}$ & Off \\
\hline $\begin{array}{l}\text { Maintenance on user roles must } \\
\text { be audited (new users, disabled } \\
\text { user, updated roles or } \\
\text { permissions) }\end{array}$ & Off \\
\hline Purge and archive events & Off \\
\hline $\begin{array}{l}\text { Domain-dependent events (e.g., } \\
\text { adding a new sensor) }\end{array}$ & Off \\
\hline REST or SOAP events & Off \\
\hline $\begin{array}{l}\text { Changes in system } \\
\text { configuration }\end{array}$ & Off \\
\hline Organizational changes & Off \\
\hline $\begin{array}{l}\text { External project ownership I } \\
\text { management changes }\end{array}$ & Off \\
\hline
\end{tabular}




\section{Security and Privacy \\ Use Case 2-2}

Requirements are externally set, Off

e.g., by $\mathrm{PCl}$ compliance

Domain-specific events (patient Off

death in a drug trial)

Other:

Yes

Other text

None

\subsection{Application Provider}

Security

4.9.1 Describe Application

Provider Security *

There is a security mechanism Off

implemented at the application

level

The app provider level is aware Off

of PII or privacy data elements

The app provider implements Off

audit and logging

The app provider security relies Off

on framework-level security for

its operation

Does not apply to our Yes

application

Other: Off

Other text

4.10 Framework Provider Security

4.10.1 Describe the

framework provider

security *

Security is implemented at the Off

framework level

Roles can be defined at the Off

framework level

The framework level is aware of Off

PII or related sensitive data

Does not apply in our setting Yes

Is provided by the Big Data tool Off

Other:

Off

Other text

\subsection{System Health}

4.11.1 Measures to

Ensure Availability * 


\section{Security and Privacy \\ Use Case 2-2}

\begin{tabular}{|ll|}
\hline $\begin{array}{l}\text { Deterrents to man-in-the-middle } \\
\text { attacks }\end{array}$ & Off \\
\hline $\begin{array}{l}\text { Deterrents to denial of service } \\
\text { attacks }\end{array}$ & Off \\
\hline $\begin{array}{l}\text { Replication, redundancy or } \\
\text { other resilience measures }\end{array}$ & Off \\
\hline $\begin{array}{l}\text { Deterrents to data corruption, } \\
\text { drops or other critical big data } \\
\text { components }\end{array}$ & Off \\
\hline $\begin{array}{l}\text { Other: } \\
\text { Other text }\end{array}$ & Yes \\
\hline $\begin{array}{l}\text { 4.12 Pystem resources are provided by the NASA Advanced } \\
\text { Cases }\end{array}$ & $\begin{array}{l}\text { Supercomputing Division (NAS) for the use case; NAS has } \\
\text { responsibility for system availability. }\end{array}$ \\
\hline $\begin{array}{l}\text { 4.12.1 Describe } \\
\text { Domain-specific Limitations } \\
\text { on Use }\end{array}$ & None \\
\hline $\begin{array}{l}\text { 4.12.2 Paywall } \\
\text { A paywall is in use at some } \\
\text { stage in the workflow }\end{array}$ & Off \\
\hline Not applicable & Yes \\
\hline
\end{tabular}

\section{Classify Use Cases with Tags}

Use Case 2-2

\subsection{DATA: Application Style and Data sharing and acquisition}

\begin{tabular}{|ll|}
\hline Uses Geographical Information Systems? & Off \\
\hline Use case involves Internet of Things? & Off \\
\hline Data comes from HPC or other simulations? & Off \\
\hline Data Fusion important? & Off \\
\hline Data is Real time Streaming? & Off \\
\hline $\begin{array}{l}\text { Data is Batched Streaming (e.g. collected remotely } \\
\text { and uploaded every so often)? }\end{array}$ & Yes \\
\hline $\begin{array}{l}\text { Important Data is in a Permanent Repository (Not } \\
\text { streamed)? }\end{array}$ & Off \\
\hline Transient Data important? & Off \\
\hline Permanent Data Important? & Yes \\
\hline
\end{tabular}




\section{Classify Use Cases with Tags}

\section{Use Case 2-2}

Data shared between different applications/users? Yes

Data largely dedicated to only this use case? Off

\subsection{DATA: Management and Storage}

\begin{tabular}{|lc|}
\hline Application data system based on Files? & Yes \\
\hline Application data system based on Objects? & Off \\
\hline Uses HDFS style File System? & Off \\
\hline Uses Wide area File System like Lustre? & Yes \\
\hline Uses HPC parallel file system like GPFS? & Off \\
\hline Uses SQL? & Off \\
\hline Uses NoSQL? & Off \\
\hline Uses NewSQL? & Off \\
\hline Uses Graph Database? & Off
\end{tabular}

\subsection{DATA: Describe Other Data}

Acquisition/ Access/ Sharing/

Management/ Storage I ssues

\subsection{ANALYTI CS: Data Format and Nature of Algorithm used in Analytics}

\begin{tabular}{|ll|}
\hline Data regular? & Yes \\
\hline Data dynamic? & Off \\
\hline Algorithm O(N^2) ? & Off \\
\hline Basic statistics (regression, moments) used? & Off \\
\hline Search/Query/Index of application data Important? & Off \\
\hline Classification of data Important? & Yes \\
\hline Recommender Engine Used? & Off \\
\hline Clustering algorithms used? & Off \\
\hline Alignment algorithms used? & Off \\
\hline (Deep) Learning algorithms used? & Off \\
\hline Graph Analytics Used? & Off \\
\hline $\begin{array}{l}\text { 5.5 ANALYTI CS: Describe Other Data } \\
\text { Analytics Used }\end{array}$ & None \\
\hline 5.6 PROGRAMMI NG MODEL & \\
\hline
\end{tabular}




\section{Classify Use Cases with Tags Use Case 2-2}

Pleasingly parallel Structure? Parallel execution over Off independent data. Called Many Task or high throughput computing. MapReduce with only Map and no Reduce of this type

Use case NOT Pleasingly Parallel -- Parallelism Off involves linkage between tasks. MapReduce (with Map and Reduce) of this type

Uses Classic MapReduce? such as Hadoop Off

Uses Apache Spark or similar Iterative MapReduce? Off

Uses Graph processing as in Apache Giraph? Off

Uses MPI (HPC Communication) and/or Bulk Off Synchronous Processing BSP?

Dataflow Programming Model used? Off

Workflow or Orchestration software used? Off

Python or Scripting front ends used? Maybe used for Off orchestration

Shared memory architectures important? Off

Event-based Programming Model used? Off

Agent-based Programming Model used? Off

Use case I/O dominated? I/O time $>$ or $>>$ Compute Off time

Use case involves little I/O? Compute $\gg$ I/O Off

\subsection{Other Programming Model Tags}

$\begin{array}{ll}\begin{array}{l}\text { 5.8 Please Estimate Ratio I/ O } \\ \text { Bytes/ Flops }\end{array} & \begin{array}{l}\text { Do not have the data to develop this } \\ \text { ratio. }\end{array} \\ \begin{array}{l}\text { 5.9 Describe Memory Size or Access } \\ \text { issues }\end{array} & \text { None }\end{array}$

\section{Overall Big Data I ssues}

Use Case 2-2

\subsection{Other Big Data} Issues 


\begin{tabular}{|l|l|}
\hline $\begin{array}{l}\text { 6.2 User Interface and } \\
\text { Mobile Access I ssues }\end{array}$ & No mobile access is applicable to this use case. \\
\hline $\begin{array}{l}\text { 6.3 List Key Features } \\
\text { and Related Use Cases }\end{array}$ & \\
\hline 6.4 Project Future & $\begin{array}{l}\text { Processing will be improved with newer and updated algorithms. } \\
\text { This process may also be applied to future datasets and } \\
\text { processing systems (Landsat } 8 \text { and Sentinel-2 satellites, for } \\
\text { example). }\end{array}$ \\
\hline
\end{tabular}

\section{Workflow Processes}

\section{Use Case 2-2}

7.1 Please comment on The processing for this use case is a 32-stage pipeline. The WELDworkflow processes Overview diagram presents a five-stage high-level workflow. Workflow details are not available at this time, but may be provided in the future if time allows. A top-level workflow diagram is being emailed separately.

\subsection{Workflow details for each stage *}

\subsubsection{Workflow Details for Stage 1}

\begin{tabular}{|l|}
\hline Stage 1 Name \\
\hline Data Source(s) \\
\hline Nature of Data \\
\hline Software Used \\
\hline Data Analytics \\
\hline Infrastructure \\
\hline Percentage of Use Case Effort \\
\hline Other Comments \\
\hline 7.2.2 Workflow Details for Stage 2 \\
\hline Stage 2 Name \\
\hline Data Source(s) \\
\hline Nature of Data \\
\hline Software Used \\
\hline Data Analytics \\
\hline Infrastructure \\
\hline Percentage of Use Case Effort \\
\hline Other Comments \\
\hline 7.2.3 Workflow Details for Stage 3 \\
\hline Stage 3 Name \\
\hline Data Source(s) \\
\hline
\end{tabular}




\begin{tabular}{|l|}
\hline Nature of Data \\
\hline Software Used \\
\hline Data Analytics \\
\hline Infrastructure \\
\hline Percentage of Use Case Effort \\
\hline Other Comments \\
\hline 7.2 .4 Workflow Details for Stage 4 \\
\hline Stage 4 Name \\
\hline Data Source(s) \\
\hline Nature of Data \\
\hline Software Used \\
\hline Data Analytics \\
\hline Infrastructure \\
\hline Percentage of Use Case Effort \\
\hline Other Comments \\
\hline 7.2 .5 Workflow Details for Stages 5 and any further stages \\
\hline Stage 5 Name \\
\hline Data Source(s) \\
\hline Nature of Data \\
\hline Software Used \\
\hline Data Analytics \\
\hline Infrastructure \\
\hline Percentage of Use Case Effort \\
\hline Other Comments \\
\hline
\end{tabular}




\section{F.3 Use Case 2-3: Urban context-aware event management for Smart Cities - Public safety}

\subsection{Use Case Title *}

\subsection{Use Case Description *}

The real-world events are now being observed by multiple networked streams, where each is complementing the other with his or her characteristics, features, and perspectives. Many of these networked data streams are becoming digitalized, and some are available in public (open data initiative) and available for sense-making.

The networked data streams provide an opportunity for their link identification, similarity, and time dynamics to recognize the evolving patterns in the inter-intra-city/community. The delivered information can help to understand better how cities/communities work (some situations, behavior or influence) and detect events and patterns that can be remedied a broad range of issues affecting the everyday lives of citizens and efficiency of cities. Providing the tools that can make this process easy and accessible to the city/community representatives will potentially impact traffic, event management, disaster management systems, health monitoring systems, air quality, and city/community planning.

\section{Current Solutions:}

- Computer(System): Fixed and deployed computing clusters ranging from 10s of nodes to 100 s of nodes.

- Storage: Traditional servers

- Networking: Gigabit wired connections, Wireless including WiFi (802.11), Cellular (3g/4g), or Radio Relay.

- Software: Currently, baseline leverages 1. NLP (several variants); 2. R/Python/Java; 3. Spark/Kafka; 4. Custom applications and visualization tools

- Big Data Specific Challenges (Gaps): Data that currently exists must be accessible through a semantically integrated data space. Some data are unstructured which requires significant processing to extract entities and information. Improving analytic and modeling systems that provide reliable and robust statistical estimated using data from multiple sources.

- Big Data Specific Challenges in Mobility: The outputs of this analysis and intelligence can be transmitted onto or accessed by the city representatives.

- Security \& Privacy Requirements: Open data web portals and social networks like Twitter publicly release their data. Although, data-sources incorporate loT meta-data, therefore, some policy for security and privacy protection must be implemented as required by various legal statutes.

- Highlight issues for generalizing this use case (e.g., for ref. architecture): Definition of high-level data schema to incorporate multiple data sources and types providing structured data format. Therefore, it requires integrated complex event processing and event-based methods that will span domains.

\subsection{Use Case Contacts *}

1125

\begin{tabular}{|l|l|l|}
\hline Name & PI / Author & Primary \\
\hline Olivera Kotevska & PI & Yes \\
\hline Gilad Kusne & Author & No \\
\hline
\end{tabular}




\begin{tabular}{|l|l|l|}
\hline Daniel Samarov & Author & No \\
\hline Ahmed Lbath & Author & No \\
\hline
\end{tabular}

1.4 Domain ("Vertical") *

1127 Smart Communities and Cities

1.5 Application *

Public Safety, City Awareness, City Events Monitoring

\subsection{Current Data Analysis Approach *}

- Analytics: Pattern detection, Link analysis, Sentiment analysis, Time-series forecasting

1131

- Software: R and R Studio

- Hardware: Laptop Dell Latitude E7440

\subsection{Future of Application and Approach *}

- Analytics: Graph analysis

- Software: SparkR

- Hardware: Supercomputer

\subsection{Actors / Stakeholders}

Decision Makers - To make decision where to allocate resources in order to increase city safety

Policy Makers - To make recommendation for long term decisions to be implemented ti order to increase city safety based on the results from the analytics

\subsection{Project Goals or Objectives}

To use advanced methods to analyze complex data streams from socio-technical networks to improve the quality of urban applications.

- Detect events from various network streams

- Ability of intelligent data integration and structuring in the common format for diverse data streams

- Relationship analysis between entities in the network

- Reasoning from varied and complex data streams

- Trends in sentiment for text data streams

- Understanding how communication spreads over networks

- Support for visualization

\subsection{Use Case URL(s)}

We do not have at this point.

\subsection{Pictures and Diagrams?}




\subsection{Data Source}

The data sources are distributed for example:

\subsection{Data Destination}

After the data is collected it is saved on the file system - one file per data source.

\subsection{Volume}

\begin{tabular}{|l|l|}
\hline Size & $\begin{array}{l}\text { Depending on the sensor type and data type, some sensors can produce over a } \\
\text { gigabyte of data in the space of hours. Other data is as small as infrequent sensor } \\
\text { activations or text messages. }\end{array}$ \\
\hline Units & \\
\hline Time Period & \\
\hline Proviso & \\
\hline
\end{tabular}

Size: Quantitative volume of data handled in the use case

Units: What is measured such as "Tweets per year", Total LHC data in petabytes, etc.?

Time Period: Time corresponding to specified size.

Proviso: The criterion (e.g. data gathered by a particular organization) used to get size with units in time period in three fields above

\subsection{Velocity}

\begin{tabular}{|l|l|}
\hline Unit of measure & $\begin{array}{l}\text { New records were gathered per week or when available, except for city events when } \\
\text { the data was gathered once per month and social media when data was gathered } \\
\text { every day. }\end{array}$ \\
\hline Time Period & \\
\hline Proviso & \\
\hline
\end{tabular}

Unit of Measure: Units of Velocity size given above. What is measured such as "New Tweets gathered per second", etc.?

Time Period: Time described and interval such as September 2015; items per minute

Proviso: The criterion (e.g., data gathered by a particular organization) used to get Velocity measure with units in time period in three fields above

\subsection{Variety}

1185 Everything from text files, raw media, imagery, electronic data, human-generated data all in various

1186 formats. Heterogeneous datasets are fused together for analytical use. 


\subsection{Variability}

Continuous data streams are coming from each source. Sensor interface formats tend to be stable, while the human-based data may be in any format. Much of the data is unstructured. There is no critical variation of data producing speed or runtime characteristics variations.

\subsection{Veracity and Data Quality}

\subsection{Visualization}

Displaying in a meaningful way complex data sets using tables, geospatial maps, time-based network graph model, and visualization techniques.

\subsection{Data Types}

1201 Semi-structured datasets like numeric data (various sensors)

Unstructured datasets like text (e.g., social networks, police reports, digital documents), multimedia (pictures, digital signal data);

\subsection{Metadata}

There was a lack of metadata description but some of the datasets were easy to understand such as social media and city events.

\subsection{Curation and Governance}

\subsection{Data Analytics}

- Pattern recognition of all kind (e.g., event behavior automatic analysis, cultural patterns).

- Classification: event type, classification, using multivariate time series to generate network, content, geographical features and so forth.

- Clustering: per topic, similarity, spatial-temporal, and additional features.

- Text Analytics (sentiment, entity similarity)

- Link Analysis: using similarity and statistical techniques

- Online learning: real-time information analysis.

- Multiview learning: data fusion feature learning

- Anomaly detection: unexpected event behavior

- Visualizations based on patterns, spatial-temporal changes. 


\section{GENERAL SECURITY AND PRIVACY}

\subsection{Classified Data, Code or Protocols}

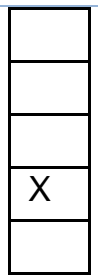

Intellectual property protections

Military classifications, e.g., FOUO, or Controlled Classified

Not applicable

Creative commons/ open source

Other:

1.25 Does the System Maintain Personally Identifiable Information (PII)? *

Other:

\subsection{Publication rights}

\begin{tabular}{|l|}
\hline$X$ \\
\hline \\
\hline \\
\hline \\
\hline \\
\end{tabular}

\section{Open publication}

Proprietary

Traditional publisher rights (e.g., Springer, Elsevier, IEEE)

"Big Science" tools in use

Other:

\subsection{Is there an explicit data governance plan or framework} for the effort?

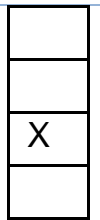

Explicit data governance plan

No data governance plan, but could use one

Data governance does not appear to be necessary

Other:

\subsection{Do you foresee any potential risks from public or private} open data projects?

Risks are known.

Currently no known risks, but it is conceivable.

Not sure

X Unlikely that this will ever be an issue (e.g., no PII, human-agent related data or subsystems.) Other: 


\subsection{Current audit needs *}

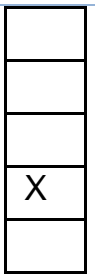

We have third party registrar or other audits, such as for ISO 9001

We have internal enterprise audit requirements

Audit is only for system health or other management requirements

No audit, not needed or does not apply

Other:

\subsection{Under what conditions do you give people access to your data?}

The data is publicly available for everyone to use it. We can share the links to the data sources.

\subsection{Under what conditions do you give people access to your software?}

\section{CLASSIFY USE CASES WITH TAGS}

\subsection{DATA: Application Style and Data sharing and} acquisition

\begin{tabular}{|c|c|}
\hline & Uses Geographical Information Systems? \\
\hline$X$ & Use case involves Internet of Things? \\
\hline & Data comes from HPC or other simulations? \\
\hline$X$ & Data Fusion important? \\
\hline & Data is Real time Streaming? \\
\hline$X$ & Data is Batched Streaming (e.g. collected remotely and uploaded every so often)? \\
\hline $\mathrm{X}$ & Important Data is in a Permanent Repository (Not streamed)? \\
\hline & Transient Data important? \\
\hline X & Permanent Data Important? \\
\hline & Data shared between different applications/users? \\
\hline & Data largely dedicated to only this use case? \\
\hline
\end{tabular}




\subsection{DATA: Management and Storage}

\begin{tabular}{|c|c|}
\hline $\mathrm{X}$ & Application data system based on Files? \\
\hline & Application data system based on Objects? \\
\hline & Uses HDFS style File System? \\
\hline & Uses Wide area File System like Lustre? \\
\hline & Uses HPC parallel file system like GPFS? \\
\hline & Uses SQL? \\
\hline & Uses NoSQL? \\
\hline & Uses NewSQL? \\
\hline & Uses Graph Database? \\
\hline
\end{tabular}

\subsection{DATA: Describe Other Data Acquisition/ Access/ Sharing/ Management/ Storage Issues}

1.35 ANALYTICS: Data Format and Nature of Algorithm used in Analytics

\begin{tabular}{|c|c|}
\hline $\bar{X}$ & Data regular? \\
\hline & Data dynamic? \\
\hline & Algorithm $\mathrm{O}\left(\mathrm{N}^{\wedge} 2\right)$ ? \\
\hline $\bar{x}$ & Basic statistics (regression, moments) used? \\
\hline & Search/Query/Index of application data Important? \\
\hline $\mathrm{X}$ & Classification of data Important? \\
\hline & Recommender Engine Used? \\
\hline $\bar{x}$ & Clustering algorithms used? \\
\hline & Alignment algorithms used? \\
\hline & (Deep) Learning algorithms used? \\
\hline $\bar{X}$ & Graph Analytics Used? \\
\hline
\end{tabular}




\subsection{PROGRAMMING MODEL}

Pleasingly parallel Structure? Parallel execution over independent data. Called Many Task or high throughput computing. MapReduce with only Map and no Reduce of this type Use case NOT Pleasingly Parallel -- Parallelism involves linkage between tasks. MapReduce (with Map and Reduce) of this type

Uses Classic MapReduce? such as Hadoop

Uses Apache Spark or similar Iterative MapReduce?

Uses Graph processing as in Apache Giraph?

Uses MPI (HPC Communication) and/or Bulk Synchronous Processing BSP?

Dataflow Programming Model used?

Workflow or Orchestration software used?

Python or Scripting front ends used? Maybe used for orchestration

Shared memory architectures important?

Event-based Programming Model used?

Agent-based Programming Model used?

Use case $\mathrm{I} / \mathrm{O}$ dominated? $\mathrm{I} / \mathrm{O}$ time $>$ or $>>$ Compute time

Use case involves little I/O? Compute $>>1 / O$

\subsection{Other Programming Model Tags}

$1250 \quad$ Web scraping with $\mathrm{R}$

\subsection{Describe Memory Size or Access issues}

\section{OVERALL BIG DATA ISSUES}




\section{WORKFLOW PROCESSES}

\subsection{Please comment on workflow processes}

1264

\subsection{Workflow details for each stage *}

\section{Description of table fields below:}

Data Source(s): The origin of data, which could be from instruments, Internet of Things, Web, Surveys, Commercial activity, or from simulations. The source(s) can be distributed, centralized, local, or remote. Often data source at one stage is destination of previous stage with raw data driving first stage.

Nature of Data: What items are in the data?

Software Used: List software packages used

Data Analytics: List algorithms and analytics libraries/packages used

Infrastructure: Compute, Network and Storage used. Note sizes infrastructure -- especially if "big".

Percentage of Use Case Effort: Explain units. Could be clock time elapsed or fraction of compute cycles

Other Comments: Include comments here on items like veracity and variety present in upper level but omitted in summary.

\subsubsection{WORKFLOW DETAILS FOR STAGE 1}

\begin{tabular}{|l|l|}
\hline Stage 1 Name & Data Collection \\
\hline Data Source(s) & $\begin{array}{l}\text { Public safety dataset (crime, traffic violations) and census dataset were } \\
\text { downloaded manually from the source. Weather, city community events, social } \\
\text { media datasets we collected by developed script. }\end{array}$ \\
\hline Nature of Data & Text, Numeric, Geo-tagged \\
\hline Software Used & $\begin{array}{l}\text { We developed a script for data collection in R Studio and used rvest, rcurl, twitteR, } \\
\text { tm libraries for web scraping. }\end{array}$ \\
\hline Data Analytics & \\
\hline Infrastructure & \\
\hline $\begin{array}{l}\text { Percentage of Use } \\
\text { Case Effort }\end{array}$ & \\
\hline Other Comments & Datasets were saved in .csv format on file system. \\
\hline
\end{tabular}

\subsubsection{WORKFLOW DETAILS FOR STAGE 2}

\begin{tabular}{|l|l|}
\hline Stage 2 Name & Data preprocessing \\
\hline Data Source(s) & Social media, City events (web scraping), Public safety - police reports \\
\hline Nature of Data & Text, Numeric, Geo-tagged \\
\hline Software Used & $\begin{array}{l}\text { Developed a code for formatting the data entries (date, time, location), selecting } \\
\text { the entries of interest from each dataset, and group them by date, time, location. }\end{array}$ \\
\hline Data Analytics & \\
\hline Infrastructure & \\
\hline $\begin{array}{l}\text { Percentage of Use } \\
\text { Case Effort }\end{array}$ & \\
\hline Other Comments & \\
\hline
\end{tabular}




\subsubsection{WORKFLOW DETAILS FOR STAGE 3}

\begin{tabular}{|l|l|}
\hline Stage 3 Name & Data analysis - Event detection \\
\hline Data Source(s) & Social media, City events (web scraping), Public safety - police reports \\
\hline Nature of Data & Text \\
\hline Software Used & $\begin{array}{l}\text { Developed a code for event detection based on topic model, frequent word and } \\
\text { associations, classification approach. Used libraries such as wordcloud, hclust, } \\
\text { kmeans, topicmodels, randomForest, ctree, e1071. }\end{array}$ \\
\hline Data Analytics & \\
\hline Infrastructure & \\
\hline $\begin{array}{l}\text { Percentage of Use } \\
\text { Case Effort }\end{array}$ & \\
\hline Other Comments & \\
\hline
\end{tabular}

\subsubsection{WORKFLOW DETAILS FOR STAGE 4}

\begin{tabular}{|l|l|}
\hline Stage 4 Name & Data analysis - Link analysis \\
\hline Data Source(s) & Social media, City events (web scraping), Public safety - police reports \\
\hline Nature of Data & Text, Numeric, Geo-tagged \\
\hline Software Used & $\begin{array}{l}\text { Developed a code for event relationship analysis. Libraries used igraph, } \\
\text { Rgraphiviz, arules, apriori, arulesViz, cmdscale, Imtest, vars, Hmisc, corrplot. }\end{array}$ \\
\hline Data Analytics & \\
\hline Infrastructure & \\
\hline $\begin{array}{l}\text { Percentage of Use } \\
\text { Case Effort }\end{array}$ & \\
\hline Other Comments & \\
\hline
\end{tabular}

\subsubsection{WORKFLOW DETAILS FOR STAGES 5 AND ANY FURTHER STAGES}

\begin{tabular}{|l|l|}
\hline Stage 5 Name & Data analysis - Prediction and Visualization \\
\hline Data Source(s) & Social media, City events (web scraping), Public safety - police reports \\
\hline Nature of Data & Text, Numeric, Geo-tagged \\
\hline Software Used & $\begin{array}{l}\text { Developed a code for event prediction and visualization of the results. Libraries } \\
\text { used forecast, arima, dtw, ggplot. }\end{array}$ \\
\hline Data Analytics & \\
\hline Infrastructure & \\
\hline $\begin{array}{l}\text { Percentage of Use } \\
\text { Case Effort }\end{array}$ & \\
\hline Other Comments & \\
\hline
\end{tabular}




\section{DETAILED SECURITY AND PRIVACY}

\section{$1284 \quad 1.46$ Roles}

$1285 \quad \mathbf{1 . 4 6 . 1} \quad$ IDENTIFYING ROLE

$1286 \quad \mathbf{1 . 4 6 . 2} \quad$ INVESTIGATOR AfFiliations

$1287 \quad \mathbf{1 . 4 6 . 3} \quad$ SPONSORS

1288 1.46.4 DECLARATIONS OF POTENTIAL CONFLICTS OF INTEREST

$1289 \quad \mathbf{1 . 4 6 . 5} \quad$ INSTITUTIONAL S/P DUTIES

$1290 \quad \mathbf{1 . 4 6 . 6}$ CURATION

$1291 \quad$ 1.46.7 Classified DATA, Code or Protocols

\begin{tabular}{|l|l|l}
\hline & Intellectual property protections \\
\hline & Military classifications, e.g., FOUO, or Controlled Classified \\
\hline & Not applicable \\
\hline $\mathrm{X}$ & $\begin{array}{l}\text { Creative commons/ open source } \\
\mathrm{On}\end{array}$ \\
& Other:
\end{tabular}

$1292 \quad \mathbf{1 . 4 6 . 8}$

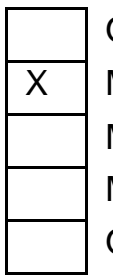

Multiple INVESTIGATORS | PROJECT LEADS *

Only one investigator | project lead | developer

Multiple team members, but in the same organization

Multiple leads across legal organizational boundaries

Multinational investigators | project leads

Other:

LEASt PRIVILEge Role-based AcCess

Yes, roles are segregated and least privilege is enforced

We do have least privilege and role separation but the admin role(s) may be too all-inclusion

Handled at application provider level

Handled at framework provider level

$X \quad$ There is no need for this feature in our application

$X \quad$ Could be applicable in production or future versions of our work Other: 


\subsubsection{Role-based Access to Data *}

\begin{tabular}{|l|l|}
\hline & Dataset \\
\hline & Data record / row \\
& Data element / field \\
\hline & Handled at application provider level \\
\cline { 1 - 1 } Handled at framework provider level \\
\cline { 1 - 1 } O Other: Not applicable at this stage.
\end{tabular}

\subsection{Personally Identifiable Information (PII)}

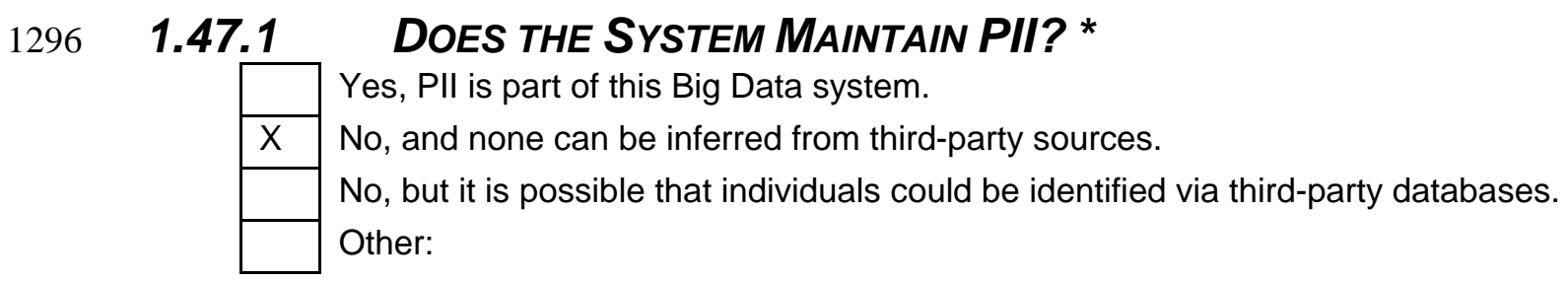

1.47.2 DESCRIBE THE PII, IF APPLICABLE

\section{1.47.3 AdDITIONAL Formal OR INFORMal PROTECTIONS FOR PII}

\subsubsection{Algorithmic / Statistical Segmentation of Human}

\section{POPULATIONS}

$\square$ Yes, doing segmentation, possible discrimination issues if abused. Please also answer the next question.

\begin{tabular}{|l} 
\\
\end{tabular} Yes, doing segmentation, but no foreseeable discrimination issues.

$\mathrm{X}$ Does not apply to this use case at all (e.g., no human subject data).

Other:

\subsubsection{PROTECTIONS AFFORDED STATISTICAL / DEEP LEARNING DISCRIMINATION}

\subsection{Covenants, Liability, Etc.}

1.48.1 IDENTIFY ANY AdDITIONAL SECURITY, ComplaACE, REgulatory REQUIREMENTS *

HHS 45 CFR 46

HIPAA

EU General Data Protection (Reference: http://bit.ly/1Ta8S1C )

COPPA

Other Transborder issues

Fair Credit Reporting Act (Reference: http://bit.ly/1Ta8XSN )

Family Educational Rights and Protection (FERPA) 
\begin{tabular}{|l|l}
\hline & $\begin{array}{l}\text { None apply } \\
\text { X }\end{array}$ \\
Other: N/A
\end{tabular}

\subsubsection{CUSTOMER PRIVACY PROMISES}

\begin{tabular}{|l|l}
\hline & $\begin{array}{l}\text { Yes, we're making privacy promises to customers or subjects. } \\
\text { We are using a notice-and-consent model. }\end{array}$ \\
\cline { 1 - 1 } & $\begin{array}{l}\text { Not applicable } \\
\text { Other: }\end{array}$
\end{tabular}

\subsection{Ownership, Identity and Distribution}

Publication Rights

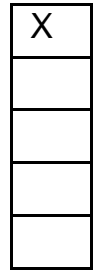

Open publication

Proprietary

Traditional publisher rights (e.g., Springer, Elsevier, IEEE)

"Big Science" tools in use

Other:

\subsubsection{ChAIN OF TRUST}

\subsubsection{DELEGATED RIGHTS}

\subsubsection{SOFTWARE LICENSE RESTRICTIONS}

- Open source software and libraries we used.

- The application was tested multiple times on different platforms and the reproducibility was proven.

RESULTS REPOSITORY

PRIVACy Notices

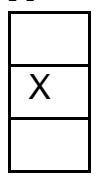

\section{Privacy notices apply}

Privacy notices do not apply Other:

KEY MANAGEMENT

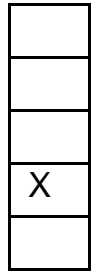

A key management scheme is part of our system.

We are using public key infrastructure.

We do not use key management, but it could have been useful.

No readily identifiable use for key management.

Other: 


\subsubsection{IS AN IDENTITY FRAMEWORK USED?}

\begin{tabular}{|l|l|}
\hline & A framework is in place. (See next question.) \\
\hline & Not currently using a framework. \\
\cline { 1 - 1 } & There is no perceived need for an identity framework. \\
\cline { 1 - 1 } Other:
\end{tabular}

Could be used, but none are available.

Not applicable

\section{DESCRIBE THE IDENTITY FRAMEWORK}

Other:

\subsection{Risk Mitigation}

ARE MEASURES IN PLACE TO DETER RE-IDENTIFICATION? *

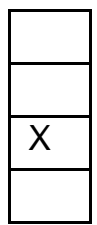

Yes, in place

Not in place, but such measures do apply

Not applicable

Other:

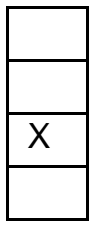

Yes, being used

Not in use, but does apply

Not applicable

Other: THE EFFORT?

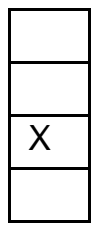

Explicit data governance plan

No data governance plan, but could use one

Data governance does not appear to be necessary

Other: 
$1330 \quad \mathbf{1 . 5 0 . 5} \quad$ Privacy-PRESERVINg Practices

1331 1.50.6 DO YOU FORESEE ANY POTENTIAL RISKS FROM PUBLIC OR PRIVATE 1332 OPEN DATA PROJECTS?

\begin{tabular}{|l|l}
\hline & Risks are known. \\
& Currently no known risks, but it is conceivable. \\
& Not sure \\
\cline { 1 - 1 } $\mathrm{X}$ & $\begin{array}{l}\text { Unlikely that this will ever be an issue (e.g., no PII, human-agent related data or subsystems). } \\
\text { Other: }\end{array}$
\end{tabular}

$1333 \quad \mathbf{1 . 5 1}$ Provenance (Ownership)

1334 1.51.1 DESCRIBE YOUR METADATA MANAGEMENT PRACTICES

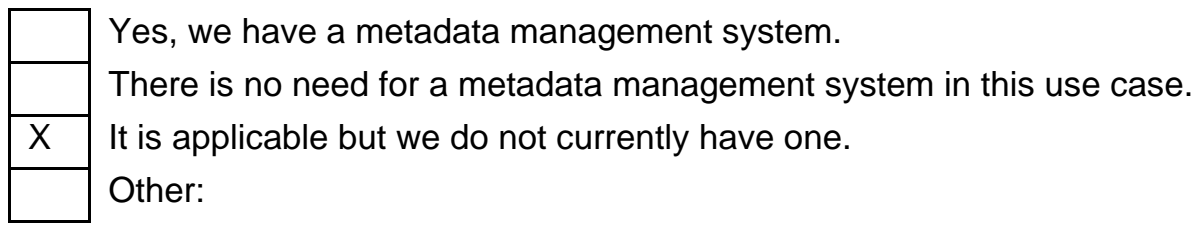

\subsubsection{IF A METADATA MANAGEMENT SYSTEM IS PRESENT, WHAT MEASURES} ARE IN PLACE TO VERIFY AND PROTECT ITS INTEGRITY?

\subsubsection{DESCRIBE PROVENANCE AS RELATED TO INSTRUMENTATION,} SENSORS OR OTHER DEVICES.

\begin{tabular}{|c|c|}
\hline & We have potential machine-to-machine traffic provenance concerns. \\
\hline & Endpoint sensors or instruments have signatures periodically updated. \\
\hline & Using hardware or software methods, we detect and remediate outlier signatures. \\
\hline & Endpoint signature detection and upstream flow are built into system processing. \\
\hline & We rely on third-party vendors to manage endpoint integrity. \\
\hline & We use a sampling method to verify endpoint integrity. \\
\hline $\mathrm{X}$ & Not a concern at this time. \\
\hline & Other: \\
\hline
\end{tabular}

$1339 \mathbf{1 . 5 2}$ Data Life Cycle

\section{1.52.1 DESCRIBE ARCHIVE PROCESSES}

Our application has no separate "archive" process.

We offload data using certain criteria to removable media which are taken offline.

We use a multi-stage, tiered archive process.

We allow for "forgetting" of individual PII on request.

Have ability to track individual data elements across all stages of processing, including archive.

Additional protections, such as separate encryption, are applied to archival data.

Archived data is saved for potential later use by applications or analytics yet to be built. 
\begin{tabular}{ll}
\hline & Does not apply to our application. \\
& Other:
\end{tabular}

\section{$1341 \quad$ 1.52.2 Describe PoInt In TIME AND Other DePENDENCY ISSUES}
$\mathrm{X}$ Some data is valid only within a point in time,
Some data is only valid with other, related data is available or applicable, such as the existence of a building, the presence of a weather event, or the active use of a vehicle.
There are specific events in the application that render certain data obsolete or unusable.
Point and Time and related dependencies do not apply.
Other:

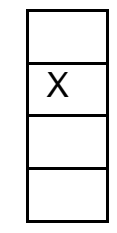

We are required to destroy or otherwise dispose of data.

Does not apply to us.

Not sure

Other:

\section{$1343 \quad 1.53$ Audit and Traceability}

\section{CURRENT AUDIT NEEDS *}

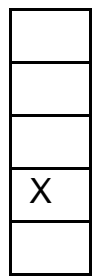

We have third-party registrar or other audits, such as for ISO 9001.

We have internal enterprise audit requirements.

Audit is only for system health or other management requirements.

No audit, not needed or does not apply.

Other:

\section{AUDITING VERSUS MONITORING}

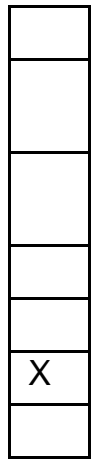

We rely on third-party or O.S. tools to audit, e.g., Windows or Linux auditing.

There are built-in tools for monitoring or logging that are only used for system or application health monitoring.

Monitoring services include logging of role-based access to assets such as PII or other resources.

The same individual(s) in the enterprise are responsible for auditing as for monitoring.

This aspect of our application is still in flux.

Does not apply to our setting.

Other:

\subsection{3}

\section{SYSTEM HEALTH TOOLS}

We rely on system-wide tools for health monitoring.

We built application health tools specifically to address integrity, performance monitoring, and related concerns.

$X \quad$ There is no need in our setting.

Other: 


\subsubsection{WHAT EVENTS ARE CURRENTLY AUDITED? *}

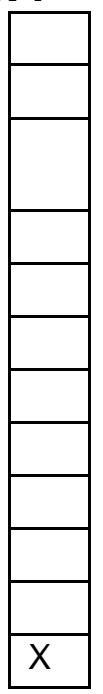

All data access must be audited.

Only selected / protected data must be audited.

Maintenance on user roles must be audited (new users, disabled user, updated roles or permissions).

Purge and archive events.

Domain-dependent events (e.g., adding a new sensor).

REST or SOAP events

Changes in system configuration

Organizational changes

External project ownership / management changes

Requirements are externally set, e.g., by $\mathrm{PCl}$ compliance.

Domain-specific events (patient death in a drug trial)

Other: Do not have at this stage.

\subsection{Application Provider Security}

\subsection{1}

\section{Describe Application Provider SeCuRITY *}

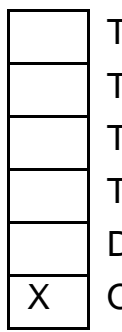

There is a security mechanism implemented at the application level.

The app provider level is aware of PII or privacy data elements.

The app provider implements audit and logging.

The app provider security relies on framework-level security for its operation.

Does not apply to our application.

Other: Do not have at this stage.

\subsection{Framework Provider Security}

\section{$1351 \quad \mathbf{1 . 5 5 . 1}$}

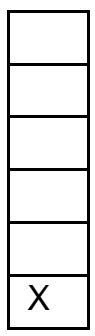

\section{DESCRIBE THE FRAMEWORK PROVIDER SECURITY *}

Security is implemented at the framework level.

Roles can be defined at the framework level.

The framework level is aware of PII or related sensitive data.

Does not apply in our setting.

Is provided by the Big Data tool.

Other: Do not have at this stage.

\subsection{System Health}

MEASURES to ENSURE AVAILABILITY *

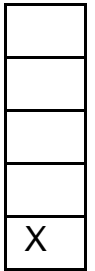

Deterrents to man-in-the-middle attacks

Deterrents to denial of service attacks

Replication, redundancy or other resilience measures

Deterrents to data corruption, drops or other critical big data components

Other: Do not have at this stage. 
NIST Big Data Interoperability Framework: Volume 3, Use CaSes and General ReQuiRements

$1354 \quad 1.57$ Permitted Use Cases

1355 1.57.1 DESCRIBE DOMAIN-SPECIFIC LIMITATIONS ON USE

$1356 \quad$ 1.57.2 PAYWALL

1357

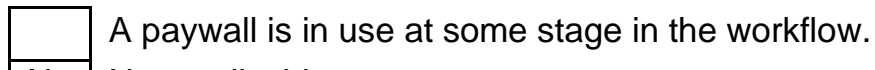

X Not applicable 


\section{Appendix G: Acronyms}

\begin{tabular}{|c|c|c|}
\hline 1360 & $2 \mathrm{D}$ and $3 \mathrm{D}$ & two- and three-dimensional \\
\hline 1361 & 6D & six-dimensional \\
\hline 1362 & AOD & Analysis Object Data \\
\hline 1363 & API & application programming interface \\
\hline 1364 & ASDC & Atmospheric Science Data Center \\
\hline 1365 & AWS & Amazon Web Services \\
\hline 1366 & $\mathrm{BC} / \mathrm{DR}$ & business continuity and disaster recovery \\
\hline 1367 & $\mathrm{BD}$ & Big Data \\
\hline 1368 & BER & Biological and Environmental Research \\
\hline 1369 & BNL & Brookhaven National Laboratory \\
\hline 1370 & CAaaS & climate analytics as a service \\
\hline 1371 & CBSP & Cloud Brokerage Service Provider \\
\hline 1372 & CCP & Climate Change Prediction \\
\hline 1373 & CERES & Clouds and Earth's Radiant Energy System \\
\hline 1374 & CERN & European Organization for Nuclear Research \\
\hline 1375 & CES21 & California Energy Systems for the $21^{\text {st }}$ Century \\
\hline 1376 & CESM & Community Earth System Model \\
\hline 1377 & CFTC & U.S. Commodity Futures Trading Commission \\
\hline 1378 & CIA & confidentiality, integrity, and availability \\
\hline 1379 & CMIP & Coupled Model Intercomparison Project \\
\hline 1380 & CMIP5 & Climate Model Intercomparison Project \\
\hline 1381 & CMS & Compact Muon Solenoid \\
\hline 1382 & CNRS & Centre National de la Recherche Scientifique \\
\hline 1383 & COSO & Committee of Sponsoring Organizations \\
\hline 1384 & $\mathrm{CP}$ & charge parity \\
\hline 1385 & CPR & Capability Provider Requirements \\
\hline 1386 & CPU & central processing unit \\
\hline 1387 & CReSIS & Center for Remote Sensing of Ice Sheets \\
\hline 1388 & CRTS & Catalina Real-Time Transient Survey \\
\hline 1389 & CSP & cloud service provider \\
\hline 1390 & CSS & Catalina Sky Survey proper \\
\hline 1391 & $\mathrm{CV}$ & controlled vocabulary \\
\hline 1392 & DCR & Data Consumer Requirements \\
\hline 1393 & DES & Dark Energy Survey \\
\hline 1394 & DFC & DataNet Federation Consortium \\
\hline 1395 & DHTC & Distributed High Throughput Computing \\
\hline 1396 & DOE & U.S. Department of Energy \\
\hline 1397 & DOJ & U.S. Department of Justice \\
\hline 1398 & $\mathrm{DPO}$ & Data Products Online \\
\hline 1399 & DSR & Data Source Requirements \\
\hline 1400 & EBAF-TOA & Energy Balanced and Filled-Top of Atmosphere \\
\hline 1401 & EC2 & Elastic Compute Cloud \\
\hline 1402 & EDT & Enterprise Data Trust \\
\hline 1403 & EHR & electronic health record \\
\hline 1404 & EMR & electronic medical record \\
\hline
\end{tabular}




\begin{tabular}{|c|c|c|}
\hline 1405 & EMSO & European Multidisciplinary Seafloor and Water Column Observatory \\
\hline 1406 & ENVRI & Common Operations of Environmental Research Infrastructures \\
\hline 1407 & ENVRI RM & ENVRI Reference Model \\
\hline 1408 & EPOS & European Plate Observing System \\
\hline 1409 & ERC & European Research Council \\
\hline 1410 & ESFRI & European Strategy Forum on Research Infrastructures \\
\hline 1411 & ESG & Earth System Grid \\
\hline 1412 & ESGF & Earth System Grid Federation \\
\hline 1413 & FDIC & U.S. Federal Deposit Insurance Corporation \\
\hline 1414 & FI & Financial Industries \\
\hline 1415 & FLUXNET & AmeriFlux and Flux Tower Network \\
\hline 1416 & FMV & full motion video \\
\hline 1417 & FNAL & Fermi National Accelerator Laboratory \\
\hline 1418 & GAAP & U.S. Generally Accepted Accounting Practices \\
\hline 1419 & GB & gigabyte \\
\hline 1420 & GCM & general circulation model \\
\hline 1421 & GEOS-5 & Goddard Earth Observing System version 5 \\
\hline 1422 & GEWaSC & Genome-Enabled Watershed Simulation Capability \\
\hline 1423 & GHG & greenhouse gas \\
\hline 1424 & GISs & geographic information systems \\
\hline 1425 & GMAO. & Global Modeling and Assimilation Office \\
\hline 1426 & GPFS & General Parallel File System \\
\hline 1427 & GPS & global positioning system \\
\hline 1428 & GPU & graphics processing unit \\
\hline 1429 & GRC & governance, risk management, and compliance \\
\hline 1430 & GSFC & Goddard Space Flight Center \\
\hline 1431 & HDF5 & Hierarchical Data Format \\
\hline 1432 & HDFS & Hadoop Distributed File System \\
\hline 1433 & HPC & high-performance computing \\
\hline 1434 & HTC & high-throughput computing \\
\hline 1435 & HVS & hosted virtual server \\
\hline 1436 & $\mathrm{I} / \mathrm{O}$ & input output \\
\hline 1437 & IaaS & Infrastructure as a Service \\
\hline 1438 & IAGOS & In-service Aircraft for a Global Observing System \\
\hline 1439 & ICA & independent component analysis \\
\hline 1440 & ICD & International Classification of Diseases \\
\hline 1441 & ICOS & Integrated Carbon Observation System \\
\hline 1442 & IMG & Integrated Microbial Genomes \\
\hline 1443 & INPC & Indiana Network for Patient Care \\
\hline 1444 & IPCC & Intergovernmental Panel on Climate Change \\
\hline 1445 & iRODS & Integrated Rule-Oriented Data System \\
\hline 1446 & ISACA & International Society of Auditors and Computer Analysts \\
\hline 1447 & isc2 & International Security Computer and Systems Auditors \\
\hline 1448 & ISO & International Organization for Standardization \\
\hline 1449 & ITIL & Information Technology Infrastructure Library \\
\hline 1450 & ITL & Information Technology Laboratory \\
\hline 1451 & JGI & Joint Genome Institute \\
\hline 1452 & KML & Keyhole Markup Language \\
\hline 1453 & kWh & kilowatt-hour \\
\hline 1454 & LaRC & Langley Research Center \\
\hline 1455 & LBNL & Lawrence Berkeley National Laboratory \\
\hline
\end{tabular}


NIST Big Data Interoperability Framework: Volume 3, Use CaSes and General ReQuiRements

\begin{tabular}{|c|c|c|}
\hline 1456 & LDA & latent Dirichlet allocation \\
\hline 1457 & LHC & Large Hadron Collider \\
\hline 1458 & LMR & Life cycle Management Requirements \\
\hline 1459 & LOB & lines of business \\
\hline 1460 & LPL & Lunar and Planetary Laboratory \\
\hline 1461 & LSST & Large Synoptic Survey Telescope \\
\hline 1462 & MERRA & Modern Era Retrospective Analysis for Research and Applications \\
\hline 1463 & MERRA/AS & MERRA Analytic Services \\
\hline 1464 & MPI & Message Passing Interface \\
\hline 1465 & MRI & magnetic resonance imaging \\
\hline 1466 & NARA & National Archives and Records Administration \\
\hline 1467 & NARR & North American Regional Reanalysis \\
\hline 1468 & NaaS & Network as a Service \\
\hline 1469 & NASA & National Aeronautics and Space Administration \\
\hline 1470 & NBD-PWG & NIST Big Data Public Working Group \\
\hline 1471 & NBDRA. & NIST Big Data Reference Architecture \\
\hline 1472 & NCAR & National Center for Atmospheric Research \\
\hline 1473 & NCBI & National Center for Biotechnology Information \\
\hline 1474 & NCCS & NASA Center for Climate Simulation \\
\hline 1475 & NEO & near-Earth \\
\hline 1476 & NERSC & National Energy Research Scientific Computing Center \\
\hline 1477 & NetCDF & Network Common Data Form \\
\hline 1478 & NEX & NASA Earth Exchange \\
\hline 1479 & NFS & network file system \\
\hline 1480 & NIKE & NIST Integrated Knowledge Editorial Net \\
\hline 1481 & NIST & National Institute of Standards and Technology \\
\hline 1482 & NLP & natural language processing \\
\hline 1483 & NRT & Near Real Time \\
\hline 1484 & NSF & National Science Foundation \\
\hline 1485 & ODAS & Ocean Modeling and Data Assimilation \\
\hline 1486 & ODP & Open Distributed Processing \\
\hline 1487 & OGC & Open Geospatial Consortium \\
\hline 1488 & OLAP & online analytical processing \\
\hline 1489 & OpenAIRE & Open Access Infrastructure for Research in Europe \\
\hline 1490 & OR & Other Requirements \\
\hline 1491 & PB & petabyte \\
\hline 1492 & PCA & principal component analysis \\
\hline 1493 & PCAOB & Public Company Accounting and Oversight Board \\
\hline 1494 & PHO & planetary hazard \\
\hline 1495 & PID & persistent identification \\
\hline 1496 & PII & Personally Identifiable Information \\
\hline 1497 & PNNL & Pacific Northwest National Laboratory \\
\hline 1498 & PR & Public Relations \\
\hline 1499 & RDBMS & relational database management system \\
\hline 1500 & $\mathrm{RDF}$ & Resource Description Framework \\
\hline 1501 & ROI & return on investment \\
\hline 1502 & RPI & Repeat Pass Interferometry \\
\hline 1503 & RPO & Recovery Point Objective \\
\hline 1504 & RTO & Response Time Objective \\
\hline 1505 & SAN & storage area network \\
\hline 1506 & SAR & Synthetic aperture radar \\
\hline
\end{tabular}


NIST Big Data Interoperability Framework: Volume 3, Use CaSes and General ReQuiRements

1507 SAR Synthetic Aperture Radar

1508 SDLC/HDLC Software Development Life Cycle/Hardware Development Life Cycle

1509 SDN software-defined networking

1510 SEC U.S. Securities and Exchange Commission

1511 SFA 2.0 Scientific Focus Area 2.0 Science Plan

1512 SIEM Security Incident/Event Management

1513 SIOS Svalbard Integrated Arctic Earth Observing System

1514 SOAP Simple Object Access Protocol

거 1515 SOX Sarbanes-Oxley Act of 2002

1516

SPADE

Support for Provenance Auditing in Distributed Environments

SPR

1518 SSH

Security and Privacy Requirements

$\begin{array}{ll}1519 & \text { SSO } \\ 1520 & \text { tf-idf }\end{array}$

$\begin{array}{ll}1519 & \text { SSO } \\ 1520 & \text { tf-idf }\end{array}$

Secure Shell

1521 TPR

Single sign-on capability

term frequency-inverse document frequency

Transformation Provider Requirements

1522 UA

University of Arizona

1523 UAVSAR

1524 UI

1525 UPS

1526 UQ

1527 vCDS

1528 VO

1529 VOIP

1530 WALF

1531 WLCG

1532 XBRL

1533 XML

1534 ZTF

Unmanned Air Vehicle Synthetic Aperture Radar

user interface

United Parcel Service

uncertainty quantification

virtual Climate Data Server

Virtual Observatory

Voice over IP

Wide Area Large Format Imagery

Worldwide LHC Computing Grid

extensible Business Related Markup Language

Extensible Markup Language

Zwicky Transient Factory 


\section{Appendix H: Bibliography}

1537

1538

1539

1540

1541

1542

1543

1544

1545

1546

1547

1548

1549

1550

1551

1552

1553

1554

1555

1556

1557

1558

1559

1560

1561

1562

1563

1564

1565

1566

1567

1568

1569

1570

[1] W. L. Chang (Co-Chair), N. Grady (Subgroup Co-chair), and NIST Big Data Public Working Group, “NIST Big Data Interoperability Framework: Volume 1, Big Data Definitions (NIST SP 1500-1 VERSION 3),” Gaithersburg MD, Sep. 2019 [Online]. Available: https://doi.org/10.6028/NIST.SP.1500-1r2

[2] W. L. Chang (Co-Chair), N. Grady (Subgroup Co-chair), and NIST Big Data Public Working Group, “NIST Big Data Interoperability Framework: Volume 2, Big Data Taxonomies (NIST SP 1500-2 VERSION 3),” Gaithersburg, MD, Sep. 2019 [Online]. Available: https://doi.org/10.6028/NIST.SP.1500-2r2

[3] W. L. Chang (Co-Chair), A. Roy (Subgroup Co-chair), M. Underwood (Subgroup Co-chair), and NIST Big Data Public Working Group, "NIST Big Data Interoperability Framework: Volume 4, Big Data Security and Privacy (NIST SP 1500-4 VERSION 3),” Gaithersburg, MD, Sep. 2019 [Online]. Available: https://doi.org/10.6028/NIST.SP.1500-4r2

[4] W. L. Chang (Co-Chair), S. Mishra (Editor), and NIST Big Data Public Working Group, “NIST Big Data Interoperability Framework: Volume 5, Big Data Architectures White Paper Survey (NIST SP 1500-5 VERSION 1),” Sep. 2015.

[5] W. L. Chang (Co-Chair), D. Boyd (Subgroup Co-chair), O. Levin (Version 1 Subgroup Co-Chair), and NIST Big Data Public Working Group, "NIST Big Data Interoperability Framework: Volume 6, Big Data Reference Architecture (NIST SP 1500-6 VERSION 3),” Gaithersburg MD, Sep. 2019 [Online]. Available: https://doi.org/10.6028/NIST.SP.1500-6r2

[6] W. L. Chang (Co-Chair), R. Reinsch (Subgroup Co-chair), D. Boyd (Version 1 Subgroup Cochair), C. Buffington (Version 1 Subgroup Co-chair), and NIST Big Data Public Working Group, "NIST Big Data Interoperability Framework: Volume 7, Big Data Standards Roadmap (NIST SP 1500-7 VERSION 3),” Gaithersburg, MD, Sep. 2019 [Online]. Available:

https://doi.org/10.6028/NIST.SP.1500-7r2

[7] W. L. Chang (Co-Chair), G. von Laszewski (Editor), and NIST Big Data Public Working Group, "NIST Big Data Interoperability Framework: Volume 8, Big Data Reference Architecture Interfaces (NIST SP 1500-9 VERSION 2),” Gaithersburg, MD, Sep. 2019 [Online]. Available: https://doi.org/10.6028/NIST.SP.1500-9r1

[8] W. L. Chang (Co-Chair), R. Reinsch (Subgroup Co-chair), C. Austin (Editor), and NIST Big Data Public Working Group, "NIST Big Data Interoperability Framework: Volume 9, Adoption and Modernization (NIST SP 1500-10 VERSION 2),” Gaithersburg, MD, Sep. 2019 [Online]. Available: https://doi.org/10.6028/NIST.SP.1500-10r1

[9] T. White House Office of Science and Technology Policy, "Big Data is a Big Deal,” OSTP Blog, 2012. [Online]. Available: http://www.whitehouse.gov/blog/2012/03/29/big-data-big-deal. 
NIST Big Data Interoperability Framework: Volume 3, Use CaSes and General ReQuiRements

1571

1572

1573

1574

1575

1576

1577

1578

1579

1580

1581

1582

1583

[Accessed: 21-Feb-2014]

[10] Multiple Federal Agencies, "Materials Genome Initiative,” The White House, President Barak Obama, 2011. [Online]. Available: https://www.mgi.gov/. [Accessed: 15-Dec-2014]

[11] Multiple Federal Agencies, “Open Government Initiative,” The White House, President Barak Obama, 2013. [Online]. Available: https://www.whitehouse.gov/open

[12] N. Allmang and J. A. Remshard, "NIKE: Integrating workflow, digital library, and online catalog systems," in Proceedings of the ACM IEEE International Conference on Digital Libraries, JCDL 2004, 2004, p. 399 [Online]. Available: http://www.scopus.com/inward/record.url?eid=2-s2.04944260950\&partnerID=40\&md5=565b010c0090e6f46b42e6b992996737

[13] J. Greenberg, K. Jeffery, R. Koskela, and A. Ball, "Metadata Standards Directory WG," Research Data Alliance. [Online]. Available: https://rd-alliance.org/group/metadata-standards-directoryworking-group.html. [Accessed: 28-Sep-2014] 